volume 8

ISSUE 2

2020 marzo-aprile

Editor in Chief Michele Meschi

Supervisor Editor Roberto Nardi

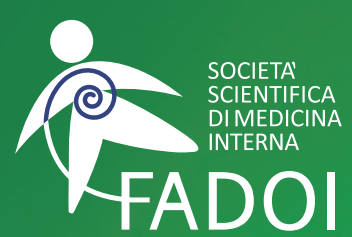

FEDERAZIONE

DELLE ASSOCIAZIONI

DEI DIRIGENTI

OSPEDALIERI

INTERNISTI

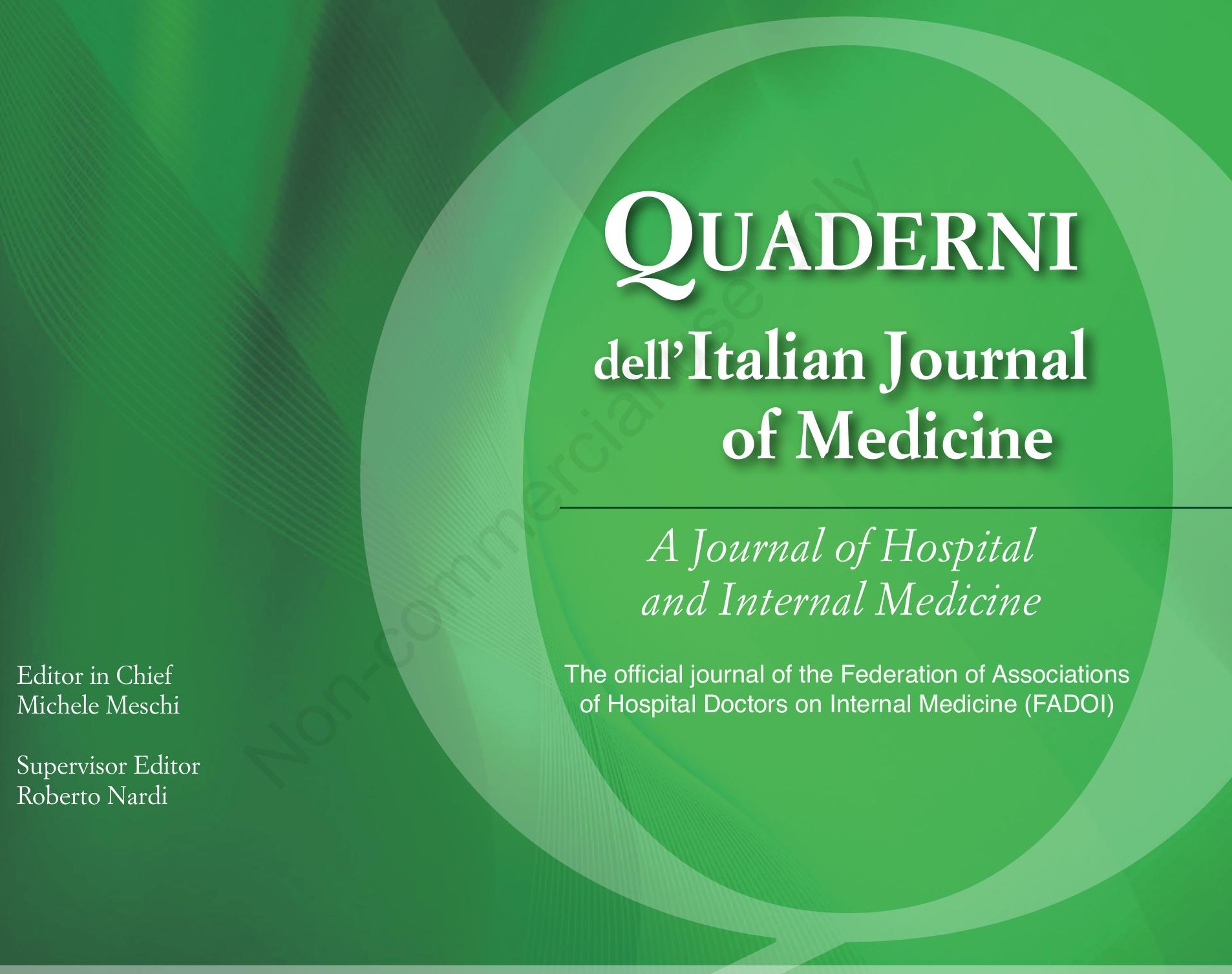

\title{
Aggiornamenti in tema di malattia cerebrovascolare: prevenzione, terapia e riabilitazione
}

Guest Editors: Fabio Bandini, Andrea Fontanella, Dario Manfellotto 
PRESIDENTE ELETTO

Francesco Dentali, Varese, Italy

\section{PAST PRESIDENT}

Andrea Fontanella, Napoli, Italy

\section{SEGRETARIO}

Paola Gnerre, Savona, Italy

\section{STAFF DI SEGRETERIA}

Maurizia Gambacorta, Todi (PG), Italy

Ada Maffettone, Napoli, Italy

Claudia Tieri, Bari, Italy

\section{TESORIERE}

Giorgio Ballardini, Rimini, Italy

\section{COORDINATORE COMMISSIONE GIOVANI}

Ombretta Para, Firenze, Italy

\section{RESPONSABILE RAPPORTI ISTITUZIONALI}

Claudio Santini, Roma, Italy

\section{RESPONSABILE RAPPORTI CON LE REGIONI}

Alberto Fortini, Firenze, Italy

\section{RESPONSABILE EVENTI E INIZIATIVE SPECIALI}

Mauro Campanini, Novara, Italy

EDITOR IN CHIEF ITALIAN JOURNAL OF MEDICINE

Giorgio Vescovo, Padova, Italy
RESPONSABILE DEI

QUADERNI DELL'ITALIAN JOURNAL OF MEDICINE EDIZIONI ON LINE

Michele Meschi, Borgo Val di Taro (PR), Italy

SUPERVISOR EDITOR DEI

QUADERNI DELL'ITALIAN JOURNAL OF MEDICINE

Roberto Nardi, Bologna, Italy

RESPONSABILE SITO WEB E COMUNICAZIONE SOCIAL

Salvatore Lenti, Arezzo, Italy

Maurizia Gambacorta, Todi (PG), Italy

WEB MANAGER E CONTENT EDITOR

Giuseppe Oteri, Milano, Italy

Davide Ghilardi, Milano, Italy

RESPONSABILE SISTEMA GESTIONE QUALITÀ

Franco Berti, Roma, Italy

DPO

Alba Sciascera, Magenta (MI), Italy

\section{CONSULTA DEI PRESIDENTI}

Sandro Fontana, Biella, Italy

Salvatore Di Rosa, Palermo, Italy

Ido Iori, Reggio Emilia, Italy

Giovanni Mathieu, Pinerolo (TO), Italy

Antonino Mazzone, Legnano (MI), Italy

Carlo Nozzoli, Firenze, Italy

Mauro Campanini, Novara, Italy

Andrea Fontanella, Napoli, Italy 


\section{Italian Journal of Medicine}

\section{COORDINATORE}

David Terracina, Roma, Italy

\section{SEGRETARIO}

Andrea Montagnani, Grosseto, Italy

\section{DIRETTORI DEL DIPARTIMENTO DELLA RICERCA CLINICA FADOI}

Filippo Pieralli, Firenze, Italy

Fulvio Pomero, Savigliano (CN), Italy

DIRETTORI DIPARTIMENTO

\section{PER LA FORMAZIONE E AGGIORNAMENTO}

Luigi Magnani, Voghera (PV), Italy

Roberta Re, Novara, Italy

\section{COORDINATORE SCIENTIFICO CENTRO STUDI FONDAZIONE FADOI}

Gualberto Gussoni, Milano, Italy

\section{DELEGATI SOCIETÀ SCIENTIFICHE COLLEGATE}

FISM Antonino Mazzone, Legnano (MI), Italy

SIF Luigi Magnani, Voghera (PV), Italy

Consulta Mauro Campanini, Novara (MI), Italy

cardiovascolare Michele Stornello, Siracusa, Italy

SIIA e ISO Michele Stornello, Siracusa, Italy Arcangelo Iannuzzi, Pomigliano d'Arco (NA), Italy

EFIM

Antonio Brucato, Milano, Italy

Lorenza Lenzi, Pomarolo (TN), Italy

Choosing wisely Roberto Frediani, Chieri (TO), Italy

\section{PROGETTI SPECIALI}

AGGIORN@FADOI

Giuliano Pinna, Marco Grandi

PROGETTO NUOVE TECNOLOGIE

Francesco Nasso, Flavio Tangianu

PROGETTO MEDICINA DI GENERE

Cecilia Politi

PROGETTO COMPETENCE

Flavio Tangianu

PROGETTO GOVERNANCE

Stefano De Carli, Andrea Montagnani, Fabrizio Colombo

PROGETTO HOSPITALIST

Francesco Orlandini

PROGETTO FINE VITA

Mauro Carbone, Fabio Gilioli

PROGETTO GASTROENTEROLOGIA \& FEGATO

Luca Fontanella, Paola Piccolo, Franco Radaelli, Giancarlo Parisi

PROGETTO NUTRIZIONE CLINICA

Roberto Risicato, Luciano Tramontano

PROGETTO MALATTIE INFETTIVE/ANTIBIOTICI

Claudio Santini, Massimo Giusti, Marco Falcone

PROGETTO ECOGRAFIA INTERNISTICA

Francesco Cipollini, Nicola Mumoli

PROGETTO MALATTIE RARE

Antonio Brucato, Antonella Paradiso

PROGETTO BPCO/NIV

Marco Candela, Giuseppe De Matthaeis, Francesco Ventrella

PROGETTO TROMBOSI

Mauro Silingardi, Matteo Giorgi Pierfranceschi, Pierpaolo Di Micco

PROGETTO SDO

Giovanni Mathieu

PROGETTO TRIAL

Giancarlo Agnelli, Antonio Ceriello, Leo Fabbri, Claudio Ferri,

Franco Radaelli, Paolo Verdecchia

\section{PRESIDENTE ANÍMO}

Gabriella Bordin, Castelfranco Veneto (TV), Italy 


\title{
QUADERNI - Italian Journal of Medicine
}

\author{
AGGIORNAMENTI IN TEMA DI MALATTIA \\ CEREBROVASCOLARE: PREVENZIONE, TERAPIA E \\ RIABILITAZIONE
}

Guest Editors: F. Bandini, A. Fontanella, D. Manfellotto

\section{INTRODUZIONE}

Introduzione

RASSEGNE

F. Bandini, P. Gnerre

Epidemiologia e classificazione

M. Stornello, E. Sanzaro

I fattori di rischio

T.M. Attardo

Prevenzione primaria

S. Spolveri

Cause rare di stroke

M. Zedde

L'attacco ischemico transitorio

M. Guarino, S. Belluoccio

Ictus ischemico: assistenza in fase acuta

F. Di Blasio

Trombolisi endovenosa

P. Candelaresi, V. Andreone

Trombectomia meccanica

P. Candelaresi, G. Leone, V. Andreone, M. Muto, M. Muto

Modelli organizzativi nella gestione dell'ictus ischemico

R. Iannacchero, A. Siniscalchi, D. Bosco

Emorragia cerebrale intraparenchimale spontanea ............ 79

M. Masato, A. Bruscagnin

Emorragia subaracnoidea

L.A.A. Lanterna, M. Pantusa, A. Montalbetti, L. Savarese, P. Gritti, M.L. Colleoni

Ictus a possibile doppia genesi. Cardioembolica o aterotrombotica? . . . 100 S. Ascione, P. Ripa, G. Grimaldi

Ictus criptogenetico: la ricerca della fibrillazione atriale e del forame ovale pervio. Come e quando. M. Botta

Le complicanze in fase acuta

C. Di Carmine, G. Francolini, S. Bedetta, L. Mucci, G. Frausini

Prevenzione secondaria dell'ictus ischemico 


\section{QUADERNI - Italian Journal of Medicine}

La riabilitazione dell'ictus .

142

P. Milia, G. Regesta

La riabilitazione post-ictus

A. Greco, F.R. Greco

Trombosi venosa cerebrale 156

A. Iannuzzi

Gestione della disfagia e prevenzione delle complicanze 168

R. Rapetti, F. Bertoncini, L. Vestito 


\title{
Introduzione
}

\author{
Fabio Bandini, ${ }^{1}$ Paola Gnerre ${ }^{2}$ \\ ${ }^{1}$ Neurologia, Savona; ${ }^{2}$ Medicina Interna, Savona, Italia
}

È ormai noto, almeno per gli addetti ai lavori, che l'ictus cerebrale (stroke nella versione anglosassone) rappresenta uno dei più importanti problemi sanitari nei paesi industrializzati, sia per dimensioni epidemiologiche che per impatto socio-economico. In ogni sede, scientifica e non, viene regolarmente sottolineato come, nel nostro paese, l'ictus costituisca la prima causa di invalidità permanente, la seconda di demenza e la terza (o la seconda, come riportano altre stime) di morte, dopo le malattie cardiovascolari e le neoplasie. Essendo inoltre l'ictus una patologia fortemente correlata all'età, l'andamento demografico occidentale fa prevedere che il peso globale delle malattie cerebrovascolari sia destinato ad aumentare nel tempo.

Dal punto di vista terapeutico, molto è stato fatto negli ultimi anni per la cura dell'ictus ischemico in fase acuta. Attualmente, e sin dalla seconda metà degli anni novanta, l'unico farmaco approvato per la terapia dell'ictus acuto ischemico è l'attivatore del plasminogeno rtPA, che può essere somministrato, tuttavia, solo in un intervallo di tempo ristretto dopo l'insorgenza dell'ictus ( 3 ore negli USA e 4,5 ore in Europa). Tra coloro che vengono sottoposti a tale trattamento, il 25$30 \%$ ottiene una adeguata ricanalizzazione arteriosa. Dopo 15-20 anni dall'introduzione dell'rtPA, la gestione acuta dell'ictus ischemico ha subito una ulteriore, importante evoluzione nel 2015, quando sette studi randomizzati hanno evidenziato, quasi simultaneamente, che la trombectomia meccanica endovascolare riduce in modo drammatico la disabilità dopo ictus ischemico causato da occlusione dei grandi vasi intracranici. L'introduzione della trombectomia mec-

Corrispondente: Fabio Bandini, Direttore Struttura Complessa Neurologia Ospedale San Paolo Savona; Presidente Società Italiana per lo Studio dello Stroke (S.I.S.S.), Italia.

E-mail: f.bandini@asl2.liguria.it

Articolo pubblicato secondo la Creative Commons Attribution NonCommercial 4.0 License (CC BY-NC 4.0).

${ }^{\circ}$ Copyright: the Author(s), 2020

Licensee PAGEPress, Italy

QUADERNI - Italian Journal of Medicine 2020; 8(2):1-3 canica dei grossi vasi cerebrali ha permesso di raggiungere tassi di ricanalizzazione che si avvicinano al $90 \%$ dei pazienti. Nel 2018, ulteriori studi hanno dimostrato che alcuni pazienti, selezionati in base all'imaging perfusionale cerebrale (imaging is brain), possono beneficiare di questa metodica ben oltre il consueto limite temporale stabilito, ossia sino a 24 ore dopo l'insorgenza dei sintomi. Sfruttando le tecniche di imaging, anche la trombolisi endovenosa può essere estesa oltre i limiti attualmente in vigore, come testimoniato da recenti metanalisi.

La consapevolezza, duplice, di avere a disposizione un nuovo potente trattamento come la trombectomia meccanica e che sia possibile espandere a più pazienti la finestra temporale per la applicazione delle tecniche di riperfusione ha sicuramente creato notevoli entusiasmi e nuove intriganti prospettive. Essa ha anche creato, tuttavia, alcuni problemi, i principali dei quali sono rappresentati dalla carenza di neuroradiologi interventisti e dal fatto che le Unità Neurovascolari attrezzate per la trombectomia meccanica sono in gran parte limitate alle grandi aree urbane. Questo fatto è molto importante, per esempio, negli USA, ove quasi la metà della popolazione vive ad oltre 60 minuti di auto dalle Unità Neurovascolari attrezzate. Studi recenti hanno mostrato che circa la metà dei pazienti che necessitano del trattamento più efficace deve essere spostata da un ospedale all'altro, con conseguente sostanziale ritardo, per non parlare dei costi e degli inconvenienti. È pertanto necessario, con gli opportuni investimenti, pensare e realizzare una re-organizzazione delle Unità Neurovascolari, in rete e in co-operazione, per fare in modo che il paziente giusto raggiunga la sede giusta nei tempi giusti.

È da sottolineare, inoltre, che non tutti i pazienti in cui i vasi arteriosi vengono ricanalizzati manifestano miglioramenti clinici e che non sempre è possibile un trattamento all'interno della finestra temporale necessaria per la trombolisi sistemica e/o la trombectomia meccanica. Ne deriva che, nonostante gli sforzi per aumentare l'uso di tali terapie di riperfusione, solo una minoranza di pazienti con ictus ischemico può ricevere tali terapie, in particolare nelle nazioni in via di sviluppo.

Ricercare un trattamento efficace per l'ictus ri- 
mane pertanto una priorità urgente. Per poter ottenere successo in una gestione più efficace dell'ictus, è necessaria una migliore comprensione di tutti i suoi aspetti, in particolare della prevenzione. Come dice il vecchio cliché, prevenire è meglio che curare, e, in effetti, la migliore arma a tutt'oggi a nostra disposizione è rappresentata dalla prevenzione, sia primaria (ossia prima che si verifichi un ictus), sia secondaria (ossia prima che si verifichi una recidiva), tenendo conto che più dell' $80 \%$ degli ictus è prevenibile e che più del $70 \%$ degli stessi rappresenta un primo evento. La strategia preventiva dell'ictus cerebrale rientra nell'ambito più generale della prevenzione e del controllo delle patologie croniche nel loro complesso. È di vitale importanza che le politiche che incidono sulla riduzione della mortalità e della morbosità delle malattie cerebrovascolari, intervenendo sui fattori di rischio modificabili (cioè correggibili apportando modificazioni allo stile di vita oppure assumendo una terapia farmacologica appropriata), siano adottate fin dalla giovane età, in modo da mantenere nel corso della vita un profilo di rischio favorevole. Molto, in verità, è stato fatto in tal senso, tanto che alcune azioni, quali abolizione del fumo di sigaretta, controllo della pressione sanguigna, della glicemia e del colesterolo, attività fisica e stili di vita salutari, stanno lentamente entrando nella quotidianità.

Molto c'è ancora da fare: si pensi, per esempio, alla terapia dell'ictus emorragico, in cui da anni non si riescono a compiere passi in avanti decisivi. Oppure alla sottovalutazione dei rischi legati alle cardiopatie emboligene, in primis la fibrillazione atriale, che rappresentano un importantissimo fattore di rischio per l'ictus ischemico con gravi carenze sia sul piano diagnostico che terapeutico, come testimoniato dalle evidenze scientifiche internazionali.

Esiste, inoltre, un oggettivo problema di informazione che riguarda sia i succitati fattori di rischio modificabili, sia il riconoscimento dei sintomi legati ad ictus: uno studio osservazionale in 16 regioni italiane ha evidenziato, infatti, che esiste un tempo eccessivamente lungo tra il momento in cui il paziente si rende conto che "c'è qualcosa che non va" e il momento in cui viene presa la decisione di recarsi in ospedale. A differenza, infatti, della patologia occlusiva coronarica, i cui sintomi cardine sono ben noti alla maggioranza della popolazione, lo stesso non può essere affermato per quanto riguarda l'ictus. L'arcinoto concetto di time is brain ha portato al centro della riflessione sull'ictus il problema del tempo che intercorre tra l'esordio sintomatologico di un ictus acuto e l'effettivo accesso del paziente alla terapia, soprattutto per quanto riguarda la trombolisi farmacologica o la trombectomia meccanica. In diverse migliaia di casi, infatti, si potrebbero azzerare o ridurre drasticamente gli effetti invalidanti dell'ictus con delle cure adeguate prestate nelle primissime ore dalla comparsa dei sintomi. Proprio in questa fase, purtroppo, si presenta uno dei problemi del Sistema Sanitario Nazionale rispetto alla patologia: in più regioni italiane, infatti, non esiste il Codice Ictus per il trasporto del paziente e quindi il personale del 118 (ora 112), pur riconoscendone i sintomi, è tenuto, in base ai protocolli vigenti, a portare il paziente al Pronto Soccorso più vicino, anche se non dotato di Unità Neurovascolare, aggiungendo quindi tempi morti a quelli che già si perdono nel riconoscimento dei sintomi. In Italia le Unità Neurovascolari, vale a dire i centri adeguatamente preparati per trattare gli ictus, sono realtà diffuse a macchia di leopardo. Il Ministero della Salute stima che dovrebbero esserne presenti oltre 300 (il numero ottimale sarebbe 350 ), mentre ne risultano operative meno di 170 , concentrate principalmente nel Nord Italia. Si va dalle 42 della Lombardia alle 5 della Sicilia. Purtroppo, la mancanza di una buona copertura nazionale, così come di una rete assistenziale integrata, fa sì che l'ictus abbia ancora conseguenze molto gravi non solo per il paziente ma anche per i suoi familiari.

L'Italia ha fatto recentemente un bel passo in avanti con il decreto del Ministro della salute n. 70 del 2 aprile 2015 (pubblicato sulla Gazzetta Ufficiale del 4 giugno 2015). Il testo del decreto declina correttamente sia gli standard ospedalieri per le varie patologie, sia l'organizzazione delle Unità Neurovascolari di primo e di secondo livello. Tali novità rappresentano sicuramente un avanzamento per quanto riguarda la causa dell'ictus, nonostante non siano ancora maturate le condizioni per l'approvazione di una legge specificamente dedicata all'argomento.

Una volta verificatosi l'evento ictale, le linee guida nazionali e internazionali sono concordi nell'indicare che, per ridurne le conseguenze, occorre necessariamente beneficiare di una valutazione precoce (entro 48 ore) del livello di disabilità post-ictus e del fabbisogno riabilitativo. Ciò allo scopo di contenere le menomazioni secondarie e di favorire il processo di recupero, sia sensorimotorio che cognitivo. Il modello organizzativo suggerito dalla letteratura per garantire adeguata continuità di presa in carico della persona con ictus fino alla fase di reinserimento (ovvero di domiciliazione o di assistenza a lungo termine), è quello indicato in letteratura come coordinated stroke care. La necessità di assicurare adeguata continuità di cura dopo la fase acuta di ospedalizzazione, e di provvedere alla definizione di un appropriato percorso riabilitativo, è sancita dall'articolo 44 del decreto del Presidente del Consiglio dei Ministri 12 gennaio 2017. Nonostante i buoni propositi, però, negli ultimi anni si è invece assistito ad un ribaltamento sulle famiglie dei costi sociali ed economici del percorso post acuto, passando da un tempo medio di riabilitazione in strutture ospedaliere di 6 mesi a 
circa 45 giorni. Un possibile compromesso potrebbe essere rappresentato dalla tele-riabilitazione domiciliare (si veda, a tal proposito, la recente indagine di Agenas Indagine conoscitiva sulla diffusione della tele-assistenza per la gestione del paziente nella riabilitazione post-ictus). Ad oggi, tuttavia, non è ancora prevista nei prontuari regionali la rimborsabilità delle tele-riabilitazione.

Questa Monografia, che ha visto la fattiva e proficua collaborazione di professionisti di alto valore scientifico, appartenenti a Società Scientifiche (FADOI, SISS e ISO) impegnate quotidianamente nella lotta all'ictus cerebrale, ha l'ambizione di fornire una revisione completa e aggiornata sui vari aspetti legati alla malattia cerebrovascolare, ossia la preven- zione, la diagnosi, il trattamento e la riabilitazione. La reale motivazione di questo volume è che sentiamo necessaria una sorta di chiamata alle armi di fronte ad una malattia così diffusa e, al contempo, così devastante e invalidante.

È possibile, visti i progressi in questo ambito, così rapidi, e a volte, senza timore di peccare di ottimismo, entusiasmanti, che, al momento di entrare in stampa, siano giunti all'attenzione della Comunità Scientifica nuovi approcci e nuove linee guida sia sul piano clinico che organizzativo. Ciò nondimeno, speriamo vivamente che la raccolta di contributi scientifici presenti in questa Monografia possa essere di aiuto a tutti coloro che affrontano quotidianamente la malattia cerebrovascolare in tutte le sue molteplici forme. 


\title{
Epidemiologia e classificazione
}

\author{
Michele Stornello, Enzo Sanzaro \\ Dipartimento di Emergenza, UOC Medicina, Unità Neurovascolare-Stroke Unit, Ospedale Umberto I, Siracusa, Italia
}

\section{Introduzione}

Nonostante i significativi miglioramenti nella prevenzione primaria e nel trattamento acuto negli ultimi decenni, l'ictus cerebrale è ancora una malattia devastante e rappresenta la prima causa di invalidità, la seconda di demenza e la terza di mortalità nei Paesi occidentali, preceduto solo dalle malattie cardiovascolari e dai tumori. Secondo l'Organizzazione Mondiale della Sanità (OMS), ogni anno 15 milioni di persone soffrono di ictus in tutto il mondo. Di questi, 5 milioni muoiono (in Europa ogni anno si registrano circa 650.000 morti per ictus) e altri 5 milioni rimangono permanentemente disabili. Pertanto, l'ictus è da ritenere una malattia di enorme importanza per la salute pubblica con gravi conseguenze economiche e sociali. ${ }^{1}$

Informazioni affidabili sull'incidenza dell'ictus in Europa provengono dai registri basati sulla popolazione. Tali registri sono gli strumenti più rilevanti per studiare l'epidemiologia dell'ictus, a condizione che rispettino criteri ben definiti, in modo da garantire, oltre alla qualità, anche l'interpretazione attendibile dei dati raccolti, consentendo così confronti internazionali tra i vari studi.Alcune razze e origini etniche sono più predisposte rispetto ad altre, particolarmente quelle di origine asiatica, africana o caraibica ed anche i fattori genetici ed i fattori legati allo stile di vita possono svolgere un ruolo nel determinismo dell'ictus.

\section{Prevalenza ed incidenza dell'ictus}

La prevalenza dell'ictus sembra spostarsi nel mondo in via di sviluppo, dove attualmente ci sono

Corrispondente: Enzo Sanzaro, Dipartimento di Emergenza, UOC Medicina, Unità Neurovascolare-Stroke Unit, Ospedale Umberto I, Siracusa, Italia.

E-mail: enzosanzaro@libero.it

Articolo pubblicato secondo la Creative Commons Attribution NonCommercial 4.0 License (CC BY-NC 4.0).

${ }^{\circ}$ Copyright: the Author(s), 2020

Licensee PAGEPress, Italy

QUADERNI - Italian Journal of Medicine 2020; 8(2):4-6
4,85 milioni di decessi per ictus e 91,4 milioni di invalidi ogni anno rispetto a 1,6 milioni di decessi e 21,5 milioni di invalidi nei paesi ad alto reddito. La prevalenza dell'ictus è nettamente più elevata nell'Europa orientale, nell'Asia settentrionale, nell'Africa centrale e nel Pacifico meridionale. Sulla base di studi recentemente pubblicati, l'incidenza di ictus in Europa all'inizio del $21^{\circ}$ secolo varia da 95 a 290 casi $/ 100.000$ all'anno e viene osservato un gradiente est-ovest e nord-sud con tassi di incidenza più elevati nei paesi orientali e tassi più bassi nei paesi meridionali. Le variazioni geografiche potrebbero essere correlate a fattori ambientali, climatici, genetici, a differenze nella distribuzione dei fattori di rischio vascolare ed agli indirizzi dettati dalle politiche sanitarie locali.

Nei paesi sviluppati, l'incidenza dello stroke sta invece diminuendo, in gran parte a causa degli sforzi effettuati per ridurre i fattori di rischio modificabili. Nella maggior parte dei Paesi dell'Europa occidentale, la morte per ictus è diminuita del $30-50 \%$ tra il 1975 ed il 2005 e ciò si è reso più evidente in paesi come Islanda, Italia, Austria e Germania. Eppure, come emerso dal recente rapporto The Burden of Stroke in Europe, prodotto dai ricercatori del King's College di Londra, i quali hanno esaminato dati provenienti da 35 Paesi europei, nonostante la mortalità sia diminuita negli ultimi quindici anni, tutte le previsioni indicano che, entro i prossimi vent'anni, a causa dell'invecchiamento della popolazione, si verificherà un aumento di oltre il $30 \%$ del numero totale di casi di ictus. ${ }^{2}$

Dopo i trent'anni, il rischio di ictus aumenta rapidamente a livello globale e, in particolare, il 95\% di tutti gli ictus si verifica nelle persone di età superiore ai 65 anni. Anche il rischio di morire a causa di un ictus aumenta con l'età e, allo stesso modo, anche la possibilità di riprendersi completamente dalla paralisi e dalla disabilità causate dall'ictus diminuisce con l'età.

I maschi sono più a rischio di stroke rispetto alle femmine. Tuttavia, con i tassi sempre crescenti di obesità, diabete, malattie cardiache e abitudini al fumo, il numero di donne che soffrono di ictus si sta avvicinando a quello dei maschi. Questo incremento di incidenza di ictus nei maschi è stato osservato dopo $\mathrm{i}$ 55-60 anni e potrebbe essere dovuto ad una maggiore prevalenza dei tradizionali fattori di rischio vascolare. 
Tuttavia, poiché l'aspettativa di vita nelle donne è maggiore e i tassi di incidenza aumentano con l'età, in realtà più donne soffrono di ictus, in termini di valore assoluto. Sono state proposte diverse spiegazioni per questa tendenza, tra cui un aumento della prevalenza di diabete, ipercolesterolemia, obesità, fumo di sigaretta, abuso di alcool e uso di droghe illecite nei giovani adulti.

In Italia, ogni anno si registrano almeno 100.000 nuovi ricoveri dovuti all'ictus cerebrale, un terzo dei quali non sopravvive ad un anno dall'evento acuto, mentre un altro terzo presenta una significativa invalidità. Attualmente, quasi un milione di persone sopravvissute all'ictus vive nel nostro Paese con esiti più o meno invalidanti. L'ictus ischemico rappresenta $1 ' 80 \%$ del numero totale degli ictus con una mortalità a 30 giorni del $20 \%$ e ad un anno del $30 \%$ circa. La mortalità a 30 giorni da un ictus emorragico si attesta, invece, attorno al $50 \%{ }^{3}$

Diversi altri aspetti degli esiti post-ictus sono stati recentemente esplorati. Oltre alla prognosi quo ad vitam, i pazienti con ictus presentano un rischio maggiore di outcome sfavorevole entro il primo anno dall'evento ictale, considerandolo ri-ospedalizzazione (33\%) ed un evento ricorrente nel 7-13\% dei casi. Inoltre, tali pazienti possono presentare anche altre malattie di origine vascolare. In una meta-analisi, il rischio di infarto miocardico è stato stimato attorno al 2,2\% e quello di danno vascolare senza ictus al 2,1\%. Da notare che dati simili si registrano anche quando si osservano i pazienti con attacco ischemico transitorio (TIA).

Dato l'invecchiamento della popolazione, la demenza post-ictus sembra essere un ulteriore ed importante problema da affrontare, con una incidenza oscillante tra il 7 e il $23 \%$ nel primo anno dall'evento acuto. Oltre alla demenza, i pazienti con ictus presentano un aumentato rischio di disturbo cognitivo lieve, la cui prevalenza è compresa tra il 35 e il $47 \%$. Tale contributo vascolare al declino cognitivo è certamente sottovalutato, poiché gli studi effettuati hanno finora considerato solo i pazienti con ictus sintomatico. Occorre ricordare che soprattutto le lesioni cerebrali silenti di origine vascolare, inclusi gli infarti territoriali, le lacune, ed i microbleeds, contribuiscono in larga mi- sura al deterioramento cognitivo di origine vascolare. Infine, i pazienti con ictus presentano un aumentato rischio di depressione (30-50\% dei pazienti nel corso del primo anno) e astenia (35-92\%), entrambi contribuendo a nuocere sulla qualità della vita e sull'autonomia funzionale.

Quasi il 50\% degli eventi cerebrovascolari potrebbero essere evitati attraverso l'adozione di stili di vita salutari e un controllo farmacologico nei soggetti ad elevato rischio cardiovascolare globale. Ad esempio, è dimostrato che l'abolizione del fumo assieme ad un'attività fisica quotidiana (almeno 150 minuti a settimana secondo l'OMS) e un'alimentazione ricca di verdura, e frutta, cereali integrali, legumi e pesce, povera di cibi ricchi di grassi saturi, colesterolo, zuccheri semplici e sale, aiuta a mantenere livelli fisiologici di pressione arteriosa, colesterolemia e glicemia. ${ }^{4}$

\section{Tipi di ictus}

Esistono diverse classificazioni dei sottotipi di stroke ischemico acuto e, tra questi, i criteri TOAST sono quelli più utilizzati. Sulla base di tali criteri, l'ictus ischemico può essere raggruppato in cinque principali tipi patologici o eziologici (Tabella 1).

Tuttavia, essendo disponibili solo dati limitati provenienti da studi europei sulla popolazione, i loro risultati mostrano grandi differenze nella distribuzione di questi sottotipi, per le quali sono possibili diverse spiegazioni. In primo luogo, importanza significativa assume la composizione della popolazione dello studio, poiché la frequenza dei sottotipi ischemici differisce in base all'età. Ad esempio, la proporzione di ictus cardioembolico aumenta drasticamente con l'età a causa dell'aumento della prevalenza della fibrillazione atriale negli anziani. Al contrario, la categoria ictus associato ad altre cause è più frequente nei giovani, data l'elevata percentuale di dissezione cervicale, l'assunzione di sostanze d'abuso, la presenza di vasculiti in questa fascia di età. In secondo luogo, le disuguaglianze etniche e di razza possono giustificare le variazioni nella distribuzione dei sottotipi di ictus ischemico, dovute alla differente prevalenza dei fattori di rischio vascolare e dallo stato socio-economico e

Tabella 1. Tipi di stroke ischemico (classificazione TOAST).

\begin{tabular}{|c|c|c|}
\hline Tipo di stroke & Cause & Percentuale \\
\hline Aterotrombotico & Placche ateromasiche delle grosse arterie del cervello che conducono ad ischemia ed infarto & $20 \%$ \\
\hline Lacunare & Malattia dei piccoli vasi cerebrali & $25 \%$ \\
\hline Cardioembolico & Associato ad aritmia cardiaca, valvulopatia e presenza di trombo in atrio sinistro & $15 \%$ \\
\hline Criptogenico & Da causa indeterminata & $5-10 \%$ \\
\hline Associato ad altre cause & Vasculiti, uso di droghe, neoplasie, ecc. & $20-25 \%$ \\
\hline
\end{tabular}


ambientale. Infine, poiché la classificazione TOAST richiede l'uso di specifiche procedure diagnostiche, le discrepanze nell'accesso alle risorse mediche e strumentali possono spiegare le differenze rappresentate nei differenti studi. Coerentemente con questa osservazione, la percentuale di ictus ischemici da causa indeterminata criptogenica può variare dal 5 al $45 \%$.

Per quanto riguarda l'ictus emorragico, esistono diversi sottotipi, tra questi l'emorragia intraparenchimale e l'emorragia subaracnoidea. La prima è il tipo più comune di emorragia intracranica non traumatica e rappresenta l' $80 \%$ delle emorragie cerebrali ed il 10 $15 \%$ di tutti gli ictus. L'emorragia intraparenchimale è principalmente causata da ipertensione non ben controllata, che conduce alla rottura di piccoli vasi e, talora, a seguito di un effetto valanga, a lesione dei vasi vicini con conseguente espansione dell'ematoma in circa il $40 \%$ dei casi. L'emorragia subaracnoidea è principalmente dovuta alla rottura di aneurismi sacculari, sebbene sia anche attribuibile alla presenza di una malformazione artero-venosa, ad una neoplasia intracranica e ad alcuni farmaci, come gli anticoagulanti. Circa il $65 \%$ dei pazienti con emorragia subaracnoidea sopravvive, ma la metà di questi rimane disabile, principalmente a causa di un grave deficit cognitivo. ${ }^{5}$

Quando l'apporto di sangue al cervello viene solo temporaneamente interrotto, si definisce una condizione chiamata attacco ischemico transitorio (TIA), che può durare alcuni minuti o persistere fino a diverse ore. I TIA dovrebbero essere identificati e trattati con urgenza, poiché spesso sono segnali di allarme che possono precedere uno stroke definitivo, il cui rischio è stato stimato essere del $5 \%$ a 7 giorni e tra il $2 \mathrm{e}$ il $5 \%$ entro l'anno.

A causa delle difficoltà metodologiche, l'incidenza del TIA è stata scarsamente studiata ed è probabilmente sottovalutata. Studi basati sulla popolazione indicano, comunque, che l'incidenza del TIA adattata alla popolazione europea varia da 28 a 59 casi/ 100.000/anno.

\section{Bibliografia}

1. The Internet Stroke Center. An independent web resource for information about stroke care and research. http://www.strokecenter.org/

2. Béjot Y, Bailly H, Durier J, Giroud M. Epidemiology of stroke in Europe and trends for the 21st century. Presse Med 2016 Dec;45(12 Pt 2):e391-e398. doi: 10.1016/j. lpm.2016.10.003.

3. Sacco S, Stracci F, Cerone D, Ricci S, Carolei A. Epidemiology of stroke in Italy. Int J Stroke 2011 Jun;6(3): 219-27 https://pubmed.ncbi.nlm.nih.gov/21557809/

4. Rapporto sull'Ictus in Italia. Una fotografia su prevenzione, percorsi di cura e prospettive. https://www.osservatorioictusitalia.it/wp-content/uploads/2018/12/Rappor to-2018-sullictus-in-Italia.pdf

5. Donkor ES. Stroke in the 21st Century: a Snapshot of the Burden, Epidemiology, and Quality of Life. Stroke Research and Treatment. https://pubmed.ncbi.nlm.nih. gov/30598741/ 


\title{
I fattori di rischio
}

\author{
Tiziana Marcella Attardo \\ UO MCAU, Ospedale Barone Lombardo, Canicattì (AG), ASP Ag, Italia
}

\section{Introduzione}

Gli studi epidemiologici hanno individuato molteplici fattori che aumentano il rischio di ictus, alcuni dei quali non possono essere modificati, principalmente l'età, ma costituiscono tuttavia importanti indicatori per definire le classi di rischio. Diversi studi hanno dimostrato che più del $90 \%$ degli ictus è attribuibile a fattori di rischio modificabili, come il fumo di tabacco, il consumo di alcool, una cattiva alimentazione, la sedentarietà e l'ipertensione arteriosa. ${ }^{1-4}$ Siccome tali fattori possono essere modificati con strategie farmacologiche e non farmacologiche, quali - in primis - le modifiche dello stile di vita, certamente averne contezza e riconoscerli rappresenta la base per una prevenzione primaria e secondaria dell'ictus. ${ }^{5}$ Inoltre il fatto che molti di questi fattori di rischio (fumo di sigaretta, cannabis, cocaina, alcool, sedentarietà, obesità, ecc.) siano già presenti nella tarda adolescenza, ne evidenzia il potenziale di una precoce identificazione e di un tempestivo intervento. ${ }^{6}$

In aggiunta, i fattori di rischio coinvolti interagiscono tra di loro in maniera fattoriale e non semplicemente addizionale per cui il rischio di ictus aumenta in maniera più che proporzionale rispetto al numero dei fattori di rischio presenti. ${ }^{5}$

La Figura $1^{5}$ riassume i fattori di rischio per ischemia cerebrale, dividendoli in modificabili e non modificabili. È evidente che il profilo dei fattori di rischio cerebrovascolare differisce a seconda dei diversi sottotipi di ictus ischemico. La fibrillazione atriale e la cardiopatia ischemica sono prevalenti nei pazienti con ictus cardioembolico; l'ipertensione arteriosa e il diabete mellito nei pazienti con ictus lacunare; l'arterio-

Corrispondente: Tiziana Marcella Attardo, UO MCAU, Ospedale Barone Lombardo, Canicattì (AG), ASP Ag, Italia. E-mail: claudia.fantucchio@alice.it

Articolo pubblicato secondo la Creative Commons Attribution NonCommercial 4.0 License (CC BY-NC 4.0).

${ }^{\circ}$ Copyright: the Author(s), 2020

Licensee PAGEPress, Italy

QUADERNI - Italian Journal of Medicine 2020; 8(2):7-18 patia periferica, l'ipertensione arteriosa, il diabete mellito, un precedente attacco ischemico transitorio (TIA) in quei pazienti con ictus aterotrombotico.

I TIA costituiscono un fattore di rischio ben documentato per ictus cerebrale ischemico, soprattutto nelle prime ore successive all'evento.

Sono stati descritti, poi, altri fattori che probabilmente aumentano il rischio di ictus, ma che al momento non appaiono completamente documentati come fattori indipendenti di rischio ${ }^{5}$ (Tabella 1).

\section{L'etnia}

I tassi di ictus sono più alti nei neri, negli ispanici e nei nativi americani rispetto ai bianchi. Ciò sembra correlato ad una maggiore incidenza di diabete mellito ed ipertensione arteriosa nelle etnie con più incidenza di eventi ictali. ${ }^{7}$

\section{L'età}

L'età rappresenta il maggiore fattore di rischio per ictus.

Certamente l'ictus può verificarsi a qualsiasi età, ma nei tre quarti dei casi l'ictus colpisce gli ultrasessantacinquenni. ${ }^{5,8} \mathrm{~L}$ 'incidenza di ictus aumenta con l'età e, a partire dai 55 anni, raddoppia per ogni decade.

L'incidenza dell'ictus aggiustata per età (principalmente ictus ischemico) negli uomini è circa il $30 \%$ più alta rispetto alle donne; ${ }^{9-11}$ una meta-analisi ${ }^{12}$ di dati stratificati in base all'età comprendente 44 studi in 19 paesi ha mostrato eterogeneità per età, con un rapporto di incidenza tra uomini e donne (IRR) del $45 \%$ in più da 45 a 64 anni, del $21 \%$ in più da 75 a 84 anni e solo del $9 \%$ in più a età superiore agli 85 anni. La disparità razziale nel rischio di ictus, con incremento negli individui neri rispetto agli individui bianchi, è più evidente nel range d'età tra i 45 e 64 anni, con un rischio di ictus 3 volte maggiore rispetto agli individui di razza caucasica. ${ }^{12}$

Si definisce ictus perinatale un evento cerebrovascolare ischemico di tipo arterioso o venoso che insorge tra la $20^{\mathrm{a}}$ settimana di vita fetale ed il $28^{\circ}$ giorno di vita post-natale; si distingue in neonatale quando le 
manifestazioni cliniche si manifestano dalla nascita fino a 28 giorni di vita; fetale quando le lesioni vascolari vengono rilevate attraverso ecografie fetali in gravidanza e fino al momento del parto. L'eziologia delle lesioni vascolari in tali epoche della vita è complessa e multifattoriale ed include fattori di rischio materni, intrapartum, e neonatali.

In tutti i casi di ictus perinatale è raccomandato eseguire l'analisi istologica placentare (alterazioni placentari, quali vasculopatia trombotica e corionamnionite, sono risultate essere associate sia a ictus arterioso che a trombosi dei seni venosi) e un dettagliato screening trombofilico, sia nel neonato che nella madre, che includa la ricerca degli anticorpi antifosfolipidi, la lipoproteina (a) ed indagini atte ad escludere una trombofilia ereditaria, in particolare il dosaggio di proteina $\mathrm{C}$, proteina $\mathrm{S}$, antitrombina III, la mutazione del fattore V Leiden e della protrombina. ${ }^{5}$

\section{Il genere}

Nel genere femminile la patologia cerebrovascolare, oltre a rappresentare la terza causa di morte (quinta nel genere maschile) e una delle principali cause di morbidità, si caratterizza per una sua peculiarità, relativamente sia ai fattori di rischio che alle manifestazioni cliniche che agli outcome. ${ }^{5}$
I fattori di rischio, infatti, presentano specificità di genere riconosciute e ben caratterizzate. ${ }^{5}$

\section{Età fertile e pre-menopausale: contraccezione orale}

Non univoci sono i risultati degli studi condotti sulla terapia estroprogestinica come fattore di rischio per ictus. ${ }^{13}$ Tuttavia, secondo la maggior parte degli studi le formulazioni a base unicamente progestinica, cosi come quelle con più alte dosi di estrogeni, sembrano associate con un aumento del rischio di stroke. ${ }^{13,14}$ Anche i contraccettivi orali (CO) di terza generazione, seppure abbiano basse dosi di estrogeni, sono anch'essi associati ad un rischio raddoppiato di ictus rispetto ai controlli. ${ }^{5}$ Ormai storica è la metanalisi di Baillargeon et al. ${ }^{15}$ condotta su una popolazione di 1,7 milioni di donne di età tra i 15 e i 49, a partire dal 1995 e seguite per 15 anni, che ha mostrato come le donne che assumevano la pillola anticoncezionale a base di estroprogestinici avevano un rischio maggiore - seppure di lieve entità - di ictus. Oltre alla pillola contraccettiva orale, altre opzioni di contraccezione ormonale includono l'anello vaginale, i cerotti transdermici, gli impianti sottocutanei e il dispositivo intrauterino a rilascio di levonorgestrel (IUD). La metanalisi ha mostrato un rischio raddoppiato nelle donne portatrici di anello vaginale rispetto ai controlli non in contraccezione ormonale. ${ }^{5,13}$ Per quanto ri-

Non modificabili:

- Età

- Fattori genetici

- Fattori etnici

- Storia di gravidanza patologica per eventi ostetrici negativi placenta-mediati (preeclampsia e/o disordini ipertensivi in gravidanza, perdite fetali, ridotto peso alla nascita e parto pretermine, diabete gestazionale)

- Menopausa precoce

Modificabili: ben documentati

- Ipertensione arteriosa

- Fibrillazione atriale

- Altre cardiopatie (infarto miocardico acuto; cardiomiopatie, valvulopatie, forame ovale pervio e aneurisma del setto interatriale, placche arco aortico)

- Ipertrofia ventricolare sinistra

- Diabete mellito

- Dislipidemia

- Obesità

- Iperomocisteinemia

- Stenosi carotidea

- Fumo di sigaretta

- Eccessivo consumo di alcool

- Ridotta attività fisica

- Dieta

- Anemia a cellule falciformi

Figura 1. Elenco dei fattori di rischio per ischemia cerebrale. ${ }^{5}$ 
guarda le formulazioni in cerotto si è evidenziata una tendenza verso l'incremento del rischio di ictus, sebbene non statisticamente significativa. ${ }^{16}$

Diversi studi hanno sottolineato che dosaggi ormonali più elevati ed un rapporto percentuale maggiore di estrogeni, aumentano il rischio di ictus; inoltre è indubbio un effetto additivo sul rischio trombotico a seguito dell'associazione tra terapia contraccettiva a base di estroprogestinici e diversi fattori di rischio convenzionali, quali il fumo di sigaretta - in primis -, l'emicrania, con e senza aura, l'obesità, l'ipertensione arteriosa, la dislipidemia, l'età (superiore ai 35 anni).$^{17}$ Donne con emicrania senza aura che utilizzano terapia ormonale, indipendentemente dalla via di somministrazione (orale, transdermica, anello vaginale), presentano un incremento del rischio di ictus ischemico da due a quattro volte, rispettivamente se di età minore o maggiore di 35 anni rispetto alle non utilizzatrici. La contemporanea presenza di un fattore di rischio cardiovascolare, quale il fumo, conferisce loro un incremento del rischio di 7 volte rispetto alle non fumatrici. Questo suggerisce che i contraccettivi orali ed il fumo abbiano un forte effetto additivo sul rischio di ictus. ${ }^{18}$ L'uso di CO in pazienti con emicrania con aura (MA) è controverso e ge-

Tabella 1. Elenco di possibili - ma ancora poco definiti fattori di rischio per ischemia cerebrale. ${ }^{5}$

\begin{tabular}{l}
\hline - Emicrania \\
\hline - Sindrome metabolica \\
\hline - Insonnia \\
\hline - Uso di contraccettivi orali \\
\hline - Terapia ormonale sostitutiva \\
\hline - Anticorpi antifosfolipidi \\
\hline - Aumento dell'apoB/apoA1 ratio \\
\hline - Aumento della lipoproteina (a) e della Lp-PLA2 \\
\hline - Alterazioni dei fattori dell'emostasi \\
\hline - Infiammazione e infezioni \\
\hline - Malattie infiammatorie intestinali \\
\hline - Uso di droghe \\
\hline - Inquinamento atmosferico \\
\hline - Lesioni vascolari neuroradiologicamente evidenti \\
\hline ma clinicamente silenti \\
\hline - Iperuricemia \\
\hline - Eccessivo stress \\
\hline - Brari di lavoro eccessivi ( $>55$ ore/settimana) \\
\hline - Depressione \\
\hline
\end{tabular}

neralmente controindicato, ${ }^{19}$ in particolare in soggetti che presentano uno o più fattori di rischio vascolari, soprattutto il fumo, l'ipertensione e l'età., 5,13

Donne portatrici di trombofilia ereditaria che utilizzano CO presentano un incremento del rischio trombotico da due a venti volte rispetto ai controlli (donne non utilizzatrici), ${ }^{20,21}$ per cui questa condizione rappresenta una controindicazione assoluta all'utilizzo di $\mathrm{CO}^{5}$ (grado di evidenza 4). È raccomandato da alcuni autori lo screening per la trombofilia prima di iniziare la terapia contraccettiva, ${ }^{22}$ seppur con uno sfavorevole rapporto costo/beneficio. Infatti le linee guida del WHO $2009^{23}$ non indicano come appropriato lo screening di test di trombofilia ereditaria in considerazione della bassa prevalenza di tale condizione nella popolazione generale.

\section{Menopausa precoce}

La menopausa precoce ( $<40$ anni, o tra 40 e 45 anni), sia spontanea che chirurgica, rappresenta un fattore che aumenta di circa due volte il rischio a lungo termine di eventi cardiovascolari e morte prematura, in misura direttamente proporzionale alla precocità dell'insorgenza della menopausa stessa. ${ }^{5,13} \mathrm{D}$ 'altro l'utilizzo di terapia ormonale sostitutiva si è visto associato ad aumento del rischio di ictus in donne sane in menopausa nel Women's Health Initiative Study, ${ }^{23}$ che ha valutato l'effetto degli estrogeni sulla prevenzione primaria dell'ictus. Una possibile spiegazione risiede nella cosiddetta timing hypothesis, per la quale si ritiene che gli estrogeni possano avere un potenziale effetto protettivo per l'ictus ischemico prima della menopausa e in donne di età $<50$ anni, mentre successivamente rappresenterebbero un fattore di rischio, particolarmente dopo i 60 anni e in corso di terapia orale a dosi elevate.

\section{Gravidanza e il puerperio}

La gravidanza e il puerperio sono associati ad aumento del rischio di eventi cerebrovascolari con incidenza variabile da 9 a 46/100.000 parti e più specificamente 3.8-18/100.000 parti per l'ictus ischemico e 9/100.000 parti per l'emorragia cerebrale, $12 / 100.000$ parti per la trombosi dei seni cerebrali. ${ }^{5,13}$ Il $5-12 \%$ di tutti i decessi materni durante la gravidanza è legato a casi rari di ictus. Il periodo del peripartum, più che la stessa alla gravidanza, specie le prime sei settimane dopo il parto, rappresenta una fase di più elevato rischio di malattia vascolare a causa delle alterazioni ormonali, dei cambiamenti emodinamici, dello stato di ipercoagulabilità e delle fluttuazioni della pressione arteriosa:; ${ }^{5,13}$ altre condizioni locali specifiche sono rappresentati dalla stasi vascolare (soprattutto nel terzo trimestre), i traumi durante il parto, possibili cause di tromboembolia paradossa, 
e la dissezione dei vasi epiaortici (principalmente l'arteria vertebrale) in soggetti predisposte. Altre condizioni possono essere la cardiomiopatia peripartum, la coagulazione intravascolare disseminata e l'embolia di liquido amniotico ${ }^{5,13}$ (Tabella 2).

In una nuova visione di medicina di genere, oltre ai fattori di rischio tradizionali o prevalenti nelle donne, si declinano fattori di rischio genere-specifici.

Tra i fattori genere specifici un ruolo peculiare è rappresentato dalla storia ostetrica, oggi considerata una finestra sul rischio cardio e cerebrovascolare futuro. Evidenze scientifiche recenti e non recenti hanno infatti dimostrato una correlazione tra eventi ostetrici negativi placenta mediati (pre-eclampsia e/o disordini ipertensivi in gravidanza, abortività, basso peso alla nascita, parto pretermine, diabete gestazionale) ed aumentato rischio cardio e cerebrovascolare nella donna. Il diabete gestazionale o una ridotta tolleranza glucidica di grado lieve durante la gravidanza rappresen-

Tabella 2. Fattori di rischio e malattie concomitanti che possono contribuire all'aumento del rischio di ictus in gravidanza. ${ }^{13}$

Fattori di rischio non modificabili

Età (>35 anni)

Razza-etnia

- Asiatica (ictus emorragico)

- Nera (ictus ischemico)

Fattori di rischio modificabili

Obesità/Diabete/Ipertensione/Fumo/Alcool/Droghe (cocaina)

Comorbidità/Complicanze

Malattie specifiche della gravidanza

- Preeclampsia

- Cardiomiopatia post-partum

- Embolia di liquido amniotico

- Coriocarcinoma

- Ipertensione

- Diabete gestionale

- Emicrania peripartum

Possibili condizioni della gravidanza

- Parto cesareo

- Multiparità

- Gestazioni multiple

Malattie ematologiche

- Trombofilia

- Lupus

- Malattie cardiache

- Anemia falciforme

- Malattia reumatica

- Pervietà del forame ovale/malformazioni arterovenose polmonari

Complicanze

- Dissecazione traumatica dei vasi epiaortici

- Infezioni post-partum

- Coagulazione intravasale disseminata

- Squilibri idroelettrolitici ed acido-base

- Trasfusioni tano fattori predittivi di maggiore rischio vascolare, in particolare in donne con una storia familiare di diabete di tipo $2.5,13$

\section{L'anemia a cellule falciformi}

Non sono chiaramente evidenti in letteratura effetti sistematici genere-correlati nel rischio ischemico cerebrale di pazienti con anemia a cellule falciformi. È documentato un incremento del rischio in alcune condizioni genere correlate, come la gravidanza e il puerperio, in cui vi sarebbe un effetto additivo dell'anemia falciforme sull'incremento fisiologico del rischio trombotico. È altresì documentato l'effetto additivo sul rischio cerebrovascolare dell'assunzione di terapia estroprogestinica in pazienti di genere femminile con anemia a cellule falciformi. Tuttavia la correzione per gli altri fattori di rischio vascolare (fumo, obesità, ipertensione arteriosa), nonché per la frequenza cardiaca e il numero di trasfusioni, non ha mostrato una differenza significativa fra utilizzatrici e non utilizzatrici di estroprogestinici relativamente all'incidenza sia di ictus ischemico che emorragico. Tali fattori rappresentano, anche in questa categoria di pazienti, un potenziale oggetto di efficaci strategie di prevenzione primaria. ${ }^{5}$

\section{La fibrillazione atriale}

Il legame tra FA e ictus ischemico è forte. Il sottotipo più comunemente associato alla FA è l'ictus cardioembolico, che è particolarmente grave e mostra $\mathrm{i}$ più alti tassi di mortalità e disabilità permanente. Una tendenza verso una maggiore prevalenza di ictus cardioembolico nei paesi ad alto reddito è probabilmente dovuta alla maggiore diffusione della FA e al migliore controllo dei fattori di rischio aterotrombotici. ${ }^{24} \mathrm{Fa}$ cendo una ricerca Pubmed/Embase di studi che riportavano incidenza di FA subclinica ed ictus in pazienti con dispositivi elettronici impiantabili cardiaci (CIED), la FA subclinica prediceva fortemente la FA clinica ed era associata ad un elevato rischio di ictus, sebbene inferiore rispetto alla FA clinica. ${ }^{25}$

Studi storici sulla prevenzione dell'ictus nella FA hanno evidenziato un rischio più elevato di ictus nelle donne rispetto agli uomini con FA. ${ }^{26}$ Pertanto nella stratificazione del rischio di ictus nei pazienti con fibrillazione atriale (FA) e le conseguenti decisioni sul trattamento anticoagulante orale (OAC), il sesso femminile è stato incluso come fattore di rischio in vari modelli di stratificazione del rischio di ictus, incluso il punteggio $\mathrm{CHA}_{2} \mathrm{DS}_{2}$-VASc. ${ }^{27}$ Nello score $\mathrm{CHA}_{2} \mathrm{DS}_{2}$-VASc, al genere femminile è, infatti, attribuito un punteggio di 1 in quanto il genere femminile è un riconosciuto fattore di rischio per tromboembolismo sistemico in pazienti con FA. Le linee guida $\mathrm{AF}$ 
europee e americane attuali raccomandano di utilizzare il punteggio $\mathrm{CHA}_{2} \mathrm{DS}_{2}$-VASc per guidare le decisioni sul trattamento anticoagulante, ma differiscono rispetto alle loro soglie per uomini e donne ${ }^{28,29}$ Studi più recenti hanno suggerito un ruolo dell'età nell'aumentato rischio di ictus delle donne con FA, vale a dire che l'aggiunta del sesso femminile è importante solo se l'età è superiore ai $65 \mathrm{o}$ se il rischio è superiore a 1 per fattori non relati al sesso; in tal modo le donne di età $<65$ anni senza altri fattori di rischio non presentano un più elevato rischio di ictus rispetto agli uomini; ${ }^{30}$ invece, le donne di età $>65$ anni o con $\geq 1$ ulteriori fattori di rischio di ictus $\mathrm{CHA}_{2} \mathrm{DS}_{2}$-VASc sembrano essere a rischio di ictus più elevato. ${ }^{31}$

Un recente studio ha confermato che il sesso femminile rappresenti un fattore di rischio di ictus nei pazienti con FA in generale, ma rivela anche che l'eccesso di rischio per le donne era particolarmente evidente tra quelli con $\geq 2$ fattori di rischio di ictus non correlati al sesso, indicando una modifica del rischio essendo donne. ${ }^{32}$

\section{L'ipertensione arteriosa}

L'ipertensione arteriosa rappresenta un importante fattore di rischio di ictus. La forte relazione tra ictus e ipertensione è stata oggetto di numerosi studi e prove. Questi studi hanno affrontato l'epidemiologia dell'ictus e dell'ipertensione, al fine di stimare la loro distribuzione mondiale e l'evoluzione del tempo, e hanno studiato gli effetti della gestione dell'ipertensione sugli esiti dell'ictus. Le prove provenienti da questi studi sono stati essenziali per pianificare servizi sanitari adeguati, ottimizzare le risorse economiche e stimare l'efficacia delle strategie terapeutiche nella prevenzione primaria e secondaria. Attualmente ulteriori suggerimenti sono necessari per adattare la gestione farmacologica dell'ipertensione alle esigenze individuali dei pazienti e per selezionare il trattamento più appropriato per evitare recidive di ictus. Inoltre, negli ultimi anni è stata data una crescente attenzione alla relazione tra la presenza di ipertensione e lo sviluppo di un danno cerebrale agli organi terminali che porta a disfunzioni cognitive precoci. ${ }^{33}$ I numerosi studi citati nella recente review di Alloubani et al. stabiliscono con certezza che l'ipertensione arteriosa e il diabete mellito sono fattori di rischio correlati con la patogenesi dell'aterosclerosi. ${ }^{34}$

Dati del National Health and Nutrition Examination Survey indicano che al di sotto di 45 anni di età è maggiore il numero degli uomini ipertesi rispetto alle donne, nella fascia 45-64 anni di età non ci sono differenze di genere, mentre da 65 anni in poi le donne hanno una maggiore frequenza di ipertensione arteriosa rispetto agli uomini. ${ }^{5,13}$

\section{Il diabete mellito e la sindrome metabolica}

Il diabete è una condizione di invecchiamento vascolare accelerato. I pazienti con diabete hanno circa il doppio del rischio di ictus rispetto ai non diabetici. L'iperglicemia è, poi, un forte fattore predittivo di peggiore outcome post - ictus, rappresentando un indicatore di scarso esito prima ancora che causa. Nonostante ciò, l'abbassamento del glucosio non ha dimostrato di essere associato ad un miglioramento della prognosi e la prevenzione a lungo termine del rischio di ictus nei pazienti diabetici non è migliorata con le terapie tradizionali ipoglicemizzanti. Il futuro, tuttavia, potrebbe essere più luminoso, con più nuovi agenti recentemente disponibili. Attendiamo l'esito di questi agenti su complicanze macrovascolari come l'ictus. ${ }^{35}$

\section{L'eccesso ponderale}

La metanalisi di Strazzullo del $2010^{36}$ ha evidenziato che l'eccesso ponderale, valutato dal body mass index (BMI), rappresenta un significativo fattore di rischio per l'ictus. ${ }^{5,13}$

È presente una chiara evidenza di una relazione dosedipendente: la metanalisi ha indicato un aumento non significativo in caso di sovrappeso e un aumento del rischio di ictus del $26 \%$ in presenza di obesità. Tali stime sono in accordo con quanto pubblicato nel successivo studio di $\mathrm{Lu}^{37}$ del 2014. Per quanto riguarda la distribuzione del grasso corporeo, esiste una significativa relazione diretta tra circonferenza vita e rischio di ictus. ${ }^{5}$

\section{L'attività fisica}

Una metanalisi di studi prospettici ${ }^{38}$ ha dimostrato un'associazione inversa tra livello di attività fisica e rischio di ictus. Tale osservazione era relativa sia ad un livello moderato che ad un livello elevato di attività fisica.

Non è stato, però, possibile identificare una relazione fra entità/tipo di attività fisica ed incidenza di ictus.

Questi risultati sono in linea con le numerosissime evidenze sperimentali circa l'effetto positivo dell'attività fisica su numerosi fattori di rischio cardiovascolare. ${ }^{5}$

\section{La dieta}

\section{Carboidrati}

I risultati di due metanalisi di studi prospettici ${ }^{39,40}$ hanno indicato una tendenza non significativa di aumentato rischio di ictus per incremento dell'indice glicemico (IG) e per un'assunzione di carboidrati (CHO) $>290 \mathrm{~g} /$ die. Diete ad alto carico glicemico (CG) si associano significativamente ad un incremento del rischio di ictus pari al $3 \%$ per ogni $50 \mathrm{U}$ di aumento del CG. ${ }^{39,40}$ 
Il meccanismo attraverso il quale un alto $\mathrm{CG}$ aumenta il rischio di ictus è attribuibile al danno vascolare indotto da un incremento cronico della glicemia e dell'insulinemia postprandiali, che agiscono sia attraverso lo stress ossidativo, con produzione di lipoproteine ossidate e AGEs, sia attraverso un'infiammazione sistemica subclinica. ${ }^{5}$

\section{Fibre alimentari}

I risultati di tre metanalisi di studi prospettici ${ }^{41-43}$ indicano che il consumo di fibra alimentare si associa a un minor rischio di ictus totale. In due delle tre metaanalisi $i^{41-42}$ l'effetto benefico era più pronunciato per l'ictus ischemico rispetto a quello emorragico, nelle donne più che negli uomini.

I risultati suggeriscono che l'effetto protettivo della fibra alimentare sull'ictus sia dose dipendente.

Il rischio di ictus, infatti, si riduce in media del $12 \%$ per ogni $10 \mathrm{~g}$ d'incremento di fibra alimentare. ${ }^{5,41-43}$

I benefici del consumo di fibra alimentare sul rischio di ictus sono dovuti in parte alla riduzione della pressione arteriosa (in particolare nei soggetti ipertesi), probabilmente mediata da un miglioramento dell'insulino-resistenza e della disfunzione endoteliale, inoltre al miglioramento del profilo lipidico con riduzione del colesterolo-LDL e della lipemia postprandiale, e agli effetti positivi sulla fibrinolisi e sui fattori dell'infiammazione.

\section{Grassi}

Due metanalisi di studi prospettici ${ }^{44,45}$ non hanno evidenziato alcuna relazione tra assunzione di acidi grassi saturi e rischio di ictus cerebrale, in particolare di ictus ischemico.

L'apparente discrepanza tra i risultati degli studi con acidi grassi e di quelli con gli alimenti che in gran parte li contengono (olio d'oliva, noci, pesce) sta ad indicare che l'effetto protettivo di questi

alimenti potrebbe essere mediato dall'interazione dei vari nutrienti in essi contenuti (acidi grassi, polifenoli, vitamine, minerali ecc.). ${ }^{5,46}$

\section{Proteine}

Una recente meta-analisi ${ }^{42}$ su un numero ristretto di studi prospettici ha evidenziato un minor rischio di ictus in presenza di più elevati apporti proteici; l'incidenza attesa si riduceva del $26 \%$ per un aumento dell'assunzione di proteine pari a $20 \mathrm{~g} /$ die.

Questa osservazione non è sostenuta, al momento, da una chiara plausibilità patogenetica. ${ }^{5}$

\section{Sodio}

Diverse metanalisi di studi prospettici $i^{47-48}$ condotti prevalentemente su campioni di popolazione adulta, concordano sull'associazione tra maggior consumo di sale e rischio di ictus cerebrale: per livelli medio-alti di consumo di sale, a una differenza di circa $5 \mathrm{~g} /$ die corrisponde una differenza nel rischio di ictus di circa il $23 \% .{ }^{46}$ Non è stato ad oggi possibile definire una precisa relazione di dose-dipendenza e non esistono dati specifici relativi a ictus ischemico ed emorragico.

La plausibilità biologica della relazione tra consumo di sale e rischio di ictus è sostenuta dalla forte relazione tra consumo di sale e pressione arteriosa e dall'efficacia della riduzione di sodio nell'abbassare la pressione arteriosa ed anche l'incidenza di ictus in modelli animali stroke-prone (anche indipendentemente dalla riduzione pressoria). ${ }^{5}$

\section{Potassio}

Quattro metanalisi di studi prospettici ${ }^{49-52}$ hanno rilevato un'associazione inversa tra apporto alimentare di potassio e rischio di ictus cerebrale. La relazione appare dose-dipendente, con una riduzione del rischio pari al $10 \%$ per un incremento del potassio della dieta pari a $1 \mathrm{~g}$ e con beneficio massimo per un'assunzione $\geq 4 \mathrm{~g} /$ die. $^{49}$ La plausibilità biologica dell'effetto protettivo del potassio è fondata in parte sull'efficacia del potassio (comprovata da numerose metanalisi di $\mathrm{RCT})^{49-52}$ nel ridurre la pressione arteriosa, ma anche dalla sua efficacia nel ridurre l'incidenza di ictus in modelli animali stroke-prone (anche indipendentemente dalla riduzione pressoria). ${ }^{5}$

\section{Fattori di rischio genetici}

Che l'ictus ischemico potesse avere un'origine almeno in parte genetica lo si è sospettato da molti anni e una storia familiare positiva per malattia cerebrovascolare è comunemente considerata un fattore di rischio per ictus. ${ }^{5}$ Esistono tre tipi di studi di epidemiologia genetica volti a valutare l'associazione fra storia familiare e insorgenza di ictus: studi di popolazione, studi familiari e studi su gemelli. Negli ultimi anni studi familiari hanno dimostrato un significativo contributo genetico all'ictus ischemico ${ }^{53}$ e i tassi di concordanza negli studi su gemelli, che danno una stima affidabile di ereditarietà, sono assai più elevati nei gemelli monocoriali che in quelli bicoriali.

Il ruolo dei fattori genetici nell'ictus cerebrale può essere diretto o mediato. Nel primo caso alterazioni genetiche possono essere di per sé legate all'insorgenza dell'ictus, nell'altro i geni possono contribuire all'ictus attraverso fattori di rischio classici o nuovi. In pratica vanno distinti i disordini monogenici che causano l'ictus dalla predisposizione poligenica e multifattoriale all'ictus. Si conoscono almeno 50 condizioni monogeniche correlate all'ictus. Sono rare 
condizioni in cui il gene conferisce un alto rischio di malattia al portatore della mutazione. I geni più verosimilmente correlati all'ictus sono quelli alla base delle angiopatie amiloidi autosomiche dominanti (geni $A P P, C S T 3$ e $B R I$ ) e del CADASIL (arteriopatia cerebrale autosomica dominante con infarti sottocorticali e leucoencefalopatia; gene NOTCH 3). In tali situazioni disponiamo di strumenti per la diagnosi molecolare e counselling genetico per la pratica clinica. Ulteriori studi invece sono necessari per precisare meglio le vie che legano genotipo e fenotipo e per sviluppare approcci terapeutici. I pazienti in cui si sospetta una di queste forme, e dunque potenziali candidati ad uno screening genetico, sono pazienti con ictus in età giovanile con assenza dei fattori di rischio classici e con storia familiare importante. ${ }^{54}$

Per quanto riguarda invece l'ictus comune esiste un'alta prevalenza ed una eterogeneità fenotipica e genetica. Numerosi alleli che conferiscono un basso rischio di malattia si combinano con effetti additivi e/o moltiplicativi assai complessi. Nuove ricerche sono necessarie per identificare tutti i geni coinvolti nella suscettibilità all'ictus, per una migliore comprensione delle vie fisiopatologiche e per identificare target terapeutici.

Un capitolo relativamente controverso al momento è quello della genetica dei fattori dell'emostasi. Diversi di questi fattori sono stati valutati in studi casocontrollo alla ricerca di un loro ruolo come fattori di rischio per ictus ischemico, ma, a tutt'oggi, i risultati degli studi sono stati piuttosto deludenti. L'iniziale speranza che tali fattori potessero contribuire alla spiegazione dello sviluppo di malattie ischemiche cerebrali non è stata soddisfatta e le aspettative legate agli iniziali rapporti di associazioni positive sono state deluse da risultati inconsistenti per quasi tutti i geni studiati. Soltanto il fattore V Leiden e la variante protrombinica potrebbero avere un ruolo come fattori di rischio in sottogruppi particolari di pazienti o in combinazione con fattori di rischio acquisiti. ${ }^{55}$

Le combinazioni di diversi polimorfismi predisponenti all'ictus con fattori di rischio modificabili possono avere effetto sinergico sul rischio globale di ictus, in particolare nei giovani individui. Un recente stu$\mathrm{dio}^{56}$ ha infatti dimostrato come il rischio di ictus in pazienti con età inferiore a 45 anni aumentava al crescere del numero di polimorfismi presenti contemporaneamente nello stesso paziente. Tale rischio era ancora più alto se il paziente era anche fumatore $o$ iperteso.

Studi caso-controllo di numerosità limitata fanno intravedere prospettive future in relazione a polimorfismi in geni codificanti componenti infiammatorie quali IL-6, MCP-1, ICAM-1, E-selettina e MMP-3, nel gene che codifica la ciclossigenasi 2 (COX-2) e nel gene codificante il peptide natriuretico atriale
(ANP) in particolare per il rischio di nuovi eventi nei soggetti portatori della variante all'elica rara del polimorfismo ANP/TC2238. ${ }^{57}$

Sono, allo stato attuale, necessari ulteriori studi per confermare che alcuni di questi marcatori genetici siano effettivamente fattori di rischio per ictus e stabilire se popolazioni che hanno una maggiore incidenza di questi marker siano da sottoporre a indagini particolari e ad una profilassi primaria più aggressiva. In particolare necessitano studi che specificamente studino le interazioni fra polimorfismi genetici candidati e fattori di rischio ambientali ma che abbiano una numerosità campionaria adeguata per essere rilevanti.

\section{L'emicrania}

Il ruolo dell'emicrania come fattore di rischio indipendente per gli eventi cardiovascolari è a tutt'oggi controverso; $;, 13$ inoltre, nella forma senz'aura che rappresenta il sottotipo più frequente di emicrania nella popolazione generale $(80 \%)$, le evidenze risultano sono ancora più ambigue. ${ }^{5,13}$

Studi epidemiologici hanno riportato un'associazione tra emicrania, per lo più con aura, ed ictus, sia emorragico che ischemico, in particolare nelle donne con età inferiore a 55 anni, eventi coronarici e mortalità globale per tutte le cause. ${ }^{5}$ Risultano rilevanti i dati relativi all'associazione tra emicrania e pre-eclampsia: questi sollevano l'ipotesi che l'ipertensione arteriosa possa rappresentare il meccanismo fisiopatologico in grado di correlare i due eventi. L'emicrania rappresenta uno dei fattori di rischio di malattia cerebrovascolare prevalenti nel genere femminile $\mathrm{e}$ in considerazione della forza delle evidenze in merito, questo fattore è stato inserito all'interno delle linee guida dell'AHA/ASA sulla prevenzione dell'ictus nella donna. ${ }^{22}$

I possibili meccanismi responsabili riguardano la patologia stessa, dal momento che un più alto numero di attacchi ed una maggior durata di malattia sembrano associarsi ad un aumentato rischio di disfunzione endoteliale, aterosclerosi, resistenza insulinica, sindrome metabolica ed assunzione cronica di antiinfiammatori non steroidei e/o triptani. Altri meccanismi sono correlati ai fattori di rischio cardiovascolare (es. fumo) e le comorbosità (es. diabete, ipertensione, sindrome metabolica, obesità e dislipidemia), che appaiono maggiormente prevalenti negli emicranici rispetto alla popolazione generale. ${ }^{5,13}$

Di recente si è colta una correlazione tra emicrania ed assetto lipidico: si sono, infatti, riscontrati livelli di colesterolo totale e LDL significativamente più elevati in pazienti emicranici (sia con che senz'aura) con alta frequenza ed intensità di crisi rispetto a quelli con bassa frequenza e intensità, 
evidenziando una correlazione lineare diretta significativa fra il profilo lipidico e la gravita di malattia. Una riduzione significativa del colesterolo totale e LDL veniva inoltre osservata dopo che una profilassi farmacologica per l'emicrania, sia con che senz'aura, effettuata con farmaci non in grado di interferire direttamente sul profilo lipidico, aveva efficacemente ridotto numero ed intensità delle crisi. Tali dati suggeriscono che un'alta severità di malattia emicranica, attraverso un incremento della disfunzione endoteliale, dello stress ossidativo e dell'infiammazione, possa portare ad uno sviluppo progressivo di un profilo lipidico sfavorevole..$^{5,13}$

È necessario che siano condotti ulteriori studi di tipo prospettico e su più ampia casistica per valutare specificamente la correlazione fra profilo lipidico ed emicrania - sia con che senz'aura- separatamente nei pazienti di sesso maschile e femminile, anche per mettere a punto strategie differenziate di prevenzione dell'ipercolesterolemia attraverso un'efficace profilassi antiemicranica. ${ }^{5,13}$

\section{L'alcool}

La relazione tra consumo di etanolo e incidenza di ictus è descritta da una curva dose-risposta di tipo $\mathrm{J}$, dove per consumi più alti (corrispondenti a più di 3-4 bicchieri di vino al giorno) si evidenzia un aumento del rischio di ictus totale ed ischemico. ${ }^{5}$ Pertanto è raccomandato limitare l'eventuale consumo voluttuario giornaliero di bevande alcoliche a non più di 1 unità alcolica per le donne $(12 \mathrm{~g}$ equivalenti a $125 \mathrm{~mL}$ di vino a media gradazione) e 2 unità per l'uomo ( $24 \mathrm{~g}$ equivalenti a $250 \mathrm{~mL}$ di vino a media gradazione) ${ }^{5}$

L'ingestione regolare di alcol è associata ad ipertensione arteriosa, emorragia intracranica fatale e non fatale, infarto cerebrale e aumento del rischio di morte per ictus.

Studi recenti - anche se non rigorosamente controllati - suggeriscono che il consumo di alcol può rappresentare un fattore di rischio per infarto cerebrale nei giovani adulti con intossicazione occasionale di etanolo e in donne di mezza età con intossicazione occasionale di alcol o consumo regolare di alcol. L'alcol può anche essere, anche, un fattore di rischio per l'emorragia subaracnoidea..$^{58}$

L'alcol può contribuire all'ictus con diversi meccanismi: induzione di aritmie cardiache e anomalie del movimento della parete cardiaca che predispongono all'embolia cerebrale; azione ipertensiva; potenziamento dell'aggregazione piastrinica e attivazione della cascata di coagulazione; riduzione del flusso sanguigno cerebrale mediante stimolazione del sistema vascolare cerebrale, contrazione della muscolatura liscia e/o alterazione del metabolismo cerebrale.

\section{Fumo di sigaretta ed uso di cannabinoidi}

Sebbene l'uso del tabacco sia diminuito sostanzialmente nel 2002 negli Stati Uniti, rimane la seconda causa principale del totale delle morti e delle disabilità. ${ }^{5}$ La percentuale di adulti fumatori di sigarette è diminuito dal 24,1\% nel 1998 al 16,9\% nel 2014; tra gli studenti delle scuole superiori, il declino è stato dal $36,4 \%$ nel 1997 al 5,6\% nel 2013. Potrebbero verificarsi cali nell'uso del tabacco negli Stati Uniti a favore delle sigarette elettroniche disponibili. Ad oggi, però, i rischi e i benefici del tabacco elettronico rimangono controversi. ${ }^{59}$

Recentemente, un crescente corpus di ricerche ha collegato l'uso di cannabis all'ictus, in particolare a quelli che si verificano prima dei 45 anni. ${ }^{60,61}$ Di recente, l'Organizzazione Mondiale della Sanità ${ }^{62}$ ha sottolineato l'urgente necessità di ulteriori studi sui potenziali rischi per la salute della cannabis (ad esempio, ictus e CVD) e la necessità di controllare meglio i potenziali problemi del fumo di tabacco. È noto che la cannabis e il tabacco sono spesso mescolati insieme e studi hanno riferito che $\leq 90 \%$ dei consumatori di cannabis sono anche fumatori di tabacco.$^{63}$ Inoltre, il tabacco viene comunemente aggiunto alle sigarette di cannabis - le cosiddette articolazioni - in modo che le due sostanze sono usate contemporaneamente. Inoltre, gli ictus associati alla cannabis si verifichino di solito in consumatori di cannabis che fumano anche tabacco, in combinazione con - o immediatamente dopo - l'uso di cannabis.

Gli effetti cerebrovascolari della cannabis evidenziati come possibili meccanismi patogenetici di ictus includono ipotensione arteriosa, alterazione della funzione vasomotoria cerebrale, vasospasmo, vasocostrizione cerebrale e oscillazioni della pressione sanguigna. Tuttavia, attualmente vi è una carenza di dati epidemiologici a supporto di un'associazione tra consumo di cannabis ed ictus e le nostre attuali conoscenze sugli ictus associati alla cannabis si basano quasi esclusivamente su report clinici, ${ }^{64-66}$ studi clinici, retrospettivi e trasversali. Infatti sebbene siano stati condotti alcuni studi, questi sono stati per lo più basati su registri ospedalieri e, pertanto, sono di natura retrospettiva e mancano della capacità di affrontare questioni di temporalità, possibili associazioni dose-risposta e l'importanza di altri fattori concomitanti (ad esempio l'uso di alcol e tabacco). Di conseguenza, non è ancora chiaro se la cannabis debba essere considerata un fattore di rischio per l'ictus.

Un recente studio del più grande database di pazienti ricoverati negli Stati Uniti ha messo in evidenza che l'uso di cannabis era associato ad un aumento del $17 \%$ del rischio di ricovero a causa di ictus ischemico acuto, ${ }^{60,67}$ anche se sia il consumo di tabacco che di anfetamine ha comportato maggiori rischi. Un prece- 
dente studio caso-controllo in Australia ha mostrato che i pazienti che manifestavano un ictus ischemico o un attacco ischemico transitorio avevano maggiori probabilità di usare cannabis rispetto ai pazienti non colpiti. ${ }^{68}$ Tuttavia, dopo l'aggiustamento per l'uso del tabacco, un'associazione tra cannabis e ictus non poteva essere confermata. Un altro studio trasversale condotto dagli Stati Uniti ha scoperto che l'abuso o la dipendenza da cannabis erano associati a ictus ischemico, ma non emorragico. ${ }^{69}$ In questo studio, però, l'uso di tabacco e cocaina erano più fortemente associati all'ictus e l'anfetamina era l'unico farmaco che realmente aumentava il rischio di morte per ictus. In un recente studio australiano che ha esaminato 7455 adulti nella comunità generale ${ }^{70}$ consumatori settimanali di cannabis hanno avuto un tasso più elevato di ictus non fatale o di attacco ischemico transitorio rispetto ai consumatori di non cannabis. Tuttavia, poiché si trattava di uno studio trasversale, non è stato possibile dedurre una relazione causale tra ictus e consumo di cannabis.

Di fatto, ancora mancano studi longitudinali nella popolazione generale. Considerando il fatto che la cannabis è la droga illecita più utilizzata in tutto il mondo, con una stima di 180 milioni di utenti annuali, l'interesse per i suoi possibili rischi per la salute sembra giustificato, tenendo conto che l'attuale ondata di depenalizzazione e liberalizzazione della marijuana medica negli Stati Uniti e in altri paesi potrebbe portare $\mathrm{a}$ un suo uso più diffuso. Questo potenziale rende importante affrontare i possibili effetti negativi sulla salute associati alla cannabis, poiché potrebbero iniziare a manifestarsi nella popolazione con maggiore frequenza. ${ }^{71}$

Un recente studio ${ }^{72}$ ha valutato una vasta coorte basata sulla popolazione comprendente 49.321 uomini svedesi e non ha trovato alcuna associazione evidente tra consumo di cannabis e ictus, incluso l'ictus prima dei 45 anni; al contrario il fumo di tabacco ha mostrato un' associazione di tipo dose-risposta con l'ictus attraverso analisi multivariate.

\section{L'uso di cocaina e anfetamine}

Evidenze sono state accumulate per due decenni a supporto di un possibile collegamento tra abuso di droghe e stroke in soggetti di giovane età. ${ }^{73-80}$

Imaging radiologici e studi post mortem, come così come i modelli di animali da laboratorio, suggeriscono che droghe stimolanti, come la cocaina e le anfetamine, potrebbero avere effetti diretti sulla circolazione cerebrale, tra cui elevata pressione sanguigna, vasculite, e vasospasmo cerebrale. La cocaina è una delle droghe più comunemente usate negli Stati Uniti e nel mondo. I dati basati sulla popolazione provenienti dalla regione di Greater Cincinnati e del Ken- tucky settentrionale indicano un triplice aumento dell'uso di cocaina tra i giovani adulti con ictus tra il 1993 e il $2005 .{ }^{81}$

Casi clinici e serie di casi 2 hanno suggerito da tempo un legame tra l'uso acuto di cocaina e l'ictus ischemico in giovani adulti. Queste osservazioni cliniche hanno una forte plausibilità biologica a causa degli effetti della cocaina sul sistema cardiovascolare. Tuttavia, pochi studi epidemiologici hanno valutato l'effetto della cocaina sul rischio di ictus; inoltre i limiti della ricerca finora effettuata sono legati alla mancata differenziazione tra ictus ischemico ed emorragico, la mancanza di dati sull'ora dell'ultimo consumo di cocaina o sulla via di utilizzo, nonché il basso numero dei dati raccolti. ${ }^{82-86}$

Un recente studio, includente più di 1000 casi di ictus giovanali, è uno dei più grandi studi caso-controllo che, ad oggi, abbia esaminato l'associazione tra uso di cocaina e rischio di ictus ischemico nei giovani adulti. ${ }^{87}$ In sintesi, i dati riscontrati evidenziano che l'uso acuto di cocaina entro 24 ore è associato a ictus ischemico nei giovani adulti.

Pertanto, poiché l'uso di cocaina può essere un fattore che contribuisce al rischio di ictus, in caso di ictus giovanile si dovrebbe prendere in considerazione il possibile recente uso di cocaina e, nel caso, eseguire uno screening tossicologico. ${ }^{88}$

La cocaina ha molteplici effetti che potrebbero predisporre all'ictus ischemico: a dosi più basse, predomina l'attività simpatica dovuta all'inibizione della ricaptazione della catecolamina ai terminali dei nervi simpatici, portando ad ipertensione e vasocostrizione; a dosi più elevate, la cocaina blocca i canali del sodio e del potassio, portando a depressione della contrattilità miocardica e possibili aritmie ventricolari. La cocaina induce, inoltre, uno stato protrombotico. ${ }^{88}$

\section{Bibliografia}

1. Feigin VL, Krishnamurthi RV, Parmar P, et al. Update on the global burden of ischemic and hemorrhagic stroke in 1990-2013: The GBD 2013 study. Neuroepidemiology. 2015; 45:161-176.

2. Sultan S, Elkind MS. The growing problem of stroke among young adults. Curr Cardiol Rep. 2013; 15:421.

3. Smajlović D. Strokes in young adults: epidemiology and prevention. Vasc Health Risk Manag. 2015; 11:157-164.

4. O'Donnell MJ, Xavier D, Liu L, et al. INTERSTROKE investigators. Risk factors for ischaemic and intracerebral haemorrhagic stroke in 22 countries (the INTERSTROKE study): a case-control study. Lancet. 2010; 376:112-123.

5. Ictus cerebrale: Linee guida italiane di prevenzione e trattamento. VIII stesura. SPREAD - Stroke Prevention and Educational Awareness Diffusion. Consultazione online 18/01/2020 https://www.osservatorioictusitalia.it/ wpcontent/uploads/2017/11/LINEEGUIDA_SPREAD_ 8a_EDIZIONE.pd 
6. Högström G, Nordström A, Eriksson M, Nordström P. Risk factors assessed in adolescence and the later risk of stroke in men: a 33-year follow-up study. Cerebrovasc Dis. 2015; 39:63-71.

7. Mozaffarian D, Benjamin EJ, Go AS, et al. Heart Disease and Stroke Statistics-2016 Update: A Report From the American Heart Association. Circulation. 2016;133 (4):e38-360.

8. The Internet Stroke Center. UT Southwestern Medical Centee. Data ultima consultazione on line 20/01/2020.

9. Benjamin EJ, Virani SS, Callaway CW, et al. Heart disease and stroke statistics-2018 update: a report from the American Heart Association. Circulation. 2018;137(12): e67-e492.

10. Howard G, Peace F, Howard VJ. The contributions of selected diseases to disparities in death rates and years of life lost for racial/ethnic minorities in the United States, 1999-2010. Prev Chronic Dis. 2014;11:E129.

11. Yang Q, Tong X, Schieb L, et al. Vital signs: recent trends in stroke death rates-United States, 2000-2015. MMWR Morb Mortal Wkly Rep. 2017;66(35):933-939.

12. Howard VJ, Madsen TE, Kleindorfer DO, et al. Sex and Race Differences in the Association of Incident Ischemic Stroke With Risk Factors. JAMA Neurol. 2019 Feb 1;76(2):179-186.

13. Zedde ML, Romana Pezzella F, Anticoli S. Position Paper Stroke and Gender. Giornale Italiano dell'Arteriosclerosi 2015; 6 (3): 7-25.

14. Allais G, Gabellari IC, Mana O et al. Migraine and stroke: the role of oral contraceptives. Neurol Sci. 2008; 29 (Suppl. 1): S12-14.

15. Baillargeon JP, McClish DK, Essah PA, Nestler JE. Association between the current use of low-dose oral contraceptives and cardiovascular arterial disease: a meta-analysis. J Clin Endocrinol Metab. 2005; 90: 38633870 .

16. Lidegaard O, Lokkegaard E, Jensen A et al. Thrombotic stroke and myocardial infarction with hormonal contraception. N Engl J Med. 2012; 366: 2257-2266.

17. Bhat VM, Cole JW, Sorkin JD et al. Dose-response relationship between cigarette smoking and risk of ischemic stroke in young women. Stroke 2008; 39: 2439-2443.

18. Bushnell C, McCullough LD, Awad IA, et al. American Heart Association Stroke Council; Council on Cardiovascular and Stroke Nursing; Council on Clinical Cardiology; Council on Epidemiology and Prevention; Council for High Blood Pressure Research. Guidelines for the prevention of stroke in women: a statement for healthcare professionals from the American Heart Association/American Stroke Association. Stroke 2014; 45: 1545-1588.

19. Medical eligibility criteria for contraceptive use, fourth edition WHO 2009 data ultima consultazione online 18/01/2020.

20. Curtis KM, Mohllajee AP, Peterson HB. Use of combined oral contraceptives among women with migraine and nonmigrainous headaches: a systematic review. Contraception 2006; 73: 189-194.

21. Blickstein D, Blickstein I. Oral contraception and thrombophilia. Curr Opin Obstet Gynecol. 2007; 19: 370-376.

22. Chang CL, Donaghy M, Poulter N. Migraine and stroke in young women: case-control study. The World Health
Organisation Collaborative Study of Cardiovascular Disease and Steroid Hormone Contraception. BMJ. 1999; 318: 13-18.

23. Wassertheil-Smoller S, Hendrix S, Limacher M, et al. Effect of Estrogen Plus Progestin on Stroke in Postmenopausal Women. The Women's Health Initiative: A Randomized Trial. JAMA. 2003;289(20):2673-2684.

24. Pistoia F, Sacco S, Tiseo C, et al. The Epidemiology of Atrial Fibrillation and Stroke. Cardiol Clin. 2016 May;34(2):255-68.

25. Mahajan R, Perera T, Elliott AD et al. Subclinical device-detected atrial fibrillation and stroke risk: a systematic review and meta-analysis. Eur Heart J. 2018 Apr 21;39(16):1407-1415.

26. Wagstaff AJ, Overvad TF, Lip GY, Lane DA. Is female sex a risk factor for stroke and thromboembolism in patients with atrial fibrillation? A systematic review and meta-analysis. QJM. 2014 Dec;107(12):955-67.

27. Lip GY, Nieuwlaat R, Pisters R, et al. Refining clinical risk stratification for predicting stroke and thromboembolism in atrial fibrillation using a novel risk factorbased approach: the euro heart survey on atrial fibrillation. Chest. 2010 Feb;137(2):263-72.

28. Kirchhof P, Benussi S, Kotecha D, et al. 2016 ESC Guidelines for the management of atrial fibrillation developed in collaboration with EACTS. Eur Heart J. 2016; 37:2893-2962.

29. January CT, Wann LS, Alpert JS, et al. 2014 AHA/ACC/HRS guideline for the management of patients with atrial fibrillation: executive summary: a report of the American College of Cardiology/American Heart Association Task Force on practice guidelines and the Heart Rhythm Society. Circulation. 2014; 130:20712104.

30. Mikkelsen AP, Lindhardsen J, Lip GY, et al. Female sex as a risk factor for stroke in atrial fibrillation: a nationwide cohort study. J Thromb Haemost. 2012; 10:1745-1751.

31. Chao TF, Liu CJ, Wang KL, et al. Should atrial fibrillation patients with 1 additional risk factor of the CHA2DS2-VASc score (beyond sex) receive oral anticoagulation? J Am Coll Cardiol. 2015; 65:635-642.

32. Nielsen PB, Thure F, Overvad F et al. Female Sex Is a Risk Modifier Rather Than a Risk Factor for Stroke in Atrial Fibrillation. Should We Use a $\mathrm{CHA}_{2} \mathrm{DS}_{2}-\mathrm{VA}$ Score Rather Than $\mathrm{CHA}_{2} \mathrm{DS}_{2}$-VASc? Circulation 2018;137:832-840.

33. Pistoia F, Sacco S, Degan D et al. Hypertension and Stroke: Epidemiological Aspects and Clinical Evaluation. High Blood Press Cardiovasc Prev. 2016;23(1):9-18.

34. Alloubani A, Saleh A, Abdelhafiz I. Hypertension and diabetes mellitus as a predictive risk factors for stroke. Diabetes Metab Syndr. 2018;12(4):577-584.

35. Hill MD. Stroke and diabetes mellitus. Handb Clin Neurol. 2014;126:167-74.

36. Strazzullo P, D'Elia L, Cairella G, et al. Excess body weight and incidence of stroke: meta-analysis of prospective studies with 2 million participants. Stroke. 2010 May;41(5):e418-26.

37. Lu J, Bi Y, Wang T, et al. The relationship between insulin-sensitive obesity and cardiovascular diseases in a Chinese population: results of the REACTION study. Int J Cardiol. 2014 Mar 15;172(2):388-94. 
38. Li J, Siegrist J. Physical activity and risk of cardiovascular disease-a meta-analysis of prospective cohort studies. Int J Environ Res Public Health. 2012 Feb;9(2): 391-407.

39. Fan J, Song Y, Wang Y, et al. Dietary glycemic index, glycemic load, and risk of coronary heart disease, stroke, and stroke mortality: a systematic review with metaanalysis. PLoS One. 2012;7(12):e52182.

40. Cai X, Wang C, Wang S et al. Carbohydrate Intake, Glycemic Index, Glycemic Load, and Stroke: A Meta-analysis of Prospective Cohort Studies. Asia Pac J Public Health. 2015 Jul;27(5):486-96.

41. Chen GC, Lv DB, Pang Z, et al. Dietary fiber intake and stroke risk: a meta-analysis of prospective cohort studies. Eur J Clin Nutr. 2013 Jan;67(1):96-100.

42. Zhang Z, Xu G, Liu D, et al. Dietary fiber consumption and risk of stroke. Eur J Epidemiol. 2013 Feb;28(2): 119-30.

43. Threapleton DE, Greenwood DC, Evans CE et al. Dietary fiber intake and risk of first stroke: a systematic review and meta-analysis. Stroke. 2013 May;44(5):1360-8.

44. Siri-Tarino PW, Sun Q, Hu FB, Krauss RM. Meta-analysis of prospective cohort studies evaluating the association of saturated fat with cardiovascular disease. Am J Clin Nutr. 2010 Mar;91(3):535-46.

45. de Souza RJ, Mente A, Maroleanu A. Intake of saturated and trans unsaturated fatty acids and risk of all cause mortality, cardiovascular disease, and type 2 diabetes: systematic review and meta-analysis of observational studies. BMJ. 2015 Aug 11;351:h3978.

46. Hooper L, Martin N, Abdelhamid A, Davey Smith G. Reduction in saturated fat intake for cardiovascular disease. Cochrane Database Syst Rev. 2015 Jun 10;(6):CD011737.

47. Strazzullo P, D'Elia L, Kandala NB, Cappuccio FP. Salt intake, stroke, and cardiovascular disease: meta-analysis of prospective studies. BMJ. 2009 Nov 24;339:b4567.

48. Graudal N, Jürgens G, Baslund B, Alderman MH. Compared with usual sodium intake, low- and excessive-sodium diets are associated with increased mortality: a meta-analysis. Am J Hypertens. 2014 Sep;27(9):1129-37.

49. D'Elia L, Barba G, Cappuccio FP, Strazzullo P. Potassium intake, stroke, and cardiovascular disease a metaanalysis of prospective studies. J Am Coll Cardiol. 2011 Mar 8;57(10):1210-9.

50. Larsson SC, Orsini N, Wolk A. Dietary potassium intake and risk of stroke: a dose-response meta-analysis of prospective studies. Stroke. 2011 Oct;42(10):2746-50.

51. Aburto NJ, Hanson S, Gutierrez H, et al. Effect of increased potassium intake on cardiovascular risk factors and disease: systematic review and meta-analyses. BMJ. 2013 Apr 3;346:f1378.

52. D'Elia L, Iannotta C, Sabino P, Ippolito R. Potassiumrich diet and risk of stroke: updated meta-analysis. Nutr Metab Cardiovasc Dis. 2014 Jun;24(6):585-7.

53. Rastenyte D, Tuomilehto J, Sarti C. Genetics of strokea review. J Neurol Sci 1998; 153: 132-145.

54. Tournier-Lasserve E. New players in the genetics of stroke. N Engl J Med 2002; 347: 1711-1712.

55. Lane DA, Grant PJ. Role of hemostatic gene polymorphisms in venous and arterial thrombotic disease. Blood 2000; 95: 1517-1532.

56. Pezzini A, Grassi M, Del Zotto E, et al. Cumulative effects of predisposing genotypes and their interaction with modifiable factors on the risk of ischemic stroke in young adults. Stroke 2005;36:533-539.

57. Cipollone F, Toniato E, Martinetti S, et al. A Polymorphism in the Cyclooxygenase 2 Gene as an Inherited Protective Factor Against Myocardial Infarction and Stroke. JAMA 2004; 291: 2221-2228.

58. Gorelick PB . Alcohol and stroke. Stroke. 1987;18: 268-271.

59. Mozaffarian D, Benjamin EJ, Go AS, et al. Heart Disease and Stroke Statistics-2016 Update: A Report From the American Heart Association. Circulation 2016; 133(4):e38-360.

60. Rumalla K, Reddy AY, Mittal MK. Recreational marijuana use and acute ischemic stroke: a populationbased analysis of hospitalized patients in the United States. J Neurol Sci. 2016; 364:191-196.

61. Rumalla K, Reddy AY, Mittal MK. Association of recreational marijuana use with aneurysmal subarachnoid hemorrhage. J Stroke Cerebrovasc Dis. 2016; 25:452-460.

62. World Health Organization (WHO). The Health and Social Effects of Nonmedical Cannabis Use.http://www. who.int/substance_abuse/publications/cannabis_report/e $\mathrm{n} /$. data ultima consultazione on line 15/01/2020

63. Agrawal A, Budney AJ, Lynskey MT. The co-occurring use and misuse of cannabis and tobacco: a review. Addiction. 2012; 107:1221-1233.

64. Wolff V, Armspach JP, Lauer V, et al. Cannabis-related stroke: myth or reality? Stroke 2013; 44:558-563.

65. Wolff V, Armspach JP, Lauer V, et al. Ischaemic strokes with reversible vasoconstriction and without thunderclap headache: a variant of the reversible cerebral vasoconstriction syndrome? Cerebrovasc Dis. 2015; 39:31-38.

66. Hackam DG. Cannabis and stroke: systematic appraisal of case reports. Stroke. 2015; 46:852-856.

67. Wolff V, Jouanjus E. Strokes are possible complications of cannabinoids use. Epilepsy Behav. 2017; 70(Pt B):355-363.

68. Hemachandra D, McKetin R, Cherbuin N, Anstey KJ. Heavy cannabis users at elevated risk of stroke: evidence from a general population survey. Aust N Z J Public Health 2016; 40:226-230.

69. Barber PA, Pridmore HM, Krishnamurthy V, et al. Cannabis, ischemic stroke, and transient is ischemic attack: a case-control study. Stroke 2013; 44(8):2327-9.

70. World drug report 2015. United Nations Office on Drugs and Crime (UNODC).https://www.unodc.org/wdr2015/. data ultima consultazione on line 15/01/2020.

71. Thomas G, Kloner RA, Rezkalla S. Adverse cardiovascular, cerebrovascular, and peripheral vascular effects of marijuana inhalation: what cardiologists need to know. Am J Cardiol. 2014; 113:187-19.

72. Falkstedt D, Wolff V, Allebeck P, et al. Cannabis, Tobacco, Alcohol Use, and the Risk of Early Stroke. A Population-Based Cohort Study of 45000 Swedish Men. Stroke 2017;48:265-270.

73. Sloan MA, Kittner SJ, Feeser BR, Gardner J, et al. Illicit drug-associated ischemic stroke in the Baltimore-Washington Young Stroke Study. Neurology 1998;50:16881693.

74. Levine SR, Welch KM. Cocaine and stroke. Stroke 1988;19: 779-783.

75. Buxton N, Conachie NS. Amphetamine abuse and intracranial haemorrhage. J R Soc Med 2000;93: 472- 477. 
76. Lichtenfeld PJ, Rubin DB, Feldman RS. Subarachnoid hemorrhage precipitated by cocaine snorting. Arch Neurol 1984;41: 223-224.

77. Levine SR, Brust JC, Futrell NH, et al. Cerebrovascular complications of the use of the "crack" form of alkaloidal cocaine. N Engl J Med 1990;323: 699-704.

78. Kibayashi K, Mastri AR, Hirsch CS. Cocaine induced intracerebral hemorrhage: analysis of predisposing factors and mechanisms causing hemorrhagic strokes. Hum Pathol 1995;26:659- 663.

79. Delaney P, Estes M. Intracranial hemorrhage with amphetamine abuse. Neurology 1980;30: 1125- 1128.

80. Yen DJ, Wang SJ, Ju TH, et al. Stroke associated with methamphetamine inhalation. Eur Neurol 1994;3416- 22.

81. Arthur N. Westover, Susan McBride, Robert W. Haley, Stroke in Young Adults Who Abuse Amphetamines or Cocaine. A Population-Based Study of Hospitalized Patients. Arch Gen Psychiatry 2007; 64(4):495-502.

82. Kaku DA, Lowenstein DH. Emergence of recreational drug abuse as a major risk factor for stroke in young adults. Annals of Internal Medicine. 1990; 113:821-827.
83. Petitti DB, Sidney S, Quesenberry C, Bernstein A. Stroke and cocaine or amphetamine use. Epidemiology. 1998; 9:596-600.

84. Westover AN, McBride S, Haley RW. Stroke in young adults who abuse amphetamines or cocaine: A population-based study of hospitalized patients. Archives of General Psychiatry. 2007; 64:495-502.

85. Qureshi AI, Suri MFK, Guterman LR, Hopkins LN. Cocaine use and the likelihood of nonfatal myocardial infarction and stroke: Data from the third national health and nutrition examination survey. Circulation. 2001; 103:502-506.

86. Qureshi AI, Akbar MS, Czander E, Safdar K, et al. Crack cocaine use and stroke in young patients. Neurology. 1997; 48:341-345.

87. Schwartz BG, Rezkallal S, Kloner RA. Cardiovascular effects of cocaine. Circulation. 2010; 122:2558-2569.

88. Yu-Ching Cheng, Kathleen A. Ryan, Saad A. Qadwai et al. Cocaine Use and Risk of Ischemic Stroke in Young Adults. Stroke. 2016; 47(4): 918-922. 


\title{
Prevenzione primaria
}

\author{
Stefano Spolveri \\ Medicina, Ospedale del Mugello, Azienda USL Toscana Centro, Borgo San Lorenzo (FI), Italia
}

\section{Introduzione}

Uno degli obiettivi principali delle organizzazioni sanitarie di tutti i paesi occidentali è quello di migliorare la salute dei cittadini attraverso la prevenzione delle malattie cardiovascolari, principalmente infarto del miocardio e ictus ischemico. Le associazioni scientifiche che si occupano della cura dell'ictusI hanno tra gli scopi dichiarati quello di educare il pubblico alla cultura della prevenzione della malattia cerebrovascolare. ${ }^{1}$ Il sistema di gestione integrata e standardizzata di cura dell'ictus (stroke systems of care) che dovrebbe essere attuato dalle organizzazioni sanitarie avanzate prevede come gradino iniziale la prevenzione primaria (e primordiale) e l'educazione della popolazione; altri passaggi, come la risposta dei servizi di emergenza-urgenza (territoriali ed ospedalieri) nella fase acuta, il trattamento acuto dell'ictus, la prevenzione secondaria, la riabilitazione ed il miglioramento continuo della qualità delle cure, sono successivi e conseguenti. ${ }^{2} \mathrm{E}$ ovvio che le politiche sanitarie di prevenzione dipendono per lo più dalle risorse (economiche) che sono allocate nei e dai sistemi sanitari regionali e nazionali.

La prevenzione primordiale comprende tutte le azioni di politica sanitaria utili ad inibire l'esposizione ai fattori di rischio e conseguentemente prevenire le malattie croniche nell'intera popolazione o per lo meno in popolazioni selezionate; ad es., i programmi che cercano di migliorare le condizioni sociali (come, ad es. prendersi cura dei cittadini con abitazioni inadeguate e difficoltà di accesso alle cure primarie), i comportamenti (invitare la popolazione con pro-

Corrispondente: Stefano Spolveri, Medicina, Ospedale del Mugello, Azienda USL Toscana Centro, viale della Resistenza, 50032 Borgo San Lorenzo (FI), Italia.

Tel.: +39.0558.451854 - Fax: +39.0558.051248.

E-mail: stefano.spolveri@uslcentro.toscana.it

Articolo pubblicato secondo la Creative Commons Attribution NonCommercial 4.0 License (CC BY-NC 4.0).

${ }^{\circ}$ Copyright: the Author(s), 2020

Licensee PAGEPress, Italy

QUADERNI - Italian Journal of Medicine 2020; 8(2):19-23 grammi di pubblica utilità ad evitare sedentarietà, fumo ed esposizione al fumo), la dieta (ridurre la produzione o vendita di cibi ad alto contenuto di grassi/sale), al fine di ridurre il rischio di sviluppare ipertensione, malattie cardiovascolari, obesità e ictus dallo sviluppo fetale sino alla vecchiaia.

La prevenzione primaria si occupa delle azioni indirizzate verso specifici fattori di rischio associati a specifiche malattie. Essa include sia le strategie indirizzate alla popolazione che quelle verso i singoli individui; in questo caso sono maggiori le responsabilità a carico degli operatori sanitari (prescrivere antiipertensivi in pazienti con alti valori di pressione arteriosa (PA), aspirina ai pazienti con fattori di rischio cardiovascolare $(\mathrm{CV})$ per lo stroke) e delle autorità sanitarie locali e regionali (centri antifumo).

\section{Campagne di prevenzione}

Nelle due decadi passate sono state lanciate negli Stati Uniti moltissime campagne per aumentare l'attenzione contro l'ictus e ridurne l'incidenza: l'Healthy People 2010 ha conseguito la riduzione del 32,3\% delle morti per ictus (da 62 a 42 morti per 100.000 dal 1999 al 2007); 3 il programma attuale Healthy People 2020 si propone di migliorare la salute cardiovascolare degli Americani del $20 \%$ e ridurre gli eventi cardiovascolari del 20\%; ${ }^{4}$ il Million Heart Programm del Dipartimento della Salute e Servizi Umani si propone di prevenire 1 milione di infarti del miocardio ed ictus attraverso programmi scientifici, di qualità e sicurezza delle cure, partnership con gruppi del settore privato, politiche sanitarie e marketing attraverso i social-media. ${ }^{5}$

In Italia lo strumento fondamentale di pianificazione del Ministero della Salute è rappresentato, a partire dal 2005, dal Piano Nazionale della prevenzione (PNP). ${ }^{6} \mathrm{Si}$ tratta di un documento di respiro strategico che a livello nazionale stabilisce gli obiettivi e gli strumenti per la prevenzione che sono poi adottati a livello regionale con i Piani regionali.

Alla completa attuazione del PNP, le Regioni e Province autonome, per gli anni 2014-2018, hanno destinato 200 milioni di euro, oltre alle risorse previste dagli accordi per la realizzazione degli obiettivi del Piano sanitario nazionale. 
Il PNP, di durata quinquennale, delinea un sistema di azioni di promozione della salute e di prevenzione, che accompagnano il cittadino in tutte le fasi della vita, nei luoghi di vita e di lavoro e deriva dalla convinzione che un investimento in interventi di prevenzione, purché basato sull'evidenza scientifica, sia in grado di contribuire a garantire, nel medio e lungo periodo, la sostenibilità del Sistema sanitario nazionale.

Tra i macro obiettivi del PNP al primo posto si trova quello di ridurre il carico prevenibile ed evitabile di morbosità, mortalità e disabilità delle malattie non trasmissibili come le malattie cardiovascolari (MCV) di origine arteriosclerotica e tra queste, quelle epidemiologicamente più rilevanti sono l'infarto del miocardio e l'ictus. Nel 2010 (ultimo dato di mortalità disponibile) in Italia si sono verificati, complessivamente, 220.539 decessi per malattie del sistema circolatorio (95.952 negli uomini e 124.587 nelle donne); di questi, 72.023 decessi sono stati attribuiti a malattie ischemiche del cuore (36.742 negli uomini e 35.281 nelle donne) e 60.586 a malattie cerebrovascolari (23.991 negli uomini e 36.595 nelle donne).

\section{Linee guida}

Le maggiori società mediche nazionali ed internazionali, come American Heart Association (AHA) e Stroke Association (ASA), European Society of Cardiology (ESC), European Society of Hypertension (ESH), American Diabetes Association (ADA) pubblicano regolarmente linee guida (LG) di prevenzione cardiovascolare. Le raccomandazioni pertanto si sovrappongono e sono aggiornate nel corso di pochi anni o mesi; pertanto non è semplice orientarsi in questo mare di letteratura. Le LG italiane dell'ictus cerebrale ISO-Spread si sono occupate di prevenzione primaria dell'ictus cerebrale nell' VIII edizione (cap. 7, luglio 2016) ${ }^{7}$ e sono attualmente in corso di aggiornamento. Nel dicembre 2014 sono state pubblicate le LG AHA/ASA per la prevenzione primaria dello stroke ${ }^{8}$ e nel febbraio 2019 le LG dell' American College of Cardiology ACC/AHA per la prevenzione primaria delle malattia cardiovascolari; 9 quest'ultime si basano su un decalogo di Takehome messages che mi servirà da guida. Le raccomandazioni a seguire sono classificate a seconda della forza $(\mathrm{I}=$ forte - beneficio $>>>$ rischio; $\mathrm{II}=$ moderata - beneficio $>>$ rischio; IIb debole - beneficio $\geq$ rischio; III= nessun beneficio; $\mathrm{III} \mathrm{H}$ - rischi $>$ beneficio) e del livello di evidenza (A evidenza fornita da più studi randomizzati e metaanalisi; B-R evidenza fornita da uno studio randomizzato; B -NR evidenza fornita da studi ben eseguiti non randomizzati, osservazionali o registri; C-LD studi con limitazioni di disegno o esecuzione; $\mathrm{C}$-EO $=$ opinione di esperti).$^{10}$

Il messaggio principale e preliminare ai successivi è quello che per prevenire le malattie vascolari atero- sclerotiche, come anche l'insufficienza cardiaca e la fibrillazione atriale è necessario promuovere un sano stile di vita per tutta la durata della vita.

È necessario un approccio di cura basato sulla partecipazione multi-professionale (team-based),(IA); i clinici dovrebbero valutare $\mathrm{i}$ determinanti sociali di salute che influenzano il singolo individuo per condividere con lui i trattamenti preventivi (I B NR). Ad es., nella prescrizione dell'esercizio fisico dovrebbe essere valutate la disponibilità di spazi o strutture sportive nelle vicinanze; insieme ai trattamenti per i diabete tipo 2 dovrebbero essere valutati i fattori ambientali, psicosociali, compresi la depressione, lo stress, la capacità di auto-trattamento per migliorare il controllo glicemico e l'aderenza al trattamento stesso; nei pazienti ipertesi dovrebbe essere considerata anche la durata e la qualità del sonno. Il Chronic Care Model di supporto alle cure primarie in diverse realtà regionali del nostro paese risponde proprio all'esigenza dell'approccio multidisciplinare nella prevenzione delle malattie cerebrovascolari e della riconciliazione terapeutica con il paziente, per la copresenza del medico di medicina generale e dell' infermiere di comunità, ed il coinvolgimento delle strutture sociali del territorio. ${ }^{11}$

È necessaria la stima del rischio cardiovascolare negli adulti tra 40 e 75 anni ed il calcolo del rischio a 10 anni usando algoritmi di coorte (IB). In Italia sono utilizzate le carte del rischio $\mathrm{CV} ;{ }^{12}$ più modernamente sono disponibili alcune applicazioni, la più scaricata delle quali è quella dell'American College of Cardiology (ASCVD Risk Calculator o Estimator Plus). ${ }^{13}$ Questi strumenti sono utili per aiutare ad identificare i soggetti che potrebbero beneficiare di interventi terapeutici e che non possono essere trattati sulla base di ogni singolo fattore di rischio. Per rischio basso si intende un rischio di evento cardiovascolare $<5 \%$ nei successivi 10 anni; rischio borderline tra il 5\% ed il $7,4 \%$; rischio intermedio tra il $7,5 \%$ ed il $19,9 \%$; alto rischio $\geq 20 \%$.

Questi algoritmi sono utili per il paziente ed il medico riguardo al possibile rischio singolo, ma la decisione di iniziare un trattamento deve tenere conto dell'intero profilo di rischio del paziente (IIb B-NR). Quindi oltre ai fattori di rischio classici che prenderemo poi in considerazione, sono da considerare anche i fattori di incremento del rischio come la storia familiare di MCV precoce (prima dei 55 anni per gli uomini e di 65 anni per le donne), l'ipercolesterolemia primaria (LDL-C 160-189 mg/dL), la sindrome metabolica (almeno 3 fattori tra aumento della circonferenza alla vita, 1trigliceridi $(\mathrm{TG})>150 \mathrm{mg} / \mathrm{dL}$, ipertensione, iperglicemia, HDL-C $<40 \mathrm{mg} / \mathrm{dL}$ negli uomini e $<50 \mathrm{mg} / \mathrm{dL}$ nelle donne), l'insufficienza renale cronica (eGFR $15-59 \mathrm{~mL} / \mathrm{min} / 1,73 \mathrm{~m}^{2}$ con o senza albuminuria), le infiammazioni croniche come psoriasi, AR, lupus o HIV/AIDS, una storia di meno- 
pausa prematura (prima dei 40 anni) o di preeclampsia, l'etnicità asiatica, i biomarkers come PCR elevata, lipoproteina a $>50 \mathrm{mg} / \mathrm{dL}$, apolipipoproteina B $>130 \mathrm{mg} / \mathrm{dL}$, indice caviglia-braccio (ankle-brachial index, $\mathrm{ABI}$ ) $<0,9$, TG elevati persistentemente ( $>175$ $\mathrm{mg} / \mathrm{dL}$ ); nei pazienti coronaropatici è utile lo score del calcio coronarico. ${ }^{9}$

\section{Dieta e obesità}

Tutti gli adulti dovrebbero consumare una dieta salutare, ricca di frutta, vegetali, noci, cereali integrali, proteine magre vegetali o animali, e pesce (I B-R) e minimizzare l'introduzione di grassi saturi (III H BNR), cibi preparati, carboidrati raffinati e bevande dolci (IIa B-NR). Nella prevenzione dell'ipertensione è raccomandato un ridotto introito di sale ed un'aumentata introduzione di potassio (IA). Le LG ESC

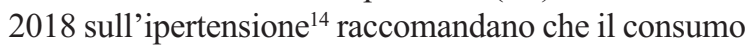
di sodio sia limitato a $2 \mathrm{~g} /$ die (equivalente a circa $5 \mathrm{~g}$ di sale al giorno). Sempre le LG ESC raccomandano di limitare il consumo di alcol (correlazione lineare con pressione arteriosa, prevalenza di ipertensione e rischio cardiovascolare) a 14 UI alcoliche la settima negli uomini e 8 unità/settimana nelle donne. Per gli adulti in sovrappeso (BMI $=25-29 \mathrm{~kg} / \mathrm{m}^{2}$ ) e obesi $\left(\mathrm{BMI}>30 \mathrm{~kg} / \mathrm{m}^{2}\right.$ ), sono consigliate la restrizione calorica e supporto psicologico, utili per raggiungere e mantenere la perdita di peso (I B-R). Per identificare i soggetti a più alto rischio cardiovascolare è utile misurare la circonferenza corporea; i valori necessari per identificare la sindrome metabolica sono $>102 \mathrm{~cm}$ nell'uomo e $>88 \mathrm{~cm}$ nella donna. Combinare la circonferenza corporea ed il BMI è l'approccio migliore per identificare il rischio correlato all'obesità. La perdita di peso è inoltre raccomandata per ridurre la PA (IA).

\section{Attività fisica}

Tutti gli adulti sani dovrebbero praticare attività fisica perché è associata alla riduzione del rischio di malattia cardiovascolare, di ipertensione, di diabete tipo 2 (IA) e di ictus (IB). Dovrebbero impegnarsi in almeno 150 minuti a settimana di attività aerobica di moderata intensità $\mathrm{o} 75$ minuti a settimana di attività aerobica intensa. Per attività moderata si intende quella che consuma 3.0-5.9 equivalenti metabolici (METs; $1 \mathrm{MET}=3,5 \mathrm{~mL}$ di ossigeno consumato per $\mathrm{kg}$ di peso corporeo al minuto); per attività intensa si intende un consumo maggiore o uguale a 6 METS. Esempi di attività fisica moderata sono la camminata a passo svelto $(3,9-6,4 \mathrm{~km} / \mathrm{h})$, il cicloturismo (8-15 $\mathrm{km} / \mathrm{h}$ ), danzare in sala da ballo, lo yoga attivo, il nuoto ricreativo; per attività intensa si intende il jogging/running, il ciclismo $(>16 \mathrm{~km} / \mathrm{h})$, un singolo di tennis, le vasche di nuoto. I comportamenti sedentari dovrebbero essere ridotti e si dovrebbe consultare regolar- mente un ambulatorio medico per ottimizzare il proprio programma di allenamento. ${ }^{9}$

\section{Diabete}

Per gli adulti con diabete mellito tipo 2 è fondamentale cambiare il proprio stile di vita, migliorando le abitudini alimentari e aderendo alle raccomandazioni sull'attività fisica. Per migliorare il controllo glicemico e ridurre il rischio $\mathrm{CV}$, al momento della diagnosi, è ragionevole iniziare come terapia di prima linea la metformina assieme ai cambiamenti di stile di vita (IIa B-R); per gli adulti che non raggiungono un adeguato controllo glicemico a dispetto del primo step, può essere ragionevole iniziare una terapia con inibitori del cotrasportatore 2 sodio-glucosio (SGLT2) - glifozina - o agonisti del recettore peptide 1 glucagon-like (GLP-1R) (IIa B-R), se presenti altri fattori di rischio $\mathrm{CV}$, scompenso cardiaco o insufficienza renale cronica. ${ }^{15}$ Questi due gruppi di farmaci per il diabete hanno mostrato sicurezza e beneficio cardiovascolare in pazienti con diabete che avevano già una malattia cardiaca e/o molteplici fattori di rischio e nelle nuove L G ESC su diabete, pre-diabete e malattie cardiovascolari ${ }^{16}$ le glifozine sono menzionate più volte nella riduzione del rischio $\mathrm{CV}$, oltre le raccomandazioni per la prevenzione dello sviluppo di malattie renali croniche e la riduzione del rischio di insufficienza cardiaca; alla luce di questi risultati in queste LG gli agonisti dei recettori GLP-1 e le gliflozine sono indicati come trattamento di prima linea in pazienti diabetici di tipo 2 con malattie cardiovascolari $\mathrm{o}$ ad alto rischio. In particolare: empagliflozin, canagliflozin, o dapagliflozin sono raccomandate nei pazienti con rischio $\mathrm{CV}$ molto alto (indipendentemente dal diabete) e pazienti con DM2 e alto rischio $\mathrm{CV}$ per ridurre il numero di eventi CV, (IA); Empagliflozin è raccomdandata nei pazienti con DM2 e malattia $\mathrm{CV}$ per ridurre il rischio di morte (IA); Liraglutide, semaglutide, o dulaglutide sono raccomandate nei pazienti con DM2 e malattia $\mathrm{CV}$, o rischio $\mathrm{CV}$ molto alto per ridurre gli eventi $\mathrm{CV}$ (IA); nei e malattia $\mathrm{CV}$ o rischio $\mathrm{CV}$ molto alto per ridurre il rischio di morte (IA).

\section{Colesterolo elevato}

Trials randomizzati con un gran numero di pazienti hanno dimostrato la riduzione del rischio CV sia con alta intensità di trattamento con statine che con moderata intensità. ${ }^{9}$ Per statine a moderata intensità si intendono la atorvastatina $10-20 \mathrm{mg}$, la sinvastatina $20-40 \mathrm{mg}$, la pravastatina $20-40 \mathrm{mg}$, la fluvastatina 80 mg RP, e sinvastatina $10 \mathrm{mg}$ + ezetimibe 10 o 20 o 40 $\mathrm{mg}$, rosuvastatina $5-10 \mathrm{mg}$; per statine ad alta intensità si intendono atorvastatina $40-80 \mathrm{mg}$ e rosuvastatina $20 \mathrm{mg}$. Un altro parametro da considerare è l'età: il rischio $\mathrm{CV}$ è presente anche nei giovani, ma nei soggetti 
ipercolesterolemici sino a 40 anni è indicato modificare lo stile di vita, mentre la terapia con statine a deve essere considerata nei pazienti con LDL-C $>160$ $\mathrm{mg} / \mathrm{dL}<190 \mathrm{mg} / \mathrm{dL}$ e storia familiare di malattia cardiovascolare precoce e in quei pazienti con LDL-C $>190 \mathrm{mg} / \mathrm{dL}$. Nei pazienti tra 40 e 75 anni con rischio $\mathrm{CV}$ intermedio $(\geq 7,5 \%$ - $>20 \%$ rischio di evento $\mathrm{CV}$ a 10 anni) è indicata la terapia con statine; è raccomandata una statina a moderata intensità (IA); le LDL$\mathrm{C}$ dovrebbero essere ridotte del $30 \%$ o più; nei pazienti ad alto rischio ( $>20 \%$ a 10 anni) i livelli dovrebbero essere ridotti del 50\% (IA); nei pazienti di 40-75 anni con diabete, indipendentemente dal rischio calcolato, dovrebbe essere utilizzata una statina a intensità moderata; nei diabetici con fattori di rischio CV multipli è ragionevole (II B-R) prescrivere una statina ad alta intensità per ridurre i livelli di LDL-C del $50 \%$ o più. Nei pazienti coronaropatici diventa importante anche la valutazione del calcio coronarico e la presenza di fattori di incremento del rischio CV. ${ }^{17}$ Nelle LG ESC 2019, nei pazienti a rischio CV molto alto, con LDL$\mathrm{C}$ persistentemente alte a dispetto di un trattamento a massimo dosaggio tollerato di statina in associazione ad ezetimibe, o nei pazienti intolleranti alle statine, è raccomandata la terapia con un inibitore del recettore PCSK9 (IA). ${ }^{16}$

\section{Ipertensione}

Le LG più seguite attualmente sono quelle dell'ESC in collaborazione con l'European Society of Hypertension del 2018. ${ }^{14}$ L'ipertensione rimane definita con il livello di pressione arteriosa al quale i vantaggi di un trattamento superano in maniera inequivocabile i suoi rischi. Si definisce un soggetto con PA normale-alta quella che ha valori di PA sistolica 130-139 e/o diastolica $85-89 \mathrm{mmHg}$; ipertensione di grado 1, 140-159 e/o 90-99 mmHg; ipertensione di grado 2, 160-179 e/o 100-109 mmHg e ipertensione di grado $3, \geq 180 \mathrm{e} / \mathrm{o} \geq 110 \mathrm{mmHg}$. Nei pazienti con PA normale-alta o ipertensione, compresi quelli che fanno un trattamento antiipertensivo sono raccomandati interventi sullo stile di vita, come perdere peso, una dieta bilanciata, la riduzione del sodio, l'integrazione alimentare con potassio, l'aumentata attività fisica con esercizi programmati, e la limitazione dell'alcol. E' indicata la valutazione del rischio $\mathrm{CV}$ e nei pazienti con rischio $>10 \%$ nei 10 anni successivi è indicato iniziare un trattamento antiipertensivo già con valori di PA normale alta (IA). Il target da raggiungere nei soggetti di età inferiore ai 65 anni deve essere 120-129 mmHg (indipendentemente da ulteriori fattori di rischio come DM2, malattia renale cronica, coronaropatia, pregresso TIA/stroke) (IB-R); sopra 65 anni 130-139 mmHg. In nessun caso la PA sistolica va abbassata al di sotto di $120 \mathrm{mmHg}$ (III-H). Vengono raccomandate la valutazione ambulatoriale della PA, l'automisura- zione domiciliare (HBPM) e il monitoraggio ambulatoriale (ABPM) di 24 ore o più. Per aumentare l'aderenza e l'immediata efficacia del trattamento è indicata la terapia iniziale con l'associazione in una compressa di due farmaci (aceinibitore o sartano + calcioantagonista o diuretico); lo step 2 la combinazione in una compressa di tre farmaci (aceinibitore o sartano + calcioantagonista + diuretico); lo step 3 (ipertensione resistente) la combinazione di tre farmaci in una compressa + spironolattone $25-50 \mathrm{mg}$ o alfabloccante o betabloccante.

\section{Fumo}

Durante ogni visita ambulatoriale dovrebbe essere chiesta l'abitudine al fumo e registrata come un segno vitale, per facilitarne la cessazione (IA). Nei fumatori, per aumentare la percentuale di cessazione è raccomandata la combinazione tra interventi comportamentali e terapia farmacologica, con sostituti della nicotina (IA). Ovviamente l'astensione dal fumo è raccomandata per ridurre il rischio cardiovascolare (I B-NR). Per aiutare il paziente a smettere di fumare, è importante usare un linguaggio chiaro e forte, non compassionevole o personalizzato. Ad es.: la cosa più importante che tu possa fare per la tua salute è smettere di fumare. Io (noi) poss(iam)o aiutarti. Il fumo passivo è conosciuto come causa di malattie CV e stroke, ed anche una breve esposizione può innescare un infarto miocardio acuto. Le sigarette elettroniche sono dispositivi che emettono un aerosol contente un particolato fine ed ultrafine, nicotina e gas tossici che possono aumentare il rischio CV e le malattie polmonari. Sono state riportate aritmie ed ipertensione; l'uso cronico è associato con l'incremento persistente dello stress ossidativo e della stimolazione simpatica nei soggetti giovani, in buona salute. ${ }^{9}$

\section{Aspirina}

L'aspirina non dovrebbe essere usata routinariamente nella prevenzione primaria delle malattie $\mathrm{CV}$ per la mancanza di evidenze di un beneficio netto, sia negli adulti sopra 70 anni che in quelli con aumentato rischio di sanguinamento (III B-R e III H). Può essere considerata, a basso dosaggio $(75-100 \mathrm{mg})$, negli adulti tra 40 e 75 anni con alto rischio cardiovascolare e basso rischio di sanguinamento (IIb A). ${ }^{9}$

\section{Raccomandazioni peculiari di prevenzione dell'ictus}

Nelle LG AHA/ASA 2014 sono riportate alcune raccomandazioni peculiari di prevenzione dell'ictus sia ischemico che emorragico. ${ }^{8}$

Alcune di queste riguardano i fattori genetici, come il trattamento della malattia di Fabry con terapia di so- 
stituzione enzimatica (IIb-C); lo screening non invasivo nel caso di scoperta accidentale di aneurisma intracranico non in fase di rottura nei pazienti con rene policistico autosomico dominante, parenti di $1^{\circ}$ grado di pazienti con rene policistico autosomico-dominante ed emorragia sub aracnoidea e/o aneurisma intracranico (IIb-C); screening non invasivo in caso di aneurisma intracranico non rotto e dsiplasia fibromuscolare (IIb-C).

Un altro gruppo di raccomandazioni riguardano l'emicrania; alle donne con emicrania con aura attiva sono raccomandate sistemi di contraccezione alternativi agli anticoncezionali orali, soprattutto quelli contenenti estrogeni (IIb-B); in generale il trattamento per ridurre la frequenza degli attacchi di emicrania è ragionevolmente indicato per ridurre il rischio di stroke (IIb-C); la chiusura del PFO non è indicata per prevenire lo stroke nei pazienti con emicrania (III-B).

\section{Bibliografia}

1. http://www.iso-stroke.it/

2. Adeoye O, Nystrom K, Yavagal D et al. Recommendations for the Establishment of Stroke Systems of Care: A 2019 Update. Stroke 2019; 50:e187-e210.

3. https://www.cdc.gov/nchs/healthy_people/hp2010/ hp2010_final_review.htm

4. https://www.healthypeople.gov/

5. https://millionhearts.hhs.gov/

6. http://www.salute.gov.it/portale/temi/p2_4.jsp?lingua= italiano\&tema $=$ Prevenzione $\&$ area $=$ prevenzione

7. http://www.iso-spread.it/

8. Meschia J, Bushnell C, Boden-Albala B et al. Guidelines for the Primary Prevention of Stroke. A Statement for Healthcare Professionals From the American Heart As-
sociation/American Stroke Association. Stroke 2014; 45:3754-3832.

9. Arnett D, Blumenthal R, Albert M et al. 2019 ACC/ AHA Guidelines on the Primary Prevention of Cardiovascular Disease. Circulation 2019;140:e596-e646. DOI: 10.1161/CIR.000000000000067.

10. Halperin JL, Levine GN, Al-Khatib SM et al. Further evolution of the ACC/AHA clinical practice guideline recommendation classification system: a report of the American college of Cardiology/American Heart Association Task Force on Clinical Practice Guidelines. Circulation 2016; 48:923-33.

11. https://www.ars.toscana.it/aree-dintervento/problemi-disalute/malattie-croniche.html

12. http://www.cuore.iss.it/valutazione/carte.asp

13. http://tools.acc.org/ASCVD-Risk-Estimator-Plus/\#!/calculate/estimate/

14. Williams B, Mancia G, Spiering W et al. 2018 ESC/ESH Guidelines for the management of arterial hypertension Hypertens 36:1953-2041.

15. American Diabetes Association. Improving Care and Promoting Health in Populations: Standards of Medical Care in Diabetes-2019. Diabetes Care 2019 Jan; 42(Supplement 1): S7-S12. https://doi.org/10.2337/dc19-S001

16. Cosentino F, Grant P, Aboyans V et al 2019 ESC Guidelines on diabetes, pre-diabetes, and cardiovascular diseases developed in collaboration with the EASD. European Heart Journal (2019) 00, 169 doi:10.1093/ eurheartj/ehz486

17. Grundy SM, Stone N, Bailey A et al. 2018 AHA / ACC / AACVPR / AAPA / ABC / ACPM / ADA / AGS / APhA / ASPC / NLA / PCNA Guideline on the Management of Blood Cholesterol. A Report of the American College of Cardiology/American Heart Association Task Force on Clinical Practice Guidelines. Journal of the American College of Cardiology 73 , June 2019 DOI: 10.1016/j.jacc.2018.11.003. 


\title{
Cause rare di stroke
}

\author{
Marialuisa Zedde \\ S.C. Neurologia, Stroke Unit, Azienda Unità Sanitaria Locale-IRCCS di Reggio Emilia, Italia
}

\section{Premessa}

La trattazione delle cause rare di patologia cerebrovascolare è virtualmente infinita, sia perché il numero e la tipologia delle malattie (spesso genetiche, ma non solo) fra le cui manifestazioni, primarie o secondarie, ci sono eventi cerebrovascolari è notevole ed in costante crescita, sia perché l'approccio diagnostico e le possibilità che la tecnologia offre sono anch'essi in continuo ampliamento, in particolare per la diagnostica di malattie monogeniche.

La definizione di raro inoltre non è mai stata realmente esplicitata per quanto riguarda la patologia cerebrovascolare, per cui, se si applica come definizione operativa quella mediata dall'Unione Europea, ${ }^{1}$ in cui viene definita rara una malattia con una prevalenza inferiore a 5 su 10.000 abitanti, il campo si delimita ulteriormente. Spesso comunque nelle classificazioni eziopatogenetiche dell'ictus ${ }^{2,3}$ nell'elenco proposto di cause rare sono presenti patologie che rare non sono (rispetto a questa arbitraria definizione) o che sono semplicemente sottodiagnosticate, come per esempio le dissecazioni dei vasi epiaortici ed intracranici, tanto da meritare in alcune classificazioni (ASCOD) ${ }^{4}$ una categoria a sé stante. Molte di queste patologie in passato rimanevano non diagnosticate ed incrementavano la percentuale di ictus da cause non determinate.

Altri preconcetti che spesso accompagnano la descrizione della patologia cerebrovascolare da cause rare sono che raro corrisponda a giovane (non vero in senso assoluto neanche per alcune condizioni monogeniche) e che raro corrisponda a ereditario, ovvero

Corrispondente: Marialuisa Zedde, S.C. Neurologia, Stroke Unit, Azienda Unità Sanitaria Locale-IRCCS di Reggio Emilia, Italia

E-mail: zedde.marialuisa@ausl.re.it; marialuisa.zedde@gmail.com

Articolo pubblicato secondo la Creative Commons Attribution NonCommercial 4.0 License (CC BY-NC 4.0).

${ }^{\circ}$ Copyright: the Author(s), 2020

Licensee PAGEPress, Italy

QUADERNI - Italian Journal of Medicine 2020; 8(2):24-33 geneticamente determinato ed in particolare monogenico (anche in questo caso ad esempio la patologia cerebrovascolare acuta indotta dalla Sindrome da Vasocostrizione Posteriore Reversibile è di per sé rara ma non genetica e colpisce qualunque fascia di età).

È inoltre difficile classificare in maniera completa tutte le condizioni che possono essere incluse nel novero delle cause rare di patologia cerebrovascolare, perché si tratta sia di malattie primitive che hanno il sistema nervoso centrale come organo bersaglio privilegiato, prevalente o esclusivo, sia di malattie sistemiche, infettive, immunomediate, protrombotiche, ecc. in cui le manifestazioni cerebrovascolari sono una delle varie componenti del quadro clinico.

Sebbene alcune di queste non siano trattabili (come ad esempio alcune delle malattie monogeniche), alcune altre, come le condizioni di ipercoagulabilità, le vasculiti, ecc. sono potenzialmente trattabili e modificabili e richiedono un approccio ed un trattamento differente.

Inoltre le modalità di interessamento cerebrovascolare, sia in forma acuta che in forma cronica (e spesso è presente la combinazione delle due componenti), sono pressoché le stesse per molte malattie, che, nella loro rarità, hanno fenocopie assai più consuete (basti pensare all'ictus ischemico lacunare in pazienti con una microangiopatia monogenica, che è spesso indistinguibile dal pattern di imaging di un ictus lacunare su encefalopatia vascolare da fattori di rischio classici, come per esempio ipertensione arteriosa e/o diabete). L'avere una condizione rara, che sia o no ereditaria, non esime dal poter avere anche i classici fattori di rischio vascolari, per cui in alcuni casi porre il sospetto diagnostico è la tappa più difficile del percorso stesso e la presenza di noti ed evidenti fattori di rischio vascolari non è una condizione di esclusione per il sospetto clinico di malattia monogenica (come abbastanza classico per le small vessel diseases - SVD - cerebrali). Un'altra caratteristica delle condizioni patologiche che colpiscono la circolazione dell'encefalo è che esse hanno comunque una doppia faccia, ischemica ed emorragica, spesso contemporaneamente presente, per cui la distinzione fra cause rare associate ad ictus ischemico e cause rare associate a ictus emorragico è spesso artificiosa. 
Nella necessaria brevità di questa trattazione è stato scelto di privilegiare le condizioni patologiche che interessano in maniera peculiare o frequente, se non esclusiva, il sistema nervoso centrale, differenziandole sulla base delle prevalenti manifestazioni ischemiche ed emorragiche, e concentrandosi sulle patologie che condividono un interessamento cerebrovascolare di tipo microangiopatico (SVD). In considerazione della molteplicità di malattie la descrizione sarà limitata alle sole condizioni mendeliane.

\section{Malattie monogeniche associate ad ictus}

Le cause monogeniche di stroke sono considerate rare, sebbene manchino dei veri e propri dati di incidenza, se non su gruppi di popolazione selezionati (secondo uno studio recente comunque circa $1,5 \%$ degli ictus lacunari apparentemente sporadici della popolazione $<70$ anni sarebbe dovuto ad una causa monogenica, con NOTCH3 al primo posto; NOTCH3, HTRA1, COL4A1, COL4A2, FOXC1, TREX1, and GLA): ${ }^{5}$ spesso tendono ad essere una componente di una malattia multisistemica, come nel caso della Malattia di Anderson Fabry, ma in alcuni casi costituiscono la manifestazione prevalente se non unica (CADASIL, CARASIL e quasi tutte le microangiopatie cerebrali).

Un approccio differente rispetto allo screening indiscriminato della popolazione selezionata per una serie identificata di malattie monogeniche è quello proposto dal gruppo GENS-Lombardia, ${ }^{6}$ in cui erano stati predefiniti dei criteri clinico-anamnestici per l'accesso allo screening per singola patologia, scegliendo cinque differenti malattie monogeniche (M. Anderson Fabry CADASIL, MELAS, HCAA, Sindrome di Marfan): con questo screening più ristretto circa il $7 \%$ dei pazienti testati aveva una di queste cinque malattie monogeniche. Questo approccio rappresenta un esempio della strategia di definizione migliore di gruppo di popolazione a rischio per ridurre i costi ed aumentare le probabilità di positività delle indagini in questione.

\section{Malattia di Anderson Fabry}

La Malattia di Anderson Fabry è una malattia lisosomiale con interessamento multisistemico, causata da mutazioni del gene della alfa-galattosidasi ( $G L A)$ localizzato sul cromosoma X. A differenza delle classiche malattie X-linked, nella malattia di Anderson Fabry la donna può presentare manifestazioni cliniche da assai lievi fino ad una severità analoga rispetto a quella del maschio emizigote. La trasmissione è quella di una malattia X-linked per cui un maschio affetto avrà figli maschi sani e figlie femmine con la mutazione responsabile della malattia, mentre una donna af- fetta eterozigote ha il 50\% di probabilità di avere figli affetti, a prescindere dal sesso. La maggior parte delle mutazioni responsabili della malattia sono mutazioni private o di singoli gruppi familiari e il numero delle mutazioni descritte è costantemente in crescita (ne sono state descritte ad oggi poco meno di 1000). Esiste per alcuni aspetti una buona correlazione genotipo-fenotipo, essendo alcune mutazioni caratteristicamente associate alla forma classica della malattia, corrispondente alle descrizioni originali, ed altre associate alla cosiddetta forma late-onset di malattia, in cui spesso, ma non sempre, le manifestazioni d'organo sono più lievi o concentrate in maniera prevalente su un organo o apparato, ma sicuramente l'età di esordio della malattia è molto più ritardata e la diagnosi viene prevalentemente effettuata in età chiaramente adulta, spesso in corrispondenza della quinta-sesta decade di vita.

La prevalenza della malattia alla nascita è stimata in vari studi di popolazione da 1:40.000 a 1:70.000 con maggiore rappresentazione di forme late-onset. La reale prevalenza e storia naturale della patologia sarà comunque definita nei programmi di screening neonatali che sono iniziati come progetto pilota in alcune regioni italiane, come ad esempio il Veneto. ${ }^{7,8}$

Nell'ambito di una malattia multisistemica le classiche manifestazioni (segni e/o sintomi di danno d'organo) sono le seguenti: lesioni cutanee (asintomatiche) chiamate angiocheratomi, che hanno dato il nome alla malattia e che spesso sono raggruppate in sedi di difficile identificazione e possono avere un corrispettivo sulle mucose, manifestazioni oculari (cornea verticillata, tortuosità vascolari retiniche) che non hanno un impatto sulle capacità visive, disfunzione renale fino all'insufficienza renale terminale e alla dialisi, interessamento cardiaco a più livelli (miocita con fenotipo ipertrofico, sistema di conduzione con blocchi atrioventricolari e aritmie ipercinetiche sopraventricolari, endocardico con valvulopatie multiple), interessamento del sistema nervoso periferico ${ }^{9}$ (neuropatia delle piccole fibre che si somma ad una componente ganglionare, ${ }^{10}$ spiccata componente autonomica, che rende ragione almeno in parte delle manifestazioni gastrointestinali dolorose, deficit del VIII n.c. con ipoacusia e disturbi dell'equilibrio) e del sistema nervoso centrale $^{9}$ (sia nella componente parenchimale con leucoencefalopatia vascolare solitamente simmetrica, eventi cerebrovascolari acuti sia con pattern lacunare che con pattern territoriale, che nella componente vascolare con arteriopatia dilatativa intracranica prevalentemente nel distretto vertebrobasilare). Solitamente le prime manifestazioni cliniche, già dall'età infantile, sono quelle dolorose a genesi neuropatica, in forma di acroparestesie urenti, spesso scatenate dall'esposizione al caldo e dall'esercizio fisico, associate ad ipoanidrosi, oppure di coliche addominali sine materia. Dall'età adolescenziale iniziano gradualmente le altre 
manifestazioni di danno d'organo, fra cui quelle cerebrovascolari (solitamente in forma di ictus ischemico, ma sono descritti casi di ictus emorragico ed alcuni isolati reports di trombosi venosa cerebrale) rappresentano la complicanza d'organo più precoce e frequente, che spesso precede temporalmente eventi in altri organi. Negli studi di screening enzimatico e genetico dei gruppi di popolazione a rischio $^{11}$ (ictus criptogenetico $<55$ anni, dializzati, cardiopatia ipertrofica) e nella revisione sistematica dei dati disponibili ${ }^{12}$ la prevalenza della malattia è rispettivamente lo $0.13 \%$ della popolazione sottoposta a screening per stroke e circa lo $0.9 \%$ della popolazione sottoposta a screening sulla base di manifestazioni cardiache e renali. La valutazione di prevalenza è complicata dal fatto che alcune varianti che inizialmente venivano considerate patogenetiche successivamente sono state declassate a polimorfismi, per cui una valutazione di prevalenza vera comprende anche la conoscenza delle singole mutazioni identificate nei differenti studi.

Per quanto riguarda le manifestazioni cerebrovascolari, i dati dei registri di patologia segnalano come in realtà una percentuale non trascurabile di pazienti nella quinta e sesta decade di vita presenta un primo ictus, ${ }^{13}$ per cui potrebbe essere utile porre il sospetto di malattia, su base individuale, anche al di fuori della categoria di pazienti con ictus giovanile.

Il danno d'organo è in gran parte determinato dall'accumulo di substrato (globotriaosil ceramide) nelle cellule bersaglio, con una componente infiammatoria potenzialmente associata. La valutazione delle mutazioni, in particolare per la forma classica e per la forma late-onset nel maschio, deve attualmente essere associata alla misura del lysoGb3 a livello plasmatico; anche in questo caso le donne tendono ad avere valori più bassi degli uomini.

Per quanto riguarda le manifestazioni cerebrovascolari esiste comunque un interessamento neuronale selettivo, potenzialmente responsabile di disfunzioni cognitive con l'aumento degli anni di malattia e di una componente atrofica. ${ }^{14}$ Inoltre non esiste un pattern di imaging in risonanza magnetica che sia patognomonico della Malattia di Anderson Fabry. ${ }^{15}$

La diagnosi è possibile nel maschio tramite il dosaggio dell'attività enzimatica della GLA su sangue periferico, con successiva analisi genetica in caso di attività enzimatica significativamente ridotta, mentre nella donna il processo di inattivazione di uno dei due cromosomi X rende sostanzialmente inattendibile per la diagnosi il dosaggio dell'attività enzimatica della GLA, per cui è necessario procedere direttamente con l'analisi genetica.

Le terapie disponibili per la malattia di Anderson Fabry sono sostanzialmente la terapia enzimatica sostitutiva con enzima ricombinante (agalsidasi alfa e beta) ${ }^{16}$ che viene somministrata con infusione per via endovenosa ogni due settimane, ed una terapia a base di una piccola molecola proteica che agisce da chaperone, ovvero potenziando l'attività enzimatica residua in un gruppo selezionato di mutazioni della GLA, il migalastat. ${ }^{17}$ Tutti e tre questi prodotti sono commercializzati nei paesi della UE.

\section{Cerebral autosomal dominant arteriopathy with subcortical infarcts and leukoencephalopathy}

Il Cerebral autosomal dominant arteriopathy with subcortical infarcts and leukoencephalopathy (CADASIL) è una malattia cerebrovascolare ad ereditarietà autosomica dominante, causata da una mutazione del gene NOTCH3 localizzato sul cromosoma 19p13.2-p13.1, caratterizzata, come da definizione, da ictus ischemico lacunare, emicrania con aura, disturbo dell'umore (depressione e apatia) spesso precedente l'esordio delle manifestazioni cerebrovascolari, con evoluzione fino alla demenza e alla completa perdita di autonomia. La prevalenza della malattia è di almeno 10.7 per 100.000 abitanti adulti ${ }^{18}$ ed è attualmente considerata la causa ereditaria più comune di ictus e demenza vascolare negli adulti. Le manifestazioni cliniche nelle casistiche classiche hanno la seguente prevalenza: ictus o TIA nel $61 \%$, emicrania nel $70 \%$, disturbo cognitivo nel $48 \%$, problemi psichiatrici (tra cui depressione e psicosi) nel $47 \%$, crisi comiziali nel $8 \%{ }^{18}$ Anche se in termini istopatologici i segni di coinvolgimento arteriolare della patologia sono riscontrabili in tutti i tessuti del corpo, ${ }^{19}$ per ragioni per lo più sconosciute sono rarissime le manifestazioni cliniche in organi differenti dal sistema nervoso centrale e la malattia sembra clinicamente limitata all'encefalo. Le manifestazioni cerebrovascolari acute (ictus, prevalentemente ischemico, e TIA) sono per lo più lacunari e si verificano fino al $60-85 \%$ dei pazienti sintomatici con una età di esordio compresa fra 49 e 57 anni su un l'intervallo che copre l'intera vita umana adulta. ${ }^{18,20} \mathrm{Il}$ CADASIL è stato riportato nel $11,1 \%$ (IC 95\% 2.5-44.5) di pazienti sotto 50 anni con ictus ischemico lacunare e leucoaraiosi ${ }^{21}$ e nel $1.5 \%$ dei pazienti con primo ictus lacunare di età inferiore a 70 anni. ${ }^{5} \mathrm{~L}$ emicrania è un segno precoce, con esordio medio nella terza decade di vita, anche se può iniziare nell'infanzia e può avere connotati clinici sostanzialmente indistinguibili rispetto alla forma primaria, senza una maggiore prevalenza di emicrania senz'aura rispetto alla popolazione generale; tuttavia, l'emicrania con aura è tipicamente presente dal $20 \%$ al $40 \%$ dei pazienti, ${ }^{20}$ mentre in un'ampia serie di pazienti pubblicata nel 2016 l'emicrania con l'aura viene riportata nel $75 \%$ dei pazienti sintomatici, ${ }^{22}$ pertanto con una prevalenza marcatamente più elevata rispetto alla popolazione generale. Una manifestazione abbastanza caratteristica è l'emicrania emiplegica, che si verifica nel $16 \%$ dei pazienti con CADASIL ed emicrania. ${ }^{22}$ Un'altra manifestazione 
acuta del CADASIL è una encefalopatia severa, con depressione della coscienza fino al coma, chiamata CADASIL coma, con associate manifestazioni emicraniche. Il CADASIL coma consiste in una forma di riduzione della coscienza prolungata (mediana di 8 giorni, range 3-17 giorni) ma in ultimo reversibile senza altre cause; in genere inizia con cefalea emicranica ed è spesso accompagnata da nausea/vomito, crisi comiziali, febbre e talora allucinazioni; essa rappresenta la prima presentazione clinica nel $10 \%$ dei pazienti CADASIL e a forte associazione con l'emicrania suggerisce una patogenesi comune..$^{23}$ Nell'imaging in risonanza magnetica con sequenze Arterial Spin Labeling (ASL) è stata evidenziata in questi casi una iperperperfusione cerebrale globale o localizzata, con successiva normalizzazione. ${ }^{24}$ Il modello murino di CADASIL dimostra una più marcata cortical spreading depression, che può spiegare la coprevalenza dell'emicrania con l'aura e il CADASIL coma, sulla cui fisiopatologia ci sono ancora elementi da chiarire. ${ }^{25}$ Anche se è stato ipotizzato che $\mathrm{i}$ pazienti con CADASIL con emicrania possano rappresentare un fenotipo più grave, uno studio ha scoperto che i pazienti con CADASIL senza emicrania avevano una maggiore incidenza cumulativa di ictus, ipotizzando che, attraverso il precondizionamento, la cortical spreading depression nel CADASIL può proteggere il cervello nei confronti di un successivo evento ischemico. ${ }^{22}$ Manifestazioni psichiatriche, sia in senso depressivo che maniacale, sono presenti in circa il 20\% dei pazienti con CADASIL, e l'apatia, indipendentemente dalla depressione, è riscontrabile nel $40 \%$ dei casi. L'esordio dei sintomi psichiatrici è solitamente attorno alla quarta decade ed essi contribuiscono alla riduzione generale della qualità della vita. Il disturbo cognitivo nel CADASIL inizia in genere con la compromissione delle funzioni esecutive e della velocità di elaborazione delle informazioni e progredisce verso una demenza più globale, spesso includendo delle manifestazioni chiaramente pseudobulbari. Il disturbo della deambulazione, che deriva anch'esso dal diffuso interessamento sottocorticale nelle indagini di imaging, è un forte predittore del successivo declino cognitivo. ${ }^{26}$ Il CADASIL è stato in passato ritenuto una patologia catastrofica, ineluttabilmente associata a demenza avanzata e severa disabilità motoria, con disfagia assoluta, ma al momento è noto che la sua espressione clinica e la sua gravità sono ampiamente variabili. I primi reports mostravano un'elevata prevalenza (oltre il $50 \%$ ) di grave disabilità in pazienti di età superiore ai 65 anni $^{27}$ con solo il $14 \%$ dei pazienti più anziani che non hanno disabilità, ma una serie pubblicata nel 2014 ha rilevato che i pazienti in vita nella fascia di età 58-75 anni erano mobili senza ausili e il $46 \%$ era completamente indipendente. ${ }^{18}$ Nella stragrande maggioranza dei pazienti, la presenza di alterazioni di segnale del parenchima cerebrale allo studio di RMN cerebrale precede l'insor- genza dei sintomi da 10 a 15 anni (fa eccezione l'emicrania con aura, che può avere un esordio precoce); pertanto, la risonanza magnetica cerebrale è un utile strumento di screening per la diagnosi sia per i pazienti sintomatici che per i pazienti presintomatici (spesso familiari del probando). Oltre agli infarti lacunari e alla leucoencefalopatia sottocorticale con tendenza alla confluenza, i segni caratteristici sono le iperintensità $\mathrm{T} 2$ che coinvolgono la sostanza bianca dei poli temporali anteriori (segno di O'Sullivan), ${ }^{28}$ riscontrabili nel 90\% dei pazienti con CADASIL, e che rappresentano un utile marcatore per distinguere il CADASIL da altre SVD sporadiche, sebbene la loro assenza non sia predittiva dell'assenza di patologia e la loro presenza non sia univocamente associata alla diagnosi genetica di CADASIL. L'iperintensità di segnale a carico della capsula esterna e del corpo calloso hanno, per contro, una assai maggiore specificità per la diagnosi di CADASIL. Il reperto, congruo con l'espressione di neuroimaging delle SVD, di microbleeds nelle sequenze gradient echo, prevalentemente profondi, ma spesso misti ed assai raramente pressochè unicamente lobari, è presente in una percentuale di pazienti fino al $69 \%,{ }^{29}$ in numero crescente con l'età e con discreta sovrapposizione rispetto ai microbleeds presenti in altre SVD, genetiche e sporadiche, come l'angiopatia amiloide cerebrale e la SVD ipertensiva. ${ }^{30,31}$ Inoltre non sono mai stati osservati nei pazienti CADASIL reperti microemorragici ritenuti più specifici dell'angiopatia amiloide cerebrale, come la siderosi corticale superficiale. ${ }^{31}$ Esistono anche reports di eventi macroemorragici associati a CADASIL, solitamente con elevato carico di marcatori di SVD nella diagnostica di neuroimaging. ${ }^{32}$ I marcatori di neuroimaging che correlano meglio con la compromissione clinica in pazienti con CADASIL sono il numero di lacune e il volume cerebrale. ${ }^{26}$ Solitamente la comorbidità aterotrombotica è minima o assente, ma non si può escludere l'associazione con molteplici fattori di rischio non ereditari nel corso della vita, per cui tale elemento non rappresenta un indicatore di minore sospetto di malattia, su base clinica.

Il gene NOTCH3 codifica un recettore transmembrana che contiene 34 ripetizioni del fattore di crescita epidermico (EGFR), una grande proteina transmembrana necessaria per la differenziazione e lo sviluppo delle cellule muscolari lisce della parete vasale. Ogni EGFR ha normalmente sei residui di cisteina. Ad oggi sono state riportate nei database pubblici 396 varianti del NOTCH3 ${ }^{33}$ Sono state riportate oltre 150 mutazioni causali, la stragrande maggioranza delle quali sono mutazioni missense e che portano a un numero dispari di residui di cisteina all'interno di un dato EGFR. Sebbene il gene NOTCH3 abbia 33 esoni, più del $90 \%$ delle mutazioni CADASIL si trovano negli esoni da 2 a 24 , con oltre il $70 \%$ delle famiglie che ospitano mutazioni negli esoni 3 e $4,{ }^{20}$ Nuove muta- 
zioni causali continuano a essere scoperte regolarmente e le mutazioni de novo non sono rare ma di frequenza sconosciuta. A questo si aggiunge che l'espressività clinica delle mutazioni sembra essere correlata alla loro posizione, essendo quelle che coinvolgono le prime 7 ripetizioni dei domini EGFR associate a maggiore gravità e mortalità rispetto a quelle che coinvolgono le ripetizioni dalla 8 alla $34 .{ }^{34}$ Esiste molta eterogeneità fenotipica inter-familiare ed intrafamiliare anche con la stessa mutazione, suggerendo fattori modificanti ambientali o epigenetici. I pazienti omozigoti per la mutazione NOTCH3 hanno dimostrato di avere un fenotipo clinico e radiologico leggermente più grave con esordio precoce rispetto ai loro membri della famiglia eterozigoti..$^{35} \mathrm{Il}$ gold standard per la diagnosi è il test genetico, con una specificità del $100 \%$ e quasi il $100 \%$ di sensibilità durante lo screening dei 23 esoni per una mutazione che porta a un numero dispari di residui di cisteina all'interno di un EGFR. A causa del gran numero di mutazioni, alcune delle quali si trovano al di fuori degli esoni solitamente sottoposti a screening, è possibile avere un risultato genetico falso negativo, per cui è necessario rivolgersi per la diagnosi genetica ad un laboratorio che effetti almeno il sequenziamento degli esoni 2-24 e non solo lo screening delle mutazioni considerate più comuni. Se ciò accade, oppure se è necessario valutare il ruolo patogenetico di varianti di incerto significato del $\mathrm{NOTCH} 3$, non coinvolgenti un residuo di cisteina, si può prendere in considerazione l'esecuzione di una biopsia cutanea che includa uno studio in microscopia elettronica per valutare la presenza di materiale granulare osmiofilo (i cosiddetti GOMs) a livello della lamina basale delle arteriole sottocutanee, reperto altamente specifico per CADASIL.

Data la sovrapposizione clinica e neuroradiologica tra CADASIL e altre SVD sporadiche, è stata sviluppata una scala CADASIL, che in alcuni casi appare utile per selezionare i pazienti da sottoporre a test genetico. ${ }^{36}$ Anche se lo studio iniziale ha mostrato un'elevata sensibilità $(97 \%)$ e specificità $(74 \%)$ in una popolazione italiana, questa precisione predittiva non è stata replicata in una popolazione cinese ${ }^{37} \mathrm{Il}$ suo utilizzo appare pertanto utile in alcuni ambiti clinici ben selezionati, ma non esclusivo, non coprendo tutte le possibilità di sospetto clinico di patologia. La decisione di sottoporre a test genetico individui asintomatici all'interno di una famiglia con un probando con diagnosi di CADASIL deve essere individualizzata e con adeguata preparazione del soggetto da sottoporre a test, considerate le implicazioni. ${ }^{38}$ Poiché il CADASIL non ha una strategia di trattamento o prevenzione nota, il test genetico non è raccomandato nei bambini asintomatici.

Pur in assenza di una strategia di trattamento specifica, è noto che l'associazione con i classici fattori di rischio vascolari (es. fumo) amplifica il rischio di evento, per cui appare necessaria una prevenzione vascolare globale aggressiva ed un trattamento di tutti i fattori di rischio associati. ${ }^{39,40}$

Sfortunatamente, a causa delle somiglianze nella presentazione clinica e nel neuroimaging, il CADASIL è talora misdiagnosticato come sclerosi multipla e trattato con farmaci immunomodulanti senza beneficio. Questo mette in evidenza l'importanza di eseguire una storia familiare dettagliata incentrata su ictus, emicrania, depressione/ansia, e declino cognitivo, nonché una corretta interpretazione dei risultati dello studio liquorale. In una analisi dei dati liquorali di 87 pazienti con CADASIL diagnosticati geneticamente, il $29 \%$ aveva proteine leggermente elevate (media 40,4 mg/dL, range da $12 \mathrm{mg} / \mathrm{dL}$ a $75 \mathrm{mg} / \mathrm{dL}$ ), solo uno aveva bande oligoclonali $(1,1 \%)$, e nessuno aveva pleocitosi. ${ }^{41}$ Inoltre il meccanismo fisiopatologico di danno della sostanza bianca nelle patologie vascolari e in quelle demielinizzanti segue due differenti patterns di attivazione delle vie dell'infiammazione e non hanno una via fisiopatologica comune. ${ }^{42}$

$\mathrm{Al}$ momento non è disponibile un trattamento specifico per CADASIL e la gestione piuttosto aggressiva dei fattori di rischio vascolari, come sopra segnalato, è correntemente suggerita, pur in assenza di studi clinici dedicati a sostegno di questa strategia. Tuttavia, uno studio osservazionale ha rilevato che fattori clinici come il fumo, l'iperlipidemia e l'ipertensione non erano di per sé associati al timing dell'insorgenza dell'ictus. Controllare l'ipertensione, peraltro, può avere altri benefici: lo stesso studio ha riscontrato una perdita di autonomia entro 16 anni in pazienti con ipertensione (età mediana 55 contro 71 anni), ${ }^{18} \mathrm{e}$ un altro studio ha identificato la sola ipertensione come fattore di rischio associato con disabilità funzionale. ${ }^{43}$ Spesso viene suggerita una terapia antiaggregante, anche se non esistono studi che abbiano affrontato in maniera dedicata il rapporto rischio/beneficio del suo utilizzo nel paziente CADASIL, ed in particolare il potenziale di aumento del rischio emorragico. ${ }^{44} \mathrm{Il}$ trattamento a breve termine con atorvastatina non migliora i parametri emodinamici nei pazienti con CADASIL, ma non ne è stato testato l'effetto nella prevenzione dell'ictus o con l'uso a lungo termine. ${ }^{45}$ Non sono disponibili informazioni sul rapporto rischio/beneficio dell'utilizzo di rtPA per ictus ischemico acuto in pazienti CADASIL, mentre esiste un unico report, peraltro favorevole..$^{32}$ Per la prevenzione dell'emicrania, i farmaci standard sono utilizzati analogamente rispetto alla gestione dell'emicrania nel paziente non CADASIL, con nessun farmaco preferito rispetto a un altro. ${ }^{22}$ Esistono reports osservazionali, tuttavia, di particolare beneficio dall'acetazolamide, probabilmente a causa dell'ipoperfusione osservata nel CADASIL. ${ }^{46,47}$ Per il trattamento dell'emicrania, i 
triptani non sono regolarmente utilizzati a causa di un presunto potenziale per aumentare il rischio di ictus, in particolare nei pazienti con emicrania complicata. Tuttavia, uno studio osservazionale sul trattamento dell'emicrania in pazienti con CADASIL ha riscontrato che 1'11\% dei pazienti aveva provato i triptani, con circa il $50 \%$ del tasso di risposta e nessun evento avverso..$^{22}$ In uno studio controllato randomizzato di pazienti con CADASIL con disfunzione cognitiva, il donepezil non ha migliorato la funzione cognitiva complessiva misurata tramite il punteggio della scala VADAS-cog (Vascular Dementia Assessment Scale cognitive subscale), ma ha fatto migliorare la funzione esecutiva, quindi a volte è prescritto. ${ }^{48}$

\section{Cerebral autosomal recessive arteriopathy with subcortical infarcts and leukoencephalopathy}

Il Cerebral autosomal recessive arteriopathy with subcortical infarcts and leukoencephalopathy (CARASIL) provoca sintomi simili a quelli del CADASIL, ma, per lo meno nella sua forma più classica, una o due decadi prima nella vita, senza associazione con l'emicrania e con l'aggiunta di alopecia e spondilosi. I pazienti con CARASIL presentano ictus lacunari ad esordio precoce (insorgenza nella terza decade di vita) in assenza di ipertensione, demenza progressiva (insorgenza nella terza-quinta decade), alopecia precoce (insorgenza negli anni dell'adolescenza) e spondilosi deformante (insorgenza nella seconda-terza decade di vita). Il pattern di neuroimaging è abbastanza simile a quello del CADASIL: microbleeds e leucoaraiosi progressiva con coinvolgimento precoce della capsula esterna, del ponte e della sostanza bianca del lobo frontale. ${ }^{49}$ Nelle fasi avanzate, una caratteristica tipica della risonanza magnetica di pazienti CARASIL è il coinvolgimento delle vie pontocerebellari, chiamato il segno dell'arco poiché appare come una lesione iperintensa a forma di arco dal ponte ai peduncoli cerebellari medi. ${ }^{49}$

La CARASIL è causato da mutazioni nel gene che codifica per la HtrA serine peptidase 1 (HTRA1) sul cromosoma 10q. ${ }^{50}$ HTRA1 lega e cliva il predominio del pro-TGF- $\beta 1$, e il prodotto del clivaggio è degradato dal reticolo endoplasmatico. Questo meccanismo regola la quantità di TGF $\beta 1$ maturo. ${ }^{51}$ Originariamente identificatonei pazienti giapponesi, il CARASIL è ora noto per essere presente in più popolazioni, compresa la popolazione occidentale. ${ }^{52}$ Infatti mutazioni HTRA1 in eterozigosi possono dare un fenotipo clinico di SVD molto simile a quello noto in pazienti CADASIL, ma con una età di esordio superiore rispetto al CARASIL, ed attualmente questa forma di malattia rappresenta la seconda causa di SVD monogenica nella popolazione occidentale, dopo il CADASIL. ${ }^{53,54}$ Inoltre in studi di popolazione con genome-wide association study (GWAS) le mutazioni patogenetiche di HTRA1 rappresentano la seconda associazione genetica come frequenza della leucoencefalopatia vascolare. ${ }^{54,55} \mathrm{Al}$ momento non è disponibile alcuna terapia specifica per la malattia.

\section{Retinal vasculopathy with cerebral leukodystrophy}

Quelle che in precedenza si pensava fossero tre malattie autosomiche con associazione di ictus e retinopatia (sindrome della vasculopatia cerebroretinica, retinopatia vascolare ereditaria e endoteliopatia ereditaria, retinopatia, nefropatia e ictus [HERNS]) sono statemappare tutte sul gene TREX1 (3p21.31) e sono ora collettivamente denominate vasculopatia retinica con leucodistrofia cerebrale. In queste sindromi, perdita del visus, ictus e demenza iniziano nella quarta-quinta decade di età, e la morte si verifica nella maggior parte dei pazienti 5-10 anni più tardi. La retinopatia è caratterizzata dalla neovascolarizzazione del disco ottico, da emorragie retiniche e da edema maculare. I deficit neurologici non visivi sono più variabili e includono episodi simili a ictus, disfunzione corticale e sottocorticale multifocale. La risonanza magnetica cerebrale mostra iperintensità multifocali della sostanza bianca e, in circa il $50 \%$ dei pazienti, una lesione pseudotumorale che risparmia la corteccia e che va in diagnosi differenziale con una neoplasia primitiva cerebrale, ma che allo studio istopatologico mostra necrosi e gliosi reattiva, di cui è stata osservata una regressione spontanea. ${ }^{56} \mathrm{I}$ pazienti con mutazioni TREX1 manifestano anche, in combinazione variabile, fenomeno di Raynaud, emicrania e disfunzione renale o epatica lieve. Il gene TREXI codifica una proteina nucleare con attività di 3' esonucleasi; le mutazioni responsabili di questa patologia provocano la sintesi di una proteina tronca che mantiene l'attività di esonucleasi ma perde la localizzazione perinucleare, avendo effetti dannosi sulle cellule endoteliali e portando ad una microangiopatia cerebrale degenerativa che causa ictus e demenza. ${ }^{57}$ I pazienti con mutazioni in omozigosi risultano affetti dalla Sindrome di Aicardi Goutieres, una grave encefalopatia con leucodistrofia; altre mutazioni dello stesso gene sono state associate a forme di lupus ereditario. La retinopatia proliferativa può rispondere al bevacizumab intravitrale. ${ }^{58}$

\section{COL4A1-A2-related cerebral small vessel disease}

Le mutazioni nel gene COL4A1, un gene che codifica la catena del collagene alfa 1 tipo IV, causano una malattia dei piccoli vasi cerebrali con ereditarietà autosomica dominante che si manifesta nella quarta decade di vita come leucoaraiosi diffusa (nel 63\% dei pazienti riportati in letteratura senza coinvolgimento del polo temporale anteriore riscontrato nel CADA- 
SIL), microbleeds (53\%) e ictus lacunari (13\%). ${ }^{59}$ Inoltre, rispetto alle altre SVD monogeniche esaminate fino ad ora, c'è una evidente suscettibilità per eventi emorragici cerebrali, superiore a quella per eventi ischemici, in particolare dopo stress ambientali come il parto vaginale, le attività sportive, l'uso di farmaci antitrombotici e i traumi cranici. ${ }^{60}$ Le manifestazioni aggiuntive delle mutazioni di COL4A1 possono includere emiparesi infantile e porencefalia congenita; presenti anche aneurismi cerebrali (44\%, multipli in circa la metà dei casi), emicrania con aura e manifestazioni sistemiche a carico di occhio (48\%, cataratta, tortuosità dei vasi della retina ed emorragie retiniche), rene (15\%) e muscoli (15\%). Come per il CADASIL, esiste una elevata variabilità fenotipica intrafamiliare. Lo screening deve essere considerato nei pazienti con uno qualsiasi dei seguenti elementi: una storia personale o familiare di emiparesi infantile o porencefalia, una storia personale di leucoencefalopatia con microbleeds ma senza ipertensione, o una storia personale di tortuosità arteriolare retinica. Sebbene non esista un trattamento noto per le mutazioni di COL4A1, la diagnosi è utile per consigliare i pazienti in merito all'evitare traumi cranici, anche lievi, e per preferire la pianificazione del parto con taglio cesareo.

Anche le mutazioni del COL4A2 sono associate ad una SVD simile a quella vista nelle mutazioni del COL4A1, con multipli eventi cerebrovascolari di tipo emorragico, poroencefalia ad esordio precoce, emiplegia congenita, aneurismi intracranici, nefropatia e miopatia. ${ }^{61}$ Le caratteristiche istopatologiche sono molto simili a quelle viste nelle mutazioni del COL4A1, con ispessimento e duplicazione della membrana basale delle arteriole cutanee alla microscopia elettronica. ${ }^{62}$

\section{SVD associate a delezione del FOXC1}

Il gene forkhead box transcription factor 1 (FOXC1) sul cromosoma 6p25 codifica un componente della famiglia di fattori di trascrizione winged/helix forkhead. FOXC1 è coinvolto in processi di sviluppo vascolare, come nella regolazione dell'angiogenesi e della differenziazione arteriosa. ${ }^{63}$ All'inizio si pensava che mutazioni del gene $\mathrm{FOXCl}$ fossero unicamente causative della sindrome di Axenfeld Rieger (ARS) e di malformazioni cerebellari in associazione al numero di copie del segmento interessato del cromosoma 6p25. ${ }^{64}$ Pazienti con ARS presentano anomalie oculari come disgenesia del segmento anteriore e glaucoma ad esordio precoce, ma anche manifestazioni non oculari come dismorfismi sistemici ed anomalie dentali ed ombelicali. ${ }^{64}$ Spesso si sovrappone un altro fenotipo, noto come malformazione di Dandy Walker, caratterizzata da ipoplasia del verme cerebellare e mega cisterna magna. ${ }^{65}$ In svariati case reports di pazienti con delezioni $6 \mathrm{p} 25$, individui con
ARS e altre anomalie di sviluppo avevano anche alterazioni di segnale della sostanza bianca alla risonanza magnetica fin dall'età di 18 mesi. ${ }^{66}$ Questi reports forniscono una chiara evidenza del coinvolgimento del gene FOXC1 nelle SVD. Una metaanalisi e uno studio dell'espressione quantitativa tramite GWAS dalla coorte del Heart and Ageing Research in Genomic Epidemiology (CHARGE) consortium ha evidenziato che 3 variazioni di singoli nucleotidi associati con iperintensità della sostanza bianca influenzano fortemente il trascritto del FOXC1. 18/18 pazienti con ARS correlata con FOXC1 nello studio CHARGE hanno inoltre mostrato la presenza di segni di SVD alla risonanza magnetica. ${ }^{67}$

\section{Hereditary cerebral amyloid angiopathy (H-CAA)}

Fra le SVD ad espressione prevalentemente emorragica, oltre alle patologie correlate con mutazioni del COL4A1-A2, un posto sicuramente importante è occupato dalle forme ereditarie di angiopatia amiloide cerebrale (CAA).

Il termine CAA viene usato per descrivere una serie di patologie accomunate dalla deposizione di fibrille di amiloide nella parete delle arterie di medio e piccolo calibro e talora nei capillari del parenchima cerebrale e delle leptomeningi. ${ }^{68} \mathrm{La}$ CAA ha sia forme sporadiche che forme ereditarie e le prime sono assai più frequenti delle seconde, ma almeno in parte le caratteristiche cliniche sono simili, sebbene con un esordio molto precoce nelle forme ereditarie. ${ }^{68}$ Solitamente la CAA ereditaria è autosomica dominante e la maggioranza dei casi è dovuta alla deposizione di beta amiloide derivante dalla proteina precursore dell'amiloide (amyloid precursor protein o APP), codificata da un gene sul cromosoma $21 .{ }^{69}$ Inoltre questo tipo di CAA ereditaria può verificarsi in associazione con demenza di Alzheimer familiare, con mutazioni dei geni APP, presenilina $1 \mathrm{e}$ 2 (PSEN1 e PSEN2) ${ }^{69}$ Esistono comunque anche un ampio numero di CAA ereditarie non correlate con la beta amiloide. ${ }^{70}$ L'amiloide si forma quando i peptidi amiloidogenici solubili vengono convertiti da spirali alfa solubili a fibrille beta insolubili, che a loro volta polimerizzano. Appaiono come formazioni color verde mela nelle preparazioni colorate con rosso Congo sotto luce polarizzata. Queste strutture si aggregano a formare depositi attorno alle cellule muscolari lisce dei vasi, che infiltrano gradualmente l'intima e sostituiscono gradualmente le cellule muscolari lisce. ${ }^{68,69} \mathrm{Le}$ pareti arteriose diventano pertanto fragili, portando a micro e macroemorragie; questi vasi sono inoltre incapaci di autoregolare il flusso ematico cerebrale, determinando delle manifestazioni ischemiche croniche (espresse da alterazioni della sostanza bianca), ed ischemie acute. ${ }^{70}$ Le diverse forme di CAA ereditaria si ma- 
nifestano con fenotipi clinici ampiamente sovrapponibili. Una caratteristica clinica comune è lo sviluppo di segni di SVD, con piccoli infarti corticali e emorragie lobari ricorrenti, come nelle forme di deposizione di beta amiloide e nella HCHWA-Icelandic type CAA. Inoltre i pazienti tendono a sviluppare un progressivo deterioramento cognitivo che progredisce fino alla demenza. ${ }^{70}$ Sul versante delle indagini di neuroimaging $i$ pazienti con CAA mostrano microbleeds lobari, una estesa e confluente microangiopatia, siderosi corticale superficiale (deposizione di emosiderina negli strati subpiali derivanti da sanguinamento di origine subaracnoidea) e infarti corticali. ${ }^{71,72}$

Non ci sono chiare correlazioni fra le forme di amiloidosi sistemica e la CAA; esiste un'unica forma di amiloidosi sistemica che può dare manifestazioni cerebrovascolari ed è l'amiloidosi da mutazioni della transtiretina, che caratteristicamente, sebbene in una minoranza di pazienti, può avere un quadro di neuroimaging caratterizzato da segni di SVD, microbleeds lobari e soprattutto siderosi corticale superficiale, ${ }^{73}$ insieme a più frequente neuropatia periferica, con recenti prospettive terapeutiche.

È importante considerare che le forma ereditarie di CAA, a parte quella da transtiretina, sono assai rare e ne sono descritti pochissimi individui o famiglie per ognuna, prendendo spesso il nome dal luogo di identificazione o di origine dei pazienti. Non è pertanto giustificato lo screening genetico per forme ereditarie nei pazienti con CAA apparentemente sporadica, senza chiara storia familiare e personale suggestiva di una forma familiare di questa patologia. ${ }^{70}$

\section{Verso una via patogenetica comune}

Le forma sporadiche e quelle monogeniche di SVD condividono per alcuni aspetti degli effettori comuni del danno cerebrale a livello molecolare, rendendo complessa l'interazione fra forme genetiche e forme sporadiche e rendendo inoltre assai difficile potere escludere dal sospetto di patologia ereditaria anche pazienti con chiara presenza di fattori di rischio vascolari.

Inoltre i meccanismi molecolari e genetici di danno si stanno costantemente ampliando nelle nostre conoscenze, come ad esempio nella identificazione, anche genetica del matrisoma come bersaglio e cofattore del danno sulla parete vasale da parte di molte SVD monogeniche, come ad esempio il CADASIL. ${ }^{74}$

\section{Bibliografia}

1. https://ec.europa.eu/health/non_communicable_diseases/rare_diseases_it

2. Arsava EM, Ballabio E, Benner T, Cole JW, DelgadoMartinez MP, Dichgans M, Fazekas F, Furie KL, Illoh $\mathrm{K}$, Jood K, Kittner S, Lindgren AG, Majersik JJ,
Macleod MJ, Meurer WJ, Montaner J, Olugbodi AA, Pasdar A, Redfors P, Schmidt R, Sharma P, Singhal AB, Sorensen AG, Sudlow C, Thijs V, Worrall BB, Rosand J, Ay H; International Stroke Genetics Consortium. The Causative Classification of Stroke system: an international reliability and optimization study. Neurology. 2010 Oct 5;75(14):1277-84.

3. Amarenco P, Bogousslavsky J, Caplan LR, Donnan GA, Hennerici MG. New approach to stroke subtyping: the A-S-C-O (phenotypic) classification of stroke. Cerebrovasc Dis. 2009;27(5):502-8.

4. Amarenco P, Bogousslavsky J, Caplan LR, Donnan GA, Wolf ME, Hennerici MG. The ASCOD phenotyping of ischemic stroke (Updated ASCO Phenotyping). Cerebrovasc Dis. 2013;36(1):1-5.

5. Tan RYY, Traylor M, Megy K, Duarte D, Deevi SVV, Shamardina O, Mapeta RP; NIHR BioResource: Rare Diseases Consortium, Ouwehand WH, Gräf S, Downes $\mathrm{K}$, Markus HS. How common are single gene mutations as a cause for lacunar stroke? A targeted gene panel study. Neurology. 2019 Nov 26;93(22):e2007-e2020.

6. Bersano A, Markus HS, Quaglini S, et al. Clinical pregenetic screening for stroke monogenic diseases: results from Lombardia GENS Registry. Stroke 2016;47(7): 1702-1709.

7. Biegstraaten M, Arngr1 'msson R, Barbey F, et al. Recommendations for initiation and cessation of enzyme replacement therapy in patients with Fabry disease: the European Fabry Working Group consensus document. Orphanet J Rare Dis 2015;10:36.

8. Burlina AB, Polo G, Salviati L, Duro G, Zizzo C, Dardis A, Bembi B, Cazzorla C, Rubert L, Zordan R, Desnick RJ, Burlina AP. Newborn screening for lysosomal storage disorders by tandem mass spectrometry in North East Italy. J Inherit Metab Dis. 2018 Mar;41(2):209-219.

9. Fellgiebel A, Mu"ller MJ, Ginsberg L. CNS manifestations of Fabry's disease. Lancet Neurol 2006;5(9):791795.

10. Tim Godel, Philipp Bäumer, Mirko Pham, Anja Köhn, Nicole Muschol, Moritz Kronlage, Jennifer Kollmer, Sabine Heiland, Martin Bendszus, Victor-Felix Mautner. Human dorsal root ganglion in vivo morphometry and perfusion in Fabry painful neuropathy. Neurology Sep 2017, 89 (12) 1274-1282.

11. Favalli V, Disabella E, Molinaro M, Tagliani M, Scarabotto A, Serio A, Grasso M, Narula N, Giorgianni C, Caspani C, Concardi M, Agozzino M, Giordano C, Smirnova A, Kodama T, Giuliani L, Antoniazzi E, Borroni RG, Vassallo C, Mangione F, Scelsi L, Ghio S, Pellegrini C, Zedde M, Fancellu L, Sechi G, Ganau A, Piga S, Colucci A, Concolino D, Di Mascio MT, Toni D, Diomedi M, Rapezzi C, Biagini E, Marini M, Rasura M, Melis M, Nucera A, Guidetti D, Mancuso M, Scoditti U, Cassini P, Narula J, Tavazzi L, Arbustini E. Genetic Screening of Anderson-Fabry Disease in Probands Referred From Multispecialty Clinics. J Am Coll Cardiol. 2016 Sep 6;68(10):1037-50.

12. Doheny D, Srinivasan R, Pagant S, Chen B, Yasuda M, Desnick RJ. Fabry Disease: prevalence of affected males and heterozygotes with pathogenic GLA mutations identified by screening renal, cardiac and stroke clinics, 1995-2017. J Med Genet. 2018 Apr;55(4):261-268.

13. Sims K, Politei J, Banikazemi M, Lee P. Stroke in Fabry 
disease frequently occurs before diagnosis and in the absence of other clinical events: natural history data from the Fabry Registry. Stroke. 2009 Mar;40(3):788-94.

14. Lelieveld IM, Böttcher A, Hennermann JB, Beck M, Fellgiebel A. Eight-Year Follow-Up of Neuropsychiatric Symptoms and Brain Structural Changes in Fabry Disease. PLoS One. 2015 Sep 4;10(9):e0137603.

15. Fazekas F, Enzinger C, Schmidt R, et al. Brain magnetic resonance imaging findings fail to suspect Fabry disease in young patients with an acute cerebrovascular event. Stroke 2015;46(6):1548-1553.

16. Mehta A, Beck M, Elliott P, et al. Enzyme replacement therapy with agalsidase alfa in patients with Fabry's disease: an analysis of registry data. Lancet 2009;374 (9706):1986-1996.

17. Germain DP, Hughes DA, Nicholls K, Bichet DG, Giugliani R, Wilcox WR, Feliciani C, Shankar SP, Ezgu F, Amartino H, Bratkovic D, Feldt-Rasmussen U, Nedd K, Sharaf El Din U, Lourenco CM, Banikazemi M, Charrow J, Dasouki M, Finegold D, Giraldo P, GokerAlpan O, Longo N, Scott CR, Torra R, Tuffaha A, Jovanovic A, Waldek S, Packman S, Ludington E, Viereck C, Kirk J, Yu J, Benjamin ER, Johnson F, Lockhart DJ, Skuban N, Castelli J, Barth J, Barlow C, Schiffmann R. Treatment of Fabry's Disease with the Pharmacologic Chaperone Migalastat. N Engl J Med. 2016 Aug 11;375 (6):545-55.

18. Moreton FC, Razvi SS, Davidson R, Muir KW. Changing clinical patterns and increasing prevalence in CADASIL. Acta Neurol Scand 2014;130(3):197Y203.

19. Ragno M, Trojano L, Pianese L, et al. Renal involvement in cerebral autosomal dominant arteriopathy with subcortical infarcts and leukoencephalopathy (CADASIL): report of a case with a six-year follow-up. Histol Histopathol 2012;27(10):1307Y1314.

20. Chabriat H, Joutel A, Dichgans M, et al. Cadasil. Lancet Neurol 2009;8(7):643Y653.

21. Dong Y, Hassan A, Zhang Z, et al. Yield of screening for CADASIL mutations in lacunar stroke and leukoaraiosis. Stroke 2003;34(1):203Y205.

22. Tan RY, Markus HS. CADASIL: migraine, encephalopathy, stroke and their inter-relationships. PLoS One 2016;11(6): e0157613.

23. Drazyk AM, Tan RYY, Tay J, Traylor M, Das T, Markus HS. Encephalopathy in a Large Cohort of British Cerebral Autosomal Dominant Arteriopathy With Subcortical Infarcts and Leukoencephalopathy Patients. Stroke. 2019 Feb;50(2):283-290.

24. Moreton FC, Santosh C, McArthur K, Muir KW. Cerebral hyperperfusion on arterial spin labeling MRI during CADASIL migrainous encephalopathy. Neurology 2015;85(24):2177Y2179.

25. Eikermann-Haerter K, Yuzawa I, Dilekoz E, et al. Cerebral autosomal dominant arteriopathy with subcortical infarcts and leukoencephalopathy syndrome mutations increase susceptibility to spreading depression. Ann Neurol 2011;69(2): 413-418.

26. Chabriat H, Herve D, Duering M, et al. Predictors of clinical worsening in cerebral autosomal dominant arteriopathy with subcorticalinfarctsandleukoencephalopathy: prospective cohort study. Stroke 2016;47(1): 4-11.

27. Dichgans M, Mayer M, Uttner I, et al. The phenotypic spectrum of CADASIL: clinical findings in 102 cases. Ann Neurol 1998; 44(5):731-739.

28. O'Sullivan M, Jarosz JM, Martin RJ, et al. MRI hyperintensities of the temporal lobe and external capsule in patients with CADASIL. Neurology 2001;56(5):628634.

29. Dichgans M, Holtmannspo "tter M, Herzog J, et al. Cerebral microbleeds in CADASIL: a gradient-echo magnetic resonance imaging and autopsy study. Stroke 2002;33(1):67-71.

30. Kleinig TJ. Associations and implications of cerebral microbleeds. J Clin Neurosci 2013; 20(7):919-927. doi:10.1016/j.jocn.2012.12.002.

31. Wollenweber FA, Baykara E, Zedde M, Gesierich B, Achmüller M, Jouvent E, Viswanathan A, Ropele S, Chabriat H, Schmidt R, Opherk C, Dichgans M, Linn J, Duering M. Cortical Superficial Siderosis in Different Types of Cerebral Small Vessel Disease. Stroke. 2017 May;48(5):1404-1407.

32. Khan MT, Murray A, Smith M. Successful use of intravenous tissue plasminogen activator as treatment for a patient with cerebral autosomal dominant arteriopathy with subcortical infarcts and leukoencephalopathy: a case report and review ofliterature. J Stroke Cerebrovasc Dis 2016;25(4):e53-e57.

33. https://databases.lovd.nl/shared/genes/NOTCH3 (aggiornato al 27 settembre 2019, accesso il 1 dicembre 2019).

34. Rutten JW, Van Eijsden BJ, Duering M, Jouvent E, Opherk C, Pantoni L, Federico A, Dichgans M, Markus HS, Chabriat H, Lesnik Oberstein SAJ. The effect of NOTCH3 pathogenic variant position on CADASIL disease severity: NOTCH3 EGFr 1-6 pathogenic variant are associated with a more severe phenotype and lower survival compared with EGFr 7-34 pathogenic variant. Genet Med. 2019 Mar;21(3):676-682.

35. Abou Al-Shaar H, Qadi N, Al-Hamed MH, et al. Phenotypic comparison of individuals with homozygous or heterozygous mutation of NOTCH3 in a large CADASIL family. J Neurol Sci 2016;367:239-243.

36. Pescini F, Nannucci S, Bertaccini B, et al. The Cerebral Autosomal-Dominant Arteriopathy With Subcortical Infarcts and Leukoencephalopathy (CADASIL) Scale: a screening tool to select patients for NOTCH3 gene analysis. Stroke 2012;43(11):2871-2876.

37. Liu X, Zuo Y, Sun W, et al. The genetic spectrum and the evaluation of CADASIL screening scale in Chinese patients with NOTCH3 mutations. J Neurol Sci 2015; 354(1-2):63-69.

38. Reyes S, Kurtz A, Herve ' D, et al. Presymptomatic genetic testing in CADASIL. J Neurol 2012;259(10): $2131 Y 2136$.

39. Chabriat H, Hervé D, Duering M, Godin O, Jouvent E, Opherk C, Alili N, Reyes S, Jabouley A, Zieren N, Guichard JP, Pachai C, Vicaut E, Dichgans M. Predictors of Clinical Worsening in Cerebral Autosomal Dominant Arteriopathy With Subcortical Infarcts and Leukoencephalopathy: Prospective Cohort Study. Stroke. 2016 Jan;47(1):4-11.

40. Adib-Samii P, Brice G, Martin RJ, Markus HS. Clinical spectrum of CADASIL and the effect of cardiovascular risk factors on phenotype: study in 200 consecutively recruited individuals. Stroke. 2010 Apr;41(4):630-4. 
41. Dichgans M, Wick M, Gasser T. Cerebrospinal fluid findings in CADASIL. Neurology 1999;53(1):233.

42. Brown RB, Traylor M, Burgess S, Sawcer S, Markus HS. Do Cerebral Small Vessel Disease and Multiple Sclerosis Share Common Mechanisms of White Matter Injury? Stroke. 2019 Aug;50(8):1968-1972.

43. Ciolli L, Pescini F, Salvadori E, et al. Influence of vascular risk factors and neuropsychological profile on functional performances in CADASIL: results from the MIcrovascular LEukoencephalopathy Study (MILES). Eur J Neurol 2014;21(1):65-71.

44. OhJH,LeeJS,KangSY,et al. Aspirin-associated intracerebral hemorrhage in a patient with CADASIL. Clin Neurol Neurosurg 2008; 110(4):384-386.

45. Peters N, Freilinger T, Opherk C, et al. Effects of short term atorvastatin treatment on cerebral hemodynamics in CADASIL. J Neurol Sci 2007;260(1-2):100-105.

46. Donnini I, Nannucci S, Valenti R, et al. Acetazolamide for the prophylaxis of migraine in CADASIL: a preliminary experience. J Headache Pain 2012;13(4):299-302.

47. Forteza AM, Brozman B, Rabinstein AA, et al. Acetazolamide for the treatment of migraine with aura in CADASIL. Neurology 2001;57(11): 2144-2145.

48. Dichgans M, Markus HS, Salloway S, et al. Donepezil in patients with subcortical vascular cognitive impairment: a randomised double-blind trial in CADASIL. Lancet Neurol 2008;7(4):310-318.

49. Nozaki H, Sekine Y, Fukutake T, et al. Characteristic features and progression of abnormalities onMRI forCARASIL. Neurology 2015;85(5):459Y463.

50. HaraK,ShigaA,FukutakeT,et al. Association of HTRA1 mutations and familial ischemic cerebral small-vessel disease. N Engl J Med 2009;360(17):1729-1739.

51. Shiga A, Nozaki H, Yokoseki A, et al. Cerebral smallvessel disease protein HTRA1 controls the amount of TGF"1 via cleavage of proTGF-"1. Hum Mol Genet 2011;20(9): 1800-1810.

52. Roeben B, Uhrig S, Bender B, Synofzik M. Teaching NeuroImages: when alopecia and disk herniations meet vascular leukoencephalopathy: CARASIL. Neurology 2016;86(15):e166-e167.

53. Verdura E, Hervé D, Scharrer E, Amador Mdel M, Guyant-Maréchal L, Philippi A, Corlobé A, Bergametti F, Gazal S, Prieto-Morin C, Beaufort N, Le Bail B, Viakhireva I, Dichgans M, Chabriat H, Haffner C, Tournier-Lasserve E. Heterozygous HTRA1 mutations are associated with autosomal dominant cerebral small vessel disease. Brain. 2015 Aug;138(Pt 8):2347-58.

54. Chung J, Marini S, Pera J, Norrving B, Jimenez-Conde J, Roquer J, Fernandez-Cadenas I, Tirschwell DL, Selim M, Brown DL, Silliman SL, Worrall BB, Meschia JF, Demel S, Greenberg SM, Slowik A, Lindgren A, Schmidt R, Traylor M, Sargurupremraj M, Tiedt S, Malik R, Debette S, Dichgans M, Langefeld CD, Woo D, Rosand J, Anderson CD. Genome-wide association study of cerebral small vessel disease reveals established and novel loci. Brain. 2019 Oct 1;142(10):3176-3189.

55. Nozaki H, Kato T, Nihonmatsu M, et al. Distinct molecular mechanisms of HTRA1 mutants in manifesting heterozygotes with CARASIL. Neurology 2016;86(21): 1964-1974.

56. Dhamija R, Schiff D, Lopes MB, et al. Evolution of brain lesions in a patient with TREX1 cerebroretinal vasculopathy. Neurology 2015;85(18):1633-1634.

57. Richards A, van den Maagdenberg AM, Jen JC, et al. Cterminal truncations in human 3'-5' DNA exonuclease TREX1 cause autosomal dominant retinal vasculopathy with cerebral leukodystrophy. Nat Genet 2007;39(9): 1068-1070.

58. Kernt M, Gschwendtner A, Neubauer AS, et al. Effects of intravitreal bevacizumab treatment on proliferative retinopathy in a patient with cerebroretinal vasculopathy. J Neurol 2010;257(7):1213-1214.

59. Lanfranconi S, Markus HS. COL4A1 mutations as a monogenic cause of cerebral small vessel disease: a systematic review. Stroke 2010;41(8):e513-e518.

60. Vahedi K, Kubis N, Boukobza M, et al. COL4A1 mutation in a patient with sporadic, recurrent intracerebral hemorrhage. Stroke 2007;38(5):1461Y1464.

61. Verbeek E, Meuwissen MEC, Verheijen FW et al (2012) COL4A2 mutation associated with familial porencephaly and small-vessel disease. Eur J Hum Genet 20:844-851.

62. Renard D, Mine' M, Pipiras E et al (2014) Cerebral small-vessel disease associated with COL4A1 and COL4A2 gene duplications. Neurology 83:1029-1031.

63. Siegenthaler JA, Choe Y, Patterson KP et al (2013) Foxc1 is required by pericytes during fetal brain angiogenesis. Biol Open 2:647-659.

64. Tu "mer Z, Bach-Holm D (2009) Axenfeld-Rieger syndrome and spectrum of PITX2 and FOXC1 mutations. Eur J Hum Genet 17:1527-1539.

65. Delahaye A, Khung-Savatovsky S, Aboura A et al (2012) Preand postnatal phenotype of 6p25 deletions involving the FOXC1 gene. Am J Med Genet A 158A:2430-2438.

66. Cellini E, Disciglio V, Novara F et al (2012) Periventricular heterotopia with white matter abnormalities associated with 6p25 deletion. Am J Med Genet A 158A:1793-1797.

67. French CR, Seshadri S, Destefano AL et al (2014) Mutation of FOXC1 and PITX2 induces cerebral small-vessel disease. J Clin Invest 124:4877-4881.

68. Revesz T, Holton JL, Lashley T et al (2009) Genetics and molecular pathogenesis of sporadic and hereditary cerebral amyloid angiopathies. Acta Neuropathol 118:115-130.

69. Di Fede G, Giaccone G, Tagliavini F (2013) Hereditary and sporadic beta-amyloidoses. Front Biosci (Landmark Ed) 18:1202-1226.

70. Biffi A, Greenberg SM (2011) Cerebral amyloid angiopathy: a systematic review. J Clin Neurol 7:1-9.

71. Linn J, Halpin A, Demaerel P et al (2010) Prevalence of superficial siderosis in patients with cerebral amyloid angiopathy. Neurology 74:1346-1350.

72. Greenberg SM, Vernooij MW, Cordonnier C et al (2009) Cerebral microbleeds: a guide to detection and interpretation. Lancet Neurol 8:165-174.

73. Garzuly F, Vidal R, Wisniewski T, Brittig F, Budka H. Familial meningocerebrovascular amyloidosis, Hungarian type, with mutant transthyretin (TTR Asp18Gly). Neurology. 1996;47(6):1562-1567.

74. Tan R, Traylor M, Rutten-Jacobs L, Markus H. New insights into mechanisms of small vessel disease stroke from genetics. Clinical Science (2017) 131 515-531. 


\title{
L'attacco ischemico transitorio
}

\author{
Maria Guarino, ${ }^{1}$ Sara Belluoccio ${ }^{2}$ \\ ${ }^{1}$ Struttura Semplice Neurologia, Azienda Ospedaliero-Universitaria S. Orsola-Malpighi, UOC Neuro-Met, IRCCS Istituto delle \\ Scienze Neurologiche, Bologna; ${ }^{2}$ Unità Operativa di Pronto Soccorso, Medicina d'Emergenza Urgenza, AUSL Romagna, Ospe- \\ dale Ceccarini, Riccione (RN), Italia
}

\section{Definizione}

Una delle prime descrizioni di un attacco ischemico transitorio (in inglese ed universalmente conosciuto come transient ischemic attack, TIA) è del 1841. Fu il romanziere francese Stendhal, che descrisse in modo accurato, in una lettera indirizzata a Domenico Fiore, i disturbi del linguaggio di breve durata che aveva ripetutamente presentato qualche mese prima di morire per un ictus fatale: all of a sudden I forget all French words...I watch myself with curiosity: apart from the ability to use words, I keep all the natural properties of the animal. Ideas are fine, but without words. It lasts eight to ten minutes. Then, slowly, the memory of words comes back, and I ambire.

Pertanto 100 anni prima che la definizione di TIA fosse inserita nella letteratura medica, si conosceva già che questi episodi transitori potevano predisporre ad eventi vascolari cerebrali maggiori. Solo nel 1958, C. Miller Fisher introdusse il termine ischemia cerebrale intermittente, definita come deficit neurologico focale improvviso di breve durata, tipicamente 10'-15' presumibilmente di origine vascolare e confinato in un'area del cervello o dell'occhio perfusi da una specifica arteria cerebrale. ${ }^{1}$ L'estensione a meno di 24 ore è stata suggerita dalla Conferenza di Princeton nel 1965 e di-

Corrispondenti: Maria Guarino, Neurologia AOU, Policlinico S.Orsola-Malpighi, Via Albertoni 15, 40138 Bologna, Italia E-mail: maria.guarino@aosp.bo.it

Sara Belluoccio, Unità Operativa di Pronto Soccorso, Medicina d'Emergenza Urgenza, AUSL Romagna, Ospedale Ceccarini, via Frosinone 1, 47838 Riccione (RN), Italia.

E-mail: sara.belluoccio@pec.libero.it

Articolo pubblicato secondo la Creative Commons Attribution NonCommercial 4.0 License (CC BY-NC 4.0).

${ }^{\circ}$ Copyright: the Author(s), 2020

Licensee PAGEPress, Italy

QUADERNI - Italian Journal of Medicine 2020; 8(2):34-46 venne la definizione ufficiale nella classificazione del National Institutes of Health nel 1975.

La tradizionale definizione di TIA è stata messa in discussione solo negli ultimi anni alla luce delle nuove evidenze fornite dalle moderne tecniche di neuroimmagini (Risonanza magnetica in diffusione-RM/DWI) che hanno dimostrato che una parte di TIA presentava un danno cerebrale permanente sovrapponibile a quello dell'ictus.

Nel 2002, un gruppo di studiosi, Albers et al., ${ }^{2}$ propose una nuova definizione di TIA basata sia sul danno tissutale che sul dato temporale. Il TIA venne ridefinito come improvvisa comparsa di segni e/o sintomi riferibili a deficit focale cerebrale o visivo attribuibile ad insufficiente apporto di sangue, di durata tipicamente inferiore a un'ora senza evidenza di ischemia acuta alle neuroimmagini.

Il gruppo di lavoro dell'American Academy of Neurology $y^{3}$ nel 2009, propose una nuova ulteriore definizione del TIA solamente tissue-based, volta ad armonizzare l'organizzazione nosologica della patologia cerebrovascolare con quella di altre condizioni vascolari ischemiche, come quelle cardiache che distingueva ad esempio l'angina (non lesionale) rispetto all'infarto del miocardio (con lesione ischemica).

Il TIA venne definito quindi episodio di disfunzione neurologica causata da ischemia focale dell'encefalo, midollo spinale o retina, in assenza di infarto acuto. La nuova definizione di TIA si riferiva solo ad episodi di ischemia cerebrale focale e transitoria non associata ad infarto cerebrale visibile con le tecniche neuroradiologiche.

Tuttavia la difficoltà di molti centri nel disporre durante la fase acuta dell'evento cerebrovascolare della RM/DWI (tecnica più sensibile della TC per il riscontro di una precoce ischemia cerebrale) ha rallentato la diffusione di questa nuova definizione e la tradizionale definizione temporale di TIA è ancora ampiamente utilizzata nella pratica clinica e nella maggior parte degli studi clinico-epidemiologici. Alcuni autori pur avvalendosi della definizione time based, utilizzano i termini TIA senza e con lesione per sottolineare l'associazione del TIA con una lesione ischemica acuta visibile alle neuroimmagini. 


\section{Messaggio chiave}

La definizione di TIA è tutt'oggi basata sul criterio time based (durata $<24 \mathrm{~h}$ ) e non su quello tissue based per la difficoltà di implementare diffusamente in fase acuta le tecniche diagnostiche di II livello come la $\mathrm{RM} / \mathrm{DWI}$ che potrebbero rilevare una lesione ischemica cerebrale acuta congrua con la sintomatologia del TIA, classificando l'evento come un ictus.

\section{Epidemiologia}

La difficoltà nella definizione del TIA e nel riconoscimento dei sintomi da parte della popolazione e del personale sanitario insieme alla apparente benignità legata alla transitorietà del quadro clinico, fanno sì che l'episodio durante la sua manifestazione clinica sia raramente valutato dal medico e che di conseguenza la reale incidenza e prevalenza possano essere sottostimate. ${ }^{3}$ La maggior parte degli studi epidemiologici si riferisce al criterio time based. L'incidenza del TIA, in modo analogo all'incidenza dell'ictus, aumenta in modo significativo con l'età, indipendentemente dal genere e dall'etnia. ${ }^{4}$ Tuttavia sembrano esserci differenze legate all'etnia: in particolare è stata osservata un'incidenza maggiore negli afroamericani (a fronte di un'incidenza di 6,4/1000 nei pazienti di età superiore a 85 anni, è riportata un'incidenza di 16/1000 nei soli soggetti neri). ${ }^{5}$ Inoltre, i TIA sono risultati più comuni negli americani messicani rispetto ai bianchi non ispanici di giovane età (da 45 a 59 anni) ma non in età più avanzata. ${ }^{6}$ Studi statunitensi riportano un'incidenza, pari a 83/100.000 abitanti. ${ }^{4}$

Negli uomini di età compresa tra 65 e 69 anni la prevalenza è del $2,7 \%$, mentre è di $1,6 \%$ nelle donne; tra $\mathrm{i}$ 75 e 79 anni sale al 3,6 \% negli uomini e al 4,1\% nelle donne. Nei soggetti di età tra i 45 e i 65 anni il tasso di prevalenza complessivo è stimato intorno allo $0,4 \%$.

L'incidenza in Italia è stata oggetto di interesse in pochi studi. Lauria et al., ${ }^{7}$ hanno valutato l'incidenza del TIA sulla popolazione della provincia di Belluno: l'incidenza rilevata di nuovi TIA è stata dello 0,80/1000/ab/anno (0,58 aggiustata per la popolazione europea). Cancelli et al. ${ }^{8}$ hanno riportato nel distretto sanitario di Udine un'incidenza di 0,52/1000/ab/anno $(0,45$ e 0,25 rispettivamente standardizzato sulla popolazione italiana e su quella europea). Un recente studio del $2017,{ }^{9}$ relativo alla popolazione dell'Aquila, riporta una incidenza del TIA di 35,2 eventi per 100.000 abitanti.

\section{Messaggio chiave}

Il tasso di incidenza del TIA varia da 52/100.000 abitanti in Italia a 83/100.000 abitanti negli Stati Uniti. La prevalenza del TIA aumenta con l'età: tra i 75 e 79 anni è pari al 3,6\% nel sesso maschile e $4,1 \%$ nel sesso femminile.

\section{Eziopatogenesi}

I principali meccanismi eziopatogenetici del TIA includono: i) embolia: di origine cardiaca (per presenza di vegetazioni valvolari, trombi murali, fibrillazione atriale-FA, altro), oppure di origine arteriosa, dall'arco aortico o da fonte sconosciuta; ii) occlusione dei piccoli vasi arteriosi penetranti o TIA lacunari; iii) patologia steno-occlusiva (in genere trombotica) delle grosse arterie con conseguente riduzione del flusso arterioso.

I sottotipi eziologici sono classificati in accordo al meccanismo fisiopatologico sottostante che è simile a quello dell'ictus ischemico e che fa riferimento alla classificazione $\operatorname{TOAST}^{10}$ (Tabella 1).

Circa il 30\% dei TIA rimane ad eziologia indeterminata nonostante uno studio esaustivo sulle possibili cause.

La patologia dei grossi vasi su base aterotrombotica rappresenta il meccanismo eziopatogenetico prevalente insieme a quello cardioembolico, ed è dovuta alla degenerazione aterosclerotica di arterie di grosso calibro a livello extra o intracranico. Nella maggior parte dei casi l'evento patologico interessa il circolo

Tabella 1. Classificazione TOAST.

\begin{tabular}{lll}
\hline Categorie eziologiche & Clinica & Neuroradiologia \\
\hline Aterosclerosi dei vasi di grosso calibro & Deficit corticali o cerebellari & Infarto corticale, cerebellare o subcorticale $>1,5 \mathrm{~cm}$ \\
\hline Cardioembolia & Deficit corticali o cerebellari & Infarto corticale, cerebellare o subcorticale $>1,5 \mathrm{~cm}$ \\
\hline Occlusione piccoli vasi & Sindrome lacunare & Infarto sub corticale/tronco cerebrale $<1,5 \mathrm{~cm}$ \\
\hline Ictus da cause diverse & $\begin{array}{l}\text { Deficit corticali o cerebellari } \\
\text { Sindrome lacunare }\end{array}$ & $\begin{array}{l}\text { Infarto corticale, cerebellare o subcorticale }>1,5 \mathrm{~cm} \text { o } \\
\text { Infarto sub corticale/tronco cerebrale }<1,5 \mathrm{~cm}\end{array}$ \\
\hline $\begin{array}{l}\text { Ictus da cause non determinate: } \\
\begin{array}{l}\text { a. } \geq 2 \text { cause } \\
\text { b. Indagini negative }\end{array}\end{array}$ & $\begin{array}{l}\text { Deficit corticali o cerebellari } \\
\text { c. Indagini incomplete }\end{array}$ & $\begin{array}{l}\text { Infarto corticale, cerebellare o subcorticale }>1,5 \mathrm{~cm} \text { o } \\
\text { Infarto sub corticale/tronco cerebrale }<1,5 \mathrm{~cm}\end{array}$ \\
\hline
\end{tabular}


carotideo. Sono a maggior rischio di eventi ischemici quei segmenti vascolari che presentano placche aterosclerotiche determinanti una stenosi significativa, maggiore del 50\%. L'occlusione di una grossa arteria, può complicarsi con trombosi murale, ulcerazione $o$ rottura della placca con embolizzazione ed emorragia intraplacca. La presenza di un circolo cerebrale collaterale insufficiente può giocare un ruolo cruciale nell'avvio dei sintomi. Le placche ateromasiche si formano più frequentemente a livello dei punti di maggior turbolenza del vaso, sottoposti a maggior stress, come le biforcazioni. L'aterosclerosi costituisce il meccanismo principale degli eventi ischemici nei soggetti di età più avanzata e nei soggetti con importanti fattori di rischio cardiovascolari (ipertensione, dislipidemia, diabete, abitudine al fumo).

Il meccanismo embolico è caratterizzato dalla migrazione di un embolo di piastrine e fibrina verso un'arteria più distale. Il focolaio emboligeno può avere sede cardiaca e associarsi alla FA, infarto miocardico acuto recente, valvulopatie/protesi valvolari, forame ovale pervio (PFO), endocarditi e miocardiopatie. In alternativa l'embolo può staccarsi da una placca ateromasica ulcerata generalmente di una arteria extracranica. L'embolo può avere origine anche a livello dell'arco aortico.

L'occlusione dei vasi penetranti profondi di piccolo calibro ( $<0.5 \mathrm{~mm}$ di spessore), che causa l'insorgenza di infarti lacunari, sembra essere correlata alla presenza di microateromi o alla lipoialinosi (un ispessimento ialino delle arteriole conseguente a uno stress emodinamico cronico, detto arteriolosclerosi ialina) della parete vascolare e si associa a fattori di rischio come l'ipertensione, il diabete mellito, il fumo.

Tra le cause più rare di eventi ischemici cerebrali specie in soggetti con età $<45$ anni vanno ricordate: l'abuso di sostanze stupefacenti, le trombofilie ereditarie, la sindrome da anticorpi anti-fosfolipidi, l'anemia falciforme, le vasculiti cerebrali primitive o secondarie, le dissezioni delle arterie cervicali su base fibrodisplasica, genetica, traumatica, malattie genetiche come la Malattia di Fabry, la CADASIL/CARASIL, malattie mitocondriali e altre malattie da accumulo. La dissecazione dei vasi extra-intracranici rappresenta la causa più frequente di ictus/TIA giovanile coprendo fino al $25 \%$ dei casi.

\section{Messaggio chiave}

L'eziologia del TIA ricalca quella dell'ictus ischemico, pertanto la classificazione TOAST può essere applicata anche al TIA. I sottotipi eziologi più frequenti includono l'aterosclerosi dei grossi vasi e il meccanismo cardioembolico. Nei soggetti con età $<45$ anni, la causa più frequente è la dissezione delle arterie cervicali, ma cause rare vanno esaustivamente ricer- cate, incluse condizioni genetiche. Nonostante l'estensiva ricerca eziologica attualmente raccomandata, circa il 30\% dei TIA rimane criptogenetico.

\section{Fattori di rischio}

I fattori di rischio per l'insorgenza dei TIA sono simili a quelli identificati per l'ictus e possono essere suddivisi in: fattori modificabili e fattori non modificabili. Il profilo dei fattori di rischio differisce a seconda dei diversi sottotipi di TIA. La FA e la cardiopatia ischemica sono prevalenti nei pazienti con TIA cardioembolico; l'ipertensione e il diabete nei pazienti con ictus lacunare; l'arteriopatia periferica, l'ipertensione, il diabete e un precedente TIA, nei pazienti con ictus aterotrombotico. Tuttavia molto spesso si riscontra un overlapping del profilo dei fattori di rischio specialmente tra l'eziologia lacunare ed aterotrombotica. Nei pazienti giovani, prevale l'assenza dei fattori di rischio tradizionali e questo induce a ricercare possibili cause rare dell'evento.

\section{Messaggio chiave}

I fattori di rischio includono fattori modificabili e fattori non modificabili e sono gli stessi dell'ictus cerebrale. Il profilo di rischio differisce in base al sottotipo eziologico di TIA. Nel paziente con età $<$ ai 45 anni spesso i fattori di rischio tradizionali sono assenti e devono essere ricercate cause rare di un evento cerebrovascolare giovanile, prime fra tutte la dissezione delle arterie cervicali.

\section{Manifestazioni cliniche}

Il TIA si caratterizza dal punto di vista clinico dall'insorgenza improvvisa, acuta, di segni e sintomi neurologici transitori. Talvolta, dal momento che il ristabilirsi della perfusione è influenzato sia da fattori sistemici (gittata cardiaca, pressione arteriosa, viscosità ematica) sia da fattori locali (sviluppo di circoli collaterali), il quadro clinico può essere fluttuante. La durata è molto variabile da 2 a 30 minuti in media, più raramente anche alcune ore, quindi $\mathrm{i}$ sintomi scompaiono senza lasciare sequele neurologiche. La coscienza, durante tutto l'episodio, rimane integra. Nella maggioranza dei casi il paziente con TIA giunge all'osservazione del medico pauci sintomatico o asintomatico: Si stima che meno del 7\% dei pazienti con TIA venga valutato e studiato in ambito specialistico all'esordio dei sintomi. Alcuni studi hanno suggerito che una durata più prolungata (in genere $>$ di $1 \mathrm{~h}$ ) si correla con una maggiore probabilità di riscontro di una lesione ischemica acuta alla RMDWI. Tuttavia una recente metanalisi mostra che la durata dei sintomi non sia un'affidabile condizione 
per predire se il TIA sarà associato o meno ad un infarto cerebrale. ${ }^{11}$

La durata della sintomatologia sembra essere correlata con l'eziologia del TIA: l'occlusione aterotrombotica dei vasi di grosso e piccolo calibro produce tipicamente episodi di breve durata (da minuti a poche ore), spesso ricorrenti e stereotipati, mentre il meccanismo embolico è associato a sintomi neurologici focali che permangono per un periodo più prolungato, di ore, e caratterizzano episodi singoli, non recidivanti. $^{12}$

Il TIA tipicamente si presenta con sintomi cosiddetti negativi, espressione di una perdita di funzione, come ad esempio emiparesi, afasia e amaurosis fugax. Sintomi positivi (come visione di linee o punti luminosi oppure episodi convulsivi) hanno una minor probabilità di essere associati ad un TIA. Un'eccezione importante è la vertigine che può essere un sintomo di ischemia del circolo posteriore, ma che raramente si presenta in forma isolata.

Si possono identificare sindromi caratteristiche del territorio di distribuzione del ramo vascolare interessato, che permettono di correlare il dato clinico a quello fisiopatologico. Sono sovrapponibili a quelle che si manifestano in corso di ictus cerebrale a cui si rimanda. Si segnalano solo alcune manifestazioni rare ma che per la transitorietà e la durata sono da prendere in considerazione nel contesto di un TIA e non di un ictus quali i cosiddetti limb-shaking-transient-ischemic-attacks, caratterizzati da movimenti involontari degli arti di breve durata, definiti come trembling, shaking, twisting, drawing up, or moving irregularly, ${ }^{13}$ che coinvolgono tipicamente il braccio e la mano e che sono spesso correlati ad una stenosi critica della carotide interna. Inoltre ricordiamo le sindromi lacunari dovute all' ischemia delle arterie penetranti che irrorano i nuclei della base, il talamo, la capsula interna, il tronco dell'encefalo e che possono configurarsi nella cosidetta capsular warning syndrome di cui si parlerà più avanti. I principali quadri semeiologici lacunari sono quelli descritti da Fisher: ${ }^{14}$ la sindrome sensitivomotoria, la sindrome motoria pura, la sindrome sensitiva pura, l'emiparesi atassica, disartria pura o associata ad adiadococinesia della mano, emicoreaemiballismo, distonie focali.

\section{Messaggio chiave}

Le manifestazioni cliniche sono tipicamente negative (perdita di una funzione), di breve durata e dipendono dal territorio vascolare coinvolto. Sono sovrapponibili a quelle dell'ictus ischemico cerebrale. Peculiari del TIA sono però i sintomi che annunciano la cosidetta capsular warning syndrome (TIA lacunari stereotipati e recidivanti) e rare manifestazioni quali limb-shaking-transient-ischemic-attacks che correlano con una stenosi critica carotidea.

\section{Diagnosi di TIA}

La diagnosi di TIA è essenzialmente una diagnosi clinica ma a differenza di quella di ictus non è semplice, perché si fa quasi sempre quando il disturbo neurologico si è risolto. Richiede expertise e competenza specialistica neurologica. Infatti solo il $40-50 \%$ delle diagnosi di TIA effettuate da medici non neurologi, viene poi confermata. Gli elementi clinici peculiari suggestivi di una diagnosi di TIA sono: l'esordio improvviso, la focalità dei sintomi, la possibilità di attribuirli ad una regione del Sistema Nervoso Centrale e ad un territorio vascolare, la transitorietà e la durata $<24 \mathrm{~h}$ (secondo la definizione classica). È pertanto importante interrogare il paziente (ed eventualmente i familiari o i testimoni dell'episodio) sulla natura focale o non focale degli attacchi, sulla semeiologia dei sintomi, la durata, la presenza di fattori scatenanti e di sintomi associati tenendo anche in considerazione l'età e il sesso del paziente.

La classificazione clinica dell'ictus proposta da Bamford et al ${ }^{15}$ che correla i disturbi neurologici con il territorio vascolare coinvolto, viene utilizzata anche per il TIA (Tabella 2).

Accanto alla valutazione neurologica e all'anamnesi recente, riveste estrema importanza la ricerca accurata di precedenti eventi cardio e cerebrovascolari, il rilievo di comorbidità e delle terapie in corso e la ricerca dei fattori di rischio cerebrovascolari.

La fase diagnostica include esami ematochimici, rilievo della pressione arteriosa, elettrocardiogramma (ECG), TC cerebrale, studio dei vasi extracranici ed intracranici ed edecocardiogramma. Gli esami di laboratorio eseguiti routinariamente comprendono: emocromo completo, coagulazione (tempo di protrombina, tempo di tromboplastina parziale attivata, INR), funzione renale, elettroliti, funzione epatica, glicemia, assetto lipidico. Non ci sono test di laboratorio diagnostici per TIA e tali esami sono utili per escludere i TIA mimics (es. da ipoglicemia) e possono indirizzare verso cause più rare di eventi trombotici (es. policitemia vera), nonchè rilevare alcuni fattori di rischio non noti. In pazienti di giovane età si deve estendere la ricerca eziologica a cause più rare quali alterazioni trombofiliche, malattie genetiche, malattie da accumulo, dissezione delle arterie cervicali. La diagnostica neuroradiologica con TC cerebrale o RM cerebrale deve essere eseguita il più precocemente possibile entro le 24 ore dall'esordio. La TC rappresenta l'indagine di prima istanza grazie alla sua diffusione in tutti i PS, alla rapidità di esecuzione e all'elevata sensibilità nel riconoscere l'emorragia cerebrale e anche per effettuare la diagnosi differenziale con altre patologie. La RM con DWI è più sensibile della TC cerebrale nell'identificare alterazioni di segnale indicative di una ischemia in atto. 
Lo studio dei vasi intra ed extracranici idealmente dovrebbe essere eseguito il più rapidamente possibile. Lo studio dei vasi extracranici può essere effettuato con eco-Doppler dei tronchi sovraortici (ECD-TSA) angio-RM e angio-TC. L'ECD-TSA rappresenta il primo approccio diagnostico allo studio dei vasi epiaortici in quanto si tratta di una tecnica non invasiva, ripetibile, di facile esecuzione e dotata di elevata accuratezza diagnostica (anche se operatore dipendente). Mediante lo studio ecografico è possibile visualizzare stenosi e placche aterosclerotiche, mentre con il color Doppler si possono stimare la velocità, la direzione, la morfologia del flusso e calcolare gli indici di resistenza e di pulsatilità. All'ecografia è possibile anche rilevare caratteristiche strutturali delle placche carotidee per identificare eventuali condizioni di rischio (placche con aspetto soft, placche ulcerate, o che presentano vascolarizzazione, placche anecogene indice di sovrapposto materiale trombotico recente). ${ }^{16}$ Lo studio dei vasi intracranici può essere effettuato mediante eco-Doppler transcranico, angio$\mathrm{TC}$, angio-RM e angiografia cerebrale (che rappresenta il gold-standard ma che non è di facile applicazione, considerata l'invasività della metodica). Il Doppler transcranico consente di individuare le stenosi intracraniche (con alto valore predittivo negativo e basso valore predittivo positivo), rileva segnali microembolici, che rappresentano un marker di rischio in quanto indice di embolizzazione periferica di natura cardiogena (presenza di PFO) o non (placche carotidee instabili, placche arco aortico). ${ }^{3,17}$ L'angio-TC è senz'altro l'esame di prima scelta nella diagnosi di dissecazione delle arterie cervicali per l'accuratezza diagnostica a carico anche delle anomalie di parete del vaso e per l'alta sensibilità sulla valutazione del circolo vertebrale. E' inoltre raccomandata per la caratterizzazione delle stenosi intracraniche e nella valutazione pre-operatoria della tromboendoarteriectomia anche dopo ECD-TSA.

La valutazione cardiaca è particolarmente importante nei pazienti nei quali si sospetta una genesi cardioembolica del TIA. Oltre all'ECG a 12 derivazioni, nei pazienti con TIA criptogenetico e con sintomi congrui con il sospetto di un origine cardioembolica è indicato un monitoraggio Holter prolungato o l'eventuale impianto di un loop-recorder. La maggiore

Tabella 2. Classificazione di Bamford.

\begin{tabular}{|c|c|c|}
\hline Sindromi lacunari (LACS) & $\begin{array}{l}\text { Ictus (o TIA) senza afasia, disturbi visuo-spaziali, } \\
\text { e senza compromissione definita del tronco } \\
\text { encefalico e della vigilanza }\end{array}$ & $\begin{array}{l}\text { Categorie: } \\
\text { - ictus motorio puro: deficit motorio puro che deve } \\
\text { coinvolgere almeno metà faccia e l'arto superiore } \\
\text { o l'arto superiore e quello inferiore } \\
\text { - ictus sensitivo puro: deficit sensitivo, anche solo } \\
\text { soggettivo, che deve coinvolgere almeno metà } \\
\text { faccia e l'arto superiore o l'arto superiore e quello } \\
\text { inferiore } \\
\text { - ictus sensitivo-motorio: ictus sensitivo + ictus } \\
\text { motorio } \\
\text { - emiparesi atassica: (incluse la sindrome della mano } \\
\text { goffa-disartria e la sindrome atassia omolaterale } \\
\text { paresi crurale) }\end{array}$ \\
\hline $\begin{array}{l}\text { Sindromi del circolo posteriore } \\
\text { (POCS) }\end{array}$ & $\begin{array}{l}\text { Uno dei seguenti } \\
\text { N.B. I casi con disturbi di funzione corticale } \\
\text { ed uno dei punti elencati devono essere } \\
\text { considerati POCS }\end{array}$ & $\begin{array}{l}\text { - paralisi di almeno un nervo cranico omolaterale con } \\
\text { deficit motorio e/o sensitivo controlaterale } \\
\text { - deficit motorio e/o sensitivo bilaterale } \\
\text { - disturbo coniugato di sguardo (orizzontale o } \\
\text { verticale) } \\
\text { - disfunzione cerebellare senza deficit di vie lunghe } \\
\text { omolaterale (come visto nell'emiparesi atassica) } \\
\text { - emianopsia isolata o cecità corticale }\end{array}$ \\
\hline $\begin{array}{l}\text { Sindrome completa del circolo } \\
\text { anteriore (TACS) }\end{array}$ & Tutti i seguenti: & $\begin{array}{l}\text { - emiplegia controlaterale alla lesione } \\
\text { - emianopsia controlaterale alla lesione } \\
\text { - nuovo disturbo di una funzione corticale superiore } \\
\text { (per esempio afasia o disturbo visuo-spaziale }\end{array}$ \\
\hline $\begin{array}{l}\text { Sindrome parziale del circolo } \\
\text { anteriore (PACS) }\end{array}$ & $\begin{array}{l}\text { Uno dei seguenti } \\
\text { N.B. Quando sono presenti più deficit essi devono } \\
\text { sempre essere riferibili ad uno stesso emisfero }\end{array}$ & $\begin{array}{l}\text { - deficit sensitivo/motorio + emianopsia } \\
\text { deficit sensitivo/motorio + nuova compromissione } \\
\text { - nuova compromissione di una funzione corticale } \\
\text { superiore + emianopsia } \\
\text { - deficit motorio/sensitivo puro meno esteso di una } \\
\text { sindrome lacunare (per esempio la monoparesi) } \\
\text { - deficit di una nuova funzione corticale superiore } \\
\text { isolata }\end{array}$ \\
\hline
\end{tabular}


durata del monitoraggio correla con la maggiore probabilità di riscontrare episodi di fibrillazione atriale parossistica. ${ }^{18-20}$ L'ecocardiografia è di fondamentale importanza per il rilievo di endocardite, patologie valvolari, forale ovale pervio, aterosclerosi dell'arco aortico o trombi transmurali, masse/tumori che possono essere responsabili del TIA cardioembolico. ${ }^{3}$

Indagini diagnostiche di secondo livello che includono l'ecocardiogramma transesofageo, la RM con DWI, l'angioRM cerebrale, l'angiografia, indagini genetiche, ricerca di malattie da accumulo o mitocondriali sono indicate in base al sospetto clinico, in paziente con TIA che rimane criptogenetico dopo la diagnostica di I livello.

\section{Messaggio chiave}

La diagnosi di TIA è prevalentemente clinica. La classificazione di Bamford può essere utile per suggerire il territorio vascolare coinvolto. Elementi clinici fondamentali a favore del TIA includono: l'esordio improvviso, la focalità dei sintomi, la possibilità di attribuirli ad un territorio vascolare cerebrale, la transitorietà e la durata $<24 \mathrm{~h}$. Gli accertamenti diagnostici di routine indirizzati ad identificare l'eziologia dell'evento, includono la visita neurologica, la misurazione della pressione arteriosa, l'ECG, esami ematochimici con assetto lipidico, la TC cerebrale, lo studio dei vasi extra-intracranici e lo studio cardiologico (ecocardiogramma, monitoraggio del ritmo cardiaco).

\section{Diagnosi differenziale}

Il TIA entra in diagnosi differenziale con una notevole varietà di patologie, che vengono considerate dei veri e propri imitatori, i TIA mimics o Transientneurological-attack (TNA). ${ }^{21}$ Con il termine TNA si definisce un improvviso disturbo neurologico che si risolve completamente nelle 24 ore. Numerosi studi hanno dimostrato che la diagnosi differenziale tra TIA e TNA non ischemici (NI-TNA) può risultare estremamente difficoltosa e che esiste un'ampia variabilità operatore-dipendente nell'inquadramento di tali disturbi. Circa il 50-60\% dei pazienti che afferisce al PS con presunto TIA non riceve una diagnosi finale di TIA, ma presenta invece un NI-TNA. ${ }^{22,23}$ Secondo le Linee Guida Italiane ISO SPREAD ${ }^{24}$ non è da considerare TIA la presenza isolata di: perdita di coscienza, vertigine isolata, amnesia globale transitoria, drop attack, astenia generalizzata, stato confusionale, incontinenza sfinterica.

A favore del TIA si può considerare: i) la presenza di sintomi negativi focali (perdita di una funzione); ii) la durata $<1$ ora; iii) la presentazione in cluster nell'arco di giorni e settimane; iv) alcuni fattori precipitanti come ipotensione, rapidi passaggi posturali, assunzione di farmaci ipotensivi, associazione con una stenosi dei grossi vasi extra o intracranici.

Le patologie che più spesso si confondono con un TIA includono: aura emicranica, deficit neurologici post-critici, crisi epilettiche focali, e i transient tumor attack. ${ }^{25}$ I tumori cerebrali possono manifestarsi con sintomi e segni neurologici focali anche ad esordio acuto, legati all'effetto massa compressivo sul parenchima cerebrale e sui vasi o ad emorragia intratumorale. Anche l'ematoma subdurale può provocare la comparsa di deficit neurologici transitori, dovuti al meccanismo compressivo sulle aree cerebrali adiacenti. Le neuroimmagini generalmente consentono un rapido inquadramento diagnostico di queste condizioni. In alcuni casi i pazienti affetti da sclerosi multipla presentano brevi e transitori deficit neurologici che entrano in diagnosi differenziale con il TIA. L'angiopatia amiloide cerebrale si può presentare con episodi di breve durata (amiloid spells) ${ }^{26}$ stereotipati, ricorrenti di ipostenia, ipoestesia, parestesia o altri sintomi corticali. Infine i disturbi funzionali, attacchi di panico e di ansia, si manifestano spesso con deficit neurologici transitori. In questo caso a supportare la diagnosi di disturbo funzionale è spesso l'inconsistenza tra i sintomi riferiti dal paziente e l'esame obiettivo, la comparsa in soggetti giovani senza fattori di rischio vascolare e l'interessamento prevalente del lato non dominante.

\section{Messaggio chiave}

Molte condizioni possono mimare un TIA. Tra le principali, ricordiamo l'aura emicranica, l'ipoglicemia, il deficit post-critico. Alcuni TIA mimics si associano a neoplasia cerebrale o ematoma subdurale o all'amiloidosi. Anche sindromi funzionali/sindromi ansiose possono presentarsi con manifestazioni cliniche simili ai TIA.

\section{Stratificazione del rischio}

Dal 2000 in avanti, diversi studi di popolazione hanno dimostrato che il rischio di ictus cerebrale dopo un TIA è molto più alto di quanto si stimasse in precedenza. Il rischio di stroke precoce è infatti pari al 9,9\%, $13,4 \%$, e 17,3\% a 2, 30, 90 giorni rispettivamente (Tabella 3). In particolare il rischio di recidiva di stroke dopo un TIA è molto alto nei primi giorni, fino al $50 \%$ entro 48 ore. ${ }^{27}$ Il TIA, inoltre, si associa ad un incremento del rischio cardiovascolare: a 3 mesi dopo il TIA, infatti, si registra un rischio di infarto cardiaco del 2$4 \%$ e morte per infarto cardiaco o ictus del $25 \% .{ }^{28-32}$

Alla luce di questi dati, numerosi studi hanno tentato di stratificare il rischio di ictus dopo TIA, cercando quali elementi clinici potessero predire il rischio di ictus. Sono state identificate cinque variabili: età 
$>60$ anni, diabete mellito, durata dei sintomi superiore a 10 minuti, ipostenia unilaterale e disturbo di linguaggio. Dalla combinazione di questi elementi nel 2005 è stato proposto dal gruppo di lavoro di Rothwell ${ }^{33} \mathrm{lo}$ score ABCD (acronimo di Age, Blood Pressure, Clinical features and Duration). Due anni dopo, è stata proposta da Johnston e Rothwell ${ }^{34}$ una nuova versione con l'inserimento del diabete mellito come ulteriore item, trasformandolo nel più noto $\mathrm{ABCD}^{2}$ score con un punteggio variabile da 0 a 7 in grado di definire il rischio di recidiva ischemica a $2,7,30$ e 90 giorni. Negli anni successivi è emerso come, anche in pazienti con $\mathrm{ABCD}^{2}$ score $<4$, punteggio che definiva un basso rischio, fossero presenti in circa il $25 \%$ dei casi, condizioni cliniche quali la stenosi carotidea $>50 \%$ o una potenziale fonte emboligena, correlabili ad un alto rischio di nuovi eventi ischemici. ${ }^{35,36}$

Inoltre, anche altri fattori non considerati nel$1^{\prime} \mathrm{ABCD}^{2}$ score condizionano il rischio di ictus a breve termine quali: TIA recidivanti, stenosi carotidea critica, presenza di lesione ischemica acuta alla TC encefalo.

$\mathrm{Nel} 2010,{ }^{37} 1^{1} \mathrm{ABCD}^{2}$ score venne quindi integrato con altri items configurando l' $\mathrm{ABCD}^{3}$ score, in cui venivano aggiunti due punti in presenza di un TIA nei 7 giorni precedenti (Dual TIA), e $\mathrm{ABCD}^{3}$-I score che oltre al Dual TIA, includeva altri 2 punti in presenza di una stenosi sintomatica della carotide interna $>50 \%$ e altri 2 punti se lo studio in acuto con RM in diffusione documentava precoci segni di ischemia.

Attualmente si è diffusa la consapevolezza che tutti i pazienti con TIA devono effettuare una diagnostica completa, includendo lo studio dei vasi, in tempi rapidi indipendentemente dall'applicazione degli scores di rischio sopra riportati. Le linee guida britanniche NICE, ${ }^{38}$ pubblicate nel 2019, raccomandano infatti di evitare l'utilizzo di scores di rischio (come $\mathrm{ABCD}^{2}$ ) sia per valutare il rischio di recidiva che per decidere la tempistica del percorso diagnostico del TIA.

$\mathrm{L}^{\prime} \mathrm{ABCD}^{2}$ score può trovare forse ancora un ruolo per individuare quei pazienti che necessitano di una osservazione clinica in ospedale di almeno 24 ore. Un
$\mathrm{ABCD}^{2}$ score $\geq 4$ identifica comunque pazienti con multipli fattori di rischio per una possibile recidiva ischemica precoce. Pertanto nonostante vi siano ancora incertezze, l'ospedalizzazione di almeno 24 ore per questa categoria di pazienti può essere più protettiva anche per favorire l'immediata possibilità di accedere alla trombolisi/trombectomia nel caso di comparsa di un ictus precoce. ${ }^{36,39}$

\section{Messaggio chiave}

Il TIA si associa ad un alto rischio di ictus precoce, dal $5 \%$ a $48 \mathrm{~h}$ al $17 \%$ a 3 mesi. Sono stati identificati vari score clinici $\left(\mathrm{ABCD}^{2}\right.$ score è il più noto) per predire il basso o alto rischio di ictus che non sono però risultati attendibili sia per predire il rischio di recidiva nella categoria a cosiddetto basso rischio che per decidere il percorso diagnostico del TIA e la sua tempistica. Attualmente le Linee Guida evidence based raccomandano che tutti i pazienti con TIA devono effettuare una diagnostica completa, includendo lo studio dei vasi, in tempi molto rapidi, indipendentemente dall'applicazione degli scores di rischio.

\section{Un caso particolare: il TIA ricorrente}

Nell'ambito del TIA merita un particolare attenzione il caso del TIA multiplo o ricorrente. Si tratta di una condizione caratterizzata dalla manifestazione di 2 o più TIA entro 7 giorni dall'evento indice. ${ }^{40}$ Solo due studi basati su popolazione $e^{40,41}$ hanno focalizzato l'attenzione su questo tema e riportano un'incidenza del TIA ricorrente di circa il 20\%. Nella nostra casistica (1035 pazienti valutati al Policlinico S. OrsolaMalpighi, Bologna), l'incidenza del TIA multiplo è risultata pari al $21 \%$ con un numero di episodi di TIA compreso tra i 2 e i 10 . Il rischio di ictus ischemico è risultato significativamente più elevato nel gruppo TIA multiplo versus il TIA singolo per tutto il follow up (60 mesi) con una frequenza a $48 \mathrm{~h}$ del $6 \%$ versus $0,4 \%$ e a 60 mesi del $10 \%$ versus $4 \%$ rispettivamente nel gruppo TIA multiplo verso quello singolo. Per

Tabella 3. Rischio di ictus dopo TIA.

\begin{tabular}{|c|c|c|c|c|c|}
\hline Studio/anno & $\mathbf{N}^{\circ}$ & Ictus a 7 giorni & Ictus a 30 giorni & Ictus a 90 giorni & $\begin{array}{l}\text { Morte per ictus o } \\
\text { infarto cardiaco }\end{array}$ \\
\hline California, $2000^{28}$ & 1707 & $\begin{array}{c}4 \%-24 \mathrm{~h} \\
5.2 \%-2 \text { giorni }\end{array}$ & I & $10,5 \%$ & $25,1 \% 3$ mesi \\
\hline Alberta, $2004^{29}$ & 2285 & / & $6,7 \%$ & $9,5 \%$ & $21,8 \% 12$ mesi \\
\hline Oxfordshire Stroke projects, $2003^{30}$ & 209 & $\begin{array}{c}4 \%-24 \mathrm{~h} \\
8.6 \%-7 \text { giorni }\end{array}$ & $12 \%$ & / & / \\
\hline Oxfordshire Stroke projects, $2004^{31}$ & 315 & $8 \%$ & $11,5 \%$ & $17,3 \%$ & / \\
\hline Wu et al., $2007^{32}$ & 10.126 & $9,9 \%$ & $13 \%$ & $17 \%$ & / \\
\hline
\end{tabular}


quanto riguarda l'eziologia, il TIA multiplo si associa più frequentemente a patologia dei grossi vasi intra ed extracranici e a un'eziologia di tipo lacunare, tuttavia solo per quanto riguarda la Capsular warning Syndrome (CWS). Questi dati suggeriscono che più frequentemente alla base del TIA multiplo possa esserci da una parte una placca carotidea o intracranica instabile a rischio di trombosi e/o embolizzazione cerebrale, dall'altra la malattia dei piccoli vasi tipicamente associata alla CWS.

La cosidetta CWS è un peculiare sottotipo di TIA ricorrente. E' stata descritta per la prima volta da Donnan et al. nel $1993^{42}$ che riportò 50 pazienti con ripetuti episodi di TIA (almeno 3) entro $24 \mathrm{~h}$ con completo recupero dei sintomi tra gli episodi, ascrivibili ad una ristretta regione cerebrale: la capsula interna. Le manifestazioni cliniche erano motorie o sensitivo-motorie prevalentemente a carico della faccia, arto superiore e arto inferiore. La frequenza della CWS fra tutti i TIA risultò del 4,5\%. La CWS era seguita nel $42 \%$ dei casi da un ictus capsulare nel breve periodo. La definizione di CWS venne poi estesa ad un arco temporale più ampio: almeno 3 episodi stereotipati di TIA lacunare nell'arco di 72 ore senza l'associazione di sintomi corticali. $^{40}$

Le sindromi lacunari considerate nell'ambito della CWS sono quelle definite da Fisher: ${ }^{14}$ emiparesi pura, emisindrome sensitiva pura, emisindrome sensitivomotoria, emiparesi classica e disartria con mano impacciata. Grazie alle tecniche avanzate di neuroimaging, alcuni studi trovarono che TIA ripetuti lacunari stereotipati potevano correlarsi ad altre sedi sottocorticali oltre alla capsula interna, ad esempio il ponte, dando origine a nuove definizioni come pontine warning syndrome. ${ }^{43}$

L'esatto meccanismo fisiopatologico alla base della CWS è ancora sconosciuto. Sono stati infatti considerati diversi meccanismi tra cui la microangiopatia delle arterie perforanti (lipoialinosi), il microembolismo da parte di una singola arteria perforante, l'ipoperfusione emodinamica ${ }^{44}$ e la stenosi intracranica $^{45}$ in particolare dell'arteria cerebrale media. Quest'ultima ipotesi emerge però da studi sulla popolazione asiatica, che rispetto a quella caucasica presenta un'alta frequenza di patologia aterosclerotica intracranica. Differenti trattamenti in acuto sono stati proposti senza però un sicuro beneficio sulla prevenzione dell'ictus a breve termine: controllo della pressione arteriosa, antiaggregante singolo o doppio, anticoagulante, e trombolisi. Pertanto la migliore gestione di questi pazienti rimane ancora controversa.

\section{Messaggio chiave}

Il TIA ricorrente rappresenta una vera sindrome a parte, per l'altissimo rischio di ictus precoce specialmente nei TIA ad eziologia da CWS e patologia dei grossi vasi intra-extracranici, che richiede immediate strategie diagnostiche e terapeutiche per scongiurare l'occorrenza precoce di un ictus cerebrale.

\section{Modelli Fast Track TIA}

La gestione dei pazienti con TIA è stata completamente rivoluzionata dalla pubblicazione nel 2007 di due studi: SOS-TIA ${ }^{46}$ ed EXPRESS Study ${ }^{47} \mathrm{Nel}$ primo sono stati valutati, da parte di un neurologo esperto di patologia cerebrovascolare, 1085 pazienti con sospetto TIA in un Ospedale parigino. Entro 4 ore dall'ingresso i pazienti venivano sottoposti ai seguenti accertamenti: studio di neuroimmagini (TC o RM), valutazione dei vasi sovra-aortici e intracranici tramite ECD-TSA e ECD transcranico, ECG ed eventualmente Ecocardiogramma transtoracico o transesofageo (se sospetto clinico di fonte cardioembolica ad alto rischio di recidiva) ed esami bioumorali (profilo lipidico e metabolico). Completata la fase diagnostica, venivano dimessi con la terapia di prevenzione secondaria ed affidati con una lettera informativa al curante. Era previsto il ricovero in Stroke Unit in presenza di: stenosi di alto grado di arteria intra-extracranica, basso flusso nell'arteria cerebrale media, fonti cardioemboliche ad alto rischio di recidiva, TIA recidivante o con peggioramento dei sintomi e sospetto di FA parossistica. Nello studio EXPRESS, sono stati confrontati due modelli assistenziali a Oxford, sempre all'interno di una TIA clinic. Nella prima fase dello studio (da Aprile 2002 a Settembre 2004), i pazienti venivano segnalati dal curante, tramite fax, alla TIA clinic che li prenotava per la visita neurologica e gli accertamenti diagnostici (in genere entro pochi giorni); la TIA clinic inviava poi via fax al curante un resoconto degli accertamenti effettuati e le indicazioni terapeutiche. Nella seconda fase (da Ottobre 2004 a Marzo 2007) i pazienti erano inviati direttamente dal curante, senza appuntamento, alla TIA clinic e il trattamento veniva iniziato contestualmente all'accesso nella struttura. I pazienti erano sottoposti in entrambe le fasi a TC encefalo ed ECG al primo accesso alla TIA clinic mentre ECD-TSA ed ecocardiogramma transtoracico o transesofageo, se clinicamente indicati, venivano prenotati entro 7 giorni. Entrambi gli studi hanno dimostrato che una diagnostica più tempestiva e l'immediata applicazione della profilassi secondaria (in sintesi l'applicazione di un modello assistenziale Fast Track), determina una riduzione del rischio di stroke a 3 mesi del $80 \%$.

Negli anni successivi alcuni studi hanno implementato questo modello assistenziale fast track nel setting del Dipartimento di Emergenza-Urgenza, dimostrando che sottoporre i pazienti con TIA ad un rapido protocollo gestionale in PS, permette di ridurre il rischio di recidiva ischemica cerebrale. Gli studi più 
significativi sono: lo studio americano TWO-ACES del 2011 di Olivot ${ }^{48}$ basato sulla stratificazione del rischio secondo il punteggio $\mathrm{ABCD}^{2}$, lo studio australiano Monash del 2012 di Sanders ${ }^{49}$ e lo studio italiano Bologna-TIA di Guarino del $2015^{39} \mathrm{che}$, al contrario, non hanno utilizzato la scala di rischio $\mathrm{ABCD}^{2}$ per la scelta e la tempistica delle indagini diagnostiche preliminari. Nel primo sono stati arruolati 224 pazienti con TIA possibile o minor stroke gestiti nel PS di Stanford (US) dal giugno 2007 al settembre 2009. Il protocollo prevedeva per tutti i pazienti una TC encefalo, un ECG e una valutazione neurologica. Sulla base del punteggio $\mathrm{ABCD}^{2}$ il paziente veniva: i) dimesso con successivo accesso ad una TIA clinic entro 1-2 giorni per eseguire angio-RM intra-extracranica se $\mathrm{ABCD}^{2}$ 0-3); ii) sottoposto ad angioTC in PS (se $\mathrm{ABCD}^{2} 4-5$ ) e ricoverato se riscontro di stenosi $>50 \%$ $\mathrm{D} 5$; iii) ricovero ospedaliero immediato (se $\mathrm{ABCD}^{2} 6$ 7). Nello studio Monash è stata invece confrontata la modalità di gestione dei pazienti con TIA nel dipartimento di emergenza del Monash Medical Centre (Australia), tra il 2003 e 2004, e, dopo l'introduzione di un nuovo protocollo, tra il 2004 e 2007. In epoca preprotocollo la maggior parte dei pazienti con TIA veniva ricoverata, oppure inviata ad un follow-up neurologico ambulatoriale. Dal 2004, il protocollo prevedeva l'esecuzione per tutti i pazienti di esami bioumorali, ECG e TC encefalo, con contatto telefonico tra il medico di PS e i neurologi della Stroke Unit. Nel sospetto di TIA del circolo cerebrale anteriore veniva eseguito ECD-TSA il giorno stesso o il giorno seguente. I criteri di ricovero includevano: sintomi persistenti o recidivanti o comorbidità rilevanti. Alla dimissione, i pazienti erano poi segnalati via fax alla TIA-clinic e quindi ricontattati per il follow-up: i) se stenosi carotidea $>50 \%$ erano sottoposti a angio$\mathrm{TC} /$ angio-RM entro 24 ore e a valutazione chirurgica urgente; ii) se riscontro di FA non nota accedevano alla TIA clinic entro pochi giorni; iii) se assenza di FA e/o di reperti significativi allo studio ECD-TSA venivano rivalutati entro 4-6 settimane. Nello studio italiano sono stati riportati i risultati sia a breve che a lungo termine, ottenuti dopo l'implementazione di un percorso diagnostico-terapeutico multidisciplinare FastTrack TIA al Policlinico Universitario $S$. Orsola-Malpighi di Bolognae. Sono stati inclusi 433 pazienti consecutivi ammessi in PS con diagnosi di TIA confermato da un neurologo vascolare dall'agosto 2010 al Dicembre 2013. Il protocollo diagnostico preliminare prevedeva per tutti i pazienti: profilo ematochimico con assetto lipidico, valutazione PA, ECG, TC encefalo, ECD-TSA, Doppler tra-nscranico ed Ecocardio (in casi selezionati), valutazione neurologica nel corso della permanenza in PS o in OBI, entro comunque 24 ore. In caso di riscontro di stenosi carotidea $>50 \%$ o placca a rischio allo studio ECD-TSA veniva attivato in urgenza il chirurgo vascolare. Accertamenti di II livello (es: Holter ECG 24 h, Doppler transcranico con microbolle, Ecocardio transesofageo, screening trombofilia, RM/DWI/angioRM cerebrale, come anche indagini genetiche o ricerca di malattie rare) se indicati, venivano programmati entro 7-15 giorni in regime ambulatoriale (con posti predefiniti). Tutti i pazienti venivano rivalutati da un neurologo vascolare a distanza di 1, 3 e 12 mesi. Il ricovero era previsto per pazienti ad alto rischio di recidiva immediata quali: $\mathrm{ABCD}^{2}$ score $\geq 4$, TIA recidivanti entro $72 \mathrm{~h}$ precedenti, TIA $>1 \mathrm{~h}$, riscontro o sospetta FA e/o altra causa cardioembolica, stenosi carotidea sintomatica candidata alla chirurgia, stenosi intracranica e/o dissecazione arteriosa e per comorbidità scompensate. Tutti i pazienti avevano un follow up di almeno 3 mesi e l'outcome era valutato dal neurologo vascolare. La riduzione del rischio relativo di stroke a 3 mesi riportata in tale studio è stata del $77 \%$ rispetto all'atteso. Tutti e tre gli studi hanno riportato una significativa riduzione del rischio di stroke a breve termine ed una riduzione dei ricoveri; lo studio italiano ha inoltre mostrato una significativa riduzione del rischio di stroke, di infarto miocardico e morte vascolare, anche nel lungo termine (al follow-up a un anno), confermando i risultati dello studio SOS-TIA.

Per quanto riguarda la prognosi del TIA a lungo termine, sono stati pubblicati due studi del progetto internazionale TIA Registry di Amarenco, con followup a 1 anno $^{50}$ e a 5 anni. ${ }^{51} \mathrm{Nel}$ follow-up a 1 anno la stima del tasso di eventi (outcome composito: morte cardiovascolare, ictus non fatale, sindrome coronarica acuta non fatale) è stata del $6.2 \%$. Il tasso di ictus ai giorni $2,7,30,90$ e 365 è stato $1,5 \%, 2,1 \%, 2,8 \%$, $3,7 \%$ e $5,1 \%$ rispettivamente. Nell'analisi multivariata la presenza di infarti multipli nello studio di neuroimaging, l'eziologia aterotrombotica e un $\mathrm{ABCD}^{2}$ score di 6 o 7 erano ciascuno associato con un rischio più che raddoppiato di ictus. Nel follow-up a 5 anni ${ }^{51}$ il tasso di eventi cardiovascolari cumulativo dal secondo al quinto anno è stato del $6,4 \%$, assolutamente uguale a quello del primo anno, mentre il tasso di ictus è stato del $9,5 \%$.

\section{Messaggio chiave}

Modelli assistenziali del TIA improntati alla rapidità di gestione diagnostico-terapeutica con protocolli predefiniti e incentrati sul neurologo vascolare hanno dimostrato una significativa riduzione dell'occorrenza dell'ictus sia a breve (3 mesi), che a lungo termine.

\section{Setting assistenziale}

11 modello assistenziale per il TIA di più facile applicazione in Italia ricalca senza dubbio il modello 
dello studio bolognese. Infatti in Italia non sono attive le TIA clinic e il nostro Sistema Sanitario Nazionale vede nel PS il punto di accesso privilegiato per la gestione delle emergenze/urgenze neurologiche, che includono i sintomi neurologici focali di sospetta origine vascolare, grazie anche all'implementazione della rete per il trattamento in acuto dell'ictus ischemico.

Inoltre sia nello studio SOS-TIA che nello studio Bologna-TIA, che hanno riportato i migliori risultati in termini di riduzione del rischio di ictus a 3 mesi, $\mathrm{i}$ cardini del percorso erano rappresentati dalla valutazione neurologica che confermava la diagnosi di TIA, dall'applicazione di un work up diagnostico/terapeutico predefinito per tutti i pazienti e dalla presa in carico con monitoraggi seriati nel follow up da parte del neurologo (vascolare). Lo specialista vascolare di riferimento dovrebbe infatti valutare il paziente con TIA il più presto possibile, entro $24 \mathrm{~h}$ come raccomandato anche dalle linee guida NICE. ${ }^{38}$ Altro punto fondamentale è la garanzia di un follow up neurovascolare nel tempo, per il monitoraggio dei fattori di rischio e della compliance farmacologica.

\section{Messaggio chiave}

Il setting più idoneo per l'applicazione del modello assistenziale fast track per il TIA è il PS-OBI, dal quale si deve dipanare un clinical pathway multidisciplinare (coordinato e comunque concluso dal neurologo). La gestione ispirata al best treatment, sulla base delle evidenze disponibili, prevede che tutti i pazienti con diagnosi di TIA debbano effettuare un rapido ed esaustivo work-up diagnostico predefinito, indipendentemente dall'utilizzo di uno score di rischio.

\section{Follow up}

Alla dimissione, il curante deve ricevere il dettaglio degli accertamenti e delle terapie proposte e ai pazienti devono essere consegnate raccomandazioni sullo stile di vita e sul controllo dei fattori di rischio modificabili. Il primo follow up deve essere a 3 mesi o prima per valutare i risultati degli eventuali accertamenti di II livello effettuati nella fase post-acuta per e aggiustare gli orientamenti diagnostici e quindi anche terapeutici.

Negli studi di prognosi a lungo termine come riportato in precedenza la maggioranza degli eventi cardio-cerebrovascolari successivi al TIA ricade nel primo anno. Tuttavia, il rischio vascolare, per quanto si possa ridurre a distanza dall'evento indice, permane elevato e richiede un monitoraggio prolungato nel tempo. I molti fattori di rischio per il TIA o l'ictus si modificano con il tempo, e può inoltre cambiare il profilo di rischio globale del singolo soggetto. L'aterosclerosi, ad esempio, è una malattia che può manifestarsi acutamente ma è una condizione patologica cronica che comporta fasi di quiescenza e riesacerbazioni. Pertanto uno stretto monitoraggio nel lungo periodo è comunque raccomandato.

\section{Messaggio chiave}

Il primo anno dopo un TIA è l'intervallo di tempo in cui il rischio di ictus è più elevato e si raccomandano controlli ravvicinati per monitoraggio dei fattori di rischio e della compliance farmacologica.

Tuttavia, il rischio vascolare, anche se ridotto permane nel tempo e pertanto il monitoraggio dei fattori dio rischio deve continuare anche nel lungo periodo.

\section{Prevenzione secondaria}

La prevenzione secondaria si basa su due principali interventi: la terapia farmacologica e la modificazione dello stile di vita.

La terapia farmacologica deve essere iniziata molto precocemente ed è indirizzata sia al controllo dei fattori di rischio riscontrati: ipertensione, diabete, dislipidemia, che al trattamento del sottotipo eziologico alla base del TIA. In tutti i pazienti deve essere comunque iniziata una terapia antitrombotica. Il riscontro di una eziologia cardioembolica (in particolare la FA) pone indicazione alla terapia anticoagulante, secondo le linee guida esistenti, previa valutazione del rapporto rischio/beneficio individuale sul versante cardioembolico ed emorragico. Per tutte le altre condizioni eziologiche, riassumibili nella definizione di TIA non cardioembolico, sono sempre indicati la terapia antiaggregante e la statina.

La terapia antiaggregante iniziata precocemente (entro $24 \mathrm{~h}$ ) rappresenta il più efficace e precoce strumento per ridurre il rischio di recidive ischemiche. Di routine si utilizza il singolo antiaggregante (prima scelta 160-300 mg aspirina/die nella fase acuta, poi $100 \mathrm{mg} / \mathrm{die}$ ). La doppia antiaggregazione nel TIA è indicata in casi particolari. Due recenti studi, $\mathrm{CHANCE}^{52}$ e PINT $^{53}$ hanno testato la doppia antiaggregazione versus terapia standard in pazienti con minor stroke (NIHSS $\leq 3$ ) e TIA ad alto rischio di recidiva ( $\mathrm{ABCD}^{2}$ score $\geq 4$ ), di età $<80$ anni, rispettivamente entro 24 e 6 ore dall'esordio dei sintomi. La durata prevista della doppia antiaggregazione era di 21 giorni e di 90 giorni rispettivamente e con dose di carico di clopidogrel (300 mg nel CHANCE e $600 \mathrm{mg}$ nel POINT). In entrambi gli studi la doppia antiaggregazione si associava ad una significativa riduzione di ictus ischemico nel follow-up, a scapito di un incremento del rischio emorragico. Tuttavia il rischio significativo di un incremento di eventi emorragici si rendeva evidente solo dopo i primi 21 giorni di doppia terapia antiaggregante. 
Sulla base di questi dati nelle linee guida americane $^{54}$ e britanniche, ${ }^{55}$ la doppia antiaggregazione è stata inserita come standard di trattamento per i pazienti con minor stroke e TIA che rispettino i criteri di inclusione dei trials sopra riportati. Le linee guida italiane ISO SPREAD ${ }^{24}$ nel loro recente update (raccomandazione rapida 11/11/2018) hanno inserito una raccomandazione con grado forte a favore, circa l'utilizzo della doppia antiaggregazione: TIA con diagnosi certa ad alto rischio di recidiva $\left(A B C D^{2}\right.$ score $\geq 4$ e altre condizioni ad alto rischio come stenosi intracraniche e microembolizzazione da placca carotidea) o con ictus minore (NIHSS <4) di origine aterotrombotica, che non abbiano un'indicazione all'anticoagulazione: doppio antiaggregante (ASA $100 \mathrm{mg}+$ clopidogrel $75 \mathrm{mg}$, con eventuale carico di clopidogrel di 300-600 mg nel primo giorno) per un periodo non superiore a 4 settimane, con inizio entro le 12 ore dal TIA (ma dopo 24 ore per i pazienti sottoposti a fibrinolisi sistemica). Recenti metanalisi e revisioni ${ }^{56-58}$ supportano l'efficacia del doppio antiaggregante in questa tipologia di pazienti ma per un periodo non superiore a 21 giorni con migliore bilancio rischio-beneficio di 7 giorni. Ulteriori studi sono comunque necessari per meglio precisare la durata del trattamento con doppio antiaggregante. Contestualmente alla prevenzione secondaria su base farmacologica, va considerata la terapia chirurgica della stenosi carotidea, l'eventuale chiusura del forame ovale pervio, e altri interventi mirati alla specifica eziologia riscontrata.

Per il dettaglio sulla terapia di prevenzione secondaria si rimanda al capitolo sulla prevenzione secondaria dell'ictus, essendo sostanzialmente la stessa, oppure alle linee guida (ISO SPREAD,${ }^{24}$ AHA 2018, ${ }^{55}$ NICE 201938). Contestualmente agli interventi medici/chirurgici specifici, è di fondamentale importanza anche il cambiamento dello stile di vita.

\section{Messaggio chiave}

La prevenzione secondaria va impostata immediatamente dopo il TIA. E' finalizzata a correggere i fattori di rischio modificabili e al trattamento dei sottotipi eziologici riscontrati. Cardini della terapia farmacologica sono il doppio antiaggregante nei pazienti ad alto rischio, l'uso di statine nei TIA aterotrombotici e l'utilizzo dell'anticoagulante nel TIA cardioembolico.

\section{Bibliografia}

1. Fisher CM. Intermittent cerebral ischemia. In: Wright IS, Millikan CM, eds. CerebralVascularDisease. New York, NY: Grune\& Stratton;1958:81-97.

2. Albers GW, Caplan LR, Easton JD, et al. Transient ischemic attack: proposal for a new definition. N Engl J Med. 2002;347:1713-1716.
3. Easton JD, Saver JL, Albers GW, et al. Definition and evaluation of transient ischemic attack: a scientific statement for healthcare professionals from the American Heart Association/American Stroke Association Stroke Council; Council on Cardiovascular Surgery and Anesthesia; Council on Cardiovascular Radiology and Intervention; Council on Cardiovascular Nursing; and the Interdisciplinary Council on Peripheral Vascular Disease. The American Academy of Neurology affirms the value of this statement as an educational tool for neurologists. Stroke; a journal of cerebralcirculation 2009; 40(6): 2276-93).

4. Kleindorfer D, Panagos P, Pancioli A, et al.Incidence and short-term prognosis of transient ischemic attack in a population-based study. Stroke2005;36, 720-723.

5. Rothwell PM, Coull AJ, Giles MF, et al. Change in stroke incidence, mortality, case-fatality, severity, and risk factors in Oxfordshire, UK from 1981 to 2004 (Oxford Vascular Study). Lancet. 2004;363:1925-1933

6. Morgenstern LB, Smith MA, Lisabeth LD, et al. Excess stroke in Mexican Americans compared with non-Hispanic whites: the Brain Attack Surveillance in Corpus Christi Project. Am J Epidemiol. 2004;160:376-383.

7. Lauria G, Gentile M, Fassetta G, et al. Incidence of Transient ischemic attacks in the Belluno Province, Italy. First-year results of a community-based study. ActaNeurolScand 1996; 93: 291-296.

8. Cancelli I, Janes F, Gigli GL,et al. Incidence of transient ischemic attack and early stroke risk: validation of the ABCD2 score in an Italian population-based study. Stroke 2011;42:2751-2757.

9. Degan D, Ornello R, Tiseo C, et al. Epidemiology of Transient Ischemic Attacks Using Time-or Tissue-Based Definitions A Population-Based Study Stroke 2017;48; 530-6.

10. Trial of ORG 10172 in Acute Stroke Treatment- HP AdamsJr, B H Bendixen, L J Kappelle, J Biller, B B Love, D L Gordon and E E Marsh Stroke. 1993;24:35-41.

11. Ay H, Koroshetz WJ, Benner T, et al. Transient ischemic attack with infarction: a unique syndrome. AnnNeurol 2005; 57:679.

12. Kimura K, Minematsu K, Yasaka M, et al. The duration of symptoms in transient ischemic attack. Neurology 1999; 52(5): 976-80.

13. Baquis GD, Pessin MS, Scott RM. Limb shaking--a carotid TIA. Stroke; a journal of cerebral circulation 1985; 16(3): 444-8.

14. Fisher CM. Lacunar strokes and infarcts: a review. Neurology1982;32, 871-876.

15. Bamford J, Sandercock P, Dennis M, et al. Classification and natural history of clinically identifiable subtypes of cerebral infarction. The Lancet. 1991;337(8756):1521-6)

16. Yamashiro K, Watanabe T, Tanaka R, et al.Clustering of risk factors increases the incidence of echolucent carotid plaque in stroke patients. Cerebrovasculardiseases (Basel, Switzerland) 2006; 22(5-6): 432-8.

17. Poppert H, Sadikovic S, Sander K, et al. Embolic signals in unselected stroke patients: prevalence and diagnostic benefit. Stroke; a journal of cerebralcirculation 2006; 37(8): 2039-43.

18. Sanna T, Diener HC,Passman RS, et al.et al. "Cryptogenic Stroke and Underlying Atrial Fibrillation". The New England Journal of Medicine. 2014. 370(26):2478-2486. 
19. Gladstone DJ, Spring M, Dorian P, et al. Atrial Fibrillation in Patients with Cryptogenic Stroke. The New England Journal of Medicine. 2014;370(26):24672477.

20. Petrovičová A, Kurča E, Brozman M, et al. Detection of occult paroxysmal atrial fibrilation by implantable longterm electrocardiographic monitoring in cryptogenic stroke and transient ischemic attack population: a study protocol for prospective matched cohort study. BMC CardiovascularDisorders 2015; 15: 160.

21. Bos MJ, van Rijn MJ, Witteman JC, et al. Incidence and prognosis of transient neurological attacks. JAMA 2007; 298:2877.

22. Nadarajan V, Perry RJ, Johnson J, et al. Transient ischaemic attacks: mimics and chameleons. Practical Neurology 2014;14(1): 23-31.

23. WengV, Loh K, Tuck D et al. Outpatient management of transient ischaemic attack. Singapore Med J. 2016;57 (12):658-663.

24. ISO SPREAD - Stroke Prevention and Educational Awareness Diffusion. Ictus cerebrale: Linee guida italiane di prevenzione e trattamento. 2017 VIII edizione; www.iso-spread.it

25. Ross RT. Transient tumor attacks. Archives of neurology 1983; 40(10): 633-6.

26. Charidimou A, Law R, Werring DJ. Amyloidspellstrouble. Lancet. 2012;380:1620.

27. Chandratheva A,Mehta Z, Geraghty OC, et al. Population-based study of risk and predictors of stroke in the first few hours after a TIA. On behalf of the Oxford VascularStudy, Neurology 2009;72(22).

28. Johnston SC, Gress DR, BrownerWS, et al. Short-term prognosis after emergency department diagnosis of TIA. JAMA2000; 284:2901-2906.

29. Hill MD, Yiannakoulias N, Jeerakathil T, et al. The high risk of stroke immediately after transient ischemic attack: a population-based study. Neurology2004;62:2015-2020.

30. Lovett JK, Dennis MS, Sandercock PAG et al.Very early risk of stroke after a first transient ischemic attack. Stroke2003;34, e138-140.

31. RothwellPM, Coull AJ, Giles MF, et al. Change in stroke incidence, mortality, case-fatality, severity, and risk factors in Oxfordshire, UK from 1981 to 2004 (Oxford Vascular Study). Lancet Lond. Engl.2004;363:1925-1933.

32. Wu CM,McLaughlin K, Diane L, et al. Early risk of stroke after transient ischemic attack: a systematic review and meta-analysis. Arch. Intern. Med. 2007;167:24172422.

33. Rothwell PM, Giles MF, Flossmann E, et al. A simple score $(A B C D)$ to identify individuals at high early risk of stroke after transient ischaemic attack. Lancet. 2005;366(9479):29-36.

34. JohnstonSC, Rothwell PM, Nguyen-Huynh MN, et al. Validation and refinement of scores to predict very early stroke risk after transient ischaemic attack. Lancet 2007;27;369(9558):283-92.

35. Amarenco P, Labreuche J, Lavallée PC, et al. Does $\mathrm{ABCD} 2$ score below 4 allow more time to evaluate patients with a transient ischemic attack? Stroke 2009; 40:3091-3095.

36. Amarenco P, Labreuche J, Lavallée PC. Patients with transient ischemic attack with $\mathrm{ABCD} 2<4$ can have similar 90-day stroke risk as patients with transient ische- mic attack with $\mathrm{ABCD} 2 \geq 4$. Stroke J. Cereb. Circ. 2012;43(3):863-5.

37. Merwick Á, Albers GW, Amarenco P, et al. Addition of brain and carotid imaging to the ABCD score to identify patients at early risk of stroke after transient ischaemic attack: a multicentre observational study. Lancet Neurol. 2010;9(11):1060-9.

38. https://www.nice.org.uk/guidance/ng128/resources/ stroke-and-transient-ischaemic-attackin-over-16s-diagnosis-and-initial-management-pdf-66141665603269.

39. Guarino M, Rondelli F, Favaretto E, et al. Short- and Long-Term Stroke Risk after Urgent Management of Transient Ischaemic Attack: The Bologna TIA Clinical Pathway.EurNeurol. 2015;74(1-2):1-7.

40. Paul NL, SimoniM, Chandratheva A et al. Populationbased study of capsular warning syndrome and prognosis after early recurrent TIA. Neurology 2012;79:1356-1362.

41. Purroy F, JiménezCaballero PE, Gorospe A, et al.Recurrent transient ischaemic attack and early risk of stroke: data from the PROMAPA study. J. Neurol. Neurosurg. Psychiatry2013;84: 596-603.

42. Donnan GA, O'Malley HM, Quang L, et al. The capsular warning syndrome: pathogenesis and clinical features. Neurology. 1993;43(5):957-62.

43. Benito-Leon J. Alvarez-Linera J. Porta-Etessam J. Detection of acute pontine infarction by diffusion-weighted MRI in capsular warning syndrome. CerebrovascDis.2001;11: 350-351.

44. Staaf G, Geijer B, Lindgren A et al. Diffusion-weighted MRI findings in patients with capsular warning syndrome. Cerebrovasc. Dis. 2004;17:1-8.

45. Xu X, Wei Y, Zhan X et al. Value of higher-resolution MRI in assessing middle cerebral atherosclerosis and predicting capsular warning syndrome. JMRI 2016;44:12771283.

46. LavalléeP, Meseguer E, Abbou $\mathrm{H}$ et al. A transient ischaemic attack clinic with round-the-clock access (SOS-TIA): feasibility and effects. The Lancet Neurology 2007; 6:953-60.

47. RothwellPM, Giles MF, Chandratheva A, et al. Effect of urgent treatment of transient ischaemic attack and minor stroke on early recurrent stroke (EXPRESS study): a prospective population-based sequential comparison. Lancet 2007;370:1432-42.

48. Olivot JM, Wolford C, Castle J et al. TWO ACES. Transient Ischemic Attack Work-Up as Out-patient Assessment of Clinical Evaluation and Safety. Stroke2011; 42:1839-43.

49. Sanders LM, Srikanth VK, Jolley DJ, et al. Monash Transient Ischemic Attack Triaging Treatment. Safety of a Transient Ischemic Attack Mechanism-Based Outpatient Model of Care. Stroke 2012;43:2936-41.

50. Amarenco P, Lavallée PC, Labreuche J,et al. One-Year Risk of Stroke after Transient Ischemic Attack or Minor Stroke, N Engl J Med 2016;374:1533-1542.

51. Amarenco P. Five-Year Risk of Stroke after TIA or Minor Ischemic Stroke, N Engl J Med 2018; 378:2182-219.

52. Wang Y, Wang Y, Zhao X, et al. CHANCE Investigators. Clopidogrel with aspirin in acute minor stroke or transient ischemic attack. N Engl J Med. 2013;369(1):11-19.

53. JohnstonSC, EastonJD, FarrantM, etal. Clinical Research Collaboration, Neurological Emergencies Treatment Trials Network, and the POINT Investigators. 
Clopidogrel and Aspirin in Acute Ischemic Stroke and ih-Risk TIA. N Engl J Med 2018;379:215-25.

54. 2018 Guidelines for the Early Management of Patients With Acute Ischemic Stroke: A Guideline for Healthcare Professionals From the American Heart Association/American Stroke Association. Stroke. 2018;49:e46-e99.

55. Dual antiplatelet therapy with aspirin and clopidogrel for acute high risk transient ischaemic attack and minor ischaemic stroke: a clinical practice guideline. BMJ 2018;363:k5130.
56. Yang Y, Zhou M, Zhong, et al. Dual versus mono antiplatelet therapy for acute non-cardioembolic ischaemic stroke or transient ischaemic attack: a systematic review and meta-analysis. Stroke and Vascular Neurology 2018;3:e000168. doi:10.1136/svn-2018-000168

57. Wang $\mathrm{Y}$ et al. Acute dual antiplatelet therapy for minor ischaemic stroke or transient ischaemic attack.BMJ 2019;364:1895 doi: 10.1136/bmj.1895.

58. Stringberg A, Camden R, Qualls K et al. Update on Dual Antiplatelet Therapy for Secondary Stroke Prevention. Missouri Medicine | July/August 2019 | 116:4 | 303-307. 


\title{
Ictus ischemico: assistenza in fase acuta
}

\author{
Francesco Di Blasio \\ U.O.C. Neurologia d’Urgenza e Stroke Unit, Presidio Ospedaliero S.Spirito, Pescara, Italia
}

\section{Introduzione}

L'ictus cerebrale (Stroke) costituisce la seconda causa di morte e la terza causa di disabilità a livello mondiale. Circa il 20\% dei pazienti muore nel primo mese successivo all'evento.

La fase acuta dello Stroke è divisibile, dal punto di vista assistenziale, in due periodi: quello pre-ospedaliero e quello di ospedalizzazione in reparto per acuti.

Il periodo pre-ospedaliero è caratterizzato dagli eventi che intercorrono tra l'esordio dell'accidente cerebrovascolare ed il ricovero ospedaliero. Esso vede coinvolti, quali attori, il paziente (ed i suoi familiari) e la Rete sanitaria di Emergenza-Urgenza. La sua durata, quantificabile in ore, deve essere, in ottemperanza all'assioma time is brain, quanto più breve possibile. Per questo motivo, sono stati istituiti all'uopo protocolli assistenziali ben definiti, già ampiamente codificati, ma tuttavia continuamente implementabili. Esso culmina nell'arrivo del paziente in Pronto Soccorso, vera e propria interfaccia tra il periodo pre-ospedaliero e quello di ospedalizzazione, ove l'assistito viene studiato, attuando una sinergia tra l'Equipe sanitaria residente, il Medico di Stroke Unit, ed altre figure professionali variamente coinvolte (Radiologo, Neurochirurgo, Chirurgo Vascolare), con l'ausilio di adeguati strumenti clinici e diagnostici, per stabilire se sia candidabile, o no, a procedura di rivascolarizzazione.

Il successivo periodo di ospedalizzazione in reparto per acuti (Stroke Unit) è caratterizzato dal per-

Corrispondente: Francesco Di Blasio, Unità Operativa di Neurologia d'Urgenza e Stroke Unit, Dipartimento di EmergenzaUrgenza, Presidio Ospedaliero S. Spirito, via Fonte Romana 8,65125 Pescara, Italia

Tel.: +39.085.4252277.

E-mail: diblasio.francesco@libero.it

Articolo pubblicato secondo la Creative Commons Attribution NonCommercial 4.0 License (CC BY-NC 4.0).

${ }^{\circ}$ Copyright: the Author(s), 2020

Licensee PAGEPress, Italy

QUADERNI - Italian Journal of Medicine 2020; 8(2):47-53 corso di stabilizzazione clinica del paziente colpito da Ictus. Avvalendosi di specifici protocolli terapeutici ed assistenziali, l'Equipe sanitaria, appositamente formata ed aggiornata, cerca di accompagnare il paziente fuori dalla situazione di criticità clinica presentata all'esordio, avviandolo ad un percorso riabilitativo.

La trattazione che segue avrà l'obiettivo di descrivere nel dettaglio l'iter di assistenza di fase acuta dello Stroke.

\section{L'assistenza in fase acuta dell'ictus: periodo pre-ospedaliero}

Il percorso assistenziale del paziente colpito da Stroke inizia nello scenario in cui si verifica l'evento acuto.

In tal senso vanno implementate e diffuse in modo più capillare possibile campagne informative sulla popolazione, che educhino a riconoscere i segni/sintomi dell'Ictus sin dall'esordio, così da poter attivare tempestivamente il sistema di Emergenza Sanitaria. Esse possono essere attuate attraverso tutti i mezzi disponibili: campagne informative, convegni, media tradizionali, social media. ${ }^{1}$

Le linee guida 2018 dell' American Heart Association prestano specifica attenzione a questo aspetto, e sottolineano come vi siano ancora carenze nella conoscenza dei segni di allarme indicativi di Ictus, con differenze per età, sesso, etnia. ${ }^{2}$ Solo circa il $60 \%$ dei pazienti affetti da Stroke acuto, infatti, provvede ad attivare tempestivamente il numero dell'Emergenza Sanitaria.

Anche il Consensus Statement European Academy of Neurology/European Stroke Organization (2018) ha raccomandato con forza l'importanza di interventi educativi sulla popolazione generale, pur prendendo atto dell'attuale mancanza di chiare evidenze scientifiche nel merito ${ }^{(3)}$

Per quanto esposto, come si può facilmente comprendere, l'attivazione della cascata assistenziale è, non di rado, intempestiva, con conseguente ritardo nell'arrivo del paziente in Ospedale e nell'accessibilità del medesimo al trattamento di rivascolarizzazione.

Il personale della Centrale Operativa di Emergenza Sanitaria deve essere in grado, già al triage telefonico, 
di riconoscere un sospetto Stroke, e di attivare, qualora ravvisi l'indicazione ad una ipotetica procedura di rivascolarizzazione, il cosiddetto Codice Ictus. ${ }^{1}$

Il Codice Ictus viene è definito dai seguenti parametri: i) esordio dei sintomi da non $>4$ ore; ii) età $>18$ anni; iii) Cincinnati Pre-Hospital Stroke Scale Positiva (Tabella 1).

Il personale di soccorso, pervenuto presso lo scenario dell'evento ictale, dovrà essere in grado di riconoscere precocemente i segni dello Stroke. In tal senso, dovrà essere specificamente formato e sottoposto ad aggiornamenti continui. ${ }^{1}$

Stando alle linee guida AHA 2018, il tempo impiegato per l'inquadramento on scene dell'Ictus è ancora, in media, eccessivamente protratto (circa 15 minuti, a fronte di un tempo medio call to hospital di circa 36 minuti). ${ }^{2}$

La prima valutazione del paziente sarà volta ad escludere eventuali traumi ed a praticare un primo inquadramento diagnostico, preservando nel contempo le funzioni vitali attraverso i seguenti items: i) $\mathrm{ABC}$ (airway, breathing, circulation); ii) Parametri vitali (respiro, polso arterioso, PA, saturazione $\mathrm{O}_{2}$ ); iii) Glasgow Coma Scale (Tabella 2); iv) Cincinnati Prehospital Stroke Scale (secondo le linee guida AHA
2018, sono validate anche la FAST scale, la Los Angeles Prehospital Stroke Screen, la MASS, la MedPACS, la OPSS, la ROSIER). E' raccomandabile che il personale sanitario coinvolto nell'inquadramento della fase acuta dello Stroke abbia dimestichezza con almeno una delle scale di valutazione indicate. ${ }^{1-3}$

Qualora il paziente sia in condizioni gravi $(\mathrm{GCS}<9)$, vanno praticate dal personale di soccorso le seguenti manovre: i) assicurare la pervietà delle vie aeree; ii) somministrare ossigeno; iii) procedere, qualora necessario, a intubazione orotracheale, per proteggere il paziente dall'aspirazione di contenuti gastrici, consentendo ventilazione ed ossigenazione adeguate; iv) somministrare cristalloidi, v) proteggere le estremità paralizzate, per evitare traumi nel corso del trasporto.

Il paziente, valutato e stabilizzato, per quanto possibile, presso lo scenario iniziale dell'evento, deve essere trasportato nel più breve tempo possibile presso il Pronto Soccorso del più vicino Ospedale dotato di una Stroke Unit (Unità Neurovascolare), reparto ove dovrà essere immediatamente ricoverato, in quanto solo attivando appropriate indagini in regime di degenza, si potranno correttamente diagnosticare sede e natura del danno cerebrale, oltre a garantire all'assi-

Tabella 1. Cincinnati pre-hospital stroke scale.

\begin{tabular}{lll}
\hline Asimmetria faciale & $\mathrm{Si}$ & No \\
\hline Asimmetria arti superiori (Mingazzini) & $\mathrm{Si}$ & No \\
\hline Anomalia del linguaggio & $\mathrm{Si}$ & No \\
\hline
\end{tabular}

Qualora 1/3 di questi segni sia positivo (Si), la probabilità che uno Stroke sia in atto è del $72 \%$

Tabella 2. Glasgow coma scale.

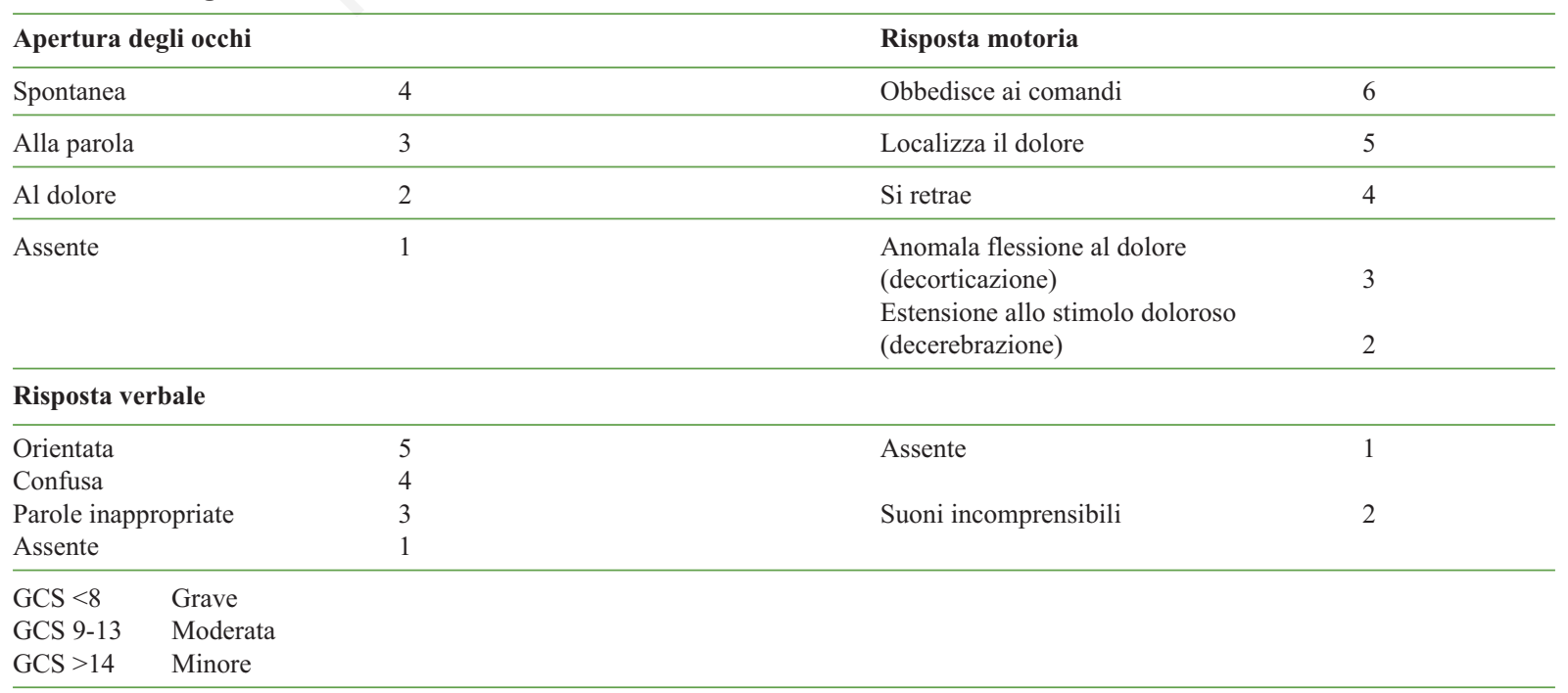


stito la gestione di eventuali complicanze internistiche, iniziando precocemente, ove richiesto, un adeguato programma di riabilitazione.

Il personale di soccorso provvederà pertanto $\mathrm{a}$ preavvisare il Pronto Soccorso, o direttamente il Medico di guardia della Stroke Unit (SU), sull'imminenza dell'arrivo di un paziente con sospetto Ictus. ${ }^{1}$

Una corretta esecuzione di queste procedure è importante, al fine di consentire la massima riduzione dei tempi del onset to imaging (dallo scenario dell'evento all'esecuzione dell'esame neuroradiologico in urgenza), onset to needle (dall'esordio dei sintomi all'inizio dell'eventuale procedura di rivascolarizzazione), e door to needle (dall'arrivo in PS all'inizio dell'eventuale procedura di rivascolarizzazione).

Si configura quindi una stretta collaborazione tra: i) il Servizio di Emergenza/Urgenza territoriale; ii) il Pronto Soccorso dell'Ospedale di destinazione dotato di SU (e/o degli Ospedali della stessa area che ne siano sprovvisti); iii) la Stroke Unit.

\section{L'assistenza in fase acuta dell'ictus: periodo di ospedalizzazione}

Giunto in Ospedale, il paziente va sottoposto ad un monitoraggio continuo delle funzioni vitali e dello stato neurologico (attraverso la National Institute of Health Stroke Scale), che deve essere condotto per almeno 48 ore, od oltre, qualora si riscontri instabilità clinica.

L'adozione di scale validate per la quantificazione del danno neurologico (specificamente, la NIHSS: Tabella 3), consente di facilitare la trasmissione di informazioni tra membri dello Stroke team, identificando i pazienti candidabili a rivascolarizzazione cerebrale, monitorando l'evoluzione clinica ed identificando i soggetti a rischio di complicanze. ${ }^{1}$

Per quanto attiene il controllo delle funzioni vitali, sono raccomandati, in maniera particolare: i) il monitoraggio ECG continuo: qualora il paziente presenti storia di cardiopatia e aritmie, instabilità della Pressione arteriosa, elementi clinici suggestivi di Insufficienza cardiaca, alterazioni dell'ECG di base; e quando lo Stroke coinvolga i territori profondi dell'Arteria cerebrale media (in particolare, la corteccia insulare); alternativamente al monitoraggio ECG continuo, sono attuabili controlli ECG seriati almeno nelle prime 24 ore; ii) il monitoraggio dello stato di ossigenazione ematica, da attuarsi almeno nelle prime 24 ore in caso di Stroke di entità medio-grave, e comunque sino a stabilità del quadro respiratorio.

La somministrazione di ossigeno è indicata, nella fase di stato, solo nei pazienti con $\mathrm{SaO}_{2}<94 \%$. ${ }^{1}$

Il supporto delle vie aeree e l'assistenza ventilatoria sono indicati nei pazienti con Stroke acuto con ridotto stato di coscienza o con disfunzione bulbare. ${ }^{2}$
Per quanto attiene al monitoraggio della Pressione arteriosa nello Stroke ischemico acuto, esso va praticato al fine di trattare in emergenza valori elevati, secondo un protocollo validato dalla American Academy of Neurology (Stroke Coding Guide, marzo 2015, successive modifiche).

Tabella 3. National Institute of Health Stroke Scale (NIHSS).

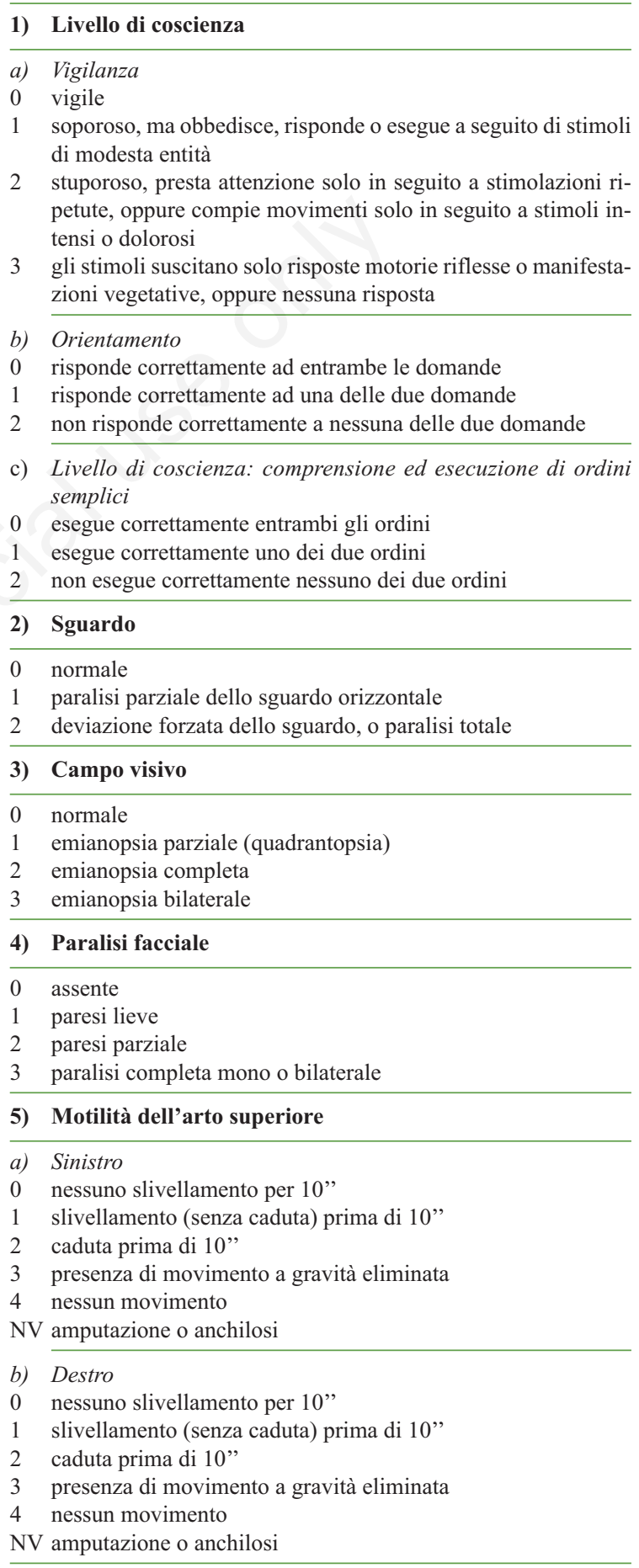

Continua nella pagina seguente 
- Se la Pressione arteriosa diastolica $(\mathrm{Pad})>140$ $\mathrm{mmHg}$ in due misurazioni successive a distanza di 5 minuti, è indicata la somministrazione continua e.v. di un agente antipertensivo (Nitroglicerina, 10$200 \mathrm{microg} / \mathrm{min}$ oppure Nitroprussiato di sodio 0,5-1 $\mathrm{mg} / \mathrm{Kg} / \mathrm{min}$ ), monitorando il rischio di sviluppo di Edema cerebrale.

- Se la Pressione arteriosa sistolica (Pas) $>220$ $\mathrm{mmHg}$, o la Pad è compresa tra 121-140 mmHg, oppure la Pressione arteriosa media $>130 \mathrm{mmHg}$ in due misurazioni successive a distanza di $20 \mathrm{mi}-$ nuti, è indicata la somministrazione di Labetalolo, $10 \mathrm{mg}$ e.v. in 1-2 minuti. Tale dose può essere ripetuta ogni 20 minuti, sino a raggiungere un dosaggio cumulativo di $300 \mathrm{mg}$. Successivamente a tale approccio iniziale, il Labetalolo può essere somministrato ogni 6-8 ore, se necessario.

Tabella 3. Segue dalla pagina precedente

\begin{tabular}{ll}
\hline 6) & Motilità dell'arto inferiore \\
\hline a) & Sinistro \\
0 & nessuno slivellamento per 5" \\
1 & slivellamento (senza caduta) prima di 5" \\
2 & caduta prima di 5" \\
3 & presenza di movimento a gravità eliminata \\
4 & nessun movimento \\
NV & amputazione o anchilosi \\
b) & Destro \\
0 & nessuno slivellamento per 5" \\
1 & slivellamento (senza caduta) prima di 5" \\
2 & caduta prima di 5" \\
3 & presenza di movimento a gravità eliminata \\
4 & nessun movimento \\
NV amputazione o anchilosi \\
\hline 7 7) Atassia degli arti \\
\hline 0 & assente \\
1 & presente all'arto superiore o inferiore \\
2 & presente all'arto superiore e inferiore \\
NV amputazione o anchilosi \\
\hline 8) & Sensibilità \\
\hline 0 & normale \\
1 & ipoestesia lieve o moderata \\
2 & ipoestesia grave \\
\hline 9) & Linguaggio \\
\hline 0 & normale \\
1 & afasia lieve-moderata \\
2 & afasia grave \\
3 & muto, afasia totale \\
\hline
\end{tabular}

\section{0) Disartria}

0 assente

1 disartria lieve-moderata

2 disartria grave

3 intubato o altro impedimento fisico all'articolazione della parola

11) Inattenzione

0 assente

1 inattenzione visiva, tattile, uditiva, spaziale o corporea

2 grave eminattenzione o estinzione a più di una modalità
- Esso è controindicato nei pazienti con asma, scompenso cardiaco, o gravi turbe della conduzione. In tali casi, l'alternativa è costituita dall'Urapidil, 10$50 \mathrm{mg}$ in bolo, ovvero in infusione $0,15-0,5 \mathrm{mg} / \mathrm{min}$.

- Se PAs=185-220 mmHg, o PAd=105-120 $\mathrm{mmHg}$, la terapia di emergenza va rimandata, tranne che nei seguenti casi: paziente con insufficienza ventricolare sn, con dissecazione aortica, con Infarto miocardico acuto. nel paziente candidabile a trombolisi endovenosa, è indicato mantenere valori di PAs $<185 \mathrm{mmHg}$ e di PAd $<110 \mathrm{mmHg}{ }^{1}$

Ovviamente, mentre si monitora, ed eventualmente si corregge, il valore di Pressione Arteriosa, è necessario tenere sotto controllo il quadro neurologico, per coglierne eventuali deterioramenti.

Nell'emorragia cerebrale, il controllo della PA è volto a raggiungere rapidamente una $\mathrm{PAs}<140 \mathrm{mmHg}$, mantenendo tale target per almeno 24 ore, e preferibilmente per i primi 7 giorni. I farmaci usati sono gli stessi adottati nello Stroke ischemico. ${ }^{1}$

Se nei primi giorni dell'evento ictale la PA risultasse $>140 / 90 \mathrm{mmHg}$, a paziente neurologicamente stabile, è ragionevole ripristinare la terapia antipertensiva precedentemente assunta, o comunque introdurne una nuova. ${ }^{2}$

Una recente review ${ }^{4}$ effettuata dall'analisi dei dati riguardanti 56.513 pazienti, sottoposti a trombolisi e.v. con tPA, ha ribadito l'importanza di un adeguato controllo pressorio nella fase acuta dello Stroke (secondo linee guida), per consentire al paziente un miglior outcome ed un'indipendenza funzionale a 3 mesi. Per contro, un'instabilità dei valori pressori è associata non solo a maggior rischio di emorragia intracranica, ma anche a minore probabilità di ricanalizzazione nei pazienti con occlusione dei grossi vasi intracranici che vengano sottoposti a trombolisi e.v. e trombectomia meccanica. Ciò avviene in relazione al fatto che l'area di penombra ischemica è più suscettibile a danno da riperfusione in caso di elevati valori pressori.

Ma il monitoraggio della Pressione arteriosa nello Stroke acuto è volto anche ad individuare e trattare eventuali valori ipotensivi. Qualora il paziente presenti segni di disidratazione e/o valori di PA significativamente inferiori a quelli usuali, andranno somministrati fluidi e.v (preferenzialmente cristalloidi: soluzione fisiologica), trattando, qualora presente, lo scompenso cardiaco congestizio e la bradicardia; eventualmente, qualora indicato, si potrà ricorrere alla somministrazione di agenti vasopressori (dopamina). ${ }^{1}$

Altro parametro da sorvegliare nel paziente affetto da Stroke acuto è la Temperatura corporea. Infatti, circa la metà dei pazienti con Ictus cerebrale presenta ipertermia nell'arco delle 48 ore dell'insorgenza dall'evento, ed essa costituisce fattore prognostico negativo nell'Ictus ischemico ed emorragico, associandosi sia a peggioramento del quadro clinico che dell'out- 
come. La Temperatura va pertanto mantenuta al di sotto dei $37^{\circ} \mathrm{C}$, adottando il paracetamolo quale farmaco antipiretico di scelta. ${ }^{1}$

Più del $50 \%$ dei pazienti con Stroke presenta valori elevati di glicemia nella fase acuta.

Secondo alcuni studi, l'iperglicemia può associarsi a maggiore gravità della lesione ischemica cerebrale, con maggiore rischio di mortalità nella prima settimana $(\mathrm{d}) .^{5}$

Pertanto, secondo le linee guida SPREAD sinora vigenti, in pazienti con Stroke acuto e glicemia $>180$ $\mathrm{mg} / \mathrm{dL}$, è indicata insulinoterapia per correggere l'iperglicemia. Un range ideale di valore glicemico è compreso tra 140 e $180 \mathrm{mg} / \mathrm{dL} .{ }^{1}$

Le linee guida ESO 2018, tuttavia, non supportano la somministrazione routinaria di insulina e.v. nello Stroke ischemico ed emorragico acuto, effettuata previo stretto controllo glicemico, al fine di migliorare l'outcome funzionale e la sopravvivenza, limitando l'estensione dell'area ischemica. Ciò alla luce della debole evidenza scientifica di tale condotta, emersa da ampia revisione della letteratura (5897 fonti), ed anche alla luce del rischio di ipoglicemia.

Resta però indicata, come negli altri pazienti critici, una terapia volta ad evitare picchi iperglicemici. ${ }^{3}$

Il range glicemico consigliato è compreso tra 7,8 e $10 \mathrm{mmol} / \mathrm{L}(141-180 \mathrm{mg} / \mathrm{dL})$. Riguardo al limite glicemico oltre il quale iniziare il trattamento, lo studio SHINE (Stroke Hyperglicemia Insulin Network Effort) propone $110 \mathrm{mg} / \mathrm{dL}$ nel paziente con Diabete già noto, e $150 \mathrm{mg} / \mathrm{dl}$ nel paziente non diabetico. ${ }^{6}$

E' altresì indicata, dalle linee guida SPREAD, la correzione dell'ipoglicemia (valore $<60 \mathrm{mg} / \mathrm{dL}$ ), ricorrendo ad infusione di glucosio in bolo e.v. (glucosio al $20-40 \%, 25-50 \mathrm{~mL}$ in infusione). ${ }^{3}$

Ciò in relazione al fatto che, in occasione di un danno cerebrale acuto, vi è un incremento della domanda metabolica (e pertanto glucidica) dell'encefalo, così che l'evenienza di ipoglicemia risulta particolarmente dannosa nella fase acuta dello Stroke.

La presenza di disfunzioni vescicali nell'Ictus acuto costituisce fattore prognostico negativo per mortalità e disabilità residua grave. Per questo motivo, il posizionamento di un catetere vescicale è indicato in caso di grave disfunzione vescicale. Anche qualora non venga attuato il cateterismo a permanenza, è indicato il controllo periodico del residuo post-minzionale, adottando, in caso positivo, la cateterizzazione sterile intermittente. ${ }^{1}$

Obiettivo importante dell'assistenza del paziente con Ictus in fase acuta è la prevenzione delle trombosi venose profonde. Essa si attua con metodiche non farmacologiche (mobilizzazione precoce, adeguata idratazione) e con metodiche farmacologiche.

In pazienti ad alto rischio (plegici, con alterazione dello stato di coscienza, e/o obesi, e/o con pregressa patologia venosa agli arti inferiori), si raccomanda l'uso di eparina a dosi profilattiche (eparina calcica non frazionata $5000 \mathrm{UI}$ x2/die, oppure eparina a basso peso molecolare: dalteparina $5000 \mathrm{UI} /$ die, enoxaparina $4000 \mathrm{UI} / \mathrm{die}$, nadroparina $3800 \mathrm{UI} / \mathrm{die}$ ), da iniziare, nello Stroke ischemico, al momento dell'ospedalizzazione, ed in quello emorragico tra il I ed il IV giorno dall'esordio, e dopo la cessazione delle attività di sanguinamento.

In pazienti selezionati, in alternativa al trattamento medico (pazienti emorragici), sono raccomandati i devices a compressione graduale.

Uno stato di malnutrizione è frequente nel paziente colpito da Stroke, tendendo ad aggravarsi nel corso del ricovero. Esso è associato ad un aumentato rischio di mortalità, morbilità (infezioni, piaghe da decubito), ed a un outcome peggiore, con recupero funzionale, nel complesso, più lento (in relazione ad ipotrofia muscolare ed a conseguente disabilità motoria).

Garantire un adeguato stato nutrizionale consente di ottenere tempi di ospedalizzazione più brevi, migliore qualità della vita, ottimizzando il percorso terapeutico e riabilitativo.

Per quanto esposto, si comprende come sia molto importante, nel paziente con Ictus, rilevare con appositi parametri lo stato nutrizionale ed il rischio nutrizionale.

Ciascun paziente, all'atto del ricovero (entro 2448 ore), andrebbe valutato individualmente per quanto attiene ai fabbisogni e al trattamento nutrizionale di fase acuta, e dovrebbe essere successivamente rivalutato in itinere, sistematicamente, durante la degenza, con l'apporto di specifiche figure professionali (Medico nutrizionista, Dietista), in relazione allo stato di nutrizione, ed alle comorbidità, complicanze e terapie.

Nel paziente in grado di mantenere la stazione eretta, sono indicati misure ed indici antropometrici quali: i) la circonferenza della vita; ii) l'Indice di Massa Corporea (IMC); iii) la stima del decremento ponderale non intenzionale.

Nel paziente non deambulante, sono indicate le seguenti misure antropometriche: i) peso corporeo; ii) semiampiezza delle braccia (in luogo dell'altezza); iii) circonferenza del braccio (alternativa, per il calcolo dell'IMC).

Nel protocollo di valutazione dello stato nutrizionale sono indicati: i) la valutazione dell'assunzione dietetica (questionario di valutazione semiquantitativo degli scarti alimentari); ii) la valutazione clinica; iii) il dosaggio dell'albumina e la conta dei linfociti sono valutazioni biochimiche essenziali.

Gli obiettivi del supporto nutrizionale in fase acuta sono la prevenzione o il trattamento di: i) malnutrizione proteico-energetica (MNPE); ii) squilibri idroelettrolitici; iii) carenze selettive (minerali, vitamine).

Il timing e la scelta della modalità di somministra- 
zione della dieta dipendono dalle condizioni cliniche, dallo stato nutrizionale e dalla capacità deglutitoria del paziente.

Si prospettano pertanto le seguenti opzioni di programma nutrizionale: i) paziente non disfagico normonutrito: alimentazione per os, secondo profilo nutrizionale del LARN (Livelli di Assunzione Raccomandata di energie e Nutrienti) e delle Linee Guida per una Sana Alimentazione; ii) paziente non disfagico con MNPE, ovvero a rischio di MN o di piaghe da decubito: alimentazione per os con aggiunta di integratori dietetici per os; iii) paziente disfagico: adattamento progressivo della dieta alla funzionalità deglutitoria ed alla capacità di preparazione del bolo o nutrizione enterale, eventualmente integrate.

In caso di apporto nutrizionale insufficiente e prolungato, è raccomandabile una supplementazione nutrizionale previo consulto del dietista.

Nel paziente non disfagico, non è dimostrata l'efficacia di una supplementazione dietetica orale (proteica, energetica, a base di integratori dietetici di routine), ai fini di un miglioramento significativo della prognosi.

Nel paziente disfagico, l'impostazione del trattamento nutrizionale richiede una valutazione preliminare della deglutizione, e va pianificata tenendo conto del rischio di polmonite ab ingestis, del grado di autonomia e dello stato nutrizionale del paziente.

Le possibilità sono costituite dalla dieta progressiva per disfagia e dalla nutrizione artificiale: in caso di disfagia completa, è indicata la sola nutrizione artificiale. E' indicata la monitorizzazione regolare degli apporti nutrizionali e del bilancio dei fluidi.

Le diete speciali, basate su alimenti e bevande a consistenza e densità motivate, devono essere prescritte sulla base di un'attenta valutazione da parte di figure professionali specializzate (nutrizionisti, dietisti e logopedisti).

La nutrizione artificiale di scelta è costituita dalla nutrizione enterale. Essa avviene attraverso Sondino naso-gastrico, secondo procedura ormai standardizzata da linee guida nazionali ed internazionali.

Quali sono i pazienti candidabili a nutrizione enterale?

I pazienti con grave stato di malnutrizione $a b$ initio e/o con stato di coscienza alterato, sottoposti a ventilazione meccanica, e/o i pazienti a rischio di disfagia protratta, poiché presentano elevato rischio di malnutrizione, di aspirazione e quindi di polmonite $\mathrm{ab}$ ingestis.

Secondo il FOOD Trial, una nutrizione enterale precoce riduce, seppur non significativamente dal punto di vista statistico, la mortalità e l'endpoint combinato morte/esito sfavorevole, da cui scaturiscono le raccomandazioni di iniziare la nutrizione enterale entro 2-3 giorni nel paziente con malnutrizione, ed entro 5-7 giorni nel paziente normonutrito.

L'alternativa al Sondino naso-gastrico è costituita dalla PEG, che, stando a quanto definito da alcuni trial randomizzati controllati, è preferibile nei pazienti in ventilazione meccanica, per i quali si preveda un'alimentazione artificiale prolungata $(>14$ giorni), in relazione alla minore incidenza di polmonite.

Il Trial FOOD-STUDY ha però stabilito che il pazienti portatori di SNG presentano, a 6 mesi, rispetto a quelli portatori di PEG, un minor rischio di mortalità e di sviluppo di piaghe da decubito, con outcome migliore.

Le linee guida SPREAD hanno sintetizzato queste evidenze scientifiche, raccomandando il posizionamento di SNG nelle prime 2-3 settimane dall'evento in presenza di disfagia severa, limitando il ricorso alla PEG ai pazienti per i quali non si preveda risoluzione della disfagia nelle successive 4-6 settimane (ventilazione meccanica).

La Nutrizione parenterale è indicata esclusivamente laddove la via enterale non sia realizzabile, o sia controindicata, o quale supplementazione alla nutrizione enterale, qualora quest'ultima non consenta di ottenere adeguata somministrazione di liquidi e di nutrienti. ${ }^{1}$

\section{Conclusioni: stabilizzazione clinica ed avvio percorso riabilitativo dello stroke}

Sin dalla fase acuta ospedaliera dell'ictus, è raccomandabile avviare un percorso riabilitativo, al fine di definire la prognosi funzionale, organizzare il percorso assistenziale, avviare attività di prevenzione di complicanze legate all'immobilità e promuovere il recupero funzionale. Vanno offerti al paziente livelli di intensità del trattamento riabilitativo adeguati alle condizioni cliniche ed alle potenzialità di recupero attraverso il Progetto Riabilitativo Individuale articolato in programmi applicati dalle figure professionali disponibili ed appropriate (medico, fisioterapista, logopedista, neuropsicologo, terapista occupazionale ed infermiere). ${ }^{1}{ }^{1}$ 'iter riabilitativo verrà successivamente proseguito, in fase di post-acuzie, presso idonea Struttura, o presso il domicilio del paziente (Assistenza Domiciliare Integrata), in relazione al setting identificato in relazione alle condizioni dell'assistito.

\section{Bibliografia}

1. Linee guida spread, viii edizione; 2017.

2. 2018 guidelines for the early management of patients with acute ischemic stroke: a guideline for healthcare professionals from the AHA/ASA. Stroke 2018;49:e46-e110.

3. EAN-ESO consensus statement and practical guidance 
for pre-hospital management of stroke. Eur J Neurol 2018;25:425-33.

4. Malhotra K, et al. Association of elevated blood pressure levels with outcomes in acute ischemic stroke patients treated with intravenous thrombolysis; a systematic review and metanalysis. J Stroke 2019;21:78-90.
5. Mc Call SJ, et al. Hyperglycaemia and the soar stroke score in predicting mortality. Vasc Dis Res 2018;15:114-21.

6. Johnston $\mathrm{KC}$, et al. Intensive vs standard treatment of hyperglycemia and functional outcome in patients with acute ischemic stroke: the shine randomized clinical trial.2019;322:326-35. 


\title{
Trombolisi endovenosa
}

\author{
Paolo Candelaresi, ${ }^{1}$ Vincenzo Andreone ${ }^{2}$ \\ ${ }^{1}$ UOSC Neurologia e Stroke Unit, AORN Antonio Cardarelli, Napoli; ${ }^{2}$ UOC Neurologia, AORN S. Anna e S. Sebastiano, \\ Caserta, Italia
}

\section{Introduzione}

Dopo circa 20 anni di trattamento trombolitico endovenoso nell'ictus ischemico, vengono riportate le maggiori evidenze a supporto di un trattamento che ha costituito e costituisce la prima linea di trattamento nella riperfusione dei territori ischemici, inizialmente limitato da una lunga lista di controindicazioni, per lo più arbitrarie, che nel corso del tempo sono state superate, garantendo l'accesso alla terapia ad una porzione di pazienti molto più consistente.

Lo scopo del trattamento trombolitico nell'ictus ischemico è ricanalizzare l'arteria occlusa al fine di garantire la riperfusione del tessuto cerebrale ischemico ed il miglioramento dei sintomi clinici.

Immediatamente dopo un'occlusione arteriosa cerebrale, si sviluppa una zona di ipoperfusione. Contestualmente si assiste all'attivazione di una circolazione collaterale di compenso, attraverso il poligono di Willis e le anastomosi corticali di Heubner, che produce un gradiente di perfusione ematica decrescente dal centro verso la periferia: ${ }^{1}$ i) nel nucleo ischemico, il flusso ematico si riduce al di sotto di $12 \mathrm{ml} / 100 \mathrm{~g}$ di tessuto cerebrale/minuto provocando una lesione anossica irreversibile (core ischemico), con necrosi cellulare in pochi minuti; ii) nell'area circostante, la circolazione collaterale fornisce un flusso sanguigno residuo compreso tra 12 e $22 \mathrm{~mL} / 100 \mathrm{~g}$ di tessuto cerebrale/minuto: in questo range di perfusione ematica - rispettivamente la soglia dell'esaurimento funzionale delle pompe ioniche di membrana e la soglia della

Corrispondente: Paolo Candelaresi, UOSC Neurologia e Stroke Unit, AORN Antonio Cardarelli, via Antonio Cardarelli 9, 80131 Napoli, Italia.

Tel.: +39.081.7472502.

E-mail: paolo.candelaresi@aocardarelli.it

Articolo pubblicato secondo la Creative Commons Attribution NonCommercial 4.0 License (CC BY-NC 4.0).

${ }^{\circ}$ Copyright: the Author(s), 2020

Licensee PAGEPress, Italy

QUADERNI - Italian Journal of Medicine 2020; 8(2):54-62 compromissione elettrica -, il tessuto cerebrale è elettricamente silente (cioè funzionalmente compromesso) ma morfologicamente integro (cioè ancora vitale) e potenzialmente salvabile (penombra ischemica); 2 iii) l'estrema periferia dell'area ischemica, infine, è caratterizzata da ipoperfusione non critica non sintomatica (oligoemia benigna).

Tale gradiente perfusivo è dipendente dall'adeguatezza del circolo collaterale e dall'avanzare del tempo. E' pertanto intuibile come l'obiettivo di tutte le terapie di fase acuta, in particolare del trattamento trombolitico, sia quello di riperfondere il territorio ischemico, nel più breve tempo possibile, salvando il tessuto in penombra.

\section{Trombolisi endovenosa: un percorso impervio tra studi clinici e mondo reale}

Ripercorrendo l'esperienza della riperfusione coronarica, tra la fine degli anni ' 70 e l'inizio degli anni ' 80 , inizia la ricerca sugli agenti trombolitici nell'ictus ischemico e sin da subito diviene evidente che gli attivatori del plasminogeno hanno la capacità di sciogliere il coagulo aumentando però il rischio emorragico. ${ }^{3}$

Negli anni ' 90 vengono pubblicate le prime esperienze multicentriche randomizzate controllate sulla trombolisi sistemica nell'ictus ischemico acuto. ${ }^{4}$ Tre studi randomizzati controllati con streptochinasi vengono interrotti prematuramente a causa di un eccesso di trasformazioni emorragiche sintomatiche e di mortalità, sia precoce che tardiva, a fronte di un'assenza di vantaggio nella riduzione della disabilità, nel braccio dei pazienti trattati rispetto a placebo. Tra le possibili motivazioni di tale fallimento sono state annoverate, più che una scarsa efficacia della molecola, la contemporanea somministrazione di acido acetilsalicilico nelle prime 24 ore dal trattamento: un'analisi di confronto post-hoc evidenziava, infatti, che tale associazione è gravata da un rischio significativamente più elevato di trasformazione emorragica severa e morte rispetto al trattamento con sola streptochinasi.

Nel 1995, lo studio no-profit finanziato dal NINDS (National Institute of Neurological Disorders and Stroke $)^{5}$ dimostra per la prima volta l'efficacia dell'at- 
tivatore tissutale del plasminogeno ottenuto con tecnica ricombinante (rt-PA), somministrato per via endovenosa, nei pazienti con ictus ischemico acuto. I criteri di inclusione nello studio erano: i) tempo ben definito di esordio dei sintomi, non superiore a 3 ore; ii) deficit neurologico misurabile alla scala NIHSS (National Institute of Health Stroke Scale); e iii) TC encefalo negativa per emorragia. La dose calcolata di farmaco era di $0,9 \mathrm{mg} / \mathrm{kg}$ di peso corporeo, il $10 \%$ del quale somministrato in bolo di 1 minuto e la restante quota in infusione di un'ora. Il trattamento con rt-PA risultava associato ad un aumento significativo delle probabilità di esito clinico favorevole (OR, 1,7;95\% CI, 1,2-2,6), definito come punteggio 0-1 alla scala Rankin modificata (mRS), con una riduzione relativa del rischio di dipendenza di circa il $30 \%$.

Il principale evento avverso associato al trattamento con rt-PA era la trasformazione emorragica: nel $6,4 \%$ dei pazienti trattati rispetto allo $0,6 \%$ dei controlli $(\mathrm{P}<0.001)$, la metà delle quali, circa, fatale. La mortalità complessiva, tuttavia, non risultava significativamente aumentata (17\% rt-PA contro $21 \%$ placebo). Il trattamento con rt-PA risultava associato ad un beneficio clinico indipendentemente dall'età del paziente, dal sottotipo eziologico di ictus, dalla gravità clinica o neuroradiologica al basale.

Sin dallo studio NINDS è parso evidente come il tempo sia la sola variabile in grado di influenzare l'effetto del trattamento: eseguita entro 90 minuti dall'insorgenza dei sintomi, la trombolisi endovenosa si associa ad una probabilità doppia di esito clinico favorevole rispetto a placebo (OR 2,11; 95\% CI, 1,33$3,55)$, che si attenua nella seconda metà della finestra terapeutica (OR 1,69; 95\% CI, 1,09-2,62 per trattamenti tra 90 e 180 minuti dall'esordio dei sintomi). Inoltre, l'intervallo di tempo tra l'inizio dei sintomi e del trattamento non si associa ad un aumento del rischio di trasformazione emorragica.

Sulla base dei risultati dello studio NINDS, nel 1996 la Food and Drug Administration (FDA) negli Stati Uniti e nel settembre 2002 l'Agenzia europea del farmaco (EMA) hanno approvato la trombolisi endovenosa con rt-PA, dando di fatto inizio ad una nuova era nel trattamento dell'ictus ischemico acuto.

Due importanti limitazioni dello studio NINDS erano la finestra terapeutica molto ristretta ( 3 ore) ed una lunga serie di controindicazioni, per lo più arbitrarie e votate a ridurre le complicanze emorragiche dopo il fallimento degli studi con streptochinasi. Ciò limitava enormemente l'accesso alla terapia, fruibile solamente per una ristretta minoranza di pazienti.

Pertanto, negli anni successivi, sono stati condotti diversi studi clinici con l'obiettivo di ampliare la finestra terapeutica per la trombolisi endovenosa, poiché la maggior parte dei pazienti colpiti da ictus si presenta all'osservazione medica oltre le tre ore dal- l'esordio dei sintomi. Gli studi ECASS I $\mathrm{I}^{6}$ II, ${ }^{7}$ ATLANTIS A e ATLANTIS $\mathrm{B}^{8}$ hanno arruolato pazienti oltre le tre ore suggerendo un potenziale beneficio dalla terapia nella finestra temporale di 3-4,5 ore.

Nel settembre 2008, lo studio europeo ECASS III (European Cooperative Acute Stroke Study III) ${ }^{9}$ riesce a dimostrare un beneficio significativo del trattamento trombolitico con rt-PA per pazienti colpiti da ictus ischemico entro 4,5 ore dall'esordio: il $52,4 \%$ dei pazienti trattati contro il $45,2 \%$ dei casi controllo raggiunge una piena indipendenza a tre mesi (OR 1,34; 95\% CI, 1,02-1,76). Anche lo studio ECASS III conferma che il trattamento trombolitico è causa di un significativo eccesso nelle trasformazioni emorragiche sintomatiche $(2,4 \%$ vs. $0,2 \%$; OR 9,$85 ; 95 \%$ CI, $1,26-$ $77,32)$ che tuttavia non impattano sulla mortalità globale (OR 0,90; 95\% CI, 0,54-1,49).

Una pooled analysis degli studi randomizzati controllati, ${ }^{10}$ nel 2010, ha dimostrato che il beneficio del trattamento, in termini di indipendenza funzionale, è strettamente tempo-dipendente ed è massimo nei primi 90 minuti dall'esordio dei sintomi [number needed to treat (NNT) 4,5 per avere un paziente indipendente in più rispetto a placebo] e decresce progressivamente con l'avanzare del tempo: NNT 9 a 180 minuti e NNT 14 a 270 minuti. Il rischio di trasformazione emorragica è dipendente solamente dall'aver ricevuto il trattamento trombolitico, ma non dal tempo intercorso tra inizio dei sintomi e terapia. Per la mortalità, infine, si osserva una più lieve tempo-dipendenza.

I registri internazionali, in particolare il SITSMOST ed il SITS-ISTR (Safe Implementation of Thrombolysis in Stroke - Monitoring Study e International Stroke Thrombolysis Register), hanno non solo confermato, nel mondo reale, il beneficio del trattamento trombolitico che si è osservato negli studi clinici, ma anzi, hanno dimostrato una magnitudine di effetto maggiore, con oltre la metà dei pazienti trattati vivi e indipendenti al follow-up. ${ }^{11}$ L'estensione della finestra terapeutica a 4,5 ore, inoltre, ha consentito di trattare il $25 \%$ dei pazienti in più, rispetto alla precedente finestra temporale di 3 ore, senza un significativo aumento dei ritardi intra-ospedalieri. E' stato infine confermato, anche nel mondo reale, come il ritardo terapeutico sia la sola variabile in grado di influenzare significativamente il beneficio atteso dal trattamento e di conseguenza l'outcome clinico: secondo i dati del registro ictus statunitense, per ogni 15 minuti risparmiati dall'esordio dei sintomi al trattamento, 18 pazienti in più vengono dimessi in grado di deambulare e 4 pazienti in meno muoiono durante il ricovero. ${ }^{12}$

Lo studio IST- $3^{13}$ (International Stroke Study - 3) nasce nel 2000, con l'obiettivo di testare l'efficacia del trattamento trombolitico in una popolazione più ampia e più eterogenea di quanto mai fatto prima. Le principali 
domande a cui l'IST-3 tenta di rispondere sono: i) la finestra terapeutica può essere estesa a 6 ore dall'esordio dei sintomi? ii) la popolazione anziana, ultraottantenne, può beneficiare del trattamento? iii) quali fattori sono in grado di predire la trasformazione emorragica? iv) esistono caratteristiche neuroradiologiche basali in grado di predire la risposta al trattamento?

Non senza difficoltà e con emendamenti al protocollo originale, dovuti per lo più all'approvazione del trattamento trombolitico nella finestra 3-4,5 ore, lo studio IST-3 randomizza 3035 pazienti in 156 centri, diventando, a tutt'oggi, il più grande studio randomizzato controllato nella storia della riperfusione dell'ictus acuto. Lo studio IST-3 manca il suo obiettivo principale: il trattamento trombolitico in una finestra terapeutica 0 6 ore, non si associa ad un guadagno significativo nella percentuale di pazienti vivi e indipendenti (mRS 0-2) a 3 mesi. Ciononostante, viene dimostrato un significativo incremento dei pazienti senza alcuna disabilità (mRS 0-1) ed una significativa riduzione della probabilità di avere una disabilità più elevata rispetto ai pazienti non trattati (ordinal shift analysis).

Questo dimostra che l'eterogeneità dell'outcome primario negli studi clinici è un problema reale, che finisce per influenzare la pratica clinica. Infatti, se lo studio IST-3 avesse conservato lo stesso obiettivo dello studio NINDS (mRS 0-1), sarebbe risultato uno studio positivo.

$\mathrm{Al}$ di là di ciò, l'IST-3 e la successiva metanalisi ${ }^{14}$ di tutti gli studi clinici randomizzati controllati confermano che esiste un beneficio dal trattamento trombolitico che è tempo-dipendente, massimo nelle prime ore e poi progressivamente minore fino a 6 ore e che il beneficio statistico si perde poco dopo le 5 ore.

Il grande risultato dello studio IST-3 è, invece, la dimostrazione che il trattamento è sicuro ed efficace anche nel paziente anziano ultraottantenne. Storicamente, questa categoria di pazienti veniva esclusa dagli studi per una percezione di maggiori rischi e diminuiti benefici. Nell'IST-3, 1617 ultraottantenni vengono randomizzati (oltre la metà dell'intera popolazione dello studio) e viene dimostrato che il beneficio per questi pazienti è almeno sovrapponibile ai pazienti più giovani, con un beneficio assoluto del $9.6 \%$ rispetto ai non trattati nella finestra $0-3$ ore e che l'età $>80$ anni non riduce la finestra di efficacia del trattamento trombolitico $(\mathrm{P} 0,08$ in direzione di un più lungo periodo di efficacia).

L'effetto globale della terapia sulla mortalità è multifasico: durante la prima settimana di follow up, la mortalità è significativamente aumentata $(11 \%$ contro $7 \%$ ) nei pazienti sottoposti a trattamento trombolitico, a causa dell'eccesso di trasformazioni emorragiche (7\% contro 1\%); al follow up di medio-termine (3-6 mesi) il dato si pareggia ( $27 \%$ in entrambi i gruppi); al follow up di lungo termine (18 mesi), si dimostra una riduzione significativa della mortalità per i pazienti trattati entro 3 ore (ARR 7,2\%; P 0,04). ${ }^{15}$

Lo studio IST-3 ha inoltre il merito di contribuire ad abbattere numerose controindicazioni relative, per lo più arbitrarie, che avevano limitato l'accesso al trattamento per molti pazienti. Lo studio, infatti, dimostra come alcune caratteristiche, quali sostanziali comorbidità internistiche ed una maggiore gravità dei sintomi in esordio, si associano ad un più elevato rischio di trasformazione emorragica, ma che il loro impatto è modesto e non mostra una significativa interazione con il beneficio netto derivante dal trattamento in termini di morte o disabilità e, pertanto, non devono costituirne una limitante. ${ }^{16}$

Allo stesso modo, in un'analisi pre-specificata, ${ }^{17}$ sono state prese in esame alcune caratteristiche neuroradiologiche basali che in precedenti analisi posthoc $^{18}$ erano state messe in correlazione con il rischio di trasformazione emorragica e di outcome sfavorevole. Nell'IST-3, la presenza di iperdensità dell'arteria occlusa, la presenza (ma non il grado di estensione) di ipodensità precoce e di lesioni ischemiche pre-esistenti correlano con un aumentato rischio di trasformazione emorragica. Ipodensità precoce, edema cerebrale precoce, iperdensità dell'arteria occlusa, atrofia e leucoaraiosi si associano, invece, ad un aumentato rischio di dipendenza al follow up. Ciononostante, nessuno di questi segni, ivi compreso un basso punteggio ASPECTS, preso singolarmente o in associazione, è in grado di modificare l'effetto del trattamento finale sull'outcome e non dovrebbe pertanto precludere alla terapia trombolitica.

\section{Attuali raccomandazioni e controindicazioni}

Se gli studi clinici randomizzati controllati, nel corso degli anni, sono stati mirati per lo più all'estensione della ristretta finestra terapeutica, i registri locali o internazionali raccogliendo dati prospettici di pazienti trattati nel mondo reale, hanno reso possibile riconsiderare la lunga lista di controindicazioni al trattamento. Storicamente, infatti, per ottimizzare al massimo il rapporto rischio-beneficio, numerose limitazioni, la maggior parte delle quali senza una forte evidenza, sono state poste al trattamento, rendendolo fruibile solo per una ristretta minoranza di pazienti.

In numerosi pazienti, trattati al di fuori delle indicazioni convenzionali, è stato dimostrato un beneficio dal trattamento trombolitico. La Tabella 1 riporta le raccomandazioni 2019 dell' American Heart Association. ${ }^{19-21}$

Per quanto riguarda pazienti con ictus ischemico $<3$ mesi, che si presentano con recidiva, il trattamento trombolitico andrebbe considerato caso per caso, in base al tempo trascorso, alla disabilità pregressa, alla gravità clinica della recidiva, al beneficio atteso e all'estensione della lesione pregressa. Purtroppo una chiara indicazione ancora non è definita. ${ }^{22}$ 
Tabella 1. Raccomandazioni 2019 American Heart Association.

\begin{tabular}{|c|c|}
\hline Segni precoci in TC & Il trattamento è indicato in caso di segni precoci in TC di lieve e moderata estensione \\
\hline Pretrattamento con antiaggreganti & Il trattamento è indicato in pazienti in terapia antiaggregante singola o doppia \\
\hline Insufficienza renale terminale & $\begin{array}{l}\text { Il trattamento è indicato in pazienti con insufficienza renale terminale o emodializzati. } \\
\text { L'allungamento del aPTT potrebbe aumentare il rischio emorragico }\end{array}$ \\
\hline Disabilità pre-esistente o demenza & $\begin{array}{l}\text { Il trattamento per pazienti con } \mathrm{mRS}>2 \text { può essere ragionevole ma la decisione deve essere } \\
\text { pesata caso per caso sul beneficio atteso }\end{array}$ \\
\hline Sintomi in miglioramento spontaneo & Il trattamento è ragionevole se i sintomi residui sono ancora disabilitanti \\
\hline Crisi epilettica in esordio & $\begin{array}{l}\text { Il trattamento è ragionevole se i sintomi residui sono secondari ad ictus (anche mediante } \\
\text { l'utilizzo di neuroimmagini avanzate) e non a stato post-critico }\end{array}$ \\
\hline Ipo- o iperglicemia & $\begin{array}{l}\text { Il trattamento è ragionevole dopo correzione di valori di glicemia }<50 \mathrm{mg} / \mathrm{dL} \text { o superiore } \\
\text { a } 400 \mathrm{mg} / \mathrm{dL}\end{array}$ \\
\hline Coagulopatia & $\begin{array}{l}\text { E' ragionevole che il trattamento non sia ritardato dall'attesa degli esami ematici, in } \\
\text { assenza di forte sospetto di alterazioni coagulative }\end{array}$ \\
\hline Terapia con eparina & $\begin{array}{l}\text { Il trattamento è indicato in pazienti in terapia con dosi profilattiche di EBPM, resta } \\
\text { controindicato per le dosi terapeutiche }\end{array}$ \\
\hline Terapia con DOAC & Il trattamento è indicato se l'ultima assunzione è $>48$ ore e la funzione renale conservata* \\
\hline Puntura lombare & $\begin{array}{l}\text { Il trattamento può essere considerato anche per pazienti che hanno ricevuto una puntura } \\
\text { lombare nei } 7 \text { giorni precedenti }\end{array}$ \\
\hline Puntura di vaso arterioso & $\begin{array}{l}\text { I rischi e i benefici derivanti dal trattamemto in pazienti con puntura di vaso arterioso non } \\
\text { comprimibile nei precedenti } 7 \text { giorni sono incerti }\end{array}$ \\
\hline Trauma maggiore (non cranico) & $\begin{array}{l}\text { Il trattamento può essere attentamente considerato pesandone rischi e benefici in pazienti } \\
\text { con trauma maggiore (non cranico) nei precedenti } 14 \text { giorni }\end{array}$ \\
\hline Chirurgia maggiore & $\begin{array}{l}\text { Il trattamento può essere attentamente considerato pesandone rischi e benefici in pazienti } \\
\text { con intervento chirurgico maggiore nei precedenti } 14 \text { giorni }\end{array}$ \\
\hline Sanguinamento gastrointestinale o genito-urinario & $\begin{array}{l}\text { Il trattamento è ragionevole in pazienti con storia di sanguinamento gastrointestinale o } \\
\text { genito-urinario, in quanto non si associa ad elevato rischio di emorragia }\end{array}$ \\
\hline Mestruazioni/menorragia & $\begin{array}{l}\text { Il trattamento è indicato in donne durante le mestruazioni, senza storia di menorragia } \\
\text { determinante significativa anemizzazione }\end{array}$ \\
\hline Dissezione & $\begin{array}{l}\text { In caso di dissezione cervicale il trattamento è indicato; in caso di dissezione intracranica } \\
\text { i rischi e i benefici del trattamento rimangono incerti }\end{array}$ \\
\hline Aneurismi cerebrali & $\begin{array}{l}\text { Il trattamento è indicato in pazienti con aneurismi asintomatici }<10 \mathrm{~mm} \text {; i rischi e i } \\
\text { benefici derivanti dal trattamento rimangono incerti per pazienti con aneurismi di } \\
\text { dimensioni maggiori }\end{array}$ \\
\hline Malformazioni artero-venose & $\begin{array}{l}\text { I rischi e i benefici derivanti dal trattamento rimangono incerti per pazienti con } \\
\text { malformazioni artero-venose cerebrali e la trombolisi endovenosa potrebbe essere } \\
\text { considerata in pazienti con sintomi molto severi e rischio di morte/disabilità talmente } \\
\text { elevati da superare il rischio di sanguinamento. I cavernomi sembrano avere un profilo } \\
\text { rischio-beneficio più favorevole }\end{array}$ \\
\hline Microsanguinamenti cerebrali (CMBs) & $\begin{array}{l}\text { Il trattamento è ragionevole in pazienti con storia di } \mathrm{CMBs}<10 \text {, dimostrati in } \mathrm{RMN} \text {; in } \\
\text { pazienti con } \mathrm{CMBs}>10 \text { il rapporto rischi/benefici rimane incerto. Non è tuttavia indicato } \\
\text { ritardare il trattamento alla ricerca di CMBs non precedentemente noti }\end{array}$ \\
\hline Neoplasie intracraniche extra-assiali & Il trattamento può essere raccomandato \\
\hline Infarto acuto del miocardio (IMA) & $\begin{array}{l}\text { Il trattamento è indicato in caso di IMA concomitante, con il dosaggio cerebrale di rt-PA, } \\
\text { seguito da PTCA+stenting se indicato }\end{array}$ \\
\hline IMA recente & $\begin{array}{l}\text { Il trattamento è ragionevole in pazienti con storia recente ( }<90 \text { giorni) di IMA, specie se } \\
\text { sottoposto a rivascolarizzazione }\end{array}$ \\
\hline Pericardite acuta & $\begin{array}{l}\text { Il trattamento è ragionevole, specie per pazienti con ictus severo; per pazienti con ictus } \\
\text { lieve-moderato il trattamento è di beneficio incerto }\end{array}$ \\
\hline Trombo in atrio/ventricolo sinistro & $\begin{array}{l}\text { Il trattamento è ragionevole, specie per pazienti con ictus severo; per pazienti con ictus } \\
\text { lieve-moderato il trattamento è di beneficio incerto }\end{array}$ \\
\hline Altre malattie cardiache & $\begin{array}{l}\text { Il trattamento è ragionevole nel mixoma atriale o nel fibroelastoma papillare, specie per } \\
\text { pazienti con ictus severo }\end{array}$ \\
\hline Ictus peri-procedurale & $\begin{array}{l}\text { Il trattamento è ragionevole per ictus in corso di procedura angiografica cardiaca o } \\
\text { cerebrale, fatte salve le ulteriori controindicazioni }\end{array}$ \\
\hline
\end{tabular}


In pazienti con pregressa emorragia cerebrale, il trattamento è da considerarsi caso per caso, a seconda del tempo trascorso dall'emorragia e dall'eziologia del sanguinamento. In caso di emorragia profonda, associata ad ipertensione arteriosa, il trattamento può essere preso in considerazione. Anche nell'esperienza degli Autori, pazienti con anamnesi di emorragia nucleo-capsulare negli anni precedenti, presentatisi con ictus ischemico acuto, hanno avuto beneficio dal trattamento senza complicanze emorragiche. Caso diverso sono le emorragie lobari, spesso sottese da una patologia malformativa, nelle quali il trattamento è più rischioso e potenzialmente dannoso.

In pazienti con trauma cranico maggiore (con lacero-contusioni, ESA o emorragie intracerebrali extrassiali) $<3$ mesi, il trattamento trombolitico resta controindicato, così come in pazienti con intervento chirurgico intracranico/spinale $<3$ mesi o che sono portatori di tumore cerebrale.

In pazienti con endocardite infettiva o dissezione dell'arco aortico, il trattamento può essere potenzialmente dannoso e non dovrebbe essere somministrato.

\section{Nuove strategie di trattamento: nuovi orizzonti temporali e nuovi farmaci}

Una delle principali limitanti al trattamento trombolitico e, più in generale, alla riperfusione acuta nell'ictus è stata la ristretta finestra temporale tra esordio dei sintomi chiaramente databile e presentazione ospedaliera. Ciò ha storicamente determinato l'esclusione da possibilità di riperfusione circa il $25 \%$ del totale dei pazienti con ictus ischemico, che si presentano in Pronto Soccorso con sintomi al risveglio (wake up stroke) o non precisamente databili (unwitnessed stroke).

Tra il 2012 e il 2017, lo studio no-profit WAKE UP, ${ }^{23}$ finanziato dall'Unione Europea, arruola pazienti con ictus al risveglio o non chiaramente databile, che si presentano oltre 4,5 ore dall'ultima volta visti in buona salute. Lo studio si basa sulla dimostrazione di mismatch DWI-FLAIR in RMN encefalo come surrogato temporale: è noto che la sequenza DWI si positivizza entro pochi minuti dall'esordio dei sintomi, mentre la sequenza FLAIR mostra la lesione recente dopo circa 4-4,5 ore dall'esordio. Pertanto la positività in DWI e la negatività in FLAIR indicano che la lesione è più recente di 4,5 ore. Pazienti con lesione DWI superiore a $1 / 3$ del territorio dell'arteria cerebrale media o con ictus molto severo con NIHSS $>25$ sono stati esclusi dalla randomizzazione. Lo studio ha arruolato 503 pazienti degli 800 previsti. L'intervallo mediano tra l'ultima volta visto in salute e la presentazione in Pronto Soccorso è stato di circa 10 ore; il punteggio NIHSS mediano 6; l'estensione mediana della lesione $2 \mathrm{ml}$; il $20 \%$ dei pazienti si presentava con occlusione di vaso maggiore intracranico. A tre mesi, il 53,3\% dei pazienti trattati contro il $41,8 \%$ dei casi controllo ha mostrato outcome favorevole definito come mRS 0-1 (P 0,02); la trasformazione emorragica è risultata più frequente nei pazienti trattati $(4 \%$ contro $0,4 \%$, P 0,03), ma senza impatto sulla mortalità $(\mathrm{P}$ $0,07)$. Lo studio apre la strada al trattamento dell'ictus al risveglio o non testimoniato e viene incluso dalle linee guida a partire dal 2018.

Altri due studi, ECASS $4^{24}$ ed EXTEND,${ }^{25}$ sono stati condotti per testare l'efficacia e la sicurezza del trattamento trombolitico endovenoso oltre le 4,5, fino a 9 ore dall'esordio dei sintomi, utilizzando rispettivamente il mismatch PWI-DWI in RMN ed il mismatch TTP-CBF in TC Perfusionale. Entrambi gli studi sono stati chiusi anzitempo: nell'ECASS 4, l'arruolamento ha registrato una brusca frenata in seguito all'approvazione degli studi DAWN e DEFUSE-3 di trombectomia meccanica, portando alla chiusura del trial con meno del 50\% del campione stimato; l'EXTEND è stato terminato dopo l'approvazione del WAKE UP con circa il $70 \%$ del campione stimato.

Tabella 1. Segue dalla pagina precedente

Tumore maligno sistemico

In pazienti con tumore maligno e aspettativa di vita $>6$ mesi, il trattamento è ragionevole, fatte salve le ulteriori controindicazioni (coagulopatia, recente intervento chirurgico o sanguinamento sistemico)

Gravidanza

Il trattamento può essere considerato per ictus moderato-severo, quando $\mathrm{i}$ benefici superino i rischi; la sicurezza ed efficacia dopo recente $(<14$ giorni) parto spontaneo non sono ancora del tutto codificati**

Malattie oftalmologiche Il trattamento è indicato in corso di retinopatie emorragiche

Anemia falciforme Adulti con ictus associato ad anemia falciforme possono beneficiarsi dal trattamento Il trattamento risulta sicuro

*Esistono case series di pazienti in terapia con Dabigatran ${ }^{20}$ che hanno ricevuto dapprima trattamento con Idarucizumab per antagonizzare l'azione anticoagulante di Dabigatran $\mathrm{e}$ a seguire trombolisi endovenosa. Anche nell'esperienza degli Autori, tale trattamento sembra sicuro. ${ }^{*}$ rt-PA non è teratogeno e non passa la barriera emato-placentare. Il rischio di complicanze emorragiche intracraniche è basso (2,8\%), il rischio di morte del feto sicuramente attribuibile a rt-PA è ad oggi stimale in meno del $10 \% .{ }^{21}$ Nell'esperienza degli Autori, il trattamento è risultato sicuro ed efficace, senza complicanze per la madre ed il feto. E' tuttavia prudente considerare rischi e vantaggi attesi dal trattamento trombolitico, avendo prontamente disponibile un'equipe ginecologica e neonatologica, per la gestione di possibili complicanze. Si deve inoltre ricordare che le tecniche endovascolari espongono madre e feto a radiazioni ionizzanti e mezzo di contrasto iodato, che correlano con il rischio di teratogenicità. 
Questo comporta che entrambi gli studi perdono potere statistico, in particolar modo l'ECASS 4.

Essendo simili nel disegno e nella finestra temporale, ECASS ed EXTEND sono stati analizzati in maniera combinata, anche con la porzione di pazienti trattata nella finestra 4,5-6 ore dello studio EPITHET (che utilizzava una finestra $0-6$ ore). La metanalisi ${ }^{26}$ ha permesso di evidenziare che il trattamento trombolitico aumenta del 7\% la probabilità assoluta di recupero funzionale completo (mRS 0-1) con un beneficio statisticamente significativo ( $36 \%$ contro $29 \%$; $95 \% \mathrm{CI}$, $1,15-2,99 ; \mathrm{P} 0,011)$. Il trattamento è associato ad un significativo incremento del rischio di trasformazione emorragica sintomatica, tuttavia comparabile con gli studi di finestra temporale ridotta (5\%), confermando che la trasformazione emorragica nel trattamento trombolitico non è tempo-dipendente. La mortalità non risulta significativamente modificata dal trattamento $(\mathrm{P}$ $0,19)$. Il beneficio sembra essere più marcato nei pazienti in cui l'analisi del mismatch viene eseguita in maniera computerizzata ed automatica.

Un punto attivo nella ricerca è individuare un'alternativa più efficace e più rapida di rt-PA. Negli anni 2000, parte della ricerca si è concentrata sul Desmoteplase, un agente trombolitico derivato dalla saliva di pipistrello, la cui lunga emivita consente un singolo bolo. Gli studi DIAS ${ }^{27}$ e DEDAS ${ }^{28}$ sembravano essere promettenti in termini di efficacia e sicurezza, tuttavia lo studio DIAS $2^{29}$ non ha confermato alcun significativo vantaggio di Desmoteplase rispetto a placebo in pazienti fino a 9 ore dall'esordio, selezionati con $m i-$ smatch PWI-DWI. Anche gli studi DIAS 3 e 4,,$^{30,31}$ condotti su pazienti con piccolo core ed evidenza di ostruzione arteriosa intracranica, non hanno mostrato nessun beneficio funzionale significativo dal trattamento con Desmoteplase, a fronte di una percentuale di ricanalizzazione di circa il $50 \%$.

Nel 2012, viene pubblicato il primo studio di confronto tra rt-PA e Tenecteplase nell'ictus ischemico. ${ }^{32}$ Il Tenecteplase, un attivatore del plasminogeno derivato da rt-PA, ha, rispetto a quest'ultimo, affinità 15 volte maggiore con la fibrina, interagisce 80 volte in meno con l'inibitore del plasminogeno ed ha emivita 6 volte superiore, consentendo un singolo bolo. Lo studio ha confrontato due dosaggi di Tenecteplase, 0,1 $\mathrm{mg} / \mathrm{kg}$ e $0,25 \mathrm{mg} / \mathrm{kg}$, contro rt-PA $0,9 \mathrm{mg} / \mathrm{kg}$, in pazienti con evidenza di mismatch perfusionale in TC ed evidenza di ostruzione vascolare, mostrando maggiore indice di ricanalizzazione e miglioramento neurologico precoce nei pazienti trattati con Tenecteplase e ridotta disabilità (differenza assoluta 14\%) nel braccio Tenecteplase $0,25 \mathrm{mg} / \mathrm{kg}$.

Il farmaco è risultato promettente anche nello studio TEMPO-1 (Tenecteplase Evaluation for Minor Ischemic Stroke With Proven Occlusion), in pazienti con sintomi minori (NIHSS $<5$ ). ${ }^{33}$
Nel 2015, nello studio ATTEST (Alteplase versus Tenecteplase for Thrombolysis after ischaemic stroke), ${ }^{34}$ in 50 pazienti entro 4,5 ore dall'esordio dei sintomi, non è stato possibile dimostrare alcun vantaggio dal Tenecteplase.

Tra il 2012 ed il 2017, 1107 pazienti norvegesi sono stati randomizzati a rt-PA contro Tenecteplase $0,4 \mathrm{mg} / \mathrm{kg}$, entro 4,5 ore dall' esordio di un ictus, nello studio NOR-TEST. ${ }^{35}$ Non è stata dimostrata la superiorità del Tenecteplase rispetto a rt-PA, nella percentuale di pazienti con outcome clinico eccellenti (mRS 0-1: $64 \%$ versus $63 \%$, OR 1.08 ; $95 \%$ CI, $0,84-1,38$ ). Tuttavia il punteggio NIHSS basale basso (mediana 4) ed un consistente numero di stroke mimics (circa $18 \%$ ) sono limitanti notevoli: difficile è, infatti, dimostrare una significativa differenza di disabilità, per eventi benigni per storia naturale. ${ }^{36}$

Diversi studi sono in corso sul Tenecteplase, con finestra terapeutica fino a 24 ore. ${ }^{37}$

\section{La trombolisi endovenosa in epoca di trombectomia: che ruolo?}

Sebbene rispetto alla trombectomia meccanica, la trombolisi endovenosa abbia una minor efficacia nel ricanalizzare trombi di grandi dimensioni, localizzati in arterie di grosso calibro, secondo gli Autori esiste a tutt'oggi un ruolo ben definito per la trombolisi sistemica.

Innanzitutto, l'obiettivo nella cura del paziente non è la ricanalizzazione, ma il recupero funzionale. Negli studi di trombectomia meccanica, la cosiddetta ricanalizzazione futile raggiunge il 50\% negli studi con finestra terapeutica di 6 ore $^{38}$ e poco meno del $30 \%$ nel DAWN ${ }^{39}$ e DEFUSE- $3 .{ }^{40}$ La trombolisi endovenosa, sebbene in misura minore rispetto alla trombectomia, si è dimostrata in grado di ridurre la disabilità anche nei pazienti con large vessel occlusion. Lo studio EXTEND-IA-TNK ${ }^{41}$ ha dimostrato che rt-PA ha una capacità globale di ricanalizzare l'occlusione di grosso vaso pari al $10 \%$, che sale al $22 \%$ con Tenecteplase. In questi pazienti, è stata inoltre dimostrata una disabilità significativamente più bassa nel braccio Tenecteplase rispetto a rt-PA. Il pre-trattamento con trombolisi potrebbe, quindi, consentire di risparmiare un trattamento invasivo e costoso quale la trombectomia meccanica, con una dimostrata efficacia funzionale.

La patologia dei grossi vasi rappresenta circa un terzo degli eventi ischemici cerebrali. Sicuramente la small vessel occlusion costituisce ancora oggi un'importante causa di disabilità fisica e cognitiva, aggredibile solamente con tecniche riperfusive sistemiche.

Un punto spesso sollevato contro il trattamento trombolitico, isolato o in associazione a trombectomia meccanica, è legato al rischio di trasformazione emorragica. Tuttavia, va ricordato che la trasformazione 
emorragica, indice di danno da riperfusione, avviene spontaneamente nella storia naturale dell'ictus, è significativamente più frequente nei pazienti sottoposti a trattamenti riperfusivi acuti, ma è indipendente dalla modalità di trattamento. Infatti, sebbene indirettamente, il confronto delle percentuali di trasformazione emorragica tra studi di trombolisi endovenosa e studi di trombectomia meccanica nel circolo anteriore dimostra che tale rischio è piuttosto simile. D'altronde, fisiopatologicamente, il danno da riperfusione è secondario alla ricanalizzazione di un'area nella quale è alterata l'integrità dell'unità neurovascolare ${ }^{42}$ - intesa come cellula endoteliale, cellula gliale e neurone. Il ripristino del flusso ematico in queste condizioni determina una attivazione di meccanismi infiammatori acuti, la formazione di radicali liberi dell'ossigeno, un overload di calcio intracellulare ed il mantenimento di meccanismi di spreading depression. A valle di questi meccanismi sono l'edema cerebrale e la trasformazione emorragica. ${ }^{43,44}$

Bisogna inoltre considerare che ricanalizzazione non è sinonimo di riperfusione, in quanto, a livello capillare, si può assistere al fenomeno del no-reflow. Mutuato dalla fisiopatologia dell' ischemia coronarica, il concetto del no-reflow è un fenomeno multifattoriale, che presuppone un' ischemia prolungata e la necrosi tissutale. Come già dimostrato nel 1975 da Kloner ${ }^{45}$ mediante microscopia elettronica, l'ischemia prolungata determina un significativo danno microvascolare con rigonfiamento e protrusione intraluminale delle cellule endoteliali, formazione locale di aggregati piastrinici e di leucociti, liberazione di radicali liberi e chinine ed embolizzazione di microparticelle (15-100 micron) di piastrine, corpi ialini e cristalli di colesterolo, contribuendo al mantenimento del no-reflow post-ischemico in quanto, oltre all'ostruzione meccanica capillare, si determina una reazione infiammatoria che peggiora la disfunzione endoteliale e la vasocostrizione.

Il parallelo che spesso viene fatto con l'infarto del miocardio, trattato con intervento percutaneo primario, è poi fisiopatologicamente scorretto: l'infarto del miocardio nasce come patologia di tipo atero-trombotico locale che poco risponde a rt-PA, richiedendo il rilascio di uno stent; l'ictus è spesso una condizione embolica dalle carotidi, dall' arco aortico o dal cuore, che possibilmente risponde meglio al trattamento farmacologico litico. La trombolisi, inoltre, quando accoppiata alla trombectomia, consentirebbe un più facile approccio all'embolo (minor numero di passaggi) e la lisi di materiale embolico migrato dal coagulo culprit. ${ }^{46}$

La trombolisi endovenosa costituisce inoltre il primo rapido intervento terapeutico per molti pazienti che non vivono nelle vicinanze di un $h u b$ per l'ictus, dotato di Neuroradiologia Interventistica. In questi pa- zienti, il sistema drip and ship, con trombolisi endovenosa erogata nell'ospedale più vicino alla scena dell'ictus, consente di ridurre i tempi al trattamento ${ }^{47} \mathrm{~A}$ tal scopo, l'utilizzo di un farmaco one shot come il Tenecteplase potrebbe risultare in maggiore rapidità nei tempi di trasporto Spoke-Hub.

Sono in corso degli studi randomizzati controllati che mirano a testare l'ipotesi di trombectomia meccanica contro approccio combinato trombolisi+trombectomia (MR CLEAN NO-IV, SWIFT-DIRECT, DIRECT-MT), ma ad oggi ogni paziente con ictus merita la migliora terapia possibile, che include la trombolisi endovenosa.

\section{Bibliografia}

1. Bandera E, Motteri M, Minelli C, et al. Cerebral blood flow threshold of ischemic penumbra and infarct core in acute ischemic stroke: a systematic review. Stroke 2006;37(5):1334-9.

2. Astrup J, Siesjö BK, Symon L. Thresholds in cerebral ischemia - the ischemic penumbra. Stroke 1981;12(6): 723-725.

3. del Zoppo GJ, Ciccone A. Trial design and reporting standards for acute intravascular cerebral thrombolysis. In: Micieli G, Amantea D, eds. Rational basis for clinical translation in stroke therapy. Boca Raton, FL, USA: CRC Press, Taylor \& Francis group, 2014: 213-248. http://www.crcpress.com/product/ isbn/9781466594975

4. Wardlaw JM, Murray V, Berge E, del Zoppo GJ. Thrombolysis for acute ischaemic stroke. Cochrane Database of Systematic Reviews 2014, Issue 7. Art. No.: CD000213. DOI: 10.1002/14651858.CD000213.pub3

5. Tissue Plasminogen Activator for Acute Ischemic Stroke The National Institute of Neurological Disorders and Stroke rt-PA Stroke Study Group. N Engl J Med 1995; 333:1581-1588.

6. Hacke W, Kaste M, Fieschi C, et al. Intravenous thrombolysis with recombinant tissue plasminogen activator for acute hemispheric stroke. The European Cooperative Acute Stroke Study (ECASS). JAMA 1995 Oct 4;274(13):1017-25.

7. Hacke W, Kaste M, Fieschi C et al. Randomised double-blind placebo-controlled trial of thrombolytic therapy with intravenous alteplase in acute ischaemic stroke (ECASS II). Second European-Australasian Acute Stroke Study Investigators. Lancet 1998 Oct 17;352 (9136):1245-51.

8. Clark WM, Wissman S, Albers GW, et al. Recombinant tissue-type plasminogen activator (Alteplase) for ischemic stroke 3 to 5 hours after symptom onset. The ATLANTIS Study: a randomized controlled trial. Alteplase Thrombolysis for Acute Noninterventional Therapy in Ischemic Stroke. JAMA 1999;282(21):2019-26.

9. Hacke W, Kaste M, Blumki E, et al. Thrombolysis with Alteplase 3 to 4.5 Hours after Acute Ischemic Stroke. N Engl J Med 2008;359:1317-29.

10. Lees K, Blumki E, von Kummer R, et al. Time to treatment with intravenous alteplase and outcome in stroke: an updated pooled analysis of ECASS, ATLANTIS, 
NINDS, and EPITHET trials. Lancet 2010;375: 1695-703.

11. Ahmed N, Wahlgren N, Grond M, et al. Implementation and outcome of thrombolysis with alteplase 3-4.5 h after an acute stroke: an updated analysis from SITS-ISTR. Lancet Neurol 2010; 9: 866-74.

12. Saver JL, Fonarow GC, Smith EE, et al. Time to Treatment With Intravenous Tissue Plasminogen Activator and Outcome From Acute Ischemic Stroke. JAMA. 2013;309(23):2480-2488.

13. The IST-3 collaborative group. The benefits and harms of intravenous thrombolysis with recombinant tissue plasminogen activator within $6 \mathrm{~h}$ of acute ischaemic stroke (the third international stroke trial [IST-3]): a randomised controlled trial. Lancet. 2012 Jun 23; 379 (9834): 2352-2363.

14. Emberson J, Lees KR, Lyden P, et al. Effect of treatment delay, age, and stroke severity on the effects of intravenous thrombolysis with alteplase for acute ischaemic stroke: a meta-analysis of individual patient data from randomised trials. Lancet 2014; 384: 1929-35.

15. The IST-3 collaborative group. Effect of thrombolysis with alteplase within $6 \mathrm{~h}$ of acute ischaemic stroke on long-term outcomes (the third International Stroke Trial [IST-3]): 18-month follow-up of a randomised controlled trial. Lancet Neurol 2013 Aug;12(8):768-76.

16. Whiteley WN, Slot KB, Fernandes P, Sandercock P, Wardlaw J. Risk factors for intracranial hemorrhage in acute ischemic stroke patients treated with recombinant tissue plasminogen activator: a systematic review and metaanalysis of 55 studies. Stroke 2012 Nov;43(11): 2904-9.

17. The IST 3 Collaborative group. Association between brain imaging signs, early and late outcomes, and response to intravenous alteplase after acute ischaemic stroke in the third International Stroke Trial (IST-3): secondary analysis of a randomised controlled trial. Lancet Neurol 2015; 14: 485-96.

18. Barber PA, Demchuk AM, Zhang J, Buchan AM. Validity and reliability of a quantitative computed tomography score in predicting outcome of hyperacute stroke before thrombolytic therapy. Lancet 2000;355:1670-74.

19. Powers WJ, Rabinstein AA, Ackerson T, et al. Guidelines for the Early Management of Patients With Acute Ischemic Stroke: 2019 Update to the 2018 Guidelines for the Early Management of Acute Ischemic Stroke: A Guideline for Healthcare Professionals From the American Heart Association/American Stroke Association. Stroke. 2019;50:e344-e418.

20. Giannandrea D, Caponi C, Mengoni A, Romoli M, Marando C, Gallina A, Marsili E, Sacchini E, Mastrocola S, Padiglioni C, Mazzoli T, Cenciarelli S, Ricci S. Intravenous Thrombolysis in stroke after dabigatran reversal with idarucizumab: case series and systematic review. J Neurol Neurosurg Psychiatry 2018;0:1-5.

21. Selim MH, Molina C. The Use of Tissue Plasminogenactivator in Pregnancy A Taboo Treatment or a Time to Think Out of the Box. Stroke. 2013;44:868-869.

22. Demaerschalk BM, Kleindorfer DO, Demchuk AN et al. Scientific Rationale for the Inclusion and Exclusion Criteria for Intravenous Alteplase in Acute Ischemic Stroke A Statement for Healthcare Professionals From the American Heart Association/American Stroke Association. Stroke. 2016;47:581-641.
23. Thomalla G, Simonsen CZ, Boutite F, et al. MRI-Guided Thrombolysis for Stroke with Unknown Time of Onset. NEJM 2018. DOI: 10.1056/NEJMoa1804355.

24. Ringleb P, Bendszus M, Bluhmki E, et al. Extending the time window for intravenous thrombolysis in acute ischemic stroke using magnetic resonance imagingbased patient selection. Int J Stroke 2019. DOI: 10.1177/1747493019840938.

25. Ma H, Campbell BCV, Parson MW et al. Thrombolysis Guided by Perfusion Imaging up to 9 Hours after Onset of Stroke. N Engl J Med 2019;380:1795-803.

26. Campbell BCV, Ma H, Ringleb P, et al. Extending thrombolysis to 4.5-9 $\mathrm{h}$ and wake-up stroke using perfusion imaging: a systematic review and meta-analysis of individual patient data. Lancet 2019. http://dx.doi.org/ 10.1016/S0140-6736(19)31053-0.

27. Hacke W, Albers G, Al-Rawi Y, et al.The Desmoteplase in Acute Ischemic Stroke Trial (DIAS). Stroke. 2005;36:66-73.

28. Furlan AJ, Eyding D, Albers G, et al. Dose Escalation of Desmoteplase for Acute Ischemic Stroke (DEDAS): evidence of safety and efficacy 3 to 9 hours after stroke onset. Stroke 2006; 37(5):1227-31.

29. Hacke W, Furlan AJ, Al-Rawi Y et al. Intravenous desmoteplase in patients with acute ischaemic stroke selected by MRI perfusion-diffusion weighted imaging or perfusion CT (DIAS-2): a prospective, randomised, double-blind, placebo-controlled study. Lancet Neurol 2009 Feb;8(2):141-5.

30. Albers GW, von Kummer R, Truelsen T, et al. Safety and efficacy of desmoteplase given 3-9 h after ischaemic stroke in patients with occlusion or high-grade stenosis in major cerebral arteries (DIAS-3): a double-blind, randomised, placebo-controlled phase 3 trial. Lancet Neurol 2015 Jun;14(6):575-84.

31. von Kummer R, Mori E, Truelsen T, et al. Desmoteplase 3 to 9 Hours After Major Artery Occlusion Stroke: The DIAS-4 Trial (Efficacy and Safety Study of Desmoteplase to Treat Acute Ischemic Stroke). Stroke 2016 Dec;47(12):2880-2887.

32. Parsons M, Spratt N, Bivard A, et al. A Randomized Trial of Tenecteplase versus Alteplase for Acute Ischemic Stroke. N Engl J Med 2012;366:1099-107.

33. Coutts SB, Dubuc V, Mandzia J, et al. Tenecteplase-Tissue-Type Plasminogen Activator Evaluation for Minor Ischemic Stroke With Proven Occlusion. Stroke. 2015;46(3):769-74.

34. Huang X, Cheripelli BK, Lloyd SM, et al. Alteplase versus tenecteplase for thrombolysis after ischaemic stroke (ATTEST): a phase 2, randomised, open-label, blinded endpoint study. Lancet Neurol 2015; 14: 368-76.

35. Logallo N, Novotny V, Assmus J, et al. Tenecteplase versus alteplase for management of acute ischaemic stroke (NOR-TEST): a phase 3, randomised, open-label, blinded endpoint trial. Lancet Neurol 2017 Oct;16(10):781-788.

36. Khatri P, Kleindorfer DO, Devlin T, et al. Effect of Alteplase vs Aspirin on Functional Outcome for Patients With Acute Ischemic Stroke and Minor Nondisabling Neurologic Deficits. The PRISMS Randomized Clinical Trial. JAMA 2018;320(2):156-166.

37. Tenecteplase in Stroke Patients Between 4 and 24 Hours (TIMELESS). https://clinicaltrials.gov/ct2/show/ NCT03785678. 
38. Goyal M, Menon BK, van Zwam WH, et al. Endovascular thrombectomy after large-vessel ischaemic stroke: a meta-analysis of individual patient data from five randomised trials. Lancet 2016;387:1723-31.

39. Nogueira R, Jadhav AP, Haussen DC, et al. Thrombectomy 6 to 24 Hours after Stroke with a Mismatch between Deficit and Infarct. NEJM 2018; 378:11-21.

40. Albers G, Marks M, Kemp S, et al. Thrombectomy for Stroke at 6 to 16 Hours with Selection by Perfusion Imaging. NEJM 2018; 378:708-718.

41. Campbell B, Mitchell P, Churilov L, et al. Tenecteplase versus Alteplase before Thrombectomy for Ischemic Stroke. NEJM 2018; 378:1573-1582.

42. DelZoppo G. The Neurovascular Unit in the Setting of Stroke. JIM 2010;267:156-171.

43. Lin L, Wang X, Yu Z. Ischemia-reperfusion Injury in the Brain: Mechanisms and Potential Therapeutic Strate- gies. Biochem Pharmacol (Los Angel)2016;5(4):doi: 10.4172/2167-0501.1000213.

44. Kalogeris T, Baines C, Krenz M, et al. Ischemia/Reperfusion. Compr Physiol;7(1):113-170. doi:10.1002/cphy. c160006.

45. Kloner RA, Ganote CE, Jennings RB, Reimer KA. Demonstration of the "no-reflow" phenomenon in the dog heart after temporary ischemia. Recent Adv Stud Cardiac Struct Metab. 1975;10:463-74.

46. Gariel F, Lapergue B, Biourcier R, et al. Mechanical Thrombectomy Outcomes With or Without Intravenous Thrombolysis. Insight From the ASTER Randomized Trial. Stroke 2018;49:2383-2390.

47. Ciccone A, Berge E, Fischer U. Systematic review of organizational models for intra-arterial treatment of acute ischemic stroke. Int J Stroke 2019 Jan;14(1):12-22. 


\title{
Trombectomia meccanica
}

\author{
Paolo Candelaresi,${ }^{1}$ Giuseppe Leone, ${ }^{2}$ Vincenzo Andreone, ${ }^{3}$ Massimo Muto, ${ }^{2}$ Mario Muto ${ }^{2}$ \\ ${ }^{1}$ UOSC Neurologia e Stroke Unit, AORN Antonio Cardarelli, Napoli; ${ }^{2}$ UOC Neuroradiologia, AORN Antonio Cardarelli, Napoli; \\ ${ }^{3}$ UOC Neurologia, AORN S. Anna e S. Sebastiano, Caserta, Italia
}

\section{Introduzione}

Negli stessi anni in cui si testa in maniera sistematica l'efficacia e la sicurezza della trombolisi endovenosa, inizia a farsi largo l'idea di una terapia endovascolare dell'ictus ischemico, similmente all'infarto del miocardio.

I presupposti teorici alla base del trattamento endovascolare sono principalmente due: i) la limitata efficacia della trombolisi endovenosa nel ricanalizzare le occlusioni arteriose prossimali - circa il 20-25\% per l'arteria cerebrale media e $10-15 \%$ per la carotide interna; ${ }^{1}$ ii) le numerose controindicazioni storiche alla terapia sistemica che la rendevano inizialmente fruibile per una quota estremamente limitata di pazienti.

Idealmente il vantaggio delle tecniche endovascolari rispetto alla trombolisi sistemica consiste nel concentrare tutta l'azione terapeutica localmente, a livello dell'occlusione; di contro, i principali svantaggi sono rappresentati da tempi più lunghi al trattamento, dai costi più elevati e dalla necessità di un intervento anestesiologico che può incidere sulla delicata omeostasi del tessuto cerebrale in un momento in cui i meccanismi di autoregolazione sono alterati.

\section{Evidenze scientifiche nel trattamento endovascolare}

Nel 1998 con il PROACT I e nel 1999 con il PROACT II, ${ }^{2}$ si stabilisce l'efficacia della trombolisi

Corrispondente: Paolo Candelaresi, UOSC Neurologia e Stroke Unit, AORN Antonio Cardarelli, via Antonio Cardarelli 9, 80131 Napoli, Italia.

Tel.: +39.081.7472502.

E-mail: paolo.candelaresi@aocardarelli.it

Articolo pubblicato secondo la Creative Commons Attribution NonCommercial 4.0 License (CC BY-NC 4.0).

${ }^{\circ}$ Copyright: the Author(s), 2020

Licensee PAGEPress, Italy

QUADERNI - Italian Journal of Medicine 2020; 8(2):63-72 loco-regionale nei pazienti con ictus ischemico acuto ed occlusione del ramo prossimale dell'arteria cerebrale media entro 6 ore dall'esordio dei sintomi. Lo studio PROACT II, in particolare, include 180 pazienti, trattati con $9 \mathrm{mg}$ di pro-urokinasi, rilasciata a livello del trombo in associazione ad eparina endovenosa (gruppo sperimentale) contro sola eparina endovenosa (gruppo controllo). A tre mesi, una percentuale di pazienti significativamente maggiore risulta indipendente (mRS 0-2: 40\% vs 25\%; P 0,04) nel braccio pro-urokinasi rispetto ai controlli. Analogamente a quanto si osserva per il trattamento trombolitico endovenoso, anche quello loco-regionale risulta gravato da un maggior rischio di trasformazione emorragica $(10 \%$ vs $2 \%$; P 0,06$)$, senza tuttavia impattare sulla mortalità $(27 \%$ vs $25 \% ; \mathrm{P} 0,80)$. Sin da subito risulta evidente quella che diventerà una costante negli studi di trattamento endovascolare: il tasso di ricanalizzazione è significativamente più elevato nei pazienti sottoposti a trattamento intra-arterioso rispetto ai casi controllo ( $66 \%$ vs $18 \%$; $\mathrm{P}<0,001)$, con una discrepanza tra ricanalizzazione e recupero clinico-funzionale. Questo concetto, noto come ricanalizzazione futile, è espressione di numerosi meccanismi fisiopatologici: i) ricanalizzazione di aree scarsamente eloquenti di tessuto cerebrale; ii) ricanalizzazione di aree già irreversibilmente danneggiate; iii) ricanalizzazione dell'arteria principale ma assenza di riperfusione capillare (no reflow); ${ }^{3}$ iv) induzione di danno da riperfusione, con attivazione di una cascata di eventi pro-infiammatori che è dipendente dalla ricanalizzazione e non dalla tecnica con cui è ottenuta. ${ }^{4}$

Sulla scorta di questi risultati, l'American Heart Association approva la fibrinolisi endovascolare con una finestra terapeutica di 6 ore nell'ictus ischemico acuto dovuto ad occlusione della cerebrale media, nei pazienti non candidabili a trombolisi sistemica. ${ }^{5}$

Seguono poi solo altri tre ${ }^{6,7}$ piccoli studi randomizzati controllati che arruolano un totale di 175 pazienti, che confermano, metanalizzati, ${ }^{8}$ il dato derivante dal PROACT II.

La trombolisi farmacologica intra-arteriosa trova tuttavia i propri limiti in una bassa percentuale di ricanalizzazioni complete (mediamente 19\%) e nei lunghi tempi di infusione (2 ore nel PROACT II). Per tale 
motivo, vengono contemporaneamente sviluppati e testati i primi dispositivi di ricanalizzazione meccanica non farmacologica. Nel 2005, negli Stati Uniti, la Food and Drug Administration (FDA) approva il primo dispositivo di ricanalizzazione meccanica per la trombectomia nell'ictus ischemico: il MERCI Retriever. Il sistema, con la forma di un cavaturaccioli, avvolge il trombo, intrappolandolo e permettendone la rimozione.

Il dispositivo viene testato in due studi profit, non randomizzati, il Merci ${ }^{9}$ ed il MultiMerci. ${ }^{10}$ Nel Merci vengono arruolati pazienti con ictus ischemico acuto con occlusione di una grossa arteria intracranica non candidabili a trombolisi endovenosa (esordio dei sintomi oltre le tre ore o presenza di altra controindicazione); nel MultiMerci si includono anche pazienti che non ricanalizzano dopo rt-PA. Il sito di occlusione è rappresentato nel $32-33 \%$ dei casi dalla carotide interna, nel $57-60 \%$ dei casi dalla cerebrale media, nel $8-10 \%$ dal tratto vertebro-basilare. Mediamente i pazienti presentano sintomi severi con NIHSS 19-20 e durata dei sintomi inferiore a 8 ore. La percentuale di ricanalizzazione arriva al 60-68\% dei trattati (con l'aggiunta di trombolitico loco-regionale), mentre un buon esito funzionale (mRS 0-2) si osserva nel $27-36 \%$. Si conferma che la ricanalizzazione completa si associa ad una più elevata probabilità di buon outcome (4649\%). Inoltre, il MultiMerci permette di notare come il pre-trattamento con trombolisi endovenosa non comporti un aumento del rischio di trasformazione emorragica, che si attesta generalmente tra il $7,8 \% \mathrm{e}$ il $9,8 \%$.

Nel dicembre 2007, sulla base di un nuovo studio profit, multicentrico, non randomizzato, la FDA approva un ulteriore dispositivo per trombectomia meccanica: il Penumbra, un sistema di frammentazione e trombo-aspirazione. Il Penumbra Stroke Trial ${ }^{11}$ arruola 125 pazienti, con NIHSS basale media di 17 e durata media di sintomi di 4 ore (entro 8 ore), con occlusione della carotide interna intracranica nel $18 \%$ dei casi, cerebrale media nel $70 \%$ dei casi, circolo vertebro-basilare nel 9\%. Nell' $81,6 \%$ si osserva ricanalizzazione parziale o completa e nell' $11,2 \%$ trasformazione emorragica sintomatica; a tre mesi la percentuale di pazienti indipendenti è del $25 \%$ (35\% in caso di ricanalizzazione completa); la mortalità è pari al 32,8\% ( $29 \%$ in caso di ricanalizzazione completa).

Nel 2012 vengono testati due nuovi sistemi di ricanalizzazione meccanica, il Solitaire ed il Trevo. Lo stent retraibile (stentriever) viene avanzato attraverso il coagulo e poi aperto, dislocando l'embolo radialmente lungo le pareti dell'arteria e permettendo di ripristinare il flusso ematico cerebrale in tempi molto rapidi. Il coagulo resta così intrappolato tra le maglie dello stent e può essere rimosso quando lo stent viene chiuso e ritirato. Negli studi SWIFT ${ }^{12}$ e TREVO $2,{ }^{13}$ il sistema Solitaire ed il Trevo vengono rispettivamente confrontati in maniera randomizzata contro il Merci Retriever. Gli stentrievers mostrano maggiori percentuali di ricanalizzazione completa o parziale, fino a $86 \%$, con un recupero funzionale a 3 mesi fino al 58\% dei casi, soppiantandone di fatto l'utilizzo.

L'anno 2013 è l'annus horribilis per la terapia endovascolare dell'ictus. Sulla necessità di colmare un gap nelle evidenze a sostegno della trombectomia meccanica, vengono svolti tre studi: il Synthesis, l'International Management of Stroke III (IMS-III) ed il Mechanical Retrieval and Recanalization of Stroke Clots Using Embolectomy (MR RESCUE).

Il Synthesis ${ }^{14}$ è uno studio no profit italiano, finanziato da AIFA, inizialmente limitato ai soli centri di Regione Lombardia (fase pilota) ${ }_{15}^{15}$ poi esteso all'intero territorio nazionale (Synthesis Expansion). Scopo dello studio è confrontare, testa a testa, la sicurezza e l'efficacia della trombolisi endovenosa ed intra-arteriosa: un paziente potenzialmente eleggibile per il trattamento sistemico entro 4,5 ore dall'esordio, viene randomizzato a ricevere trombolisi endovenosa immediatamente o terapia endovascolare nel più breve tempo possibile, entro 6 ore. Lo studio pilota viene interrotto dopo 54 pazienti, con un trend in favore del trattamento endovascolare, per iniziare la fase Expansion. La seconda fase randomizza 362 pazienti, ma fallisce nel dimostrare differenze significative tra i due trattamenti (mRS $0-1,30,4 \%$ vs $34,8 \%$ con trend in favore della trombolisi endovenosa). La successiva pooled analysis ${ }^{16}$ dei due studi su 416 pazienti - il 68\% dei pazienti nel braccio endovascolare riceve fibrinolisi farmacologica loco-regionale, mentre nel 31,5\% viene utilizzato un dispositivo di trombectomia meccanica - conferma l'assenza di differenze significative tra i due trattamenti, sia in termini di sicurezza che di efficacia. Stratificando per gravità dei sintomi in esordio, i pazienti con NIHSS $<11$ tendono ad avere maggior beneficio dalla terapia endovenosa (OR 0,67; 95\% CI 0,35-1,28; P 0,22); viceversa, nei pazienti con ictus molto severo con NIHSS $>18$, sebbene il campione sia esiguo (89 pazienti) si evidenzia un trend molto più favorevole alla terapia intra-arteriosa (OR 3,52; 95\% CI 0,83, 14-93; P 0,08).

Lo studio randomizzato controllato IMS $\mathrm{III}^{17}$ confronta, in pazienti con sintomi moderato-severi (NIHSS $>10$ ) esorditi entro 3 ore, la sola trombolisi endovenosa a dosaggio standard contro un approccio combinato rt-PA a dosaggio $0.6 \mathrm{mg} / \mathrm{kg}$ più trattamento endovascolare da iniziare entro 5 ore dall'esordio. Il trattamento endovascolare viene di volta in volta scelto tra infusione di rt-PA loco-regionale, sonotrombolisi loco-regionale mediante catetere EKOS o trombectomia meccanica con uno dei dispositivi disponibili (Merci Retriever, Penumbra, Solitaire).

In aprile 2012, dopo una interim analysis, il reclu- 
tamento è interrotto precocemente per futilità, dopo 656 dei 900 pazienti previsti: pur in assenza di un aumento delle complicanze, a tre mesi, il trattamento combinato non modifica significativamente l'outcome (mRS 0-2: 40,8\% vs 38,7\%).

Lo studio randomizzato controllato MR RESCUE $^{18}$ confronta la trombectomia meccanica con i dispositivi Merci Retriever o Penumbra contro terapia medica standard in pazienti con punteggio NIHSS compreso tra 6 e 29, esordio entro 8 ore ed evidenza di occlusione di grosso vaso del circolo anteriore. In particolare i pazienti vengono stratificati in due gruppi: uno con buona ed uno con scarsa rappresentazione del tessuto in penombra, come elaborato alle immagini TC o RMN perfusionale. Anche questo trial non mostra alcun vantaggio funzionale dalla trombectomia.

Il fallimento di questi tre trial causa un forte scetticismo nei riguardi del trattamento endovascolare. Le possibili motivazioni per il fallimento di questi studi sono diverse: i) incostante presenza di un'occlusione vascolare maggiore; ii) incostante presenza ed estensione variabile di tessuto salvabile; iii) incostante raggiungimento di adeguata, efficace e rapida ricanalizzazione; iv) sintomi in esordio non sufficientemente severi.

Nell'IMS III, ad esempio, solo il 46\% dei pazienti è studiato con AngioTC in acuto e, di questi, un terzo presenta una lesione vascolare che difficilmente trae vantaggio da un trattamento endovascolare (vasi eccessivamente distali o occlusione acuta di carotide interna extracranica). Nel Synthesis, solo il 30\% dei pazienti è sottoposto ad AngioTC in acuto ed è possibile che una quota relativamente ampia di pazienti non presenti occlusione vascolare maggiore. Sebbene l'esatta quantificazione del tessuto in penombra ischemica non sia a tutt'oggi definitivamente chiarita, nell'IMS III, circa il $42 \%$ dei pazienti presenta eccessiva estensione dell'ipodensità precoce alla TC encefalo basale, con ASPECT (Alberta Stroke Project Early Computed Tomography) Score $<7$. Ancora, nel Synthesis, il punteggio NIHSS mediano basale è risultato 13 , con il $36 \%$ dei pazienti con NIHSS $<10$ : un punteggio così basso può riflettere una occlusione distale o un ottimo compenso leptomeningeo spontaneo, che difficilmente risente di un significativo vantaggio di un trattamento endovascolare rispetto alla terapia sistemica. Il tempo al trattamento e al raggiungimento di una ricanalizzazione adeguata (completa/sub-completa) sono importanti variabili indipendenti di buon esito funzionale. Nell'IMS III, il trattamento endovascolare è iniziato anche oltre due ore dopo l'imaging basale, con il concreto rischio di un aumento della lesione ischemica iniziale. Ancora, circa il 50\% dei pazienti dell'IMS III riceve trattamento fibrinolitico loco-regionale, circa il $28 \%$ è trat- tato con il Merci Retriever, il 16\% con il sistema Penumbra e solo 1' $1,5 \%$ riceve il trattamento con Solitaire; nel Synthesis addirittura quasi i due terzi dei pazienti ricevono solo trombolisi farmacologica intraarteriosa. Ciò si riflette in una bassa possibilità di ricanalizzazione, compresa tra $23 \%$ e $44 \%$.

Con queste premesse ben salde, e garantendo inoltre il rimborso per le procedure endovascolari solo se eseguite all'interno dello studio clinico, il 1 Gennaio 2015, dopo soli 40 mesi e 500 pazienti randomizzati, viene pubblicato lo studio no profit olandese MR CLEAN $^{19}$ (Multicenter Randomized Clinical Trial of Endovascular Treatment for Acute Ischemic Stroke in the Netherlands). Lo studio diviene una pietra miliare nel trattamento dell'ictus ischemico, in quanto, per la prima volta, dimostra un vantaggio significativo del trattamento endovascolare in pazienti con provata occlusione di grosso vaso del circolo anteriore (carotide intracranica, tratto M1 eM2 della cerebrale media, tratto A1 A2 della cerebrale anteriore) entro sei ore dall'esordio dei sintomi. Circa il $90 \%$ dei pazienti randomizzati riceve un pretrattamento con rt-PA endovenoso; circa l' $80 \%$ è trattato con stentrievers. Lo studio dimostra uno shift verso un outcome migliore nel braccio endovascolare, fatta eccezione per la mortalità (OR $1,67 ; 95 \% \mathrm{CI}, 1,21-2,30)$. A tre mesi, il 32.6\% dei trattati contro il 19,1\% dei controlli è indipendente (mRS 0-2: OR 2,16; 95\% CI, 1,39-3,38). Anche il MR CLEAN conferma l'esistenza di ricanalizzazione futile, in circa la metà dei trattati: a fronte del $75 \%$ di pazienti con ricanalizzazione completa, solo la metà circa è indipendente a tre mesi. Tutti i sottogruppi, ivi compresi i pazienti ultraottantenni o con ASPECT $<$ 7, hanno beneficio dal trattamento. Il MR CLEAN conferma l'enorme tempo-dipendenza dei trattamenti endovascolari: il beneficio statistico si perde se la procedura ha inizio a 5 ore e 13 minuti o se termina oltre 6 ore, con un $7 \%$ circa di riduzione delle probabilità di indipendenza per ogni ora di ritardo. Tra i punti a favore del MR CLEAN è quello di essere uno studio pragmatico, con criteri di inclusione semplici e replicabili nella vita reale, non sponsorizzato da privati, rapido e completo. Importante, inoltre, la scelta di garantire la rimborsabilità della procedura solo se condotta all'interno dello studio: questo ha permesso di superare il selection bias e l'eccessiva durata che avevano caratterizzato gli studi precedenti.

Il MR CLEAN funge da pietra angolare: all'indomani della pubblicazione di questi risultati, gli altri studi randomizzati controllati in corso interrompono l'arruolamento in favore di interim analysis. La Tabella 1 mostra gli studi di trombectomia meccanica successivi al MR CLEAN. ${ }^{20-26}$

Ciò che appare evidente è che la selezione dei pazienti cambia nel tempo, in quanto da un criterio temporale, si passa ad una valutazione multimodale in cui 
al tempo si aggiungono parametri neuroradiologici. Nello studio ESCAPE, il paziente viene considerato eleggibile per il trattamento endovascolare se presenta: i) core ischemico di dimensioni ridotte, alla TC basale, definito da un punteggio ASPECTS $>5$; ii) occlusione di grosso vaso intracranico (primo tratto dell'arteria cerebrale media o carotide interna terminale); iii) buon circolo collaterale all'AngioTC trifasica, definito come opacizzazione di almeno il $50 \%$ della circolazione piale nel territorio di cerebrale media. Negli studi EXTEND-IA e SWIFT PRIME, invece, i criteri di selezione prevedono che, oltre alla presenza di occlusione di grosso vaso intracranico, si dimostri la presenza di tessuto potenzialmente salvabile alla TC Perfusionale.

I parametri analizzati da una TC Perfusionale sono CBV (Cerebral Blood Volume), CBF (Cerebral Blood Flow), MTT (Mean Transit Time) e TTP (Time To Peak). Il core ischemico è classicamente definito come un'area con CBV ridotto, CBF ridotto e MTT o TTP aumentato; la penombra come un'area con CBV normale, CBF ridotto e MTT o TTP aumentato. Diversamente dal concetto di mismatch PWI-DWI in RMN, il mismatch perfusionale in TC è meno intuitivo e ha subito nel tempo diverse ridefinizioni. Inizialmente il mismatch utilizzato è stato quello MTT-CBV. Abbandonato per possibile sottostima del core e fallito nella sperimentazione clinica in studi randomizzati controllati, il mismatch CBV-MTT è stato sostituito dal mismacth Tmax-CBF: un Tmax $>6$ secondi indica tutta l'area di ipoperfusione, mentre un $\mathrm{CBF}<30 \%$ del controlaterale sano corrisponde al core ischemico; la sottrazione delle due aree restituisce la penombra ischemica. Sono stati poi sviluppati software di autolettura dei dati, per rendere tale processo più semplice ed immediato, quali il software Rapid, basato sul mismatch CBF-Tmax: i criteri perfusionali di selezione dei pazienti, negli studi EXTEND-IA e SWIFT PRIME, prevedono che l'estensione del core ischemico sia $<70 \mathrm{ml}$ al CBF e che il rapporto Tmax/CBF (estensione della penombra) sia maggiore di 1,2 nel-
l'EXTEND-IA e maggiore di 1,8 nel SWIFT PRIME. Questa analisi volumetrica quantitativa automatica basata sul mismatch CBF-Tmax, pur essendo ancora migliorabile, si è dimostrata efficace per la selezione dei pazienti negli studi clinici.

Una pooled analysis di studi randomizzati controllati, ${ }^{27}$ condotta su 1287 pazienti (634 assegnati a trombectomia meccanica e 653 ai controlli), dimostra che il trattamento endovascolare conduce a minore disabilità (OR 2,49; 95\% CI, 1,76-3,53) con un NNT 2,6, per una riduzione di un punto di disabilità alla scala Rankin. Il $46 \%$ dei pazienti trattati contro il $26,5 \%$ dei pazienti controllo è funzionalmente indipendente a tre mesi (mRS 0-2). Non si osserva nei trattati un aumento del rischio di trasformazione emorragica o di mortalità; la ricanalizzazione futile si conferma all'incirca nel $50 \%$ dei trattati. Le analisi di sottogruppo dimostrano che il beneficio si mantiene in particolari popolazioni, quali gli ultraottantenni (OR 3,68; 95\% CI, 1,95-6,92), i pazienti randomizzati oltre le 5 ore e quelli non eleggibili a trombolisi sistemica.

Cosi come il trattamento endovenoso, anche quello endovascolare è strettamente tempo-dipendente. E' stato infatti dimostrato che, rispetto ai pazienti non trattati, vi è un guadagno del $25-30 \%$ nella probabilità di indipendenza a tre mesi (mRS 0-2) se il trattamento è iniziato entro 3 ore dall'esordio dei sintomi, che decresce al $10-15 \%$ se il trattamento è iniziato a 6 ore. ${ }^{28}$

Un'ulteriore rivoluzione viene apportata dagli studi DAWN e DEFUSE 3.

Questi studi rappresentano un'assoluta innovazione certificando il passaggio dalla selezione time based alla selezione tissue based. Il DAWN ${ }^{29}$ seleziona pazienti con ictus ischemico associato ad occlusione di grosso vaso del circolo anteriore (ICA terminale o M1), occorsi nelle 6-24 ore precedenti, con evidenza di mismatch clinico-radiologico definito come: i) paziente $>80$ anni, NIHSS $>10$ e core DWI o CTP $<21 \mathrm{~mL}$; ii) paziente $<80$ anni, NIHSS $>10$, core DWI o CTP $<31 \mathrm{~mL}$; iii) paziente $<80$ anni, NIHSS $>20$, core DWI o CTP compreso tra $31-50 \mathrm{~mL}$.

Tabella 1. Studi di trombectomia meccanica successivi al MR CLEAN.

\begin{tabular}{ll}
\hline Studio & $\begin{array}{l}\text { Criteri di selezione (tutti gli studi richiedono la dimostrazione di occlusione di grosso vaso definito (primo tratto } \\
\text { dell'arteria cerebrale media o carotide interna intracranica) }\end{array}$ \\
\hline ESCAPE $^{20}$ & ASPECTS $>5$, buon circolo collaterale, esordio entro 12 ore \\
\hline EXTEND-IA $^{21}$ & CBV $<70 \mathrm{ml}$, Mismatch Ratio $>1,2$ \\
\hline SWIFT PRIME $^{22}$ & ASPECTS $>6$ e buon circolo collaterale, Mismatch Ratio $>1,8$ \\
\hline REVASCAT $^{23}$ & ASPECTS $>7$, esordio entro 8 ore \\
\hline THERAPY $^{24}$ & Paziente eleggibile per IVT + Clot $>8 \mathrm{~mm}$ \\
\hline THRACE $^{25}$ & Paziente eleggibile per IVT + Occlusione di ICA intracranica/M1/basilare \\
\hline PISTE $^{26}$ & Paziente eleggibile per IVT + Occlusione di ICA intracranica/MCA \\
\hline
\end{tabular}


Lo studio permette il solo utilizzo dello Stentriever Trevo e viene interrotto precocemente dopo una interim analysis: dei 500 pazienti previsti, vengono arruolati solo 206 pazienti, con NIHSS basale mediano 17, visti per l'ultima volta in buona salute circa 12-13 ore prima. Circa il $77 \%$ dei pazienti trattati mostra ricanalizzazione adeguata. A tre mesi, il $43 \%$ dei pazienti è vivo e indipendente (mRS 0-2), nel braccio trattati, contro il 13\% dei controlli, che si traduce in un Number needed to treat (NNT) di 2,8. Le complicanze emorragiche si aggirano intorno al 6\%, confermando che la trasformazione emorragica è associata con la ricanalizzazione e non con la tecnica con cui si ottiene.

Con un intento simile al DAWN, è condotto il DEFUSE $3 .^{30}$ Lo studio multicentrico, finanziato dal NINDS, negli Stati Uniti, seleziona pazienti con esordio dei sintomi tra 6 e 16 ore precedenti, occlusione di grosso vaso del circolo anteriore (ICA terminale o $\mathrm{M} 1$ ), con evidenza di mismatch perfusionale in TC o RMN perfusionale, definito come: i) volume di ipoperfusione/Volume Core $>1,8$; ii) volume di ipoperfusione $>15 \mathrm{~mL}$; iii) core $<70 \mathrm{~mL}$

Il tipo di dispositivo utilizzato viene lasciato a discrezione dell'operatore. A 192 dei 476 pazienti previsti, lo studio viene interrotto da una interim analysis dopo la divulgazione dei risultati del DAWN, dimostrando una distribuzione più favorevole sulla scala di disabilità, per i pazienti trattati. Similmente al DAWN, la percentuale di pazienti indipendenti (mRS 0-2) a tre mesi è il $45 \%$ a fronte del $17 \%$ dei controlli (risk ratio, 2,67; 95\% CI, 1,60 to 4,48; P < 0,001). Simile anche la percentuale di trasformazione emorragica: $7 \%$.

I due studi rappresentano un'innovazione enorme e pongono ancor di più l'accento sulla corretta identificazione del paziente nel territorio e nel trasporto diretto verso un centro dotato di expertise interventistica. Si stima che circa il $25-30 \%$ degli ictus ischemici possa soddisfare i criteri DAWN.

Se per il circolo anteriore, le evidenze sono ormai robuste, altrettanto non può dirsi per il circolo posteriore. Lo studio randomizzato controllato $\mathrm{BEST}^{31}$ è il primo ad arruolare pazienti con occlusione del circolo vertebro-basilare, in cui siano previste le moderne tecniche di trombectomia. Vengono arruolati pazienti con occlusione acuta di basilare o di vertebrale intracranica con assenza di flusso residuo in basilare, entro 8 ore dall'esordio dei sintomi. Chiuso anzitempo, dopo 131/288 pazienti arruolati, il trial non dimostra una superiorità del trattamento endovascolare nella percentuale di pazienti indipendenti/con lieve disabilità (mRS 0-3, 42\% versus $32 \%$; OR 1,74 95\% CI, 0,81-3,74). Pesa tuttavia sullo studio l'elevato numero di crossover tra i due gruppi ( $22 \%$ verso il braccio endovascolare).

Tra le aree grigie è il trattamento per i pazienti con ASPECTS basso in esordio o con imaging perfusionale con evidenza di core ischemico $>70 \mathrm{~mL}$. Una pooled analysis di studi randomizzati controllat ${ }^{32}$ ha dimostrato che quanto maggiore è l'estensione del core perfusionale iniziale, tanto più basse sono le possibilità di outcome favorevole; tuttavia, anche per un core ischemico esteso, il beneficio derivante dal trattamento rimane statisticamente significativo, sebbene di magnitudine minore (mRS 0-2 per core $100 \mathrm{~mL}$ : $20 \%$ nei pazienti trattati versus $5 \%$ nei pazienti controllo). Per ogni incremento di $10 \mathrm{~mL}$ nel core ischemico, si osserva una riduzione relativa del 23\% nella probabilità di indipendenza funzionale. Vi sono studi randomizzati controllati in corso, ad esempio il TENSION e l'IN EXTREMIS, che testano l'effettiva utilità del trattamento in pazienti con ipodensità precoce estesa in esordio.

E' tuttora incerto se i pazienti con punteggio NIHSS $<6$ in esordio ed occlusione di grosso vaso siano da sottoporre a trattamento endovascolare. I dati ad oggi disponibili non sono conclusivi e sono in corso studi randomizzati controllati, tra cui ENDOLOW e IN EXTREMIS.

Altri studi sono in corso, per testare quale strategia perseguire tra trattamento combinato endovenoso + endovascolare (bridging) o trombectomia meccanica primaria in monoterapia, tra cui il MR CLEAN -NO IV e il SWIFT DIRECT e, di conseguenza se, nell'organizzazione dei sistemi territoriali, sia da preferire un trasporto diretto verso il centro hub (mothership) o verso il centro più vicino per la trombolisi endovenosa ed un successivo trasferimento verso l'hub (drip-and-ship).

Ciò che dovrebbe essere sempre considerato sono le differenze territoriali geografiche e di risorse e che il trattamento riperfusivo dovrebbe essere garantito al maggior numero di pazienti e non solo a ristrette, fortunate, minoranze.

\section{Aspetti tecnici nel trattamento endovascolare}

\section{L'accesso vascolare}

L'accesso vascolare ai tronchi sovra-aortici nel paziente con ictus ischemico acuto, candidabile a strategie riperfusive rappresenta senza dubbio la prima difficoltà tecnica dell'intervento endovascolare, in quanto la stragrande maggioranza dei pazienti è geriatrica e vasculopatica.

Lo studio Angio-TC dell'arco aortico e dei TSA deve essere necessariamente incluso nel protocollo Angio-TC multifasico, in modo da poter pianificare il migliore approccio arterioso e nel guidare la scelta dei materiali più adeguati da utilizzare.

L'approccio percutaneo transfemorale comune destro o sinistro è quello più comunemente utilizzato e si realizza mediante introduttore 8 o $9 \mathrm{~F}$, preferibilmente corto, con o senza ausilio di guida ecotomografica.

E' da considerarsi l'approccio omerale o radiale 
destro in casi con marcata tortuosità del tronco anonimo, per accesso alla vertebrale ed alla carotide interna destra e nei casi di variante bovina per origine dell'arteria carotide comune sinistra dal tronco anonimo; l'approccio omerale o radiale sinistro va scelto invece in caso di marcata tortuosità dell'arteria succlavia sinistra per l'accesso alla vertebrale sinistra.

Nel circolo anteriore si può utilizzare catetere guida $8 \mathrm{~F}$ con pallone per eseguire blocco di flusso e aspirazione durante il recupero dello stent-retriever per raggiungere l'arteria carotide interna o un introduttore lungo a punta morbida. È bene preparare un sistema coassiale con catetere intermedio diagnostico 5 o $6 \mathrm{~F}$ lungo $125 \mathrm{~cm}$ all'interno del catetere $8 \mathrm{~F}$ e guida 0,035 all'interno del catetere 5 o 6 Fr. Si possono usare diverse curve del catetere in base all'anatomia (Sim2, Bern, VTK). E' da preferirsi il sistema coassiale allo scambio su guida stiff 0.035 " o 0.038 " da $260 \mathrm{~cm}$. Per il circolo posteriore, non è di norma utilizzato il catetere guida a blocco di flusso.

\section{Tecniche endovascolari meccaniche di rivascolarizzazione intracranica}

\section{Trombectomia meccanica con stent-retrievers}

Il Merci (Concentric Medical, Mountain View, California, USA) è stato il primo retriever introdotto nella pratica clinica, approvato dalla FDA nel 2004. Da un punto di vista strutturale il dispositivo era un device a forma di spirale, che veniva introdotto nel circolo intracranico attraverso microcatetere dedicato; una volta fuoriuscito dal microcatetere, il device era posizionato oltre il trombo con la funzione di aggancio e recupero nel catetere portante; onde evitare la migrazione del trombo durante la manovra di recupero, fu successivamente introdotto uno specifico catetere guida a blocco di flusso, che presentava in corrispondenza del suo estremo distale pallone con lume separato, che consentiva il temporaneo arresto del flusso. Mediante aspirazione combinata con siringhe a pressione negativa costante in corrispondenza del catetere guida e con pallone gonfio era possibile ottenere oltre al blocco del flusso una vera e propria inversione di flusso, che permetteva di migliorare i risultati di ricanalizzazione, riducendo le embolie in nuovi territori.

Un altro retriever concettualmente simile, introdotto nel 2005 è stato il Catch (Balt, Montmorency, France) di prima generazione; tale device, distribuito solo in Europa in quanto con marchio CE, ma non negli USA, era costituito da un cestello autoespandibile di nitinol, in grado di agire distalmente al trombo.

Attualmente questi due dispositivi non hanno più alcun impiego clinico e sono stati soppiantati da stentretrievers di nuova generazione.

Tra gli stent-retrievers di nuova generazione, il So- litaire (Covidien Neurovascular, Irvine, California) è stato il primo dispositivo ad essere approvato dalla FDA (2012) per il trattamento endovascolare dell'ictus ischemico, alla luce del trial randomizzato SWIFT. ${ }^{12}$ Questo tipo di device è stato inizialmente introdotto nella pratica clinica nel 2008 come stent intracranico a distacco controllato con meccanismo elettrolitico, per il trattamento di aneurismi a colletto largo in associazione alle spirali, con tecnica di stent-assistedcoiling. Nel 2008 tuttavia esso fu utilizzato off-label per il trattamento dell'ictus ischemico come stent-retriever $^{33}$. Posizionato mediante microcatetere da 0,021 " o da 0,027 ", a cavallo del trombo, permetteva la retrazione dello stesso in specifico catetere guida, con risultati eccellenti, in particolar modo quando utilizzato con la tecnica del blocco di flusso prossimale.

Diversamente dai vecchi dispositivi Merci (Concentric Medical, Mountain View, California, USA) e Catch (Balt Extrusion, Montmorency, France) che concettualmente agivano distalmente al trombo mediante sistema di recupero a cestello, lo stent-retriever di seconda generazione agiva inglobando il trombo all'interno dello stent, favorendo il suo recupero e limitando fenomeni di embolizzazione in nuovi territori. Allo stato attuale tale dispositivo è stato modificato in versioni dotate di multipli marker radio-opachi (Solitaire Platinum e Solitaire X) onde favorire la visualizzazione e l'interazione con il coagulo durante il rilascio sotto guida fluoroscopica. Ulteriori stent-retrievers dal disegno molto simile, in termini di geometria e morfologia delle celle sono il Preset (Phenox, Bochum, Germany) e la nuova versione del Catch (Balt, Montmorency, France). I vantaggi di tali tipi di device sono senza dubbio le versioni lite o mini di basso profilo, che permettono il loro utilizzo con microcateteri di calibro inferiore allo 0.021 " quali microcateteri da 0.017 ". Tra le principali alternative al Solitaire, il dispositivo Trevo (Stryker Neurovascular, Kalamazoo, MI) è senza dubbio il secondo stent-retriever più studiato e con risultati dimostrati da trial clinici randomizzati. Similmente al Solitaire, il Trevo è risultato superiore al Merci Retriever nello studio randomizzato TREVO, ottenendo l'approvazione dall'FDA nell'Agosto 2012. Il principale vantaggi di tale stent-retriever consiste nella totale radio-opacità, che permette di identificare la corretta espansione e posizionamento del device onde verificare l'interazione stent-coagulo prima della retrazione. E' infine lo stent-retriever con specifica indicazione al trattamento endovascolare nell'ictus in finestra temporale ampia (6-24 h), in quanto utilizzato nello studio clinico randomizzato e controllato DAWN. I più recenti stent-retrievers introdotti nella pratica clinica sono stati realizzati con strutture complesse e modulari, tra questi si rammenta l'Embotrap (Cerenovus, Galway, Ireland), il Neva (Vesalio, Nashville, Tennessee) e l'E- 
ric (Microvention, Tustin, California). Embotrap è uno stentriever realizzato in nitinol e composto da uno stent interno per il ripristino immediato del flusso e uno stent esterno con un design modulare segmentato per l'integrazione e l'intrappolamento di trombi. Anche il Neva ha una struttura simile, con disegno modulare e porzione centrale funzionale con estremo distale chiuso, in assenza tuttavia di un canale centrale. L'Eric diversamente ha una struttura modulare con un numero variabile microsfere interconnesse in nitinolo, con la funzione di integrare e catturare il coagulo. Con l'eccezione dell'Embotrap, i cui risultati sono stati validati in studio clinico randomizzato (ARISE II), ${ }^{34} \mathrm{gli}$ altri due stent-retriever non presentano allo stato evidenze cliniche sufficienti di sicurezza ed efficacia, in quanto il loro utilizzo è limitato a serie retrospettive monocentriche.

A prescindere dello stent utilizzato è possibile sintetizzare le principali manovre di trombectomia meccanica con stent-retriever come di seguito: per quanto concerne le occlusioni del circolo anteriore viene posizionato il catetere guida, con o senza blocco di flusso nella carotide interna, preferibilmente nel tratto cervicale pre-petroso mentre per quanto concerne le occlusioni del circolo posteriore viene posizionato catetere guida senza blocco di flusso nel tratto V2 distale dell'arteria vertebrale. Si oltrepassa il punto di occlusione con una navigazione alla cieca; è consigliabile la formazione di un ampio loop alla punta della microguida onde evitare l'ingaggio di rami collaterali. Una volta superata l'occlusione, viene fatto avanzare il microcatetere: è importante assicurarsi che il microcatetere abbia superato completamente l'occlusione e che si trovi in un tratto di vaso regolarmente canalizzato. $\mathrm{Si}$ procede quindi a iniezione di mezzo di contrasto dal microcatetere per confermare la posizione a valle dell'occlusione, indi si rimuove la microguida e si inserisce lo stent-retriever, che viene posizionato a cavallo del coagulo. Per gli stent-retriever a struttura modulare (Embotrap o Neva), il marker prossimale dello stent deve coincidere con l'estremo prossimale del clot, in quanto in questi dispositivi è la struttura centrale quella deputata alla interazione e cattura del coagulo. Lo stent viene quindi lasciato in loco per 35 minuti e, a discrezione dell'operatore, può essere eseguita una serie angiografica a stent aperto dal catetere guida, onde valutare l'eventuale effetto bypass. È consigliabile effettuare la rimozione dello stentriever con blocco di flusso e in aspirazione continua, utilizzando una siringa da $60 \mathrm{cc}$ vacuum-locked o con l'ausilio di una pompa automatica; secondo questa tecnica, il palloncino posto all'estremità del catetere portante viene gonfiato mediante iniezione di mezzo di contrasto prima d'iniziare il recupero dello stentriever che viene ritirato lentamente insieme al microcatetere; è regola ferma rimuovere lo stentriever ancora aperto, in quanto un eventuale passaggio dello stesso nel microcatetere provocherebbe frammentazione e migrazione distale del trombo.

\section{Sistemi di tromboaspirazione}

Il primo device di aspirazione sviluppato è invece stato il Penumbra, utilizzato con approvazione FDA negli USA dal 2008. Il dispositivo nella sua versione originale era formato da tre diverse componenti: un catetere di riperfusione, un separatore e un anello utilizzato per la rimozione del trombo. Questo dispositivo poteva agire tramite due diversi meccanismi: frammentazione e aspirazione del trombo ed eventuale estrazione meccanica del materiale rimanente. In questa procedura il catetere Penumbra veniva fatto avanzare attraverso un catetere guida in un punto appena prossimale all'occlusione; una microguida chiamata separatore veniva ripetutamente fatta passare attraverso attraverso il trombo per frammentarlo ed infine si procedeva all'aspirazione del trombo e di eventuali frammenti. Il sistema Penumbra è stato successivamente modificato con cateteri intermedi ad alta flessibilità e di grosso calibro, per la navigazione intracranica effettuata su microcateteri, in modo da ottenere l'aspirazione diretta del coagulo. Ad oggi sono disponibili diversi cateteri di aspirazione distale, prodotti da diverse ditte, con caratteristiche peculiari che li differenziano per navigabilità, capacità di aspirazione e lume interno. Oltre alla famiglia ACE, MAX e JET della Penumbra, si ricorda il Catalyst (Stryker Neurovascular, Kalamazoo, MI), il Sofia (Microvention, Tustin, California), il React (Medtronic, Dublin, Ireland), il Titan (Balt, Montmorency, France). Questi cateteri a lume singolo, presentano rigidezza variabile lungo la loro lunghezza per la loro struttura modulare in nylon ed uretano; presentano difatti una struttura rinforzata con parte prossimale armata con fili intrecciati in acciaio inossidabile e coil distale in nitinolo con segmento flessibile, il che li rende atti a navigare nel circolo intracranico. Presentano altresì in genere marker radio-opaco distale in platino, coating esterno per ridurre l'attrito durante la navigazione e coating interno in PTFE per migliorare la navigabilità dei device inseriti con lunghezze di lavoro da $105 \mathrm{a} 160 \mathrm{~cm}$ (in genere $131 \mathrm{~cm}$ ) e diametri interni $0,038 / 0,058 / 0,072(4 / 5 / 6$ F). Per quanto concerne il lume di lavoro possiamo schematizzare come di seguito: cateteri per la tromboaspirazione distale, con diametro interno tra 0,035 " e 0,058 ", cateteri da 0,060 " e 0.064 " per arterie di medio calibro e cateteri a grande lume $(0,068-0,072$ ") per i vasi intracranici prossimali. A prescindere dal catetere di aspirazione utilizzato i passaggi della tecnica ADAPT (direct aspiration first pass technique) possono essere riassunti come di seguito. Viene posizionato catetere guida con lume interno di $0,084-0,088^{\prime \prime}$ in carotide interna sino 
al tratto pre-petroso. Sebbene con la tromboaspirazione semplice non sia necessario ricorrere al catetere guida con blocco di flusso prossimale, alcuni recenti studi hanno dimostrato che il catetere guida a blocco di flusso possa ridurre il rischio di embolizzazione anche con la tecnica ADAPT, ${ }^{35}$ che nella pratica clinica sembra essere maggiore rispetto all'uso del solo stent-retriever, soprattutto per occlusioni sostenute da coaguli $>1 \mathrm{~cm}$ di lunghezza (elevato clot burden). Il catetere di aspirazione di calibro più grande viene selezionato per ciascun caso, compatibilmente alla sede dell'occlusione, di solito 0,068-0,072", per ICA distale, arteria cerebrale media (M1) occlusioni basilari, 0,060 e di calibro inferiore per M2-M3. Il catetere di aspirazione viene fatto avanzare sino al livello del trombo, di solito coassialmente su un microcatetere dedicato e specifica microguida. Altri cateteri di piccolo calibro, come il 3MAX (Penumbra, Alameda, California) possono anche essere veicolati nel circolo intracranico direttamente su microguida compatibile. Laddove possibile è consigliabile non far avanzare il microcatetere di supporto oltre il punto di occlusione, onde evitare frammentazione ed embolizzazioni distali; nella pratica tuttavia, soprattutto in sifoni tortuosi, appare necessario portare il microcatetere di supporto assai distalmente al trombo, onde favorire la navigazione del catetere di tromboaspirazione di grosso calibro (0,068-0,072"). Con il catetere di aspirazione affrontato al trombo, l'aspirazione veniva applicata con una siringa da $60 \mathrm{~mL}$ vacuum-locked $\mathrm{o}$, più frequentemente mediante una pompa di aspirazione. L'assenza di flusso all'interno del sistema di aspirazione conferma il contatto con il trombo. A questo punto, il catetere è stato fatto avanzare delicatamente per 1-2 $\mathrm{mm}$ per garantire un solido ingaggio con il trombo. L'aspirazione viene lasciata in pompa per un periodo variabile (3-5 minuti) e se non si assiste ad alcun flusso attraverso il sistema, il catetere viene lentamente ritirato. Se l'aspirazione fallisce, denunciato dal ritorno del sangue nel sistema vacuum-locked, il catetere di aspirazione viene reinserito fino al livello del coagulo e si ripete l'aspirazione. Sul catetere guida viene in genere posizionata ulteriore siringa da $60 \mathrm{cc}$ vacuum-locked, onde evitare embolizzazione distale, nella manovra di recupero del catetere di aspirazione all'interno del catetere guida. La tecnica ADAPT prevede l'utilizzo della tromboaspirazione per poi passare all'impiego di stentriever solo in caso di fallimento. Questa tecnica è stata proposta al fine di ridurre il tempo della procedura ed i costi legati allo stent-retriever. Lo studio randomizzato controllato ASTER, ${ }^{36}$ che ha confrontato la tromboaspirazione e la trombectomia tramite l'uso di stentriever come tecniche di primo approccio, non ha dimostrato differenze significative tra le due in termini di tassi di rivascolarizzazione al termine della procedura, di outcome clinico e di eventi avversi, di fatto però non raggiungendo l'endpoint primario, ovvero la dimostrazione della superiorità della tromboaspirazione rispetto alla trombectomia meccanica con stent-retriever.

\section{Tecniche combinate di rivascolarizzazione}

Le tecniche di trombectomia meccanica di tromboaspirazione e stent-retriever possono essere utilizzate in combinazione, onde ottenere maggiori possibilità di ricanalizzazione efficace al primo passaggio. La ricanalizzazione al primo passaggio sì è difatti dimostrata fattore cruciale nella determinazione del buon outcome clinico in diversi recenti studi. L'utilizzo di uno stent-retriever in combinazione alla tromboaspirazione permette difatti di ridurre il rischio di perdita del materiale trombotico, con abbattimento della percentuale di embolie in nuovi territori. Sono state proposte diverse varianti di tecnica, denominate ARTS, SAVE e SOLUMBRA, con o senza utilizzo di catetere a blocco di flusso. Tutte si sono dimostrate tutte efficaci e sicure, ${ }^{37}$ in assenza di chiara superiorità dell'una rispetto all'altra.

\section{Trattamento delle occlusioni carotidee acute e delle lesioni tandem}

Le occlusioni tandem rappresentano tra il 9 ed il $20 \%$ delle casistiche dei principali trial e possono rendere tecnicamente più impegnativa la procedura di rivascolarizzazione intracranica. L'occlusione della carotide al collo si realizza propriamente mediante due meccanismi fisiopatologici, ovvero la trombosi su placca o la dissezione; in entrambe le situazioni si vengono a delineare tre distinti scenari: i) assenza di concomitante occlusione intracranica in compenso emodinamico; ii) assenza di occlusione intracranica senza compenso emodinamico; iii) presenza di concomitante occlusione intracranica. In caso di assente occlusione intracranica maggiore e compenso del circolo intracranico, per attivazione vicariante del sistema delle comunicanti, l'occlusione in acuto della carotide viene ben tollerata ed il paziente può presentarsi del tutto asintomatico; non vi è pertanto indicazione clinica al trattamento endovascolare. Diversamente in assenza di concomitante occlusione intracranica, con scarso o assente compenso emodinamico, il paziente risulterà sintomatico per sofferenza ischemica dei territori di confine, configurando, in assenza di trattamento, la comparsa di infarti degli ultimi prati (o watershed). Lo studio perfusionale in questi casi realizzato mediante TC o RM può essere utile e mostra estese aree di ipoperfusione non critica (area in MTT o TMAX $>6 \mathrm{sec}$ ), alla convessità emisferica; in tali casi la sintomatologia potrà essere dipendente dai valori di pressione arteriosa sistemica ed il paziente dovrà essere candidato a rivascolarizzazione carotidea in acuto 
mediante tromboendoarterectomia in urgenza o angioplastica e stenting carotideo. Nel caso di occlusione intracranica maggiore, la priorità assoluta è rappresentata dalla più celere rivascolarizzazione del circolo cerebrale. Nelle occlusioni carotidee su base aterotrombotica, una volta posizionato il catetere guida in carotide comune, mediante microguida da 0.014 " da $200 \mathrm{~cm}$ è in genere possibile oltrepassare l'occlusione. Nel caso ciò risulti particolarmente difficoltoso è possibile oltrepassare l'occlusione con microcatetere da 0.017 " e guida 0.014 da scambio da 300 $\mathrm{cm}$; una volta valicata l'occlusione viene rimosso il microcatetere lasciando la microguida in sede e vengono eseguite multiple manovre di PTA con palloni per angioplastica monorail di diametro crescente sino a guadagnare un lume accettabile da permettere il passaggio del catetere guida. La scelta di posizionare uno stent carotideo in acuto è variabile e dipendente dallo specifico scenario clinico. In particolar modo, se da un lato lo stent permette di stabilizzare la placca e garantisce un accesso stabile al circolo intracranico, dall'altra parte il suo rilascio impone l'inizio di un trattamento con doppio antiaggregante in acuto o antiaggregante in urgenza (Tirofiban). Tale terapia andrebbe iniziata in caso di ictus ischemico acuto alla luce di un limitato core evidenziato alla TC perfusionale o alla $\mathrm{RM}$ in diffusione, onde minimizzare il rischio di infarcimento emorragico. Sulla tempistica dello stenting, ovvero se questo debba essere praticato prima o dopo la ricanalizzazione del circolo cerebrale, l'orientamento della letteratura attuale non è univoco, stabilendo tuttavia la sostanziale equivalenza tra due pratiche, a patto che le manovra endovascolari al collo non ritardino la rivascolarizzazione del circolo cerebrale. Nel caso in cui il trattamento endovascolare venga effettuato dopo la procedura di trombectomia meccanica o tromboaspirazione è consigliabile trattare la carotide al collo con dispositivo di protezione antiembolia o con blocco di flusso prossimale. In caso di dissezione della carotide appare necessario di guadagnare il lume vero con microguida e microcatetere; in tal caso è consigliabile utilizzare un microcatetere da 0,017 " e microguida 0,014 "da scambio da $300 \mathrm{~cm}$. Una volta guadagnato il lume vero, dimostrata dalla visualizzazione microseriografica del circolo intracranico, viene rimosso il microcatetere e si procede a multiple PTA per riguadagnare il lume carotideo. La scelta di posizionare uno stent carotideo al collo ed eventualmente al passaggio intra-extra-cranico deve essere dettata dalle medesime considerazioni esplicate nelle occlusioni aterotrombotiche. Tuttavia è consigliabile sottoporre a stenting le dissezioni cervicali solo in situazioni di estrema necessità, come nei casi di dissezione progressivamente occlusale in concomitante insufficienza del circolo del Willis, tenendo a mente che, in tutte le altre situazioni il gold standard per tali lesioni risulta la terapia medica. Una volta guadagnato l'accesso al circolo intracranico si procede a trombectomia con stent- retriever, tromboaspirazione o metodica combinata. Nel caso di utilizzo di stent-retriever e rilascio di stent carotideo al collo è necessario far avanzare il catetere guida oltre lo stent carotideo, onde evitare che lo stent-retriever nel suo recupero possa incastrarsi nello stent carotideo.

\section{Complicanze delle manovre meccaniche di rivascolarizzazione cerebrale}

Le complicanze correlate alla procedura di trattamento endovascolare dell'ictus ischemico possono essere correlate alla somministrazione di mezzo di contrasto o alle manovre endovascolari. Nei maggiori trial clinici positivi recenti erano riportate in circa il $15 \%$ delle procedure, ma molte di queste non hanno influito sull'outcome clinico. Le complicanze osservate più frequentemente sono state perforazioni vascolari $(0,9-4,9 \%)$, emorragie intracraniche sintomatiche (3,6-9,3\%), emorragie subaracnoidee $(0,6-4,9 \%)$, dissezioni arteriose $(0,6-3,9 \%)$, vasospasmo e complicanze a carico del sito di accesso vascolare. ${ }^{38}$ Non trascurabile appare infine il rischio di embolie in nuovi territori che sono riportati in circa il $2-8,6 \%$ dei nuovi trial. Pur con il limite della relativa traumaticità dell'atto endovascolare, la trombectomia non sembra aumentare il rischio di emorragia intraparenchimale o d'infarcimento emorragico nei giorni successivi alla ricanalizzazione e questo dato è confermato dai trial del 2015 , dove l'incidenza varia tra 0 e il 7,7 per cento ( $v s$ 1,9-6,4\% nei pazienti sottoposti a sola terapia medica) con sovrapposizione del rischio di emorragie sintomatiche simili a quelle osservate nel solo trattamento fibrinolitico sistemico. Anche la mortalità risulta paragonabile tra i pazienti sottoposti a trattamento endovascolare (9-18,9\%) rispetto ai pazienti in terapia medica $(12,2-18,4 \%)$.

\section{Bibliografia}

1. Rha JH, Saver JL. The impact of recanalization on ischemic stroke outcome: a meta-analysis. Stroke. 2007; 38:967-973.

2. Furlan A, Higashida R, Wechsler L, et al. Intra-arterial prourokinase for acute ischemic stroke. The PROACT II study: a randomized controlled trial. J Am Med Assoc. 1999; 282: 2003-11.

3. Rezkalla SH, Kloner RA. The no reflow phenomenon. Circulation. 2002;105:656-662.

4. Kalogeris T, Baines C, Krenz M, and Korthuis R. Ischemia/Reperfusion. Compr Physiol;7(1):113-170. doi:10. 1002/cphy.c160006.

5. Adams HP, Del Zoppo G, Alberts M, et al. Guidelines for the early management of adults with ischemic stroke. Stroke 2007. 38; 1655-1711.

6. Ogawa A, Mori E, Minematsu K et al. Randomized trial 
of intra-arterial infusion of urokinase within 6 hours of middle cerebral artery stroke: the Middle cerebral artery Embolism Local fibrinolytic intervention trial (MELT) Japan. Stroke 2007; 38: 2633-39.

7. Macleod M, Davis S, Mitchell P, et al. Results of a multicentre, randomized controlled trial of intra-arterial urokinase in the treatment of acute posterior circulation ischemic stroke. Cerebrovasc Dis 2005; 20: 12-17.

8. Lee M, Hong KS, Saver J. Efficacy of intra-arterial fibrinolysis for acute ischemic stroke. Meta-analysis of randomized controlled trials. Stroke 2010; 41: 932-937.

9. Smith WS, Sung G, Starkman S, et al. Safety and efficacy of mechanical embolectomy in acute ischemic stroke: results of the Merci trial. Stroke 2005; 36: 1432-38.

10. Smith WS, Sung G, Saver J, et al. Mechanical thrombectomy for acute ischemic stroke: final results of the Multi Merci trial. Stroke 2008; 39: 1205-12.

11. Penumbral Pivotal Stroke Trial Investigators. The penumbra pivotal stroke trial: safety and effectiveness of a new generation of mechanical devices for clot removal in intracranial large vessel occlusive disease. Stroke 2009; 40: 2761-68.

12. Saver JL, Jahan R, Levy E, et al. Solitaire flow restoration device versus the Merci Retriever in patients with acute ischemic stroke (SWIFT): a randomized, parallelgroup, non-inferiority trial. Lancet 2012; 380: 1241-49.

13. Nogueira RG, Lutsep H, Guota R, et al. Trevo versus Merci retrievers for thrombectomy revascularization of large vessel occlusions in acute ischemic stroke (TREVO 2): a randomized trial. Lancet 2012; 380 : 1231-40.

14. Ciccone A, Valvassori L, Nichelatti M, et al. Endovascular treatment for acute ischemic stroke. NEJM 2013;368:904-13.

15. Ciccone A, Valvassori L, Ponzio M. Intra-arterial or intravenous thrombolysis for acute ischemic stroke? The SYNTHESIS pilot trial. JNIS 2010; 2:74-9.

16. Candelaresi P, Ciccone A, Cotrufo R. Trombolisi endovenosa o intra-arteriosa nell'ictus ischemico acuto: una pooled analysis degli studi Synthesis. Unpublished data.

17. Broderick JP, Palesch YY, Demchuk A, et al. Endovascular therapy after intravenous t-PA versus t-PA alone for stroke. NEJM 2013 DOI: 10.1056/NEJMoa1214300.

18. Kidwell CS, Jahan r, Gornbein J, et al. A trial of imaging selection and endovascular treatment for ischemic stroke. NEJM 2013 DOI: 10.1056/NEJMoa1212793.

19. Berkhemer O, Fransen P, Beumer D, et al. A Randomized Trial of Intraarterial Treatment for Acute Ischemic Stroke. N Engl J Med 2015; 372:11-20.

20. Goyal M, Demchuk A, Menon B, et al. Randomized Assessment of Rapid Endovascular Treatment of Ischemic Stroke. N Engl J Med 2015; 372:1019-1030.

21. Campbell B, Mitchell P, Kleinig T, et al. Endovascular Therapy for Ischemic Stroke with Perfusion-Imaging Selection. N Engl J Med 2015; 372:1009-1018.

22. Saver J, Goyal M, Bonafe A, et al. Stent-Retriever Thrombectomy after Intravenous t-PA vs. t-PA Alone in Stroke. N Engl J Med 2015; 372:2285-2295.

23. Jovin T, Chamorro A, Cobo E, et al. Thrombectomy within 8 Hours after Symptom Onset in Ischemic Stroke. N Engl J Med 2015; 372:2296-2306.

24. Mocco J, Zaidat O, von Kummer R, et al. Aspiration Th- rombectomy After Intravenous Alteplase Versus Intravenous Alteplase Alone. Stroke. 2016;47:2331-2338.

25. Bracard S, Ducrocq X, Mas JL, et al. Mechanical thrombectomy after intravenous alteplase versus alteplase alone after stroke (THRACE): a randomised controlled trial. DOI: https://doi.org/10.1016/S1474-4422(16)30177-6.

26. Muir K, Ford G, Messow CM, et al. Endovascular therapy for acute ischaemic stroke: the Pragmatic Ischaemic Stroke Thrombectomy Evaluation (PISTE) randomised, controlled trial. Journal of Neurology, Neurosurgery \& Psychiatry 2017;88:38-44.

27. Goyal M, Menon B, Zwam W, et al. Endovascular thrombectomy after large-vessel ischaemic stroke: a metaanalysis of individual patient data from five randomised trials. https://doi.org/10.1016/S0140-6736(16)00163-X.

28. Saver JL, Goyal M, van der Lugt A et al. Time to Treatment With Endovascular Thrombectomy and Outcomes From Ischemic Stroke: A Meta-analysis. JAMA. 2016 Sep 27;316(12):1279-88.

29. Nogueira RG, Jadhav AP, Haussen DC, et al; DAWN Trial Investigators. Thrombectomy 6 to 24 Hours after Stroke with a Mismatch between Deficit and Infarct. N Engl J Med. 2018 Jan 4;378(1):11-21.

30. Albers GW, Marks MP, Kemp S et al Thrombectomy for Stroke at 6 to 16 Hours with Selection by Perfusion Imaging. N Engl J Med. 2018 Feb 22;378(8):708-718. doi: 10.1056/NEJMoa1713973.

31. Mistry EA, Sucharew H, Mistry AM et al. Blood Pressure after Endovascular Therapy for Ischemic Stroke (BEST): A Multicenter Prospective Cohort Study. Stroke. 2019 Dec;50(12):3449-3455. doi: 10.1161/STROKEAHA.119. 026889.

32. Ryu WHA, Avery MB, Dharampal N, Allen IE, Hetts SW. Utility of perfusion imaging in acute stroke treatment: a systematic review and meta-analysis. J Neurointerv Surg. 2017 Oct;9(10):1012-1016.

33. Pérez MA, Miloslavski E, Fischer S, Bäzner H, Henkes H. Intracranial thrombectomy using the Solitaire stent: a historical vignette. J Neurointerv Surg. 2012 Nov;4(6):e32. doi: 10.1136/neurintsurg-2011-010149.

34. Zaidat OO, Bozorgchami H, Ribó M, Saver JL, et al. Primary Results of the Multicenter ARISE II Study (Analysis of Revascularization in Ischemic Stroke With EmboTrap). Stroke. 2018 May;49(5):1107-1115.

35. Chueh JY, Kang DH, Kim BM, Gounis MJ. Role of Balloon Guide Catheter in Modern Endovascular Thrombectomy. J Korean Neurosurg Soc. 2020 Jan;63(1):14-25.

36. Lapergue B, Blanc R, Gory B, et al. Effect of endovascular contact aspiration vs stent retriever on revascularization in patients with acute ischemic stroke and large vessel occlusion: the ASTER randomized clinical trial. JAMA 2017;318:443-52.

37. Maus V, Behme D, Kabbasch C, et al. Maximizing firstpass complete reperfusion with SAVE. Clin Neuroradiol 2018;28:327-338.

38. Evans MRB, White P, Cowley P, Werring DJ. Revolution in acute ischaemic stroke care: a practical guide to mechanical thrombectomy. Pract Neurol 2017;17:252- 65. 


\title{
Modelli organizzativi nella gestione dell'ictus ischemico
}

\author{
Rosario Iannacchero, ${ }^{1}$ Antonio Siniscalchi, ${ }^{2}$ Domenico Bosco ${ }^{1}$ \\ ${ }^{1}$ S.O.C. Neurologia + Stroke Unit, Azienda Ospedaliera Pugliese-Ciaccio, Catanzaro; ${ }^{2}$ S.O.C. Neurologia+Stroke Unit, Azienda \\ Ospedaliera di Cosenza, Italia
}

\section{Introduzione}

Il riconoscimento precoce di un insulto vascolare ischemico e il suo trattamento in strutture dedicate, le Stroke Unit (SU), dotate di equipe multidisciplinari (neurologi, neuroradiologi interventisti, neurosonologi, internisti, cardiologi, fisiatri, fisioterapisti, infermieri dedicati) in grado di predisporre un trattamento integrato medico-riabilitativo, riduce il rischio di mortalità e di invalidità. La realizzazione e la diffusione di queste strutture dedicate era l' obiettivo dell' $A c$ cordo Stato - Regione del 3 /2/2005 linee di indirizzo per la definizione del percorso assistenziale ai pazienti con ictus cerebrale. Tale accordo, prodigo di dettagli operativi e organizzativi, ma di limitata cogenza, impegnava i Dipartimenti delle Sanità Regionali alla costruzione di reti per l'assistenza integrata allo Stroke e favorire la formazione di aree di assistenza dedicate di maggiore intensità di cura negli ospedali regionali HUB dotati delle competenze di Neuroradiologia Interventistica e Neurochirurgia e aree di minor intensità di cura negli ospedali spoke territoriali, pur in assenza della disciplina di Neurochirurgia ma collegati con procedure di telemedicina con la struttura ospedaliera Neurochirurgica di riferimento territoriale e, con personale medico-infermeriestico polivalente e dedicato. La rete Stroke viene pianificata e disegnata nell'accordo Stato-Regione 2005 mediante un modello integrato circolare di governance territoriale, che ruota intorno alla figura del paziente colpito da insulto vascolare ischemico; l'obiettivo era creare un link operativo virtuoso tra territorio ed ospedale, con azioni

Corrispondente: Rosario Iannacchero, SOC Neurologia+Stroke Unit, Azienda Ospedaliera Pugliese-Ciaccio Catanzaro, Italia. E-mail: rosarioiann@tiscali.it

Articolo pubblicato secondo la Creative Commons Attribution NonCommercial 4.0 License (CC BY-NC 4.0).

${ }^{\circ}$ Copyright: the Author(s), 2020

Licensee PAGEPress, Italy

QUADERNI - Italian Journal of Medicine 2020; 8(2):73-78 sinergiche e consequenziali degli operatori sanitari coinvolti, mentre in precedenza quest'ultimi erano autonomi nella loro pratica clinica e quindi con limiti operativi. Nella fase di pre-evento ischemico vi è la necessità di realizzare campagne formative e divulgative con l'assistenza territoriale primaria (medici di medicina generale e medici di continuità assistenziale) e le associazioni dei pazienti per la prevenzione dell'ictus sui fattori di rischio delle malattie cardio-cerebrovascolari (fumo, ipertensione, controllo glicemia, obesità) e gli stili di vita (attività fisica quotidiana, alimentazione sana e mirata). Nella fase dell' insulto ischemico la rete Stroke, usufruendo dei Dipartimenti Emergenza -Urgenza di I e II livello e delle loro reti (SUEM 118 - Medicina d' Urgenza - Pronto Soccorso), assicura un tempestivo intervento sul paziente con l' applicazione di tecniche rianimatorie e specifiche scale di valutazione (scale di Cincinnati, del Coma di Glasgow, NIHSS). La possibilità da parte degli operatori sanitari della rete di emergenza - urgenza di individuare la natura dell' insulto vascolare ischemico (TIA e/o ictus ischemico o emorragico) favorisce l' individuazione del contesto ospedaliero più appropriato per il paziente ed il ricovero nelle SU di I o II livello per il trattamento specifico di trombolisi e/o endovascolare ed avvio precoce ad una fase riabilitativa. Il modello organizzativo della Rete Stroke aiuta ad incrementare il numero di pazienti eleggibili per la trombolisi endovenosa entro la finestra terapeutica di 4-1/2 ore dall'evento nelle strutture autorizzate Hub o Spoke $\mathrm{d}$ in funzione o meno della presenza delle discipline della Neuroradiologia Interventistica e della Neurochirurgia. Nella fase post-acuzie si deve attivare un link diretto con le strutture riabilitative, individuate e calibrate sulle esigenze di cura del paziente (riabilitazione intensiva/estensiva) e non solo sulla disponibilità dei posti letto; bisogna favorire i piani terapeutici individuali che favoriscono il reinserimento nel tessuto sociale - familiare - lavorativo, condividendo il percorso clinico del paziente intrapreso nella struttura ospedaliera con quello pianificato nei servizi territoriali riabilitativi socio-assistenziali . Il passaggio dalla best evidence alla best practice, con la diffusione di cambiamenti strutturali nei metodi di cura dei pazienti, è tuttavia molto difficile, poiché incontra barriere cul- 
turali e organizzative; è necessario creare gruppi di lavoro e di studio tra i soggetti implicati nella pratica di cura medica per acuti e le strutture riabilitative di riferimento nell' area territoriale in cui è ubicata la Stroke Unit per condividere un approccio integrato o e di presa in carico del paziente colpito da insulto vascolare ischemico .

\section{Aree di degenza dedicate}

In molti casi l'orientamento del degli indirizzi regionali delle Reti Stroke è quello di promuovere l'attivazione di strutture dedicate (Stroke Unit o SU). La regione Lazio, per esempio, ha definito i servizi ospedalieri dedicati alla gestione clinico-assistenziale dei pazienti con ictus quali Unità di trattamento Neuro Vascolare; si tratta di trattamenti di terapia semi-intensiva distinti in due livelli (UTN I e UTN II), e sono definite le priorità per l'accesso rapido e preferenziale alle UTN II secondo protocolli prestabiliti e strumentazioni diagnostiche - terapeutiche adeguate (Neuroradiologia Interventistica). La Lombardia, invece, ha un piano cardio-cerebrovascolare regionale che prevede l'attivazione della rete di UCV - Stroke Units. Le UCV sono aree di degenza specializzata per la cura dell' ictus, collocate nell' ambito di reparti di neurologia o in strutture di degenza a caratterizzazione neurologica. Riguardo alla definizione dei requisiti delle aree di degenza in acuzie o SU, alcune regioni prevedono dei contesti ospedalieri definiti per i pazienti con ictus (Friuli Venezia Giulia, Valle d'Aosta, Piemonte e Lazio), mentre altre modalità organizzative collocano le SU nell'area medica con valenza interdipartimentale (Basilicata), oppure prevedono che tali aree dedicate siano individuate nei reparti di neurologia o in strutture di degenza a caratterizzazione neurologica (Lombardia, Marche, Toscana, Abruzzo e Calabria).

\section{Team multidisciplinare}

A prescindere del modello organizzativo scelto dalle singole realtà regionali, viene esortata l'attivazione di un team multidisciplinare e multi professionale con personale medico ed infermieristico adeguatamente preparato e aggiornato che garantisca continuità dell'assistenza durante tutto il percorso diagnostico-terapeutico, dalla fase acuta ospedaliera a quella post-acuta. In alcuni casi è prevista anche l'attivazione di un team itinerante presso alcuni ospedali periferici sede di Pronto Soccorso oppure, qualora non sia presente la degenza neurologica, si prevede (es. in Basilicata ) di attivare un'equipe multidisciplinare nell'ambito delle Unità Operative di Medicina Interna. Il team multidisciplinare, oltre alle attività assistenziali, ha il compito di promuovere protocolli diagnostici - terapeutici per la patologia cerebrovascolare da validare in sede regionale, di offrire la propria consulenza ad altri presidi ospedalieri e di gestire un ambulatorio dedicato alle malattie cerebrovascolari per favorire il follow-up dei pazienti ricoverati nei letti dedicati.

\section{Modello organizzativo preospedaliero e ospedaliero per ictus acuto}

Nella cura dell'ictus ischemico, la riperfusione rapida è essenziale per migliorare la sopravvivenza libera da disabilità ed è tempo dipendente (time is brain). ${ }^{1}$ L'ictus ischemico acuto viene trattato con modalità di rivascolarizzazione attraverso la somministrazione sistemica di alteplase per via endovenosa nei centri di riferimento dello stroke (primary stroke center, PSC; Stroke Unit I livello), mentre nelle occlusioni dei grandi vasi cerebrali il trattamento combinato o il solo trattamento endovascolare, o la necessita di un trattamento chirurgico vascolare o neurochirurgico, vengono effettuati nei centri stroke di eccellenza (comprehensive stroke center, CSC; o Stroke Unit II livello). Stante questa possibilità di scelta di varie opzioni terapeutiche della fase acuta dell'Ictus ischemico, è essenziale l'organizzazione in rete pre-ospedaliera che permetta rapidi trasferimenti da PSC a CSC qualora si identifichi una condizione clinica che richieda l'intervento di procedure e tecniche altrimenti non disponibili. ${ }^{2}$ La possibilità di approcci innovativi nella gestione dell'ictus pone peraltro problematiche organizzative non indifferenti. ${ }^{2}$ Infatti, se da una parte i nuovi trattamenti dell'ictus assicurano una significativa riduzione della mortalità e dell'invalidità residua dell'ictus, dall'altra parte, a volte, assistiamo anche impotenti al ritardo con cui i pazienti arrivano in ospedale, o i loro familiari decidono di muoversi verso l'ospedale, rendendo di fatto indisponibile una decisiva possibilità di trattamento terapeutico. Esistono differenti modelli organizzativi pre-ospedalieri nel trattamento dell'ictus acuto: i) mother-ship; ii) drip and ship; iii) mobile interventionist; e iv) mobile stroke units (MSU) model. ${ }^{3} \mathrm{Nel}$ modello mother-ship, i pazienti vengono trasportati direttamente al centro CSC, by-passando qualsiasi centro di PSC. La caratteristica distintiva del modello drip and ship, invece, è che il trattamento trombolitico endovenoso viene avviato nel $\mathrm{PSC}$ più vicino, seguito successivamente dal trasporto al CSC in caso di occlusione dei grossi vasi cerebrali. Il trasporto del paziente, in caso di distanze superiori a 60 minuti ad una PSC, come negli Stati Uniti, ed in assenza di infrastrutture tecnologiche (telemedicina), potrebbe avvenire tramite anche l'utilizzo dell'elicottero. ${ }^{4}$ Il trasporto in elicottero per trombolisi sistemica, rispetto ad altre procedure di intervento medico come l'endoarteriectomia, da uno studio americano, è risultato 
essere meno impegnativo nel consumo di risorse e favorisce una riduzione della finestra terapeutica per trattamento fibrinolitico con minore disabilità residua, anche se il limite al suo utilizzo può essere rappresentato dall'area geografica e, a volte, dalle condizioni metereologiche. ${ }^{5,6} \mathrm{Nel}$ modello mobile interventionist, l'interventista endovascolare viene trasportato nel centro PSC, al fine di eseguire il trattamento endovascolare. Infine, nel modello di mobile stroke unit (MSU), presente in alcuni paesi come Germania e Stati Uniti, il paziente viene gestito in un'ambulanza di unità di stroke mobile, dotata di un sistema di imaging, un laboratorio di assistenza e una connessione telemedicina al CSC. Le unità di stroke mobile (MSU) forniscono una preziosa risorsa pre-ospedaliera in contesti rurali e remoti in cui i pazienti potrebbero non avere un facile accesso alle cure per l'ictus in ospedale. ${ }^{7}$ La strategia MSU ha dimostrato di essere efficace nel facilitare le decisioni di triage nell'ictus con un risparmio nel tempo. Il modello MSU attraverso utilizzo mobile delle indagini neuro radiologiche ( TC cranio e Angio - TC vasi cranici) determina se un paziente deve essere portato in un PSC per il trattamento standard o in un CSC per il trattamento dell'ictus (terapia intra-arteriosa o trattamento endovascolare). Inoltre, per i pazienti con ictus emorragico, il triage basato sulla MSU consente il trasporto verso ospedali con servizi di neurochirurgia (CSC), bypassando gli ospedali senza tali opportunità. ${ }^{8}$ La diagnosi effettuata nel MSU riduce i tempi fisiologici morti che si possono verificare durante il trasporto del paziente nei dipartimenti di emergenza, e il trasporto secondario (interospedaliero) ed aumenta la finestra terapeutica per il paziente. Le MSU possono essere adattate alle esigenze locali, soprattutto in contesti rurali e remoti, con adeguamenti del personale, della configurazione delle ambulanze e dei modelli di trasporto. Inoltre, con imaging avanzato e ulteriori capacità diagnostiche, le MSU forniscono una preziosa piattaforma per la telemedicina (teleradiologia e telestroke) in queste aree sottoservite. Ci sono, però, alcune limitazioni nel loro uso. Per primo, il trattamento dell'ictus acuto in una MSU è un esercizio complesso che prevede l'esecuzione di più attività parallele da parte di diversi operatori sanitari all'interno dello spazio limitato di una MSU. Ciò include la valutazione neurologica, il monitoraggio dei segni vitali, il posizionamento del paziente, la gestione del comfort del paziente e l' interazione telematica tra ambulanza mobile e ospedale dove eseguire il trattamento terapeutico specifico, condividendo con gli operatori sanitari ospedalieri la scansione TC, i test di laboratorio e la preparazione e la somministrazione dei farmaci. ${ }^{9}$ Anche se, in futuro, i miglioramenti della tecnologia potranno consentire unità TC CT più piccole, più leggere e più robuste. Inoltre, la decisione clinica sull'opportunità di som- ministrare farmaci trombolitici richiede addestramento, esperienza e un attento giudizio clinico e, quindi, la necessità di un neurologo vascolare. Un altro problema, ad esempio negli Stati Uniti, e che alcune MSU non sono gestite da medici.

In Italia, per la maggior parte, nello scenario preospedaliero, si pone il problema se è più favorevole trasportare un paziente dapprima ad un PSC per una trombolisi sistemica e, successivamente, ad un CSC per un trattamento endovascolare (drip-and-ship) o il trasporto diretto del paziente al CSC, dove potrà essere sottoposto ad una trombolisi sistemica e se necessario a un successivo trattamento endovascolare (mothership). La maggiore possibilità e disponibilità di un trattamento endovenoso e l'introduzione del trattamento endovascolare ha portato a una rivalutazione del modello di drip-and-ship..$^{10}$ Il modello mother-ship è stato usato per la maggior parte dei pazienti negli studi randomizzati e controllati di trattamento intraarterioso/endovascolare. ${ }^{11}$ Gli studi osservazionali hanno mostrato che il modello drip and ship viene frequentemente utilizzato in ambito clinico pratico in molti paesi rispetto al mother-ship (Stati Uniti, Corea del Sud, Spagna, Germania, Italia e Francia), mentre nella letteratura medica ci sono pochi dati sull'unità di $M S U$ e sui modelli di mobile interventionist. Una recente metanalisi non ha riportato differenze nei risultati clinici o radiologici tra i modelli di drip and ship e mother-ship..$^{12}$ Altri studi, invece, in cui sono stati confrontati i due modelli drip and ship e mother-ship sono stati riscontrati tempi di inizio trattamento più brevi nel modello mother-ship rispetto al modello drip and ship. ${ }^{12}$ Più sorprendentemente, $\mathrm{i}$ tempi di arrivo in PS ed inizio trattamento (onset-to-needle times) sono stati anche più brevi nel modello mother-ship.

Secondo le linee guida europee, il trasporto del paziente al PSC per il trattamento trombolitico endovenoso è indicato se la distanza è inferiore di 30-45 minuti rispetto al CSC e studi di modellistica statistica, ipotizzando arrivo in PS ed inizio trattamento (doorto-needle time) di massimo 60 minuti Le raccomandazioni dell'American Heart Association/American Stroke Association per servizi medici di emergenza è ancora più restrittivo, raccomandando il trasporto diretto ad un CSC per i pazienti preospedalieri con sospetta occlusione dei grossi vasi cerebrali mediante una scala di gravità dell'ictus (NIHSS), se il tempo necessario per raggiungere il CSC è inferiore a 15 minuti aggiuntivi rispetto al tempo di percorrenza fino al PSC più vicino. È quindi difficile valutare se sia ragionevole utilizzare il limite di 30-45 minuti per il processo decisionale nella pratica clinica. Gli effetti clinici dei diversi modelli dipenderanno dalla qualità di questi modelli. Ad esempio, affinché il modello drip and ship funzioni, la valutazione iniziale e l'inizio del trattamento trombolitico nel PSC devono essere rapidi e gli 
studi di neuroimaging complessi devono essere evitati. Allo stesso modo, per valorizzarla bontà del modello mother-ship, l'accuratezza della diagnosi preospedaliera di occlusione dell'arteria di grandi dimensioni è importante, in particolare laddove il tempo di trasporto è lungo e, pertanto, diventa necessario l'uso di punteggi clinici o della telemedicina. ${ }^{12}$ Il recente studio RACECAT, sviluppato a Barcellona, in Spagna, condotto su pazienti con ictus con sospetta occlusione di grandi vasi, valutati dai servizi medici di emergenza, ha dimostrato tassi più elevati di esito favorevole quando gli stessi vengono trasferiti direttamente a un centro endovascolare (mothership), rispetto ad un iniziale trasporto al PSC (drip-and-ship). ${ }^{13}$ Questo percorso provoca un' allungamento della finestra terapeutica con ritardo nel trasferimento dei pazienti con occlusione di grandi vasi negli ospedali senza competenze di trattamento endovascolare diminuisce la probabilità che i pazienti ricevano un trattamento intra-arterioso del 2,5\%. Non si può affermare che un singolo modello organizzativo è superiore in efficacia rispetto all'altro, poichè la strategia di scegliere un percorso diagnostico-terapeutico è influenzato da aspetti logistici ambientali ( tempi di trasporto e presenza di competenze di neuroradiologia interventista) e caratteristiche del paziente (finestra terapeutica dall'esordio dell'ictus e eleggibilità ammissibilità ai metodi di ricanalizzazione endovenosa e intra-arteriosa). Ad esempio, nelle aree metropolitane, con tempi di trasporto verso un PSC inferiore a 30-45 minuti, è possibile raccomandare il modello di mothership, con l'uso del modello drip and ship quando i tempi di trasporto sono più lunghi. Una strategia ottimale per l'interventista mobile (mobil interventionist) è quella in cui lo stroke team interventistico raggiunge il paziente, nella struttura ospedaliera PSC, dove lo stesso è sottoposto a trombolisi endovenosa . Infine, il modello di MSU, con l'uso della diagnostica avanzata e della medicina remota in ambulanza, può offrire vantaggi in aree con lunghi tempi di trasporto alla $\mathrm{SU}$ più vicina. E' necessario realizzare degli studi randomizzati controllati sui diversi modelli organizzativi preospedalieri per individuare il percorso ottimale per il paziente colpito da ictus acuto.;la scelta tra i modelli più frequentemente utilizzati (mother-ship e drip- andship), è sostenuta dall'utilizzo di una valutazione clinica preospedaliera, attraverso scale di valutazione per l'ictus, che identificano il territorio ischemico cerebrale causa l'occlusione di grandi arterie sia del circolo anteriore, che il circolo posteriore con la possibilità di utilizzare le indagini neurosologiche (Doppler sonografia transcranica). In conclusione, non ci sono prove da studi di alta qualità a supporto dell'adozione di un modello organizzativo universale preospedaliero per la cura dell' ictus, ma la scelta del modello per la cura del paziente colpito da insulto va- scolare ischemico è in funzione delle caratteristiche cliniche del paziente e dal contesto organizzativo della rete ospedaliera territoriale per lo stroke

\section{Riabilitazione post-acuzie}

Nei contesti organizzativi ospedalieri dove è presente sia l'area di degenza Stroke Unit che l'area di degenza ordinaria neurologica, quando la situazione clinica risulti stabilizzata, di norma mai prima di 4-6 giorni, il paziente viene trasferito nei letti di regime ordinario della degenza neurologica In alcune strutture ospedaliere, evento poco frequente, vi è la possibilità di avere dei reparti di riabilitazione intensiva e nella fattispecie il paziente viene trasferito dall'area di degenza dedicata alla cura dell'ictus alla area di degenza riabilitativa. Nei pazienti in cui vi sia indicazione, la riabilitazione va iniziata precocemente, già durante la degenza in $\mathrm{SU}$, ma deve estrinsecarsi nei reparti di riabilitazione post-evento acuto. Il passaggio dalla fase acuta a quella riabilitativa post-acuta o alla tipologia di setting riabilitativo più appropriato nella fase post-ospedaliera è stabilito essenzialmente dal grado di disabilità, di comorbilità del paziente $\mathrm{e}$ dalla relativa potenzialità $\mathrm{a}$ trarre giovamento dal livello assistenziale riabilitativo più appropriato (intensivo/estensivo). Alcune regioni hanno concentrato l'interesse prevalentemente sulla fase riabilitativa del percorso assistenziale, come ad esempio la Regione Umbria; essa ha infatti individuato la necessità di sviluppare la funzione riabilitativa chiedendo alle Aziende Sanitarie un appropriato sviluppo della riabilitazione territoriale e di quella in regime di degenza. Un gruppo di lavoro multidisciplinare aziendale in regione Umbria ha collaborato alla stesura di una linea guida diagnostico-terapeutica per la riabilitazione della persona con ictus cerebrale. Lo scopo è assistere medici, operatori sanitari delle strutture ospedaliere e territoriali nella gestione del paziente con ictus dal momento dell'evento al ritorno nella migliore qualità di vita possibile, individuando il percorso più idoneo alle sue caratteristiche di malattia.

\section{Conclusioni}

L'attuale sistema sanitario nazionale si sta fortemente regionalizzando, mantenendo sempre delle tendenze comuni; in particolare l'orientamento di modello organizzativi a rete per le grandi patologie (rete oncologica, emergenza-urgenza, cardiologica, traumatologica) e l'integrazione di queste reti cliniche con le opportunità offerte al paziente in termini socio-sanitari. Il modello della rete Stroke integrata evidenzia la tendenza a costruire un modello organizzativo ad intensità di cura, che si determina sul territorio per gli aspetti clinici di prevenzione secondaria e riabilitativa e sulle 
strutture ospedaliere per gli aspetti acuti, con logiche di concertazione e cooperazione tra opinion leader che sono presenti nella pratica clinica ospedaliera/territoriale. L'implementazione del modello a rete nel servizio sanitario nazionale implica d'altronde il superamento di settorialismi e specializzazioni che caratterizzano il nostro sistema sanitario e l'inserimento del processo assistenziale in un'ottica di governance di un contesto di cura geograficamente ben limitato e definito, ed in cui è necessaria la compresenza, il dialogo e la complementarietà tra attori di natura differente (pubblici, privati, no profit e profit, associazione dei pazienti). La centralità è assegnata al territorio ed al paziente che, assieme ai familiari, diventa parte attiva del processo di costruzione del percorso assistenziale, in un'ottica di cittadinanza attiva e di legittimazione; diventa fondamentale anche la responsabilità, che spinge verso i processi di accreditamento ed al monitoraggio di qualità. Il processo di managerializzazione del sistema sanitario, iniziato dagli anni Ottanta, ha comportato un'attenzione maggiore al contenimento della spesa ed all'allocazione delle risorse in termini di efficacia, efficienza ed economicità. Il ridimensionamento e la razionalizzazione delle strutture ospedaliere risponde alla necessità di diminuire i costi, ma esprime la necessità di superare una visione tradizionale dell'assistenza, offrendo servizi destandardizzati, più qualificati, attraverso un potenziamento dei servizi territoriali. L' analisi delle differenze di implementazione dell'Accordo Stato-Regioni del 2005 in materia di Ictus ha messo in luce la centralità dei fattori di politica sanitaria nel determinare le modalità in cui viene pianificata e realizzata la Rete Stroke. Per esempio, la centralità del modello organizzativo dell' equità in Toscana e della concorrenza in Lombardia si riflettono in un'approccio diametralmente opposto alla rete: nel primo caso essa risulta strutturata gerarchicamente in quanto i nodi sono incorporati negli obiettivi di politica sanitaria pubblica ed hanno scarsa autonomia decisionale. Nel secondo caso la rete è definita attraverso un percorso, in cui i diversi nodi sono in competizione costruttiva tra di loro. Le caratteristiche socio-ambientali dei vari territori possono ostacolare o favorire le strategie di implementazione: per esempio la cultura individualista e poco incline alla collaborazione genera chiusura e frammentazione ostacolando la condivisione necessaria al buon funzionamento della rete dello stroke. Al contrario la cultura dell'associazionismo e volontariato rappresenta una risorsa particolarmente utile che, tuttavia non è spesso valorizzata e in alcuni casi si trasforma in vincoli. Più specificamente, sebbene tutte le Regioni abbiano deliberato in materia di Reti Stroke, si registra un rallentamento sul piano della realizzazione. Anche le Regioni più attente e sensibili a tale problematica, faticano a gestire tutta la filiera prevista dalla rete, soprattutto in relazione all' integrazione con i servizi socio-sanitari nelle fasi di post-acuzie. La costruzione della rete Stroke è al momento schiacciata sulla gestione delle acuzie dell' insulto vascolare ischemico, mentre sono ancora poco sviluppate gli aspetti di riabilitazione intensiva; critico è il collegamento tra strutture ospedaliere per acuti e quelle riabilitative, spesso non dotate di livelli di competenza. Altro punto cruciale è il riordino del Sistema Emergenza-Urgenza: laddove la riorganizzazione di questo settore è più avanzata, anche la rete Stroke è più sviluppata e a regime; quindi Rete Stroke e Rete Emergenza - Urgenza devono coordinarsi e condividere obiettivi comuni e non avere conflittualità operativa. In alcuni regioni, in particolare nel sud Italia, le Stroke Unit sono state attivate con modalità artigianali, grazie ad un strategia cooperativa tra neurologi particolarmente motivati e il management delle Aziende Ospedaliere. Purtroppo un vincolo fortissimo alla realizzazione delle Stroke Unit sono i divieti di assunzione, particolarmente nelle Regioni Commissariate, prevalentemente nel centro Sud Italia motivo che difficilmente una SU si comporrà di personale specializzato e multidisciplinare; ciò allontana il modello organizzativo reale da quello consigliato dalla normativa ministeriale vigente. Infine vanno sottolineate le difficoltà collegate alle tensioni relazionali tra gli operatori sanitari della rete Stroke, legate a dinamiche di potere, sospetto e/o scetticismo; gli stessi specialisti neurologici devono modificare l'immagine di neurologi come specialisti delle patologie neurologiche croniche-degenerative verso una nuova cultura dello specialista neurologo che è quella dell'emergenza-urgenza. In altri casi bisogna considerare l'ostilità culturale di alcuni neurologi per i modelli di assistenza clinica di intensità di cura. Tutte queste problematiche possono essere alla base della non completa realizzazione delle Reti Stroke regionali. Il deficit comunicativo può essere superato attivando percorsi formativi cooperativi e condivisi con gli operatori sanitari dei vari nodi della rete; non percorsi formali, ma collaborazioni sostanziali in grado di attivare processi decisionali condivisi e organizzativi partecipati condivisi e dal basso, in cui tutti i soggetti interessati dalla filiera di cura siano coinvolti e maturino percorsi culturali condivisi.

\section{Bibliografia}

1. Holodinsky JK, Patel AB, Thornton J, et al. Drip and ship versus direct to endovascular thrombectomy: The impact of treatment times on transport decision-making. Eur Stroke J. 2018;3:126-35.

2. Détraz L, Ernst M, Bourcier R. Stroke Transfer and its Organizational Paradigm. Clin Neuroradiol 2018;28: 473-80.

3. Ciccone A, Berge E, Fischer U. Systematic review of organizational models for intra-arterial treatment of acute ischemic stroke. Int J Stroke 2019;14:12-22.

4. SalerM , SwitzerJA, HessDC. Use of telemedicine and 
helicopter transport to improve stroke care in remote locations. Curr Treat Options Cardiovasc Med. 2011;13:215-24.

5. Silbergleit R, Scott PA, Lowell MJ, et al. Cost-effectiveness of helicopter transport of stroke patients for thrombolysis. Acad Emerg Med 2003;10:966-72.

6. SalerM, SwitzerJA, HessDC. Use of telemedicine and helicopter transport to improve stroke care in remote locations. Curr Treat Options Cardiovasc Med. 2011;13:215-24.

7. Mathur S, Walter S, Grunwald IQ, et al. Improving Prehospital Stroke Services in Rural and Underserved Settings With Mobile Stroke Units. Front Neurol. 2019;10:159.

8. Rajan SS, Baraniuk S, Parker S, et al. Implementing a mobile stroke unit program in the United States: why, how, and how much? JAMA Neurol 2015;72:229-34.

9. Ismail M, Armoiry X, Tau N, et al. Mothership versus drip and ship for thrombectomy in patients who had an acute stroke: a systematic review and meta-analysis. Neurointerv Surg. 2019;11:14.
10. Abilleira S, Pérez de la Ossa N, Jiménez X, et al. Transfer to the Local Stroke Center versus Direct Transfer to Endovascular Center of Acute Stroke Patients with Suspected Large Vessel Occlusion in the Catalan Territory (RACECAT): Study protocol of a cluster randomized within a cohort trial. Int J Stroke 2019;14:734.

11. Froehler MT, Saver JL, Zaidat OO, et al. Interhospital Transfer prior to thrombectomy is associated with delayed treatment and worse outcome in the STRATIS registry. Circulation 2017;13:2311-21.

12. Weber R, Reimann G, Weimar C, et al. Outcome and periprocedural time management in referred versus directly admitted stroke patients treated with thrombec- tomy. Ther Adv Neurol Disord 2016;9:79-84.

13. Abilleira S, Pérez de la Ossa N, Jiménez X, et al. Transfer to the Local Stroke Center versus Direct Transfer to Endovascular Center of Acute StrokePatients with Suspected Large Vessel Occlusion in the Catalan Territory (RACECAT): Study protocol of a cluster randomized within a cohort trial. Int J Stroke. 2019;14:734-44. 


\title{
Emorragia cerebrale intraparenchimale spontanea
}

\author{
Maela Masato, ${ }^{1}$ Andrea Bruscagnin ${ }^{2}$ \\ ${ }^{1}$ UOC Neurologia, Ospedale Mirano-Venezia; ${ }^{2}$ UOC Radiologia, Ospedale Mirano-Venezia, Italia
}

\section{Introduzione}

L'emorragia cerebrale intraparenchimale spontanea (intracerebral hemorrhage, ICH) rappresenta un'emergenza medica ad alto rischio per la vita che richiede cure immediate. ${ }^{1-41}$

La prognosi è grave quod vitam et valetudinem. ${ }^{4}$

Si tratta di una raccolta di sangue circoscritta all'interno del parenchima cerebrale, che può aprirsi all'interno dei ventricoli cerebrali.

In base alla sede e all'estensione dell'emorragia si possono avere vari sintomi e segni neurologici.

Le cause sono suddivise in due gruppi: i) emorragie primitive: principalmente dovute all'ipertensione arteriosa e all'arteriopatia amiloidotica cerebrale (cerebral amyloid angiopathy, CAA); ii) emorragie secondarie: dovute a tumori, malformazioni vascolari, aneurismi.

Questa trattazione è rivolta alle emorragie intraparenchimali spontanee e quindi primitive con cenno a quelle dovute ad abuso di sostanze e a situazioni predisponenti.

\section{Epidemiologia}

L'emorragia intracranica è un evento medico rilevante e riscontrabile nel $15-20 \%$ degli ictus cerebrali. L'incidenza dell'emorragia cerebrale spontanea è di 10-30 casi su 100.000 persone per anno e aumenta nell'età dai 55 anni in poi.

Le emorragie primitive costituiscono il 78\% della totalità delle emorragie cerebrali.

La mortalità a 30 giorni è di circa il 40-50\%.

Corrispondente: Maela Masato, Neurologia, Ospedale MiranoVenezia, via G. Sartor 4, 30035 Mirano-Venezia, Italia. E-mail: maela.masato@aulss3.veneto.it

Articolo pubblicato secondo la Creative Commons Attribution NonCommercial 4.0 License (CC BY-NC 4.0).

${ }^{\circ}$ Copyright: the Author(s), 2020

Licensee PAGEPress, Italy

QUADERNI - Italian Journal of Medicine 2020; 8(2):79-93
Il $75 \%$ delle persone che sopravvivono presentano un'invalidità e solamente $20 \%$ di esse raggiungono una parziale indipendenza.

L'ipertensione arteriosa è la causa principale, seguita dall'arteriopatia amiloidotica cerebrale, dall'utilizzo di anticoagulanti, dall'abuso di alcool e dalla assunzione di droghe. ${ }^{12}$

\section{Prognosi}

Volume e sede dell'ematoma sono i fattori critici per la prognosi sia per quanto riguarda la sopravvivenza sia per l'esito funzionale.

Le dimensioni dell'ematoma possono essere misurate con la tomografia computerizzata (TC) cerebrale diretta utilizzando la formula $\mathrm{V}=\mathrm{ABC} / 2$ :

$\mathrm{A}=\mathrm{il}$ diametro maggiore della lesione;

$\mathrm{B}=$ il diametro maggiore della lesione a 90 gradi dalla linea $\mathrm{A}$;

$\mathrm{C}=$ numero di slides $\mathrm{TC}$ nel piano verticale dove sia

visibile l'immagine dell'emorragia moltiplicato per

lo spessore della singola slide (in genere $0,5 \mathrm{~cm}$ ).

La recidiva di emorragia avviene prevalentemente nelle prime 6 ore dopo il primo sanguinamento indicando una prognosi sfavorevole. . $^{1522}$

Un basso valore di Glasgow Coma Scale (GCS) e la presenza di comorbidità peggiorano la prognosi (Figura 1).

\section{Patofisiologia}

L'emorragia intraparenchimale spontanea è presumibilmente dovuta alla malattia dei piccoli vasi intracerebrali. ${ }^{14}$

Le due principali malattie dei piccoli vasi sono l'arteriopatia ipertensiva che predilige le piccole arterie perforanti dei nuclei grigi profondi, del tronco encefalico e della sostanza bianca e l'angiopatia amiloidea che causa emorragie lobari per rottura delle piccole arterie superficiali corticali e leptomeningee.

Quindi le due principali categorie di emorragie intraparenchimali spontanee sono: i) non lobari: profonde o infratentoriali (Figura 2); ii) lobari: interessamento dei lobi cerebrali (Figura 3). 


\section{Classificazioni e strumenti di definizione}

Esistono criteri di classificazione e di definizione delle emorragie spontanee:

- secondo la definizione neuroradiologica delle malattie dei piccoli vasi: STRIVE (STandard for ReportIng Vascular changes on nEuroimaging), position paper, che valuta le lesioni definite alla RM distinguendo la sede, la dimensione e le sequenze RM per identificarle e il microsanguinamento. ${ }^{40}$

- la classificazione anatomica delle emorragie intra- parenchimali: CHARTS (Cerebral Haemorrhage Anatomical RaTing inStrument) che definisce tre principali categorie: ${ }^{8}$ i) lobare; ii) profonda e infratentoriale; iii) incerta: per l'estensione risulta difficile distinguere tra lobari e non lobari. Nella descrizione viene considerata anche l'estensione intraventricolare o subaracnoidea (Figura 4).

- la classificazione che prende in considerazione le possibili cause: $\mathrm{SMASH}-\mathrm{U}(\mathrm{S}=$ =structural vascular lesion, $\mathrm{M}=$ medication, $\mathrm{A}=$ amyloid angiopathy, $\mathrm{S}=$ sistemi disease, $\mathrm{U}=$ undetermined).

\begin{tabular}{|c|c|c|}
\hline \multicolumn{3}{|c|}{ GLASGOW COMA SCORE } \\
\hline \multirow{4}{*}{ Apertura degli occhi } & spontaneamente & 4 \\
\hline & alla parola & 3 \\
\hline & al dolore & 2 \\
\hline & non apre gli occhi & 1 \\
\hline \multicolumn{3}{|c|}{ 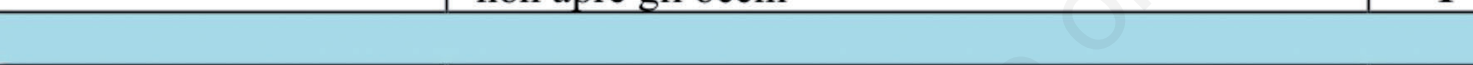 } \\
\hline \multirow{5}{*}{ Risposte verbali } & $\begin{array}{l}\text { orientata, cioè il paziente relaziona con } \\
\text { l'ambiente, capisce e risponde }\end{array}$ & 5 \\
\hline & confusa & 4 \\
\hline & $\begin{array}{l}\text { parole non appropriate, parole a casaccio, urla, } \\
\text { bestemmia, cose insensate, anche se pronunciate } \\
\text { bene }\end{array}$ & 3 \\
\hline & suoni incomprensibili, per esempio farfuglia & 2 \\
\hline & nessuna & 1 \\
\hline \multirow{6}{*}{ Risposte motorie } & obbedisce ai comandi & 6 \\
\hline & $\begin{array}{l}\text { localizza il dolore, se non vi è risposta ai comandi si } \\
\text { applica uno stimolo doloroso che viene mantenuto finché non } \\
\text { si abbia il massimo della risposta: inizialmente si applica la } \\
\text { pressione al letto ungueale con il risultato di estensione o } \\
\text { flessione del gomito; se vi è una di queste risposte allora lo } \\
\text { stimolo viene effettuato al collo o al tronco per ricercare la } \\
\text { "localizzazione" che si intende effettuata quando gli arti si } \\
\text { muovono per tentare di rimuovere lo stimolo doloroso. }\end{array}$ & 5 \\
\hline & $\begin{array}{l}\text { si retrae, flette normalmente ma non localizza il } \\
\text { dolore. }\end{array}$ & 4 \\
\hline & $\begin{array}{l}\text { Anormale flessione allo stimolo doloroso } \\
\text { (decorticazione) }\end{array}$ & 3 \\
\hline & $\begin{array}{l}\text { Estensione allo stimolo doloroso, si ha quando la } \\
\text { risposta è in adduzione delle braccia, rotazione interna e } \\
\text { pronazione dell'avambraccio nel modello stereotipato della } \\
\text { decerebrazione. (decerebrazione) }\end{array}$ & 2 \\
\hline & nessuna & 1 \\
\hline \multicolumn{2}{|r|}{ RISULTATO } & \\
\hline Grave, con GCS $\leq 8$ & Moderata, GCS 9-13 & \\
\hline
\end{tabular}

Figura 1. Glasgow coma scale. 
Questi strumenti possono esse utilizzati in piattaforme create per raccogliere dati in modo standardizzato.

Ipertensione (vasculopatia delle arterie profonde perforanti)

L'ipertensione determina multipli cambiamenti patologici nelle arteriole cerebrali e rappresenta la più

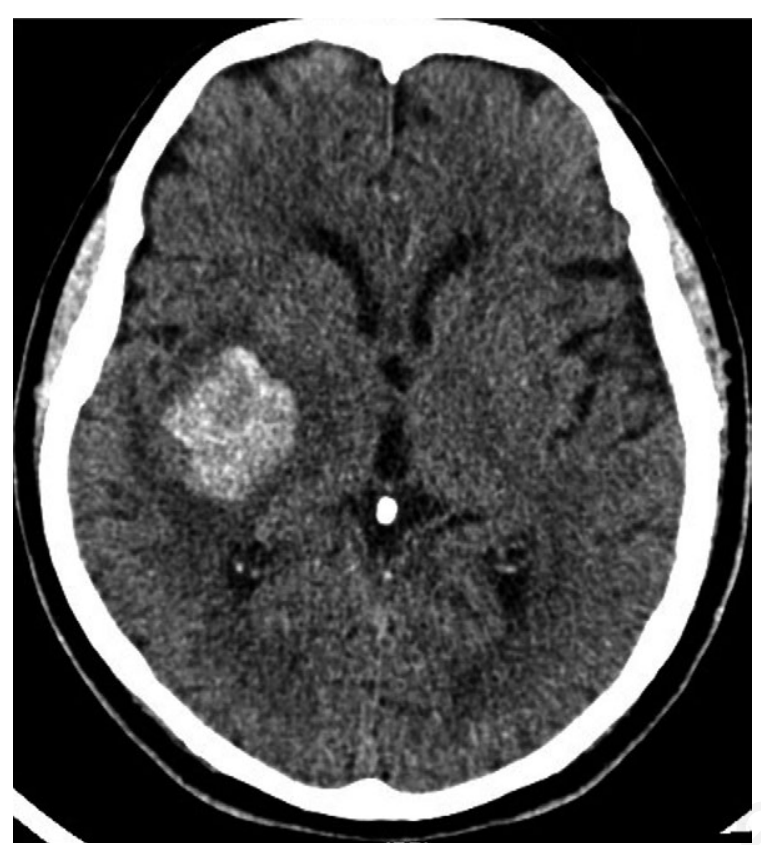

Figura 2. Tomografia computerizzata: emorragia cerebrale profonda. comune causa di emorragia cerebrale spontanea.

L'ipertensione cronica produce una malattia dei piccoli vasi con cambiamenti strutturali della parete delle arteriole di diametro di 50-200 $\mu \mathrm{m}$ caratterizzati da lipoialinosi, necrosi fibrinoide e sviluppo di aneurismi di Charcot-Bouchard.

I vasi colpiti sono le arteriole penetranti nel cervello: i) arterie lenticolostriate che originano dalla ar-

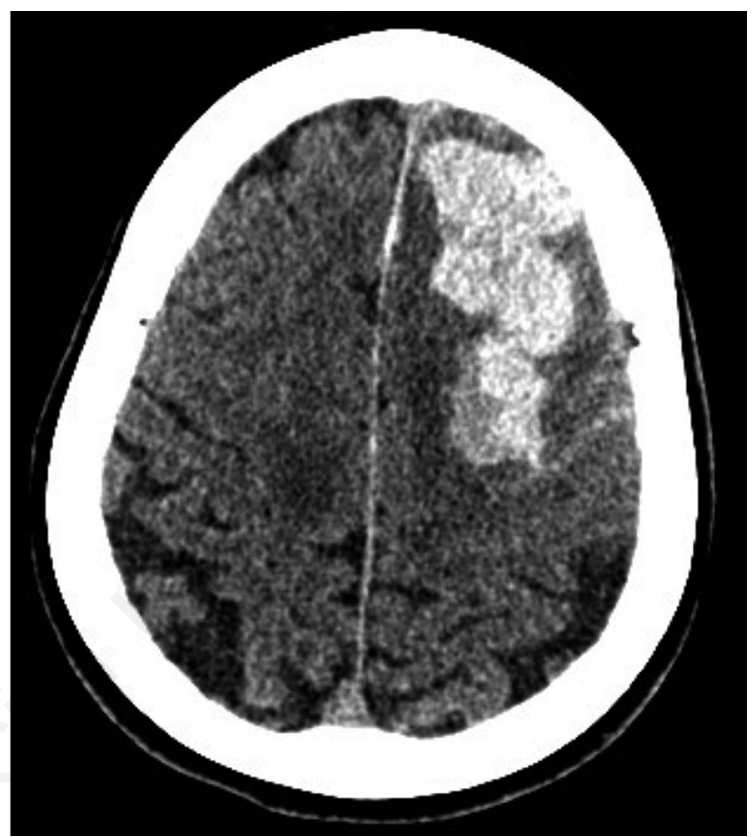

Figura 3. Tomografia computerizzata: emorragia lobare.

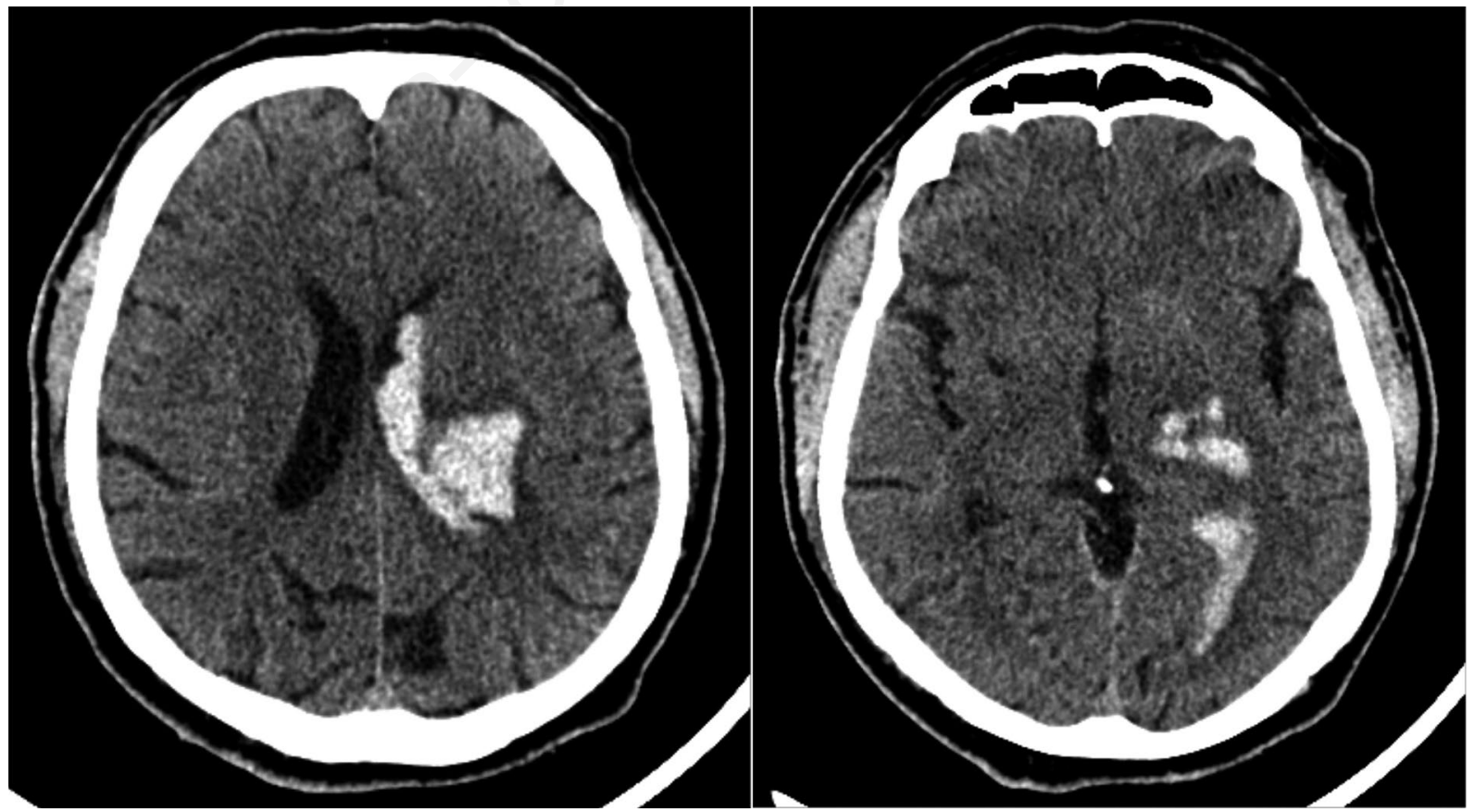

Figura 4. Tomografia computerizzata: apertura dell'ematoma in ventricolo. 
teria cerebrale media (ACM): nell' $80 \%$ circa; ii) arterie talamo perforanti che originano dalle arterie comunicanti posteriori e cerebrali posteriori (ACP): nel 5\% circa; iii) branche paramediane dell'arteria basilare (AB): nel $5 \%$ circa; iv) branche paramediane delle arterie cerebellari superiori (ACS) e delle arterie cerebellari anteriori inferiori (AICA): nel 10\% circa (Figura 5).

\section{Angiopatia amiloidea cerebrale}

L'angiopatia amiloidotica cerebrale si riscontra nella popolazione anziana, generalmente con più di 70 anni di età. ${ }^{14} \mathrm{E}$ ' comunemente di tipo lobare, generalmente occipitale e parietale (Figure 6 e 7).

Il paziente sviluppa nel tempo emorragie cerebrali multiple. Ci sono forme sporadiche e forme familiari.

La deposizione di amiloide coinvolge le arteriole corticali e leptomeningee, capillari e vene.

La $\beta$-amiloide si deposita all'interno della tunica media e dell'avventizia con progressiva formazione di microaneurismi.

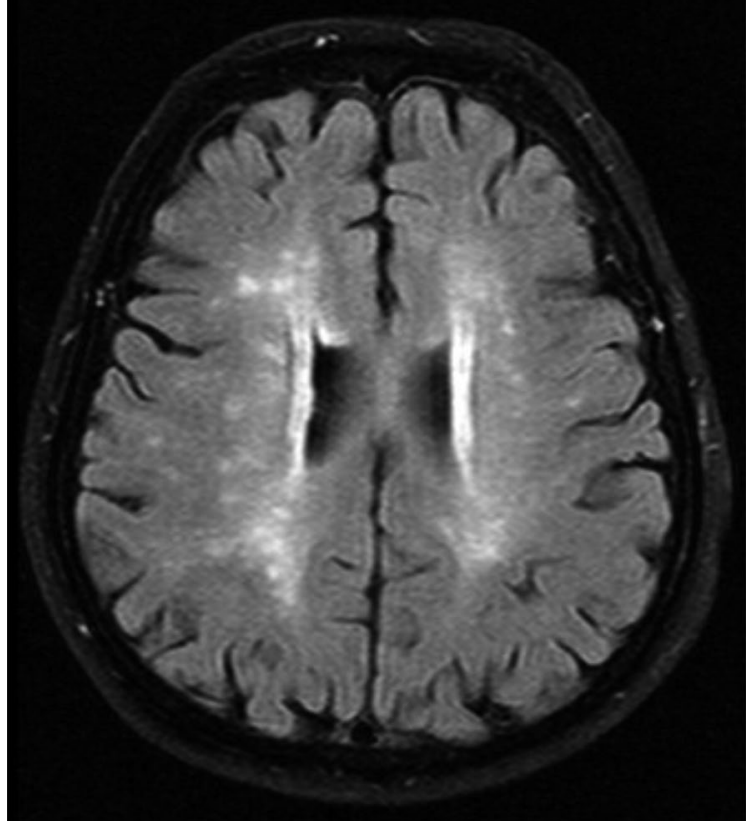

Figura 5. RM FLAIR: malattia dei piccoli vasi.

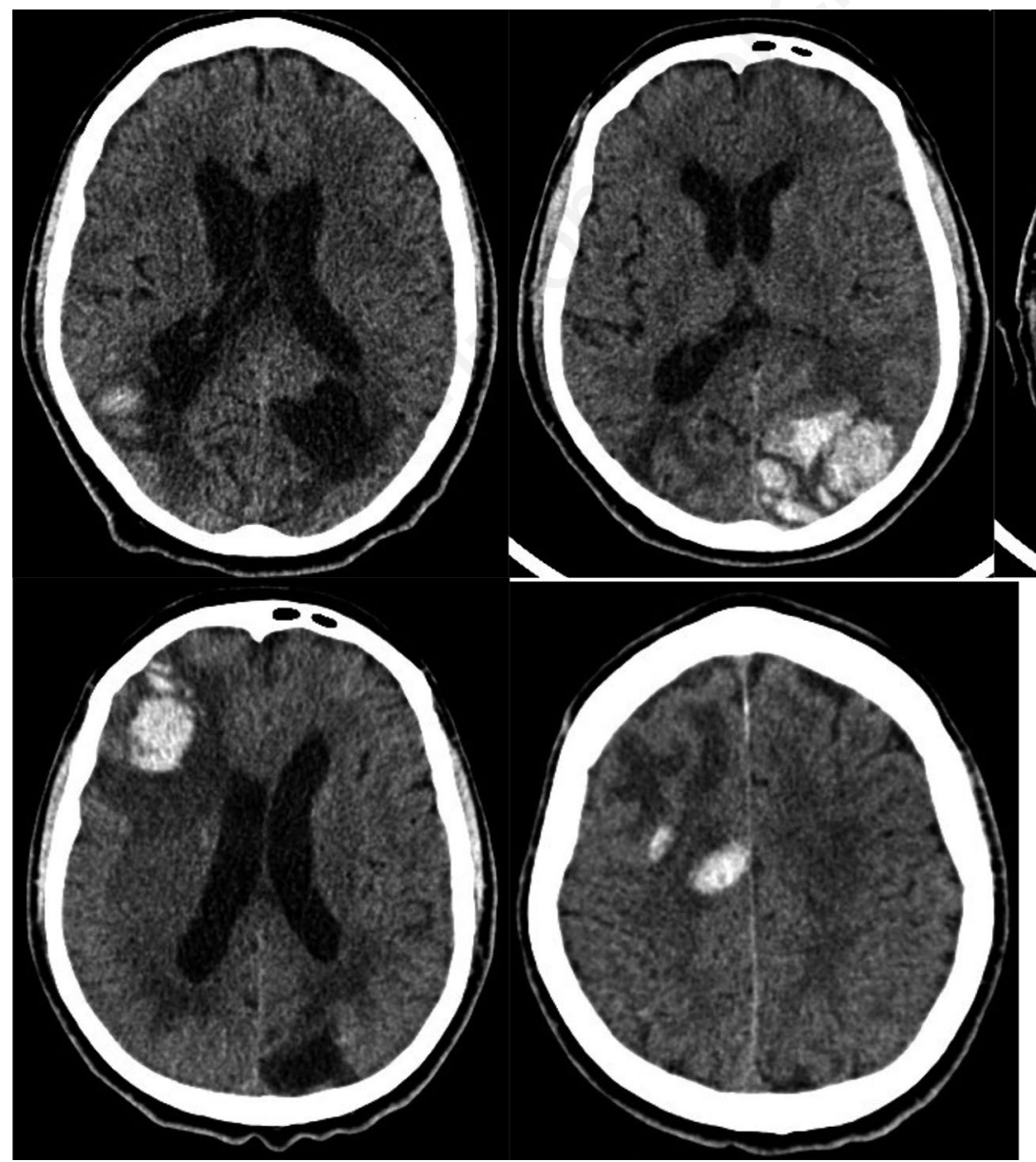

Figura 6. Angiopatia amiloidea cerebrale: emorragie multiple alla tomografia computerizzata. 


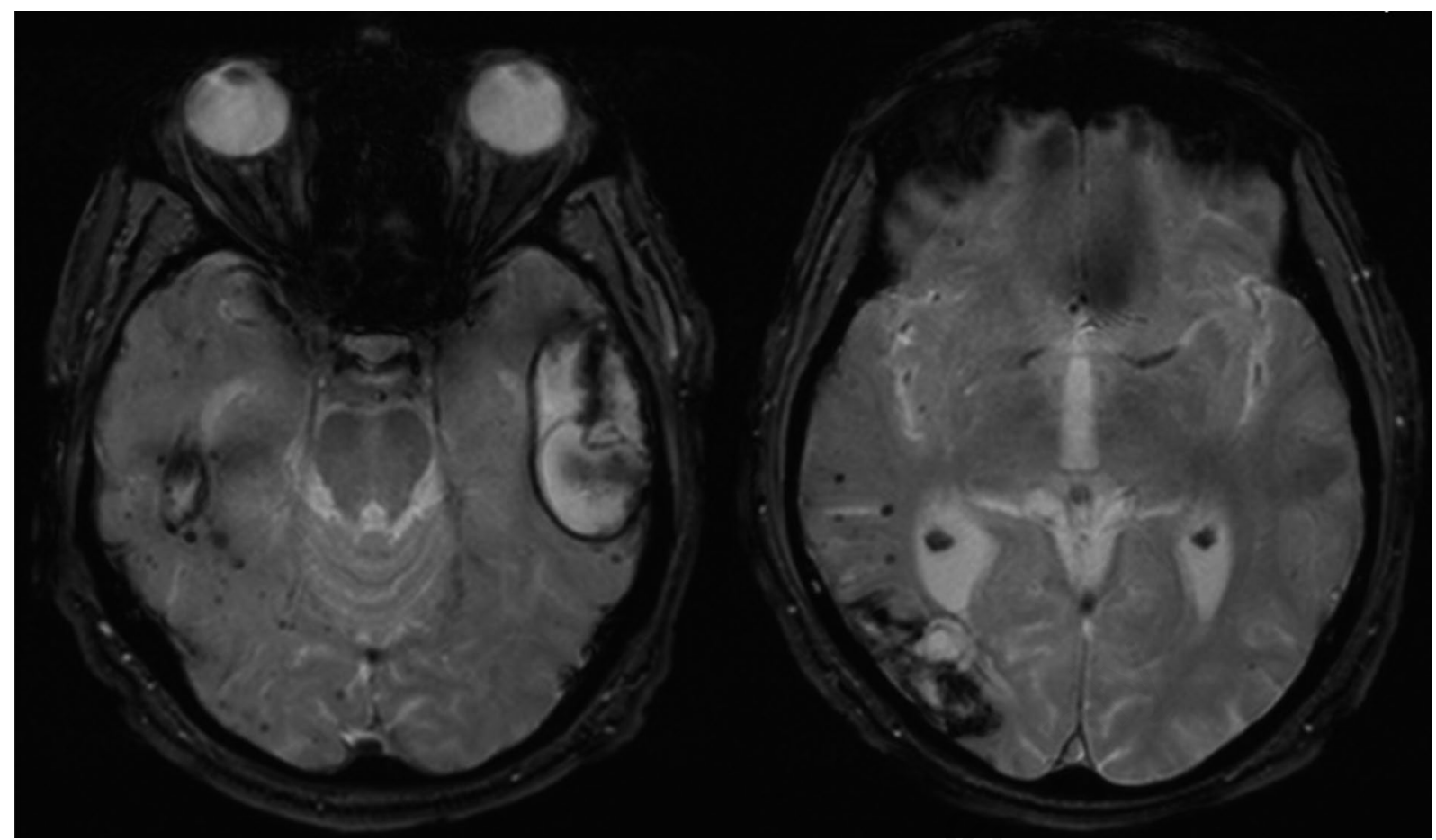

Figura 7. Risonanza magnetica sequenze GRE: angiopatia amiloidea cerebrale con microbleeding.

Per classificarla si usano i Criteri di Boston (Figura 8).

\section{Terapia anticoagulante e antiaggregante}

L'uso di warfarin aumenta il rischio di sviluppare emorragia cerebrale. Le sedi più frequenti sono quelle cerebellari e lobari. La percentuale di mortalità è di circa il 60-65\%.

La recente introduzione degli anticoagulanti orali diretti (direct oral anticoagulants: DOACs) ha ridotto la percentuale di rischio di emorragia cerebrale, inoltre per il Dabigatran è possibile utilizzare l'antidoto idarucizumab. In futuro altri antidoti consentiranno di neutralizzare tutti i DOACs: andexanet alfa per gli xabani e ciraparantag, antidoto considerato universale. ${ }^{6}$

Il rischio di emorragia cerebrale da assunzione di antiaggreganti è minore di quello da anticoagulanti, tuttavia è difficile contrastare l'azione terapeutica antiaggregante.

\section{Alterazione dell'emostasi}

Pazienti con diatesi emorragica sono esposti anche all'insorgenza di emorragia cerebrale.

Nei pazienti con emorragia cerebrale e piastrine $<50.000$ è raccomandata la trasfusione delle piastrine. $^{28}$

\section{Abuso di farmaci e sostanze}

L'emorragia cerebrale spontanea nei giovani adulti è rara, ma è possibile tra coloro i quali fanno uso illecito di amfetamine e cocaina.

La stimolazione da simpatico-mimetici causa transitori aumenti dei valori pressori che portano alla rot-

\begin{tabular}{|c|c|}
\hline AAC Definita & $\begin{array}{l}\text { Rilievo autoptico di: } \\
\text { - emorragia lobare, corticale o sottocorticale } \\
\text { - grave AAC } \\
\text { - } \text { assenza di altre lesioni }\end{array}$ \\
\hline $\begin{array}{l}\text { AAC } \\
\text { Probabile con } \\
\text { riscontro A.P. }\end{array}$ & $\begin{array}{l}\text { Dati clinici e tessuto patologico indicativi di: } \\
\text { - emorragia lobare, corticale o sottocorticale } \\
\text { - campione istologico positivo per AAC } \\
\text { - assenza di altre lesioni }\end{array}$ \\
\hline $\begin{array}{l}\text { AAC } \\
\text { Probabile }\end{array}$ & $\begin{array}{l}\text { Dati clinici e RM o TC indicativi di: } \\
\text { - emorragie multiple limitate alle sedi lobare, } \\
\text { corticale o sottocorticale } \\
\text { - età } \geq 55 \text { anni } \\
\text { - assenza di altre possibili cause di emorragia }\end{array}$ \\
\hline $\begin{array}{l}\text { AAC } \\
\text { Possibile }\end{array}$ & $\begin{array}{l}\text { Dati clinici e RM o TC indicativi di: } \\
\text { - un'unica emorragia lobare, corticale o } \\
\text { sottocorticale } \\
\text { - età } \geq 55 \text { anni } \\
\text { - assenza di altre possibili cause di emorragia }\end{array}$ \\
\hline
\end{tabular}

Figura 8. Criteri di Boston. 
tura dei vasi. L'abuso cronico di farmaci per via intravenosa (eroina) provoca cambiamenti di tipo vasculitico che esitano in debolezza della parete vasale fino alla sua rottura.

Queste emorragie possono essere profonde o lobari, subaracnoidee o intraventricolari.

\section{Eclampsia}

Nelle donne in gravidanza bisogna considerare l'eclampsia una situazione predisponente all'emorragia cerebrale.

\section{Localizzazione dell'emorragia cerebrale intraparenchimale spontanea}

Le sedi principali dell'emorragia cerebrale intraparenchimale sono: i) emorragia putaminale (la sede più frequente, associata ad ipertensione); ii) emorragia talamica ( $15 \%$ di tutte le emorragie); iii) emorragia del caudato ( $5-7 \%$ di tutte le emorragie); iv) emorragia lobare (la maggior parte di esse è probabilmente causata da AAC); v) emorragia cerebellare (5-10\% di tutte le emorragie); vi) emorragia tronco-encefalica (dovuta alla rottura delle branche perforanti dell'arteria basilare o arterie lunghe circonferenziali); vii) emorragia intraventricolare (comunemente dovuta all'estensione di una emorragia cerebrale intraparenchimale).

\section{Sintomi}

I sintomi si suddividono in: i) non specifici, quali: cefalea, alterazione della coscienza. La cefalea è molto frequente; ii) focali, illustrati nel paragrafo successivo.

\section{Sintomi focali}

- Emorragia putaminale: grave cefalea associata o meno a nausea e vomito.

Quando l'espansione dell'emorragia persiste, si sviluppano altri sintomi: emiparesi, disturbi sensitivi lateralizzati, emianopsia omonima, deviazione forzata dello sguardo verso la sede della lesione. Se è interessato l'emisfero dominante può comparire afasia. Se è interessato l'emisfero non dominante si può sviluppare hemineglect.

Emorragie estese possono causare alterazione della coscienza con comparsa di letargia fino al coma.

- Emorragia talamica: la cefalea è il primo sintomo e i sintomi neurologici dipendono dalla misura dell'ematoma. L'estensione della lesione può essere: i) laterale: nella capsula interna o nella corona radiata con emiparesi controlaterale; ii) mediale: nel sistema ventricolare con il rischio di ostruzione della circolazione liquorale e idrocefalo; iii) infe- riore: nel tronco encefalico con il rischio d'importanti deficit neurologici e coma.

La sintomatologia oculare è caratterizzata da paralisi dello sguardo, pupille miotiche e areagenti, nistagmo in retrazione e skew deviation.

- Emorragia del caudato: cefalea, nausea e vomito con disorientamento.

L'estensione nel corno frontale del ventricolo laterale espone il paziente al rischio di ostruzione del drenaggio liquorale e d'idrocefalo.

L'estensione nel talamo provoca transitorio deficit di memoria a breve termine.

- Emorragia lobare: varietà di sintomi in relazione al lobo interessato e all'estensione della lesione emorragica. Cefalea, vomito e crisi epilettiche si osservano frequentemente.

Ematomi periferici si associano a bassa incidenza di coma all'esordio, invece se evolvono possono comprimere le strutture adiacenti con la sovrapposizione di nuovi sintomi (Figura 9).

- Emorragia cerebellare: cefalea con nausea e vomito seguiti da vertigine, disartria e rigidità collonucale. Col progressivo deterioramento clinico compaiono: atassia, paralisi periferica del facciale, paralisi del sesto nervo cranico, nistagmo con alto rischio di sviluppare coma.

- Emorragia del tronco encefalico: cefalea, nausea e vomito, emiparesi, tetraparesi, diplopia, deficit sensitivi, progressiva insorgenza di coma con postura decerebrata, respiro patologico, pupille puntiformi, ocular bobbing, movimenti dello sguardo orizzontale assenti e sindrome uno e mezzo.

- Emorragia intraventricolare: rischio di idrocefalo acuto o di idrocefalo comunicante tardivo.

\section{Le emorragie intracraniche al neuroimaging}

E' possibile suddividere complessivamente le emorragie intracraniche in: i) emorragie traumatiche: subaracnoidea, ematoma epidurale, ematoma subdurale, contusione parenchimale, microemorragie; ii) emorragia intraparenchimale: dovuta a ipertensione arteriosa o ad angiopatia amiloidea cerebrale; iii) conversione in emorragia di un infarto cerebrale; iv) emorragia subaracnoidea da rottura di aneurisma cerebrale; v) emorragia da rottura di malformazione artero-venosa (MAV); vi) emorragia da fistola artero-venosa durale; vii) emorragia dovuta a trombosi di seno venoso o di vene corticali; viii) emorragia conseguente a vasculiti; ix) emorragia da aneurisma micotico; $x$ ) emorragia su tumore.

Di esse, in questo lavoro, vengono prese in considerazione quelle intraparenchimali spontanee da ipertensione arteriosa e da angiopatia amiloidea cerebrale, delle quali vengono descritte le caratteristiche neuro radiologiche. 


\section{Emorragia da ipertensione arteriosa}

Avviene più frequentemente tra la sesta e settima decade di vita e ha una mortalità di circa il 30-50\%. Le sedi prevalentemente interessate: gangli basali, cervelletto e lobi occipitali.

Nei pazienti con età inferiore a 50 anni bisogna tenere in considerazione altre possibili cause di sanguinamento quali neoplasie e malformazioni vascolari.

L'emorragia iniziale varia per dimensioni da una piccola emorragia (meno di $1-2 \mathrm{~cm}$ ) senza significativo effetto massa a un grande ematoma con effetto massa ed erniazione cerebrale o estensione intraventricolare.

E' importante eseguire controlli neuroradiologici seriati per valutare l'andamento nel tempo e l'eventuale espansione al fine di stabilire l'eventuale necessità d'intervento di decompressione o evacuazione.

L'angio-TC può rilevare la presenza dello spot sign (immagine iperdensa che depone per stravaso di sangue in atto), che indica un'espansione possibile dell'ematoma ed è segno predittivo sfavorevole.

L'angio-TC o la risonanza magnetica (RM) cerebrale possono essere utilizzate per identificare altre eventuali cause di emorragia.

\section{Emorragia da angiopatia amiloidea cerebrale}

Può manifestarsi con microemorragie, emorragia

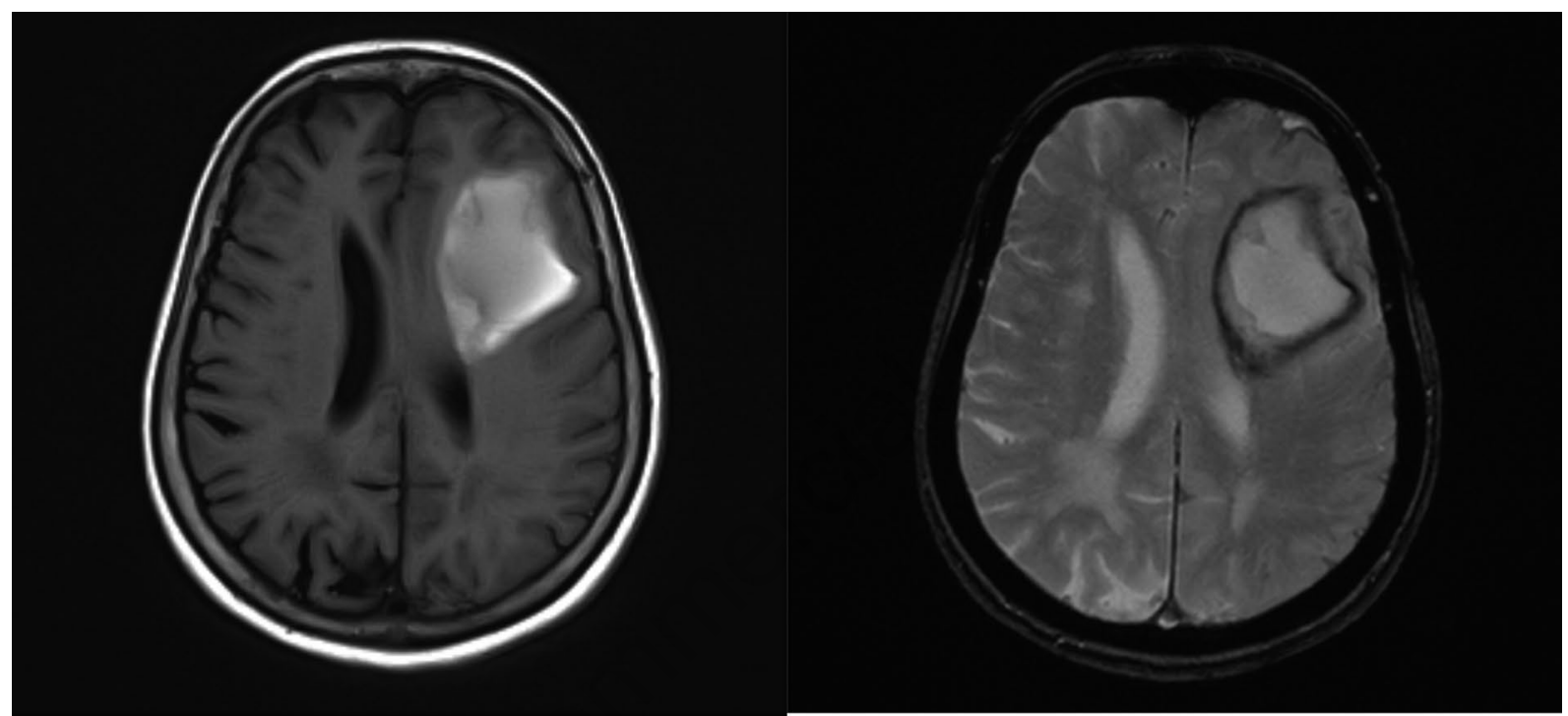

Figura 9. Risonanza magnetica: emorragia cerebrale con compressione sulle strutture sottostanti. 
subaracnoidea sulcale o grandi emorragie lobari che si sviluppano tipicamente nella sostanza bianca adiacente alla corteccia cerebrale e risparmia i nuclei della base, la fossa cranica posteriore e il tronco encefalico.

Si riscontrano diffuse alterazioni della sostanza bianca e piccoli foci di suscettibilità alle sequenze gradient echo (GRE) e susceptibility-weighted imaging (SWI).

\section{Monitoraggio dell'evoluzione dell'emorragia}

Per il monitoraggio delle lesioni emorragiche, secondo le linee guida SPREAD, è indicato eseguire TC e RM cerebrali.

La RM cerebrale, in particolare con mdc, può evidenziare una causa secondaria di emorragia. Le sequenze GRE sono in grado di documentare gli esiti emosiderinici in fase subacuta e cronica di un'emorragia anche a distanza di anni dall'evento e per questo rappresentano un marker di pregresso sanguinamento.

\section{Risanguinamento}

Il $38 \%$ dei pazienti presenta risanguinamento nelle prime 24 ore, il $26 \%$ nelle prime 6 ore.

Fattore predittivo di risanguinamento è considerato lo spot sign, presente in $1 / 4$ dei pazienti nella TC iniziale. Nello studio PREDICT questo segno, riscontrato all'angio-TC cerebrale, viene considerato indice di prognosi evolutiva. ${ }^{15}$

Uno score formato da un algoritmo a 5 punti è stato proposto per i pazienti ad alto rischio di espansione dell'emorragia basato sulla TC senza mezzo di contrasto: il BAT score. Questo attribuisce 1 punto per il blend sign (area di ipo e iperattenuazione a margini ben definiti), 2 punti per ogni ipodensità riscontrata, 2 punti se il timing di esecuzione della TC senza mdc è $<2,5$ ore. ${ }^{27}$

\section{Emorragia cerebrale o infarcimento emorragico?}

Non è facile differenziare un'emorragia cerebrale spontanea da un infarcimento emorragico cerebrale (hemorrhagic transformation: HT) di un'ischemia cerebrale.

Non ci sono criteri diagnostici di certezza per differenziarle, tuttavia, secondo lo Stroke and ageing research group, alcune caratteristiche al neuroimaging possono orientare la diagnosi.

La trasformazione emorragica dopo un infarto cerebrale avviene in circa $1 / 3$ dei casi, tipicamente entro le prime due settimane dall'ischemia e nel $9 \%$ circa nelle prime 24 ore.

Se essa avviene subito dopo l'infarto con l'aspetto di petecchie emorragiche nel territorio di pertinenza del vaso occluso la diagnosi di HT è abbastanza agevole.
Se, invece, per vari motivi, il paziente viene sottoposto ad indagini neuroradiologiche non in fase acuta, ma successivamente all'esordio dei sintomi, è possibile confondere HT con ICH.

HT avviene più comunemente nei pazienti anziani e in quelli con infarto esteso oppure in alcuni pazienti diabetici e ipertesi sottoposti a trombolisi sistemica o dopo avere iniziato la terapia anticoagulante orale per ischemia cardioembolica e talora anche in coloro $\mathrm{i}$ quali sono stati sottoposti a endoarteriectomia o stenting carotideo (Figura 10).

HT è correlato alla rottura della lamina basale dei microvasi dovuta all'attività della matrice delle metalloproteinasi come conseguenza dell' ischemia prolungata ed esacerbata dalla ricanalizzazione del vaso occluso (Figura 11).

\section{Caratteristiche neuroradiologiche: diagnosi differenziale tra ICH e HT}

In base alla sede è possibile cercare di stabilire se si tratti di emorragia o infarcimento emorragico. Nel 1993 era stata proposta una classificazione delle HT in base alla topografia e alterazione della intensità del sanguinamento.

\section{Conversione emorragica di ischemia cerebrale e suo grading}

In base all'estensione della trasformazione emorragica si distinguono: i) petecchie emorragiche; ii) petecchie confluenti (H1); iii) ematoma intraparenchimale (H2).

La topografia del coinvolgimento delle strutture cerebrali profonde alle neuroimmagini e l'ipodensità che circonda la zona emorragica possono aiutare nella diagnosi differenziale.

L'ICH comunemente non coinvolge il caudato, mentre l'interessamento del caudato e del putamen suggeriscono un interessamento ischemico delle arterie lenticolo-striate con successivo infarcimento piuttosto che una emorragia spontanea.

Inoltre, nella ICH l'edema che circonda l'emorragia non segue la distribuzione topografica tipica di un territorio arterioso.

Nel caso di HT il centro dell'ematoma da infarcimento corrisponde alla zona maggiormente infartuata e negli infarti venosi con infarcimento emorragico l'ipodensità da edema può raggiungere la superficie corticale.

La sequenza RM cerebrale in DWI può evidenziare una regione di restrizione della diffusione lontano dalla regione emorragica compatibile con ischemia.

L'angio-RM time of flight (TOF) può dimostrare la presenza di occlusione arteriosa.

La RM in perfusione suggerisce la diagnosi di HT se mostra un deficit di perfusione oltre la regione dell'ematoma. 

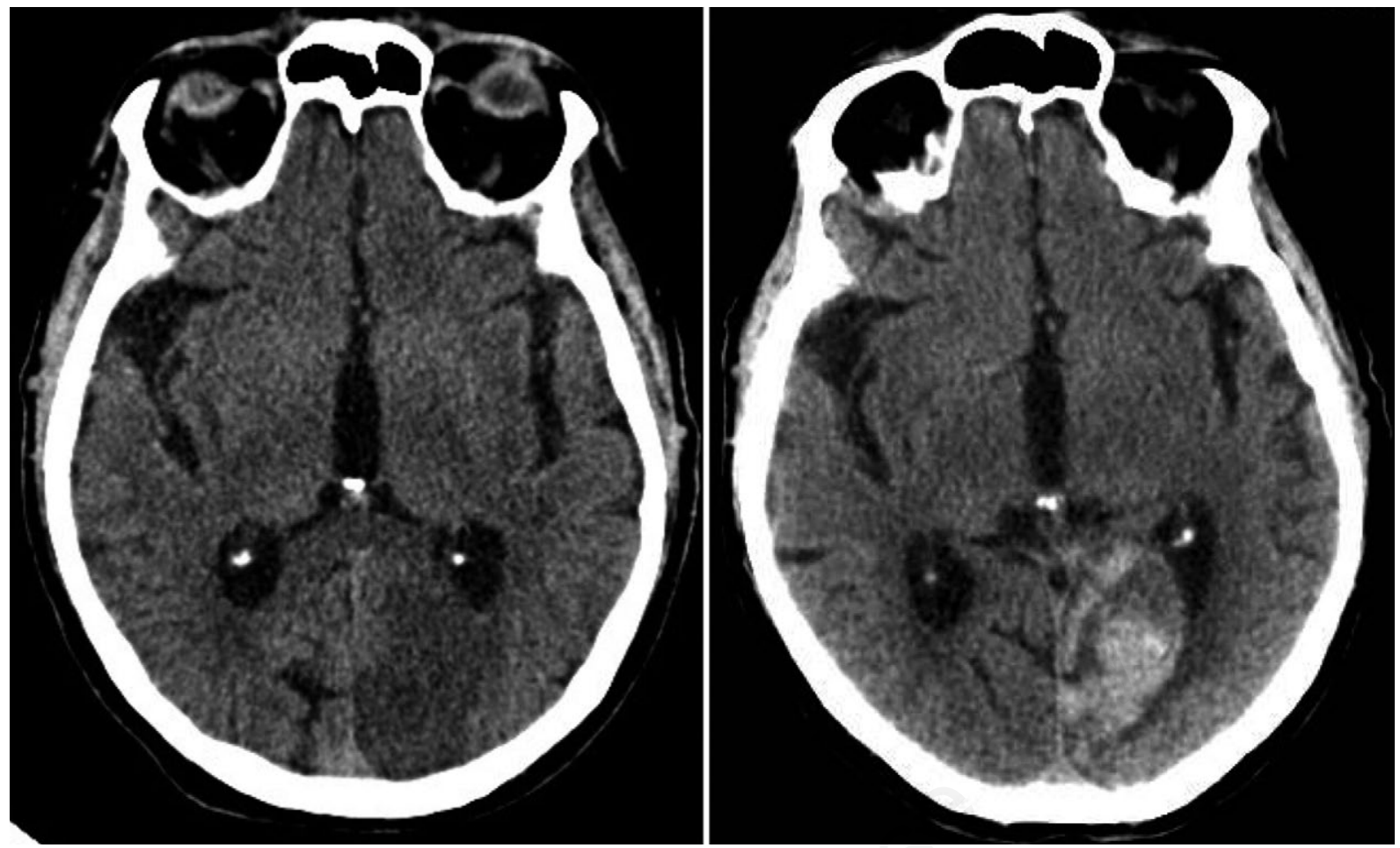

Figura 10. Tomografia computerizzata: ischemia cerebrale e infarcimento emorragico dopo terapia con warfarin.

La presenza di micro sanguinamento (microbleeds) nelle sequenze RM GRE o SWI non necessariamente indicano se si tratti di ICH o di HT.

Nelle emorragie cerebrali lobari, in particolare posteriori, lo studio PET-amiloide può essere di aiuto. L'angio-TC può rivelare la presenza di occlusione vasale oppure spot sign in caso di rottura vasale da ICH. La TC in perfusione può evidenziare, come la RM in perfusione, un difetto ampio di perfusione o un mismatch compatibile con lesione ischemica.

\section{Altre cause di emorragia intraparenchimale}

Frequenti: emorragia da trombosi dei seni venosi o delle vene corticali

L'ostruzione del deflusso venoso da trombosi cerebrale può provocare infarcimento ematico.

Si tratta di una patologia non comune e la cui diagnosi può comportare alcune difficoltà.

La trombosi dei seni venosi è più frequente rispetto a quella delle vene corticali.

L'emorragia che si forma non ha la tipica distribuzione lungo il decorso di un vaso arterioso e si forma prevalentemente nella giunzione tra sostanza bianca e grigia e sulla corteccia.

Tra le cause troviamo stati di ipercoagulabilità, infezioni della base cranica, disidratazione e meningiomi o altri tumori durali.

\section{Meno frequenti}

- Vasculiti: si può formare un'emorragia subaracnoidea acuta nei solchi della convessità cerebrale (ESA sulcale) e microemorragie della giunzione cortico-midollare.

- Leucoencefalite acuta emorragica: è una rara forma di malattia demielinizzante.

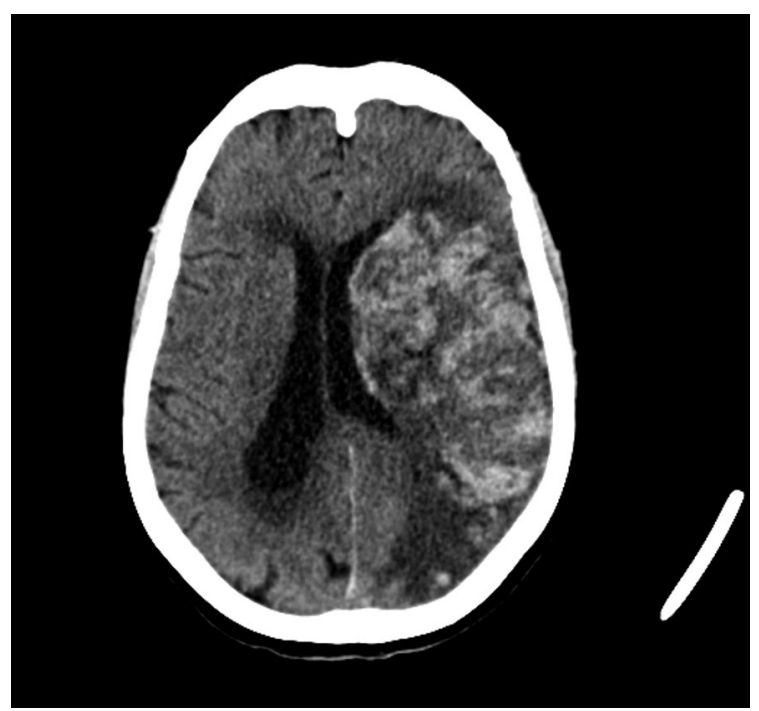

Figura 11. Infarcimento emorragico. 
- COL4A1 (braccio lungo del cromosoma 13): è un gene che codifica per la catena alfa 1 del collagene tipo 4. La sua mutazione può causare una malattia dei piccoli vasi cerebrali autosomica dominante monogenica, la cui sintomatologia è data da emorragia sottocorticale nei centri grigi profondi o nel tronco encefalico, emorragia subaracnoidea, infarti lacunari, emicrania e crisi epilettiche.

- CADASIL: si tratta di una microvasculopatia autosomica dominante con infarti ischemici lacunari sottocorticali, emicrania e demenza vascolare con esordio in età giovane-media (30-50 anni di età). La mutazione coinvolge il gene NOTCH3 del cromosoma $19 \mathrm{p} 13.12$ e la sintomatologia è caratterizzata da attacchi ischemici transitori, emicrania, psicosi e depressione. Caratteristica è la deposizione di materiale osmiofilico in stretta relazione con la muscolatura liscia vasale. Microemorragie possono essere presenti nel $45 \%$ circa dei casi senza distribuzione tipica.

- CARASIL: malattia a trasmissione recessiva dovuta a mutazione del gene HTRA1 nel cromosoma 10q. Interessa i piccoli vasi, la colonna vertebrale e i follicoli piliferi dei capelli. Si tratta di malattia molto rara caratterizzata da sintomi Stroke-like nella terza decade di vita, alopecia e spondilosi, inoltre insorgenza di epilessia e demenza. Presenta un rischio potenziale di sanguinamento cerebrale.

\section{Scale diagnostiche}

Le scale diagnostiche utilizzate nell'emorragia cerebrale che si ritrovano in letteratura mettono in relazione i valori pressori con lo stato di coscienza e le comorbidità cardiologiche e sono le seguenti: i) Guy Hospital Score (Figura 12); ii) Siriraj Stroke Score (Figura 13).

\section{Score prognostici}

ICH Score correla con la mortalità a 30 giorni $\mathrm{e}$ con l'outcome funzionale ad un anno (Figura 14).

\section{Test biologici}

È importante studiare la coagulazione nei pazienti con emorragia spontanea.

\section{Gestione dell'emorragia cerebrale}

E' fondamentale il monitoraggio del paziente per la possibilità di un peggioramento clinico entro le prime $24-48$ ore e valutare l'evoluzione al neuroimaging nelle prime ore e nelle fasi successive.

La temperatura corporea e i valori della pressione arteriosa (PA) del paziente possono essere osservati presso un'Unità di Cura Intensiva oppure Subintensiva (Stroke Unit) in base alla presentazione clinica.

Presso una Stroke Unit è possibile seguire l'andamento della pressione arteriosa, della frequenza cardiaca, della saturazione di ossigeno, dell'elettrocardiogramma (ECG) e dello stato neurologico.

\section{Ruolo della pressione arteriosa}

Ridurre i valori della pressione arteriosa se elevata è chiaramente indicato, ma quanto bisogna ridurla?

Lo standard di riduzione della PA non è ancora chiaramente definito. Da tempo si sostiene che un abbassamento eccessivo possa portare ad insulto ischemico cerebrale delle regioni di penombra periematoma.

Trials clinici randomizzati, invece, hanno dimostrato che ridurre la PA sistolica a valori $<150 \mathrm{mmHg}$ non diminuisce il flusso sanguigno periematoma alle immagini TC di perfusione.

Recentemente sono stati condotti alcuni trials randomizzati per valutare la sicurezza della riduzione della PA in fase acuta: INTERACT 1 (2008) e ATACH (2007) che hanno confermato la possibilità di una riduzione della PA sistolica in fase acuta a $140 \mathrm{mmHg}$ e successivamente anche INTERACT 2 (2013) e ATACH II (2016).

Il trial INTERACT2 ha dimostrato che il trattamento intensivo della PA al di sotto di $140 \mathrm{mmHg}$ entro 1 ora, mantenendo questo target per 7 giorni, migliora l'outcome funzionale in modo significativo rispetto all'abbassamento al di sotto di $180 \mathrm{mmHg}$.

Esso, inoltre è associato a minore mortalità e minore dipendenza ed è associato a maggiore riduzione dell'espansione dell'ematoma a 24 ore.

Nello studio ATACH II viene evidenziato che un target di PA sistolica di 110-130mmHg verso 140-179 $\mathrm{mmHg}$ non aumenta la mortalità, ma non porta beneficio sull'outcome clinico e le oscillazioni rapide di pressione andrebbero evitate.

Pazienti dello studio ATACH II che avevano eseguito angio-TC erano stati analizzati nello studio SCORE-IT per valutare il significato prognostico dello spot sign in relazione ai valori pressori, si tratta di uno studio ancillare dell'ATACH II che conferma la validità dello spot sign come indice prognostico di evoluzione dell'emorragia e indica che una riduzione importante della PA sistolica non migliora l'outcome e né riduce il rischio effettivo di espansione. Quindi lo spot sign può essere usato nella pratica clinica per stratificare i pazienti candidabili a terapie anti-ipertensive aggressive.

Nel 2015 le linee guida AHA/ASA hanno raccomandato di abbassare la PA sistolica a valori target di $140 \mathrm{mmHg}$ nei pazienti che si presentano con valori di PA sistolica 150-220 mmHg e senza controindicazione al trattamento antipertensivo in acuto e si consiglia di 
utilizzare farmaci e.v. monitorando l'andamento clinico nei pazienti che si presentano con $\mathrm{PA}>220 \mathrm{mmHg}$.

Nelle linee guida ESC 2018 si preferisce non abbassare rapidamente la PA sistolica se $<220 \mathrm{mmHg}$, ma, se i valori sono $>220 \mathrm{mmHg}$, ridurre la PA con farmaci somministrati per via e.v. per portarla verso valori di $180 \mathrm{mmHg}$.

Le linee guida italiane SPREAD 2016 raccomandano di iniziare il trattamento intensivo dell'iperten- sione per raggiungere valori di PA sistolica inferiori a $140 \mathrm{mmHg}$.

Non c'è chiara indicazione su quali farmaci antipertensivi e.v. utilizzare, nel nostro paese vengono utilizzati più comunemente il Labetalolo e l'Urapidil.

Lo studio SCAST ha evidenziato che l'uso di Candesartan nel sottogruppo con emorragia cerebrale non portava beneficio prognostico a 6 mesi su morte da cause vascolari, infarto del miocardio o stroke. ${ }^{33}$

\begin{tabular}{|c|c|c|c|}
\hline Variable & Clinical feature & & Score \\
\hline $\begin{array}{l}\text { Apoplectic onset } \\
\text { - Loss of consciousness }\end{array}$ & One or none of these & $=$ & 0 \\
\hline $\begin{array}{l}\text { - Headache within } 2 \\
\text { hours } \\
\text { - Vomiting } \\
\text { - Neck stiffness }\end{array}$ & Two or more & $=$ & +21.9 \\
\hline \multirow{3}{*}{$\begin{array}{l}\text { Level of consciousness ( } 24 \\
\text { hours after onset) }\end{array}$} & Alert & $=$ & 0 \\
\hline & Drowsy & $=$ & +7.3 \\
\hline & Unconscious & $=$ & $=+14.6$ \\
\hline \multirow[t]{2}{*}{ Plantar responses } & Both flexor/single extensor & $=$ & 0 \\
\hline & Both extensor & & $=+7.1$ \\
\hline $\begin{array}{l}\text { Diastolic blood pressure ( } 24 \\
\text { hours post-onset) }\end{array}$ & $\mathrm{BP}$ in $\mathrm{mm}$ of mercury & $=$ & $+\left(\mathrm{BP}^{\star} 0.17\right)$ \\
\hline Atheroma markers & None & $=$ & 0 \\
\hline $\begin{array}{l}\text { - angina, claudication, } \\
\text { history of diabetes }\end{array}$ & One or more & $=$ & -3.7 \\
\hline \multirow[t]{2}{*}{ History of hypertension } & Not present & $=$ & 0 \\
\hline & Present & $=$ & -4.1 \\
\hline \multirow[t]{2}{*}{$\begin{array}{l}\text { Previous event (stroke or } \\
\text { TIA) }\end{array}$} & None & $=$ & 0 \\
\hline & Any number & $=$ & -6.7 \\
\hline \multirow[t]{9}{*}{ Heart disease } & None & $=$ & 0 \\
\hline & Aortic/mitral murmur & $=$ & -4.4 \\
\hline & Cardiac failure & $=$ & -4.3 \\
\hline & Cardiomyopathy & $=$ & -4.3 \\
\hline & Atrial fibrillation & $=$ & -4.3 \\
\hline & Cardiomegaly (chest $\mathrm{X}$-ray) & $=$ & -4.3 \\
\hline & $\begin{array}{l}\text { Myocardial infarct within } 6 \\
\text { months }\end{array}$ & $=$ & -4.3 \\
\hline & Constant & $=$ & -12.6 \\
\hline & Total & $=$ & $\overline{ }$ \\
\hline
\end{tabular}

Interpretation of score:

-30 to $0=95$ per cent probability of being infarct

25 to $50=95$ per cent probability of being haemorrhage

See original paper for a more accurate graph

References Allen (1983); Sandercock et al. (1985)

Comment

Figura 12. Guy's hospital score for hemorrage. 


\section{Gestione del paziente con emorragia da anticoagulanti}

\section{Emorragie da vitamina K antagonisti (VKA)}

Nei pazienti che sviluppano emorragia cerebrale in corso di terapia con warfarin è necessario sospendere immediatamente l'anticoagulante ed inoltre: i) controllare INR; ii) somministrare Vitamina $\mathrm{K}$ e.v. $5-10 \mathrm{mg}$; iii) somministrare plasma fresco congelato (meno raccomandato); iv) complessi concentrati di protrombina (PCCs); v) complessi a 4 fattori (FEIBA).

\section{Emorragie da non vitamina $\mathrm{K}$ antagonisti (direct oral anticoagulants DOACs)}

La gestione del paziente in DOACs è più difficile non essendoci possibilità di un monitoraggio ematologico di routine all'interno dei comuni esami ematochimici.

Dabigatran: il riscontro di aPTT alto indica coagulazione in atto. Utilizzare Idarucizumab.

Xabani (rivaroxaban, apixaban, edoxaban): PT e aPTT non danno indicazioni certe, è necessario ricercare gli Anti Fattore X.

Adexanet potrà utilizzato in futuro per rivaroxaban e apixaban e ciraparantag per tutti gli xabani, dabigatran, le eparine e fondaparinux.

\section{Ripresa dell'anticoagulante: timing}

Non è ancora ben stabilito quando riprendere l'anticoagulante dopo un'emorragia cerebrale.

In generale la ripresa entro il primo mese è a rischio di ricorrenza di emorragia.

Una ripresa precoce è indicata nei pazienti con val- vole cardiache meccaniche per l'alto rischio cardioembolico.

La ripresa dell'anticoagulante nei pazienti con emorragia lobare è più rischiosa di quella nei pazienti con emorragia profonda.

Valida alternativa per la ripresa di anticoagulante dopo emorragia cerebrale nei soggetti che assumevano VKA è quella di passare all'assunzione di DOACs.

In caso di emorragia cerebrale da angiopatia amiloidea riprendere la terapia anticoagulante è una controindicazione assoluta, in questi casi si potrà prendere in considerazione la chiusura percutanea dell'auricola sinistra.

Le linee guida italiane SPREAD per la ripresa del trattamento anticoagulante dopo un'emorragia cerebrale tengono in considerazione che: i) il rischio emorragico annuo è del 2,1-3,7\%; ii) la ripresa della terapia anticoagulante aumenta il rischio di sanguinamento cerebrale di 5 volte, ma diminuisce il rischio di eventi ischemici del $90 \%$.

Le controindicazioni alla ripresa dell'anticoagu-

\begin{tabular}{|l|l|c|}
\hline Variable & Clinical feature & Score \\
\hline Level of consciousness & Alert & 0 \\
& $\begin{array}{l}\text { Drowsy/stuporous } \\
\text { Semiconscious/ } \\
\text { comatose }\end{array}$ & 1 \\
& Absent & 2 \\
History of vomiting after the onset & Present & 0 \\
History of headache within 2 hours & Absent & 1 \\
of onset & Present & 0 \\
Diastolic blood pressure (mmHg) & & 1 \\
Atheroma markers (angina, & None & 0 \\
claudication, diabetes) & One or more & 1 \\
\hline
\end{tabular}

Figura 13. Siriraj stroke scoring system.

\begin{tabular}{|ll|}
\hline ICH Score & Points \\
\hline GCS score * & \\
$3-4$ & 2 \\
$5-12$ & 1 \\
$13-15$ & 0 \\
ICH volume ** & \\
$\geq 30 \mathrm{~cm}^{3}$ & 1 \\
$<30 \mathrm{~cm}^{3}$ & 0 \\
IVH *** & \\
Yes & 1 \\
No & 0 \\
Infratentorial origin of ICH & \\
Yes & 1 \\
No & 0 \\
Age & \\
$\geq 80$ & 1 \\
$<80$ & 0 \\
ICH Total Score & $0-6$ \\
\hline
\end{tabular}

The ICH Score is a clinical grading scale that allows for risk stratification of patients presenting with ICH. The 5 categories are independent predictors of 30-day mortality. Mortality rises as the ICH Score increases. The use of the ICH Score could improve standardization of treatment protocols and clinical research studies in ICH.

* GCS on initial

presentation (or after resuscitation)

**ICH volume, volume on initial CT calculated using $\mathrm{ABC} / 2$ method

*a** IVH, presence of any IVH on initial CT

Figura 14. ICH score. 
lante orale (ACO) dopo emorragia cerebrale sono le seguenti: ${ }^{28}$ i) controindicazioni assolute: emorragia lobare correlabile ad angiopatia amiloidea; ii) ripresa della ACO dopo tre settimane: nel paziente a rischio trombo embolico elevato (CHADVASC $>=5$ o CHADs $>=4$ ), protesi valvolare meccanica mitralica, trombosi delle valvole cardiache, tromboembolismo venoso o arterioso <30 giorni; iii) ripresa dell' ACO dopo la trentesima settimana: pazienti ad alto rischio emorragico per presenza di microbleeds multiple alla RM GRE, leucoaraiosi, emorragie lobari non correlabili ad angiopatia amiloidea; iv) in tutti gli altri casi: ripresa dell'ACO tra la decima e la trentesima settimana.

Lo studio RESTART non ha tratto sostanziali conclusioni circa la ripresa dell'anticoagulante o della doppia antiaggregazione, lo studio SoSTART in corso è possibile possa offrire maggiori indicazioni. ${ }^{3}$

\section{Emorragia cerebrale in pazienti in terapia antiaggregante}

Non è chiaro l'outcome dopo emorragia in terapia con antiaggreganti piastrinici.

E' buona norma, comunque, sospendere l'uso di antiaggreganti in corso di emorragia cerebrale. La trasfusione di piastrine è indicata solamente in caso di piastrinopenia o disfunzione piastrinica.

\section{Tromboprofilassi nei pazienti con emorragia cerebrale}

Si tratta di un problema complicato per la coesistenza di rischio emorragico e trombo embolico. $\mathrm{Si}$ preferisce la compressione pneumatica intermittente degli arti inferiori da iniziare precocemente. Il rischio di tromboembolismo varia dallo $0,5 \%$ al $13 \%$ e l'embolia polmonare dallo $0,5 \%$ al $5 \%$.

La profilassi con eparina a basso peso molecolare andrebbe iniziata dopo le prime 48 ore.

Secondo le linee guida SPREAD in caso di trombosi venosa profonda (TVP) e/o embolia polmonare (EP) è raccomandato il posizionamento di filtro cavale $\mathrm{o}$, se possibile, ripresa della terapia con ACO. La scelta tra due possibilità va considerata caso per caso in base all'avvenuto riassorbimento dell'emorragia cerebrale o il tempo trascorso tra evento emorragico ed esordio della TVP e/o EP, alle cause dell'emorragia e alle condizioni cliniche del paziente.

\section{Febbre}

Si ha alta incidenza di febbre nelle emorragie sopratentoriali e in particolare nelle emorragie intraventricolari.

Se la febbre dura 72 ore dopo l'ospedalizzazione la prognosi è peggiore.

Si consiglia di usare antipiretici e cercare eventuali foci infettivi.

\section{Glicemia}

L'iperglicemia all'ingresso in Ospedale nei pazienti non diabetici o diabetici è indice di gravità clinica, va monitorata e trattata con insulinoterapia.

Iperglicemia e ipoglicemia vanno evitate.

\section{Crisi epilettiche}

La registrazione prolungata dell'elettroencefalogramma (EEG) evidenzia che $1 / 3$ dei pazienti presenta crisi epilettiche, che nel $16 \%$ dei casi si manifestano entro la prima settimana dopo l'emorragia.

Le emorragie corticali sono quelle a rischio di sviluppare crisi comiziali.

La presenza di crisi epilettiche cliniche o elettroencefalografiche in pazienti con interessamento della coscienza devono essere trattate farmacologicamente, mentre è da evitare l'uso di farmaci a scopo di profilassi.

\section{Complicanze mediche}

Polmoniti e infezioni delle vie urinarie sono tra le più frequenti complicanze.

Aritmie cardiache e scompenso cardiaco sono da tenere in considerazione particolarmente in pazienti anziani.

E' necessario controllare costantemente la funzionalità renale e gli elettroliti.

\section{Ipertensione endocranica ed edema cerebrale}

\section{Terapia medica}

Il trattamento medico dell'ipertensione endocranica consiste nel mantenere la testa elevata a $30 \%$, lieve sedazione e terapia iperosmolare (mannitolo), infusione di soluzione salina ipertonica, iperventilazione e sedazione fino al coma barbiturico.

I corticosteroidi potrebbero esporre a rischio d'infezione.

Secondo le linee guida SPREAD non esistono evidenze certe di quale sia il miglior trattamento dell' $i$ pertensione endocranica in corso di emorragia cerebrale spontanea. La maggior parte delle indicazioni sono derivate dalle evidenze relative all'emorragia cerebrale post-traumatica.

Non è indicato l'uso di corticosteroidi nel trattamento dell'ipertensione endocranica secondaria all'emorragia cerebrale spontanea.

\section{Terapia medica dell'ematoma}

Lo studio TICH 2: valuta la somministrazione di acido tranexamico entro 8 ore nell'emorragia cere- 
brale intraparenchimale spontanea con dose di carico di $1 \mathrm{gr}$ in $100 \mathrm{cc}$ di soluzione fisiologica seguita da $1 \mathrm{gr}$ in $250 \mathrm{cc}$ di soluzione fisiologica in 8 ore ed i risultati paiono essere favorevoli. ${ }^{36}$

\section{Terapia chirurgica dell'ematoma}

\section{Derivazione ventricolare}

Lo stravaso dell'emorragia nei ventricoli è fattore prognostico negativo con mortalità che varia dal 50 all' $80 \%$. La derivazione ventricolare è utile per ridurre il volume dell'ematoma e dell'ipertensione endocranica.

\section{Svuotamento dell'ematoma}

L'intervento di evacuazione dell'ematoma di 10$100 \mathrm{ml}$ entro le prime 48 ore è stato messo in paragone con il trattamento conservativo nello studio STICH II. Questo approccio non aumenta la percentuale di decessi o di disabilità a 6 mesi, invece potrebbe avere una piccola ma clinicamente rilevante percentuale di sopravvivenza nei pazienti con emorragia cerebrale superficiale senza spandimento intraventricolare.

\section{Chirurgia mini-invasiva}

La craniotomia convenzionale frequentemente si associa a ulteriore danno tessutale. Per ridurre questo danno sono state sviluppate tecniche di chirurgia miniinvasiva.

Si tratta di evacuazione endoscopica e aspirazione stereotassica con fibrinolisi: la chirurgia mini-invasiva guidata dall'imaging (MISTIE III).

Lo studio MISTIE III negli ematomi maggiori o uguali $30 \mathrm{ml}$ valuta se l'utilizzo di trombolisi intralesionale riduce la dimensione dell'ematoma.

La maggior parte del volume dell'emorragia viene ridotto durante la chirurgia, per un buon outcome si indica la soglia di riduzione al di sotto di $15 \mathrm{~mL}$. La maggior esperienza del neurochirurgo si associa a maggior riduzione del volume residuo. Purtroppo questo studio dimostra che questa chirurgia mini-invasiva non riesce a influenzare gli esiti funzionali, ma offre uno spunto alla comunità scientifica per cogliere informazioni al fine di migliorare il trattamento dell'emorragia cerebrale.

Alcuni studi di chirurgia mini-invasiva (ENRICH, MIND e INVEST) sono in atto e sulla base di dati preliminari queste tecniche sembrerebbero essere efficaci nel rimuovere il coagulo e potrebbero incidere maggiormente sull'outcome funzionale rispetto al MISTIE III. ${ }^{20}$

\section{Bibliografia}

1. ACR Appropriateness Criteria Cerebrovascular Disease Expert Panel on Neurologic Imaging. J AM Coll Radiol 2017;14 S34-S61.
2. AHA/ASA Guidelines (2017) for the prevention, detection, evaluation and management of high blood pressure in adults.

3. R Al-Shahi Salman, MD Dennis, GD Murray et al. RESTART collaboration. The REstart or STop Antithrombotics Randomized Trial (RESTART) after stroke due to intracerebral haemorrhage. Trial May, 302019.

4. Peter S Amenta, Jacques J Morcos Nonlesional Spontaneous Intracerebral Hemorrhage. Neurological Surgery 2017 Part 3-375: 3186-3197.

5. JS Balami, AM Buchan Complication of intracerebral haemorrhage. Lancet Neurol 2012; 11:101-18.

6. C Becattini, A Sembolini, M Paciaroni Resuming anticoagulant therapy after intracerebral bleeding. Vascular Pharmacology 84(2016) 15-24.

7. J Alfredo Caceres, Joshua N Goldstein Intracranial Hemorrhage. Emerg Med Clin N Am 30 (2012) 771-794.

8. Andreas Charidimou, Anne Schmitt, Duncan Wilson, Yusuke Yakushiji, Simone M Gregoire, Zoe Fox, Hans R Jager, David J Werring The Cerebral Haemorrhage Anatomical RaTing inStrument (CHARTS) J Neurol Sci 372 (2017)178-183.

9. PMC Choi, JV Ly, V Srikanth, H Ma, W Chong, M Holt, TG Phan Differentiation between Hemorrhagic Infarct and Parenchimal Intracerebral Hemorrhage. Radiology Research and Practice 2012: 1-11.

10. HK Chopra, C Venkata, S Ram Recent Guidelines for Hypertension. A Clarion Call for Blood Pressure Control in India. Circ Res 2019;29: 984-986.

11. H Christensen Antiplatelets after intracerebral haemorrhage: treat the patient, not the brain imaging. The Lancet Neurol Vol 18 july 2019.

12. TJ Crocco, William J Meurer Rosenn's Emergency Medicine 2018 Chapter 91: 1241-1255.

13. CK Dastur, W Yu Current Management of spontaneous intracerebral haemorrhage. BMJ SVN 2017: 21-29.

14. CV De Simone, J Graff-Radford, MA El-Harasis, et al. Cerebral Amyloid Angiopathy and implications for atrial fibrillation management. Lancet Neurol Vol 390 July 1, 2017; 9-11.

15. AM Demchuk, D Dowlatstshahi, D Rodriguez-Luna et al. Prediction of haematoma growth and out come in patients with intracerebral haemorrhage using the CT-angiography spot sign (PREDICT): a prospective observational study. Lancet Neurol 2012 Apr11(4):307-14.

16. Renan Domingues, Costanza Rossi, Charlotte Cordonnier Diagnostic Evaluation for Nontraumatic Intracerebral Hemorrhage. Neurol clin 33 (2015) 315-328.

17. ESC 2018 Guidelines for the management of arterial hypertension.

18. JN Goldstein, HB Browers, JM Romero et al. SCOREIT: the Spot Sign score in restricting ICH growth - an ATACH-II ancillary study. J Vascular Interventional Neurology 2012: 20-25.

19. A Gonzalez-Perez, ME Saez, S Johansson, A Himmelmann, LAG Rodriguez Incidence and Predictors of Hemorrhagic Stroke in Users of Low-Dose Acetylsalicylic Acid. J Stroke and Cerebrovascular Diseases Vol 24 No 10 (October) 2015: 2321-2328.

20. DF Hanley, RE Thompson, M Rosenblum, G Yenokyan, $\mathrm{K}$ Lane, $\mathrm{N}$ McBee, et al. for the MISTIE III investigators. Efficacy and safety of minimally invasive surgery with trombolysis in intracerebral hemorrhage evacuation (MI- 
STIE III): a randomised controller, open label, blinded endpoint phase 3 trial. Lancet Vol 393, 2019; 1021-1032.

21. Jeremy J Heit, Michael Iv, Max Wintermark Imaging of Intracranial Hemorrhage. JOS 2017;19(1):11-27.

22. HE Hinson, DF Hanley, WC Ziai Management of intraventricular hemorrhage. Curr Neurol Neuroci Rep 2010; 10(2):73-82.

23. Jun Yup Kim, Hee-Joon Bae Spontaneous Intracerebral Hemorrhage: Management J of Stroke. 2017;19(1):28-39.

24. AD Mendelow, BA Gregson, EN Rowan, GD Murray, A Gholkar, PM Mitchell for the STICH II investigator. Early surgery versus initial conservative treatment in patients with spontaneous supratentorial lobar intracerebral haematomas (STICH II): a randomised trial. Lancet 2013 Vol 382: 397-408.

25. M Morioka and K Orito Management of spontaneous intracerebral hematoma. Neurol Med Chir 57, 563-574, 2017.

26. A Morotti, HB Brouwers, JM Romero et al. Intensive Blood Pressure Reduction and Spot Sign in Intracerebral Hemorrhage. A secondary Analysis of a Randomized Clinical Trial. JAMA Neurol. 2017 74(8): 950-960.

27. A Morotti, D Dowlatshahi, G BBoulois et al. Predicting Intracerebral Hemorrhage Expansion with non-contrast CT: the BAT score. Stroke 2018 May; 49(5): 1163-1169.

28. A Morotti, Joshua N Goldstein Diagnosis and Management of Acute Intracerebral Hemorrhage. Emerg Med Clin N Am 34 (2016) 883-899.

29. S Osawa, T Shimizu, T Kano, R Shintoku, H Fujimaki, K Asakura Risk Factors for hemorrhage and cardioembolic complications of intracerebral hemorrhage associated with anticoagulants. J Stroke and Cerebral Diseases Vol 28 No 2 (February) 2019: 325-329.

30. AI Qureshi, YY Palesh, WG Barsan e al. Intensive Blood-Pressure Lowering in Patients with Acute Cerebral Hemorrhage. N J Med 2016; 375: 1033-1043.

31. A Rabinstein Intracerebral haemorrhage: no good treatment but treatment helps. Lancet Neurol Vol 389 February 11, 2017:575-576.
32. B Riveros Gilardi, JI Munos Lopez, AC Hernandez Villegas, JA Garay Mora, OC Rico Rodriguez, R Chavez Appendini, M De la Mora Malvaez, JA Higuera Galleja Types of Cerebral Herniation and their imaging features. Radiographics 2019 Vol 9 n.6: 1598-1610.

33. EC Sandset, PMW Bath, G Boysen et al. Treatment of acute stroke (SCAST): a randomised, placebo controller, double-blind trial. The Lancet Feb 26, 2011 Vol 377; issue 9767: 741-750.

34. M Scott, R Low, D Swan, J Thachil Reintroduction of anticoagulant therapy after intracranial haemorrhage: if and when? Blood Reviews, 32 (2018) 256-263.

35. SPREAD Guidelines 2016.

36. N Sprigg, K Flaherty, JP Appleton et al. Tranexamic acid for hyperacute primary Intracerebral Haemorrage (TICH-2): an International randomized, placebo-controlled, phase 3 superiority trial. The Lancet Neurol May 16, 2018: 1-9.

37. K Suzuki, J Aoki, Y Sakamoto, S Suda, S Okubo, T Nagao, K Kimura Low risk of ICH after reperfusion therapy in acute stroke patients treated with direct oral anticoagulant. J Neurol Sci 379 (2017) 207-211.

38. RG Victor, P Libby Systemic Hypertension: Management. Brawnwald's Hearts disease 2015: 928-956.

39. W-H Wang, Y-C Hung, S PC Hsu, C-F Lin, H-H Chen, Y-H Shih, C-C Lee Endoscopic hematoma evacuation in patients with spontaneous supratentorial intracerebral hemorrhage. J Chi Medical Association 78 (2015) 101-107.

40. JM Wardlow, EE Smith Neuroimaging standards for research into small vessel disease and its contribution to ageing and neurodegeneratione (STRIVE). Lancet Neurol 2013; 12(8): 822-838.

41. SM Zuurbier, CR Hickman, LA Rinkel et al. Long-term antithrombotic therapy and risk of intracranial haemorrhage from cerebral cavernous malformations: a populaltion based-cohort study, systematic review and meta.analysis. Lancet Neurol 2019;18:935-41. 


\title{
Emorragia subaracnoidea
}

\author{
Luigi Alberto Andrea Lanterna, ${ }^{1}$ Maria Pantusa, ${ }^{2}$ Andrea Montalbetti, ${ }^{3}$ Luciano Savarese, ${ }^{4}$ Paolo Gritti, ${ }^{5}$ \\ Maria Luisa Colleoni ${ }^{6}$
}

${ }^{1}$ UOC Neurochirurgia, ASST Papa Giovanni XXIII, Bergamo; ${ }^{2}$ UOC Neurologia e Stroke Unit, Presidio Ospedaliero San Giovanni di Dio, Crotone; ${ }^{3}$ UOC Neurochirurgia, Fondazione IRCCS Policlinico San Matteo, Pavia; ${ }^{4}$ UOC Neurochirurgia, Ospedale Casa Sollievo della Sofferenza, San Giovanni Rotondo (FG); ${ }^{5}$ UOS Neurorianimazione, ASST Papa Giovanni XXIII, Bergamo; ${ }^{6}$ UOC Neuroradiologia, ASST Papa Giovanni XXIII, Bergamo, Italia

\section{Introduzione}

La definizione anatomo-patologica di emorragia subaracnoidea (ESA) è la presenza di sangue nello spazio anatomico meningeo tra aracnoide e pia madre. Diverse sono le cause che possono essere causa della presenza di sangue nello spazio subaracnoideo. Si suole distinguere tra ESA da causa traumatica ed ESA spontanea. La causa più frequente di ESA spontanea è la rottura di aneurisma cerebrale. ${ }^{1}$ Altre possibili, ma molto rare cause di ESA spontanea sono le malformazione artero-venose cerebrali, le fistole artero-venose durali, le vasculiti e, a livello di case-report, le trombosi venose cerebrali e I tumori. ${ }^{1}$ Il 5-10\% delle ESA spontanee non ha invece un'evidente causa sottostante e si parla in questi casi di ESA sine materia o perimesencefalica o ancora, non-aneurysmal subarachnoid hemorrhage. ${ }^{1,2}$ Le emorragie sine materia hanno una prognosi tipicamente benigna, un rischio minimo di recidiva e hanno un'incidenza di circa 0,5 casi per 100.000 per anno. ${ }^{2}$ Il sangue si localizza pressoché costantemente anteriormente al ponte e mesencefalo con, solitamente, minima estensione nella parte più prossimale della cisterna silviana. La patogenesi dell'ESA sine materia non è nota ma l'ipotesi più accreditata è che sia correlata a rottura di piccole vene perimesencefaliche. ${ }^{1,2}$

Vista la netta predominanza epidemiologica, la gra-

Corrispondente: Luigi Alberto Andrea Lanterna, UOC Neurochirurgia, ASST Papa Giovanni XXIII, Bergamo, Italia.

E-mail:1.lanterna@gmail.com

Key words: Emorragia subaracnoidea; aneurisma cerebrale.

Articolo pubblicato secondo la Creative Commons Attribution NonCommercial 4.0 License (CC BY-NC 4.0).

${ }^{\circ}$ Copyright: the Author(s), 2020

Licensee PAGEPress, Italy

QUADERNI - Italian Journal of Medicine 2020; 8(2):94-99 vità clinica e I complessi risvolti in termini di gestione e trattamento, la nostra attenzione, così come è quella della letteratura scientifica e dell'everyday practice, è rivolta principalmente all'ESA da aneurisma.

\section{Emorragia subaracnodea da rottura di aneurisma cerebrale}

\section{Epidemiologia}

L'ESA da aneurisma ha un'incidenza variabile da circa 2 casi per 100.000 per anno fino a oltre 20 casi per 100.000 per anno. ${ }^{3}$ L'estrema variabilità del dato di incidenza è maggiormente dovuta a fattori di tipo metodologico e in parte anche al fatto che diverse popolazioni possono avere una differente prevalenza di aneurismi cerebrali e un differente rischio di sanguinamento degli stessi. In particolare, le popolazioni che sembrano essere a maggior rischio sono la popolazione finlandese e la popolazione giapponese con valori di incidenza di ESA che raggiungono i 20-23 casi/100.000/anno. L'età media della popolazione studiata e la distribuzione di eventuali fattori di rischio così come la frequenza nell'uso della TAC e la frequenza di autopsie condizionano la variabilità dei dati di incidenza. L'età media della popolazione studiata è metodologicamente importante perché l'incidenza di ESA aumenta con l'età e l'età media normalmente è superiore a 50 anni. La frequenza nell'uso della TAC è importante perché negli studi più datati la diagnosi di ESA era prevalentemente clinica con il conseguente rischio di includere pazienti con altre cause di emorragia cerebrale o altre patologie con simile presentazione clinica. Infine, la frequenza di riscontri autoptici influenza I risultati perché il 12-15\% dei pazienti decede prima di raggiungere l'ospedale e quindi prima di eseguire qualunque accertamento neuroradiologico.,4

La media degli studi di popolazione e alcuni studi eseguiti sulla popolazione americana ha dato valori di incidenza attorno a 10 casi/100.000/anno. Uno studio virtuale in cui si supponeva che la TAC venisse usata nel $100 \%$ dei pazienti dava un'incidenza di circa 6 
casi/100.000/anno, che poco si discosta da 6.9 casi/100.000/anno della popolazione dell'Olmsted county studiata per 30 anni. ${ }^{1,2,4}$

La frequenza di ESA è superiore nel sesso femminile con incidenze che sono circa di 1.2 volte superiori rispetto a quelle del sesso maschile. I maggiori fattori di rischio noti sono la familiarità, il fumo, l'ipertensione arteriosa, l'importante uso di alcool e l'uso di sostanze simpaticomimetiche come la cocaina. Condizioni predisponenti possono essere le malattie del connettivo (Ehlers-Danlos tipo IV), il rene policistico o avere aneurismi cerebrali non trattati.

L'ESA da aneurisma è una malattia grave. Il casefatality rate varia dal $35 \%$ al $67 \%$ con un valore medio pesato di circa il $51 \%$. Negli ultimi anni sembra che il case-fatality rate si stia riducendo con una decrescita stimata di circa l'1\%/anno. In particolare, da uno studio sulla popolazione USA, dalla metà degli anni ' 70 alla metà degli anni ' 80 il case fatality rate si è ridotto da circa il $60 \%$ al $42 \%$. Il mortality rate mediano varia nelle diverse popolazioni con range dal $45 \%$ della popolazione europea al 27 della popolazione giapponese. Parte della variabilità è però dovuta al fatto che non tutti gli studi prendono in considerazione la frequenza di morti prima di raggiungere l'ospedale che si attesta attorno al $10-15 \%$ con conseguente sottostima. ${ }^{5}$

Dei pazienti che sopravvivono all'emorragia subaracnoidea, molti hanno deficit neurologici e/o deficit cognitivi che condizionano l'autonomia nella vita quotidiana e la qualità della vita. Dal $10 \mathrm{al} 20 \%$ dei pazienti sono dipendenti nella vita quotidiana. I dati dei recenti non-population based trial (ISATs) ${ }^{6}$ ha mostrato che fino al $12 \%$ dei pazienti hanno significative restrizioni successivamente all'ESA (Modified Rankin Scale 3) e il 6,5\% sono completamente dipendenti (Modified Rankin Scale 4-5) a 1 anno dal sanguinamento. Deficit cognitivi colpiscono circa il $20 \%$ dei pazienti che sopravvivono all'emorragia e I disturbi cognitivi si associano a un peggior recupero funzionale e una peggiore qualità della vita. Frequenti sono inoltre I disturbi dell'umore (ansietà e depressione), disturbi del sonno e profonda astenia/faticabilità. Solo circa il $20 \%$ dei pazienti indipendenti non hanno un peggioramento della qualità della vita a 4 mesi dell'emorragia subaracnoidea. La rivalutazione a 1 anno mostrava un netto miglioramento ma, ancora solo il $31 \%$ del totale dei pazienti indipendenti non denunciavano una riduzione della qualità della vita. ${ }^{1,45}$

In conclusione solo una piccola parte dei pazienti ha un recupero completo funzionale e cognitivo e con una qualità della vita non ridotta successivamente all'emorragia subaracnoidea. L'importanza socio-sanitaria di questo dato è ulteriormente sottolineata dalla giovane età del paziente con ESA (circa 50 anni) rispetto a quella degli altri stroke.

\section{Presentazione clinica}

La presentazione clinica dell'emorragia subaracnoidea è molto eterogenea. Alcuni pazienti si presentano svegli e con solo cefalea, altri pazienti giungono in ospedale in stato di coma, altri decedono prima di giungere all'osservazione medica. Poiché la presentazione clinica condiziona la prognosi e le scelte di trattamento, sono state proposte diverse scale cliniche per descrivere il paziente con ESA. Le scale più utilizzate sono la scala Hunt and Hess $(\mathrm{HH})^{7}$ e la scala della world federation (WFNS) ${ }^{8}$ Entrambe, seppur con modalità differenti valutano il livello di coscienza del paziente e la presenza o meno di deficit focali. La scala $\mathrm{HH}$ descrive il paziente e può così essere riassunta: grado 1-2 il paziente è sveglio con cefalea di variabilità intensità e rigor nucalis e non ha deficit focali salvo eventualmente deficit di nervi cranici, il grado 3 include pazienti confusi, disorientati, soporosi, o con lievi deficit, I pazienti in grado 4 o 5 sono in stato di coma e possono avere emiparesi di grado moderato o severo. La WFNS considera anch'essa 2 fattori: il livello di coscienza e la presenza di deficit focali. Diversamente dalla scala $\mathrm{HH}$, nella scala WFNS il livello di coscienza è definito in base alla scala GCS. Nella scala WFNS I pazienti in grado 1 si presentano in GCS 15 e non hanno deficit, I gradi 2-3 hanno una lieve riduzione del livello di coscienza corrispondente a GCS 13-14 e quello che differenzia i gradi 2 dai 3 è, rispettivamente, l'assenza o la presenza di deficit focali, I poor grades (gradi 4 e 5) sono caratterizzati da una riduzione del livello di coscienza importante (grado 4: GCS 7-12; grado 5: GCS $<7$ ) indipendentemente dalla presenza o meno di deficit focali. Il deficit focale è quindi fattore dominante solo nei pazienti con lieve riduzione del livello di coscienza (grado 2-3). ${ }^{7,8}$

Il sintomo più distintivo dell'ESA nei pazienti che giungono svegli e possono raccontarlo, è la cefalea. Nell' $80 \%$ dei pazienti che possono raccontare la propria storia, la cefalea da ESA è descritta come la peggior cefalea della mia vita. ${ }^{1,4}$ E' una cefalea improvvisa che raggiunge immediatamente la massima intensità (thunderclap headache) e che interessa la regione occipito-nucale. Nel $10-40 \%$ dei pazienti questa cefalea è preceduta da una cefalea sentinella che tipicamente si verifica 2-8 settimane prima dell'ESA vera e propria ed è considerata espressione di un microsanguinamento (warning leak). Sintomi associati sono la nausea, il vomito, la fotofobia e la rigidità nucale (espressione di irritazione meningea). Sebbene sia talvolta descritta in seguito a sforzi fisici importanti, nella maggior parte dei casi l'ESA si verifica durante le comuni attività della vita quotidiana. L'ESA rappresenta solo 1'1\% delle cefalee valutate in pronto soccorso. Una cefalea sentinella ha quindi un alto rischio di essere sottovalutata o misdiagnosticata 
e non investigata. Importante da sapere è che un paziente con una cefalea sentinella non diagnosticata ha un rischio di morte o disabilità 4 volte superiore rispetto al paziente in cui viene diagnosticata. Importante è quindi avere una alto indice di sospetto soprattutto quando: i) è una cefalea di nuova insorgenza o definita come la peggiore della propria vita (la cefalea sentinella può non essere intensa come in caso di franca ESA); ii) ha un andamento temporale tipo thunderclap; e iii) vi si associano segni meningei come la rigidità nucale (tipica dell'ESA franca, più rara in caso di cefalea sentinella). Il $20 \%$ dei pazienti con ESA si presenta anche con crisi epilettiche che si verificano soprattutto quando vi è un'estensione del sanguinamento nel parenchima cerebrale. ${ }^{1}$

\section{Imaging - diagnostica}

Se la clinica fa sospettare un'emorragia subaracnoidea, l'esame di prima scelta è la TAC dell'encefalo senza mezzo di contrasto. L'alta capacità diagnostica della TAC deriva dalla caratteristica elevata iperdensità del sangue appena stravasato. La distribuzione del sangue può inoltre suggerire la sede di un possibile aneurisma o la presenza di altre, più rare cause di ESA. ${ }^{4,9}$

Come ogni metodica diagnostica anche la TAC può avere dei falsi positivi o dei falsi negativi. Esiste la rara possibilità di falsi positivi in contesti di grave e generalizzato edema cerebrale dove la congestione venosa negli spazi subaracnoidei può mimare la presenza di sangue. Il problema dei falsi negativi è invece più frequente e complesso: nei primi 3 giorni la sensibilità della TAC nella diagnosi di ESA è attorno al 98$100 \%$, ma la sensibilità si riduce più passano I giorni dal momento del sanguinamento. Ad esempio, a 7 giorni dal sanguinamento la sensibilità diagnostica della TAC si riduce a circa il $50 \%{ }^{4,9}$

Il problema è quindi su come approfondire la diagnostica nei casi in cui la clinica suggerisce un'ESA ma la TAC è giudicata negativa, cosa che può accadere nell' $1-2 \%$ dei casi per ESA di minima entità o nei casi in cui il paziente si è presentato in pronto soccorso diversi giorni dopo l'insorgenza della sintomatologia. La puntura lombare è considerata come procedura diagnostica tra le linee guida dell'American Heart Association in caso di clinica suggestiva di ESA e TAC encefalo negativa. ${ }^{4}$ La prima regola da considerare quando si prende in considerazione l'ipotesi di eseguire una puntura lombare è che siano passate almeno 6-12 ore dall'insorgenza della cefalea affinché si abbia il tempo di avere la lisi dei globuli rossi e la conseguente classica pigmentazione del supranatante dopo centrifugazione (xanthocromia). La xanthocromia del supranatante dopo centrifugazione del liquor è elemento importante perché non si verifica in caso di puntura traumatica. In altre parole, dopo puntura lombare traumatica - se la centrifugazione del liquor ematico viene fatta subito la xanthocromia del supranatante non è visibile. Il test delle 3 provette non è invece attendibile a distinguere un'ESA vera da una puntura lombare traumatica. ${ }^{1}$ Poiché la xanthocromia continua ad essere presente anche dopo 15-18 giorni dal sanguinamento, la puntura lombare è elemento diagnostico importante soprattutto quando per motivi temporali la sensibilità diagnostica della TAC si riduce, cioè quando il paziente si presenta in pronto soccorso diversi giorni dopo l'insorgenza della cefalea. La RM encefalo (sequenze FLAIR, densità protonica, gradient-echo) può identificare il sangue subaracnoideo quando la TAC non lo mostra per l'elevata sensibilità della RM all'heme. Tuttavia, secondo le linee guida dell'American Heart Association, la negatività della RM non consente di ovviare alla puntura lombare. ${ }^{1,4,9}$

Una volta fatta diagnosi di ESA bisogna indagarne la causa, o più propriamente escludere o meno la presenza di un aneurisma. Attualmente, l'angioTAC viene fatta immediatamente dopo la TAC di base e permette di individuare la maggior parte degli aneurismi con pochi limiti: aneurismi $<3 \mathrm{~mm}$, forme rare di aneurisma (dissecanti e blister) o rari casi di aneurisma completamente trombizzato. I dettagli anatomici dell'angioTAC sono tali da definire anche la sede e le dimensioni dell'aneurisma e, più grossolanamente, la sua morfologia. ${ }^{4} \mathrm{Nei}$ casi in cui l'angioTAC è negativa con TAC diagnostica per ESA è opportuno approfondire la diagnostica con il gold standard che è rappresentato dall'angiografia digitale. L'angiografia cerebrale e soprattutto la sua ricostruzioni tridimensionale permette di identificare anche gli aneurismi di minori dimensioni e fornisce il miglior dettaglio anatomo-morfologico della sacca aneurismatica. Secondo alcuni autori, sebbene vi siano molte controversie a riguardo, un'angioTAC negativa può concludere la diagnostica in caso sia sicura la diagnosi di ESA peri-mesencefalica. ${ }^{1,49} \mathrm{Nel}$ caso invece di un'ESA cisternale diffusa un' angioTAC negativa deve essere seguita da un'angiografia digitale. Se anche l'angiografia digitale fosse negativa, in presenza di ESA diffusa (non perimesencefalica), è opportuno ripeterla perché la seconda angiografia ha una probabilità fino al $14 \%$ di identificare un piccolo aneurisma passato inosservato alla prima angiografia. Il timing della seconda angiografia è tipicamente dopo 10-15 giorni dalla prima per escludere l'effetto confondente del vasospasmo ma, in casi molto particolari, può essere ripetuta anche pochi giorni dopo la prima (es. sospetto blister). L'angioTAC e ancor meglio l'angiografia permettono di avere I dati anatomici sufficienti a decidere come trattare l'aneurisma. In tal senso, il limite che alcuni studi riportano circa l'angioTAC è che può portare ad una sovrastima del col- 
letto per effetto di volume parziale. Tuttavia, l'evoluzione tecnologica (numero/spessore delle slices e data processing) ha ridotto le distorsioni e nettamente migliorato la definizione anatomica dell'aneurisma. ${ }^{1,4,9}$

\section{Misure mediche per ridurre il rischio di risanguinamento}

Il rischio di risanguinamento di un aneurisma è del $4-14 \%$ nelle prime 24 ore. Un terzo dei risanguinamenti avvengono nelle prime 3 ore e circa la metà nelle prime 6 ore.$^{10} \mathrm{Il}$ risanguinamento è associato ad un incremento del rischio di morte o disabilità. Il maggior fattore di rischio di risanguinamento è il trattamento ritardato dell'aneurisma. Sono fattori di rischio di risanguinamento anche le dimensioni dell'aneurisma (aneurismi di maggiori dimensioni hanno un rischio di sanguinamento superiore), un grado elevato alle scale cliniche (HH, WFNS) e una pressione arteriosa non controllata $\left(\mathrm{Pa}_{\max }>160 \mathrm{mmHg}\right){ }^{4}$

Sebbene non vi siano dati che definiscano precisamente I valori ideali di pressione arteriosa da tenere, c'è un generale consenso che l'ipertensione arteriosa debba essere controllata farmacologicamente (tenere una $\mathrm{Pa}_{\max }<160 \mathrm{mmHg}$ ) finché l'aneurisma non viene trattato. Storicamente, quando gli aneurismi venivano trattati a distanza dal sanguinamento, si usavano gli antifibrinolitici per ridurre il rischio di risanguinamento. ${ }^{4}$ Questo però si accompagnava ad un maggior rischio di ischemia cerebrale tardiva. Recentemente, uno studio riguardante l'uso degli antifibrinolitici per periodi limitati ( $<72$ ore), ha documentato una riduzione del rischio di risanguinamento dall' $11 \%$ al $2 \%$ senza incremento del rischio di ischemia cerebrale tardiva. Le linee guida dell'American Heart Association considerano l'uso dell'antifibrinolitico per meno di 72 ore in pazienti il cui aneurisma non può essere trattato acutamente. ${ }^{11}$

\section{Il trattamento dell'aneurisma}

La letteratura scientifica e la pratica clinica hanno dimostrato come il trattamento dell'aneurisma elimini il rischio di risanguinamento e che il trattamento è una procedura sicura ed efficace. ${ }^{1,4}$

Prima del 1991 il trattamento dell'aneurisma era rappresentato esclusivamente dal trattamento chirurgico. Il trattamento chirurgico implica una craniotomia, la dissezione delle cisterne aracnoidee, l'esposizione dell'aneurisma e dell'arteria parente e quindi il posizionamento di una clip in titanio a livello del colletto dell'aneurisma in modo da escludere l'aneurisma dal circolo e, contemporaneamente, salvaguardare il flusso nel vaso parente e I suoi collaterali. Nel 1991 iniziava l'uso in clinica delle Guglielmi de- tachable coils e con esse il trattamento endovascolare. Il trattamento endovascolare comporta la navigazione con cateteri dei vasi arteriosi fino a livello del colletto dell'aneurisma e il rilascio di spirali metalliche all'interno della sacca dell'aneurisma in modo da modificarne il flusso all'interno ed indurne la trombosi. ${ }^{4,9}$ Più recentemente sono stati introdotti altri devices come gli stent che però, salvo eccezioni, vengono usati solo una volta superata la fase acuta dell'emorragia subaracnoidea.

Sono stati pubblicati 2 studi randomizzati che hanno confrontato rischio ed efficacia del trattamento chirurgico rispetto al trattamento endovascolare con coils. Lo studio ISAT ${ }^{6}$ ha randomizzato 2143 pazienti su un totale di 9559 pazienti seguendo il principio secondo cui 2 consultant (un chirurgo ed un endovascolare) valutavano l'aneurisma e questo veniva randomizzato solo se l'aneurisma era considerato trattabile da entrambi gli specialisti. Lo studio ha identificato una differenza assoluta a favore del trattamento endovascolare del 7\% (morte o disabilità del 31\% nel braccio chirurgico e del $24 \%$ nel braccio endovascolare). Il braccio endovascolare aveva però un maggior rischio di cross-over, un maggior rischio di riabitazione dell'aneurisma, un maggior rischio di chiusura incompleta e di risanguinamento dell'aneurisma. Il braccio chirurgico era gravato in piccola parte da maggiori complicanze ma sopratutto da un maggior rischio di mortalità pretrattamento da risanguinamento, cioè di pazienti il cui aneurisma ha risanguinato prima di essere sottoposti al trattamento. ${ }^{9}$ Ad un' analisi as treated (anziché intention-to-treat: tenendo conto dei cross-over e della mortalità pretrattamento) non vi era differenza tra I due gruppi. Diversamente dallo studio ISAT, nello studio BRAT ${ }^{12,13}$ gli aneurismi venivano allocati ai due bracci di trattamento indipendentemente dal principio di equipoise tra I due consultant. Considerando tutti I tipi di aneurismi (sia aneurismi sacculari che I rari aneurismi fusiformi), lo studio identificava un rischio inferiore nel braccio endovascolare. Considerando solo gli aneurismi sacculari che rappresentano gli aneurismi inclusi nello studio ISAT nonché la stragrande maggioranza degli aneurismi della pratica clinica - non vi erano differenze statisticamente significative tra I due gruppi. L'analisi post-hoc per sede mostrava che l'unica localizzazione in cui vi era una differenza statisticamente significativa a favore del trattamento endovascolare era per gli aneurismi dell'arteria vertebrale all'origine dell'arteria cerebellare postero-inferiore.

Sebbene vi siano delle eccezioni, e l'argomento sia un work-in-progress, l'American Heart Association ha così riassunto I principi generali di gestione degli aneurismi rotti: ${ }^{4}$ i) il paziente con aneurisma sanguinante deve essere trattato il prima possibile per ridurre il rischio di risanguinamento; ii) la decisione su come 
trattare l'aneurisma deve essere multidisciplinare (chirurgo ed endovascolare) considerando sia le caratteristiche del paziente che quelle dell'aneurisma; iii) per quegli aneurismi che sono egualmente gestibili con le due metodiche il trattamento endovascolare dovrebbe essere preferibile; iv) il trattamento chirurgico è da preferire in caso di aneurismi della cerebrale media e pazienti con ematomi. Secondo alcuni autori anche nei pazienti giovani $(<40$ anni) per il minor rischio di riabitazione e minor rischio di risanguinamento. Il trattamento endovascolare è da preferire negli aneurismi della basilare, nei pazienti con WFNS 4 o 5 (senza ematoma) e nel paziente anziano; v) l'uso degli stent in fase acuta è associato ad un maggior rischio e dovrebbe essere considerato solo quando le opzioni meno rischiose e più time-tested (coiling semplice o chirurgia) sono state escluse.

\section{I primi 15 giorni post-ESA: ischemia cerebrale tardiva}

Il termine vasospasmo significa restringimento del lume di un'arteria e, nel caso dell'emorragia subaracnoidea, è sinonimo di restringimento delle grosse arterie del circolo di Willis o delle loro maggiori diramazioni. Il 70\% dei pazienti con emorragia subaracnoidea ha vasospasmo angiograficamente dimostrabile. ${ }^{1,49}$ Il vasospasmo, se emodinamicamente significativo, può correlarsi ad una riduzione del flusso ematico cerebrale e causare ischemia cerebrale. Il vasospasmo è una causa possibile di ischemia cerebrale tardiva. Inizialmente era l'imputato principale se non l'unico dell'ischemia cerebrale tardiva. L'ischemia cerebrale tardiva è una delle principali cause di mortalità e morbilità tra I pazienti che sopravvivono al primo sanguinamento. ${ }^{1,4,9}$

Molta letteratura è stata dedicata all'eziopatogenesi e fisiopatologia dell'ischemia cerebrale tardiva e del vasospasmo nell'ESA. I dati sono proteiformi, spesso eterogenei e contrastanti. Quello che è sicuro è che il vasospasmo tipicamente si verifica tardivamente cominciando in $3^{\circ}-4^{\circ}$ giornata, raggiunge un picco tra la $9^{\circ}$ e $12^{\circ}$ giornata per poi risolversi spontaneamente verso la $21^{\circ}$ giornata. ${ }^{1,4,9} \mathrm{Il}$ vasospasmo dei grossi vasi dle Willis da solo non sempre è causa del danno ischemico tardivo perchè molti pazienti con vasospasmo non sviluppano ischemie cerebrali tardive e molti pazienti che sviluppano ischemie tardive non hanno avuto vasospasmo dei grossi vasi. L'ipotesi è che diversi fattori, assieme al vasospasmo dei grossi vasi, possano concorrere nella patogenesi del danno ischemico tardivo. Tra questi, l'azione contemporanea di diversi fattori come lo spasmo del microcircolo (cioè che il vasospasmo possa interessare anche il microcircolo oltre che i grossi vasi del circolo di Willis), la perdita/esaurimento dell'autoregolazione, l'infiam- mazione disregolata, l'accumulo di radicali liberi, la disfunzione elettrica cellulare (cortical spreading depression), le microembolie e, non ultimo, la riduzione della perfusione cerebrale da ipertensione endocranica. A sostegno di un quadro fisiopatologico complesso, multifattoriale e non vasospasmo-centrico è non solo il fatto che il danno ischemico tardivo può svilupparsi anche in pazienti che non hanno avuto vasospasmo dei grossi vasi ma anche il fatto che l'unico farmaco che ha mostrato efficacia nel miglioramento della prognosi del paziente con ESA (nimodipina) non ha effetto sul vasospasmo dei grossi vasi. ${ }^{1,4,9}$

Sebbene l'ischemia cerebrale tardiva rappresenti una forma di stroke potenzialmente prevedibile e che molta ricerca sia stata fatta sia in campo sperimentale che clinico, le potenzialità di prevenzione e trattamento rimangono limitate. ${ }^{4}$ Attualmente, la nimodipina e il mantenimento dell'euvolemia sistemica rappresentano gli unici approcci preventivi per l'ischemia cerebrale tardiva post-ESA. ${ }^{4}$ Nonostante molte aspettative fossero state riposte verso altri farmaci come gli antiaggreganti, le statine, il magnesio solfato e il clazosentan (antagonisti dell'endotelina-1), studi randomizzati e/o metaanalisi non hanno mostrato la loro efficacia clinica. ${ }^{4}$ Anche l'uso profilattico della tripla H (terapia emodinamica caratterizzata dall'induzione farmacologica di ipertensione arteriosa, ipervolemia ed emodiluizione) o dell'angioplastica profilattica non hanno mostrato un miglioramento del mortality/morbidity. ${ }^{4}$ Una volta che il paziente sviluppa l'ischemia cerebrale tardiva poche sono le armi per impedire che sviluppi una danno neurologico definitivo.

Le linee guida dell'American Heart Association in merito al trattamento del paziente che sviluppa una clinica secondaria ad ischemia cerebrale tardiva, suggeriscono l'induzione dell'ipertensione (salvo che la funzionalità cardiaca non lo precluda o che il paziente non sia già spontaneamente iperteso) (Classe I, livello di evidenza B) e l'angioplastica se il paziente ha un vasospasmo dei grossi vasi congruo con il deficit clinico e che non risponde all'ipertensione indotta (Classe IIa, livello di evidenza B). ${ }^{4}$

\section{Bibliografia}

1. van Gijn J, Rinkel GJE. Subarachnoid hemorrhage: diagnosis, causes and management. Brain 2001;124:249278.

2. van Gijn J, van Dongen KJ, Vermeulen M, Hijdra A. Perimesencephalic hemorrhage: a nonaneurysmal and benign form of subarachnoid hemorrhage. Neurology 1985;35:493-497.

3. de Rooij NK, Linn FH, van del Plas JA, Algra A, Rinkel GJ. Incidence of subarachnoid hemorrhage: a systematic review with emphasis on region, age, gender and time trends. J Neurol Neurosurg Psychiatry 2007;78:13651372. 
4. Connolly ES Jr, Rabinstein AA, Carhuapoma JR, et al. Guidelines for the management of aneurysmal subarachnoid hemorrhage: a guideline for healthcare professionals from the American Heart Association. Stroke 2012;43:1711-1737.

5. Nieuwkamp DJ, Setz LE, Algra A, et al. Changes in case fatality of aneurysmal subarachnoid hemorrhage over time, according to age, sex and region: a meta-analysis. Lancet Neurol 2009;8:635-642.

6. Molyneux A, Kerr R, Stratton I, et al. International Subarachnoid Aneurysm Trial (ISAT) of neurosurgical clipping versus endovascular coiling in 2143 patients with ruptured intracranial aneurysms: a randomized trial. Lancet 2002;360:1267-1274.

7. Hunt WE, Hess RM. Surgical risk as related to time of intervention in the repair of intracranial aneurysms. J Neurosurg 1968;28:14-20.

8. Report of World Federation of Neurological Surgeons
Committee on a universal Subarachnoid Haemorrhage Grading Scale. J Neurosurg 1988;68:985-986.

9. Lawton MT, Vates GE. Subarachnoid hemorrhage. N Engl J Med 2017;377:257-266.

10. Kassell NF, Torner JC. Aneurysmal rebleeding: a preliminary report from the Cooperative Aneurysm Study. Neurosurgery 1983;479-481.

11. Starke RM, Kim GH, Fernandez A, et al. Impact of a protocol for acute antifibrinolytic therapy on aneurysm rebleeding after subarachnoid hemorrhage. Stroke 2008;39:2617-2621.

12. Spetzler RF McDougall CG, Albuquerque FC, et al. The Barrow Ruptured Aneurysm Trial: 3-year results. J Neurosurg 2013;119:146-157.

13. Spetzler RF McDougall CG, Zabramski JM, et al. The Barrow Ruptured Aneurysm Trial: 6-year results. J Neurosurg 2015;123:609-617. 


\title{
Ictus a possibile doppia genesi. Cardioembolica o aterotrombotica?
}

\author{
Salvatore Ascione, Patrizia Ripa, Giacinta Grimaldi \\ U.O.C. di Neurologia, Ospedale del Mare, ASL Napoli 1 Centro, Napoli, Italia
}

\section{Ictus ischemico cardioembolico}

Numerose patologie cardiache presentano tra le loro complicanze l'ictus ischemico, il cui meccanismo etiopatologico è rappresentato da un embolismo di origine cardiaca e pertanto definito come ictus cardioembolico. ${ }^{1}$ Si stima che $15-20 \%$ di tutti gli ictus ischemici abbia un origine cardioembolica. ${ }^{2}$ Il substrato cardiologico che più frequentemente si associa all'embolismo, soprattutto nella popolazione anziana, è rappresentato dalla fibrillazione atriale che è in grado di spiegare dalla metà fino ai $3 / 4$ di tutti i fenomeni cardioembolici cerebrali in occidente.

\section{Cardiopatie e rischio emboligeno: sinossi}

Tra le altre condizioni cardiache ad elevato potenziale emboligeno sono da considerare l'infarto acuto del miocardio, l'endocardite infettiva, la stenosi mitralica su base reumatica, le protesi valvolari meccaniche, la cardiomiopatia dilatativa e i tumori intracardiaci. Sono invece a basso rischio emboligeno il prolasso della mitrale, la calcificazione dell'anulus mitralico della valvola aortica, la stenosi aortica calcifica, l'infarto del miocardio distale, l'aneurisma del ventricolo sinistro, la cardiomiopatia ipertrofica, il forame ovale pervio, l'aneurisma del setto atriale e il flutter atriale. Nella popolazione pediatrica invece le malattie congenite cardiache rappresentano la maggiore causa di cardioembolismo ${ }^{3} 1^{1}$ 'incremento di sopravvivenza di tale popolazione di piccoli pazienti ha portato conseguentemente ad un aumento dell'inci-

Corrispondente: Giacinta Grimaldi, U.O.C. di Neurologia, Ospedale del Mare, ASL Napoli 1 Centro, via delle Metamorfosi, 80147 Napoli, Italia.

E-mail: neurologia.odm@aslnapoli1centro.it

Articolo pubblicato secondo la Creative Commons Attribution NonCommercial 4.0 License (CC BY-NC 4.0).

${ }^{\circ}$ Copyright: the Author(s), 2020

Licensee PAGEPress, Italy

QUADERNI - Italian Journal of Medicine 2020; 8(2):100-110 denza di ictus ischemici pediatrici, nonostante la correzione chirurgica. ${ }^{4}$

L'embolismo di origine cardiaca può essere silente oppure provocare severi deficit neurologici o la morte. Sebbene la maggior parte delle patologie cardiache possono provocare embolismo cerebrale, alcune patologie sono a particolare rischio emboligeno (Tabella 1).

\section{Ictus cardioembolici e clinica}

Tipiche caratteristiche degli ictus cardioembolici sono l'estesione, la bilateralità, l'aspetto cuneiforme delle lesioni, la presenza di più territori vascolari coinvolti, in particolare la territorialità vascolare della divisione inferiore dell' arteria cerebrale media, e la rapida trasformazione emorragica. Ictus ischemici con una probabile fisiopatogenesi embolica clinicamente non sono differenziabili dagli ictus ischemici da altre cause anche se in genere sintomi quali afasia di Wernicke, emianopsia laterale omonima senza emiparesi e/o deficit sensitivo controlaterale o un'aprassia ideomotoria sono più suggestivi di un ictus cardioembolico. ${ }^{5}$

\section{Ictus cardioembolici e diagnostica differenziale}

Queste caratteristiche vanno sempre e comunque ricercate alle neuroimmagini, per una corretta diagnosi differenziale di ictus cardioembolico. L'individuazione di una fonte emboligena da sola, in effetti, non è in grado di stabilire con certezza l'origine cardioembolica di un ictus ischemico, dal momento che l'aterosclerosi cerebrale è di per sé legata a numerose malattie cardiache (Figura 1) oltre che alcune lesioni ischemiche possono essere aritmogene come ad esempio le lesioni ischemiche in regione insulare. ${ }^{6} \mathrm{La} \mathrm{Fi}-$ gura 1 esemplifica le più frequenti patologie cardiche emboligene ma che di fatto non escludono una comorbidità con un'aterosclerosi pluridistrettuale.

\section{Patologie cardiache emboligene}

Un ictus ischemico acuto si verifica nell' $1 \%$ dei pazienti ospedalizzati per infarto del miocardio, ${ }^{7}$ circa il 
$50 \%$ degli infarti anteriori si associano a formazione di trombi ventricolari sinistri, meno del $4 \%$ per gli infarti inferiori. Quasi la totalità dei fenomeni cardioembolici si verificano entro il primo trimestre con 1' $85 \%$ dei fenomeni embolici che si verificherebbero nelle prime 4 settimane dall'infarto acuto. Una ridotta frazione di eiezione è un fattore di rischio indipendente di ictus ischemico in seguito ad infarto miocardico acuto. Una cardiomiopatia dilatativa o congestizia, sia essa di natura ipertensiva infiammatoria, infettiva, immunologica, metabolica,o tossica o dovuta a patologie neuromuscolari predispone alla formazione di trombi a causa della stasi dovuta alla complessiva compromissione della performance ventricolare. Occasionalmente tali pazienti presentano anche fibrillazione atriale, ad aumentare il rischio emboligeno. Talora l'ictus ischemico può rappresentare la manifestazione iniziale di un'insufficienza cardiaca. Le encefalomiopatie mitocondriali $^{8}$ (MELAS, MERRF, sindrome di Kearns Sayre) sono talvolta associate ad un quadro di cardiomiopatia dilatativa di cui l'ictus ischemico è una complicanza. La cardiomiopatia di Chagas è talvolta complicata da aneurismi atipici con risultante embolismo cerebrale. La gran parte dei quadri di stenosi mitralica $^{9}$ è legata alla malattia reumatica cardiaca. Emboli sistemici si verificano nel 9-14\% dei pazienti con stenosi mitralica, con un $60-75 \%$ di questi che presentano ictus ischemici cerebrali su base cardioembolica. Anche la stenosi subaortica ipertrofica idiopatica può manifestarsi con un quadro di ictus cerebri, così come nei pazienti portatori di valvole aortiche bicuspidi ${ }^{10}$ anche se più raramente I pazienti con calcifica-

Tabella 1. Cardiopatie emboligene.

\begin{tabular}{lc}
\hline Patologia cardiaca & Rischio emboligeno \\
\hline Infarto acuto del miocardio & $*$ \\
\hline Cardiomiopatia dilatativa & $* *$ \\
\hline Aritmie cardiache & $*$ \\
\hline Fibrillazione atriale & $* *$ \\
\hline Sindrome del seno malato & $* *$ \\
\hline Malattia valvolare cardiaca & $*$ \\
\hline Valvulopatia mitralica reumatica & $* *$ \\
\hline Endocardite infettiva & $* * *$ \\
\hline Endocardite trombotica asettica & $* * *$ \\
\hline Protesi valvolari meccaniche & $* * *$ \\
\hline Tumori intracardiaci & $* *$ \\
\hline Forame ovale pervio & $*$ \\
\hline Aneurisma del setto interatriale & $*$ \\
\hline Encefalomiopatie mitocondriali & $* *$ \\
\hline Cardioversione per la fibrillazione atriale & $*$ rischio; medio rischio;**alto rischio. \\
\hline
\end{tabular}

zione anulare della valvola mitrale (MAC) hanno un rischio doppio rispetto alla popolazione generale di stroke ma in assoluto i fenomeni embolici cerebrali restano rari. Il prolasso della valvola mitrale, che riguarda il 3-4\% della popolazione occidentale adulta, quando non complicato non sembra incrementare il rischio relativo di ictus ischemico. Eventi ischemici cerebrali di natura cardioembolica sembrano essere più frequenti in pazienti di sesso maschile di età superiore a 50 anni con reperti auscultatori di un murmure sistolico e di lembi mitralici ispessiti all'ecocardiogramma transtoracico. Fenomeni tromboembolici complicanti l'endocardite infettiva ${ }^{11}$ possono essere sistemici (endocardite del cuore sinistro) o polmonari (endocardite del cuore destro). Le vegetazioni sono in genere documentate dall'ecocardiogramma transtoracico nella maggior parte dei pazienti con endocardite e sono correlate ad un maggior rischio cardioembolico. Emboli sistemici possono verificarsi nel $50 \%$ dei pazienti affetti da endocardite trombotica non batterica, una condizione caratterizzata dalla presenza di multiple vegetazioni sterili con coinvolgimento della valvola mitrale o aortica. Il rischio di tromboembolismo è più elevato nei portatori di protesi valvolari meccaniche ${ }^{12}$ rispetto a quelle biologiche in particolare nella posizione mitralica rispetto a quella aortica. Il tasso di embolismo sistemico nei portatori di valvole meccaniche che ricevono terapia anticoagulante è del $4 \%$ annuo nella posizione mitralica e del $2 \%$ annuo nella posizione aortica. Il riscontro occasionale all'ecocardiogramma di banderelle filamentose adese alla valvola mitrale in giovani pazienti sembra essere un fattore di rischio indipendente per embolismo sistemico. La fibrillazione atriale non valvolare ${ }^{13}$ (Figura 2), l'aritmia cardiaca più frequentemente documentata nei pazienti ospedalizzati in Italia, ha un tasso di incidenza di tromboembolismo del $4-7.5 \%$ annuo rappresentando la principale fonte di cardioembolismo nella popolazione anziana. I pazienti con fibrillazione atriale hanno un rischio di ictus ischemico di 5-6 volte superiore alla po-

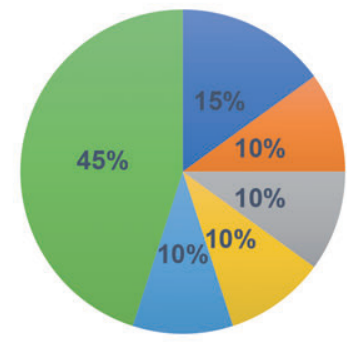
- Infarto miocardico acuto Aneurisma ventricolare
- Malattia reumatica cardiaca = Infarto miocardico acuto
- Aneurisma ventricolare Malattia reumatica cardiaca

Figura 1. Etiologia cardioembolismo. 
polazione generale con un richio cumulativo del $35 \%$ nel corso di tutta la vita. I pazienti con fibrillazione atriale valvolare (reumatica) ${ }^{14}$ hanno rischio relativo di ictus ischemico cardioembolico di 17 volte superiore alla popolazione generale. I pazienti con meno di 65 anni e con sola fibrillazione atriale, tuttavia, hanno un basso rischio cardioembolico soprattutto se non associato ad ipertensione arteriosa, insufficienza cardiaca congestizia, diabete mellito di tipo II ed una precedente storia di TIA o ictus. La mortalità nei pazienti con fibrillazione atriale è più elevata con un incremento della prevalenza di fibrillazione atriale che si verifica con l'età, da uno 0.2 casi per 1000 abitanti tra i 30-39 anni a 39 casi per 1000 abitanti tra gli 80-89 anni con un proporzionale trend di crescita di ictus ischemici cardioembolici provocati dalla fibrillazione atriale da un $6.7 \%$ di tutti gli stroke tra i 50-59 anni al 36.2\% di tutti gli stroke tra gli 80-89 anni. Analogo l'incremento di rischio tra i pazienti con fibrillazione atriale ed ipertiroidismo. Un quadro di embolismo sistemico e/o cerebrale può verificarsi nel corso una sindrome del seno malato (sick sinus syndrome). ${ }^{15}$ Tra costoro, i pazienti a maggior rischio di embolizzazione presentano in genere un quadro di braditachiaritmia. Un ecocontrasto spontaneo come reperto ecocardiografico accessorio (a livello dell'atrio sinistro) ed una ridotta eiezione atriale incrementano ulteriormente il rischio embolico. Tali pazienti possono sperimentare un'ischemia cerebrale o embolismo sistemico anche dopo l'inserzione di un pacemaker. I mixomi atriali ${ }^{16}$ sono tumori cardiaci rari complicati da sincope posturale e manifestazioni emboliche sistemiche. I mixomi possono provocare un ictus attraverso diversi meccanismi ovvero attraverso l'embolizzazione di materiale mixomatoso o trombotico. Le manifestazioni emboliche possono rappresentare il primo sintomo in un terzo dei pazienti affetti da mixoma atriale. In alternativa aneurismi periferici e cerebrali multipli sono stati descritti in seguito alle iniziali manifestazioni emboliche del mixoma. Il trattamento del mixoma consiste nella rapida exeresi chirurgica della massa. I rabdomiomi sono invece associati alla sclerosi tuberosa con un embolismo siste-

\section{Fibrillazione atriale non valvolare (parossistica, cronica, permanente)}

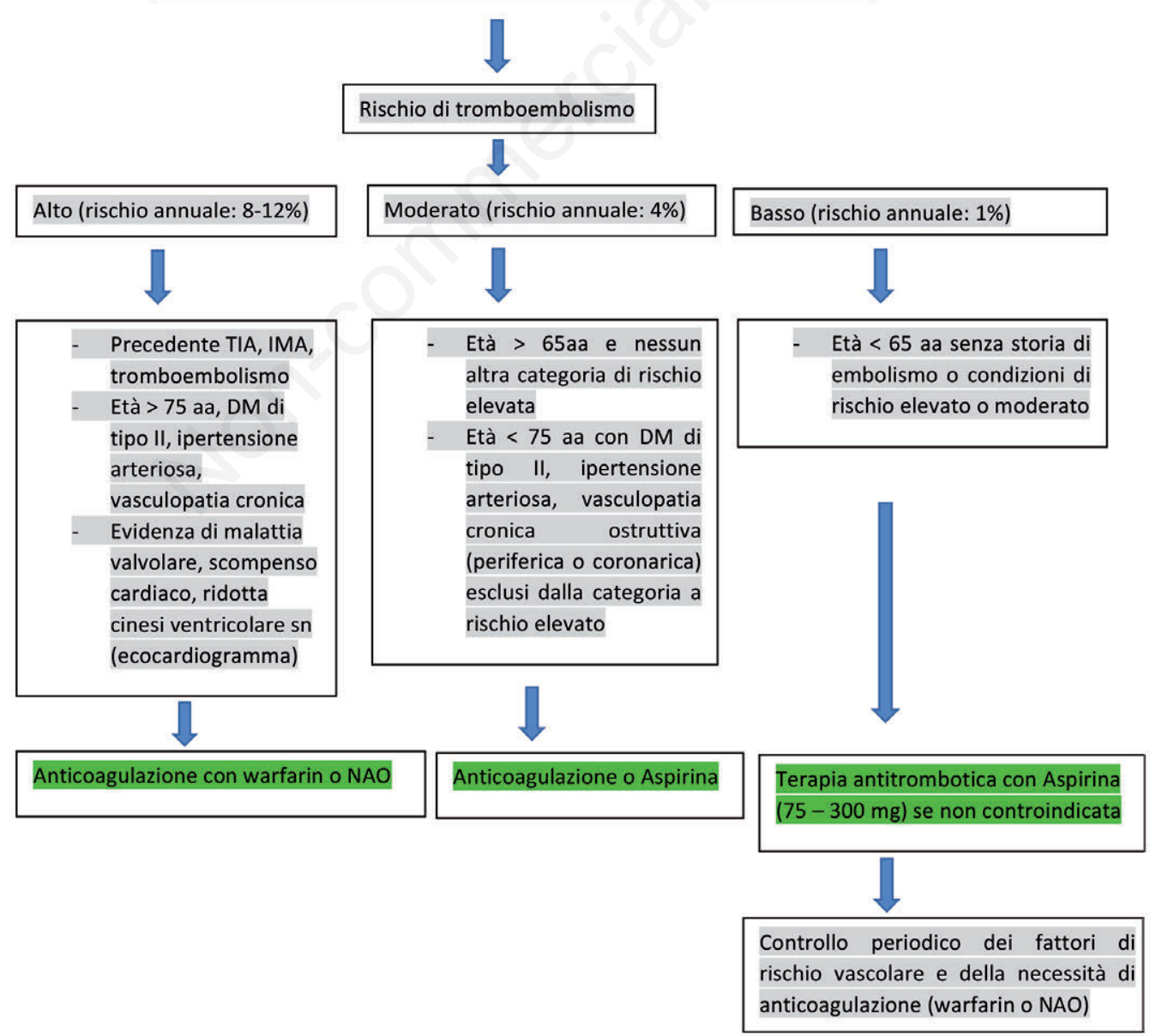

Figura 2. Fibrillazione atriale non valvolare e rischio emboligeno. 
mico piuttosto raro. Il fibroelastoma papillare della mitrale infine è raramente associato a complicanze emboliche cerebrali, causa di stroke cardioembolico.

\section{Forame ovale pervio}

Un embolismo paradosso causato da uno shunt destra-sinistra associato a forame ovale pervio o aneurisma del setto interatriale ${ }^{17}$ può essere responsabile di ictus. Un forame ovale pervio crea le circostanze per uno shunt destra-sinistra nelle condizioni di incremento della pressione atriale destra. Si tratta di una variante anatomica presente nel $35 \%$ delle popolazione normale tra i 0-29 anni, nel 25\% tra i 30-79 anni, nel 20\% tra gli 80-99 anni. Il forame ovale pervio è più comunemente documentato nei pazienti con ictus ischemico rispetto alla popolazione generale. Sono stati pertanto raccomandati come strategie terapeutiche: terapia antiaggregante, anticoagulante, chiusura transcateterale con patch del forame ovale pervio. Nonostante ciò il reale beneficio della chiusura con patch rispetto alla terapia medica di fatto non è stata ancora ben definita. Tra l'altro le complicanze cerebrali della chiusura percutanea del forame ovale pervio possono essere embolismo gassoso, tromboembolismo, fibrillazione atriale acuta, endocardite infettiva ed infine ictus cardioembolico tardivo legato alla trombizzazione intorno al device. I trials preliminari come vedremo, non hanno trovato differenze significative nel tempo fino al primo endpoint tra aspirina e warfarin (INR medio di 2,04) e soprattutto un vantaggio del patching del PFO sulla terapia medica. La presenza di un aneurisma del setto (ASA) tra l'altro associato al forame ovale pervio può inoltre un fattore di rischio indipendente per l'embolismo. Solo uno studio ha riportato che la coesistenza di ASA e forame ovale pervio incrementano il rischio di ictus cardioembolico. In sostanza per l'ictus criptogenetico associato al forame ovale pervio in assenza di altre indicazioni alla terapia antiaggregante, la terapia con acido acetisalicilico 100-325 mg cp è raccomandata dalle attuali linee guida. I risultati relativi allo studio CLOSURE $\mathrm{I}^{18} \mathrm{e}$ RESPECT pubblicati tra il 2012-2013 non sembravano dimostrare, come già sostenuto sopra, una differenza statisticamente significativa tra chiusura percutanea del forame ovale pervio e la terapia medica antitrombotica (antiaggregazione singola e/o doppia antiaggregazione) con durata di follow up oltre i 4 anni. Inoltre dagli stessi trials sarebbe emerso un maggior rischio nel gruppo sottoposto a chiusura del forame ovale pervio con device Amplatzer di fibrillazione atriale di nuova insorgenza. Questi dati, dopo il 2017 sono stati contraddetti dal completamento del follow up dello studio RESPECT (5,9 anni vs 2,6 anni dalla precedente pubblicazione). Nel trial RESPECT $^{19}$ infatti (pazienti affetti da ictus e forame ovale pervio) che ha confrontato un gruppo sottoposto a chiusura del forame ovale pervio + ASA/clopidogrel per un mese, ASA per 5 mesi, poi terapia antitrombotica a discrezione degli investigatori con un gruppo a terapia medica (ASA o clopidogrel/dipiridamolo e $\mathrm{TAO}$ ) ha dimostrato un tasso di recidiva più basso nel gruppo sottoposto a chiusura rispetto a quello con sola terapia medica ed un'incidenza di fibrillazione atriale sovrapponibile. Nello studio CLOSE sono stati selezionati pazienti con grandi shunts ed aneurisma del setto associato, con un analogo disegno dei bracci di trattamento, con i medesimi risultati ma con una più netta efficacia nel gruppo sottoposto a chiusura del forame ovale pervio ed altresì una maggiore incidenza in questo ultimo di fibrillazione atriale di nuova insorgenza. Risultati sovrapponibili allo studio CLOSE riguardano lo studio REDUCE. Adesso per spiegare questa inversione di tendenza ci sono diversi BIAS da considerare: i) nei primi studi c'era un elevato tasso di drop-out (bias di selezione); ii) l'arruolamento dei pazienti ad una certa distanza dall'evento ha indice ha escluso quegli stessi pazienti a rischio di una recidiva precoce; iii) la durata del follow-up mediamente più lunga nel RESPECT ha comportato che verosimilmente la terapia medica ha un vantaggio che si perde nel lungo periodo; iv) Nello studio CLOSE la caratteristiche anatomiche del PFO (shunts di grandi dimensioni ed aneurisma del setto interatriale) hanno favorito il braccio sottoposto a chiusura in quanto questo presentava una maggiore indicazione alla chiusura; v) infine la doppia antiaggregazione precoce (nel primo mese) potrebbe avere protetto maggiormente il gruppo sottoposto a chiusura rispetto alla terapia medica standard (singola antiaggegazione). In conclusione si può dire che la pur esigua riduzione del rischio di recidiva ischemica ottenuta con la chiusura del PFO determina una beneficio clinico rilevante in quanto si tratta di soggetti più giovani. La FDA ha approvato la procedura ma la selezione dei pazienti deve essere collegiale (neurologi/cardiologi).

\section{Cause rare di cardioembolismo e procedure chirurgiche}

Le malformazioni arterovenose possono talvolta essere un fonte di embolismo paradosso alla base di una ischemia cerebrale e si verificano nel $15-20 \%$ dei pazienti con malattia di Rendu Osler. Il contrasto ecocardiografico spontaneo è in genere associato con elevati livelli di fibrinogeno e di viscosità plasmatica ed è un fattore di rischio potenziale per lo stroke cardioembolico. Il rischio per eventi cerbrovascolari è incrementato in pazienti con malattie cardiache congenite cianogene ${ }^{20}$ in presenza di ipertensione arteriosa, fibrillazione atriale, storia di flebotomia ed in particolare in pazienti con microcitosi. La prevalenza 
di ictus ischemico del circolo posteriore in seguito a cateterizzazione cardiaca non ha spiegazioni. In corso di grafting da bypass aortocoronarico, l'incidenza di ictus ischemico è compresa tra il 1-5\%. I due terzi degli ictus si verificano nel secondo giorno del post operatorio e coinvolgono in maniera predominante gli emisferi cerebrali mentre gli infarti lacunari e del tronco sono in genere più rari. La causa dell'ictus in post operatorio è multifattoriale; ipoperfusione, trombi ventricolari ed emboli sono i probabili fattori causali. Il clampaggio stesso durante le manovre dell'intervento di bypass può favorire il distacco di trombi aterosclerotici dalle placche ateromatose. L'ecografia epiaortica ha evidenziato un dato scontato di un incremento del rischio di ictus ischemico nei pazienti con aterosclerosi più severa. Contrariamente a questo dato, una occlusione della carotide ma non una stenosi carotidea si associano ad aumentato rischio di ictus in seguito a bypass aortocoronarico. Fenomeni cardioembolici possono complicare interventi cardiochirurgici utilizzando il bypass cardiopolmonare con ipotermia spinta ed arresto cardiaco. L'ictus ischemico è una potenziale complicanza della cardioversione per la fibrillazione atriale. L'embolismo cerebrale può complicare inoltre gli interventi di valvulolpastica; il rischio è in genere più elevato per la valvuloplastica aortica piuttosto che per quella mitralica. L'ictus può ancora, complicare il trapianto cardiaco, l'utilizzo dei sistemi di supporto ventricolare, i cuori artificiali ed infine dell'utilizzo dell'ossigenatore extracorporeo membranaceo. La mielopatia è una rara complicanza del posizionamento del palloncino a pompa intraortico. Una dissecazione o un ematoma può portare ad un'occlusione di un ramo radicolare o dell'occlusione locale dell'arteria di Adamkiewicz.

\section{Ictus ischemico aterotrombotico}

Numerosi fattori predisponenti all'aterosclerosi sono stati identificati come possibili fattori per l'ictus ischemico aterotrombotico (Tabella 2).

L'aterosclerosi di fatto è una patologia multifattoriale con comorbidità che spesso si sovrappongono fra di loro ed in tal modo i fattori di rischio esercitano tra di loro un effetto sinergico.

\section{Ictus ischemico aterotrombotico e profilo di rischio}

Per esempio l'ipertensione arteriosa è spesso associata alla dislipidemia mista, iperglicemia, elevati livelli di fibrinogeno, obesità ed ipertrofia ventricolare sinistra all'ECG. Un rapporto a riposo fianchi - caviglie inferiore di 0,90 è generalmente indicativo di ateroscle- rosi diffusa. Allo stesso modo pazienti che sono stati colpiti da un ictus sono ad elevato rischio di sviluppare altre complicanze vascolari. Dopo un ictus infatti, la probabilità di sviluppare un evento tromboembolico fatale è di circa il $25 \%$ in 3 anni, molti dei quali in relazione ad un'infarto del miocardio.

\section{Ictus aterotrombotico: fisiopatogenesi}

L'ictus aterotrombotico riconosce come eventi fisiopatogenetici un'embolizzazione artery-to-artery da lesioni aterosclerotiche complicate (placche ulcerate ed instabili) con conseguente occlusione di vasi intracranici ovvero lesioni stenotiche aterosclerotiche e aterotrombotiche a livello dei vasi cervicali extracranici e a livello delle grosse arterie intracraniche, con conseguente ipoperfusione distale alla lesione. Tale trombosi in situ con coinvolgimento del tratto prossimale della carotide interna, della vertebrale distale ovvero il tratto prossimale o medio dell'a. vertebrale resta sempre alla base di tali fenomeni ipoperfusivi. Altresì un coinvolgimento aterosclerotico della porzione intracranica del sistema vertebrobasilare frequentemente si verifica invece in tandem ed è un comune meccanismo alla base della sindrome da insufficienza del territorio vertebrobasilare. Un'ipoperfusione secondaria ad alterazioni emodinamiche può innescare tali eventi.

\section{Ictus aterotrombotico ed occlusioni in tandem}

Le lesioni in tandem ${ }^{21}$ nell'ictus ischemico acuto (intra ed extracraniche) sono causa di circa il 10-20\% degli ictus aterotrombotici e sono correlate ad una prognosi terapeutica sfavorevole e con un tasso di ricanalizzazione del 30\%, dopo trombolisi. I casi pubblicati sono per la maggior parte relativi alla presenza per esem-

Tabella 2. Fattori di rischio aterotrombotico.

\begin{tabular}{ll}
\hline Non modificabili & Modificabili \\
\hline Età & Ipertensione arteriosa \\
\hline Sesso & TIA \\
\hline Etnia & Pregresso stroke \\
\hline Familiarità & Stenosi carotidea asintomatica \\
\hline Genetica & Malattia cardiaca \\
& Ateromatosi dell'arco aortico \\
& Diabete mellito di tipo II \\
& Fumo di sigaretta \\
& Consumo alcolico \\
& Attività fisica \\
& Iperomocisteinemia \\
& Bassi livelli di folati \\
& Contraccettivi orali \\
& Obesità \\
\hline
\end{tabular}


pio nel 25\% dei casi delle occlusioni dell'ACM, un'occlusione di ACI omolaterale ed allo stesso tempo e nel $50 \%$ delle occlusioni di ACI un'occlusione dell'ACM distalmente. Pazienti con lesioni in tandem possono beneficiare della trombectomia entro $6 \mathrm{~h}$ dall'inizio dei sintomi con un tasso di ricanalizzazione tra il 60-80\%. Gli approcci possibili sono anterogrado, caratterizzato da rivascolarizzazione dell'ACI e successivamente trombectomia meccanica della carotide ovvero inverso, con angioplastica a palloncino per accedere prima alla circolazione anteriore per eseguire la trombectomia ed ottenere la rivascolarizzazione intracranica, seguita poi da una completa angioplastica della carotide. Talora la rivascolarizzazione prossimale potrebbe portare a ricanalizzazione spontanea dell'occlusione intracranica. ${ }^{22}$ In genere con la rivascolarizzazione prossimale dell'occlusione in associazione con la trombectomia, il tasso di ricanalizzazione è del $80 \%$ con mortalità del $13 \%$ ed emorragia intraparenchimale sintomatica del 4\% (circolo anteriore con stent retriever thrombectomy, 'SRT'). L'unico aspetto più rilevante è che nelle tandem occlusions con occlusione extracranica dell'ACI, una necessità di stenting urgente dell'ACI potrebbe aumentare il rischio di emorragia intracranica sintomatica legata alla doppia antiaggregazione fino al 7\%. A questo punto la questione del timing diventa fondamentale. Associando al trattamento endovascolare delle occlusioni in tandem, anche un trattamento trombolitico venoso, l'outcome (in termini di ricanalizzazione precoce) migliora fino all' $80 \%$ vs il $10 \%$ del solo approccio endovascolare. Lo stenting carotideo in acuto per le tandem occlusion non è invece supportato da evidenze scientifiche. Al contrario, un approccio conservativo (clinical evidence based) prevede una dilatazione con palloncino della stenosi dell'ACI con trombectomia distale in acuto e solo successivamente alla stabilizzazione del quadro clinico e della lesione ischemica lo stenting di carotide e/o endoarterectomia. Evidenze scientifiche invece supportano il trattamento in acuto con stenting in emergenza di un'ACI extracranica occlusa. La patogenesi dell'ictus da stenosi intracranica è simile a quello legato a patologia arteriosa extracranica. ${ }^{23}$

\section{Ictus aterotrombotico: aterosclerosi complicata}

Nei pazienti che presentano fattori di rischio aterotrombotico, una stria lipidica endoteliale ricca in colesterolo può facilmente progredire in una placca fibrosa e quindi eventualmente in una placca complicata da un'emorragia intraplacca, necrosi, calcificazione e successiva trombosi. L'infiltrazione per esempio della placca fibrosa da parte di cellule infiammatorie può contribuire alla rottura della placca aterosclerotica carotidea. L'aterosclerosi è spesso segmentale e le lesioni più precoci tendono a localizzarsi nelle regioni di elevato stress emodinamico laminare, in particolare a livello del bulbo carotideo (Figura 3). Il processo aterosclerotico in genere interessa primariamente le arterie extracraniche ed intracraniche di diametro maggiore, come la biforcazione della carotide comune ed il tratto prossimale della carotide interna, il sifone carotideo, la triforcazione dell'ACM, l'origine delle arterie vertebrali (V1), il segmento intracranico delle arterie vertebrali (V4) e dell'arteria basilare. La distribuzione dell'aterosclerosi cerebrale può essere differente in taluni gruppi etnici. Per esempio la stenosi delle maggiori arterie intracraniche è in genere prevalente tra i sottogruppi etnici degli afroamericani, indiani ed popolazioni estremorientali.

\section{Neuroimmagini nell'ictus cardiembolico ed aterotrombotico}

Le neuroimmagini hanno un ruolo critico nella diagnosi precoce dell'ictus. Inoltre i pattern ischemici documentati indirizzano verso il corretto inquadramento fisiopatogenetico dell'ictus ed influenzano le scelte terapeutiche sia in termini di trattamento immediato che di prevenzione seconadaria.
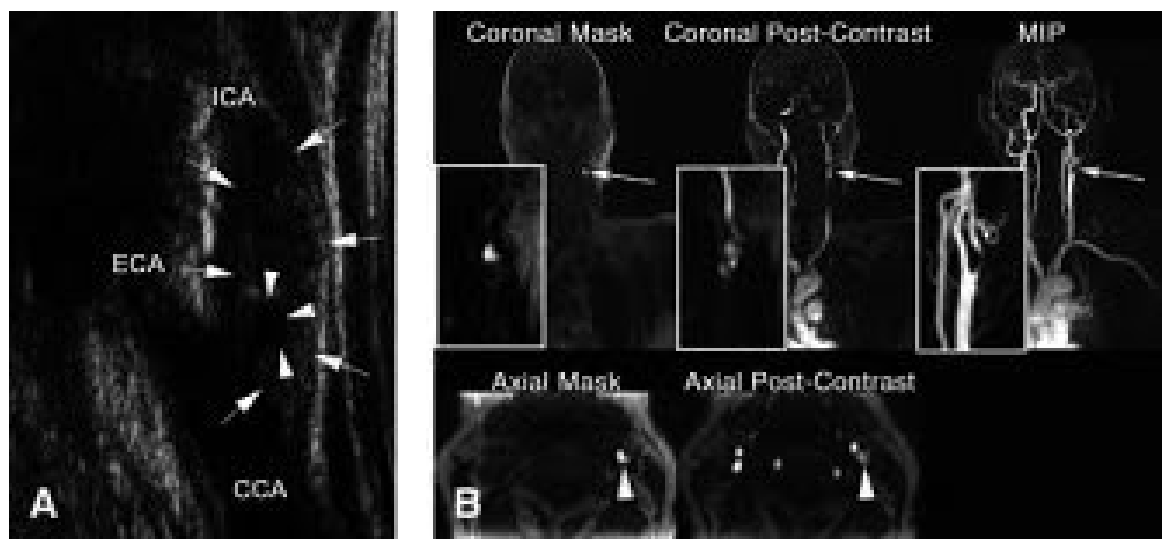

Figura 3. Angiografica di placca carotidea 'soft' complicata. 


\section{Neuroimmagini ed ictus aterotrombotico}

Lesioni ischemiche più estese di $15-20 \mathrm{~mm}$ che coinvolgono la corteccia, il cervelletto, il tronco enecefalo e le regioni sottocorticali, sono generalmente causate da aterosclerosi dei grossi vasi (epiaortici e/o intracranici) comprendendo la maggiore causa di ictus ischemico (30-43\%). La valutazione dell'albero vascolare extra ed intracranico è essenziale quando viene sospettata l'aterosclerosi come causa dell'ictus ischemico acuto. Il meccanismo dell'infarto secondario ad un fenomeno di aterosclerosi delle grosse arterie extracraniche è una combinazione di rallentamenti di flusso e di propagazione di emboli con una migrazione di tipo artery to artery.

\section{Neuroimmagini e circolo anteriore}

Lesioni ischemiche del circolo anteriore legate ad aterosclerosi della carotide più comunemente coinvolgono l'ACM risultando in un infarto territoriale che coinvolge la sostanza grigia profonda e la corteccia. Tali infarti generalmente coinvolgono la porzione centrale dell'encefalo, incluso il lobo parietale anteriore, la parte posteriore del lobo frontale e la parte superiore del lobo temporale. Il territorio dell'arteria cerebrale anteriore viene generalmente risparmiato in relazione ad un circolo di Willis intatto. La malattia vascolare intracranica prossimale è spesso anch'essa legata ad un processo di aterosclerosi dei grossi vasi che causa eventi ischemici secondo diversi meccanismi fisopatogenetici qualiembolismo artery-to-artery, tromboembolismo in situ ed infine la compromissione emodinamica.

\section{Neuroimmagini e circolo posteriore}

L'aterosclerosi dei grossi vasi che coinvolge il sistema vertebrobasilare può inoltre produrre un particolare pattern di neuroimmagini. Per esempio, all'inizio dell'occlusione della porzione prossimale o media della basilare, estese lesioni ischemiche sono in genere documentabili a livello del ponte così come a livello cerebellare, della porzione inferomediale dei lobi temporali, lobi occipitali e porzione posteriore dei talami. Le occlusioni distali della basilare coinvolgono il mesencefalo e i talami ed in questo caso si tratta di uno dei pochi esempi in cui infarti bilaterali possono essere documentati nel contesto di un'aterosclerosi dei grossi vasi. Un infarto della porzione laterale del bulbo dovrebbe spingere alla valutazione del flusso nella porzione distale dell'a.vertebrale omolaterale dal momento che può essere legato all'occlusione dell'arteria cerebellare posteriore inferiore (PICA), risultando clinicamente in una sindrome laterale del bulbo (s. di Wallenberg).

\section{Neuroimmagini ed ictus nei territori di confine}

Gli infarti delle zone di confine esterne, definite $u l$ timi prati sono generalmente localizzati in territori vascolari di confine tra l'arteria cerebrale anteriore, media e posteriore e sono in genere legati ad una combinazione di meccanismi di embolismo ed ipoperfusione. Quest'ultima potrebbe di fatto risultare da una compromissione emodinamica nel contesto di una stenosi vascolare oppure legata al fatto che queste regioni cerebrali sono già di base ipoperfuse. Tale condizione può compromettere la ricanalizzazione in caso di fenomeno embolico distale in un territorio di confine. $\mathrm{Al}$ contrario, gli infarti profondi nei territori di confine sono più frequentemente causati da stenosi prossimali o compromissione emodinamica dal momento che $\mathrm{i}$ vasi lenticolostriati che suppliscono in genere queste regioni hanno la più bassa pressione di perfusione. Infarti dei territori di confine a livello del circolo posteriore sono meno comuni e quando sono unilaterali sono più frequentemente causati da una fonte embolica a partenza dal circolo anteriore nel contesto di una origine fetale dell'arteria cerebrale posteriore (Figura 4).

\section{Neuroimmagini e sequenze oltre le diffusion weighted images}

In presenza di tali pattern neuroradiologici occorre studiare l'albero vascolare al fine di documentare patologia aterosclerotica a carico dei grossi vasi come principale causa dell'ictus in atto. Tale valutazione neuroradiologia può essere effettuata con angio TC (CTA), angio RM dei vasi intracranici (MRA) o angiografia a sottrazione digitale (DSA). Inoltre, il ricorso a sequenze che vadano oltre le sequenze diffusion weighted imaging (DWI) può portare ulteriore luce sull'inquadramento etiopatogenetico dell'ictus. Per esempio, una iperintensità alle sequenze T2

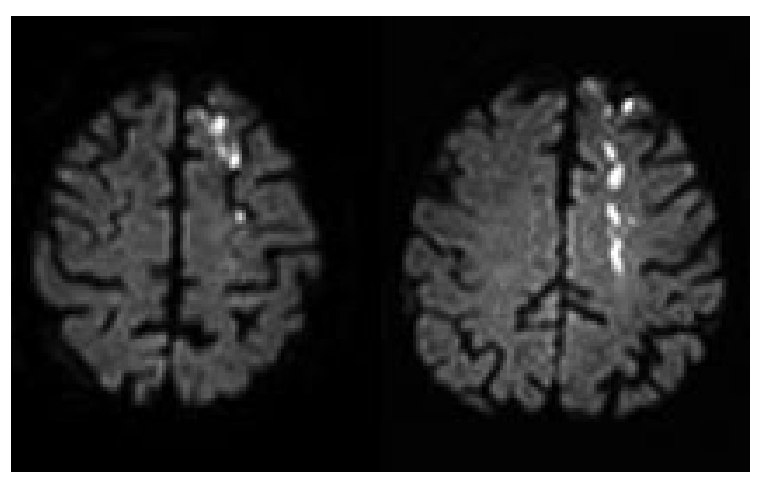

Figura 4. Neuroimmagini di un ischemia degli ultimi prati. 
fluid attenuated inversion recovery (FLAIR) relativamente ai vasi intracranici può suggerire un rallentamento di flusso provocato da una malattia vascolare prossimale. Una lesione ipointensa di tipo serpiginoso vista nelle sequenze gradient possono indicare un processo tromboembolico in un vaso distale.

\section{Neuroimmagini ed ictus cardioembolico}

Le fonti cardioemboliche sono responsabili del 20$30 \%$ degli ictus acuti. Tali lesioni ischemiche si presentano generalmente alle immagini di RM come aree multiple ristrette in DWI o in TAC (Figura 5) che coinvolgono diversi distretti vascolari e sono generalmente bilaterali. Quando viene identificato un pattern con tali caratteristiche diventa critica un'accurata valutazione del cuore, tenendo conto di tutte le fonti emboligene discusse precedentemente. Allo stesso tempo quando una lesione isolata nel territorio dell'a. cerebrale anteriore viene documentata alle neuroimmagini, una fonte cardioembolica dovrebbe essere considerata per quanto venga dibattuta la possibilità di un processo aterosclerotico che coinvolge l'arteria cerebrale anteriore in sé (frequente nella popolazione asiatica, per esempio). Gli infarti isolati dell'a. cerebrale anteriore sono in genere meno comuni degli ictus dell'arteria cerebrale media. Se ad essere coinvolti invece sono entrambi i territori dell'arteria cerebrale anteriore e dell'arteria cerebrale media, la causa è spesso cardioembolica.

\section{Diagnosi differenziale precoce tra ictus ischemico cardioembolico ed aterotrombotico}

Una precoce diagnosi differenziale (Figura 6) tra ictus cardioembolico ed aterotrombotico è necessaria ai fini di un corretto approccio terapeutico dell'ictus. Purtroppo dati clinici che ci permettano di differenziare gli ictus embolici da quelli aterotrombotici mancano. In genere viene acquisito dalla letteratura scientifica che la presenza di fibrillazione atriale e la comparsa improvvisa di un deficit neurologico sono fattori di rischio indipendenti significativamente associati ad ictus ischemico cardioembolico, laddove in-

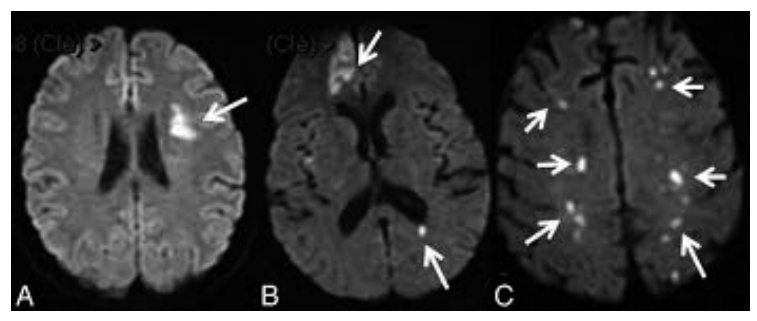

Figura 5. Neuroimagini di un ictus ischemico cardioembolico biemisferico. vece ipertensione arteriosa, $\mathrm{BPCO}$, diabete mellito di tipo II, dislipidemia e l'età avanzata sono variabili cliniche indipendenti, associate con l'ictus aterotrombotico. Tale schema però rappresenta una estrema semplificazione del problema diagnostico differenziale. Ai fini pratici potrebbe essere utile un Cardioembolic score a scopo decisionale terapeutico. In una serie di pazienti $(164$, ndr $),{ }^{24}$ con un gruppo di FA cronica in $101(61,6 \%)$ e 63 parossistica $(38,4 \%)$ sono stati considerati i fattori direttamente ed indirettamente correlati alla FA (età superiore a 75 aa, sesso femminile, dilatazione atriale sinistra all'ecocardiografia, lesioni cortico sottocorticale in un singolo territorio o in più di un territorio vascolare e la trasformazione emorragica della lesione) o indirettamente correlati alla FA (dislipidemia, stenosi carotidea non emodinamica ovvero compresa tra il 50-100\%, stenosi intracranica non significativa). A questo punto l'inserimento in una equazione di regressione che tenga conto di tali variabili si può considerare:

$\mathrm{L}$ odds $=\beta$ età $x \chi+\beta$ sesso $+\beta$ dilatazione atriale sinistra $+\beta$ territori vascolari multipli $+\beta$ lesioni cortico sottocorticali $+\beta$ trasformazione emorragica + Bstenosi intracranica o occlusione $x \chi_{\mathrm{L}} 7$ costante dell'equazione.

La $P$ può essere calcolata come segue:

$P=$ odds $/(1+o d d s)=e^{\eta^{\eta} \text { odds }} /\left(1+e^{l^{\eta} \text { odd }}\right)$

Da questa equazione ${ }^{25}$ di regressione risulta evidente quanto variabili rilevanti quali età, sesso, localizzazione cortico sottocorticale delle lesioni ischemiche (coinvolgimento del circolo terminale), tendenza alla trasformazione emorragica (da ricanalizzazione per lisi dell'embolo) e grado di severità di una stenosi intracranica siano indipendentemente as-

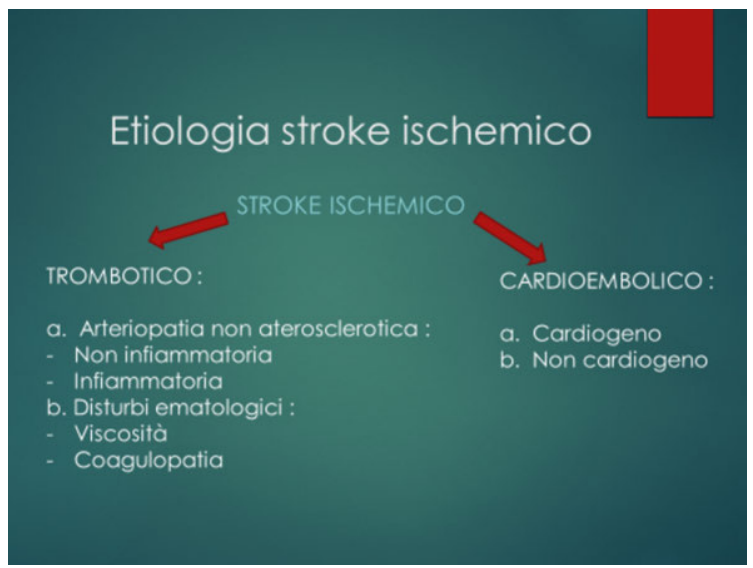

Figura 6. Work up diagnostico differenziale ictus ischemico aterotrombotico vs cardioembolico. 
sociate al rischio relativo di una fisiopatogenesi cardioembolica.

Per esempio, una paziente con una dilatazione atriale sinistra, trasformazione emorragica della lesione ischemica, sesso femminile, età superiore a 75 anni ha una probabilità secondo questo modello del $92 \%$ di avere un ictus cardioembolico legato alla fibrillazione atriale. Questa formula oltre che riconoscere un profilo cardioembolico permette di stimare il rischio individuale di ictus cardioembolico legato alla FA e quindi di individuare eventualmente pazienti ad rischio di una FAP, altrimenti classificati come da causa indeterminata, candidati ad un monitoraggio Holter prolungato.

\section{Fibrillazione atriale nell'ictus cardioembolico ed aterotrombotico}

Per quanto la FA rappresenti il principale disturbo del ritmo cardiaco nella diagnosi dell'ictus cardioembolico nei paesi industrializzati, essa può essere osservata nell'ictus aterotrombotico non in quanto fonte embolica ma piuttosto come marker di altre condizioni che hanno condotto all'ictus quale l'aterosclerosi. In tal caso rappresenta un epifenomeno e/o manifestazione clinica di malattia aterosclerotica. Da questo punto di vista non tutti gli ictus ischemici nei pazienti fibrillanti hanno un'origine cardioembolica. ${ }^{26}$ In una cospicua serie di pazienti, la FA è stata diagnosticata nel $16.5 \%$ dei pazienti con occlusione trombotica e/o stenosi arteriosa $>70 \%$, presumibilmente responsabile dell'ictus ischemico in sé. In tali casi per esempio, alcuni reperti ecocardiografici legati al cardioembolismo come l'insufficienza cardiaca oppure l'incremento del volume dell'atrio sn o infine la disfunzione del ventricolo sinistro erano assenti. Questo dato è in contrasto con una serie di Bogousslavsky che ha dimostrato che nel $76 \%$ dei pazienti con ictus ischemico nel territorio carotideo affetti da FA, il meccanismo fisiopatologico presunto fosse il cardioembolismo dal momento che non era possibile documentare una patologia arteriosa significativa. ${ }^{27}$ Solo 1'11\% era aterosclerotico dal momento che era stato possibile documentare una stenosi arteriosa o occlusione completa ed il restante $13 \%$, l'ictus ischemico era spiegabile con un meccanismo di occlusione dei rami perforanti dell'ACM (lacunare) in associazione con l'ipertensione. In conclusione La diagnosi differenziale tra ictus cardioembolico ed aterotrombotico può essere difficile da stabilire nei pazienti con FA all'esordio dei sintomi neurologici. Nell'esperienza tratta da una serie di 2000 pazienti con malattia cerebrovascolare cronica, $1712(85.6 \%)$ ha presentato un ictus ischemico nelle 48 ore precedenti. Un totale di $347(17,4 \%)$ sono stati classificati come ictus cardioembolici, 452 $(22,6 \%)$ come aterotrombotici. ${ }^{28}$ Pazienti con ictus cardioembolico ed FA hanno rappresentato il 76,6\% $(=226)$ mentre i pazienti con ictus aterotrombotico ed FA il $16,5 \%(=75)$.

\section{Ictus cardioembolico con e senza fibrillazione atriale}

Quando sono stati confrontati pazienti con ictus cardioembolico con e senza FA, ${ }^{29}$ il sesso femminile, la storia di insufficienza cardiaca e l'improvvisa comparsa di segni neurologici, alterazione della coscienza, deficit motori, sensitivi e visivi oltre che la localizzazione corticale (parietale, ndr) sono stati più frequentemente registrati nei pazienti con ictus cardioembolico con FA..$^{30}$ Malattia ischemica coronarica, fumo di sigaretta, e topografia capsulare sono stati invece più frequenti nei pazienti con ictus cardioembolico senza fibrillazione atriale. La mortalità intra ospedaliera è stata del $31,6 \%$ nei pazienti con FA e del $14,8 \%$ in quelli senza FA. ${ }^{31}$

\section{Ictus aterotrombotico con e senza fibrillazione atriale}

Nel confronto dei pazienti con ictus aterotrombotico con e senza fibrillazione atriale, quelli con FA erano più vecchi, con predominanza del sesso femminile, con maggiore prevalenza di malattia coronarica e valvolare, esordio improvviso dei deficit neurologici, disturbi sensitivi, motori, visivi, topografia parietale, temporale, occipitale ed ischemia nel territorio dell'ACM. ${ }^{32}$ Negli ictus aterotrombotici senza fibrillazione atriale, il fumo di sigaretta, coinvolgimento dei nervi cranici ed una topografia del territorio vascolare vertebrale sono stati molto più frequentemente documentati. Un assenza di compromissione funzionale neurologica alla dimissione è stata inoltre molto più frequente. La mortalità intra ospedaliera è stata del $29,3 \%$ nei pazienti con fibrillazione atriale e del $18,8 \%$ in quelli senza FA.

\section{Ictus cardioembolici ed ictus aterotrombotici con fibrillazione atriale}

Quando i predittori dell'ictus cardioembolico o aterotrombotico con fibrillazione atriale sono stati valutati in una analisi multivariata, la malattia valvolare reumatica $(\mathrm{OR}: 4,6)$ e la comparsa improvvisa di sintomi (OR:1,8) sono stati associati in maniera indipendente con l'ictus cardioembolico laddove invece l'esordio subacuto della sintomatologia ictale (OR:8,01), BPCO (OR:5,2), ipertensione arteriosa (OR:2,26) sono stati indipendentemente associati all'ictus aterotrombotico. ${ }^{33}$ Andrebbe notato che la fi- 
brillazione atriale presenta un impatto negativo sull'outcome sia negli ictus cardioembolici che aterotrombotici. È stato ipotizzato che l'outcome peggiore associato alla FA sia legato ad una maggiore prevalenza di insufficienza cardiaca e di cardiopatia ischemica cronica. Tale ipotesi coincide in parte con i risultati, dal momento che una maggiore incidenza di insufficienza cardiaca nei pazienti con ictus cardioembolico ed una maggiore incidenza di cardiopatia ischemica cronica nei pazienti con ictus aterotrombotico è stata osservata. Ciò potrebbe contribuire ad una riduzione del flusso ematico cerebrale ed una compromissione dei meccanismi di autoregolazione che nell'area ischemica sono già alterati. Altri autori suggeriscono che la fibrillazione atriale cronica possa provocare una significativa riduzione del flusso ematico regionale che può normalizzarsi altresì dopo conversione a ritmo sinusale. ${ }^{34}$ Altri ancora ritengono che l'incremento della mortalità possa essere spiegato dall'età più avanzata dei pazienti, un maggiore volume della lesione oppure dalla maggiore gravità del deficit neurologico iniziale nei pazienti con fibrillazione atriale. In sintesi, la malattia cerebrovascolare nell'ictus ischemico cardioembolico o in quello aterotrombotico è più severa in presenza di fibrillazione atriale rispetto ai pazienti in ritmo sinusale.

\section{Conclusioni}

Dalla disamina accurata dei fattori di rischio associati all'ictus aterotrombotico e cardioembolico risulta evidente quanto la semplice associazione di una sindrome neurologica acuta legata ad un'ischemia cerebrale in un paziente eventualmente affetto da ipertensione arteriosa, DM di tipo II, dislipidemia mista ed aterosclerosi plurididstrettuale non è di per sé sufficiente a porre diagnosi di ictus aterotrombotico dal momento che altre cause di ictus andrebbero escluse. Una lesione ischemica estesa infatti (anche per localizzazioni anatomiche ascrivibili ad un ictus dei grossi vasi) in un paziente con comorbidità per una patologia cardioemboligena come la fibrillazione atriale nonostante la coesistenza di una diffusa aterosclerosi intra ed extracranica andrebbe inquadrata come una lesione ischemica a fisiopatogenesi cardioembolica per la presenza di una fonte embolica cardiaca rilevante. Va pertanto sensibilizzato il neurologo clinico sul fatto che un esteso work up diagnostico, esemplificato nei paragrafi precedenti, associato eventualmente al ricorso all'algoritmo (più in senso clinico che statistico) del cardioembolic score andrebbe applicato a tutti i pazienti affetti da ictus con anamnesi positiva per fattori di rischio cerebrovascolari tradizionali per ictus aterotrombotico allo scopo di escludere o confermare la coesistenza di una fonte emboligena cardiaca che altrimenti sarebbe sottostimata.

\section{Bibliografia}

1. Zhang H, Li Z, Dai Y, et al. Ischaemic stroke etiological classification system: the agreement analysis of CISS, SPARKLE and TOAST. Stroke Vasc Neurol. 2019 Jun 2;4(3):123-128.

2. Pillai AA, Tadi P, Kanmanthareddy A. Cardioembolic Stroke. Treasure Island (FL): StatPearls Publishing; 2019 Jan-.2019 Oct 1.

3. Donahue MJ, Dlamini N, Bhatia A, Jordan LC. Neuroimaging Advances in Pediatric Stroke. Stroke. 2019 Feb;50(2):240-248.

4. Donahue MJ, Dlamini N, Bhatia A, Jordan LC. Neuroimaging Advances in Pediatric Stroke. Stroke. 2019 Feb;50(2):240-248.

5. Rovira A, Grivé E, Rovira A, Alvarez-Sabin J. Distribution territories and causative mechanisms of ischemic stroke. Eur Radiol. 2005 Mar;15(3):416-26. Epub 2005 Jan 19.

6. Amarenco P, Bogousslavsky J, Caplan LR, Donnan GA, Hennerici MG. Classification of stroke subtypes. Cerebrovasc Dis. 2009;27(5):493-501. doi: 10.1159/ 000210432. Epub 2009 Apr 3.

7. Hornung $\mathrm{M}$ et al. Cardioembolic Stroke and Postmyocardial Infarction Stroke. Cardiol Clin. (2016)

8. Finsterer J et al. Stroke-like episodes, peri-episodic seizures, and MELAS mutations. Eur J Paediatr Neurol. (2016)

9. Ha AC, Verma A, Verma S. Oral anticoagulation for stroke prevention amongst atrial fibrillation patients with valvular heart disease: an update. Curr Opin Cardiol. 2017 Mar;32(2):174-180.

10. Mahajan N, Khetarpal V, Afonso L.J Cardiol. Stroke secondary to calcific bicuspid aortic valve: case report and literature review. 2009 Aug;54(1):158-61.

11. Sotero FD, Rosário M, Fonseca AC, Ferro JM. Neurological Complications of Infective Endocarditis. Curr Neurol Neurosci Rep. 2019 Mar 30;19(5):23.

12. Vink R, Van Den Brink RB, Levi M. Management of anticoagulant therapy for patients with prosthetic heart valves or atrial fibrillation. Hematology. 2004 Feb;9(1):1-9.

13. Campbell BCV, De Silva DA, Macleod MR, et al. Ischaemic stroke. Nat Rev Dis Primers. 2019 Oct 10;5(1):70.

14. Mtwesi V, Amit G. Stroke Prevention in Atrial Fibrillation: The Role of Oral Anticoagulation. Med Clin North Am. 2019 Sep;103(5):847-862.

15. De Ponti R et al. SickSinusSyndrome. Card Electrophysiol Clin. (2018)

16. Yuan SM, Humuruola G. Stroke of a cardiac myxoma origin. Rev Bras Cir Cardiovasc. 2015 Mar-Apr;30(2): 225-34.

17. Osteraas ND, Vargas A, Cherian L, Song S. Role of PFO Closure in Ischemic Stroke Prevention. Curr Treat Options Cardiovasc Med. 2019 Nov 14;21(11):63.

18. Furlan AJ, et al. Closure or medical therapy for cryptogenic stroke with patent foramen ovale. N Engl J Med 2012;366(11):991-999.

19. Carroll JD, et al. Closure of patent foramen ovale versus medical therapy after cryptogenic stroke. N Engl J Med 2013. 368(12):1092-1100

20. Mojadidi MK, Ruiz JC, Chertoff J, et al. Patent Foramen 
Ovale and Hypoxemia. Cardiol Rev. 2019 Jan/Feb;27 (1): 34-40.

21. Mbabuike $\mathrm{N}$ et al. Revascularization of tandem occlusions in acute ischemic stroke: review of the literature and illustrative case. Neurosurg Focus. (2017)

22. Goyal M, et al. Randomized assessment of rapid endovascular treatment of ischemic stroke. . N Engl J Med 2015. 372(11):1019-1030.

23. Qureshi AI, Caplan LR. Intracranial atherosclerosis. Lancet. 2014 Mar 15;383(9921):984-98.

24. Chiti A et al. Cardioembolicprofile" in patients with ischemic stroke: data from the analysis of 1037 cases. J Stroke Cerebrovasc Dis. (2015)

25. Chiti A et al. Cardioembolicprofile" in patients with ischemic stroke: data from the analysis of 1037 cases. J Stroke Cerebrovasc Dis. (2015)

26. Arboix A, Obach V, Sánchez MJ, Massons J. Complementary examinations other than neuroimaging and neurosonology in acute stroke. World J Clin Cases. 2017 Jun 16;5(6):191-202.

27. Amarenco P, Bogousslavsky J, Caplan LR, et al. Classification of stroke subtypes. Cerebrovasc Dis. 2009;27 (5):493-501.

28. Sugumar H, Nanayakkara S, Prabhu S, et al. Pathophy- siology of Atrial Fibrillation and Heart Failure: Dangerous Interactions. Cardiol Clin. 2019 May;37(2): 131138.

29. Arboix A. Cardiovascular risk factors for acute stroke: Risk profiles in the different subtypes of ischemic stroke. World J Clin Cases. 2015 May 16;3(5):418-29.

30. Kostopoulou A, Zeljko HM, Bogossian H, et al. Atrial fibrillation-related stroke in women: Evidence and inequalities in epidemiology, mechanisms, clinical presentation, and management. REF INCOMPLETA

31. Arboix A, Alio J. Acute cardioembolic cerebral infarction: answers to clinical questions. Curr Cardiol Rev. 2012 Feb;8(1):54-67.

32. Depoorter L, Sels L, Deschodt M, et al. Clinical Outcomes of Rate vs Rhythm Control for Atrial Fibrillation in Older People: A Systematic Review and Meta-Analysis. Drugs Aging. 2019 Nov 20.

33. Campbell BCV, De Silva DA, Macleod MR, et al. Ischaemic stroke. Nat Rev Dis Primers. 2019 Oct 10;5(1):70.

34. D'Souza A, Butcher KS, Buck BH. The Multiple Causes of Stroke in Atrial Fibrillation: Thinking Broadly. Can J Cardiol. 2018 Nov;34(11):1503-1511. 


\title{
Ictus criptogenetico: la ricerca della fibrillazione atriale e del forame ovale pervio. Come e quando
}

\author{
Marco Botta \\ S.C. Cardiologia-UTIC, Ospedale San Paolo, Savona, Italia
}

\section{Introduzione}

L'ictus ischemico costituisce circa il $75-80 \%$ di tutti gli ictus ed è fisiopatologicamente eterogeneo, per cui occorre classificarlo in base ai diversi sottotipi patogenetici (Tabella 1), che presentano peculiarità cliniche e strumentali tali da orientare verso un corretto approccio diagnostico.

La possibilità di differenziare questi meccanismi dipende, ovviamente, dall'accuratezza delle indagini eseguite; ad esempio, la distinzione tra meccanismo embolico artero-arterioso da patologia dei grossi vasi e meccanismo embolico a partenza cardiaca dipende dalla sensibilità delle indagini nel ricercare, da un lato, alterazioni aterosclerotiche dei vasi epiaortici, dall'altro, cardiopatie potenzialmente emboligene (Tabella 2).

Nonostante il ricorso alle moderne indagini strumentali, è frequente non riuscire a stabilire con esattezza l'eziologia, allorché nessun fattore di rischio venga identificato (infarto da causa sconosciuta o criptogenetico) o quando siano contemporaneamente presenti più meccanismi, ad esempio, fibrillazione atriale e stenosi carotidea (eziologia indeterminata) come spesso si osserva nei soggetti anziani.

Si definisce pertanto criptogenetico un ictus in cui esista la dimostrazione della sua natura ischemica, ma la ricerca delle cause sia negativa. La prevalenza dell'ictus criptogenetico nella popolazione con ictus ischemico può arrivare al $30 \%$. La causa dello stroke può essere sconosciuta perché transitoria e reversibile, o perché non ben indagata, o perché non ancora nota.

Lo stroke criptogenetico, comunque, presenta so-

Corrispondente: Marco Botta, S.C. Cardiologia-UTIC, Ospedale San Paolo, Savona, Italia.

E-mail: marcobotta.mb@gmail.com

Articolo pubblicato secondo la Creative Commons Attribution NonCommercial 4.0 License (CC BY-NC 4.0).

${ }^{\circ}$ Copyright: the Author(s), 2020

Licensee PAGEPress, Italy

QUADERNI - Italian Journal of Medicine 2020; 8(2):111-117 litamente un maggior numero di recidive e una prognosi peggiore.

Le controversie maggiori in letteratura sulla definizione eziopatogenetica in questo setting clinico riguardano la ricerca e la diagnosi dei due principali fattori imputati nell'embolia cardiogena: la fibrillazione atriale (FA) ed il forame ovale pervio (FOP).

\section{Fibrillazione atriale}

La fibrillazione atriale (FA) è la più comune aritmia sopraventricolare sostenuta. ${ }^{1}$ La sua incidenza aumenta con l'aumentare dell'età della popolazione, quindi ci si aspetta che nei prossimi anni il numero dei malati crescerà. ${ }^{2}$ La FA è associata ad un aumento di circa 5 volte del rischio di ictus ${ }^{3}$ e gli eventi tromboembolici legati alla FA determinano un più alto rischio di mortalità e di comorbilità. ${ }^{4}$ Per queste ragioni la prevenzione degli ictus correlati alla FA deve essere una priorità della sanità pubblica. Gli ictus dovuti alla FA sono comuni e spesso devastanti (tra il $70 \%$ e $1 ' 80 \%$ dei pazienti muore o ha invalidità permanenti), ${ }^{5,6}$ ma anche largamente prevenibili con la terapia anticoagulante che garantisce il $64 \%$ di riduzione del rischio di ictus e il $25 \%$ di riduzione della mortalità. ${ }^{7}$ Tuttavia, siccome la FA è spesso intermittente ed asintomatica, essa può rappresentare un fattore di rischio silente, facilmente non diagnosticato. ${ }^{8,9}$

D'altra parte anche episodi di breve durata aumentano il rischio tromboembolico clinico ${ }^{10}$ e silente. ${ }^{11}$ Nello studio ASSERT (Asymptomatic Atrial Fibrillation and Stroke Evaluation in Pacemaker Patients and the Atrial Fibrillation Reduction Atrial Pacing Trial) qualsiasi tachicardia atriale, anche silente, che durasse più dei 6 min aumentava di 2,5 volte il rischio di ictus od embolia sistemica. ${ }^{8}$ Nello studio MOST (Mode Selection Trial) un qualsiasi evento di tachicardia atriale di durata $>5$ min aumentava di 2.8 volte il rischio di ictus o morte. ${ }^{12}$

Un ruolo di primo piano, nell'inquadramento del problema in caso di ictus criptogenetico, è dato quindi dalla ricerca e dalla valutazione degli episodi di fibrillazione atriale asintomatica. 
Nello studio AFFIRM ${ }^{13}$ i pazienti con FA asintomatica avevano più eventi cerebrovascolari, durata più lunga degli episodi, frequenza cardiaca media più bassa, funzione ventricolare sinistra migliore e mortalità ed eventi maggiori totali simili ai pazienti con FA sintomatica.

La FA asintomatica, quindi, ha complicanze paragonabili alla FA sintomatica ed è presente in un numero di casi almeno paragonabile a questa, per cui un'accurata identificazione dei pazienti con episodi asintomatici è fondamentale per guidare una corretta anticoagulazione, soprattutto in coloro che hanno elevati score di rischio trombo embolico..$^{10,11,14}$

E' stato quindi dimostrato, come anche pochi minuti della forma subclinica di fibrillazione atriale aumentino il rischio di ictus cerebrale. Inoltre un paziente potrebbe avere degli episodi subclinici che portano all'ictus ma che al momento dell'ospedalizzazione non vengono identificati.

Questi scenari, purtroppo comuni nella pratica clinica quotidiana, rappresentano un reale rischio in quanto la non diagnosi di fibrillazione atriale si può tradurre in una scelta terapeutica antitrombotica potenzialmente sub-ottimale, non viene cioè utilizzata in questi pazienti la terapia anticoagulante orale a lungo termine che è supportata da maggiori e solide evidenze come trattamento più efficace nella prevenzione secondaria dell'ictus cerebrale associato a cardioembolismo. I criteri di valutazione del rischio cardio-embolico con il va- lidato score $\mathrm{CHA}_{2} \mathrm{DS}_{2}$-VASc riportano nelle più attuali Linee Guida l'indicazione alla terapia anticoagulante orale nei pazienti con anamnesi di FA clinica (in ogni sua forma) e Score $\geq 2 .{ }^{15}$

L'inquadramento clinico del paziente in questi casi specifici verte anche su altri aspetti eziopatogenetici: sulla valutazione del AF burden, ossia definire quale è la durata (secondi ?, minuti ?, ore ?) che rende potenzialmente emboligeno un episodio di FA. Secondo alcuni studi la durata minima è 6 minuti, secondo altri è 24 ore, specialmente in un contesto in cui spesso l'aritmia non ha relazione temporale diretta con l'evento neurologico, mettendo in discussione quindi lo stesso rapporto di causalità. L'atteggiamento attuale più condiviso sembra essere quello ancora più aggressivo di trattare con terapia anticoagulante a lungo termine tutti i pazienti che presentano episodi di almeno 30 secondi di durata e hanno un Score maggiore o uguale a 2, con aspetti controversi anche nelle Linee Guida sulle decisioni da prendere nel paziente a più basso rischio, ossia con $\mathrm{CHA}_{2} \mathrm{DS}_{2}$-VASc Score maggiore o uguale a 1. ${ }^{15,16}$

Sempre in questi casi stanno inoltre emergendo dati ulteriori per cui sarebbe anche la stessa miocardiopatia atriale una potenziale condizione determinante per favorire la formazione di trombi: dimensioni dell'atrio, funzione dell'auricola e markers bioumorali potrebbero diventare le spie del rischio tromboembolico più che la durata della fibrillazione atriale stessa. Sono già stati pubblicati studi che suggeriscono la pos-

Tabella 1. Ictus ischemico - Classificazione fisiopatologica (TOAST).

\begin{tabular}{|c|c|c|}
\hline Ictus ischemico & 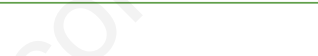 & \\
\hline - Patologia dei grossi vasi & Ictus aterotrombotico & \\
\hline - Patologia dei piccoli vasi & Ictus lacunare & \\
\hline - Cardioembolismo & & \\
\hline - Altre cause (cause rare) & & \\
\hline - Meccanismo indeterminato & & \\
\hline Più fattori causali & Accertamento insufficiente & Ictus criptogenetico \\
\hline
\end{tabular}

Tabella 2. Ictus cardio-embolico: patologie cardiache potenzialmente emboligene.

\begin{tabular}{ll}
\hline Rischio elevato & Rischio minore o incerto \\
\hline Fibrillazione atriale & Prolasso mitralico \\
\hline Stenosi mitralica & Calcificazioni Annulus mitralico \\
\hline Protesi valvolari & Pevietà forame ovale \\
\hline Recente IMA & Aneurisma del setto interatriale \\
\hline Trombosi ventricolare sinistra & Stenosi aortica calcifica \\
\hline Mixoma atriale & \\
\hline Endocardite batterica & \\
\hline Cardiopatia dilatativa &
\end{tabular}


sibilità di sospettare una genesi embolica dell'ictus in fase acuta utilizzando markers quali BNP (o NTproBNP), CPK o Troponina. ${ }^{15,16}$

La Fibrillazione Atriale va quindi ricercata con tutti i mezzi a disposizione per tutti i pazienti con ictus criptogenetico e specialmente per quelli che presentano caratteristiche cliniche di aumentato rischio per lo sviluppo di tale aritmia. ${ }^{15,16}$

\section{Fattori di rischio per lo sviluppo di fibrilla- zione atriale}

Il $70 \%$ delle FA sono associate a cardiopatia organica e solo nel $2 \%$ dei pazienti con FA non si trova nessun fattore predisponente, né cardiaco né extracardiaco.

I fattori di rischio (FdR) per lo sviluppo di FA si distinguono in maggiori e minori, come illustrato nei seguenti sotto-paragrafi. ${ }^{17}$

\section{Maggiori}

- Ipertensione arteriosa

- Alterazioni strutturali del cuore (dilatazione e/o ipertrofia ventricolare sinistra, atriomegalia, valvulopatie, mio-pericarditi)

- Diabete non controllato

- Scompenso cardiaco

- Cardiopatia ischemica

- Età avanzata

\section{Minori}

- Obesità

- Anamnesi di aritmie atriali

- Malattie polmonari

- Malattie tiroidee

- Fumo

- Disionie

\section{Metodi di indagine della fibrillazione atriale}

Il monitoraggio del ritmo cardiaco diventa quindi parte cardine dello standard di diagnosi e cura del paziente che sviluppa evento ischemico cerebrale al fine di effettuare un corretto inquadramento clinico delle cause e di guidare la corretta strategia terapeutica.

La capacità di documentare l'aritmia da FA dopo un evento cerebrale è correlata a:

- durata del monitoraggio

- definizione di evento significativo e diagnosi differenziale con altre cause

- intervallo temporale tra evento indice e inizio della registrazione

- tipologia dei pazienti studiati (età e fattori di rischio trombotici)
Solitamente il paziente ricoverato con diagnosi di ictus cerebrale viene sottoposto a monitoraggio elettrocardiografico per 24-48 ore, ove disponibile Centro Ictus con disponibilità di letti monitorizzati. Diversi studi suggeriscono che un monitoraggio prolungato può identificare la fibrillazione atriale non diagnosticata nel 10\% dei pazienti. Tuttavia allo stato attuale non è possibile definire una durata ottimale del monitoraggio utile ad individuare episodi di FA. ${ }^{18}$

Lo studio Cryptogenic Stroke and Underlying AF (CRYSTAL AF) e lo studio 30-Day Cardiac Event Monitor Belt for Recording Atrial Fibrillation after Cerebral Ischemic Event (EMBRACE) hanno arruolato pazienti con ictus criptogenetico o con TIA (attacco ischemico transitorio) senza una storia di fibrillazione atriale. ${ }^{19,20}$ Prima dell'inizio dello studio i pazienti erano stati sottoposti a monitoraggio ECG continuo di 24 ore che non aveva rilevato nessun segno di fibrillazione atriale. Nello studio CRYSTAL $\mathrm{AF}$ metà dei pazienti sono stati monitorati per sei mesi con loop-recorder impiantabile e l'altra metà con monitoraggio Holter standard (gruppo di controllo). In questo studio il monitoraggio continuo ha permesso nell'arco di 6 mesi la rilevazione della FA nell' $8,9 \%$ dei pazienti versus 1 ' $1,4 \%$ dei pazienti del gruppo di controllo (hazard ratio, 6,4; 95\% intervallo di confidenza [CI], da 1,9 a 21,7, P<0,001). Nell'arco di 12 mesi la fibrillazione atriale è stata rilevata nel 12,4 dei pazienti del gruppo con loop recorder e nel 2,0\% dei pazienti con monitoraggio standard (hazard ratio, 7,3; $95 \%$ CI, da 2,6 a 20,8, P<0,001).

Nello studio EMBRACE invece è stato confrontato il monitoraggio con loop-recorder esterno per 30 giorni con il monitoraggio Holter standard. In questo studio il monitoraggio con loop-recorder esterno ha rilevato eventi di fibrillazione atriale della durata di 30 secondi in $16,1 \%$ pazienti a fronte del' $1,4 \%$ pazienti del gruppo di controllo (differenza assoluta, 12,9 punti percentuali; 95\% CI, da 8,0 a 17,6; $\mathrm{P}<0,001)$. Episodi di fibrillazione atriale della durata di 2,5 minuti o superiori sono stati registrati nel $9,9 \%$ dei pazienti con loop-recorder esterno versus il $2,5 \%$ dei pazienti del gruppo di controllo (differenza assoluta, 7,4 punti percentuali; $95 \% \mathrm{CI}$, da 3,4 a 11,3; $\mathrm{P}<0,001$ ). Nell'arco di tre mesi gli anticoagulanti orali sono stati prescritti in un maggior numero di pazienti sottoposti a monitoraggio continuo (18,6\% versus $11,1 \%$; differenza assoluta, 7,5 punti percentuali, $95 \% \mathrm{CI}$, da 1,6 a 13,3 ; $\mathrm{P}=0,01$ ).

I risultati di entrambi gli studi hanno dimostrato che il monitoraggio per lunghi periodi con loop-recorder è superiore al monitoraggio Holter standard nel rilevare episodi di FA nei pazienti con stroke criptogenetico. Molti dei pazienti con ictus criptogenetico o TIA, anche sulla base della stratificazione di rischio generale dovrebbero essere sottoposti almeno 
ad alcune settimane di monitoraggio elettrocardiografico per poter intervenire con una terapia ottimizzata per la prevenzione dello stroke. Su quale modalità di monitoraggio adottare, andranno tuttavia considerate anche successive valutazioni di costo-efficacia. ${ }^{19,20}$

$\mathrm{Al}$ dato pratico è chiaro come l'efficacia dello screening sia ovviamente fortemente influenzata dalla sua durata. Fermo restando che per i pazienti già portatori di Device (Pace Maker o ICD) resta fondamentale l'interrogazione dei device stessi e l'implementazione dei sistemi di monitoraggio remoto, nelle altre categorie di pazienti il Loop-Recorder (esterno o impiantabile) assicura in questo tipo di pazienti una più elevata specificità e sensibilità nella diagnosi. ${ }^{18}$

Le raccomandazioni di EHRA indicano inoltre ulteriori sistemi di monitoraggio per lo screening della Fibrillazione Atriale silente con risultati dimostratisi anch'essi più sensibili del tradizionale ECG dinamico sec. Holter (solitamente eseguibile limitatamente alle sole $24 \mathrm{~h}$ ) nel rilevare episodi di aritmia silente. $\mathrm{Si}$ tratta di patch, cinture, orologi o altri dispositivi collegati a software di registrazione ECG che possono garantire un monitoraggio fino a 14 giorni.

Per i pazienti in grado di utilizzarlo le nuove tecnologie sviluppatesi negli smartphone e dispositivi di registrazione di singola traccia ECG ad essi correlati tramite applicazioni dedicate consentono inoltre di prolungare ulteriormente il monitoraggio (su base volontaria) in caso di presenza od assenza di sintomi con accuratezza diagnostica sull'interpretazione del ritmo pari alla registrazione di ECG a 12 derivazioni. Restano da considerare comunque anche il controllo periodico del polso e la misurazione pressoria periodica con apparecchi automatici.

In conclusione, allo stato attuale delle conoscenze, un monitoraggio aggressivo a lungo termine per l'individuazione di episodi di FA silente risulta raccomandabile in tutti i pazienti con ictus criptogenetico ${ }^{21} \mathrm{e}$ comunque nei pazienti ad alto rischio per i quali la documentazione dell'aritmia comporterebbe l'immediata prescrizione di una terapia specifica e di documentata efficacia (la terapia anticoagulante orale). Se il monitoraggio vada esteso anche alla popolazione generale, invece, nonostante l'introduzione di nuove promettenti tecniche di monitoraggio della FA, meno invasive e molto sensibili bisognerà attendere ulteriori evidenze riguardanti il rapporto costo-efficacia. ${ }^{22,23}$

\section{Forame ovale pervio}

L'associazione tra ictus ischemico e forame ovale pervio, in combinazione o meno ad aneurisma del setto interatriale (ASI), rappresenta una delle problematiche più controverse in ambito neurologico e cardiologico. In circa la metà dei casi di ictus ischemico l'eziologia resta sconosciuta, ancor di più se guardiamo ai soggetti giovani. L'associazione tra ictus ischemico criptogenico e pervietà del forame ovale è tematica dibattuta da oltre vent'anni, supportata da numerose dimostrazioni di emboli in transito attraverso il forame, il più delle volte in concomitanza con un'embolia polmonare o con tromboembolismo venoso in genere.

Il forame ovale pervio è riscontro particolarmente frequente nella popolazione generale, con caratteristiche estremamente variabili, e anche la dimostrazione di uno shunt destro-sinistro in particolari condizioni non è eventualità rara. ${ }^{24} \mathrm{La}$ possibilità oggi di chiudere efficacemente la comunicazione tra le due cavità atriali, rimuovendo in via teorica la causa di una consistente quota di eventi cerebrovascolari idiopatici, offre un'importante alternativa terapeutica alla sola terapia medica con antiaggreganti o anticoagulanti.

Non esistono tuttavia dati conclusivi che permettano di assumere una posizione definita.

L'embolia paradossa è definita dalla concomitante presenza di: i) trombosi venosa profonda (TVP) e/o embolia polmonare (EP); ii) una comunicazione intracardiaca che permetta uno shunt destro-sinistro; iii) un episodio di embolia sistemica, dopo averne escluso altre possibili fonti. ${ }^{25,26}$

Essa è quindi dovuta al passaggio di trombi o emboli dal sistema venoso al circolo sistemico, come descritto da Cohnheim nel 1877, e rappresenta il meccanismo attraverso il quale il FOP può dar luogo a ictus ischemico, ma nel contempo risulta spesso una diagnosi incompleta in uno o più dei suoi criteri sopracitati, sia in letteratura che nella pratica clinica quotidiana.

\section{Questioni aperte}

L'impatto reale del forame ovale pervio tra le cause di ictus ischemico ad eziologia sconosciuta nella popolazione generale non è stato definito in maniera univoca. Inoltre la frequenza delle recidive emboliche riportata in letteratura in questo tipo di pazienti sembra essere inferiore rispetto alle altre eziologie. Permane estrema generalizzazione nella diagnosi di pervietà del forame ovale, in assenza di criteri diagnostici univoci e senza che venga sottolineata l'importanza della valutazione delle specifiche caratteristiche anatomiche. Il profilo di rischio cerebrovascolare dei pazienti inseriti nei diversi studi è estremamente eterogeneo e questo porta in alcuni casi a sottostimare l'impatto dell'embolia paradossa ed in altri a sovrastimare l'efficacia della tecnica percutanea. Sebbene i risultati immediati siano particolarmente incoraggianti, non vi sono a disposizione casistiche con periodi di followup sufficientemente lunghi da poter definitivamente escludere fallimenti e complicanze della chiusura transcatetere nel lungo termine. Infine, anche se appare 
consolidata l'efficacia della procedura, non ne è stata dimostrata la significativa superiorità sulla sola terapia medica. ${ }^{24}$

\section{Le ipotesi}

La crescente disponibilità di studi clinici controllati e randomizzati ci fornirà forse maggiori certezze nei confronti di una problematica circondata ancora da numerosi lati oscuri.

Indipendentemente da questi è comunque attraverso un iter diagnostico puntualmente completo e approfondito che risulta possibile classificare correttamente un evento, caratterizzare dal punto di vista anatomico ciascun forame ovale e stratificare il rischio cerebrovascolare dei pazienti, indipendentemente dalla presenza o meno di un difetto così frequente da potersi quasi considerare parafisiologico. ${ }^{24}$

Negli ultimi anni si sono tuttavia maggiormente definite le strategie terapeutiche ed i percorsi con la pubblicazione di 3 studi randomizzati (CLOSE, REDUCE e PFO-DEFENCE, anni 2017-2018) che, insieme ad un follow-up a lungo termine dello studio RESPECT (anno 2013), hanno aggiunto prove sufficienti per avere un accettabile grado di evidenza sulla terapia.

I 4 trial hanno dimostrato che, nelle popolazioni studiate, il trattamento con chiusura percutanea associato a terapia medica era superiore alla sola terapia medica nella prevenzione secondaria dell'ictus di origine indeterminata. ${ }^{27}$

I dati pubblicati si sono aggiunti a quelli dei tre studi precedenti, molto più incerti, ed è stato così definitivamente chiarito che il $\mathrm{PFO}$ può essere implicato nei processi fisiopatologici alla base dell'ictus criptogenetico e che non tutti i PFO hanno un uguale rischio. Queste evidenze hanno consentito di pubblicare un documento di posizione condiviso da ben 8 società scientifiche europee sulla gestione dei pazienti con trombo embolia del circolo di sinistra associata a PFO..$^{28,29}$

I nuovi indirizzi in breve di questo documento comprendono: i) una personalizzazione delle indicazioni e dei percorsi; ii) scelte di cura condivise con il paziente con consenso informato personalizzato; iii) test di primo livello: da preferire Doppler transcranico con test alle micro bolle; iv) loop-recorder per 6 mesi in pazienti ad alto rischio di Fibrillazione atriale e screening aritmico di primo livello negativo; v) offrire chiusura percutanea per pazienti con alta probabilità di FOP causale e alta probabilità di recidiva (valutazione interdisciplinare); vi) se scelta terapia medica: terapia anticoagulante orale nei pazienti a basso rischio emorragico; vii) terapia medica post-chiusura: duplice terapia antiaggregante per 1-6 mesi, poi singolo antiaggregante per 5 anni; viii)- ricerca orientata ad individuare i sottogruppi: scienza dei sistemi e medicina di precisione. ${ }^{28-30}$

\section{Tecniche diagnostiche}

Il test diagnostico di primo livello suggerito è il Doppler transcranico con test alle microbolle (DTCC) che, nella meta-analisi di 29 studi ha mostrato la migliore accuratezza per la diagnosi di PFO (sensibilità del 94\% e specificità del 92\%, con un'area sotto la curva del $0,97 \%) .{ }^{27}$ Per ragioni logistiche e di opportunità è anche possibile fare in prima istanza un esame Ecocardiografico color-Doppler trans toracico con test alle microbolle, con l'accortezza in caso di risultato dubbio o negativo di fare comunque un DTC$\mathrm{C}$ prima di escludere la presenza di PFO.

Nel caso l'esame di primo livello sia risultato positivo, l'Ecocardiografia trans esofagea con test alle microbolle (ETE-C) serve per confermare la presenza del PFO e rilevarne le caratteristiche utili alla stratificazione del rischio e all'eventuale indicazione a terapia interventistica.

L'ETE-C, nonostante sia stata utilizzata come gold standard diagnostico per lungo tempo, è invece un test con una sensibilità largamente inferiore a quanto auspicabile e non dovrebbe essere quindi utilizzata come esame di primo livello. ${ }^{31} \mathrm{E}$ ' invece indicata un'ETE nel caso sia necessario escludere altre fonti emboli gene cardiovascolari.

Anche nel paziente con FOP documentato e caratteristiche di elevato profilo di rischio per Aritmia da FA in cui lo screening standard sia stato negativo, rimane fortemente proposto l'impianto di un Loop recorder da monitorare per almeno 6 mesi. In caso di positività di questo esame, è però fondamentale sempre valutare, ancora una volta in modo interdisciplinare, il bilancio tra il rischio emboligeno degli episodi di FA e quello del PFO. Sulla base di tale valutazione si deciderà in merito alla possibile chiusura percutanea o alla terapia medica, valutando i criteri di rischio collegati sia al burden aritmico, sia al punteggio $\mathrm{CHA}_{2} \mathrm{DS}_{2}$-VASc, per poi fare scelte in merito al PFO valutando la probabilità di un beneficio ulteriore di un'eventuale chiusura.

Altro aspetto importante nell'iter diagnostico è la definizione degli elementi per stimare la probabilità di un ruolo causale del PFO (Tabella 3) e per stimare la probabilità di recidiva (Tabella 4). Vi sono indicazioni ad una posizione forte a favore della chiusura percutanea aggiunta ad una terapia medica nei casi di tromboembolia del circolo sinistro solo nei pazienti di età tra 18 e 65 anni, se è presente un alto rischio attribuibile al PFO e valutando attentamente i rapporti tra probabilità di nesso causale, rischio di recidiva e rischio emorragico, oltre ad effettuare con il paziente una scelta condivisa di cura. ${ }^{27}$ Nei pazienti con PFO considerati a basso rischio, invece non vi sono differenze statisticamente significative nella recidiva di ictus tra chiusura e terapia medica. ${ }^{29}$ 
Tabella 3. Elementi per stimare la probabilità di ruolo causale del FOP.

\begin{tabular}{ll}
\hline Alto Rischio & Da valutare anche \\
\hline- Aneurisma del setto interatriale & - Caratteristiche imaging dell'ictus (corticale vs profondo) \\
\hline- Shunt moderato-severo & - Dimensioni FOP e del tunnel \\
\hline- Ipermobilità del setto & - Rete di Chiari e/o valvola di Eustachio prominente \\
\hline- Concomitante TEP o TVP & - Età $<55$ anni \\
\hline & - Fattori di rischio per ictus \\
\hline & $\begin{array}{l}\text { Elementi clinici (viaggi lunghi, immobilizzazione, sforzi isometrici, sindrome apnee ostruttive } \\
\text { del sonno, recente chirurgia maggiore, pregresse TEP o TVP }\end{array}$ \\
\hline & - Score RoPE \\
\hline
\end{tabular}

Tabella 4. Elementi per stimare la probabilità di recidiva ischemica.

\begin{tabular}{ll}
\hline Alto Rischio & Da valutare anche \\
\hline- Aneurisma del setto interatriale & - Età avanzata \\
\hline- Alterazioni della coagulazione & - Ampiezza del FOP \\
\hline & - Necessità di terapia anticoagulante $v s$ antipiastrinica \\
\hline & - Ictus $v s$ TIA come evento indice \\
\hline & - Ictus insorto in corso di terapia anticoagulante orale o antipiastrinica
\end{tabular}

In conclusione, in un ambito ancora molto controverso, la ricerca del Forame Ovale Pervio e la sua attenta definizione diagnostica in termini anatomo-clinici è un punto cardine del work-up diagnostico nei pazienti con ictus criptogenetico; la decisione in merito al rapporto causale e al possibile e validato trattamento interventistico richiede una attenta valutazione multidisciplinare tra cardiologi, neurologi ed internisti per decidere quale sia la migliore strategia terapeutica in base alle caratteristiche del singolo paziente. ${ }^{29-32}$

\section{Bibliografia}

1. Kirkhof P, Benussi S, Kotecha D et al. 2016 ESC Guidelines for the management of Atrial Fibrillation. Eur Heart Journal V 37 I38: 2893-2962.

2. Naccarelli GV, Varker H, Lin J, Schulman KL. Increasing prevalence of atrial fibrillation and flutter in the United States. Am J Cardiol 2009;104:1534-9.

3. Grau AJ, Weimar C, Buggle F, et al. Risk factors, outcome, and treatment in subtypes of ischemic stroke: the German stroke data bank. Stroke 2001;32:2559-66.

4. Jorgensen HS, Nakayama H, Reith J, Raaschou HO, Olsen TS. Acute stroke with atrial fibrillation. The Copenhagen Stroke Study. Stroke 1996;27:1765-9.

5. Gladstone DJ, Bui E, Fang J, et al. Potentially preventable strokes in high-risk patients with atrial fibrillation who are not adequately anticoagulated. Stroke 2009;40:235-40.

6. Saposnik G, Gladstone D, Raptis R, Zhou L, Hart RG; Investigators of the Registry of the Canadian Stroke Network (RCSN) and the Stroke Outcomes Research
Canada (SORCan) Working Group. Atrial fibrillation in ischemic stroke: predicting response to thrombolysis and clinical outcomes. Stroke 2013;44:99-104.

7. Hart RG, Pearce LA, Aguilar MI. Meta-analysis: antithrombotic therapy to prevent stroke in patients who have nonvalvular atrial fibrillation. Ann Intern Med 2007; 146:857-67.

8. Healey JS, Connolly SJ, Gold MR, et al.; ASSERT Investigators. Subclinical atrial fibrillation and the risk of stroke. N Engl J Med 2012;366:120-9.

9. Camm AJ, Corbucci G, Padeletti L. Usefulness of continuous electrocardiographic monitoring for atrial fibrillation. Am J Cardiol 2012;110:270-6.

10. Hohnloser SH, Pajitnev D, Pogue J, et al.; ACTIVE W Investigators. Incidence of stroke in paroxysmal versus sustained atrial fibrillation in patients taking oral anticoagulation or combined antiplatelet therapy: an ACTIVE W substudy. J Am Coll Cardiol 2007;50:2156-61.

11. Gaita F, Corsinovi L, Anselmino M, et al. Prevalence of silent cerebral ischemia in paroxysmal and persistent atrial fibrillation and correlation with cognitive function. J Am Coll Cardiol 2013;62: 1990-7.

12. Glotzer TV, Hellkamp AS, Zimmerman J, et al.; MOST Investigators. Atrial high rate episodes detected by pacemaker diagnostics predict death and stroke: report of the Atrial Diagnostics Ancillary Study of the MOde Selection Trial (MOST). Circulation 2003;107:1614-9.

13. Wyse DG, Waldo AL, Di Marco JP et al. A comparison of rate control and rhythm control in patients with atrial fibrillation (AFFIRM). N Engl J Med 2002; 347: 1825-33.

14. Flaker GC, Belew K, Beckman K et al. Asymptomatic atrial fibrillation: demographic features and prognostic information from the Atrial Fibrillation Follow-up Investigation of Rhythm Management (AFFIRM) study. Am Heart J 2005; 149: 657-663. 
15. Kirkhof P, Benussi S, Kotecha D et al. 2016 ESC Guidelines for the management of Atrial Fibrillation. Eur Heart Journal V 37 I38: 2893-2962.

16. Mairesse GH, Moran P et al. Screening for atrial fibrillation: a European Heart Rhythm Association (EHRA) consensus document endorsed by the Heart Rhythm Society (HRS), Asia Pacific Heart Rhythm Society (APHRS), and Sociedad Latinoamericana de Estimulacion Cardiaca y Electrofisiologia (SOLAECE). Europace (2017) 19, 1589-1623.

17. Schoonderwoerd B, Smith MD, Pen L et al. New risks factors for atrial fibrillation: cause of "not-so-lone" atrial fibrillation. Euro pace 2008; 10: 668-673.

18. Kamel H. Heart-Rhythm Monitoring for Evaluation of Cryptogenic Stroke. N Engl J Med 2014; 370:25322533.

19. Gladstone DJ, Spring M, Dorian P, et al. Atrial Fibrillation in Patients with Cryptogenic Stroke. N Engl J Med 2014; 370: 2467-77.

20. Sanna T, Diener HC, Passman RS, et al. Cryptogenic Stroke and Underlying Atrial Fibrillation. N Engl J Med 2014; 370: 2478-86.

21. Kishore A, Vail A, Majid A, et al. Detection of atrial fibrillation after ischemic stroke or transient ischemic attack: a systemic review and meta-analysis. Stroke 2014;45:520-6.

22. McManus DD, Lee J, Maitas O, et al. A novel application for 25. the detection of an irregular pulse using an iPhone $4 \mathrm{~S}$ in patients with atrial fibrillation. Heart Rhythm 2013;10:315-9.

23. Marazzi G, Iellamo F, Volterrani M, et al. Comparison of Mi-26. crolife BP A200 Plus and Omron M6 blood pressure monitors to detect atrial fibrillation in hypertensive patients. Adv Ther 2012; 29:64-70.

24. Stolfo D, Piazza M, Cassin R. Forame ovale pervio ed ictus cardioembolico, l'eterno dilemma. G Ital Cardiol 2012;13(7-8):474-489

25. Meacham RR 3rd, Headley AS, Bronze MS, Lewis JB,
Rester MM. Impending paradoxical embolism. Arch Intern Med 1998; 158:438-48.

26. Johnson BI. Paradoxical embolism. J Clin Pathol 1951;4:316-32.

27. Pristipino C. Cosa c'è di nuovo nelle indicazioni alla chiusura del forame ovale pervio? G Ital Cardiol Vol 20 Suppl 1 al n3 2019.

28. Pristipino C, Sievert H, D'Ascenzo F et al. European position paper on the management of patients with patent foramen ovale. General Approach and left circulation thromboembolism. Eur Heart J 2018 Vol 40 I 38 3182-3195.

29. Pristipino C, Anzola GP, Ballerini L, et al.; Italian Society of Invasive Cardiology (SICI-GISE); Italian Stroke Association (ISAAIS); Italian Association of Hospital Neurologists, Neuroradiologists, Neurosurgeons (SNO); Congenital Heart Disease Study Group of Italian Society of Cardiology; Italian Association of Hospital Cardiologists (ANMCO); Italian Society of Pediatric Cardiology (SICP); Italian Society of Cardiovascular Echography (SIEC); Italian Society of Hemostasis and Thrombosis (SISET). Management of patients with patent foramen ovale and cryptogenic stroke: a collaborative, multidisciplinary, position paper. Catheter Cardiovasc Interv 2013;82:E38-51.

30. Tamarina E, et al. Diagnosis and quantification of patent foramen ovale. Which is the reference technique? Simultaneous study with transcranial Doppler, transthoracic and transesophageal echocardiography. Rev Esp Cardiol 2011;64:133-9.

31. Pepi M, Evangelista A, Nihoyannopou-46. los P, et al.; European Association of Echocardiography. Recommendations for echocardiography use in the diagnosis and management of cardiac sources of embolism: European Association of Echocardiography (EAE) (a registered branch of the ESC). Eur J Echocardiogr 2010;11:461-76.

32. Madden KP, Karanjia PN, Adams HP, and the TOAST investigators. Accuracy of the initial stroke subtype diagnosis in the TOAST study. Neurology 1995; 45: 1975-79. 


\title{
Le complicanze in fase acuta
}

\author{
Caterina Di Carmine, ${ }^{1}$ Giancarlo Francolini, ${ }^{1}$ Samuele Bedetta, ${ }^{2}$ Luciano Mucci, ${ }^{2}$ Gabriele Frausini ${ }^{2}$ \\ ${ }^{1}$ Stroke Unit, Fano (PU); ${ }^{2}$ Medicina Interna, Fano (PU), Italia
}

\section{Introduzione}

Le complicanze dello stroke ischemico in fase acuta sono comuni e costituiscono una delle principali cause di mortalità e morbidità di questa patologia, pertanto è necessario prevenirle e trattarle tempestivamente.

Si possono pertanto distinguere complicanze di ordine neurologico (edema cerebrale, trasformazione emorragica, crisi epilettiche, disfagia) e complicanze di ordine medico (polmonite da aspirazione, trombosi venosa profonda ed embolia polmonare, ischemia miocardica, aritmie).

Scopo di questo capitolo è la revisione delle principali complicanze internistiche dello stroke ischemico in fase acuta, presentandone le basi fisiopatologiche, la clinica, le indicazioni diagnostiche e terapeutiche.

\section{Edema cerebrale}

Nella fase precoce dell'ictus ischemico compare precocemente l'edema citotossico ( $0-6 \mathrm{~h})$ causato dalla disfunzione della pompa sodio-potassio con ingresso di acqua intracellulare; successivamente il danno della barriera ematoencefalica con passaggio di macromolecole, proteine ed acqua nell'interstizio determina la comparsa di edema vasogenico (6-24 h). La distinzione tra le due forme di edema è possibile anche grazie alle metodiche si risonanza magnetica dove le sequenze pesate in diffusione (DWI) documentano la presenza precoce di edema citotossico (entro pochi minuti) mentre le sequenze FLAIR riflettono la comparsa di edema vasogenico dopo circa 6 ore dall'esordio.

Corrispondente: Gabriele Frausini, UOC Medicina Interna Fano-Pesaro, Azienda Ospedaliera Ospedali Riuniti Marche Nord, Fano (PU), Italia.

E-mail: medicinafano@libero.it

Articolo pubblicato secondo la Creative Commons Attribution NonCommercial 4.0 License (CC BY-NC 4.0).

${ }^{\circ}$ Copyright: the Author(s), 2020

Licensee PAGEPress, Italy

QUADERNI - Italian Journal of Medicine 2020; 8(2):118-127
L'incidenza di edema cerebrale nell'ischemia da occlusione del circolo anteriore è del $10-20 \%$ e si verifica tra la $2^{\circ}$ e la $5^{\circ}$ giornata. ${ }^{1,2}$ L'edema può svilupparsi pre- $^{\prime}$ cocemente, fino a $20 \mathrm{~h}$ dopo l'insorgenza dei sintomi, in caso di infarto maligno con coinvolgimento dell'intero territorio dell'arteria cerebrale media: questa sindrome correla con un'elevata mortalità (fino l' $80 \%$ nei pazienti trattati con sola terapia medica). ${ }^{3}$

Le complicanze potenzialmente fatali sono dovute alla comparsa di effetto massa con aumento della pressione intracranica e successiva compressione delle strutture del tronco encefalico deputate al controllo del respiro e della funzione cardiocircolatoria.

Gli elementi predittivi per la comparsa di un infarto maligno della media sono: giovane età, elevato punteggio del National Institute of Health Stroke Scale (NIHSS) all'ingresso, segni precoci di ischemia in $>50 \%$ del territorio della media, disturbo precoce dello stato di coscienza, coinvolgimento associato di altri territori vascolari (a. cerebrale anteriore e posteriore).

L'occlusione dell'arteria carotide interna e la presenza di scarsi circoli collaterali sono ulteriori indicatori prognostici negativi. L'Enhanced Detection of Edema in Malignant Anterior Circulation (EDEMA) score è uno strumento clinico predittivo del rischio di edema cerebrale maligno e combina diverse variabili quali cancellazione delle cisterne basali, shift della linea mediana, glicemia e procedure di rivascolarizzazione mediante trombolisi o trombectomia. ${ }^{4}$

Anche in corso di ischemia cerebellare l'edema può determinare un rapido deterioramento clinico, con un picco di incidenza in $3^{\circ}$ giornata, per effetto compressivo sulle strutture troncoencefaliche, compressione del IV ventricolo, erniazione transforaminale/transtentoriale ed idrocefalo ostruttivo. ${ }^{5}$

La gestione iniziale del paziente con edema cerebrale si basa sull'elevazione della testata del letto di $30^{\circ}$ per facilitare il drenaggio venoso. Inoltre vanno identificati e corretti tutti i fattori aggravanti quali ipertermia, iperglicemia (target 140-180 mg/dL), ipossia ed ipercapnia. L'impiego di farmaci anti-ipertensivi vasodilatatori, come i nitroderivati, va evitato per il potenziale effetto aggravante l'ipertensione endocranica. La terapia profilattica con diuretici osmotici è sconsigliata ma è invece indicata dopo comparsa di edema cerebrale ed 
effetto massa. Il farmaco di prima scelta è mannitolo e.v.: infusioni di $0,25-0,5 \mathrm{~g} / \mathrm{kg}$ ogni $4-8 \mathrm{~h}$ possono essere precedute da un bolo iniziale di $1-1,5 \mathrm{~g} / \mathrm{kg}$ in caso di edema maligno. In caso di trattamento prolungato o ad alto dosaggio sono consigliati controlli seriati della funzionalità renale, della sodiemia e dell'osmolarità plasmatica (target $<320 \mathrm{mOsm} / \mathrm{kg}$ ). Un miglior parametro per la prevenzione del danno renale da mannitolo è il calcolo del gap osmolare ovvero della differenza tra l'osmolarità plasmatica misurata (che riflette il contributo del mannitolo) e quella calcolata, con un target $<18-20 \mathrm{mOsm} / \mathrm{kg}$.

E' consigliato il monitoraggio della diuresi mediante posizionamento di catetere di Foley e l'idratazione con soluzioni isotoniche per prevenire l'ipovolemia.

Possibile alternativa ai diuretici osmotici è la soluzione salina ipertonica al $3 \%$ in boli ripetuti o infusione continua. Non esistono protocolli condivisi circa dosaggio e modalità di somministrazione ma nel trattamento del ipertensione da trauma cerebrale sono utilizzati boli di $2,5-5 \mathrm{~mL} / \mathrm{kg}$ (in 5-20 minuti) mantenendo una sodiemia target $145-155 \mathrm{mEq} / \mathrm{L} .{ }^{6} \mathrm{Il}$ controllo seriato della natriemia è raccomandato per prevenire la sindrome da mielinolisi osmotica. ${ }^{7}$

I pazienti con rapido peggioramento dello stato di coscienza sono candidati ad intubazione orotracheale e ventilazione meccanica al fine di proteggere le vie aeree dal rischio di aspirazione. Una modica iperventilazione $\left(\mathrm{PCO}_{2}\right.$ target di $\left.30-35 \mathrm{mmHg}\right)$ determina una transitoria riduzione dell'ipertensione endocranica attraverso un meccanismo di vasocostrizione cerebrale. L'iperventilazione più aggressiva $o$ prolungata rischia al contrario di peggiorare l'ischemia cerebrale.

In questi casi l'unico trattamento indicato è l'emicraniectomia decompressiva che consiste nella rimozione di un'ampia superficie della calotta cranica e dura madre $(>12 \mathrm{~cm})$ con il razionale di ridurre il danno cerebrale da effetto massa e aumentare la perfusione cerebrale attraverso i circoli collaterali. Una recente metanalisi di 7 studi randomizzati controllati ha dimostrato una riduzione della mortalità nei pazienti trattati con chirurgia decompressiva al prezzo di un aumento della quota di soggetti con severa disabilità residua (mRS 4-5). ${ }^{8}$

In questi pazienti è indicata la sospensione temporanea della terapia antiaggregante per le prime $48 \mathrm{~h}$, fino a quando non sia stata escluso il secondario infarcimento emorragico o l'opzione chirurgica.

I candidati a chirurgia decompressiva entro $48 \mathrm{~h}$ dall'esordio sono i pazienti giovani ( $<60$ anni), con deficit neurologici severi (NIHSS $>16$ ), deterioramento dello stato di coscienza ( $>1$ punto nell'item 1a della scala NIHSS), segni di ischemia nel territorio dell'arteria cerebrale media con o senza coinvolgimento associato di altri territori vascolari, volume le- sionale $>145 \mathrm{~cm}^{2}$ nelle sequenze DWI, autonomia funzionale pre-stroke $(\mathrm{mRS}<2)$.

Alla luce dell'elevato rischio di disabilità residua e dipendenza in caso di infarto maligno cerebrale, l'indicazione alla decompressione chirurgica deve essere preceduta da una attenta discussione dei benefici e dei rischi della procedura e la scelta deve tener conto delle e preferenze del paziente, laddove possibile, o dello stato di necessità.

\section{Trasformazione emorragica}

L'infarcimento emorragico per danno della barriera ematoencefalica nel contesto di una lesione ischemica può avvenire spontaneamente o come conseguenza del trattamento fibrinolitico nel 2-7\% dei casi (OR 5,55 per emorragia sintomatica e 7,14 per emorragia fatale rispetto a placebo). ${ }^{9,10}$

I principali fattori di rischio per infarcimento emorragico sono: età avanzata, gravità del deficit neurologico, iperglicemia, ipertensione, diabete mellito, scompenso cardiaco congestizio, insufficienza renale, fibrillazione atriale, utilizzo di antiaggreganti, leucoaraiosi e alterazioni ischemiche precoci alla TC cerebrale basale. ${ }^{11}$

La classificazione utilizzata nello studio ECASS distingue due forme di infarcimento emorragico in base alle caratteristiche neuroradiologiche: l'infarto emorragico (hemorrhagic infarction, HI) in caso di emorragie petecchiali isolate (HI-1) o confluenti (HI2) ed ematoma parenchimale (parenchymal hematoma, $\mathrm{PH}$ ) con distinzione tra $\mathrm{PH}-1$ e $\mathrm{PH}-2$ secondo il grado di estensione del contesto dell'area infartuale (PH-1 $<30 \%$, PH-2 $>30 \%) .{ }^{12}$

L'infarcimento può decorrere in forma asintomatica o manifestarsi con un improvviso peggioramento del quadro neurologico, cefalea, nausea e vomito o deterioramento dello stato di coscienza (symptomatic intracranial hemorrhage, $\mathrm{sICH})$.

Un peggioramento di almeno 4 punti nella scala NIHSS costituisce un'indicazione alla sospensione temporanea della fibrinolisi sistemica e all'esecuzione di una TC cerebrale urgente per escludere il sanguinamento acuto sebbene in caso elevato livello di disabilità neurologica all'ingresso (NIHSS $>12$ ), anche peggioramenti di minor entità possono essere la spia di un infarcimento emorragico. ${ }^{13,14}$

L'emivita dell'unico farmaco fibrinolitico approvato per l'uso clinico (alteplase) è breve (@4 minuti) con eliminazione del $75 \%$ del farmaco dopo 8 minuti, tuttavia l'ipofibrinogemia secondaria determina una coagulopatia che si protrae per le prime $24 \mathrm{~h}$. Quindi un infarcimento emorragico che insorga dopo $36 \mathrm{~h}$ dal trattamento è improbabile sia dovuto agli effetti dell'alteplase.

Sono stati proposti diversi punteggi predittivi per la stratificazione del rischio di infarcimento emorra- 
gico come ad esempio SEDAN score, il cui acronimo comprende dati clinici, laboratoristici e strumentali di facile rilievo all'ingresso del paziente (Sugar Early infarct sign Dense artery sign Age NIHSS) ma l'uso di tali strumenti è indicato solo per valutazioni prognostiche ed eventualmente per decidere la frequenza del monitoraggio clinico, laddove anche i pazienti a rischio più elevato traggono un beneficio potenziale dal trattamento medico. ${ }^{15}$

La prevenzione dell'emorragia intracranica sintomatica si basa su uno stretto monitoraggio clinico e sul controllo della pressione arteriosa sistemica con target $<180 / 105 \mathrm{mmHg}$ nelle prime $24 \mathrm{~h}$ dopo il trattamento. Le emorragie sintomatiche che si verificano nelle prime $24 \mathrm{~h}$ dopo fibrinolisi o in corso di ipofibrinogemia rappresentano un'indicazione al trattamento.

Le opzioni terapeutiche sono riassunte in Tabella 1.

La terapia di prima scelta nella coagulopatia da alteplase è la somministrazione di crioprecipitato, derivato del plasma fresco congelato che contiene fibrinogeno, fattore VIII, fattore VXIII e fattore di von Willebrand. Il dosaggio iniziale empirico è di $10 \mathrm{U}$ eventualmente ripetibili in caso di fibrinogemia $<150$ $\mathrm{mg} / \mathrm{dL}$ (10 U di crioprecipitato aumentano i livelli plasmatici di fibrinogeno di circa $50 \mathrm{mg} / \mathrm{dL}$ ). Gli svantaggi sono il potenziale rischio di reazioni trasfusionali e la necessità di scongelamento prima dell'utilizzo.

Nel caso il crioprecipitato non sia immediatamente disponibile o ci siano controindicazioni al suo impiego, gli agenti antifibrinolitici come l'acido tranexamico o l'acido $\varepsilon$-aminocaproico rappresentano l'alternativa terapeutica. L'utilizzo di plasma fresco congelato, complesso protrombinico concentrato o trasfusione di concentrati piastrinici trova impiego solo in caso di coagulopatia nota da dicumarolici o piastrinopenia preesistente.

Infine, in caso di sICH da ematoma intraparenchimale l'evacuazione chirurgica rappresenta un'alternativa terapeutica che va riservata ai pazienti con: emorragia sopratentoriale e deterioramento neurologico, disturbo dello stato di coscienza, shift della linea mediana o segni di ipertensione endocranica refrattari alla terapia medica massimale. E' indicata inoltre nei casi di emorragia cerebellare con compressione delle strutture del tronco e progressione del deficit neurologico.

\section{Crisi epilettiche}

Le crisi comiziali secondarie a patologia cerebrovascolare si distinguono in: crisi epilettiche acute sintomatiche (acute symptomatic seizures, ASS) e crisi non provocate (unprovoked seizures, US) in base all'epoca di insorgenza ( $<0>7$ giorni dall'esordio). Il meccanismo patogenetico delle crisi acute sintomatiche risiede nel danno neuronale acuto, nella eccitotossicità da glutammato e nella disfunzione dei canali ionici. Al contrario gliosi, alterazioni delle plasticità sinaptica, neuroinfiammazione e degenerazione sottendono la comparsa di crisi epilettiche non provocate. Tale classificazione ha pertanto delle importanti implicazioni prognostiche e terapeutiche.

L'incidenza delle ASS è del 3-6\% in fase acuta ed i principali fattori di rischio sono coinvolgimento corticale, ischemia totale del circolo anteriore, ictus disabilitanti ed emorragia intracranica. ${ }^{16,17}$

$\mathrm{Al}$ contrario l'incidenza di crisi non provocate è del $10-12 \%$ in un follow-up di 5-10 anni e raggiunge il $50 \%$ nei casi di infarto maligno dell'arteria cerebrale media sottoposti a craniectomia.

La diagnosi è clinica ma la compromissione dello stato neurologico conseguente all'ictus può mascherare il riconoscimento delle crisi. L'esordio è focale nel $72 \%$ delle crisi con secondaria generalizzazione in quasi un quarto dei casi. ${ }^{18}$ Talora gli unici segni clinici dell'attività critica consistono in fini mioclonie faciali, episodi di deviazione tonica di sguardo o jerks nistagmoidi, parestesie focali, peggioramento del deficit motorio, arresto dell'eloquio o perdita di contatto ambientale. In questi casi l'unico strumento per fare diagnosi è l'elettroencefalogramma (EEG). Inoltre esistono molte condizioni cliniche che mimano l'attività comiziale nei pazienti con ictus in fase acuta quali disturbi ipercinetici

Tabella 1. Opzioni terapeutiche della emorragia intracranica sintomatica.

\begin{tabular}{|c|c|c|}
\hline Trattamento & Indicazioni & Dosaggio \\
\hline Crioprecipitato & Tutte le sICH & $\begin{array}{l}\text { Trasfusione empirica di } 10 \mathrm{U} \text { (eventualmente ripetibili } \\
\text { in caso di fibrinogemia }<150 \mathrm{mg} / \mathrm{dL})\end{array}$ \\
\hline Plasma fresco congelato & Coagulopatia da dicumarolici & $25-50 \mathrm{U} / \mathrm{kg}$ in base al livello di INR \\
\hline Vitamina $\mathrm{K}$ & Coagulopatia da dicumarolici & $10 \mathrm{mg}$ e.v. \\
\hline Concentrato piastrinico & Piastrinopenia $<100.000 / \mathrm{mm}^{3}$ & Pool di 8-10 U \\
\hline Agenti antifibrinolitici: & Tutte le sICH & \\
\hline - Acido tranexamico & & $10 \mathrm{mg} / \mathrm{kg} \mathrm{x} \mathrm{3-4/die}$ \\
\hline - Acido aminocaproico & & $4 \mathrm{~g}$ nella prima ora seguiti da $1 \mathrm{~g}$ ogni $8 \mathrm{~h}$ \\
\hline
\end{tabular}

sICH, symptomatic intracranial hemorrhage. 
del movimento (emicorea, emiballismo), asterixis, movimenti simil-convulsivi nell'occlusione acuta di circolo vertebro-basilare e la limb-shaking-syndrome. Quest'ultima si manifesta con tremore o movimenti involontari limitati ad un arto ed è espressione di un attacco ischemico transitorio da ipoperfusione cerebrale in presenza di severa patologia steno-ostruttiva della carotide interna controlaterale.

Non esistono sufficienti evidenze scientifiche a favore dell'utilizzo di anticomiziali in profilassi primaria per prevenire le crisi epilettiche acute sintomatiche e non provocate pertanto il loro impiego non è raccomandato. ${ }^{17,19}$ Anche nel caso di una singola ASS generalmente la terapia anticomiziale va valutata caso per caso in quanto non esistono forti raccomandazioni a favore ed il rischio di ricorrenza a 10 anni è del $30 \%$ circa; pertanto, laddove si opti per la terapia anticomiziale, è indicata la graduale sospensione dopo la fase acuta. ${ }^{20}$

Al contrario, l'alto rischio di recidiva delle crisi non provocate (fino al $70 \%$ ) definisce una diagnosi di epilessia post-stroke anche in caso di singola US. ${ }^{21}$ Pertanto se l'impiego di antiepilettici non è indicato per prevenire le US, deve essere invece considerato in prevenzione secondaria per ridurre il rischio di ricorrenza delle stesse.

Allo stato dell'arte sono stati condotti pochi studi che abbiano indagato l'efficacia di singoli farmaci anticomiziali nell'epilessia post-stroke; non esistono pertanto raccomandazioni definitive sul farmaco di prima scelta. Le indicazioni attuali derivano infatti dalle evidenze scientifiche sul trattamento delle forme di epilessia focale. La scelta dell'anticomiziale andrà pertanto individualizzata sulla base delle caratteristiche della crisi, della comorbidità, delle preferenze del paziente e delle interazioni farmacologiche. In uno studio randomizzato non sono emerse differenze significative in termini di efficacia tra carbamazepina e levetiracetam a fronte di una minor incidenza di effetti collaterali per quest'ultimo. ${ }^{21}$ Lamotrigina e levetiracetam sono i farmaci di più largo impiego per il favorevole profilo di sicurezza e tollerabilità; fenitoina, valproato e carbamazepina rappresentano valide alternative terapeutiche.

Interessanti per il loro ridotti effetti collaterali ed interazioni farmacologiche, sebbene siano necessari ulteriori studi a riguardo, sono i nuovo farmaci antiepilettici quali: oxcarbazepina, eslicarbazepina, lacosamide e perampanel. ${ }^{21}$

\section{Disfagia}

La disfagia è una complicanza comune in corso di ictus (37-78\%). ${ }^{22}$ Secondo una recente revisione sistematica, l'ampia variabilità dei tassi di incidenza riportati in letteratura dipende dagli strumenti diagnostici utilizzati a seconda che si basi su valutazioni di scree- ning (37\%-45\%), test clinici validati (water swallow test; 51\%-55\%) o metodiche strumentali (esame endoscopico a fibre ottiche o videofluoroscopia; 64\%$78 \%)^{22}$ La videofluoroscopia rappresenta il gold standard diagnostico, tuttavia la diagnosi strumentale sovrastima l'incidenza di disfagia clinicamente significativa e nella pratica quotidiana i test di screening standardizzati come il test all'acqua hanno un sufficiente valore predittivo positivo.

L'atto deglutitorio è costituito da una complessa sequenza di movimenti coordinati il cui controllo volontario è dato dall'area motrice primaria, l'opercolo rolandico, la corteccia premotoria, l'insula ed il giro cingolato anteriore. Suddette area sono poi collegate direttamente od indirettamente tramite le vie discendenti corticobulbari con i nuclei bulbari del troncoencefalo quali ad esempio il nucleo del trigemino o del tratto solitario.

In corso di ischemia cerebrale, la disfagia è causata da una disfunzione della fase orofaringea dovuta a un rallentamento dei movimenti della lingua, ipomobilità dello sfintere laringeo ed esofageo superiore con conseguente ingresso di materiale nel laringe e rischio di aspirazione. Difatti, il rischio di polmonite $a b$ ingestis è superiore nei pazienti disfagici (RR 3,17) ed in particolar modo in presenza di episodi di aspirazione (RR 11,56). ${ }^{22}$

Sesso maschile, età avanzata, gravità del quadro clinico (misurato secondo le scale NIHSS, mRS, Barthel), lesioni ischemiche bilaterali o estese, compromissione dello stato di coscienza aumentano il rischio di disfagia in corso di ictus acuto. ${ }^{23,24}$

La prognosi a breve termine è generalmente favorevole con il 50\% dei casi che presentano un recupero entro 1 settimana, ed il $90 \%$ a due settimane; tuttavia in alcuni pazienti permane una disabilità a lungo termine. ${ }^{25}$

Possibili complicanze della disfagia come disidratazione, polmonite da aspirazione, denutrizione correlano con peggior outcome funzionale, rischio di istituzionalizzazione e aumento della mortalità.

Il FOOD trial, uno studio randomizzato controllato in cui sono stati arruolati 5033 pazienti con ischemia cerebrale acuta ha indagato l'outcome funzionale dei pazienti disfagici in funzione della ripresa dell'alimentazione enterale. Lo studio ha evidenziato come la ripresa della nutrizione enterale precoce si associ ad un decremento non significativo della mortalità $(5,8 \%) \mathrm{e}$ dell'outcome combinato morte/esito sfavorevole $(1,2 \%)$. Inoltre il confezionamento precoce della gastrostomia percutanea endoscopica (PEG) determina un aumento della mortalità non significativo $(1 \%){ }^{26}$

Le attuali indicazioni sono pertanto a favore di una ripresa precoce dell'alimentazione enterale, optando per il posizionamento di sondino naso-gastrico (SNG) qualora le aspettative di recupero della disfagia entro 3-4 settimane dall'ictus siano favorevoli.

Nella diagnosi e gestione della disfagia è importante 
un approccio multidisciplinare che comprenda le figure del medico, infermiere, logopedista e dietista. E' indispensabile una valutazione della funzione deglutitoria prima della ripresa della terapia farmacologica orale e dell'alimentazione per mantenere in sicurezza il paziente e ridurre il rischio di complicanze.

Il test di screening di più largo impiego è il water swallow test: consiste nella somministrazione di $10 \mathrm{ml}$ di acqua mediante un cucchiaio da minestra per 3 volte consecutive e, a seguire, di $50 \mathrm{~mL}$ di acqua in un bicchiere. Dopo ogni deglutizione si verifica la comparsa di tosse immediata o ritardata, voce gorgogliante ed alterazione della fonazione.

Nei pazienti capaci di deglutire $10 \mathrm{~mL}$ di acqua è possibile introdurre l'alimentazione orale utilizzando degli accorgimenti: controllo della struttura della dieta (dieta semisolida e di consistenza omogenea), stazione eretta del busto con capo e collo appoggiati, dimensione del bolo inferiore a un cucchiaino da tè, colpo di tosse dopo ogni atto deglutitorio, deglutizioni multiple anche per piccoli boli al fine di svuotare completamente il faringe, restrizione di liquidi ed utilizzo di bicchiere invece della cannuccia. L'utilizzo di un test di screening formale riduce il rischio di polmonite da aspirazione (OR 0,1). ${ }^{27} \mathrm{La}$ videofluoroscopia o l'esame endoscopico a fibre ottiche (fibreoptic endoscopic evaluation of swallowing, FEES) sono invece indicate, a stabilizzazione clinica, per quantificare il grado di disfagia. La FEES ha il vantaggio di poter essere eseguita anche a letto del paziente, anche in caso di impossibilità al mantenimento della posizione seduta e senza esposizione a radiazioni ionizzanti. E' infine appropriato un programma riabilitativo precoce per la disfagia con esercizi per implementare la mobilità linguale, velare, faringea e cervicale..$^{28}$

\section{Polmonite da aspirazione}

La polmonite da aspirazione rappresenta una delle complicanze più frequenti e una causa comune di febbre nelle prime $48 \mathrm{~h}$ dall'esordio dell'ictus con un tasso di mortalità significativamente più alta rispetto alle altre forme di polmonite $(29,4$ vs $11,6 \%) .{ }^{29} \mathrm{La}$ patogenesi è data dall'aspirazione di materiale a provenienza gastrica e orofaringea nelle vie aeree inferiori e si può manifestare come polmonite chimica, determinata dalla reazione infiammatoria provocata dal contenuto gastrico sterile a basso $\mathrm{PH}(<2,5)$, o polmonite da inalazione secondaria alla penetrazione nelle vie aeree di batteri a partenza dalla flora orofaringea o gastrica. Nella maggioranza dei casi l'episodio di aspirazione non è testimoniato.

Nei pazienti con ictus i fattori di rischio riconosciuti sono: età $>65$ anni, disfagia, riflesso della tosse inefficace, disturbo dello stato di coscienza, sovradosaggio di farmaci sedativi, nutrizione enterale tramite sondino naso gastrico (SNG), ictus con severa disabilità neurologica. La disfagia è il singolo fattore di rischio più importante nei pazienti con patologia cerebrovascolare (OR 12,9), pertanto una diagnosi precoce e un approccio multidisciplinare nel paziente disfagico sono cruciali per ridurre il rischio di aspirazione. ${ }^{30}$ In caso di disfagia post-ictus, è stato dimostrato che l'alimentazione enterale sia in ogni caso preferibile a quella parenterale e la nutrizione enterale tramite SNG correli con miglior outcome funzionale e minor mortalità rispetto al posizionamento precoce di una gastrostomia percutanea endoscopica (PEG). ${ }^{31}$ Anche l'utilizzo di inibitori di pompa protonica e anti$\mathrm{H} 2$ correla con il rischio di polmonite da aspirazione in quanto l'aumento del PH gastrico favorisce la crescita di batteri gram-negativi causando quindi l'incremento della carica batterica dell'inoculo in caso di aspirazione, pertanto il loro uso andrebbe limitato in caso di alto rischio per ulcera gastrica e da stress. Al contrario una metanalisi di 8693 pazienti avrebbe dimostrato un minor rischio di aspirazione nei pazienti in trattamento con ACE inibitori (OR 0,6) attribuibile all'aumento della sostanza $\mathrm{P}$ e del riflesso della tosse nell'ictus in fase acuta. ${ }^{32}$

Clinicamente la polmonite da inalazione può esordire in forma acuta o subacuta, a seconda della carica e virulenza del germe patogeno, con severo distress respiratorio, ipossiemia, tachicardia, rantoli crepitanti e broncospasmo diffuso. Tosse produttiva con escreato maleodorante sono caratteristici delle infezioni da anaerobi a provenienza dalla flora batterica del cavo orale (Peptostreptococchi, Prevotella sp., Bacteroides, Fusobacterium sp., etc.).

La diagnosi di polmonite ab ingestis è una prima di tutto una diagnosi clinica poiché il reperto radiologico risulta in molti casi tardivo. Nella fase precoce la radiografia del torace è negativa in una percentuale fino al $28 \%$ dei casi. ${ }^{33}$ Le caratteristiche radiografiche tipiche consistono in aree di consolidazione a carico dei segmenti superiori dei lobi inferiori o segmenti basali lobi inferiori a seconda che l'episodio di aspirazione sia avvenuto in posizione supina o semi-seduta. Solitamente il lobo inferiore destro è più frequentemente interessato per l'angolo acuto con asse tracheale che favorisce la penetrazione diretta del materiale aspirato per gravità, tuttavia la localizzazione è variabile, anche per il frequente riposizionamento dei pazienti con ictus.

La terapia si basa inizialmente su un'antibioticoterapia empirica sulla scorta dei dati epidemiologici locali e antibiogrammi cumulativi delle singole aziende sanitarie. Nel corso degli ultimi anni è in atto uno shift dei micro-organismi isolati da prevalenza di anaerobi ad aerobi che ha condizionato un cambiamento nella scelta del regime antibiotico iniziale. ${ }^{34}$

Nel caso di forme precoci non complicate le op- 
zioni terapeutiche comprendono: penicillina protetta (amoxicillina/acido clavulanico; ampicillina/sulbactam), cefalosporine di II o di III generazione (cefuroxime; cefotaxime; ceftriaxone) fluorochinolonici (levofloxcina, moxifloxacina) in associazione con agenti attivi contro anaerobi, soprattutto in presenza di fattori di rischio come severa patologie parodontale, ostruzione intestinale (clindamicina; metronidazolo). Uno studio clinico ha dimostrato la superiorità di clindamicina rispetto a metronidazolo nel trattamento delle polmoniti da anaerobi. ${ }^{35}$ In caso di polmoniti ad esordio tardivo o con fattori di rischio per germi multiresistenti le alternative terapeutiche includono: carbapenemici (meropenem; imipenem), cefalosporine di IV generazione (cefepime), beta-lattamici ad ampio spettro (piperacillina/tazobactam; ticarcillina/clavulanato) eventualmente associati ad aminoglucosidi (amikacina) o polimixine (colistina). Solo nei casi con fattori di rischio o colonizzazione nota per stafilococco meticillino-resistente (MRSA) è indicata l'ag-

Tabella 2. Antibioticoterapia della polmonite da aspirazione.

\begin{tabular}{|c|c|}
\hline \multicolumn{2}{|c|}{ Forme precoci } \\
\hline Amoxicillina/acido clavulanico & $875 \mathrm{mg} \times 2$ p.o2,2 g x 3 e.v. \\
\hline Ampicillina/sulbactam & $1,5-3 \mathrm{~g} \mathrm{x} 4$ e.v. \\
\hline Cefuroxime & $1,5 \mathrm{~g} \mathrm{x} 3$ e.v. \\
\hline Ceftrixone & 2 g e.v. \\
\hline Cefotaxime & $2 \mathrm{~g} \mathrm{x} 3$ e.v. \\
\hline $\begin{array}{l}\text { Levofloxacina } \\
\text { e.v. }\end{array}$ & $500 \mathrm{mg} \times 2$ p.o. /e.v. $750 \mathrm{mg}$ \\
\hline Moxifloxacina & 400 mg p.o. /e.v. \\
\hline \multicolumn{2}{|c|}{ Con } \\
\hline Clindamicina & $600 \mathrm{mg} \times 3$ e.v. \\
\hline Metronidazolo & $500 \mathrm{mg}$ x 3-4 p.o. /e.v. \\
\hline \multicolumn{2}{|c|}{ Forme tardive } \\
\hline Meropenem & $1 \mathrm{~g} \mathrm{x} 3$ e.v. \\
\hline Imipenem & 500 mg x 4 e.v. \\
\hline Cefepime & $2 \mathrm{~g} \mathrm{x} 2$ e.v. \\
\hline \multicolumn{2}{|c|}{ Oppure } \\
\hline Piperacillina/tazobactam & 4,5 g x 3-4 e.v. \\
\hline Ticarcillina/acido clavulanico & $3,2 \mathrm{~g} \mathrm{x} 4$ e.v. \\
\hline \multicolumn{2}{|c|}{ Con } \\
\hline Amikacina & $15 \mathrm{mg} / \mathrm{kg}$ e.v. \\
\hline \multicolumn{2}{|l|}{ Colistina } \\
\hline \multicolumn{2}{|c|}{$\begin{array}{l}\text { Se fattori di rischio per Stafilococco meticillino-resistente } \\
\text { (MRSA) }\end{array}$} \\
\hline Linezolid & 600 mg x 2 p.o./e.v. \\
\hline Vancomicina & $15 \mathrm{mg} / \mathrm{kg} \times 2$ e.v. \\
\hline
\end{tabular}

giunta di linezolid o vancomicina al regime antibiotico (Tabella 2).

La durata della terapia antibiotica varia da un minimo di 5-7 giorni a 2-3 settimane in caso di infezioni complicate, coinvolgimento multilobare, localizzazione extrapolmonare, evoluzione ascessuale o condizioni cliniche scadute. In questi caso il drenaggio ed esame colturale devono guidare la modifica della terapia antibiotica in atto.

In caso di polmonite chimica da aspirazione invece la gestione iniziale del paziente mira al mantenimento della pervietà delle vie aeree ed alla gestione dell'edema e broncospasmo causato dall'inalazione del materiale gastrico. A seconda della gravità del quadro clinico le opzioni terapeutiche comprendono la broncoaspirazione, bronscopia con toilette bronchiale, intubazione e ventilazione meccanica invasiva. La chemioprofilassi antibiotica non è invece indicata in fase acuta ma è va considerata caso per caso nei pazienti fragili, con condizioni cliniche generali scadute e in terapia con inibitori di pompa protonica, rivalutando l'appropriatezza terapeutica a 3 giorni in base all'evoluzione clinica.

\section{Trombosi venosa profonda ed embolia polmonare}

Nei pazienti con stroke ischemico acuto il tromboembolismo rappresenta una delle principali complicanze, tanto da condizionarne l'outcome in termini di morbidità e mortalità. In assenza di tromboprofilassi fino al $25 \%$ delle morti precoci è dovuto ad embolia polmonare la quale ha un tasso di mortalità pari al $50 \% \cdot{ }^{36}$ In questi pazienti, tra i fattori di rischio per tromboembolismo venoso, oltre a neoplasia e pregresso evento tromboembolico vanno ricordati l'età avanzata ed i bassi punteggi del Barthel Index $(\leq 9) .37,38$ Nella descrizione delle caratteristiche cliniche, alcuni dati della letteratura rilevano come solo in 1 paziente su 3 il tromboembolismo sia sintomatico ed, in caso di trombosi venosa profonda sia colpito l'arto plegico in 4 casi su $5 .{ }^{39}$ Le strategie di profilassi tromboembolica includono:

- profilassi meccanica: i) calze elasto-compressive; ii) compressione pneumatica intermittente;

- profilassi farmacologica: i) eparina non frazionata; ii) eparina a basso peso molecolare; iii) fondaparinux;

- mobilizzazione precoce.

\section{Profilassi meccanica}

I Trial CLOTS hanno valutato l'efficacia della profilassi meccanica per eventi tromboembolici in pazienti con stroke acuto. Nel CLOTS-1 l'impiego di elasto- 
compressione applicata alla radice della coscia non appare efficace nel prevenire l'outcome combinato di trombosi venosa profonda prossimale asintomatica e tromboembolismo venoso asintomatico. Nel CLOTS2 gli eventi tromboembolici (definiti con lo stesso outcome del CLOTS-1) sono risultati meno frequenti nei pazienti con elastocompressione applicata a livello della radice della coscia rispetto a quella sotto il ginocchio. ${ }^{40}$ Il CLOTS-3 ha rilevato l'efficacia della compressione pneumatica intermittente nella prevenzione degli eventi tromboembolici in pazienti con stroke. ${ }^{41}$

\section{Profilassi farmacologica}

Va ricordato che nei più importanti trial che hanno testato le eparine a basso peso molecolare (MEDENOX, PREVENT) e il fondaparinux (ARTEMIS) per la profilassi del tromboembolismo in pazienti medici erano stati esclusi i soggetti con ictus ischemico nei 3 mesi precedenti. In letteratura abbiamo a disposizione solo pochi studi sulla profilassi tromboembolica farmacologica in pazienti con ictus ischemico acuto, con l'impiego di 3 eparine a basso peso molecolare: certoparina, dalteparina, enoxaparina. ${ }^{42-44}$ Per quest'ultima, somministrata entro 48 ore dall'evento ictale alla dose di $40 \mathrm{mg}$ sc/od, lo studio PREVAIL ha dimostrato una significativa superiorità rispetto all'eparina non frazionata nella prevenzione del tromboembolismo venoso, in presenza, però, di una maggiore incidenza di sanguinamenti extracranici. ${ }^{45} \mathrm{~L}$ 'impiego di una profilassi farmacologica determina una riduzione di 38 eventi tromboembolici e l'incremento di 5 eventi emorragici sintomatici (di cui 3 endocranici) per 1000 pazienti con stroke ischemico e ridotta mobilità. ${ }^{46}$ Pertanto le Linee Guida dell'European Stroke Organization (ESO) suggeriscono l'impiego della compressione pneumatica intermittente, e dell'EBPM qualora i benefici di un'aumentata protezione per eventi tromboembolici superino i rischi di un'aumentata incidenza di eventi emorragici. ${ }^{47}$ In trials retrospettivi il Fondaparinux impiegato nella profilassi tromboembolica del paziente con stroke ischemico ha dimostrato uguale efficace e sicurezza rispetto all'eparina non frazionata. ${ }^{48}$

Per quanto riguarda la durata della terapia farmacologica per la profilassi tromboembolica, in una sottoanalisi del trial EXCLAIM un trattamento di durata extended (28 \pm 4 giorni) rispetto allo standard $(10 \pm 4$ giorni) ha ridotto l'incidenza di eventi tromboembolici aumentando, però, il rischio di emorragie maggiori. ${ }^{49}$ Da quanto detto si può concludere che in caso di ictus ischemico la scelta terapeutica per la profilassi tromboembolica va individualizzata, valutando il rischio trombotico ed emorragico del paziente ed il rischio di sanguinamento della lesione ischemica cerebrale, di cui sono stati riconosciuti alcuni predittori quali: estensione ed etiologia cardioembolica. ${ }^{50}$

\section{Complicanze cardiache}

Esiste una stretta correlazione tra ictus ischemico e patologia cardiaca come dimostrato dai fattori di rischio ampiamente sovrapponibili e dall'alta incidenza di complicanze cardiache nella fase acuta dell'ischemia cerebrale. La necessità di una diagnosi e trattamento tempestivo delle complicanze cardiache è testimoniato dal tasso di mortalità per cause cardiache nei primi 3 mesi dopo ictus ischemico, pari al $4 \%{ }^{51}$

In caso di danno lesionale dei network neurali che presiedono al controllo del sistema nervoso autonomo, in particolare del lobo dell'insula, si ha un improvviso squilibro tra tono orto e parasimpatico, con l'attivazione prevalente del primo che si può manifestare con crisi ipertensive, alterazioni elettrocardiografiche, aritmie, infarto del miocardio, cardiomiopatia da stress, scompenso cardiaco congestizio e morte cardiaca improvvisa.

Talora l'unico indice di danno miocardico consiste in un aumento indici di miocardionecrosi in assenza di patologia coronarica. Inoltre i livelli di troponina $\mathrm{T}$ in fase acuta correlano con l'outcome funzionale e mortalità a 1,3 e 6 mesi. I possibili meccanismi fisiopatologici del rialzo di tale enzima in fase acuta sono: i) patologia coronarica concomitante; ii) ischemia da discrepanza per aumentato consumo di ossigeno; iii) stunning miocardico neurogenico: la scarica catecolaminergica causa un sovraccarico intracellulare di calcio, disfunzione contrattile e miocitolisi con aree di necrosi e microemorragie subendocardiche in corrispondenza dei nervi epicardici. Lo stunning miocardico neurogenico è simile all'infarto del miocardio e si presenta con alterazioni ECG, rialzo degli enzimi, ridotta contrattilità miocardica e alterazioni della cinetica segmentale in assenza di patologia coronarica. Le alterazioni sopradescritte sono reversibili. Esistono dei criteri di diagnosi differenziale con l'ischemia miocardica ma sono stati validati solo per le forme di cardiomiopatia da stress in corso di emorragia subaracnoidea e quindi non sono completamente applicabile all'ictus ischemico: pertanto l'evoluzione clinica rappresenta un punto cruciale per la corretta classificazione diagnostica. ${ }^{52}$

L'incidenza di casi di concomitante infarto del miocardio e ictus ischemico impone un attento monitoraggio clinico, soprattutto nei pazienti ad alto rischio (diabete, patologia coronarica pre-esistente, vasculopatia periferica, ictus disabilitanti). Inoltre le indicazioni dell'AHA/ASA (American Heart Association/American Stroke Association) suggeriscono uno studio non invasivo della riserva coronarica nei pazienti con ictus ischemico e multipli fattori di rischio cardiovascolari o una classe di rischio cardiovascolare $>20 \%$ secondo lo score di Framingham. ${ }^{53}$ 
Anche le alterazioni elettrocardiografiche sono frequenti, con un'incidenza del $69 \%$ in fase acuta. ${ }^{54} \mathrm{Le}$ più comuni sono: sottoslivellamento del tratto ST, onde $\mathrm{T}$ invertite, prolungamento e dispersione del QT. In particolare, il prolungamento del QTc è un fattore di rischio per aritmie maligne (ex. torsione di punta) e correla in modo indipendente con aumento della mortalità. ${ }^{55}$

Le stesse aritmie cardiache sono riportate nel 25\% dei pazienti nelle prime $24-72 \mathrm{~h}$ e determinano un aumento della mortalità per instabilità emodinamica $\mathrm{e}$ morte cardiaca improvvisa. ${ }^{56,57}$ Le più comuni sono: fibrillazione atriale, tachicardia sopraventricolare, battiti ectopici ventricolari e tachicardia ventricolare ma anche asistolia, blocco seno-atriale, blocco atrioventricolare di alto grado e fibrillazione atriale a bassa risposta ventricolare. ${ }^{57}$ In alcuni casi il ruolo della fibrillazione atriale in fase acuta è dibattuto laddove evidenze scientifiche suggeriscono come la stessa possa essere un epifenomeno del transitorio squilibrio nel sistema nervoso autonomo nei pazienti con ischemia insulare..$^{58}$ I fattori predittivi per l'insorgenza di aritmie sono principalmente l'età e la gravità del quadro neurologico inteso come NIHSS all'ingresso pertanto questi indici dovrebbero essere usati per la stratificazione di rischio e la selezione dei candidati a monitoraggio ECG prolungato.

Le attuali linee guida indicano l'appropriatezza del monitoraggio continuo nelle prime $48 \mathrm{~h}$, ed in particolare nei pazienti con: cardiopatie preesistenti, storia di aritmie, pressione arteriosa instabile, insufficienza cardiaca, alterazioni dell'ECG di base. Tuttavia un monitoraggio prolungato $(>72 \mathrm{~h})$ è preferibile per la ricerca di FA occulta e mandatorio in caso di instabilità emodinamica. ${ }^{59-61}$

Nei casi in cui non sia disponibile il monitoraggio ECG continuo è opportuno eseguire controlli seriali dell'ECG almeno nelle prime $24 \mathrm{~h}$. Da quanto illustrato le complicanze dello stroke ischemico in fase acuta sono comuni e presentano un importante significato prognostico negativo, aumentando in maniera significativa la disabilità residua e la mortalità; per questo devono essere riconosciute e trattate tempestivamente e, laddove possibile, prevenute.

Va ricordato che se è mandatorio trattare condizioni patologiche che mettono a rischio la vita del paziente è altrettanto fondamentale prevenire le importanti disabilità che hanno un forte impatto sulla qualità di vita del singolo e sull'impiego di risorse della collettività; per questo sono essenziali: percorsi dedicati (per la disfagia), un'attenta gestione della terapia farmacologica (per le complicanze infettive, per le crisi comiziali o per la profilassi trombo embolica) e una stretta e chiara collaborazione mutidisciplinare per affrontare situazioni anche urgenti (infarcimento emorragico sintomatico, aritmie).
La complessità di queste condizioni patologiche richiede competenze multidisciplinari e prevede l'interazione di diversi Specialisti; questo giustifica e rafforza il concetto di una Stroke Unit dove l'Internista può avere un ruolo centrale nell'orientare il percorso diagnostico-terapeutico e nel coordinare i diversi Specialisti.

\section{Bibliografia}

1. Alexandrov AV, Grotta JC. Arterial reocclusion in stroke patients treated with intravenous tissue plasminogen activator. Neurology 2002; 59: 862-67.

2. Krieger DW, Demchuk AM, Kasner SE, et al. Early clinical and radiological predictors of fatal brain swelling in ischemic stroke. Stroke 1999; 30: 287-92.

3. Hacke W, Schwab S, Horn M, et al. 'Malignant' middle cerebral artery territory infarction: clinical course and prognostic signs. Arch Neurol 1996; 53: 309-15.

4. Ong CJ, Gluckstein J, Laurido-Soto O, et al. Enhanced detection of Edema in Malignant Anterior Circulation Stroke (EDEMA) score: a risk prediction tool. Stroke 2017;48:1969-72.

5. Jauss M, Krieger D, Homing C, et al. Surgical and medical management of patients with massive cerebellar infarctions: results of the German-Austrian Cerebellar Infarction Study. J Neurol 1999; 246: 257-64.

6. White H, Cook D, Venkatesh B. The use of hypertonic saline for treating intracranial hypertension after traumatic brain injury. Anesth Analg 2006 Jun;102.6:18361846.

7. Aiyagari V, Deibert E, Diringer MN. Hypernatremia in the neurologic intensive care unit: how high is too high? J Crit Care 2006;21.2:163-172.

8. Alexander P, Heels-Ansdell D, Siemieniuk R, et al. Hemicraniectomy versus medical treatment with large MCA infarct: a review and meta-analysis. BMJ Open 2016; 6:e014390.

9. Seet RC, Rabinstein AA. Symptomatic intracranial hemorrhage following intravenous thrombolysis for acute ischemic stroke: a critical review of case definitions. Cerebrovasc Dis 2012;34:106-114.

10. Seet RC, Rabinstein AA. Symptomatic intracranial hemorrhage following intravenous thrombolysis for acute ischemic stroke: a critical review of case definitions. Cerebrovasc Dis 2012;34:106-114.

11. Whiteley WN, Slot KB, Fernandes P, et al. Risk factors for intracranial hemorrhage in acute ischemic stroke patients treated with recombinant tissue plasminogen activator: a systematic review and meta-analysis of 55 studies. Stroke 2012;43:2904-2909.

12. Larrue V, von Kummer R R, Müller A, Bluhmki E. Risk factors for severe hemorrhagic transformation in ischemic stroke patients treated with recombinant tissue plasminogen activator: a secondary analysis of the European-Australasian Acute Stroke Study (ECASS II). Stroke 2001;32.2:438-41.

13. Seners P, Turc G, Tisserand M, et al. Unexplained early neurological deterioration after intravenous thrombolysis: incidence, predictors, and associated factors. Stroke. 2014;45:2004-2009 
14. Yaghi S, Willey JZ, Cucchiara B, et al. Treatment and Outcome of Hemorrhagic Transformation After Intravenous Alteplase in Acute Ischemic Stroke: A Scientific Statement for Healthcare Professionals From the American Heart Association/American Stroke Association. Stroke 2017;48.12:e343-e361

15. Strbian D, Engelter S, Michel P, et al. Symptomatic intracranial hemorrhage after stroke thrombolysis: the SEDAN score. Annals of neurology 2012; 71.5: 634-641.

16. Strzelczyk A, Haag A, Raupach H, et al. Prospective evaluation of a post-stroke epilepsy risk scale. J Neurol 2010;257:1322-6.

17. Holtkamp M, Beghi E, Benninger F, et al. European Stroke Organisation guidelines for the management of post-stroke seizures and epilepsy. European stroke journal 2017;2.2:103-115.

18. Stefanidou M, Das RR, Beiser AS, et al. Incidence of seizures following initial ischemic stroke in a community-based cohort: The Framingham Heart Study. Seizure 2017;47:105-10.

19. Hemphill JC III, Greenberg SM, Anderson CS, et al. Guidelines for the management of spontaneous intracerebral hemorrhage: A guideline for healthcare professionals from the American Heart Association/ American Stroke Association. Stroke 2015;46:2032-60.

20. Hesdorffer DC, Benn EK, Cascino GD, et al. Is a first acute symptomatic seizure epilepsy? Mortality and risk for recurrent seizure. Epilepsia 2009; 50:1102-1108.

21. Xu MY. Poststroke seizure: optimising its management. Stroke and Vascular Neurology 2019;4: e000175.

22. Martino R, Foley N, Bhogal S, et al. Dysphagia after stroke. Incidence, diagnosis, and pulmonary complications. Stroke 2005;36: 2756-63.

23. Crary MA, Carnaby-Mann GD, Miller L, et al. Dysphagia and nutritional status at the time of hospital admission for ischemic stroke. J Stroke Cerebrovasc Dis 2006;15:164-71

24. Langdon PC, Lee AH, Binns CW. Dysphagia in acute ischaemic stroke: severity, recovery and relationship to stroke subtype. Journal of Clinical Neuroscience 2007;14.7: 630-634.

25. Smithard DG, O'Neill PA, England RE, et al. Natural history of dysphagia following a stroke. Dysphagia 1997; 12:188-93.

26. Dennis MS, Lewis SC, Warlow C. FOOD Trial Collaboration. Effect of timing and method of enteral tube feeding for dysphagic stroke patients (FOOD): a multicentre randomised controlled trial. Lancet 2005; 365 : 764-72

27. Hinchey JA, Shephard T, Furie K, et al. Formal dysphagia screening protocols prevent pneumonia. Stroke 2005;36.9:1972-1976.

28. Robbins J, Kays SA, Gangnon RE, et al. The effects of lingual exercise in stroke patients with dysphagia. Arch Phys Med Rehabil 2007; 88.2:150-158.

29. Lindenauer PK, Strait KM, Grady JN et al. Variation in the Diagnosis of Aspiration Pneumonia and Association with Hospital Pneumonia Outcomes. Ann Am Thorac Soc. 2018;15.5:562-569.

30. van der Maarel-Wierink CD, Vanob- bergen JN, Bronkhorst EM, et al. Meta-analysis of dysphagia and aspiration pneumonia in frail elders. J Dent Res 2011;90:1398-404.
31. Dennis MS, Lewis SC, Warlow C. FOOD Trial Collaboration. Effect of timing and method of enteral tube feeding for dysphagic stroke patients (FOOD): a multicentre randomised controlled trial. Lancet 2005; 365: 764-72.

32. Shinohara Y, Origasa H. Post-stroke pneumonia prevention by angiotensin-converting enzyme inhibitors: results of a meta-analysis of five studies in Asians. Adv Ther. 2012;29.10:900-912.

33. Miyashita N, Kawai Y, Tanaka T, et al. Detection failure rate of chest radiography for the identification of nursing and healthcare-associated pneumonia. J Infect Chemother 2015;21.7:492-6.

34. Mandell LA, Niederman MS. Aspiration pneumonia. NEJM 2019, 380.7: 651-663.

35. Perlino CA. Metronidazole vs clindamycin treatment of anerobic pulmonary infection: failure of metronidazole therapy. Arch Intern Med 1981;141:1424-1427.

36. Kamphuisen PW, Agnelli G, Sebastianelli M Prevention of venous thromboembolism after acute ischemic stroke. J Thromb Haemost 2005; 3.6:1187-1194

37. Pongmoragot J, Rabinstein AA., Nilanont $Y$, et al. Pulmonary embolism in ischemic stroke: clinical presentation, risk factors and outcome. J Am Heart Assoc 2013;2.6:e000372

38. Kelly J, Rudd A, Lewis RR, et al. Venous thromboembolism after acute ischemic stroke: a prospective study using magnetic resonance direct thrombus imaging. Stroke 2004; 35.10:2320-2325.

39. Dennis M, Mordi N, Graham C, et al. CLOT trial collaboration. The timing, extent, progression and regression of deep vein thrombosis in immobile stroke patients: observational data from CLOTS multicenter randomized trials. J Thromb Haemost 2011; 9.11: 2193

40. Kearon C, O'Donnell M. Graduated compression stockings to prevent venous thromboembolism in hospital: evidence from patients with acute stroke. Pol Arch Med Wewn 2011; 121.1-2:40-43.

41. Denni M, Sandercock P, Reid J, et al. Effectiveness of intermittent pneumatic compression in reduction of risk of deep vein thrombosis in patients who have had a stroke (CLOTS 3): a multicenter randomised controlled trial. Lancet 2013;382.9891:516-24.

42. Diener HC, Ringelstein EB, von Kummer R, et al. Prophylaxis of thrombotic and embolic events in acute ischemic stroke with the low-molecular-weight heparin ertoparin: results of the PROTECT Trial. Stroke 2006; 37.1:139-144.

43. Sandset PM, Dahl T, Stiris M, et al. A double-blind and randomized placebo-controlled trial of low molecular weight heparin once daily to prevent deep-vein thrombosis in acute ischemic stroke. Semin Thromb Hemost 1990; 16:25-33

44. Hillborn M, Erila T, Sotaniemi K, et al. Enoxaparin vs heparin for prevention of deep-vein thromboembolism in acute stroke: a randomized, double-blind study. Acta Neurol Scand 2002; 106.2: 84-92

45. Sherman DG, Albers GW, Blandin C, et al. The efficacy and safety of enoxaparin versus unfractionated heparin for the prevention of venous thromboembolism after ischemic stroke (PREVAIL Study): an open-label randomised comparison. Lancet 2007 ; 369.9570: 1347-55.

46. Lansberg MG, O’Donnell MJ, Khatri P, et al. Antithrombotic and thrombolytic therapy for ischemic 
stroke: Antithrombotic Therapy and Prevention of Thrombosis, 9th ed: American College of Chest Physicians Evidence-Based Clinical Practice Guidelines. Chest 2012; 141.2: e601s-e636s.

47. Dennis M, Caso V, Kappelle LJ, et al. European Stroke Organisation (ESO) guidelines for prophylaxis for venous thromboembolism in immobile patients with acute ischaemic stroke. Eur Stroke J 2016; 1: 6-19.

48. Hackett CT, Ramanathan RS, Malhotra K, et al. Safety of venous thromboembolism prophylaxis with fondaparinux in ischemic stroke. Throm Res $2015 ; 135.2: 249-54$

49. Turpie AG, Hull RD, Sghellong SM, et al. EXCLAIM Investigator. Venous thromboembolism risk in ischemic stroke patients receiving extended-duration enoxaparin prophylaxis: results from the EXCLAIM study. Stroke 2013; 44.1: 249-51.

50. Paciaroni M, Agnelli G, Corea F, et al. Early hemorrhagic transformation of brain infarction: rate, predictive factors, and influence on clinical outcome: results of a prospective multicenter study. Stroke 2008;39.8:2249-56.

51. Prosser J, MacGregor L, Lees KR, et al. Predictors of early cardiac morbidity and mortality after ischemic stroke. Stroke 2007; 38: 2295-302.

52. Bulsara KR, McGirt M, et al. Use of the peak troponin value to differentiate myocardial infarction from reversible neurogenic left ventricular dysfunction associated with aneurysmal subarachnoid hemorrhage. Journal of neurosurgery, 2003, 98.3: 524-528.

53. Adams RJ, Chimowitz MI, Alpert JS, et al. Coronary risk evaluation in patients with transient ischemic attack and ischemic stroke: a scientific statement for healthcare professionals from the Stroke Council and the Council on Clinical Cardiology of the American Heart Association/American Stroke Association. Circulation, 2003, 108.10: 1278-1290.

54. Tatschl C, Stollberger C, Matz K, et al. Insular involvement is associated with QT prolongation: ECG abnormalities in patients with acute stroke. Cerebrovasc Dis 2006; 21: 47-53.

55. Wong KY, Mac Walter RS, Douglas D, et al. Long QTc predicts future cardiac death in stroke survivors. Heart 2003; 89: 377-81.

56. Prosser J, MacGregor L, Lees KR, et al. Predictors of early cardiac morbidity and mortality after ischemic stroke. Stroke. 2007;38:2295-2302.

57. Kallmünzer B, Breuer L, Kahl N, Bobinger T, RaazSchrauder D, Huttner HB, Schwab S, Köhrmann M. Serious cardiac arrhythmias after stroke: incidence, time course, and predictor: a systematic, prospective analysis. Stroke, 2012, 43.11: 2892-2897.

58. Sposato LA, Riccio PM, Hachinski V. Poststroke atrial fibrillation: cause or consequence? Critical review of current views. Neurology, 2014, 82.13: 1180-1186.

59. Kirchhof P, Benussi S, Kotecha D, et al. 2016 ESC Guidelines for the management of atrial fibrillation developed in collaboration with EACTS. European journal of cardio-thoracic surgery, 2016, 50.5: e1-e88.

60. Warner JJ, Harrington RA, Sacco RL, Elkind MSV. Guidelines for the Early Management of Patients With Acute Ischemic Stroke: 2019 Update to the 2018 Guidelines for the Early Management of Acute Ischemic Stroke. Stroke. 2019; STROKEAHA119027708.

61. (2017) Linee guida SPREAD per prevenzione e trattamento dell'ictus (versione italiana) 


\title{
Prevenzione secondaria dell'ictus ischemico
}

\author{
Luana Gentile
}

Dipartimento di Neuroscienze Umane, Unità di Trattamento Neurovascolare, Policlinico Umberto I, Università La Sapienza, Roma, Italia

\section{Introduzione}

Nei pazienti andati incontro ad ischemia cerebrale, il rischio di recidiva di ictus è stimato essere in media di circa il 5\% per anno. Negli Stati Uniti si verificano ogni anno circa 795.000 ictus, di cui 691.000 sono ischemici e circa 185.000 ricorrenti. Nei pazienti con attacco ischemico transitorio (TIA) è stata stimata una percentuale di rischio di ictus ischemico del $19 \%$ a 10 anni. ${ }^{1}$ Il rischio di recidiva aumenta in presenza di età avanzata e di più di due fattori di rischio per eventi cerebrovascolari, come evidenziato dallo studio Framingham. ${ }^{2}$ In questa prospettiva, appare di fondamentale importanza l'attuazione di specifiche strategie di prevenzione secondaria, che richiedono la definizione del rischio globale del paziente e la corretta identificazione dei meccanismi eziopatogenetici sottostanti. Il rischio di ricorrenza di ictus, così come il migliore algoritmo terapeutico, sono infatti strettamente correlati al meccanismo responsabile dell'ictus. ${ }^{3}$

Gli interventi di prevenzione secondaria dell'ischemia cerebrale possono pertanto strutturarsi su due livelli: i) strategie generali di prevenzione secondaria, che implicano modifiche dello stile di vita e controllo dei fattori di rischio associati; ii) strategie di prevenzione secondaria in relazione all'eziologia dell'ictus ischemico, che comportano, in aggiunta alle strategie generali, un sapiente utilizzo dei farmaci attualmente disponibili (soprattutto antiaggreganti/anticoagulanti).

Corrispondente: Luana Gentile, Dipartimento di Neuroscienze Umane, Unità di Trattamento Neurovascolare, Policlinico Umberto I, Università La Sapienza, Roma, Italia.

Fax: +39.06.49979526.

E-mail: luana.gentile@uniroma1.it

Articolo pubblicato secondo la Creative Commons Attribution NonCommercial 4.0 License (CC BY-NC 4.0).

${ }^{\circ}$ Copyright: the Author(s), 2020

Licensee PAGEPress, Italy

QUADERNI - Italian Journal of Medicine 2020; 8(2):128-141

\section{Strategie generali di prevenzione secondaria dell'ictus}

\section{Cambiamenti dello stile di vita}

Includono:

- cessazione dell'abitudine tabagica: il fumo di sigaretta è responsabile del $12 \%-37 \%$ di tutti gli ictus, con una azione dose-dipendente. ${ }^{4,5}$ Lo studio REGARDS $^{6}$ evidenzia inoltre come anche l'esposizione al fumo passivo rappresenti un fattore di rischio per ictus, aumentandone il rischio di circa il 30\% rispetto ai non esposti. Contrariamente alla ben nota associazione tra fumo di sigaretta e rischio di prima occorrenza di ictus, i dati sull'associazione con la recidiva di ictus sono limitati, tanto che le linee guida dell'American Heart Association $(\mathrm{AHA})^{3}$ sulla prevenzione secondaria dell'ictus, raccomandano, con Classe I ma livello di evidenza $\mathrm{C}$, la cessazione dell'abitudine tabagica nei pazienti già andati incontro a ictus o TIA. Il Cardiovascular Health Study $y^{7}$ ha tuttavia evidenziato una importante associazione tra abitudine tabagica e aumentato rischio di recidiva di ictus nei più anziani e una recente sottoanalisi dei pazienti arruolati nel trial IRIS (Insulin Resistance Intervention After Stroke) ha evidenziato come la cessazione del fumo di sigaretta entro sei mesi dall'ictus ischemico cerebrale comporti una riduzione del rischio relativo di ricorrenza del $34 \%$ e assoluto del $6,9 \%$ a 4,8 anni dall'evento. ${ }^{5}$

- dieta bilanciata: è noto come una sana alimentazione risulti fondamentale nella prevenzione primaria dell'ictus ischemico e in particolare come l'aderenza ad un regime alimentare Mediterraneo sia in grado di determinare una riduzione del rischio di ictus a 5 anni del $30 \% .{ }^{8}$ Tuttavia, anche in questo caso i dati relativi a specifici pattern alimentari e prevenzione secondaria sono limitati e non vi sono attualmente trial clinici al riguardo. Le linee guida $\mathrm{AHA}^{3}$ suggeriscono come raccomandazione di Classe II e livello di evidenza $\mathrm{C}$ nei pazienti andati incontro a ictus ischemico o TIA una riduzione dell'introito alimentare di sodio a $<2,4$ $\mathrm{g} /$ die e una dieta di tipo Mediterraneo che prediliga 
alimenti di origine vegetale quali frutta, verdura, cereali integrali e legumi, pesce, olio di oliva.

- regolare attività fisica: diversi studi e metanalisi hanno evidenziato come una regolare attività fisica sia in grado di determinare una riduzione del rischio di ictus dal $25 \%$ al $30 \%,{ }^{9,10}$ anche in considerazione degli effetti protettivi verso lo sviluppo di malattie metaboliche e cardiovascolari. ${ }^{11}$ I pazienti sopravvissuti ad una ischemia cerebrale, tuttavia, a causa spesso delle importanti sequele neurologiche e della disabilità residua, non risultano in grado di seguire le raccomandazioni internazionali sullo svolgimento di una regolare attività fisica. Il trial PREVENT ${ }^{12}$ potrebbe ulteriormente chiarire e confermare $\mathrm{i}$ benefici dell'attività fisica in prevenzione secondaria.

- controllo del peso corporeo: Vari studi epidemiologici hanno dimostrato che a partire da un body mass index (BMI) di $20 \mathrm{~kg} / \mathrm{m}^{2}$, per ogni unità di aumento di BMI il rischio di ictus ischemico aumenta di circa il $5 \% .{ }^{10}$ Se ciò è vero per la prevenzione primaria degli eventi cerebrovascolari, il beneficio della perdita di peso nei pazienti obesi andati incontro a ictus ischemico o TIA sembra essere controverso, tanto che in letteratura si parla di obesity paradox. Recenti studi hanno infatti evidenziato come pazienti obesi andati incontro a ictus avessero un minore rischio di ricorrenza di eventi vascolari maggiori rispetto ai non obesi. ${ }^{3,12,13}$

- riduzione del consumo alcolico: la relazione tra consumo di bevande alcoliche e rischio di ictus dipende dalla quantità di alcol e dal sottotipo eziologico di ictus. Infatti, per quanto riguarda l'ictus ischemico vari studi hanno dimostrato una associazione protettiva tra consumo moderato di alcol ( $\leq 2$ drinks al giorno per gli uomini e $\leq 1$ drink al giorno per le donne) e ictus ischemico, mentre un elevato introito di alcol è correlato ad aumentato rischio di ischemia cerebrale. La correlazione tra assunzione di alcol e ictus è invece lineare per l'ictus emorragico. ${ }^{14}$ Pochi studi hanno in realtà esaminato la relazione tra assunzione di alcol e prevenzione secondaria: modiche quantità di alcol sembrerebbero avere un'azione protettiva anche in relazione alla recidiva di ictus, tuttavia l'abuso alcolico aumenterebbe il rischio di ricorrenza. ${ }^{15}$

\section{Ipertensione arteriosa}

Il trattamento dell'ipertensione arteriosa (IPA) è probabilmente uno dei più importanti interventi nell'ambito della prevenzione secondaria dell'ictus ischemico. La prevalenza di IPA tra i soggetti andati incontro a ictus è di circa il $70 \%,{ }^{3}$ tanto che le linee guida italiane ${ }^{11}$ raccomandano in tali pazienti il miglior controllo possibile dei valori pressori, con predilezione di farmaci agenti sul sistema renina-angiotensina, calcio-antagonisti e diuretici. Il primo studio a dimostrare il beneficio del trattamento dell'ipertensione arteriosa è stato il Post-Stroke Antihypertensive Treatment Study (PATS) ${ }^{16}$ studio cinese randomizzato (placebo versus indapamide). I risultati sono stati confermati dal trial clinico Perindopril Protection Against Recurrent Stroke Study (PROGRESS), ${ }^{17}$ che ha randomizzato 6015 pazienti ipertesi o non ipertesi con pregresso ictus o TIA a trattamento antipertensivo con perindopril $4 \mathrm{mg}$ più indapamide (aggiunto a discrezione del curante) o placebo. Dopo 4 anni di follow up nel gruppo sottoposto a trattamento si è evidenziata una riduzione di ricorrenza di ictus del 28\% (95\% IC, 17\%-38\%). Una metanalisi del $2009,{ }^{18}$ che ha incluso dieci trial randomizzati che comparavano terapia antipertensiva contro placebo, ha confermato che il trattamento antipertensivo nei pazienti andati incontro a ictus o TIA riduce il rischio di ricorrenza di eventi cerebrovascolari. Relativamente ai pazienti non in trattamento antipertensivo antecedente l'evento ischemico cerebrale, è ragionevole iniziare il trattamento farmacologico se, a stabilizzazione dei valori pressori facenti seguito l'evento acuto, questi fossero $\geq 140 \mathrm{mmHg}$ di sistolica e $\geq 90 \mathrm{mmHg}$ di diastolica. ${ }^{3}$ Relativamente al target pressorio ottimale da raggiungere in prevenzione secondaria non vi sono al momento sufficienti evidenze e questo dovrebbe essere individualizzato: le linee guida dell' $\mathrm{AHA}^{3}$ suggeriscono il raggiungimento di valori pressori $<140$ $\mathrm{mmHg}$ di sistolica e $<90 \mathrm{mmHg}$ di diastolica. Il trial Secondary Prevention of Small Subcortical Strokes (SPS3) $)^{19}$ effettuato su 3000 pazienti con ictus ischemico lacunare ha evidenziato una riduzione di recidive di ictus non significativa per valori di sistolica $<130$ $\mathrm{mmHg}$ contro valori $<140 \mathrm{mmHg}$. Il trial randomizzato Action to Control Cardiovascular Risk in Diabetes blood pressure (ACCORD BP $)^{20}$ ha inoltre evidenziato che nei pazienti diabetici ad alto rischio non vi è alcun beneficio nel raggiungimento di valori di sistolica $<120 \mathrm{mmHg}$ (trattamento intensivo) contro i $140 \mathrm{mmHg}$.

\section{Diabete mellito}

Il diabete mellito (DM) costituisce indubbiamente uno dei principali fattori di rischio per ictus, anche in relazione alle frequenti comorbidità che ad esso si accompagnano ed è responsabile di circa il $60 \%$ delle ricorrenze di ictus nella popolazione più anziana [hazard ratio (HR) 1,59; intervallo di confidenza (IC) $1,07-2,3] .{ }^{21}$ Non vi sono sicure evidenze sul management del DM nei pazienti andati incontro a ischemia cerebrale, per cui la gestione di tali pazienti è basata sui risultati di trial in popolazione non affetta da ictus. Il controllo glicemico intensivo, definito da valori di emoglobina glicata $<6 \%$ non è stato associato ad una riduzione significativa dell'incidenza di ictus, dati pe- 
raltro in accordo con una recente metanalisi. ${ }^{22} \mathrm{~L}^{\prime} \mathrm{Ame}$ rican Diabetes Association (ADA) ${ }^{23}$ suggerisce, nei pazienti diabetici con associate comorbidità vascolari tra cui l'ictus, terapia con statine con un target di colesterolo $\mathrm{LDL}<100 \mathrm{mg} / \mathrm{dL}$. Controverso è invece il raggiungimento di un accordo sul target pressorio ottimale nei pazienti diabetici. In pazienti selezionati (come ad esempio i più giovani) l'ADA suggerisce il beneficio del raggiungimento di valori pressori $<130$ $\mathrm{mmHg}$ di sistolica e $80 \mathrm{mmHg}$ di diastolica.

\section{Dislipidemia}

Il controllo dell'ipercolesterolemia costituisce un altro importante target nelle strategie di prevenzione secondaria. Il trial Stroke Prevention by Aggressive Reduction in Cholesterol Levels (SPARCL) ${ }^{24}$ ha incluso 4731 pazienti con TIA o ictus non cardioembolico, colesterolo LDL tra 100 e $190 \mathrm{mg} / \mathrm{d}$ e anamnesi negativa per patologia coronarica, evidenziando, in seguito a quasi 5 anni di trattamento con atorvastatina $80 \mathrm{mg}$ contro placebo, una riduzione del rischio assoluto di ictus e TIA del 2,2\% a favore dei trattati. Ulteriori sottoanalisi del trial SPARCL hanno confermato la sicurezza ed efficacia di atorvastatina $80 \mathrm{mg} /$ die in prevenzione secondaria, nella riduzione delle recidive di ictus e TIA non cardioembolico ed eventi vascolari cardiaci, indipendentemente dal sottotipo di ictus. ${ }^{11}$ Sia lo studio SPARCL che l'Heart Protection Study ${ }^{25}$ (che aveva evidenziato il beneficio del trattamento con simvastatina $40 \mathrm{mg} /$ die nella riduzione della mortalità cardiovascolare nei pazienti ad alto rischio, indipendentemente dal valore iniziale di colesterolemia) avevano mostrato un piccolo aumento delle emorragie cerebrali nel gruppo trattato con statine. Tuttavia, una meta-analisi di 31 studi randomizzati controllati non ha successivamente evidenziato differenze nell'incidenza di emorragia cerebrale in 91588 pazienti in trattamento con statine rispetto a 91.215 controlli. ${ }^{11}$ I risultati del trial Treat Stroke to Target (TST) ${ }^{26}$ interrotto precocemente, hanno mostrato un trend a favore di una riduzione aggressiva dei livelli di LDL $(<70$ $\mathrm{mg} / \mathrm{dL}$ ), rispetto a una più modesta riduzione (90-110 $\mathrm{mg} / \mathrm{dL}$ ) nei pazienti con patologia cardiovascolare aterosclerotica ed evidenza di ictus ischemico o TIA, con tassi di emorragia cerebrale lievemente più alti, ma statisticamente non significativi.

\section{Prevenzione secondaria dell'ictus in relazione all'eziologia}

La corretta classificazione eziologica dell'ictus ischemico è indispensabile per la migliore strategia terapeutica di prevenzione secondaria. Possiamo dividere i sistemi classificativi in due categorie: fenotipica e causativa. ${ }^{27}$ Rientrano nel primo gruppo i sistemi di classificazione Causative Classification System (CCS), ${ }^{28}$ Atherosclerosis, Small-Vessel Disease e Cardiac Source, Other Cause (A-S-C-O). ${ }^{29}$ L'approccio fenotipico prende in considerazione esclusivamente il riscontro di anomalie nei test diagnostici effettuati, per cui il limite principale è rappresentato dal fatto che un paziente può essere categorizzato in più di un sottotipo eziologico. Ad esempio, un ictus cerebrale in cui si ha riscontro di una stenosi carotidea $>50 \%$ e di contemporanea fibrillazione atriale viene categorizzato come aterosclerosi dei grossi vasi plus embolismo cardiaco e ad ogni possibile eziologia viene inoltre attribuito un diverso grado di probabilità. I sistemi di classificazione causativa, invece, assegnano ciascun paziente con ictus ischemico in una unica categoria eziologica, mediante l'integrazione delle caratteristiche cliniche, epidemiologiche e dei risultati dei test diagnostici. ${ }^{27}$ Appartengono a questo gruppo i sistemi: Trial of Org10172 in Acute Stroke Treatment (TOAST) ${ }^{30}$ e la sua revisione (SSS-TOAST) ${ }^{31}$ e CCS. ${ }^{28}$ Una corretta integrazione dei due concetti classificativi risulta indispensabile per un corretto approccio clinico. Le principali caratteristiche dei sistemi classificativi sopra elencati sono riassunte nella Tabella 1. Ai fini di un corretto approccio terapeutico risulta innanzitutto fondamentale la distinzione dell'ictus ischemico in due macrogruppi: ictus cardioembolico e non cardioembolico.

\section{Ictus cardioembolico}

L'ictus cardioembolico rappresenta oggi il 20\%$30 \%$ di tutte le forme di ictus ischemico ed è generalmente associato ad una maggiore gravità clinica, con una probabilità di morte entro il primo anno del 50\%, rispetto al $27 \%$ degli ictus a diversa eziologia. Il tasso di ricorrenza a 5 anni è del $21,5 \%$, con percentuali di dipendenza funzionale del $26 \%$ circa. ${ }^{32,33} \mathrm{~L}$ 'incidenza di ictus cardioembolico si è triplicata rispetto alle decadi passate e, secondo le proiezioni del Regno Unito, aumenterà ancora fino a tre volte entro il $2050,,^{34,35}$ probabilmente anche in relazione ai progressi in ambito diagnostico. L'ictus cardioembolico rappresenta un'entità eterogenea, riconoscendo diverse possibili cause sottostanti, di cui la più frequente è indubbiamente la fibrillazione atriale (FA).

\section{Fibrillazione atriale}

In presenza di fibrillazione atriale (FA) la prevenzione, sia primaria che secondaria, degli eventi cerebrovascolari è affidata all'utilizzo dei farmaci anticoagulanti. Di notevole importanza a tal proposito sono da un lato la stratificazione del rischio di ictus di ciascun individuo e di recidiva dello stesso, dall'altro 
la scelta del farmaco anticoagulante più appropriato. La stratificazione del rischio di ictus nei soggetti con FA si avvale di scale cliniche, di cui la più utilizzata è lo score $\mathrm{CHA}_{2} \mathrm{DS}_{2}$-VASc (Tabella 2), calcolato attribuendo 1 punto per età compresa tra 65 e 74 anni, sesso femminile, scompenso cardiaco, ipertensione arteriosa, diabete e vasculopatie e 2 punti per età $\geq 75$ anni e per pregresso evento cerebrovascolare. Lo score è stato validato dall'Euro Heart Survey on AF population su una coorte di 1084 pazienti non anticoagulati. ${ }^{36}$ Secondo tale score il rischio di incorrere in un ictus viene stratificato in tre gruppi a rischio crescente: score uguale a $0,1 \mathrm{e} \geq 2$, con un tasso medio annuale di ictus rispettivamente dello $0,2 \%, 0,6 \%$ e $2,2 \%$. Il rischio di ricorrenza di ictus in caso di ischemia cerebrale correlata a FA è dell' $8 \%$ nelle prime due

Tabella 1. Principali sistemi classificativi ictus ischemico.

\begin{tabular}{|c|c|c|c|c|c|}
\hline Classificazione & $\begin{array}{l}\text { Tipo di } \\
\text { classificazione }\end{array}$ & Tipo di ictus & Grado di evidenza & Vantaggi & Svantaggi \\
\hline TOAST & Causativa & $\begin{array}{l}\text { 1. Aterosclerosi di grosso } \\
\text { vaso } \\
\text { 2. Cardioembolismo } \\
\text { 3. Occlusione di piccolo } \\
\text { vaso } \\
\text { 4. Causa indeterminata } \\
\text { - Due o più cause } \\
\text { - Accertamenti } \\
\text { negativi } \\
\text { - Accertamenti } \\
\text { incompleti }\end{array}$ & $\begin{array}{l}\text { Probabile } \\
\text { Possibile }\end{array}$ & $\begin{array}{l}\text { - Ampiamente usata } \\
\text { - Utile da usare nella } \\
\text { pratica clinica }\end{array}$ & $\begin{array}{l}\text { - Affidabilità e validità } \\
\text { influenzata } \\
\text { dall'esperienza del } \\
\text { valutatore } \\
\text { - I gradi di evidenza sono } \\
\text { spesso ignorati } \\
\text { - Sovrastima del gruppo } \\
\text { indeterminato } \\
\text { - Bassa affidabilità per gli } \\
\text { ictus minori }\end{array}$ \\
\hline SSS-TOAST & Causativa & $\begin{array}{l}\text { 1. Aterosclerosi di grosso } \\
\text { vaso } \\
\text { 2. Cardioembolismo } \\
\text { 3. Occlusione di piccolo } \\
\text { vaso } \\
\text { 4. Altre cause } \\
\text { 5. Causa indeterminata } \\
\text { - Non noto } \\
\text { a) Embolismo } \\
\text { criptogenico } \\
\text { b) Altri criptogenici } \\
\text { c) Accertamenti } \\
\text { incompleti } \\
\text { - Non classificati }\end{array}$ & $\begin{array}{l}\text { Evidente } \\
\text { Probabile } \\
\text { Possibile }\end{array}$ & $\begin{array}{l}\text { - Migliore affidabilità } \\
\text { e criteri aggiornati } \\
\text { rispetto al TOAST } \\
\text { - Riduzione dei numeri } \\
\text { di casi classificati } \\
\text { come indeterminati } \\
\text { - Identificazione } \\
\text { dell'embolismo } \\
\text { criptogenico come } \\
\text { entità separata }\end{array}$ & $\begin{array}{l}\text { - Schema classificativo } \\
\text { complicato } \\
\text { - Difficile da usare nella } \\
\text { pratica clinica }\end{array}$ \\
\hline $\mathrm{CCS}$ & $\begin{array}{l}\text { Causativa e } \\
\text { fenotipica }\end{array}$ & Stesso di SSS-TOAST & $\begin{array}{l}\text { Evidente } \\
\text { Probabile } \\
\text { Possibile }\end{array}$ & $\begin{array}{l}\text { - Simile a SSS-TOAST } \\
\text { - Algoritmo } \\
\text { computerizzato } \\
\text { - Affidabilità e validità } \\
\text { eccellenti } \\
\text { - Specificazione di } \\
\text { dettagli fenotipici }\end{array}$ & $\begin{array}{l}\text { - Simile ad SSS-TOAST } \\
\text { - Necessità di internet }\end{array}$ \\
\hline ASCO & Fenotipica & $\begin{array}{l}\text { 1. Aterotrombosi } \\
\text { 2. Patologia di piccolo } \\
\text { vaso } \\
\text { 3. Patologia cardiaca } \\
\text { 4. Altre cause }\end{array}$ & $\begin{array}{l}\text { 0: assenza di malattia } \\
\text { 1: potenzialmente } \\
\quad \text { causale } \\
\text { 2: Nesso causale } \\
\quad \text { incerto } \\
\text { 3: Nesso causale } \\
\quad \text { improbabile } \\
\text { 9: work-up insufficiente }\end{array}$ & $\begin{array}{l}\text { - Criteri esaustivi } \\
\text { - Comprensivo di tutte le } \\
\text { potenziali patologie } \\
\text { coinvolte nella } \\
\text { patogenesi } \\
\text { dell' ictus } \\
\text { - Adatto a studi di } \\
\text { registro e clinici } \\
\text { - Buona validità }\end{array}$ & $\begin{array}{l}\text { - Schema classificativo } \\
\text { complesso } \\
\text { - Difficile da utilizzare } \\
\text { nella pratica clinica }\end{array}$ \\
\hline ASCO-D & Fenotipica & $\begin{array}{l}\text { 1. Aterotrombosi } \\
\text { 2. Patologia di piccolo } \\
\text { vaso } \\
\text { 3. Patologia cardiaca } \\
\text { 4. Altre cause } \\
\text { 5. Dissecazione }\end{array}$ & $\begin{array}{l}\text { 0: assenza di malattia } \\
\text { 1: potenzialmente } \\
\quad \text { causale } \\
\text { 2: Nesso causale } \\
\quad \text { incerto } \\
\text { 3: Nesso causale } \\
\quad \text { improbabile } \\
\text { 9: work-up insufficiente }\end{array}$ & $\begin{array}{l}\text { - Simile ad ASCO } \\
\text { - Criteri aggiornati } \\
\text { Identificazione della } \\
\text { dissecazione arteriosa } \\
\text { come eziologia a sé stant } \\
\text { - Work-up diagnostico } \\
\text { definito }\end{array}$ & - Simili ad ASCO \\
\hline
\end{tabular}


settimane. ${ }^{37}$ Tuttavia, il beneficio del precoce utilizzo di terapia anticoagulante deve indubbiamente tenere conto del rischio di trasformazione emorragica della lesione ischemica, evidenziando alcuni studi come un inizio precoce ( $<48$ ore) sia associato ad aumentato rischio di emorragia intracranica sintomatica. ${ }^{38}$ In conseguenza di ciò nei pazienti con FA, il timing ottimale per l'inizio della terapia anticoagulante dopo un ictus cardioembolico è ancora oggetto di discussione. Attualmente si fa riferimento alla regola "1-3-6-12" proposta dall'European Society of Cardiology, ${ }^{39}$ che suggerisce l'inizio della terapia anticoagulante rispettivamente ai giorni 1, 3, 6 e 12 in relazione rispettivamente all'occorenza di TIA, ictus lieve, moderato o severo, questi ultimi definiti sulla base del punteggio National Institutes of Health Stroke Scale (NIHSS) e dell'estensione dell'infarto cerebrale. Quattro grandi trials randomizzati, OPTIMAS, TIMING, START ed ELAN stabiliranno il beneficio dell'inizio precoce della terapia anticoagulante $(<4$ giorni) nei pazienti con ischemia cerebrale correlata a FA..$^{40}$

\section{Terapia anticoagulante orale}

Distinguiamo gli Antagonisti della Vitamina K (AVK), quali il warfarin, e gli anticoagulanti orali diretti (AOD), distinti a loro volta in inibitori della trombina (Dabigatran) e inibitori del fattore $\mathrm{X}$ attivato (Rivaroxaban, Apixaban, Edoxaban). La scelta della terapia anticoagulante si basa innanzitutto sulla classica distinzione della FA in: i) valvolare: presenza di stenosi valvolare mitralica o valvola cardiaca meccanica; ii) non valvolare: assenza delle suddette condizioni. ${ }^{41}$
Si tratta di una distinzione fondamentale, in quanto molti di questi pazienti sono stati esclusi dai recenti trial sugli AOD. Gli AOD, di conseguenza, sono stati testati e trovano indicazione solo nei pazienti con FA definita non valvolare, benché non vi sia un unanime consenso nei criteri di esclusione adottati in relazione alla definizione di FA valvolare. ${ }^{42}$

L'efficacia del warfarin in prevenzione secondaria è stata dimostrata dall'European Atrial Fibrillation Trial (EAFT), ${ }^{43}$ che ha randomizzato 669 pazienti con FA non valvolare a warfarin (INR target $=3$ ), aspirina o placebo. Nel braccio warfarin si è osservata una riduzione del rischio di ictus dal $12 \%$ al $4 \%$, con profilo di sicurezza comparabile ad aspirina e placebo. Per quanto riguarda gli AOD, comparati al warfarin, hanno una maggiore rapidità di azione, una più breve emivita, nessuna interazione con cibo, minori interazioni con terapie mediche e non richiedono un periodico monitoraggio ematico di attività anticoagulante come l'INR per gli AVK. Tutti i trial di fase III hanno evidenziato la non inferiorità degli AOD rispetto al warfarin nella prevenzione di ictus ed embolismo sistemico, a fronte di un tasso di emorragia intracranica dimezzato, ${ }^{42,44-46}$ tanto che le linee guida italiane ${ }^{11}$ raccomandano in caso di ictus ischemico o TIA attribuibile a FA non valvolare il ricorso agli AOD per la loro uguale efficacia e la loro maggiore sicurezza rispetto agli AVK, con l'esclusione dei casi di controindicazione assoluta (valvola prostetica meccanica, stenosi severa della mitrale in genere su base reumatica, compromissione renale severa) e cautela in quelli con controindicazioni relative (concomitante neoplasia, scarsa aderenza terapeutica del paziente) ${ }^{47}$ Peraltro, una re-

Tabella 2. Principali scores di stratificazione del rischio di ictus ischemico $\left(\mathrm{CHA}_{2} \mathrm{DS}_{2}-\mathrm{VASc}\right.$ e ATRIA) e di sanguinamento (HAS-BLED) nei pazienti con fibrillazione atriale.

\begin{tabular}{|c|c|c|c|c|c|c|}
\hline \multicolumn{2}{|c|}{$\mathrm{CHA}_{2} \mathrm{DS}_{2}-\mathrm{VASc}$ score } & \multicolumn{2}{|l|}{ HAS-BLED } & \multicolumn{3}{|c|}{ ATRIA } \\
\hline Fattori di rischio & Score & Fattori di rischio & Score & Fattori di rischio & $\begin{array}{l}\text { ore in assenza } \\
\text { storia di ictus }\end{array}$ & $\begin{array}{l}\text { Score in presenza } \\
\text { di pregresso ictus }\end{array}$ \\
\hline Scompenso cardiaco & 1 & Ipertensione arteriosa & 1 & Età $<65$ & 0 & 8 \\
\hline Ipertensione arteriosa & 1 & Disfunzione renale & 1 & Età 65-74 & 3 & 7 \\
\hline Età $\geq 75$ anni & 2 & Epatopatia & 1 & Età 75-84 & 5 & 7 \\
\hline Diabete & 1 & Pregresso ictus & 1 & Età $\geq 85$ & 6 & 9 \\
\hline Ictus/TIA & 2 & INR labile & 1 & Sesso femminile & 1 & 1 \\
\hline Vasculopatia & 1 & Età $>65$ & 1 & Diabete mellito & 1 & 1 \\
\hline Età $\geq 65$ anni & 1 & $\begin{array}{l}\text { Farmaci che predispongono al } \\
\text { sanguinamento (Aspirina, } \\
\text { Clopidogrel, FANS) }\end{array}$ & 1 & Scompenso cardiaco & 1 & 1 \\
\hline \multirow[t]{2}{*}{ Sesso Femminile } & 1 & Abuso alcolico & 1 & Ipertensione arteriosa & 1 & 1 \\
\hline & & $\begin{array}{l}\text { Pregressa emorragia maggiore o } \\
\text { predisposizione al sanguinamento }\end{array}$ & 1 & $\begin{array}{l}\text { Proteinuria } \\
\text { Filtrato glomerulare (MDRD) } \\
<45\end{array}$ & ) & $\begin{array}{l}1 \\
1\end{array}$ \\
\hline
\end{tabular}


cente analisi dell'esito clinico dei pazienti arruolati nel trial ENGAGE-AF-TIMI-48 con cancro, ha evidenziato il beneficio dell'Edoxaban, suggerendo che l'utilizzo degli AOD in questo gruppo di pazienti necessiti di ulteriori indagini. ${ }^{48}$ Per quanto riguarda i soggetti di età avanzata, un'analisi secondaria dei trial ARISTOTLE, ${ }^{49}$ ROCKET-AF ${ }^{50}$ e RELY, ${ }^{51}$ ha evidenziato che questi beneficiano del trattamento anticoagulante con $\mathrm{AOD}$ al pari dei più giovani, ${ }^{52,53}$ sebbene l'utilizzo del Dabigatran al dosaggio di $150 \mathrm{mg}$ due volte al giorno sia associato con elevato rischio di emorragia extracranica nei soggetti di età maggiore agli 80 anni. ${ }^{54}$ Nei soggetti ad elevato rischio di sanguinamento sulla base degli score HAS-BLED o ATRIA (Anticoagulation and Risk Factors in Atrial Fibrillation) (Tabella 2), o in presenza di controindicazioni alla terapia anticoagulante, le alternative utilizzabili sono rappresentate da aspirina, clopidogrel o entrambi. L'Atrial Fibrillation Clopidogrel Trial With Irbesartan for Prevention of Vascular Events (ACTIVE A) $)^{55}$ ha comparato aspirina (ASA) versus ASA più clopidogrel in 7550 pazienti fibrillanti che non potevano assumere warfarin. Dopo un follow up di 3,6 anni si è osservata una riduzione dell'incidenza di ictus, a scapito di una maggior percentuale di sanguinamenti maggiori, nei pazienti trattati con doppia antiaggregazione. L'aggiunta di clopidogrel ad ASA appare comunque di beneficio modesto, rispetto alla singola terapia antiaggregante. Nel 2015, in seguito ai risultati dei trial PROTECT $\mathrm{AF}^{56}$ e PREVAIL, ${ }^{57}$ il WATCHMAN Left Atrial Appendage Closure Device è stato approvato negli USA per il trattamento della FA nei pazienti con elevato rischio emorragico o controindicazione a terapia anticoagulante. Tuttavia, l'utilizzo di tale dispositivo implica che il paziente sia in grado di tollerare la terapia con warfarin più ASA per almeno 45 giorni e doppia antiaggregazione per almeno 6 mesi. ${ }^{57}$

\section{Altre condizioni associate a cardioembolismo}

\section{Valvole protesiche cardiache}

Si stima che il rischio di cardioembolismo nei pazienti con valvola meccanica è di circa il $4 \%$ per anno in assenza di terapia antiaggregante/anticoagulante, $2 \%$ con l'uso di ASA e $1 \%$ con anticoagulanti. ${ }^{58,59} \mathrm{Gli}$ AOD sono controindicati in questo sottogruppo di pazienti, per i quali trovano invece indicazione gli AVK. Il range di INR richiesto dipende dal tipo e localizzazione della valvola meccanica, da eventi embolici antecedenti o seguenti la sostituzione valvolare cardiaca. In linea generale, in accordo con le linee guida dell'AHA, ${ }^{3}$ per i pazienti con valvola meccanica aortica e pregresso ictus/TIA è ragionevole un INR di 2,5 (range 2,0-3,0), mentre per la valvola mitrale in pre- senza di pregresso ictus l'INR ottimale è di 3,0 (2,53,5). Il recente trial RE-ALIGN (Randomized Phase II Study to Evaluate the Safety and Pharmacokinetics of Oral Dabigatran Etexilate in Patients After Heart Valve Replacement),${ }^{60}$ che comparava warfarin versus dabigatran in pazienti con valvola meccanica cardiaca, è stato interrotto precocemente senza dimostrazione del beneficio del dabigatran.

\section{Scompenso cardiaco}

Lo scompenso cardiaco rappresenta il secondo fattore di rischio maggiormente associato a ischemia cerebrale, dopo la fibrillazione atriale, condizione peraltro spesso concomitante. Il meccanismo fisiopatologico più frequentemente responsabile dell'ictus ischemico è quello tromboembolico, in seguito alla formazione di trombi intracavitari conseguenti alla stasi sanguigna favorita dall'alterata cinetica ventricolare. ${ }^{61}$ La prevenzione secondaria deve tener conto del tipo di scompenso cardiaco (classificato sulla base della frazione di eiezione (FE) in scompenso cardiaco a $\mathrm{FE}$ compromessa se $\mathrm{FE}<40 \%$, o conservata se $\mathrm{FE} \geq 50 \%$ o moderatamente compromessa se FE compresa tra il $40 \%-49 \%{ }^{62}$ ) e dalla concomitante presenza di FA. Se in quest'ultimo caso infatti, l'utilizzo della terapia anticoagulante rappresenta il trattamento di prima scelta, con predilezione per gli AOD per il profilo di maggiore sicurezza e pari efficacia rispetto al warfarin, ${ }^{63}$ più controversa è la decisione terapeutica in presenza di scompenso cardiaco con ritmo sinusale. Una recente metanalisi ${ }^{64}$ di quattro trial (WARCEF, ${ }^{65}$ WATCH ${ }^{66}{ }^{6 E L A S},{ }^{67} \mathrm{WASH}^{68}$ ) che avevano indagato l'utilizzo del warfarin versus antiaggreganti e/o placebo in pazienti con scompenso cardiaco con compromessa $\mathrm{FE}$ in assenza di FA, e del più recente trial COMMANDER ${ }^{69}$ (che ha indagato la sicurezza ed efficacia del Rivaroxaban $5 \mathrm{mg} /$ die nel ridurre il rischio di ictus, infarto del miocardio e morte nei pazienti con scompenso cardiaco a FE compromessa e ritmo sinusale, ma con associata patologia coronarica) ha infatti evidenziato che l'utilizzo di terapia anticoagulante, pur riducendo il rischio di ictus del $37 \%$, determina un aumento delle emorragie di 1.8 volte, in assenza peraltro di vantaggi in termini di mortalità. Non vi sono trial che abbiano invece indagato in maniera sistematica il possibile beneficio dell'anticoagulazione in pazienti con scompenso cardiaco in ritmo sinusale e FE preservata.

\section{TIA e ictus non cardioembolico}

Rientrano in questo gruppo l'ictus aterotrombotico da aterosclerosi dei grossi vasi intra ed extracranici) e aterotrombotico lacunare. In aggiunta alle strategie di prevenzione generale, la terapia di prevenzione secon- 
daria si fonda sull'utilizzo di antiaggreganti piastrinici, con le dovute differenze in relazione alla classe eziopatogenetica dell'ictus.

\section{Aspirina}

Il gruppo ISO-SPREAD ${ }^{11}$ raccomanda nella prevenzione secondaria del TIA e ictus non cardioembolico la terapia antiaggregante con ASA 100-325 $\mathrm{mg} /$ die, utilizzando ASA $100 \mathrm{mg} /$ die per il trattamento prolungato. Nel 2002 la metanalisi dell'Antithrombotic Trialists Collaboration (ATC) ${ }^{70}$ ha analizzato 195 trial randomizzati che comparavano la terapia antiaggregante (ASA soprattutto) versus placebo per la prevenzione di ictus, infarto del miocardio e morte in pazienti ad alto rischio. Nel gruppo di pazienti con pregresso evento cerebrovascolare l'utilizzo di antiaggreganti piastrinici ha determinato una riduzione del rischio di ricorrenza di ictus, infarto del miocardio e morte per eventi vascolari del $22 \%$, indipendentemente da età, sesso, diabete e ipertensione. Dosaggi di ASA inferiori a $75 \mathrm{mg} /$ die, tuttavia, non raggiungevano la significatività statistica in termini di riduzione degli eventi cardiovascolari. Nei pazienti con ictus non cardioembolico che erano già in terapia con ASA prima dell'evento, il gruppo ISO SPREAD ${ }^{11}$ suggerisce di sostituire ASA con altro antiaggregante piastrinico, quale Clopidogrel $75 \mathrm{mg} /$ die o Dipiridamolo a lento rilascio $400 \mathrm{mg} / \mathrm{die}$ e $A S A \geq 50 \mathrm{mg} / \mathrm{die}$, dopo aver valutato che gli eventuali fattori di rischio concomitanti siano stati trattati adeguatamente.

\section{Clopidogrel}

Lo studio CAPRIE (Clopidogrel versus Aspirin in Patients at Risk of Ischaemic Events $)^{71}$ ha confrontato in 19.185 pazienti con recente evento vascolare (ictus, infarto acuto del miocardio o arteriopatia periferica sintomatica, equamente rappresentati) l'efficacia del clopidogrel $(75 \mathrm{mg} / \mathrm{die})$ con quella dell'ASA (325 $\mathrm{mg}$ /die). Il gruppo trattato con clopidogrel ha evidenziato, rispetto al gruppo ASA, una riduzione dell'incidenza dell'endpoint composito primario di ictus ischemico, infarto del miocardio e morte per cause vascolari (5,3\% vs 5,8\% annuo, rispettivamente), con una riduzione del rischio relativo del 8,7\% (95\% IC $0,3-16,5)$. Peraltro, la forza di questa evidenza è limitata dal fatto che il maggiore beneficio è stato riscontrato nel gruppo con arteriopatia periferica sintomatica, mentre nessuna differenza statisticamente significativa tra clopidogrel e ASA è emersa nei gruppi con recente ictus o infarto acuto del miocardio.

\section{Associazione ASA+Dipiridamolo}

L'efficacia dell'associazione è stata valutata nel trial ESPS-2 (European Stroke Prevention Study-2) ${ }^{72}$ che ha randomizzato 6602 pazienti con recente ictus ischemico o TIA in quattro bracci: dipiridamolo formulazione retard $200 \mathrm{mg} \times 2 /$ die; ASA $25 \mathrm{mg} \times 2 /$ die; associazione dipiridamolo retard $200 \mathrm{mg} \times 2 \mathrm{e}$ ASA 25 $\mathrm{mg} \times 2$; placebo.

Nella prevenzione delle ricorrenze di ictus ischemico l'associazione è risultata più efficace dei due singoli farmaci e del placebo (OR 0,59, 95\%, IC 0,48-0,73).

In relazione ai risultati della metanalisi ATC è stato tuttavia ipotizzato che il vantaggio dell'associazione ASA+dipiridamolo rispetto ad ASA in monoterapia fosse dovuto al basso dosaggio di ASA utilizzato nell'ESPS-2 (inferiore a $75 \mathrm{mg} /$ die), per tale motivo le linee guida italiane ${ }^{11}$ suggeriscono di utilizzare almeno $100 \mathrm{mg}$ di ASA, anche in associazione con dipiridamolo.

Lo studio PROFESS ${ }^{73}$ che ha incluso 20332 pazienti con pregresso TIA o ictus ischemico non ha evidenziato differenze statisticamente significative nella prevenzione delle recidive di ictus a fronte di un lieve incremento delle complicanze emorragiche nel gruppo trattato con associazione ASA-dipiridamolo versus clopidogrel.

\section{Associazione ASA+Clopidogrel}

Nella prevenzione delle recidive di ictus ischemico la combinazione di ASA+clopidogrel non ha evidenziato un beneficio rispetto alla singola antiaggregazione, a fronte di un aumento del rischio emorragico. Queste evidenze provengono dai risultati del trial MATCH (Management of Atherothrombosis With Clopidogrel in High-Risk Patients With Recent Transient Ischemic Attacks or Ischemic Stroke), ${ }^{74}$ CHARISMA (Clopidogrel for High Atherothrombotic Risk and Ischemic Stabilization, Management, and Avoidance) $^{75}$ ed SPS3 (Secondary Prevention of Small Subcortical Strokes). ${ }^{76}$ Il trial MATCH ${ }^{74}$ ha arruolato 7600 pazienti con recente TIA o ictus ischemico con associate ulteriori patologie definite ad alto rischio, quali pregresso infarto miocardico, ictus, angina, arteriopatia periferica sintomatica. I pazienti sono stati randomizzati ad associazione clopidogrel $75 \mathrm{mg} / \mathrm{die}$ più ASA $75 \mathrm{mg} /$ die versus clopidogrel $75 \mathrm{mg} / \mathrm{die}$ in monoterapia. I risultati hanno evidenziato per il gruppo associazione una riduzione non significativa degli eventi ischemici, a fronte di un aumento delle complicanze emorragiche maggiori dopo tre mesi di trattamento. Il CHARISMA ${ }^{75}$ non ha invece evidenziato differenze di beneficio né di rischio nel gruppo associazione ASA-clopidogrel rispetto alla sola ASA (RR, 0,93; 95\% CI, 0,83-1,05; $\mathrm{P}=0,22$ ).

Lo studio SPS $3^{76}$ ha valutato in 3000 pazienti con ictus ischemico lacunare l'associazione clopidogrel 75 mg +ASA $325 \mathrm{mg}$ versus ASA $325 \mathrm{mg}$. I risultati non hanno mostrato alcuna riduzione della ricorrenza di ictus nella terapia protratta di associazione clopidogrel 
$75 \mathrm{mg}+$ ASA $325 \mathrm{mg}$ rispetto alla sola ASA $325 \mathrm{mg}$ ( $2,5 \%$ vs $2,7 \%$ anno), con un aumento significativo delle complicanze emorragiche e della mortalità.

Sulla base dei risultati di questi trial non vi sono evidenze a favore della doppia antiaggregazione a lungo termine nel ridurre il rischio di ictus.

\section{Indicazioni specifiche per sottogruppi}

\section{Ictus minore di origine aterotrombotica e TIA ad alto rischio}

L'ictus minore è stato definito in vari modi: ictus cerebrale con basso punteggio alla National Institute of Health Stroke Scale (NIHSS), ${ }^{77-78}$ in assenza peraltro di un univoco valore soglia NIHSS, o ictus cerebrale caratterizzato dall'assenza di un deficit neurologico persistente disabilitante. ${ }^{79}$ Il TIA ad alto rischio viene tradizionalmente definito da uno score $\mathrm{ABCD} 2 \geq 4 .{ }^{80}$ In questo sottogruppo di pazienti è particolarmente elevato il rischio di recidiva precoce, cioè entro i 3 mesi successivi, per cui sono stati oggetto di trial sulla doppia antiaggregazione a breve termine.

Il trial $\mathrm{POINT}^{81}$ ha randomizzato 4881 pazienti, entro 12 ore dall'esordio di un ictus minore o TIA ad alto rischio, a clopidogrel (dose di carico di $600 \mathrm{mg}$, seguito da $75 \mathrm{mg} /$ die per 90 giorni) + Aspirina versus Aspirina+Placebo per 90 giorni. In entrambi i gruppi la dose di aspirina era da 50 a $325 \mathrm{mg}$. A 90 giorni l'outcome composito di eventi ischemici maggiori (ictus ischemico, infarto del miocardio o morte per eventi ischemici) è risultato inferiore nel gruppo clopidogrel-aspirina $(5,0 \%$ versus $6,5 \%$, HR $0,75,95 \%$, IC 0,59-0,95), così come l'outcome ictus ischemico o emorragico ( $4,8 \%$ versus $6,4 \%$, HR $0,74,95 \%$ IC $0,58-0,94)$, a scapito tuttavia di un incremento del tasso di emorragie maggiori ( $0,9 \%$ versus $0,4 \%$, HR $2,32,95 \%$, IC 1,10-4,87). Nel trial CHANCE ${ }^{82}$ sono stati invece randomizzati 5170 pazienti Cinesi entro 24 ore dall'esordio di un ictus minore o TIA ad alto rischio a clopidogrel (300 mg dose di carico, poi 75 mg per 90 giorni) +aspirina (75 mg per i primi 21 giorni) versus aspirina $75 \mathrm{mg}+$ placebo per 90 giorni. A 90 giorni si è registrata una significativa riduzione dell'incidenza dell'ictus nel gruppo clopidogrel+aspirina $(8,2 \%$ versus $11,7 \%$, riduzione assoluta del rischio $3,5 \%$, HR $0,68,95 \%$, IC $0,57-0,81$ ). Il tasso di trasformazione emorragica è stato basso in entrambi $\mathrm{i}$ gruppi. Tuttavia i risultati del trial CHANCE, e in misura minore del POINT, non sono generalizzabili a tutti i pazienti con ictus minore o TIA ad alto rischio: il trial POINT ha escluso i pazienti candidabili a trombolisi endovenosa, trombectomia meccanica e endoarterectomia carotidea; la popolazione cinese inclusa nel CHANCE, aveva una maggiore prevalenza di stenosi intracraniche su base aterosclerotica e minore preva- lenza dei convenzionali fattori di rischio vascolari, rispetto al resto della popolazione mondiale; inoltre entrambi i trials hanno escluso pazienti con alterazioni isolate della sensibilità, del visus e vertigini isolate. Alla luce di tali risultati il gruppo ISO SPREAD, ha suggerito, in una recente raccomandazione online, ${ }^{11}$ nei pazienti con ictus minore o TIA ad alto rischio, il ricorso alla doppia antiaggregazione (ASA 100 $\mathrm{mg}+$ clopidogrel $600 \mathrm{mg}$ il primo giorno, poi 75 $\mathrm{mg} /$ die) preferibilmente entro 12 ore dall'esordio dei sintomi (o 24 ore in caso di trombolisi endovenosa), limitando il trattamento preferibilmente ai primi 30 giorni dall'evento ischemico cerebrale e comunque non prolungandolo oltre i 90 giorni, a causa di un significativo aumento delle complicanze emorragiche.

Il trial SOCRATES (Acute Stroke or Transient Ischaemic Attack Treated with Aspirin or Ticagrelor and Patient Outcomes ${ }^{83}$ ha invece comparato l'azione di Ticagrelor, inibitore diretto, reversibile e competitivo del recettore piastrinico P2Y12, al dosaggio di 90 $\mathrm{mg}$ due volte al giorno versus ASA $100 \mathrm{mg}$, in più di 13.000 pazienti con ictus ischemico o TIA ad alto rischio, non raggiungendo l'enpoint primario di efficacia (ictus, infarto del miocardio, morte). Infine, il trial THALES (Acute Stroke or Transient Ischemic Attack Treated with Ticagrelor and Aspirin for Prevention of Stroke and Death), ${ }^{84}$ i cui risultati non sono ancora stati pubblicati, ha comparato nei pazienti con ictus minore o TIA ad alto rischio l'efficacia dell'associazione Ticagrelor (90 mg due/die)-Aspirina (300-325 $\mathrm{mg} / \mathrm{die}$ ) versus Aspirina (300-325 mg/die)-Placebo.

\section{Stenosi intracraniche}

Il miglior trattamento di prevenzione secondaria nell'ictus determinato da stenosi intracraniche è stato indagato da vari trial. Il trial WASID (Warfarin and Aspirin Symptomatic Intracranial Disease ${ }^{85}$ ha comparato warfarin versus ASA nei pazienti con stenosi del $50-99 \%$ di grosso vaso intracranico (carotide, arteria cerebrale media, vertebrale o basilare). Il trial è stato interrotto precocemente per l'elevato tasso di eventi avversi nel gruppo trattato con warfarin. Il trial SAMMPRIS (Stenting and Aggressive Medical Menagement for Preventing Recurrent Stroke in Intracranial Stenosis), ${ }^{86}$ che ha confrontato terapia medica e angioplastica con stenting in casi di stenosi sintomatica di grado elevato (70-90\%) di una arteria intracranica, è stato interrotto dopo l'arruolamento di 451 casi ( $59 \%$ dei previsti) per la netta prevalenza $(14 \%$ vs $5,8 \%)$ di ictus cerebrale o decesso nei 30 giorni successivi a trattamento con stenting. Una analisi combinata ${ }^{87}$ dei due trial sopra citati suggerisce per i pazienti con stenosi intracranica sintomatica $<70 \%$ o con stenosi sintomatica $>70 \%$ e ictus o TIA verificatosi da più di 30 giorni, l'utilizzo di ASA 325 $\mathrm{mg} /$ die in associazione al controllo dei fattori di ri- 
schio associati; per i pazienti con stenosi sintomatica del 70-99\% ed ictus verificatosi nei 30 giorni precedenti, l'associazione ASA (325 mg/die)-Clopidogrel $(75 \mathrm{mg} / \mathrm{die})$ e controllo dei fattori di rischio associati. Le linee guida Americane ${ }^{3}$ suggeriscono, come Classe I e livello di evidenza B, nei pazienti con ictus o TIA determinato da una stenosi di grosso vaso intracranico (50-99\%) l'utilizzo di ASA al dosaggio di $325 \mathrm{mg} /$ die rispetto al warfarin; entro i primi trenta giorni dall'evento viene suggerita come Classe II e livello di evidenza B, l'associazione Clopidogrel 75 mg e Aspirina per 90 giorni.

\section{Stenosi extracraniche}

Per quanto riguarda le stenosi carotidee, secondo le linee guida italiane ${ }^{11}$ vengono definite sintomatiche se l'ultimo episodio ischemico cerebrale o retinico congruo si è verificato nei 3 mesi precedenti. L'indicazione al trattamento chirurgico con endoarteriectomia viene posta nel caso di stenosi sintomatiche $\geq 70 \%$ se il rischio perioperatorio di morte e ogni tipo di ictus è inferiore al $6 \%$. In caso di stenosi carotidea inferiore al $50 \%$ non è raccomandata l'endoarteriectomia carotidea. Per stenosi carotidee sintomatiche tra il $50 \%$ e il $69 \%$, se il rischio perioperatorio di morte per ogni tipo di ictus è inferiore al $6 \%$, è indicata endoarteriectomia in presenza di almeno una tra le seguenti condizioni: ischemia recente $(<2$ mesi dal sintomo), sintomo cerebrale e non oculare, placca ulcerata - vulnerabile, sesso maschile, assenza di diabete. ${ }^{11}$ È stato dimostrato che il maggior beneficio della endoarteriectomia carotidea si ha se eseguita entro 2 settimane dall'ictus. ${ }^{88}$ Per quanto riguarda invece la migliore strategia terapeutica tra stenting versus CEA nella stenosi carotidea sintomatica, le evidenze ${ }^{89-92}$ hanno per ora dimostrato la non inferiorità dello stenting rispetto alla CEA, ma sono necessari ulteriori studi. Lo stenting viene tuttavia raccomandato in presenza di importanti comorbidità quali scompenso cardiaco congestizio e/o disfunzione ventricolare sinistra, intervento cardiochirurgico nelle sei settimane precedenti, infarto miocardico nelle quattro settimane precedenti, angina instabile. Sia in attesa che dopo CEA è raccomandata la monoterapia antiaggregante piastrinica. Dopo stenting carotideo è indicata la doppia antiaggregazione piastrinica per i primi 3 mesi, in seguito la monoterapia. ${ }^{11}$ Per quanto riguarda la stenosi vertebrale extracranica le linee guida dell' $\mathrm{AHA}^{3}$ suggeriscono il trattamento chirurgico o lo stenting, solo in presenza di sintomi nonostante la migliore terapia medica (antiaggreganti, statine).

\section{Ictus associato a forame ovale pervio}

Il forame ovale pervio (PFO) è una anomalia congenita presente nel $25 \%$ circa della popolazione, con una prevalenza del $66 \%$ nei soggetti di età $<55$ anni con ictus criptogenetico. ${ }^{93}$ La prevenzione secondaria nei pazienti con ictus ischemico correlato a PFO è stata oggetto di importante dibattito negli ultimi anni, soprattutto in riferimento all' annosa questione del beneficio della chiusura percutanea del PFO rispetto alla sola terapia medica. Infatti, in seguito ai risultati dei trial CLOSURE, ${ }^{94}$ RESPECT ${ }^{95}$ e PC $^{96}$ (circa 2300 soggetti di età $\leq 60$ anni, con ictus ischemico o TIA criptogenetici e riscontro di PFO all'ecocardiogramma transesofageo in assenza di altre potenziali cause di ictus), che non avevano evidenziato, in termini di recidiva di ictus ischemico o TIA, alcuna differenza statisticamente significativa tra la chiusura percutanea del PFO con device e la sola terapia medica antitrombotica (antiaggregante singola o doppia oppure anticoagulante), una successiva analisi del RESPECT $^{97}$ e tre più recenti trial (CLOSE, ${ }^{98}$ REDUCE $^{99}$ e DEFENSE-PFO ${ }^{100}$ ) sono invece giunti a conclusioni opposte. I risultati del follow-up a 5.9 anni del trial RESPECT (che aveva arruolato 980 pazienti di età $\leq 60$ anni con PFO e ictus criptogenetico, randomizzandoli a chiusura PFO con il device Amplatzer più terapia antitrombotica versus sola terapia medica) hanno infatti mostrato, a differenza della precedente pubblicazione con follow-up di 2,6 anni, un tasso di ricorrenza di ictus nei pazienti trattati con chiusura del PFO significativamente inferiore rispetto al gruppo terapia medica $(0,58$ vs 1,07 eventi per 100 pazienti/anno, HR 0,55, 95\%, IC 0,31-0,99; $\mathrm{P}=0,046$ ). Il trial $\mathrm{CLOSE}^{98}$ ha randomizzato soggetti di età compresa tra 16 e 60 anni, con ictus ischemico attribuito al PFO (in presenza di aneurisma del setto interatriale o di shunt di grandi dimensioni) a chiusura percutanea del PFO più terapia antiaggregante per l'intera durata del follow-up, o a sola terapia antiaggregante (ASA o clopidogrel o ASA/dipiridamolo), oppure a terapia anticoagulante (warfarin o AOD). Dopo un follow-up medio di 5,3 anni la ricorrenza di ictus nei pazienti sottoposti a chiusura percutanea del PFO è risultata significativamente inferiore rispetto al gruppo terapia antitrombotica (HR 0,03, 95\% IC 0,0-0,26), con una più alta incidenza, tuttavia, di fibrillazione atriale di nuova insorgenza $(4,6 \%$ vs $0,9 \%, \mathrm{P}=0,02)$, peraltro transitoria nella maggior parte dei casi. Ad analoghi risultati è giunto il trial REDUCE, ${ }^{99}$ che ha arruolato 664 pazienti di età compresa tra 18 e 59 anni, affetti da ictus ischemico con riscontro di PFO, randomizzati con un rapporto di 2:1 a chiusura percutanea del PFO più terapia antiaggregante somministrata per l'intera durata del follow-up versus sola terapia antiaggregante. Dopo un follow-up medio di 3,2 anni il tasso di recidiva di ictus si è mostrato inferiore nel gruppo sottoposto a chiusura percutanea del PFO (1,4\% versus $5,4 \%$, HR $0,23,95 \%$ IC $0,09-0,62)$. Il rischio di fibrillazione atriale si è tuttavia dimostrato 
significativamente più alto nel gruppo sottoposto a chiusura del $\mathrm{PFO}$ rispetto all'altro gruppo di trattamento $(6,6 \%$ vs $0,4 \%, \mathrm{P}<0,001)$, soprattutto entro 45 giorni dalla procedura; ma anche in questo caso la fibrillazione è stata per lo più transitoria. Il recente trial DEFENCE-PFO ${ }^{100}$ ha randomizzato 120 pazienti con ictus ischemico e associato PFO ad alto rischio (definito da associazione con aneurisma del setto interatriale, escursione del setto $>10 \mathrm{~mm}$ o dimensioni del $\mathrm{PFO} \geq 2 \mathrm{~mm}$ ) a chiusura percutanea del PFO versus sola terapia medica, dimostrando, dopo una media di 2,8 anni di follow up, la superiorità della chiusura del PFO in termini ricorrenza di ictus. Il recente consensus paper europeo, ${ }^{101}$ sulla base della metanalisi dei 6 sopracitati trial, suggerisce la chiusura percutanea del PFO in soggetti di età compresa tra $\mathrm{i} 18$ e i 65 anni, con TIA o ictus criptogenetico ed elevata probabilità di associazione causale con PFO (sulla base di caratteristiche cliniche e diagnostiche strumentali). L'incidenza di FA di nuovo riscontro sembra essere ridotta con utilizzo dell'AMPLATZER PFO Occluder, rispetto agli altri device.

\section{Conclusioni e raccomandazioni}

- La prevenzione secondaria dell'ictus ischemico si basa sia su strategie generali di prevenzione (modifiche dello stile di vita e dei principali fattori di rischio associati, soprattutto ipertensione, diabete e dislipidemia) che sull'utilizzo di terapia farmacologiche mirate in relazione all'eziologia dell'ischemia cerebrale.

- Nell'ictus cardioembolico associato a FA non valvolare è raccomandato l'utilizzo di terapia anticoagulante orale, con maggior predilezione per gli AOD. Per ictus cardioembolico associato a patologia valvolare cardiaca è indicato l'utilizzo di warfarin con target di INR da correlare al tipo di valvulopatia sottostante.

- Nell'ictus cardioembolico associato a scompenso cardiaco a bassa FE e ritmo sinusale, in relazione alle evidenze ad ora disponibili, non è raccomandato l'utilizzo di terapia anticoagulante per l'elevato rischio di complicanze emorragiche. Non sono ancora disponibili dati su scompenso cardiaco a FE preservata e ritmo sinusale. Nei pazienti con scompenso cardiaco associato a FA, indipendentemente dalla $\mathrm{FE}$, è raccomandata la terapia con anticoagulanti orali, preferendo gli AOD per il maggior profilo di efficacia e sicurezza rispetto al warfarin.

- Nella prevenzione secondaria di ictus ischemico o TIA non cardioembolico ASA, clopidogrel, o l'associazione ASA-dipiridamolo LR sono valide opzioni. L'associazione ASA-Clopidogrel per oltre 90 giorni, non riduce l'incidenza di ricorrenza di ictus, a fronte di un aumento delle complicanze emorragiche.

- Nei pazienti con ictus minore o TIA ad alto rischio è raccomandato l'utilizzo dell'associazione ASAclopidogrel per i primi 30 giorni. Oltre 90 giorni è preferibile l'utilizzo di antiaggregante in monoterapia.

- Nei pazienti con ictus ischemico da stenosi di grosso vaso intracranico la terapia medica associata ad angioplastica con stenting non si è dimostrata superiore rispetto alla sola terapia medica.

- Nei pazienti con ictus ischemico da stenosi carotidea extracranica $\geq 70 \%$ è indicata la CEA. Le evidenze ad oggi disponibili hanno mostrato la non inferiorità dello stenting rispetto alla CEA, ma sono necessari ulteriori studi. Sia in attesa che dopo CEA è raccomandata la monoterapia antiaggregante piastrinica. Dopo stenting carotideo è indicata la doppia antiaggregazione piastrinica peri primi 3 mesi, in seguito la monoterapia.

- Nell'ictus ischemico associato a PFO le attuali evidenze suggeriscono la superiorità della chiusura percutanea del PFO rispetto alla sola terapia medica in pazienti di età compresa tra i 18 e 65 anni con ictus criptogenetico ed esclusione di altre potenziali fonti emboligene.

\section{Bibliografia}

1. Mozaffarian D, Benjamin EJ, Go AS, et al. American Heart Association Statistics Committee; Stroke Statistics Subcommittee. Heart disease and stroke statistics-2016 update: a report from the American Heart Association. Circulation, 2016; 133: e38-e360.

2. Wolf PA, D'Agostino RB, Belanger AJ, et al. Probability of stroke: a risk profile from the Framingham Study. Stroke, $1991 ; 22: 312-18$.

3. Kernan WN, Ovbiagele B, Black HR, et al; American Heart Association Stroke Council, Council on Cardiovascular and Stroke Nursing, Council on Clinical Cardiology, and Council on Peripheral Vascular Disease. Guidelines for the prevention of stroke in patients with stroke and transient ischemic attack: a guideline for healthcare professionals from the American Heart Association/American Stroke Association. Stroke. 2014; 45:2160-2236.

4. O'Donnell MJ, Chin SL, Rangarajan S, et al. Global and regional effects of potentially modifiable risk factors associated with acute stroke in 32 countries (INTERSTROKE): a case-control study. Lancet 2016;388: 761-75.

5. Epstein KA, Viscoli CM, Spence JD, et al. Smoking cessation and outcome after ischemic stroke or TIA. Neurology 2017;89:1723-29.

6. Malek A, Cushman M, Lackland D et al. Secondhand smoke exposure and stroke: the Reasons for Geographic and Racial Differences in Stroke (REGARDS) study. Am J Prev Med 2015, 49: e89-e97.

7. Kaplan RC, Tirschwell DL, Longstreth WT Jr, et al. Va- 
scular events, mortality, and preventive therapy following ischemic stroke in the elderly. Neurology 2005; 65:835-42.

8. Estruch R, Ros E, Martínez-González MA. Mediterranean diet for primary prevention of cardiovascular disease. N Engl J Med. 2013; 369:676-77.

9. Meschia JF, Bushnell C, Boden-Albala B, et al. Guidelines for the primary prevention of stroke: a statement for healthcare professionals from the American Heart Association/American Stroke Association. Stroke 2014; 45:3754-832.

10. Guzik A, C. Bushnell. Stroke Epidemiology and Risk Factor Management. Continuum 2017; 23:15-39.

11. ISO-SPREAD (Stroke Prevention and Educational Awareness Diffusion). Ictus cerebrale: linee guida italiane di prevenzione e trattamento. VIII edizione. 21 luglio 2016.

12. MacKay-Lyons M, Gubitz G, Giacomantonio N, et al. Program of rehabilitative exercise and education to avert vascular events after non-disabling stroke or transient ischemic attack (PREVENT Trial): a multi-centred, randomised controlled trial. BMC Neurol. 2010; 10:122.

13. Vemmos K, Ntaios G, Spengos K, et al. Association between obesity and mortality after acute first-ever stroke: the obesity-stroke paradox. Stroke 2011; 42: 30-36.

14. Mazzaglia G, Britton A, Altmann D et al. Exploring the relationship between alcohol consumption and non-fatal or fatal stroke: a systematic review. Addiction 2001, 96 : 1743-56.

15. Ois A, Gomis M, Rodríguez-Campello A, et al. Factors associated with a high risk of recurrence in patients with transient ischemic attack or minor stroke. Stroke. 2008; 39:1717-21.

16. PATS Collaborating Group. Post-stroke Antihypertensive Treatment Study: a preliminary result. Chin Med J (Engl). 1995; 108:710-17.

17. PROGRESS Management Committee. PROGRESS: Perindopril Protection Against Recurrent Stroke Study: characteristics of the study population at baseline. J Hypertens. 1999; 17:1647-55

18. Liu L, Wang Z, Gong L, Zhang Y, et al. Blood pressure reduction for the secondary prevention of stroke: a Chinese trial and a systematic review of the literature. Hypertens Res. 2009; 32:1032-40.

19. Benavente OR, McClure LA, Coffey CS, et al. The Secondary Prevention of Small Subcortical Strokes (SPS3) trial: results of the blood pressure intervention. Presented at: International Stroke Conference 2013; February 8, 2013; Honolulu, HI. Abstract LB8.

20. ACCORD Study Group; Cushman WC, Evans GW, Byington RP, ET AL. Effects of intensive blood-pressure control in type 2 diabetes mellitus. N Engl J Med. 2010; 362:1575-85

21. Kaplan R, Tirschwell D, Longstreth W et al. Vascular events, mortality, and preventive therapy following ischemic stroke in the elderly. Neurology, 2005; 65: 835-42.

22. Ray KK, Seshasai SR, Wijesuriya S, et al. Effect of intensive control of glucose on cardiovascular outcomes and death in patients with diabetes mellitus: a metaanalysis of randomised controlled trials. Lancet. 2009; 373:1765-72.
23. American Diabetes Association. Standards of medical care in diabetes: 2013. Diabetes Care 2013; 36: S11S66.

24. Amarenco P, Bogousslavsky J, Callahan, et al.Stroke Prevention by Aggressive Reduction in Cholesterol Levels (SPARCL) Investigators. High-dose atorvastatin after stroke or transient ischemic attack. N Engl J Med. 2006; 355:549-59.

25. Collins R, Armitage J, Parish S, et al. Heart Protection Study Collaborative Group. Effects of cholesterollowering with simvastatin on stroke and other major vascular events in 20536 people with cerebrovascular disease or other high-risk conditions. Lancet. 2004; 363:757-67.

26. Amarenco P, Kim JS, Labreuche MJD. A Comparison of Two LDL Cholesterol Targets after Ischemic Stroke. N Engl J Med 2020; 382:9-19.

27. Radu R, Terecoasă E, Băjenaru $\mathrm{O}$ et al. Etiologic classification of ischemic stroke: Where do we stand? Clin Neurol Neurosurg. 2017; 159:93-106.

28. Ay H, Benner T, Arsava EM. A computerized algorithm for etiologic classification of ischemic stroke: The Causative Classification of Stroke System. Stroke 2007; 38: 2979-84.

29. Amarenco P, Bogousslavsky J, Caplan LR, et al. New approach to stroke subtyping: The A-S-C-O (phenotypic) classification of stroke. Cerebrovasc Dis 2009; 27: 502-508.

30. Adams H, Bendixen B, Kapplle LJ, et al. Classification of subtype of acute ischemic stroke. Definitions for use in a multicenter clinical trial. TOAST. Trial of Org 10172 in Acute Stroke Treatment. Stroke 1993;24: 35-41.

31. Ay H, Furie KL, Singhal A, et al.An Evidence-Based Causative Classification System for Acute Ischemic Stroke. Ann Neurol 2005, 58:688-697.

32. Pistoia F, Sacco S, Tiseo C. The Epidemiology of Atrial Fibrillation and Stroke Cardiol Clin 2016, 34: 255-268.

33. O'Carroll C, Barrett K. Cardioembolic Stroke. Continuum 2017; 23 :111-132.

34. Kamel H, Healey J. Cardioembolic Stroke. Circ Res. 2017, 120: 514-526.

35. Yiin GSC, Howard DPJ, Paul NLM, et al.Age-specific incidence, outcome, cost, and projected future burden of atrial fibrillation-related embolic vascular events: a population-based study. Circulation 2014, 130: 1236-1244.

36. Lip G, Nieuwlaat R, Pisters R, et al. Refining clinical risk stratification for predicting stroke and thromboembolism in atrial fibrillation using a novel risk factorbased approach: the Euro Heart survey on atrial fibrillation. Chest 2010; 137:263-272.

37. Berge E, Abdelnoor M, Nakstad PH, Sandset PM; on behalf of the HAEST Study Group. Low molecular-weight heparin versus aspirin in patients with acute ischaemic stroke and atrial fibrillation: a double-blind randomised study. Lancet. 2000; 355:1205-10.

38. Paciaroni M, Agnelli G, Micheli S, et al. Efficacy and safety of anticoagulant treatment in acute cardioembolic stroke: a meta-analysis of randomized controlled trials. Stroke 2007, 38:423-430.

39. Kirchhof P, Benussi S, Kotecha D, et al. 2016 ESC Guidelines for the management of atrial fibrillation develo- 
ped in collaboration with EACTS. Eur Heart J 2016, 37:893-962.

40. Seiffge D, Werring D, Paciaroni M, et al. Timing of anticoagulation after recent ischaemic stroke in patients with atrial fibrillation. Lancet Neurol 2019, 18:117-126.

41. Pistoia F, Sacco S, Tiseo C et al. The Epidemiology of Atrial Fibrillation and Stroke. Cardiol Clin 2016, 34 : 255-68.

42. Connolly SJ, Ezekowitz MD, Yusuf S, et al. Dabigatran versus warfarin in patients with atrial fibrillation. $\mathrm{N}$ Engl J Med 2009; 361:1139-1151.

43. EAFT (European Atrial Fibrillation Trial) Study Group. Secondary prevention in non-rheumatic atrial fibrillation after transient ischaemic attack or minor stroke. Lancet 1993; 342:1255-62.

44. Granger CB, Alexander JH, McMurray JJ, et al. Apixaban versus warfarin in patients with atrial fibrillation. $\mathrm{N}$ Engl J Med. 2011; 365:981-92.

45. Giugliano RP, Ruff CT, Braunwald E, et al. Edoxaban versus warfarin in patients with atrial fibrillation. N Engl J Med. 2013; 369:2093-2104.

46. Patel MR, Mahaffey KW, Garg J, et al. Rivaroxaban versus warfarin in nonvalvular atrial fibrillation. N Engl J Med. 2011; 365:883-891.

47. Heidbuchel H, Verhamme P, Alings M, et al. EHRA practical guide on the use of new oral anticoagulants in patients with non-valvular atrial fibrillation: executive summary. Eur Heart J. 2013;34:2094-2106.

48. Fanola CL, Ruff CT, Murphy SA, et al. Efficacy and Safety of Edoxaban in Patients With Active Malignancy and Atrial Fibrillation: Analysis of the ENGAGE AF TIMI 48 Trial. J Am Heart Assoc. 2018;7:e008987.

49. Lopes RD, Alexander JH, Al-Khatib SM, et al. Apixaban for reduction in stroke and other ThromboemboLic events in atrial fibrillation (ARISTOTLE) trial: design and rationale [published correction appears in Am Heart J. 2010 Jun;159(6):1162]. Am Heart J. 2010;159: 331-339.

50. Hori M, Matsumoto M, Tanahashi N, et al. Rivaroxaban vs. warfarin in Japanese patients with atrial fibrillation - the J-ROCKET AF study -. Circ J. 2012;76:2104-2111.

51. Ezekowitz MD, Connolly S, Parekh A, et al. Rationale and design of RE-LY: randomized evaluation of longterm anticoagulant therapy, warfarin, compared with dabigatran. Am Heart J. 2009;157:805-810.e8102.

52. Halvorsen S, Atar D, Yang H, et al. Efficacy and safety of apixaban compared with warfarin according to age for stroke prevention in atrial fibrillation: observations from the ARISTOTLE trial. Eur Heart J. 2014; 35: 1864-1872.

53. Halperin JL, Hankey GJ, Wojdyla DM, et al. Efficacy and safety of rivaroxaban compared with warfarin among elderly patients with nonvalvular atrial fibrillation in the Rivaroxaban Once Daily, Oral, Direct Factor Xa Inhibition Compared With Vitamin K Antagonism for Prevention of Stroke and Embolism Trial in Atrial Fibrillation (ROCKET AF) [published correction appears in Circulation. 2018 Dec 18;138(25): e783]. Circulation. 2014; 130:138-146.

54. Lauw MN, Eikelboom JW, Coppens M, et al. Effects of dabigatran according to age in atrial fibrillation. Heart. 2017; 103:1015-1023.

55. ACTIVE Investigators, Connolly SJ, Pogue J, et al. Ef- fect of clopidogrel added to aspirin in patients with atrial fibrillation. N Engl J Med. 2009; 360:2066-78.

56. Reddy VY, Holmes D, Doshi SK, et al. Safety of percutaneous left atrial appendage closure: results from the Watchman Left Atrial Appendage System for Embolic Protection in Patients with AF (PROTECT AF) clinical trial and the Continued Access Registry. Circulation. 2011; 123:417-24.

57. Holmes DR Jr, Kar S, Price MJ, et al. Prospective randomized evaluation of the Watchman Left Atrial Appendage Closure device in patients with atrial fibrillation versus long-term warfarin therapy: the PREVAIL trial. J Am Coll Cardiol. 2014; 64:1-12.

58. Cannegieter SC, Rosendaal FR, Briët E. Thromboembolic and bleeding complications in patients with mechanical heart valve prostheses. Circulation. 1994;8 9:635-41.

59. Esenwa C, Gutierrez J. Secondary stroke prevention: challenges and solutions. Vasc Health Risk Manag. 2015; 11:437-50.

60. Van de Werf F, Brueckmann M, Connolly SJ, et al. A comparison of dabigatran etexilate with warfarin in patients with mechanical heart valves: THE Randomized, phase II study to evaluate the safety and pharmacokinetics of oral dabigatran etexilate in patients after heart valve replacement (RE-ALIGN). Am Heart J. 2012; 163:931-37.e1.

61. Schumacher K, Kornej J, Shantsila E, Lip GYH. Heart Failure and Stroke. Curr Heart Fail Rep. 2018; 15: 287-96.

62. Kim W, Kim EJ. Heart Failure as a Risk Factor for Stroke. J Stroke. 2018; 20:33-45.

63. Xiong Q, Lau YC, Senoo K, et al. Non-vitamin K antagonist oral anticoagulants (NOACs) in patients with concomitant atrial fibrillation and heart failure: a systemic review and meta-analysis of randomized trials. Eur J Heart Fail. 2015; 17:1192-1200.

64. Beggs SAS, Rørth R, Gardner RS, et al. Anticoagulation therapy in heart failure and sinus rhythm: a systematic review and meta-analysis. Heart. 2019; 105:1325-34.

65. Pullicino P, Thompson JL, Barton B, et al. Warfarin versus aspirin in patients with reduced cardiac ejection fraction (WARCEF): rationale, objectives, and design. J Card Fail. 2006; 12:39-46.

66. Massie BM, Collins JF, Ammon SE, et al. Randomized trial of warfarin, aspirin, and clopidogrel in patients with chronic heart failure: the Warfarin and Antiplatelet Therapy in Chronic Heart Failure (WATCH) trial. Circulation. 2009; 119:1616-24.

67. Cokkinos DV, Haralabopoulos GC, Kostis JB, et al. HELAS investigators. Efficacy of antithrombotic therapy in chronic heart failure: the HELAS study. Eur J Heart Fail. 2006;8(4):428-432.

68. Cleland JG, Findlay I, Jafri S, et al. The Warfarin/Aspirin Study in Heart failure (WASH): a randomized trial comparing antithrombotic strategies for patients with heart failure. Am Heart J. 2004; 148:157-64.

69. Zannad F, Anker SD, Byra WM, et al. Rivaroxaban in Patients with Heart Failure, Sinus Rhythm, and Coronary Disease. N Engl J Med. 2018;379(14):1332-42.

70. Antithrombotic Trialists' Collaboration. Collaborative meta-analysis of randomised trials of antiplatelet therapy for prevention of death, myocardial infarction, and 
stroke in high risk patients [published correction appears in BMJ 2002 Jan 19;324(7330):141]. BMJ. 2002; 324:71-86.

71. CAPRIE Steering Committee. A randomised, blinded, trial of clopidogrel versus aspirin in patients at risk of ischaemic events (CAPRIE). CAPRIE Steering Committee. Lancet. 1996; 348:1329-39.

72. Diener HC, Cunha L, Forbes C, et al. European Stroke Prevention Study. 2. Dipyridamole and acetylsalicylic acid in the secondary prevention of stroke. J Neurol Sci. 1996;143(1-2):1-13.

73. Diener HC, Sacco RL, Yusuf S, et al. Effects of aspirin plus extended-release dipyridamole versus clopidogrel and telmisartan on disability and cognitive function after recurrent stroke in patients with ischaemic stroke in the Prevention Regimen for Effectively Avoiding Second Strokes (PRoFESS) trial: a double-blind, active and placebo-controlled study [published correction appears in Lancet Neurol. 2008 Nov;7(11):985]. Lancet Neurol. 2008; 7:875-84.

74. Diener HC, Bogousslavsky J, Brass LM, et al. Aspirin and clopidogrel compared with clopidogrel alone after recent ischaemic stroke or transient ischaemic attack in high-risk patients (MATCH): randomised, double-blind, placebo-controlled trial. Lancet. 2004; 364:331-37.

75. Bhatt DL, Fox KA, Hacke W, et al. CHARISMA Investigators. Clopidogrel and aspirin versus aspirin alone for the prevention of atherothrombotic events. N Engl J Med. 2006; 354:1706-1717.

76. SPS3 Investigators; Benavente OR, Hart RG, McClure LA, et al. Effects of clopidogrel added to aspirin in patients with recent lacunar stroke. N Engl J Med. 2012; 367:817-25.

77. Coutts SB, Hill MD, Simon JE, et al. Silent ischemia in minor stroke and TIA patients identified on MR imaging. Neurology. 2005; 65:513-17.

78. Fischer U, Baumgartner A, Arnold M, et al. What is a minor stroke? Stroke 2010; 41:661-66.

79. Re-examining Acute Eligibility for Thrombolysis (TREAT) Task Force; Levine SR, Khatri P, et al. Review, historical context, and clarifications of the NINDS rt-PA stroke trials exclusion criteria: Part 1: rapidly improving stroke symptoms. Stroke. 2013; 44:2500-2505.

80. Easton JD, Saver JL, Albers GW, et al. Definition and evaluation of transient ischemic attack: a scientific statement for healthcare professionals from the American Heart Association/American Stroke Association Stroke Council; Council on Cardiovascular Surgery and Anesthesia; Council on Cardiovascular Radiology and Intervention; Council on Cardiovascular Nursing; and the Interdisciplinary Council on Peripheral Vascular Disease. The American Academy of Neurology affirms the value of this statement as an educational tool for neurologists. Stroke. 2009; 40:2276-93.

81. Johnston SC, Easton JD, Farrant M, et al. Clopidogrel and Aspirin in Acute Ischemic Stroke and High-Risk TIA. N Engl J Med. 2018; 379:215-25.

82. Wang Y, Wang Y, Zhao X, et al. Clopidogrel with aspirin in acute minor stroke or transient ischemic attack. N Engl J Med. 2013; 369:11-19.

83. Johnston SC, Amarenco P, Albers GW, et al. Ticagrelor versus Aspirin in Acute Stroke or Transient Ischemic Attack. N Engl J Med. 2016; 375:35-43.
84. Johnston SC, Amarenco P, Denison H, et al. The Acute Stroke or Transient Ischemic Attack Treated with Ticagrelor and Aspirin for Prevention of Stroke and Death (THALES) trial: Rationale and design. Int J Stroke. 2019; 14: 745-51.

85. Chimowitz MI, Lynn MJ, Howlett-Smith H, et al; Warfarin-Aspirin Symptomatic Intracranial Disease Trial Investigators. Comparison of warfarin and aspirin for symptomatic intracranial arterial stenosis. $\mathrm{N}$ Engl $\mathrm{J}$ Med. 2005; 352:1305-16.

86. Derdeyn CP, Chimowitz MI, Lynn MJ, et al; Stenting and Aggressive Medical Management for Preventing Recurrent Stroke in Intracranial Stenosis Trial Investigators. Aggressive medical treatment with or without stenting in high-risk patients with intracranial artery stenosis (SAMMPRIS): the final results of a randomised trial. Lancet. 2014; 383:333-341.

87. Holmstedt CA, Turan TN, Chimowitz MI. Atherosclerotic intracranial arterial stenosis: risk factors, diagnosis, and treatment. Lancet Neurol. 2013; 12:1106-14.

88. Ferguson GG, Eliasziw M, Barr HW, et al. The North American Symptomatic Carotid Endarterectomy Trial: surgical results in 1415 patients. Stroke. 1999;30:175158.

89. Liu ZJ, Fu WG, Guo ZY, et al. Updated systematic review and meta-analysis of randomized clinical trials comparing carotid artery stenting and carotid endarterectomy in the treatment of carotid stenosis. Ann Vasc Surg. 2012; 26:576-90.

90. Rantner B, Goebel G, Bonati LH, et al. Carotid Stenting Trialists' Collaboration. The risk of carotid artery stenting compared with carotid endarterectomy is greatest in patients treated within 7 days of symptoms. J Vasc Surg. 2013; 57:619-26.e2.

91. International Carotid Stenting Study Investigators; Ederle J, Dobson J, Featherstone RL, et al. Carotid artery stenting compared with endarterectomy in patients with symptomatic carotid stenosis (International Carotid Stenting Study): an interim analysis of a randomised controlled trial [published correction appears in Lancet. 2010; 376:90]. Lancet 2010; 375:985-97.

92. Carotid Stenting Trialists' Collaboration; Bonati LH, Dobson J, Algra A, et al. Short-term outcome after stenting versus endarterectomy for symptomatic carotid stenosis: a preplanned metaanalysis of individual patient data. Lancet. 2010; 376:1062-73.

93. Khairy P, O'Donnell CP, Landzberg MJ. Transcatheter closure versus medical therapy of patent foramen ovale and presumed paradoxical thromboemboli: a systematic review. Ann Intern Med. 2003; 139:753-60.

94. Furlan AJ, Reisman M, Massaro J, et al. Closure or medical therapy for cryptogenic stroke with patent foramen ovale. N Engl J Med. 2012; 366:991-99.

95. Carroll JD, Saver JL, Thaler DE, et al. Closure of patent foramen ovale versus medical therapy after cryptogenic stroke. N Engl J Med. 2013; 368:1092-1100.

96. Meier B, Kalesan B, Mattle HP, et al. Percutaneous closure of patent foramen ovale in cryptogenic embolism. N Engl J Med. 2013; 368:1083-1091.

97. Saver JL, Carroll JD, Thaler DE, et al. Long-Term Outcomes of Patent Foramen Ovale Closure or Medical Therapy after Stroke. N Engl J Med. 2017; 377: 1022-1032. 
98. Mas JL, Derumeaux G, Guillon B, et al. Patent Foramen Ovale Closure or Anticoagulation vs. Antiplatelets after Stroke. N Engl J Med. 2017 ;377:1011-21.

99. Søndergaard L, Kasner SE, Rhodes JF, et al. Patent Foramen Ovale Closure or Antiplatelet Therapy for Cryptogenic Stroke. N Engl J Med. 2017; 377:1033-42.

100. Lee PH, Song JK, Kim JS, et al. Cryptogenic Stroke and High-Risk Patent Foramen Ovale: The DEFENSEPFO Trial. J Am Coll Cardiol. 2018;71(20):2335-2342.

101. Pristipino C, Sievert H, D'Ascenzo F, et al. European position paper on the management of patients with patent foramen ovale. General approach and left circulation thromboembolism. EuroIntervention. 2019; 14:1389-1402. 


\title{
La riabilitazione dell'ictus
}

\author{
Paolo Milia, ${ }^{1}$ Giovanni Regesta ${ }^{2}$ \\ ${ }^{1}$ Istituto Prosperius Tiberino Area Alta Complessità Neuroriabilitazione Robotica, Università di Perugia; ${ }^{2}$ Centro di Riabilitazione \\ Gruppo Fides srl, Genova, Italia
}

\section{Introduzione}

Nonostante gli importanti risultati ottenuti negli ultimi vent'anni nel trattamento dell'ictus acuto, in termini di riduzione della mortalità e di deficit residui, grazie alla gestione in stroke unit e, limitatamente all'ictus ischemico, alla trombolisi endovenosa e alla trombectomia meccanica, ancora oggi circa due terzi dei pazienti sopravvissuti necessitano di un trattamento riabilitativo destinato a durare nel tempo. L'ictus rimane pertanto la prima causa di disabilità neurologica a lungo termine. Infatti, la diminuzione del tasso di incidenza dell'ictus correlato all'età osservato negli anni recenti, da attribuirsi ad una migliorata attenzione nei confronti della prevenzione primaria, non ha determinato una riduzione dell'incidenza cumulativa che, al contrario, è destinata ad aumentare in misura rilevante a causa del progressivo invecchiamento della popolazione nel mondo occidentale. Le proiezioni indicano che nel 2035 vi sarà un aumento del $35 \%$ del numero complessivo di ictus in Europa rispetto al 2015. ${ }^{1}$

Già oggi una elevata percentuale di pazienti trova difficoltà ad intraprendere un percorso riabilitativo personalizzato e aggiornato alle recenti conoscenze, vanificando così in gran parte i risultati ottenuti nella fase acuta. Tale situazione di insufficiente offerta riabilitativa risente in qualche modo di una percezione, tuttora persistente, dell'ictus come evento acuto anziché come malattia cronica ad esordio acuto che richiede terapie adeguate e attento monitoraggio per la sua intera durata. Esiste quindi l'impellente necessità di un potenziamento quali- quantitativo

Corrispondente: Giovanni Regesta, Centro di Riabilitazione Gruppo Fides srl, Via Bolzano 1, 16166 Genova, Italia.

E-mail: giovanniregesta@gruppofides.it

Articolo pubblicato secondo la Creative Commons Attribution NonCommercial 4.0 License (CC BY-NC 4.0).

${ }^{\circ}$ Copyright: the Author(s), 2020

Licensee PAGEPress, Italy

QUADERNI - Italian Journal of Medicine 2020; 8(2):142-152 dell'assistenza riabilitativa, ospedaliera e territoriale, anche mediante investimenti in tecnologie innovative, in grado di offrire risposte stato dell'arte ad una domanda in costante crescita. Infatti, la tecnologia è oramai entrata a far parte in modo significativo del percorso del paziente, garantendo una riabilitazione ad alta intensità e alta frequenza.

\section{Fase acuta}

L'importanza di iniziare precocemente il trattamento riabilitativo, entro 48 ore dall'esordio, viene sottolineato da varie linee guida ${ }^{2-4}$ anche se il tempo ottimale non può essere definito, richiedendo una valutazione del singolo caso, soprattutto nei soggetti molto anziani o con ictus severo nei quali un inizio molto precoce, entro 24 ore, risulta potenzialmente pericoloso. L'inizio precoce si basa sul presupposto che nelle prime 4-6 settimane dopo l'ictus la plasticità neuronale post-lesionale è particolarmente attiva (finestra di neuroplasticità) attraverso una serie di eventi cellulari e molecolari che consentono alle strutture cerebrali rimaste indenni di riorganizzarsi e di consentire il recupero funzionale. ${ }^{5}$ Sino a non molto tempo fa la convinzione comune che i neuroni che muoiono con l'età o a seguito di una lesione cerebrale non potessero essere rimpiazzati aveva determinato un atteggiamento quasi fatalistico nei confronti delle conseguenze dell'ictus cerebrale e delle possibilità di recupero. In anni più recenti, la dimostrazione della crescita successiva a danno cerebrale di nuovi dendriti e in alcuni casi di assoni, con formazione di nuove sinapsi anche nella corteccia controlaterale; ${ }^{6,7}$ la comparsa precoce nella penombra ischemica di angiogenesi capace di favorire la migrazione e la differenziazione di cellule staminali neurali nella zona peri-infartuale $;{ }^{8}$ la dimostrazione basata su numerosi studi fMRI e PET che una funzione rappresentata nella zona danneggiata possa migrare alla regione omologa controlaterale ed eventualmente ritornare in un'area perilesionale dell'emisfero leso (riorganizzazione corticale); ${ }^{6,9}$ la possibilità di modulare la plasticità neurale, favorire l'apprendimento e il recupero con metodi innovativi di stimolazione multisensoriale; ${ }^{10}$ la 
recente scoperta di aumento post ictus delle concentrazioni di BDNF (Brain derived neurotrophic factor) dopo esercizio aerobico in modelli animali e nell'uomo, ${ }^{11}$ sono state le principali acquisizioni che hanno evidenziato la necessità di un inizio precoce della riabilitazione. ${ }^{12}$

Il trattamento consiste inizialmente nella mobilizzazione passiva e possibilmente attiva, quando le condizioni del paziente lo consentono, secondo tutto il range di movimento (ROM) delle articolazioni. Il programma prosegue con il corretto posizionamento del paziente seduto sul bordo del letto per il controllo posturale del tronco e del capo e con il successivo trasferimento su sedia o su comoda, tenendo conto che nelle prime fasi la ridotta mobilità, con conseguente difficoltà a modificare il carico, è spesso causa di insofferenza o anche di dolore. La posizione corretta della spalla e la corretta gestione dei trasferimenti posturali sono indispensabili per prevenire la sublussazione dell'articolazione gleno-omerale, con conseguente insorgenza della Sindrome della spalla dolorosa, una complicanza frequente dei pazienti emiplegici che si osserva prevalentemente nella fase ipotonica flaccida e condiziona negativamente il percorso riabilitativo. $\mathrm{Si}$ passa quindi alla verticalizzazione con l'assunzione della stazione eretta, avvalendosi dello standing (stabilizzatore per statica), al caricamento del peso ed al cammino. ${ }^{3}$

\section{Valutazione del deficit e progetto riabilitativo individuale}

La presa in carico del paziente richiede l'elaborazione di un Progetto Riabilitativo Individuale (PRI), a cui partecipano tutti i componenti del team, basato su una valutazione clinico-funzionale effettuata mediante un esame obiettivo accurato e l'impiego di scale di valutazione standardizzate, con indicazione degli obiettivi, delle modalità e del tempo giornaliero di erogazione del trattamento, e definizione delle misure di esito. Il PRI deve essere condiviso con il paziente e con la famiglia ed aggiornato periodicamente.

Le scale di valutazione devono essere facili e veloci da utilizzare, accettate dal paziente, sensibili a variazioni cliniche significative, riproducibili con valutazioni ripetute dallo stesso osservatore o da osservatori diversi. Le scale maggiormente utili e più comunemente usate sono le seguenti.

National Institute of Health Stroke Scale (NIHSS). Strumento fondamentale per la valutazione del deficit e della prognosi in fase acuta, viene impiegata anche nella valutazione successiva per quantificare l'evoluzione del quadro neurologico. E' composta da 15 item ognuno dei quali valuta una funzione con un punteggio da 0 (nessuna compromissione) a 3 o 4 punti (compromissione grave). Il punteggio massimo è 42. Un limite della scala è rappresentato dal peso maggiore attribuito a lesioni dell'emisfero dominante rispetto a quello non dominante a parità di volume della lesione e dal peso più limitato assegnato alle lesioni del circolo posteriore rispetto a quelle del circolo anteriore.

Scandinavian Stoke Scale (SSS). Rappresenta una alternativa alla NIHSS. La versione per l'impiego a lungo termine è composta da 7 item valutati con un punteggio da 0 (compromissione grave) ad un massimo variabile da 2 a 12 (nessuna compromissione) secondo la funzione esplorata. Il punteggio massimo totale è 48 .

Sia la NIHSS che la SSS, progettate per essere impiegate in trial clinici, sono successivamente entrate nell'uso clinico corrente. Entrambe semplici da somministrare, richiedono pochi minuti e presentano una buona riproducibilità tra osservatori.

Specificamente progettata per la riabilitazione è invece la Scala di Fugl-Mayer. E' la scala più usata per la valutazione quantitativa del recupero sensorimotorio nei pazienti con ictus ed è divisa in 5 sezioni: funzione motoria (100 punti: 66 arto superiore, 34 arto inferiore), funzione sensitiva (24 punti), equilibrio (14 punti), ampiezza di movimento articolare (ROM) espressa in gradi (44 punti), dolore articolare (44 punti). Punteggio massimo 226. La somministrazione della scala completa richiede circa 30 minuti, con eccellente riproducibilità tra esaminatori.

Scala di Tinetti. E' lo strumento clinimetrico maggiormente usato per valutare l'equilibrio, l'andatura e il rischio di caduta. Si compone di due parti: la prima riguarda l'equilibrio con un punteggio da 0 a 16, la seconda si riferisce all'andatura con un punteggio da 0 a 12 . Si ottiene così un punteggio totale da 0 a 28. Un punteggio inferore a 20 esprime rischio di cadute. Valida ed affidabile, richiede pochi minuti.

Scala di Berg (Berg Balance Scale). Largamente utilizzata per rilevare i disturbi dell'equilibrio nei pazienti post ictus, consiste di 14 compiti che richiedono equilibrio valutati con un punteggio da $0 \mathrm{a}$ 4. Il punteggio massimo è $56 \mathrm{e}$ un punteggio di 45 viene considerato il cut-off per rischio di caduta. Richiede circa 15 minuti, è valida ed affidabile con eccellente riproducibilità.

Scala di Ashworth modificata (mAS). E' la scala normalmente usata per la valutazione qualitativa della spasticità ed è basata sull'esame della resistenza del muscolo all'allungamento passivo. L'aumento del tono muscolare viene rappresentato con un punteggio da 0 a 5 agli arti superiori (spalla, gomito, polso) e agli arti inferiori (anca, ginocchio, caviglia). Un limite è costituito dal fatto che la valutazione non è conforme al concetto di spasticità come aumento del tono velocità- dipendente e viene influenzata da risposte riflesse allo stiramento (stretch reflex).

Indice di Barthel modificato (mBI). Fornisce un 
punteggio indicativo dell'autonomia e del livello di indipendenza valutato nelle dieci principali attività della vita quotidiana (ADL), con un punteggio complessivo massimo di 100 per la maggiore indipendenza. Un punteggio inferiore a 50 esprime una condizione di dipendenza importante. E' lo strumento di più largo impiego nella riabilitazione dell'ictus, utile per monitorare il recupero. Semplice da somministrare, richiede pochi minuti, è affidabile e riproducibile.

Scala di Rankin modificata (mRS). Valuta il livello globale di inabilità nelle attività della vita quotidiana (ADL) con un punteggio da 0 (assenza di sintomi) a 5 (disabilità grave). Di facile impiego, disponendo di 6 punti complessivi è tuttavia scarsamente responsiva a modificazioni del quadro clinico e può presentare variabilità tra osservatori.

Test dei 9 pioli (Nine-hole pegboard test). Progettato per l'utilizzo nella terapia occupazionale, valuta la destrezza motoria della mano e delle dita. Il paziente deve inserire nove piccoli pioli contenuti in una vaschetta in altrettanti fori disposti ordinatamente su una tavoletta e, dopo averli inseriti, rimuoverli e riporli nella vaschetta. L'esaminatore cronometra il tempo.

Nelle lesioni dell'emisfero dominante è necessaria una valutazione dell'afasia da eseguire con un Test di fluenza verbale (Novelli et al. 1986) che permette di analizzare la capacità di evocazione delle parole. L'esaminatore chiede al paziente di dire in un minuto tutte le parole che iniziano con una determinata lettera dell'alfabeto o tutte le parole che appartengono ad una determinata categoria semantica. Il Test dei gettoni $o$ Token test (De Renzi e Vignolo, 1962) valuta invece la comprensione; il soggetto deve operare con gettoni diversi per forma, colore e grandezza eseguendo gli ordini di difficoltà crescente impartiti dall'esaminatore. Il Test di Aachen- AAT (Huber, 1983; Versione italiana di Luzzatti, 1991), il più completo, molto affidabile, permette di accertare il tipo e la gravità dell'afasia e di seguirne l'evoluzione; può risultare impegativo per il paziente e richiede di essere valutato da personale specializzato.

E' inoltre necessario indagare la presenza di una aprassia ideomotoria, generalmente bilaterale, spesso associata ad afasia, chiedendo al paziente, sempre che non siano presenti disturbi afasici di comprensione, di eseguire gesti che hanno un significato simbolico (saluto militare, segno della croce, ciao) e gesti transitivi connessi con l'uso di oggetti comuni (forbici, fiammifero).

Il metodo più corretto è però quello di far ricorso a prove di imitazione, in quanto non richiedono al paziente la rappresentazione mentale del movimento che per definizione è conservata in questo tipo di aprassia mentre risulta compromessa nella meno frequente aprassia ideatoria.
Nelle lesioni dell'emisfero non dominante occorre invece ricercare la presenza di una sindrome di emidisattenzione spaziale (neglect), un disturbo fortemente disabilitante di cui i pazienti presentano spesso una consapevolezza limitata o assente (anosognosia), o nei confronti della quale manifestano indifferenza (anosodiaforia). Con il Test di barrage di linee (Albert, 1973), di cui esistono varie versioni, al paziente viene richiesto di barrare con una matita tutte le linee rappresentate su un foglio A4 posto di fronte a lui. Nel Test delle campanelle (Gauthier, 1989) il paziente deve cancellare le campanelle rappresentate su un foglio insieme ad altri simboli con funzione di distrattori. I test consentono la diagnosi differenziale nei confronti di una emianopsia, che in taluni casi può coesistere, in quanto i pazienti emianoptici superano il loro deficit con movimenti compensatori degli occhi e del capo.

Il processo di riabilitazione viene influenzato dalla eventuale presenza di deficit cognitivi per cui è necessario eseguire anche un test sul funzionamento cognitivo globale quale il MMSE-Mini Mental State Examination.

\section{Metodologie riabilitative tradizionali}

Vari sono i metodi impiegati nella riabilitazione dell'ictus che possono essere suddivisi in due grandi gruppi principali, tradizionali e innovativi. I primi, formulati sulla base di principi neurofisiologici, del controllo motorio e del rinforzo muscolare, ed in alcuni casi anche sulle teorie dell'apprendimento, si distinguono tra loro per il diverso approccio teorico ed includono, tra i più diffusi, i seguenti.

Il metodo Bobath ipotizza una relazione fra spasticità e movimento basata sulla conoscenza dei meccanismi riflessi delle risposte posturali e consiste essenzialmente nel cercare di inibire l'aumento del tono con mobilizzazioni passive, iniziando dai cingoli per proseguire distalmente, con l'obiettivo di normalizzare il pattern di movimento ed il controllo posturale. Sviluppato negli anni 60 e diffusosi in Europa, è stato successivamente aggiornato con un approccio problem-solving rivolto alle interazioni tra paziente e ambiente circostante.

Il metodo Kabat, noto anche come Proprioceptive Neuromuscular Facilitation (PNF, di Knott e Voss), introdotto in Italia negli anni 70 e rielaborato come Riequilibrio Modulare Progressivo, è basato sulla stimolazione propriocettiva di muscoli e articolazioni; tende a ricostruire il movimento e la funzione mediante pattern specifici, con direzione diagonale o spirale, e mediante la stimolazione di riflessi da stiramento per aumentare il reclutamento di unità motorie.

Il metodo Perfetti è una tecnica sensori-motoria sviluppata negli anni 70 , volta a determinare la 
riprogrammazione del movimento a livello corticale (motor relearning technique). Inizia con la ricognizione di diversi stimoli tattili e cinestesici derivanti dal movimento per passare all'utilizzo passivo di muscoli e articolazioni e quindi all'utilizzo attivo. A differenza dei metodi precedenti che utilizzano stimoli semplici in grado di provocare una risposta riflessa, senza una partecipazione vera e propria da parte del paziente (c.d. metodi sherringtoniani), questo tipo di approccio considera essenziale il ruolo di processi cognitivi (c.d. metodi corticalisti).

I metodi e le tecniche rivolte al rafforzamento e alla funzionalità dell'azione muscolare, basati sulle teorie dell'apprendimento motorio e del controllo (motor control and learning), prevedono l'esecuzione di movimenti finalizzati e di aggiustamenti posturali mediante distribuzione selettiva della tensione muscolare e sono finalizzati ad acquisire la capacità di eseguire con sufficiente destrezza azioni utili nella vita quotidiana (task oriented) come trasferimenti di posizione, alzarsi e sedersi ripetutamente (sit-tostand), equilibrio, cammino. ${ }^{13}$ Sit-to-stand (STS) è un'abilità chiave nella vita quotidiana, fisicamente impegnativa in quanto condizionata oltreché dalla forza muscolare, dalla resistenza, dall'equilibrio e dalle possibili alterazioni dei diversi tipi di sensibilità. Viene valutata mediante il Test Sit-to-stand (Bohannon, 2006) che misura il tempo impiegato per 5 alzate oppure per 10 alzate o per numero di alzate in $30 \mathrm{sec}^{14}$

E' utile a questo punto considerare che la possibilità di svolgere attività fisica è direttamente correlata con la capacità aerobica che corrisponde alla capacità del sistema cardiorespiratorio di fornire ossigeno e alla capacità del sistema muscoloscheletrico di utilizzarlo. Una prolungata inattività fisica comporta una riduzione della capacità aerobica (decondizionamento) che può essere anche in parte correlata con una pre-esistente insufficienza cardiorespiratoria. Pertanto, migliorare l'attività aerobica è necessario per migliorare il recupero postictus, come è dimostrato da trial clinici randomizzati. I metodi più usati sono l'esecizio del cammino su tappeto scorrevole (treadmill) con parziale sostegno del peso e la riabilitazione in acqua (idroriabilitazione) che offre una discreta resistenza al movimento ed una riduzione di peso sugli arti inferiori. ${ }^{15,16}$

Non esistono evidenze in grado di dimostrare la superiorità di un metodo riabilitativo rispetto agli altri e nessun tipo di trattamento può essere applicato ad ogni paziente, per cui è spesso vantaggioso utilizzare una combinazione di approcci diversi. ${ }^{17}$ Esiste oggi consenso sul fatto che il trattamento debba essere orientato all'esecuzione di compiti di rilievo per le necessità del paziente (task specific) in quanto la semplice ripetizione del movimento senza la comprensione della sua funzione non è in grado di favorire la riorganizzazione corticale necessaria all'apprendimento motorio (motor learning) e, quindi, al miglioramento funzionale. ${ }^{17} \mathrm{Il}$ training task oriented è attualmente considerato il tipo di approccio maggiormente efficace per migliorare le attività della vita quotidiana (ADL) dopo l'ictus e per prevenire il fenomeno del non-uso appreso (learned non-use) che determina l'inibizione del comportamento motorio nell'arto leso in seguito al fallimento dei tentativi di attività.

Nuove metodologie di trattamento sono state sviluppate negli anni recenti con efficacia dimostrata da trial clinici di alta qualità, la cui diffusione rimane purtroppo, a tutt'oggi, estremamente limitata ma il cui uso, combinato con le metodologie tradizionali, è indispensabile per facilitare e massimizzare il recupero funzionale dell'ictus.

\section{Metodologie innovative}

\section{Constraint-induced movement therapy}

Introdotta da Taub e Miltner (1999), è basata sulla teoria derivata dalla ricerca sperimentale del non-uso appreso (learned non-use), comportamento reso reversibile con l'immobilizzazione dell'arto sano. La versione modificata (mCIMT), meno impegnativa e meglio accettata dal paziente, comporta un training ripetitivo task-oriented dell'arto superiore paretico, con impedimento all'uso della mano indenne mediante una manopola imbottita. Gli studi randomizzati controllati sono concordi nel sostenere l'efficacia della tecnica che è indicata in particolare per pazienti con deficit motorio dell'arto superiore di grado moderato ed insorgenza recente, con limitata compromissione cognitiva. ${ }^{18}$ Studi mediante fMRI dimostrano un'attivazione uso-dipendente delle riorganizzazione corticale conseguente alla CIMT. ${ }^{19,20}$

\section{Mirror therapy, action observation treatment, motor imagery}

Queste tecniche innovative utilizzano il ruolo potenziale del meccanismo mirror nel motor learning per il recupero motorio. La scoperta casuale dei neuroni specchio avvenuta da parte di Giacomo Rizzolatti e del suo gruppo, a Parma, all'inizio degli anni 90 nella scimmia e successivamente confermata nell'uomo, costituisce senza dubbio una delle più importanti scoperte nel campo della neurobiologia ed ha permesso interessanti applicazioni nell'ambito della riabilitazione, soprattutto dei pazienti colpiti da ictus. Si tratta di una classe di neuroni visuo-motori che si attivano sia quando viene compiuta una determinata azione, sia quando si osserva la stessa azione compiuta da un altro individuo, sia anche quando l'azione venga solo 
immaginata (neuroni bimodali), ${ }^{21}$ favorendo in tal modo i processi di riorganizzazione neurale.

\section{Mirror therapy}

Consiste nel far eseguire al paziente, a cui sia stato posto sul piano sagittale uno specchio che nasconda l'arto paretico, un movimento con l'arto sano in modo che, proiettato sullo specchio, dia l'illusione di essere compiuto con l'arto paretico. Ne deriva la stimolazione dei neuroni specchio dell'emisfero controlaterale con conseguente eccitabilità cortico spinale che facilita il recupero dell'arto leso. L'efficacia del trattamento (in media $30 \mathrm{~min}$. per sessione, per 4 settimane) che si mantiene oltre i sei mesi, viene evidenziata da una recente revisione sistematica della letteratura. ${ }^{22} \mathrm{La}$ Mirror therapy è altresì in grado di migliorare il recupero dell'emidisattenzione.

\section{Action observation treatment}

Viene richiesto al paziente di osservare sullo schermo di un PC il video di una comune azione della vita quotidiana (es. prendere un caffè) scomposta nelle diverse fasi motorie. Dopo aver osservato un singolo atto, viene richiesto al paziente di imitarlo. Il trattamento, semplice da applicare, richiede attenzione e compliance. I risultati positivi in termini di efficacia sono dimostrati da trial clinici randomizzati. Studi con fMRI confermano la riattivazione di aree motorie all'interno del network dei neuroni specchio. ${ }^{23}$

\section{Motor imagery}

Un trattamento riabilitativo basato sulla capacità di rappresentare mentalmente un atto della vita quotidiana è in grado di attivare le aree cerebrali motorie coinvolte nell'esecuzione reale dell'atto e di favorire il processo riabilitativo. ${ }^{24}$

\section{Realtà virtuale e exergames}

La motivazione è uno degli elementi che maggiormente influenzano i risultati della riabilitazione per cui è importante offrire al paziente che tende a risentire della monotonia del trattamento, la possibilità di affiancare al metodo tradizionale una tecnica avanzata maggiormente gratificante e coinvolgente.

L'uso della Realtà Virtuale non immersiva e, in particolare, della tecnologia dei videogiochi si sta sempre più affermando in neuroriabilitazione come una strategia innovativa ed efficace che permette al paziente di interagire con un ambiente simulato multisensoriale e di ricevere in tempo reale un feedback visivo e/o uditivo della prestazione effettuata. ${ }^{25,26}$ Gli Exergames sono esercizi motori mascherati da videogiochi, specificamente progettati per la riabilitazione, che usano sensori di movimento (Kinect, Leap motion) in grado di catturare i movimenti del paziente. Il sistema dispone di un ampio set di esercizi tra i quali il fisiatra sceglie i più utili in base al tipo e al grado di disabilità, per l'arto superiore, l'arto inferiore, l'equilibrio, l'emidisattenzione spaziale. Il feedback immediato è di grande importanza in quanto determina reazioni istantanee di aggiustamento del controllo motorio task-oriented e favorisce il motor learning e la plasticità neurale. Il sistema, in grado di acquisire dati e informazioni durante lo svolgimento degli exergames, fornisce al fisiatra uno strumento per la valutazione oggettiva e per il monitoraggio continuo della performance. I benefici di tale trattamento sono riportati da trial clinici randomizzati. La presentazione dell'esercizio come gioco, di difficoltà crescente con il migliorare delle prestazioni, stimola il paziente a continuare per superare il proprio record personale e contribuisce a migliorare le funzioni cognitive generali. ${ }^{27,28}$

\section{Stimolazione cerebrale non invasiva}

La Stimolazione cerebrale non invasiva (NIBS) costituisce una strategia terapeutca complementare in grado di modulare l'attività cerebrale e di contribuire alla riabilitazione. ${ }^{29}$ Sia la Stimolazione Magnetica Transcranica ripetitiva (rTMS) che la Stimolazione Elettrica Transcranica a Corrente Contnua (tDCS) favoriscono i meccanismi di plasticità sinaptica mediante potenziamento a lungo termine (LTP) della trasmissione del segnale tra i neuroni attraverso la via dipendente dal recettore per l'NMDA e mediante aumento della secrezione di BDNF.

La stimolazione delle regioni perilesionali con corrente anodica (A-tDCS) o con rTMS ad alta frequenza ha dimostrato effetti positivi nel recupero dell'afasia, confermati da revisioni sistematiche della letteratura. ${ }^{30}$ La tDCS presenta il vantaggio di essere sicura, facile di usare e di richiedere apparecchiature poco costose.

La lesione dell'emisfero sinistro può anche alterare il controllo interemisferico mediato dalle connessioni transcallose, in misura variabile tra le diverse fasi successive all'ictus, con conseguente iperattivazione dell'emisfero destro che si rivela utile se in misura compensatoria ma di danno se in misura elevata e, quindi, a sua volta inibitoria sull'emisfero sinistro (plasticità maladattiva). ${ }^{31}$ In questi casi sono stati dimostrati gli effetti positivi della stimolazione inibitoria con corrente catodica (C-tDCS) sull'area omologa dell'area di Broca. ${ }^{32}$

L'impiego della NIBS può altresì contribuire a migliorare il recupero di varie attività motorie anche se da una revisione della letteratura l'efficacia risulta sinora limitata, probabilmente per la mancanza di protocolli validati. ${ }^{33}$ 


\section{Stimolazione elettrica nervosa transcutanea}

Impiegata generalmente per l'arto inferiore, stimola i recettori cutanei e i propriocettori e la contrazione muscolare, aumenta l'eccitabilità corticale, favorisce la plasticità cerebrale e l'impulso corticale motorio, con risultati sulla performance sensitivo-motoria. Può ridurre la spasticità con conseguente aumento dell'angolo di movimento dell'articolazione (ROMRange of motion) e ridurre il dolore, rallentando la trasmissione sinaptica degli impulsi nocicettivi e stimolando l'aumento della produzione di endorfine. ${ }^{34,35}$

\section{La riabilitazione robotica}

I robot implicati nella riabilitazione possono essere classificati in assistivi quando intervengono compensando il deficit motorio e terapeutici quando invece si caratterizzano per un training con tasks e obiettivi specifici ${ }^{36}$ La tecnologia applicata può essere classificata in end-effector e esoscheletri. ${ }^{37} \mathrm{Nel}$ sistema end-effector lo svolgimento dell'esercizio arriva direttamente dalla parte distale dell'arto garantendo la normale cinematica del movimento, rendendolo più fisiologico e con ottima compliance del paziente. L'esoscheletro si caratterizza invece per avvolgere l'arto e garantendo, tramite dispositivo, una ripetibilità del movimento.

\section{Training del passo (gait training)}

\section{End-effector robot}

Gli studi effettuati su ictus cronico $^{38}$ hanno evidenziato effetti simili tra terapia assistita con robot e fisioterapia convenzionale, sottolineando il concetto che la terapia con end-effector non può sostituire la terapia convenzionale. L'attività riabilitativa nella fase subacuta $^{39}$ ha invece evidenziato che il trattamento robotico associato a terapia convenzionale si caratterizza per un miglior recupero del paziente in termini di deambulazione anche con una significatività superiore rispetto alla terapia convenzionale. ${ }^{40,41}$ Si può concludere che la terapia con end-effector associata a terapia convenzionale è raccomandata nella fase subacuta dell'ictus nel recupero del cammino.

\section{Esoscheletri}

I primi studi condotti con esoscheletri su un numero limitato di pazienti hanno evidenziato una superiorità della terapia con esoscheletro rispetto alla terapia convenzionale. ${ }^{42}$ I trial successivi hanno fornito risultati contrastanti, ${ }^{43}$ tuttavia oggetto della discussione non è la supposta superiorità della terapia robotica bensì il maggior recupero del paziente quando questa sia utilizzata in associazione alla terapia convenzionale. Un recente studio multicentrico ha valutato l'effetto di un esoscheletro in pazienti subacuti, con training del cammino di circa un'ora, 35 volte alla settimana, dimostrando un miglioramento significativo della capacità di camminare anche in pazienti con grave quadro motorio. ${ }^{44,45} \mathrm{~L}$ 'esoscheletro si caratterizza inoltre per una buona compliance del paziente come mostrato in un recente studio che ha evidenziato con scale specifiche la facilità di utilizzo sia nei pazienti con grave lesione midollare sia nei pazienti con ictus. ${ }^{45}$

\section{Riabilitazione dell'arto superiore e della mano}

\section{End-effector}

Una metanalisi Cochrane del 2012 ha evidenziato che la riabilitazione robot-assistita dell'arto superiore si caratterizzava per un miglioramento delle funzioni motorie. ${ }^{46}$ Studi successivi hanno rilevato una maggiore efficacia della terapia robot-assistita con o in assenza di terapia convenzionale sia nei pazienti in fase sub-acuta sia nei pazienti con esiti cronici di ictus. In particolare nei pazienti cronici si è osservato che il recupero è direttamente proporzionale all'intensità di terapia con robot. ${ }^{47}$ Relativamente all'utilizzo della robotica nella riabilitazione della mano gli studi hanno dimostrato l'efficacia della terapia robot -assistita, sebbene non ci siano studi adeguati nel sostenerne la maggiore efficacia rispetto le terapie convenzionali. In particolare uno studio focalizzato sull'utilizzo di un dispositivo tipo guanto ha riscontrato un recupero in termini di motilità funzionale ma scarsi effetti sulla spasticità. ${ }^{48}$ Per una migliore definizione dell'efficacia della terapia endeffector sulla mano sono necessari trials randomizzati.

\section{Esoscheletri}

Il trattamento con esoscheletri nella riabilitazione dell'arto superiore evidenzia un processo di recupero che va di pari passo con il concetto di neuroplasticità. In particolare la capacità dell'esoscheletro di garantire intensità, interattività, flessibilità e adattabilità alle performance del paziente, incrementa la produttività del percorso riabilitativo. Tuttavia la capacità dei vari trials di modificare il corso delle attività cliniche si dimostra con i risultati del trial RATULS ${ }^{49}$ che conclude come il trattamento amplificato per l'arto superiore e il trattamento robotico nei pazienti con esiti di ictus non migliorano l'attività motoria dell'arto superiore rispetto alla terapia convenzionale.

\footnotetext{
Afasia

Non diversamente da quanto avviene per la riabilitazione motoria, nel corso degli anni si sono susseguiti vari tipi di approccio alla rieducazione
} 
dell'afasia, riconducibili ad alcuni filoni principali legati alle teorie interpretative del disturbo afasico sostenute dalle diverse scuole sorte a partire dagli anni ' 70 .

La scuola classica o sindromica di Boston (Goodglass e Kaplan) riconosce due gruppi principali di pazienti afasici, non-fluenti e fluenti, e forme diverse all'interno di entrambi i gruppi.

Nel primo gruppo rientrano l'afasia globale, l'afasia di Broca e l'afasia transcorticale motoria con ripetizione conservata. Nel secondo, l'afasia di Wernicke, l'afasia transcorticale sensoriale con ripetizione conservata, l'afasia di conduzione e l'afasia anomica. Il principio di base del trattamento è quello della stimolazione volta a determinare risposte corrette e raggruppa trattamenti diversi in riferimento alla diagnosi sindromica del disturbo.

L'approccio neurolinguistico, sviluppato principalmente dalla scuola di Aachen (Poeck e Huber), si basa sul concetto che il linguaggio ha un'organizzazione interna che può essere descritta da un sistema di regole e lo analizza secondo i principi della linguistica teorica. Il programma riabilitativo sarà quindi volto a far superare al paziente il deficit linguistico specifico fonologico, lessicale-semantico o sintattico-grammaticale.

L'approccio cognitivo, iniziato dalla scuola di Cambridge (Howard e Hatfield), nasce dalla considerazione che nella maggior parte dei casi non è possibile attribuire i sintomi presenti ad un'unica categoria sindromica e, d'altra parte, i sintomi che definiscono una sindrome spesso non coesistono nel singolo caso. Inoltre, i pazienti con ictus emisferico sinistro ed afasia possono presentare deficit cognitivi associati, in particolare della memoria di lavoro e dell'attenzione, ed esiste una chiara associazione tra questi e la gravità dell'afasia. Solo una diagnosi cognitiva permette quindi di programmare un percorso terapeutico razionale.

L'approccio pragmatico è maggiormante interessato al potenziamento delle abilità comunicative residue del paziente afasico (terapia compensativa) rispetto alla correttezza della produzione. Il metodo più diffuso è il $P A C E$ Promoting Aphasics' Communicative Effectiveness, sviluppato da Davis e Wilcox in USA.

Mentre appare evidente che tutti i pazienti con afasia post-ictale beneficiano della logoterapia, specie se attuata mediante un trattamento intensivo, non risulta dimostrata la superiorità di un metodo rispetto ad altri. ${ }^{50}$ Negli anni più recenti si è assistito allo sviluppo di nuove modalità di approccio terapeutico, tra cui la Stimolazione cerebrale non invasiva ed altre di seguito brevemente illustrate.

La Constraint-Induced Language Therapy (CILT), elaborata sul modello della CIMT impiegata nella rieducazione motoria, è stata studiata come mezzo per migliorare la produzione verbale in pazienti con comprensione conservata. Prevede l'osservazione di figure che il paziente deve denominare escludendo ogni altra possibilità di comunicazione. E' un programma terapeutico intensivo che consente un miglioramento nella produzione di lessemi in un breve periodo. ${ }^{51,52}$

La Stimolazione cerebrale non invasiva costituisce una strategia terapeutica complementare in grado di modulare l'attività cerebrale e di apportare miglioramenti significativi, utilizzata in associazione al trattamento logopedico. Sia la Stimolazione magnetica transcranica ripetitiva (rTMS) che la Stimolazione elettrica transcranica a corrente continua (tDCS) favoriscono i meccanismi di plasticità sinaptica con potenziamento a lungo termine della trasmissione del segnale tra i neuroni attraverso la via dipendente dal recettore per l'NMDA e mediante aumento della secrezione di BDNF. La stimolazione delle regioni perilesionali con corrente anodica (AtDCS) o con rTMS ad alta frequenza ha dimostrato effetti positivi nel recupero dell'afasia confermati da revisioni sistematiche della letteratura. La tDCS presenta il vantaggio di essere facile da usare e di richiedere apparecchiature meno costose. ${ }^{46}$ La lesione dell'emisfero sinistro può anche alterare il controllo interemisferico mediato dalle connessioni transcallose, in misura variabile tra le diverse fasi successive all'ictus, con conseguente iperattivazione dell'emisfero destro che si rivela utile se in misura compensatoria ma di danno se in misura elevata e quindi a sua volta inibitoria sull'emisfero sinistro (plasticità maladattiva). ${ }^{47} \mathrm{Nei}$ casi in cui gli stessi meccanismi alla base della plasticità possono deviare in cambiamenti maladattivi, sono stati dimostrati gli effetti positivi della stimolazione inibitoria con corrente catodica (C-tDCS) sull'area omologa dell'area di Broca. ${ }^{48}$

La Terapia di intonazione melodica, basata sulla capacità relativamente conservata di unirsi al canto, battendo il ritmo con la mano sinistra non affetta da deficit, si rivela utile in pazienti con scarsa espressione verbale ma con buona comprension. ${ }^{53}$ Studi con fMRI hanno dimostrato per la parola intonata (canto), per la percezione del ritmo e della melodia, una rappresentazione biemisferica ma prevalente nell'emisfero destro, in particolare nella circonvoluzione temporale superiore, circonvoluzione frontale inferiore e opercolo centrale. ${ }^{54}$

\section{Aprassia}

Esiste un duplice motivo per cui la riabilitazione dell'aprassia non è mai stata considerata con attenzione. Il primo è connesso con una tendenza al miglioramento spontaneo che si può osservare in una 
certa percentuale di casi nei primi mesi; il secondo è rappresentato dalla dissociazione automaticovolontaria, presente in taluni casi, che rende in qualche misura meno pesanti le ricadute del disturbo nelle ADL. Di conseguenza, non esistono ancora oggi metodologie riabilitative consolidate per il paziente aprassico. Tutti i pazienti non trattati continuano comunque a commettere errori sia nei gesti transitivi che in quelli intransitivi e sono fortemente svantaggiati nella riabilitazione motoria dell'emiparesi. L'approccio riabilitativo cognitivo ipotizza l'esistenza di un magazzino dei movimenti appresi che verrebbe attivato dalla richiesta del gesto secondo la modalità verbale o su imitazione e dovrebbe così essere eseguito con l'organizzazione di singoli movimenti a formare la sequenza del gesto. L'intervento terapeutico viene programmato in base alla sede del danno funzionale mediante test in riferimento al modello di funzionamento normale..$^{55}$ Risultati molto promettenti in termini di miglioramento generale delle abilità gestuali, sono stati ottenuti da uno studio randomizzato che prevedeva un training riabilitativo intensivo di una ampia varietà di gesti transitivi, rilevanti sul piano funzionale e proposti all'interno di situazioni contestuali differenti, oltreché di gesti intransitivi simbolici e non simbolici. ${ }^{56}$

\section{Negligenza spaziale unilaterale o eminattenzione}

Presente in oltre il $50 \%$ dei pazienti con ictus emisferico destro, può ritrovarsi anche nel $10 \%$ dei casi di ictus a carico dell'emisfero dominante. Gli approcci riabilitativi appartengono essenzialmente a due categorie. La modalità top-down richiede l'attiva partecipazione del paziente, incoraggiato a prestare attenzione e ad esplorare lo spazio dal lato ignorato come avviene nel Training di scanning visuo-spaziale (Pizzamiglio, 1992) che opera su PC collegato ad un sistema di proiezione di stimoli su maxischermo con parametri variabili di comparsa, eventualmente accompagnati da stimolazione acustica. Con la stessa modalità agiscono la Realtà Virtuale immersiva, che consente al paziente di interagire con un ambiente simile all'esperienza della vita reale, e gli Exergames. Grazie a queste tecnologie avanzate il paziente può essere valutato $\mathrm{e}$ rieducato in maniera più coinvolgente ed efficace rispetto ai metodi tradizionali. La modalità bottom-up agisce invece mediante stimolazioni sensoriali allo scopo di favorire la rappresentazione dello spazio controlesionale e di riattivare l'equilibrio attentivo interemisferico.

Appartengono a questo tipo la stimolazione propriocettiva dell'arto superiore, la stimolazione optocinetica e l'adattamento prismatico. ${ }^{57}$
La stimolazione cerebrale non invasiva mediante rTMS o tDCS si dimostra molto utile nel rimodulare l'equilibrio tra i due emisferi e ridurre i sintomi del neglect mediante applicazione di stimoli inibitori sulla corteccia parietale posteriore dell'emisfero sano. La mirror therapy si è rivelata efficace nel migliorare i sintomi del neglect in trial clinici controllati e randomizzati. ${ }^{58}$

\section{Spasticità}

La spasticità è una delle complicanze più frequenti dell'ictus, presente nel $30 \%-40 \%$ dei pazienti soprattutto con emiparesi di entità medio-grave. In circa la metà dei casi compare entro il primo mese potendosi manifestare anche nei primi giorni, tende ad aumentare progressivamente nel tempo, interessa i muscoli antigravitari, peggiora la motilità, causa dolore e limita ulteriormente le attività della vita quotidiana.

Gli studi sulle correlazioni tra lesioni di specifiche strutture cerebrali e lo sviluppo della spasticità, eseguiti mediante MRI e mappatura lesione-sintomo basata sui voxel, concordano nell'identificare il ruolo delle lesioni dell'insula, della corteccia premotoria, del braccio posteriore della capsula interna, del talamo, dei nuclei della base, della corona radiata, e del fascicolo longitudinale superiore, mentre resta da chiarire il ruolo svolto dal complesso network neurale interessante le diverse aree. ${ }^{59}$

La classica definizione di Lance (1980), frequentementemente citata, descrive la spasticità come un disturbo motorio caratterizzato da un aumento velocità-dipendente dei riflessi tonici da stiramento (stretch reflex) e quindi del tono muscolare, conseguente a lesione del motoneurone superiore, che altera l'equilibrio fra gli input eccitatori ed inibitori causando una situazione di disinibizione dei riflessi spinali. La spasticità viene in tal modo descritta secondo il significato fisiologico di ipertonia piramidale ma la spasticità che compare dopo l'ictus è un fenomeno più complesso in termini clinici, in quanto espressione di fattori concomitanti quali la rigidità muscolare (stiffness) dovuta ai cambiamenti delle proprietà visco-elastiche del muscolo, alla fibrosi del connettivo e alle retrazioni muscolo-tendinee ${ }^{60}$ oltreché quale risultato del possibile interessamento di altre strutture encefaliche.

La spasticità a carico dell'arto inferiore, se di entità lieve, può avere un effetto compensativo in quanto contribuisce a sostenere il peso corporeo e la postura del tronco nella stazione eretta e nella deambulazione. La spasticità dell'arto superiore comporta invece una significativa compromissione funzionale riducendo l'angolo di movimento e la velocità di movimento.

Il trattamento riabilitativo può essere riferito a due approcci principali, di tipo neurofisiologico o di tipo 
motor learning. Il primo tende a diminuire la spasticità con mobilizzazioni passive e ad agire sulle sinergie patologiche per ottenere sequenze motorie corrette; il secondo prevede invece il coinvolgimento attivo del paziente con l'impiego di compiti motori e relativi feedback per favorire l'apprendimento. Esiste forte evidenza che la TENS, come terapia aggiuntiva, risulta efficace nel ridurre la spasticità all'arto inferiore. ${ }^{36}$ Può essere utile, in molti casi, associare l'impiego di ortesi e soprattutto di splint statici o dinamici.

Nei casi in cui la spasticità è causa di sofferenza e di dolore è indicato l'uso di farmaci in grado di agire sul tono muscolare come il baclofene e il gabapentin per via orale ma i risultati migliori si ottengono con l'iniezione di tossina botulinica.

\section{Disturbi del controllo posturale e dell'equilibrio (balance)}

La compromissione del controllo posturale costituisce uno dei principali fattori che riducono l'indipendenza nelle attività della vita quotidiana (ADL) per l'elevato rischio di cadute e non sempre al miglioramento della funzione motoria dell'arto inferiore paretico corrisponde un miglioramento del balance che dipende da una costante elaborazione dei parametri dell'attività muscolare e dalla sede della lesione cerebrale. Non esistono pertanto tecniche utilizzabili in tutti i casi ma esiste evidenza preliminare che l'esercizio sit-to-stand ripetitivo con biofeedback o associato a exergames e gli esercizi di andatura su tappeto scorrevole sono in grado di migliorare le prestazioni. ${ }^{27,61} \mathrm{La}$ consolle Wii Fit Balance Board, in grado di eseguire il rilevamento della postura eretta e del movimento in tre direzioni, si dimostra uno strumento efficace e a basso costo, utile in particolare per proseguire il trattamento a domicilio.

\section{Conclusioni}

I progressi nella conoscenza dei meccanismi della plasticità neurale, dell'eccitabilità corticale e della neuromodulazione, la dimostrazione del ruolo fondamentale dell'integrazione sensitivo-motoria e dell'integrazione cerebrale di segnali multisensoriali per il recupero della funzione motoria, oltre che del ruolo altrettanto importante del coinvolgimento clinico del sistema dei neuroni specchio nella riabilitazione motoria, dell'afasia e dell'emidisattenzione visuospaziale, hanno favorito l'introduzione nella pratica riabilitativa di strategie innovative. Questo tipo di evoluzione che ha portato a importanti risultati nella riabilitazione dell'ictus, ha favorito il sorgere e l'affermarsi di tecnologie avanzate in continuo sviluppo che consentono di raggiungere risultati sempre maggiori.

Nell'elaborazione del progetto riabilitativo individuale e dei programmi per la sua realizzazione il team assistenziale deciderà la metodologia di approccio più appropriata o la combinazione di approcci diversi, se ritenuta utile nel caso in esame, considerando il livello di compromissione e le diverse fasi della sua evoluzione.

E' chiaro ormai che una maggiore intensità della terapia è associata ad un significativo incremento dell'attività motoria dopo ictus. La riabilitazione convenzionale si basa prevalentemente su un rapporto uno-a-uno tra terapista e paziente, soggetto a costi e limitazioni rappresentate da mancanza di alta intensità e alta frequenza di ripetizioni. Inoltre, le terapie manuali si diversificano in termini di intra- ed intervariabilità. Una recente metanalisi ha evidenziato come il percorso riabilitativo classico si caratterizzi per i tempi insufficienti per raggiungere un recupero ottimale. Le evidenze dei benefici ottenibili da una mobilizzazione precoce, dall'impiego di terapie innovative e di tecnologie avanzate, associate alle terapie convenzionali, ne raccomandano fortemente l'uso. Anche le terapie neurocognitive effettuate attraverso software particolari o addirittura mediante una sintesi tra i diversi programmi digitali, si caratterizzano per un miglior recupero del paziente affetto da lesione ischemica frontale. ${ }^{62}$ La terapia robotica può inoltre essere adattata alla telemedicina permettendo al paziente un suo utilizzo al di fuori del centro di riabilitazione ma sotto il controllo telematico di un fisiatra o di un fisioterapista.

\section{Bibliografia}

1. The Burden of Stroke in Europe. Report. King's College London for the Stroke Alliance for Europe (SAFE) 2017. http://strokeeurope.eu

2. Winstein CJ, Stein J, Arena R, et al. Guidelines for adult stroke rehabiltation and recovery: A guideline for healthcare professionals from the American Heart Association/American Stroke Association. Stroke 2016;47 e98-169.

3. Hebert D, Lindsay MP, McIntyre A, et al. Canadian stroke best practice recommendations: stroke practice guidelines. Int J Stroke 2016;11:459-84.

4. Quinn TJ, Paolucci S, Sunnerhagen KS, et al. Evidencebased stroke rehabilitation: an expanded guidance document from the European Stroke Organization guidelines for management of stroke. J Rehabil Med 2009;41:99-115.

5. Dancause N, Nudo RJ. Shaping plasticity to enhance recovery after injury. Prog Brain Res 2011;19: 273-95.

6. Carmichael ST, Kathirvelu B, Schweppe CA, et al. Molecular, cellular and functional events in axonal sprouting after stroke. Exp Neurol. 2017;287(Pt3):384-94. 
7. Nemirovich-Danchenko NM, Khodanovich MY. New neurons in the post-ischemic and injured brain. Front Neurosci. 2019; 13: 588.

8. Hatakeyama M, Ninomiya I, Kanazawa M. Angiogenesis and neuronal remodeling after ischemic stroke. Neural Regen Res. 2020; 15: 16-19.

9. Saur D, Lange R, Baumgaertner A, et al. Dynamics of language reorganization after stroke. Brain 2006; 129(Pt6): 1371-84.

10. Johansson BB. Multisensory stimulation in stroke rehabilitation. Front Hum Neurosci 2012; 6: 60

11. Mang CS, Campbel KL, Ross CJ, et al. Promoting neuroplasticity for motor rehabilitation after stroke: considering the effects of aerobic exercise and genetic variation on brain derived neurotrophic factor. Phys Ther. 2013; 93: 1707-16.

12. Coleman ER, Moudgal R, Lang K, et al. Early rehabilitation after stroke: a narrative review. Curr Atheroscler Rep. 2017; 19: 59.

13. Krakauer JW. Motor learning: its relevance to stroke recovery and neurorehabilitation. Curr Opin Neurol. 2006; 19: 84-40.

14. Kerr A, Dawson J, Robertson C, et al. Sit to stand activity during stroke rehabilitation. Top Stroke Rehabil. 2017; 24: 562-6.

15. Saunders DH, Sanderson M, Hayes S, et al. Physical fitness training for stroke patients. Cochrane Database Syst Rev. 2016; 3: CD003316.

16. Pollock A, Baer G, Campbell P, et al. Physical rehabilitation approaches for the recovery of function and mobility following stroke (Review). Cochrane Database Syst Rev. 2014: CD001920

17. Langhorne P, Bernhardt J, Kwakkel G. Stroke rehabilitation. Lancet 2011; 377: 1693-702.

18. Kwakkel G, Veerbeek JM, van Wegen EEH, et al. Constraint-induced movement therapy after stroke. Lancet Neurol. 2015; 14: 224-34.

19. Mark VW, Taub E, Morris DM. Neuroplasticity and Constraint-Induced Movement Therapy. Eura.Medicophys. 2006; 42: 269-84.

20. Taub E, Uswatte G, Mark VW. The functional significance of cortical reorganization and the parallel development of Constraint Induced Therapy. Front Hum Neurosci. 2014; 8: 396.

21. Rizzolatti G, Fadiga L, Gallese V, et al. Premotor cortex and recognition of motor actions. Brain Res Cogn Brain Res. 1996; 3: 131-41.

22. Thieme H, Morkish N, Mehrholz J, et al. Mirror therapy for improving motor function after stroke. Cochrane Database Syst Rev. 2018: CD008849.

23. Buccino G. Action observation treatment: a novel tool in neurorehabilitation. Philos Trans Soc Lond B Biol Sci. 2014; 369: 20130185.

24. Machado TC, Carregosa AA, Santos MM, et al. Efficacy of motor imagery additional to motor-based therapy in the recovery of motor function of the upper limb in poststroke individuals: a systematic review. Top Stroke Rehabil. 2019; 26: 548-53.

25. Saposnik G, Levin M. Virtual reality in stroke rehabilitation: a meta-analysis and implication for clinicians. Stroke 2011; 42: 1380-6.

26. Henrique PPB, Colussi EL, DeMarchi ACB. Effects of exergame on patients' balance and upper limb motor function after stroke: a randomized controlled trial. J Stroke Cerebrovasc Dis. 2019; 28: 2351-7.

27. Norouzi-Gheidari N, Hernandez A, Archambault PS, et al. Feasibility, safety and efficacy of a virtual reality system to supplement upper extremity rehabilitation post-stroke. Int J Environ Res Public Health 2020; 17: 113.

28. Kannan L, Vora J, Bhatt T, et al. Cognitive-motor exergaming for reducing fall risk in people with chronic stroke: a randomized controlled trial. Neurorehabil. 2019; doi:10.3233.

29. Webster BR, Celnik PA, Cohen LG. Non-Invasive Brain Stimulation in stroke rehabilitation. NeuroRx 2006; 3: 474-81.

30. Biou E, Cassoudesalle H, Cogné M. Transcranial direct current stimulation in post-stroke aphasia rehabilitation: a randomized controlled trial. Ann Phys Rehabil Med. 2019: 62: 104-21.

31. Xerri C, Zennou-Azogui Y, Sadlaoud K, et al. Interplay between intra- and interhemispheric remodeling of neural networks as a substrate of functional recovery after stroke: Adaptive vs maladaptive reorganization. Neuroscience 2014; 283: 178-201.

32. Kang EK, Kim YK, Sohn HM, et al. Improved picture naming in aphasia patients treated with cathodal tDCS to inhibit the right Broca's homologous area. Restor Neurol Neurosci 2011; 29: 141-52.

33. Morishita T, Hummel FC. Non-invasive brain stimulation in motor recovery after stroke: concepts to increase efficacy. Curr Behav Neurosci Rep. 2017: 4: 280-9.

34. Ng SS, Hui-Chan CWY. TENS combined with taskrelated training improves lower limb functions in subjects with chronic stroke. Stroke 2007; 38: 2953-9.

35. Mahmood A, Veluswamy SK, Hombali A. Effect of TENS on spasticity in adult stroke: A systematic review and meta-analysis. Arch Phys Med Rehabil. 2019; 100: 751-68.

36. Lum PS, Godfrey SB, Brokaw EB, et al. Robotic approaches for rehabilitation of hand after stroke. Am J Med Rehabil. 2012; 91: S242-S254.

37. Mehrholz J, Pohl M. Electromechanical-assisted gait training after stroke: a systematic review comparing end-effector and esoskeleton devices. J Rehabil Med. 2012; 44: 193-99.

38. Dias D, Lains J, Pereira A, et al. Can we improve gait skills in chronic emiplegics? A randomized controlled trial with gait trainer. Eura Medicophys. 2007; 43: 499504.

39. Peurala SH, Tarkka IM, Pitkanen K, et al. The effectiveness of body weight-supported training and floor walking in patients with chronic stroke. Arch Phys Med Rehabil 2005; 86: 1557-64.

40. Marone G, Bragone M, Iosa M, et al. Who may benefit from robotic-assisted gait training? A randomized controlled trial in patients with subacute stroke. Neurorehabil Neural Repair 2011; 25: 636-44.

41. Peurala SH, Airaksinen O, Huuskonen P, et al. Effects of intensive therapy using gait trainer of floor walking exercises early after stroke. J Rehabil Med. 2009; 41: 166-73.

42. Husemann B, Muller F, Krewer C, et al. Effects of locomotion training with assistance of a robot-driven gait 
orthosis in emipartic patients after stroke: a randomized controlled pilot study. Stroke 2007; 38: 349-54.

43. Westlake KP, Patten C. Pilot study of Lokomat versus manual-assisted treadmill training for locomotor recovery post-stroke. J Neuroeng Rehabil. 2009; 6: 18.

44. Goffredo M, Guanziroli E, Pournajaf S, et al. Italian EksoGait Study Study Group. Overground wearable powered exoskeleton for gait training in subacute stroke subjects: clinical and gait assessments. Eur J Phys Rehabil Med. 2019; 55: 710-21.

45. Milia P, De Salvo F, Peccini MC, et al. Exoskeleton in neurorehabilitaton process: Neuropsychological effects in patients affected by spinal cord injury and stroke. Digit Med 2018; 4: 180-3.

46. Mehrholz J, Hadrich A, Platz T, et al. Electromechanical and robot-assisted arm training for improving generic activities of daily living, arm function, and arm muscle strength after stroke. Cochrane Database Syst Rev. $2012 ; 6$.

47. Lo AC, Guarino PD, Richards LG, et al. Robot assisted therapy for long-term upper-limb impairment after stroke. N Engl J Med. 2010; 362: 1772-83.

48. Milia P, Peccini MC, De Salvo F, et al. Rehabilitation with robotic glove (Gloreha) in poststroke patients. Digit Med. 2019; 5: 62-7.

49. Rodgers H, Bosomworth H, Krebs HI, et al. Robotassisted training for the upper limb after stroke (RATULS): a multicenter randomized controlled trial. Lancet 2019; 394: 51-62.

50. Brady MC, Kelly L, Godwin J. Speech and language therapy for aphasia following stroke. Cochrane Database Syst Rev. 2016; CD000425.

51. Pulvermuller F, Berthier ML. Aphasia therapy on a neuroscience basis. Aphasiology 2008;22:563-99

52. Zhang J, Yu J, Bao Y, et al. Constraint-Induced Language Therapy in post-stroke rehabilitation: A systematic review and meta-analysis of randomized controlled trials. PloS One 2017; 12: e0183349.

53. Haro-Martinez AM, Lubrini G, Madero-Jarabo R, et al. Melodic intonation therapy in post-stroke nonfluent aphasia: a randomized controlled trial. Clin Rehabil. 2019; 33: 44-53.

54. Ozdemir E, Norton A, Schlaugh G. Shared and distinct neural correlates of singing and speaking. Nuroimage 2006; 33: 628-35.

55. Cavatorta S. La riabilitazione delle aprassie. In Mazzucchi A. (ed.) La riabilitazione neuropsicologica. Milano, Masson, 2000; pp. 241-261.

56. Smania M, Girardi F, Domenicali C, et al. The rehabilitation of limb apraxia: a study in left brain damaged patients. Arch Phys Med Rehabil. 2000; 81: 379-88.

57. Azouvi P, Jaquin-Courtois S, Luauté J. Rehabilitation of unilateral neglect: evidence-based medicine. Phys Rehab Med Ann. 2017; 60: 191-7.

58. Pandian JD, Arora R, Kaur P, et al. Mirror therapy in unilateral neglect after stroke (MUST trial). Neurology 2014; 83: 1012-7.

59. Lee KB, Hong BY, Kim JS, et al. Which brain lesions produce spasticity? An observational study on 45 stroke patients. PloS One 2019; 14: e0210038.

60. Kuo CL, Hu GC. Post-stroke spasticity: a review of epidemiology, pathophysiology, and treatments. Int J Gerontol. 2018; 12: 280-4.

61. van Duijnhoven HJR, Heeren A, Peters MAM et al. Effect of exercise on balance capacity in chronic stroke. Systematic review and meta-analysis. Stroke 2016; 47 : 2603-10.

62. De Salvo F, Milia P, Peccini MC, et al. Computerized neuropsychological rehabilitation of a frontal lobe stroke: Hierarchical attention training. Digit Med. 2018; 4: 191-6. 


\title{
La riabilitazione post-ictus
}

\author{
Antonio Greco, Francesca Romana Greco \\ UOC Geriatria, IRCCS Casa Sollievo della Sofferenza, San Giovanni Rotondo (FG), Italia
}

\section{Introduzione}

L'ictus ha colpito circa 800.000 persone nel 2017 configurandosi come la quinta causa di morte negli Stati Uniti. ${ }^{1}$ Nonostante la diffusione di questa patologia la sua mortalità si è ridotta di circa il $35 \%$ dal 2001 al 2011 soprattutto in relazione all'introduzione della trombolisi ed all'evoluzione delle tecniche di imaging in grado di rendere più agevole e rapida la diagnosi. Numerosi pazienti, tuttavia, non ricevono un completo beneficio dai trattamenti subiti residuando spesso un'importante disabilità. Almeno il 75\% dei pazienti che sopravvivono allo stroke ricevono uno specifico trattamento riabilitativo. Tenendo conto dei circa sei milioni di persone colpite da ictus e sopravvissute, la stima dei costi annuali sostenuti negli Stati Uniti è stata di circa 18 miliardi di dollari. ${ }^{2}$

Nonostante gli importanti costi sostenuti, lo stroke rimane, un'importantissima causa di disabilità. L'ictus conta in Italia circa 200.000 casi ogni anno, di cui $1 ' 80 \%$ sono nuovi episodi e il $20 \%$ recidive, che riguardano soggetti precedentemente colpiti. In Italia l'ictus è la terza (la seconda, stando ad alcune stime) causa di morte dopo le malattie cardiovascolari e le neoplasie (il 10-12\% di tutti i decessi per anno si verifica dopo un ictus) e rappresenta la principale causa d'invalidità. Nel nostro Paese il numero di soggetti che hanno avuto un ictus e sono sopravvissuti, con esiti più o meno invalidanti, è pari a circa 913.000. Ad 1 anno circa dall'evento acuto, un terzo dei soggetti sopravviventi ad un ictus - indipendentemente dal fatto che sia ischemico o emorragico - presenta un grado di disabilità elevato, tanto da poterli definire totalmente dipendenti. Il fenomeno è in costante crescita a causa

Corrispondente: Antonio Greco, UOC Geriatria, IRCCS Casa Sollievo della Sofferenza, San Giovanni Rotondo (FG), Italia. E-mail: a.greco@operapadrepio.it

Articolo pubblicato secondo la Creative Commons Attribution NonCommercial 4.0 License (CC BY-NC 4.0).

${ }^{\circ}$ Copyright: the Author(s), 2020

Licensee PAGEPress, Italy

QUADERNI - Italian Journal of Medicine 2020; 8(2):153-155 dell'invecchiamento della popolazione. Si stima che l'evoluzione demografica porterà in Italia, se l'incidenza rimanesse costante, ad un aumento dei casi di ictus fino a 207.000 nel 2018. Il tasso di prevalenza di ictus nella popolazione anziana (età 65-84 anni) italiana è del $6,5 \%$, leggermente più alta negli uomini $(7,4 \%)$ rispetto alle donne $(5,9 \%)$.

L'incidenza dell'ictus aumenta progressivamente con l'età raggiungendo il valore massimo negli ultra ottantacinquenni. Il 75\% degli ictus, quindi, colpisce i soggetti di oltre 65 anni. $^{3}$

\section{La riabilitazione}

Le Guidelines for Adult Stroke Recovery and Rehabilitation dell'American Heart Association/American Stroke Association (AHA/ASA) definiscono in maniera precisa i contorni organizzativi della riabilitazione post ictus. Si tratta di un sforzo coordinato di un team multidisciplinare che comprende medici, infermieri, fisioterapisti, logopedisti, psicologi e che coinvolge nel piano di trattamento i pazienti e le loro famiglie. La comunicazione ed il coordinamento dei membri del team sono fondamentali per l'efficienza e l'efficacia dell'intervento riabilitativo. Senza questo, gli eventuali sforzi isolati per riabilitare coloro che sopravvivono allo stroke non riusciranno a raggiungere il risultato atteso. ${ }^{4}$

Il gruppo multidiscipinare AHA/ASA raccomanda in particolare l'integrazione delle competenze del neurologo e del fisiatra per meglio intercettare i bisogni del paziente cercando di superare le parcellizzazioni organizzative del processo di cura, che vedono spesso separati gli attori del processo ponendo il paziente al centro del setting di cura. In accordo con quanto definito dal gruppo multidisciplinare la durata della degenza nel setting per acuti non dovrebbe superare i quattro giorni per l'ictus non complicato per poi proseguire in riabilitazione. ${ }^{5}$ L'assessment per la riabilitazione dovrebbe cominciare durante la fase acuta parallelamente alla rilevazione della scala NIHSS ${ }^{6}$ la quale dovrebbe predire l'intensità riabilitativa da erogare ed il possibile successo della stessa. Score minori di 7 predicono un'elevata probabilità di successo, valori compresi tra 7-16 risultati variabili, e valori mag- 
giori di 16 scarso successo. Pazienti con buono stato funzionale possono essere affidati ad una riabilitazione ambulatoriale od in strutture a bassa intensità di cura. Condizioni di maggiore gravità ed instabilità clinica posso essere gestite in strutture in grado di affrontare la cronica criticità come ad esempio gli ospedali per la Long Term Acute Care (LTAC). Qui, accanto alle facilities riabilitative, sono presenti quelle proprie dell'area intensivistica/rianimatoria. ${ }^{7}$

Le linee guida internazionali raccomandano che i pazienti con stroke siano trattati mediante un approccio multiprofessionale e multidisciplinare secondo il setting più idoneo preferendo, ove possibile, strutture a bassa intensità clinica ma ad alto impegno riabilitativo coordinate dal fisiatra.

In relazione all'elevato consumo di risorse necessario per supportare quanto indicato dalle linee guida sono state elaborate delle strategie riabilitative alternative quali ad esempio la teleriabilitazione. Questo approccio sembrerebbe inoltre venire incontro anche alle esigenze dei pazienti e delle loro famiglie spesso vincolate dai limiti del trasporto pubblico verso le strutture riabilitative. Sono state ipotizzate un teleriabilitazione domiciliare realizzabile attraverso la televisione o lo smartphone e condotte dal fisiatra e dai fisioterapisti per le singole persone disabili che vivono al proprio domicilio o per gruppi di persone che soggiornano in strutture situate in zone rurali lontane dai centri hub. In una metanalisi condotta da Chen e coll. ${ }^{8}$ la teleriabilitazione si è dimostrata non inferiore al trattamento convenzionale in particolare per le persone con elevata compromissione corticale ed alterazione importante delle attività del vivere quotidiano.

\section{La prevenzione delle complicanze legate alle comorbilità}

\section{Prevenzione delle ulcere da pressione e delle contratture muscolari}

Il deficit motorio, le alterazioni della sensibilità somatica e propriocettiva associate alle possibili alterazioni dello stato di coscienza mettono il paziente a rischio di spasticità, anchilosi articolare ed ulcere da pressione. Queste ultime si associano a disturbi del microcircolo, a età avanzata e a incontinenza sia vescicale che rettale. Il regolare controllo della cute e l'uso di scale oggettive come la Braden consentono il corretto approccio al fine di evitare il rischio di sviluppare decubito durante le fasi riabilitative.

In conseguenza dello stroke con emiparesi il 60\% dei pazienti sviluppa nel primo anno spasticità con particolare riguardo alle articolazioni della mano della quale si perde frequentemente l'uso.

Questo deficit produce importanti ripercussioni funzionali sulle attività del vivere quotidiano come l'i- giene personale o la capacità di vestirsi. L'impiego di tutori per la prevenzione della spasticità risulta ancora controverso nelle linee guida internazionali. ${ }^{9}{ }^{10}$ Più certo appare l'impiego dell'ortesi piede-caviglia per favorire la ripresa della deambulazione. ${ }^{11}$

Uno strategia alternativa prevede l'impiego della tossina botulinica. In una recente metanalisi la tossina botulinica si è dimostrata efficace nel controllo della spasticità degli arti superiori nei pazienti con stroke e per questo motivo viene indicata nelle linee guida come un importante strumento della strategia riabilitativa post ictale. E' tuttavia verosimile che l'effetto della tossina si esplichi soprattutto favorendo la possibilità di un trattamento passivo della motilità dell'arto piuttosto che di un movimento attivo con recupero dello skill di motricità. ${ }^{12}$

\section{L'impairment cognitivo post ictus}

L'impairment cognitivo residuale può mostrare un rapido miglioramento con gli interventi riabilitativi. La valutazione neuropsicologica può essere importante per definire gli interventi atti a consentire un precoce reinserimento in ambito lavorativo, favorire il ripristino all'autonomia nella guida dell'auto e, più in generale, una completa indipendenza nelle attività del vivere quotidiano. Le strategie d'intervento per il recupero dai disturbi del linguaggio appaiono più complesse e lunghe e quindi con maggior onere per il paziente. Le nuove tecnologiee assistenziali dedicate al linguaggio ed alla comprensione del test scritto sembrano fornire una valida opzione rendendo disponibile un numero maggiore di ore di riabilitazione riducendo lo stress per i pazienti ed i care giver. ${ }^{2}$

\section{Attività fisica post ictus}

Superata la fase di acuzie, in cui l'intervento dei fisioterapisti è massimale, $\mathrm{e}$ in cui le attività motorie attive e passive sono orientate al pieno recupero funzionale dei distretti colpiti in molti dei piani di trattamento, manca tuttavia un preciso indirizzo su come proseguire l'attività fisica ed il movimento per periodi che generalmente durano mesi od addirittura anni. Le linee Guida ASA/AHA suggeriscono di accelerare quanto più possibile la ripresa dell'esercizio fisico per prevenire le complicanze tardive in questi pazienti. In particolare, l'esercizio fisico aerobico sembra produrre benefici effetti assumendo nelle linee guida il livello di raccomandazione piuttosto che di semplice suggerimento. ${ }^{4}$

\section{Conclusioni}

La comunicazione ed il coordinamento nel processo di cura del paziente con ictus condotta da team 
multidisciplinari e multi professionali si avvantaggia dalla presenza di uno specialista in neuroriabilitazione piuttosto che di altri specialisti compenti nella solo area fisiatrica. Le raccomandazioni suggerite dalle linee guida più recenti rendono mandatoria la presenza di un approccio multidisciplinare ben organizzato e strutturato dedicando le facilities intraospedaliere solo ai casi più gravi e raccomandando un approccio extraospedaliero per i pazienti meno compromessi. I trattamenti riabilitativi dovranno poi proseguire col trasferimento del paziente al domicilio a secondo della necessità del paziente per un periodo almeno di qualche mesi e talora superiori ad un anno. E' verosimile che l'integrazione ospedale-territorio dovrebbe essere massimizzata in questo contesto favorendo anche l'adozione di un sistema di finanziamento che guardi all'intero processo di recupero funzionale piuttosto che alla sola fase ospedaliera.

\section{Bibliografia}

1. Mozaffarian D, Benjamin EJ, Go AS, et al.; on behalf of the American Heart Association Statistics Committee and Stroke Statistics Subcommittee. Heart disease and stroke statistics - 2015 update: a report from the American Heart Association [published corrections appear Circulation. 2015;131:e29-e322.

2. Burris J. Stroke Rehabiliation Current American Stroke Association Guidelines, Care, and implication for practice. Missouri Medicine 2017 .114: 40-44.

3. Ictus: I numeri in Italia. Available at www: siia.it accessed on November 2019.

4. Winstein CJ, Stein J, Arena R, et al; on behalf of the American Heart Association Stroke Council, Council on Cardiovascular and Stroke Nursing, Council on Clinical Cardiology, and Council on Quality of Care and Outco- mes Research. Guidelines for adult stroke rehabilitation and recovery: a guideline for healthcare professionals from the American Heart Association/ American Stroke Association. Stroke. 2016;47:XXX-XXX. DOI: 10.1161/STR.0000000000000098.

5. Harold P. Adams, Gregory del Zoppo, Mark J. Alberts, et al. Guidelines for the Early Management of Adults With Ischemic Stroke; http:// dx.doi.org/10.1161/ STROKEAHA.107.181486 Stroke. 2007;38:1655-1711.

6. Ortiz G.A Sacco RL National Institutes of Health Stroke Scale (NIHSS) https://doi.org/10.1002/9781118445112. stat06823.

7. Makam AN, Tran T, Miller ME, et al. The Clinical Course after Long-term Acute Care Hospital Admission among Older Medicare Beneficiares J Am Geriatr Soc. 2019 Nov;67(11):2282-2288. doi: 10.1111/jgs.16106. Epub 2019 Aug 26.

8. Chen J, Jin W, Zhang XX, et al. Telerehabilitation approaches for stroke patients: systematic review and meta-analysis of Randomized Controlled Trials. J Stroke Cerebrovasc Dis. 2015; 24(12):2660-8. [PubMed: 26483155].

9. Pressure Ulcer Prevention and Treatment Protocol: Health Care Protocol. Bloomington, MN; Institute for Clinical Systems Improvement (ICSI); 2012.

10. Harvey L, de Jong I, Goehl G, Mardwedel S. Twelve weeks of nightly stretch does not reduce thumb webspace contractures in people with a neurological condition: a randomised controlled trial. Aust J Physiother. 2006;52:251-258.

11. Mayer NH, Harvey RL. Use of a resting hand orthosis for the hemi- paretic hand after stroke. PM R. 2014;6:188-195. doi: 10.1016/j. pmrj.2014.01.012.

12. Shaw LC, Price CI, van Wijck FM, et al; BoTULS Investigators. Botulinum Toxin for the Upper Limb after Stroke (BoTULS) Trial: effect on impairment, activity limitation, and pain. Stroke. 2011;42:1371-1379. doi: 10.1161/STROKEAHA.110.582197. 


\title{
Trombosi venosa cerebrale
}

\author{
Angela Iannuzzi \\ Medicina Interna, UOSC PS/OBI AORN Cardarelli, Napoli, Italia
}

\section{Cenni di anatomia}

Il sistema venoso cerebrale consta di un sistema superficiale e di un sistema profondo che drena il sangue di entrambi gli emisferi nelle vene giugulari destra e sinistra. Il sistema superficiale è formato dal seno sagittale superiore, dal seno sagittale inferiore e dalle vene corticali superficiali; il sistema profondo dal seno trasverso, dal seno retto, dal seno sigmoidale e dalle vene corticali profonde.

Tipicamente, il flusso venoso cerebrale inizia con le vene corticali superficiali che drenano nel seno sagittale superiore e inferiore. Il seno sagittale inferiore decorre lungo il margine libero della falce cerebrale e drena nel seno retto che a sua volta giunge al confluente dei seni. Il seno sagittale superiore decorre lungo il margine aderente della falce cerebrale e drena nel confluente dei seni da cui poi partono il seno trasverso destro e sinistro che sfociano nel seno sigmoideo destro e sinistro per terminare nelle vene giugulari interne destre e sinistre. Il seno sagittale superiore drena le porzioni supero-laterali degli emisferi cerebrali bilateralmente ricevendo le vene diploiche, meningee e emissarie. Ciò è di importanza clinica nelle infezioni del cuoio capelluto e del liquido cefalorachidiano in quanto lo stato protrombotico indotto dall'infezione stessa può determinare la formazione di trombi all' interno del seno sagittale superiore. Il seno sagittale inferiore drena gli emisferi cerebrali mediali bilaterali e la falce cerebrale unendosi alla grande vena di Galeno a formare il seno retto. La grande vena di Galeno è formata dalla vena cerebrale interna (a sua

Corrispondente: Angela Iannuzzi, Medicina Interna, Dirigente Medico presso UOSC PS/OBI AORN Cardarelli, via Antonio Cardarelli 9, 80131 Napoli, Italia

Tel.: +39.081.7472888.

E-mail: angela.iannuzzi@aocardarelli.it ;

angelaiannuzzi8@gmail.com

Articolo pubblicato secondo la Creative Commons Attribution NonCommercial 4.0 License (CC BY-NC 4.0).

${ }^{\circ}$ Copyright: the Author(s), 2020

Licensee PAGEPress, Italy

QUADERNI - Italian Journal of Medicine 2020; 8(2):156-167 volta formata dalla vena talamostriata, dalla vena settale e dalla vena corioidea) e dalla vena basale di Rosenthal (formata dalla vena cerebrale anteriore e media e dalla vena striata) che drenano i gangli della base e la sostanza bianca profonda bilateralmente. I seni laterali (trasversi e sigmoidei) ricevono flusso direttamente dagli emisferi cerebrali posteriori, dal tronco encefalico e dal cervelletto bilateralmente. L'importanza clinica di tali strutture consiste nella loro vicinanza alle cellette aeree mastoidee, aumentando la suscettibilità alla formazione di trombi in caso di infezioni quali otiti e mastoiditi (Figura 1).

\section{Key message: Anatomia del circolo venoso cerebrale}

- Il sistema venoso cerebrale consiste di due sistemi, uno superficiale ed uno profondo, che drenano il sangue di tutte le strutture cerebrali nelle vene giugulari destra e sinistra.

- Le anastomosi che si creano a livello dei vasi cerebrali possono temporaneamente sopperire alla riduzione di flusso in un determinato vaso venoso.

\section{Eziologia}

Le cause che predispongono alla trombosi venosa cerebrale (TVC) sono legate alla classica triade di Virchow che comprende variazioni emodinamiche, disfunzione endoteliale e ipercoagulabilità. I fattori di rischio vengono generalmente distinti in genetici ed acquisiti (Tabella 1).

\section{Condizioni protrombotiche ereditarie}

Tra i fattori genetici vanno annoverate le trombofilie. Lo studio International Study on Cerebral Vein and Dural Sinus Thrombosis (ISCVT) ha analizzato un campione di 624 pazienti con TVC riscontrando condizioni protrombotiche ereditarie o acquisite nel $34 \%$ dei casi. ${ }^{1}$ Le mutazioni clinicamente più rilevanti sono quelle che conducono a deficit di antitrombina III, di proteina $\mathrm{C}$ e di proteina $\mathrm{S}$. La resistenza alla proteina $\mathrm{C}$ attivata è principalmente causata dalla mutazione del fattore $\mathrm{V}$ Leiden che è un disordine trombofilico ereditario alquanto comune. La muta- 
zione del gene della protrombina (mutazione G20210A) è presente nel $2 \%$ degli individui di razza caucasica e causa aumento dei livelli di protrombina circolante predisponendo in tal modo a tromboembolismo venoso. L'iperomocisteinemia è un fattore di rischio indipendente per TVC ed è presente nel $27-43 \%$ dei pazienti. ${ }^{2}$ I bassi livelli plasmatici di folati, secondari all'iperomocisteinemia, determinano un rischio più evidente durante la gravidanza. Diversi studi hanno dimostrato l'associazione tra anticorpi antifosfolipidi e anticardiolipina e TVC; lo studio ISCVT ${ }^{1}$ riporta una prevalenza del 5.9\% nei pazienti con positività a tali anticorpi.

Numerosi studi, infine, hanno indagato l'associazione tra trombofilia e rischio di trombosi venosa cerebrale; una metanalisi ha revisionato 26 studi caso-controllo dimostrando che le mutazioni dei geni che maggiormente sono correlati a trombosi riguardano il fattore V Leiden/G1691A e il gene della protrombina. ${ }^{3}$ La mutazione del gene della protrombina G20210A e la mutazione del fattore V Leiden sono le più frequenti, seguite dalla positività degli anticorpi anticardiolipina e antifosfolipidi, dal deficit di proteina $\mathrm{C}$ e proteina $\mathrm{S}$ e dal deficit di antitrombina III.

\section{Condizioni protrombotiche acquisite}

Tra i fattori acquisiti vanno menzionati la gravidanza ed il puerperio, l'utilizzo di farmaci quali contraccettivi orali e terapie ormonali, gli stati infiammatori (malattie autoimmuni), le infezioni e gli stati settici, le patologie tumorali, la disidratazione, il trauma cranico.

Dati epidemiologici tratti dallo studio ISCVT dimostrano che più del $44 \%$ dei soggetti affetti da TVC presentano più di un fattore di rischio; in questo studio, la terapia contraccettiva orale rappresenta quello più frequente (54\%), seguito dalle trombofilie (34\%), dal puerperio (14\%), dalle infezioni (12\%), dal cancro $(7,4 \%)$ e dalla gravidanza $(6 \%) .{ }^{4}$ La gravidanza ed il puerperio sono comuni cause acquisite di trombosi e

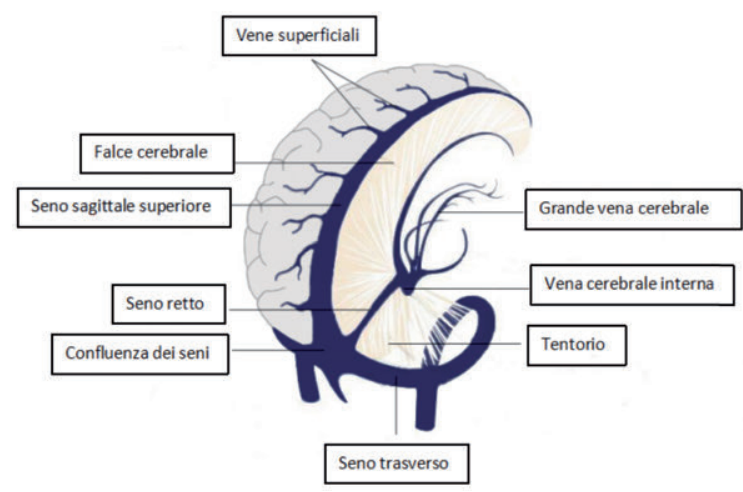

Figura 1. Anatomia del circolo venoso cerebrale. circa il $2 \%$ degli ictus in gravidanza è attribuibile a TVC. La frequenza è stimata a 12 casi/ 100.000 parti e il rischio è più alto nel terzo trimestre, in quanto più importanti sono le alterazioni ormonali in tale periodo. Allo stesso modo, durante il puerperio (6-8 settimane dopo il parto) il rischio tromboembolico è notevolmente aumentato. Sebbene vi sia un'esperienza limitata, i fattori associati al puerperio sono l'iperomocisteinemia, il parto cesareo, l'ipertensione materna e le infezioni.

L'utilizzo di contraccettivi orali aumenta il rischio relativo di TVC con un OR calcolata di 5,59 e l'effetto è sinergico in associazione a trombofilie genetiche quali la mutazione del fattore $\mathrm{V}$ Leiden o del gene della protrombina G20210A (OR 149,3 95\% CI 31,0$711,0) .{ }^{5}$ Pertanto, è dimostrato ampiamente che di per sé l'utilizzo di contraccettivi orali aumenta il rischio di TVC, che la maggior parte delle giovani donne non gravide che presentano trombosi utilizzano tali farmaci e che l'associazione tra contraccettivi orali e mutazioni genetiche trombofiliche aumenta drammaticamente il rischio di TVC.

Uno stato di ipercoagulabilità acquisita comune

Tabella 1. Fattori di rischio per trombosi venosa cerebrale (ISCVT STUDY).

\begin{tabular}{lc}
\hline Nessun fattore di rischio identificato & $12,5 \%$ \\
\hline Trombofilia & $34,1 \%$ \\
Genetica & $22,4 \%$ \\
Acquisita & $15,7 \%$ \\
\hline Anticorpi antifosfolipidi & $5,9 \%$ \\
Iperomocisteinemia & $4,5 \%$ \\
Sindrome nefrotica & $0,6 \%$ \\
\hline Tumori & $7,4 \%$ \\
SNC & $2,2 \%$ \\
Tumori solidi extracranici & $3,2 \%$ \\
Tumori ematologici & $2,9 \%$ \\
\hline Disordini del SNC & $1,9 \%$ \\
\hline Malattie ematologiche & $12 \%$ \\
\hline Malattie autoimmunitarie & $3 \%$ \\
\hline Altri disordini sistemici infiammatori & $1,8 \%$ \\
\hline Altri disordini sistemici & $2,4 \%$ \\
\hline Gravidanza & $6,3 \%$ \\
\hline Puerperio & $13,8 \%$ \\
\hline Infezioni & $12,3 \%$ \\
Sistema nervoso centrale & $2,1 \%$ \\
\hline Orecchio, seni, bocca, faccia, collo & $8,2 \%$ \\
\hline Traumi & $4,5 \%$ \\
\hline Farmaci & $7,5 \%$ \\
\hline Hormonetivi orali & $54,3 \%$ \\
\hline Chirurgia & $4,3 \%$ \\
\hline Disidratazione & $2,7 \%$ \\
\hline & $1,9 \%$ \\
\hline
\end{tabular}


nel setting della TVC è dato dai tumori e circa il 7,4\% delle trombosi venose cerebrali è associato a cancro. Diversi meccanismi sottendono a alla formazione del trombo nel sistema venoso, inclusi l'invasione delle cellule tumorali nei seni e la loro compressione, lo squilibrio dei mediatori dell'infiammazione, la chemioterapia e la terapia ormonale. I tumori più frequentemente associati sono quelli del sistema nervoso centrale $(2,2 \%)$, metastasi di tumori solidi $(3,2 \%)$ e neoplasie ematologiche $(2,9 \%)$.

Altre cause non comuni di TVC sono le infezioni, responsabili dell' $8,2 \%$ dei casi adulti, in special modo quelle a localizzazione parameningea (orecchio, seni, bocca e collo), più frequenti in età pediatrica, e cause minori riportate in Tabella 1.

\section{Key message: Eziologia della TVC}

- I fattori di rischio della TVC vengono distinti in genetici ed acquisiti.

- Tra i fattori genetici, la mutazione del gene della protrombina G20210A e la mutazione del fattore $\mathrm{V}$ Leiden sono le più frequenti e quelle maggiormente correlate a trombosi venosa cerebrale.

- Tra i fattori acquisiti, l'utilizzo di contraccettivi estroprogestinici, il puerperio, le infezioni, il cancro e la gravidanza predispongono a maggior rischio di TVC.

\section{Fisiopatologia}

La trombosi venosa cerebrale è causata da uno squilibrio sistemico o locale dei processi protrombotici e trombolitici che porta a formazione di trombo e quindi ad ostruzione dei seni venosi e delle vene corticali e profonde. Ciò conduce ad un aumento della pressione venosa e a una ridotta perfusione capillare. In un primo momento, il circolo venoso, grazie alle sue numerose anastomosi tra il circolo superficiale e quello profondo, fornisce un sufficiente circolo collaterale atto a compensare tali cambiamenti pressori. In questi casi non si sviluppa un vero e proprio infarto, ma soltanto edema cerebrale di entità variabile. Quando però il reclutamento di circoli collaterali diviene insufficiente, si ha stasi venosa, aumento della pressione intramurale e danno a carico della barriera emato-encefalica con conseguente edema vasogenico ed infarti venosi che possono andare da petecchie a ematomi cerebrali. ${ }^{6}$ Gli infarti venosi avvengono nel $60 \%$ circa dei pazienti con TVC e differiscono consistentemente dagli infarti arteriosi in quanto sono nei $2 / 3$ dei casi di tipo emorragico. ${ }^{7}$ In aggiunta a ciò, la ridotta perfusione cerebrale causa edema citotossico ed un ulteriore aumento della pressione intracranica. I seni durali, infine, giocano un ruolo vitale nell'assorbimento del fluido cerebrospinale, processo me- diato dai villi aracnoidei componenti le pareti dei seni. La disfunzione di tali strutture risulta in un ridotto assorbimento del liquido cerebrospinale e in un ulteriore aumento della pressione intracranica.

\section{Key message: Fisiopatologia della TVC}

- I meccanismi fisiopatologici della TVC sono rappresentati dall'aumento della pressione venosa che consegue ad ostruzione di flusso e dalla ridotta perfusione tissutale della zona coinvolta.

- Ciò causa stasi venosa, aumentata pressione intramurale con edema vasogenico e infarti venosi (petecchie fino ad emorragie intracraniche).

\section{Epidemiologia}

La TVC, in termini assoluti, è una patologia rara ed è responsabile dello $0.5-1 \%$ di tutti gli ictus. Tra gli adulti ha un'incidenza di 1,3-1,6/100.000 persone/ anno, è più frequente nelle femmine che nei maschi ( $74 \%$ vs $26 \%$ ) e colpisce maggiormente nella fascia di età di 30-50 anni. ${ }^{8}$

\section{Presentazione clinica}

La TVC si presenta con un corteo di segni e sintomi alquanto variabile, entrando peraltro in diagnosi differenziale con molti altri disturbi neurologici (Tabella 2). La clinica è riconducibile ai due meccanismi fisiopatologici di ipertensione endocranica, attribuibile ad un alterato drenaggio venoso, e di danno focale cerebrale dovuto all'infarto venoso. Molti pazienti hanno segni e sintomi relati ad entrambi i meccanismi sia alla presentazione che alla progressione della patologia sottostante. L'aumentata pressione intracranica è responsabile della cefalea, del papilledema e dei disturbi visivi con cui spesso i soggetti affetti da TVC si presentano all'osservazione medica. Il 90\% lamenta cefalea descritta come ingravescente, presente da giorni a settimane, raramente con caratteristiche a colpo di pugnale, scarsamente responsiva alla terapia analgesica. I sintomi clinici del papilledema possono essere perdita o calo transitorio del visus, perdita della visione periferica, spesso associati a nausea e/o vomito. La diplopia è spesso orizzontale se causata da compressione di uno o entrambi i nervi abducenti.

Il secondo meccanismo relato alla TVC è l'ipoperfusione cerebrale con associato danno ischemico o emorragico. Ciò conduce a differenti sindromi focali correlati alle sede dove si è verificato il danno trombotico, sebbene siano meno stereotipate di quelle osservate negli ictus arteriosi. Le sindromi più frequenti sono le seguenti: i) trombosi del seno sagittale superiore: tipica presentazione acuta, con deficit motori spesso bi- 
laterali, crisi epilettiche correlate al danno emisferico corticale ed alterazioni comportamentali legate al coinvolgimento del lobo frontale. Meno comunemente ipertensione endocranica isolata; ii) trombosi del seno cavernoso: segni oculari con dolore orbitario, chemosi, proptosi e deficit nell'oculomozione; iii) trombosi del seno trasverso: afasia o neglect, in base alla specializzazione emisferica. Tipica è l'ipertensione endocranica isolata; iv) trombosi delle vene profonde: clinica variabile da lievi deficit focali fino ad alterazione della coscienza e coma se vi è coinvolgimento del talamo bilateralmente; v) trombosi delle vene corticali: crisi epilettiche più o meno associate a deficit neurologici, frequentemente sensitivo-motori. ${ }^{9}$

La clinica è spesso influenzata dall'età, dal tempo intercorrente tra l'inizio dei sintomi e l'ingresso in ospedale, dalla sede della TVC e dalla presenza o meno di lesioni parenchimali. I pazienti con un decorso cronico o ritardato presentano più spesso papilledema, segno meno frequente nei casi acuti.

La possibilità di cefalea isolata come unico sintomo di presentazione della TVC è stata recentemente enfatizzata, in quanto nei soggetti che presentano esclusivamente questo sintomo la diagnosi è particolarmente difficoltosa, per l'assenza di deficit neurologici, di segni di irritazione meningea o di ipertensione endocranica e con TC encefalo e rachicentesi negative. Ciò si riscontra frequentemente nella trombosi dei seni trasversi; a questo si aggiunge la particolare conformazione anatomica di tali seni che risultano spesso asimmetrici e dove la diagnosi differenziale tra trombosi ed ipoplasia non è spesso agevole in diagnostica TC senza mezzo di contrasto. ${ }^{10}$

Le crisi epilettiche, infine, sono comuni poiché vi

Tabella 2. Sintomi e segni di presentazione della TVC.

\begin{tabular}{l}
\hline Sintomi e segni comuni \\
\hline Ipertensione endocranica isolata \\
\hline Sindromi focali \\
\hline Encefalopatia diffusa \\
\hline Combinazioni di più segni su riportati \\
\hline Sintomi e segni rari \\
\hline Sindrome del seno cavernoso \\
\hline Emorragia subaracnoidea \\
\hline Cefalea a colpo di pugnale \\
\hline Emicrania con aura \\
\hline Cefalea isolata \\
\hline Attacchi ischemici transitori \\
\hline Tinniti \\
\hline Sintomi psichiatrici isolati \\
\hline
\end{tabular}

Paralisi isolata o multipla dei nervi cranici è spesso un'alterazione di barriera emato-encefalica con formazione di edema corticale. Le crisi possono essere focali con o senza secondaria generalizzazione, con possibile evoluzione verso lo stato di male epilettico. Nello studio ISCVT le crisi epilettiche sono presenti nel $40 \%$ dei soggetti affetti da TVC. ${ }^{1}$

La diagnosi ritardata è di frequente riscontro nelle trombosi cerebrali con una mediana dall'inizio dei sintomi all'ingresso in ospedale di 4 giorni e dall'inizio dei sintomi alla diagnosi di 7 giorni. Pertanto, lo studio ISCVT distingue proprio sulla base del tempo di insorgenza dei sintomi una TVC acuta ( $<48$ ore), subacuta ( 48 ore -30 giorni) e cronica $>30$ giorni.

\section{Key message: Presentazione clinica della TVC}

- I segni e sintomi della TVC sono variabili, legati ai due meccanismi di ipertensione endocranica e di danno focale cerebrale.

- L'ipertensione endocranica è responsabile di cefalea, papilledema e disturbi visivi.

- L'infarto venoso conduce a differenti sindromi focali correlate alle sede dove si è verificato il danno trombotico.

- La cefalea isolata come unico sintomo di presentazione della TVC è un'importante sfida per il clinico, pertanto in coloro che presentano uno o più fattori di rischio per trombosi venosa, ulteriori indagini sono mandatorie.

\section{Diagnosi}

\section{Neuroimaging}

La diagnosi di TVC si basa sulle neuroimmagini ma, a differenza degli ictus arteriosi, le lesioni non rispettano un preciso territorio vascolare e nel $25-30 \%$ dei casi risultano assenti. ${ }^{9}$ La chiave per la diagnosi della TVC è pertanto il sospetto clinico che guida lo studio del sistema venoso.

In caso di sospetto clinico, fattori di rischio o condizioni predisponenti, il primo esame che generalmente viene eseguito in Pronto Soccorso è la tomografia computerizzata (TC) dell'encefalo. In TC è possibile distinguere segni indiretti e segni diretti di TVC. Tra i segni indiretti vi è l'ipodensità della sostanza bianca, segno di edema cerebrale presente nel $75 \%$ dei casi, spesso bilaterale, associata nel $10-50 \%$ dei casi ad iperdensità ematiche intraparenchimali o subaracnoidee. Tra i segni diretti, il principale è l'iperdensità di una vena corticale o dei seni durali, presente però soltanto in $1 / 3$ dei casi di TVC.

In caso di trombosi della porzione posteriore del seno sagittale superiore, dopo somministrazione di mezzo di contrasto, si può osservare il caratteristico segno del delta pieno presente in fase precoce nel $2 \%$ dei casi. Allo stesso modo la TC con mezzo di contra- 
sto mostra un difetto di riempimento del seno venoso, dando luogo al segno del delta vuoto in cui un'ipodensità centrale dovuta all'assenza di flusso all'interno dei seni è circondata da impregnazione del mdc a forma triangolare. Questo segno può non comparire per diversi giorni dall'inizio dei sintomi ma persiste per diverse settimane.

Indicativo di trombosi delle vene corticali, è, invece, il segno della corda presente in circa il $2-25 \%$ dei casi.

La TC è tuttavia una metodica a bassa sensibilità e con una percentuale elevata di falsi negativi sino al $30 \%$ dei casi.

La risonanza magnetica $(\mathrm{RMN})$ è più sensibile nella diagnosi di TVC consentendo di identificare meglio segni indiretti quali l'edema e la componente emorragica dell'infarto venoso. L'insidia delle sequenze angioRM risiede nella difficoltà a distinguere tra trombosi ed ipoplasia di un seno venoso, un dilemma diagnostico tipico per i seni trasversi. In tutti i casi dubbi, in particolare per le trombosi corticali, il gold standard diagnostico rimane l'angiografia cerebrale.

I segni diretti evidenziati dalla RMN variano a seconda del tempo di presentazione del paziente. Nella prima settimana il trombo fresco appare isointenso in T1 e ipointenso in T2 grazie all'aumentato contenuto di deossiemoglobina e risulta molto difficile da differenziare dai normali vasi venosi.

Dalla seconda settimana il trombo contiene metaemoglobina e viene pertanto visualizzato come iperintenso in $\mathrm{T} 1 \mathrm{e}$ in $\mathrm{T} 2$ con una sensibilità del $70-80 \%$. Solitamente, ciò corrisponde ad assenza di segnale del seno venoso nelle sequenze angio.

I segni indiretti di TVC in RM sono simili a quelli visti in TC e, quindi, edema cerebrale visualizzabile come iperintensità nelle sequenze T2/FLAIR ed infarti venosi con componente emorragica, visualizzabile nelle sequenze Gradient Echo o SWI come disomogenea ipointensità, con una sensibilità del 90\% (Figure 2-6). ${ }^{11-13}$

In angiografia cerebrale, la fase angiografica venosa mostra la trombosi come difetto di riempimento. Secondo le linee guida AHA/ASA, TC o RM con sequenze angio sono gli esami diagnostici di primo livello (Classe I, Livello di evidenza C); in caso di negatività, se il sospetto clinico è elevato, è autorizzato il ricorso ad angiografia cerebrale (Classe IIa, Livello di evidenza C). ${ }^{14}$

In Tabella 3 sono elencati i vantaggi e gli svantaggi della TC encefalo e della RM encefalo.

\section{Esami di laboratorio}

Data la varietà e la non specificità dei sintomi e segni con cui si presenta la TVC, sarebbe utile e di particolare interesse un test di facile esecuzione in emergenza che consenta di escludere un'eventuale trombosi venosa. Diversi studi hanno testato il valore

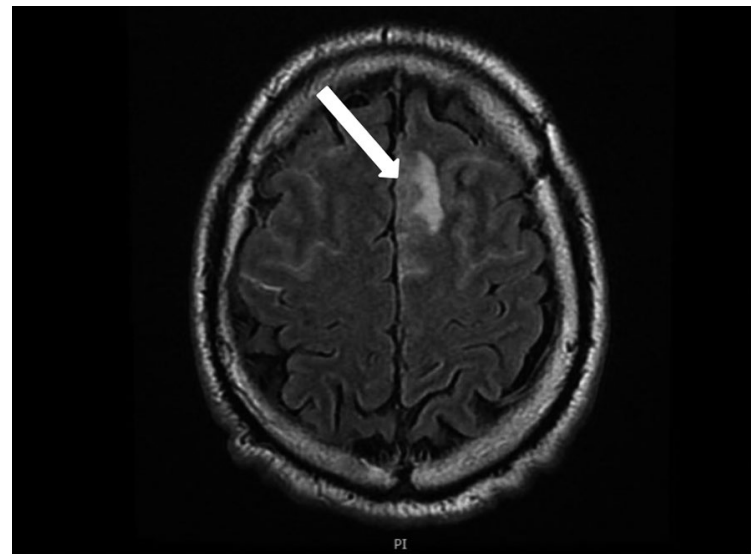

Figura 2. RMN encefalo, sequenza T2 FLAIR: iperintensità da edema vasogenico secondario a trombosi venosa corticale sinistra (freccia bianca) con iperintensità sulcale da stravaso ematico subaracnoideo (freccia).

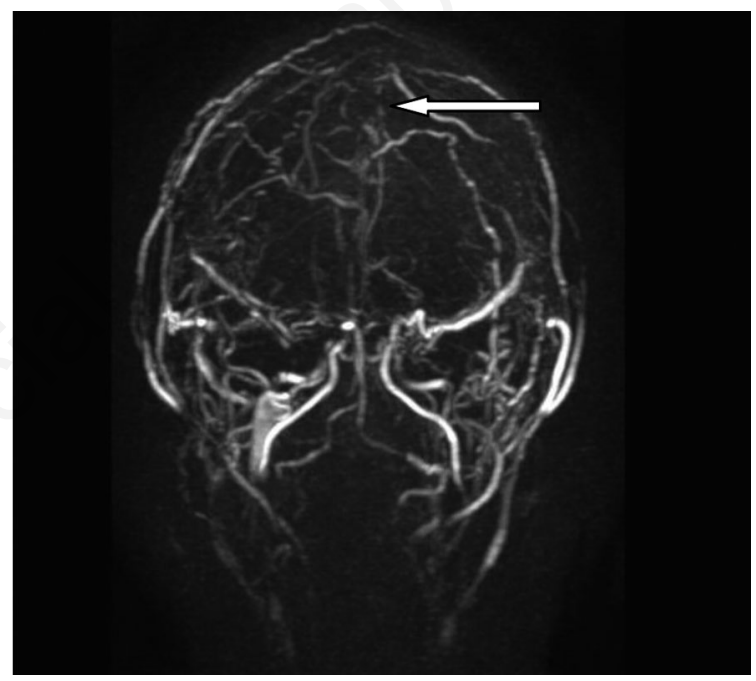

Figura 3. Angio-RMN encefalo: trombosi del seno sagittale superiore (mancata visualizzazione del seno: freccia).

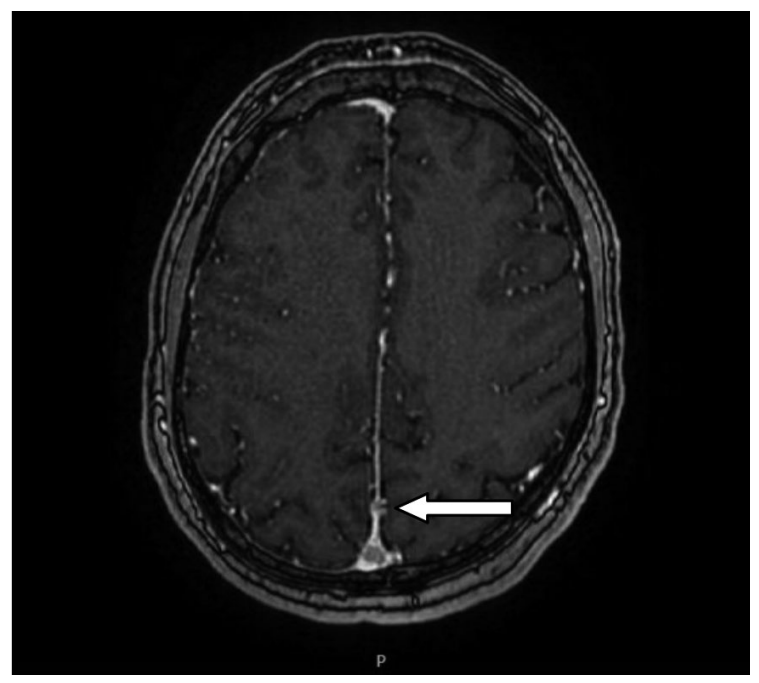

Figura 4. RMN encefalo (FLAIR): empty delta sign (freccia). 
di D-dimero basandosi su quanto è valido per la trombosi venosa profonda degli arti, in quanto una negatività dello stesso è in grado di escludere una trombosi con un elevato valore predittivo negativo. In un recente studio prospettico multicentrico, di 343 pazienti che accedevano al dipartimento di emergenza con segni suggestivi di TVC, un livello positivo di D-dimero (cut-off $>500 \mathrm{mcg} / \mathrm{L}$ ) è stato riscontrato in $34 / 35$ soggetti con diagnosi confermata di TVC e in 27/308 soggetti senza TVC. Ciò determina una sensibilità del $97,1 \%$ e una specificità di $91,2 \%$, un valore predittivo negativo dei $99.6 \%$ e un valore predittivo positivo di $55,7 \% .{ }^{15} \mathrm{Il}$ ruolo del D-dimero viene messo in discus-

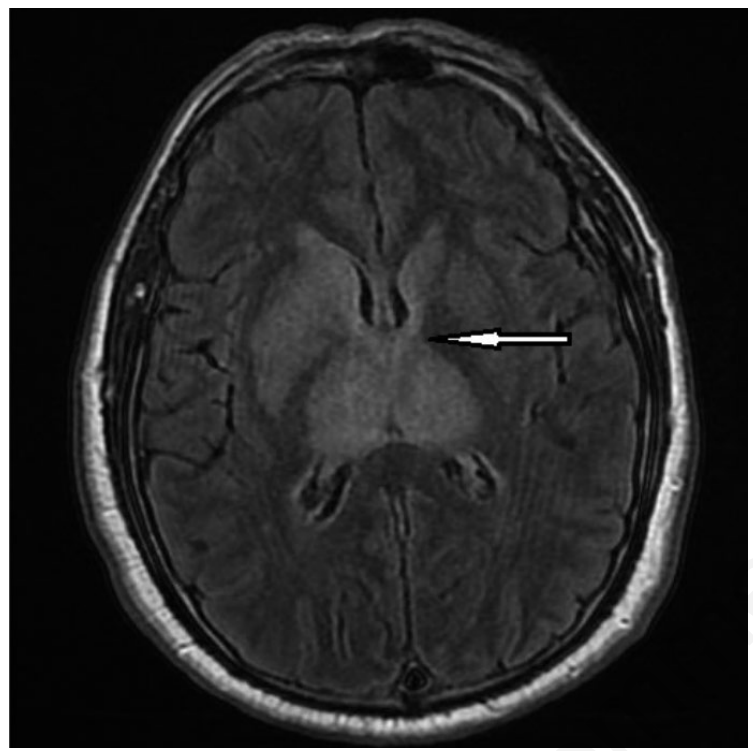

Figura 5. RMN encefalo (FLAIR): edema da trombosi del seno retto (freccia). sione nei pazienti con cefalea isolata dove fino al $26 \%$ può risultare negativo. ${ }^{16}$

Allo stesso modo pazienti che abbiano sintomi subacuti o cronici (> una settimana) sono più suscettibili nell'avere livelli negativi di D-dimero, in quanto questi si riducono col passare dei giorni dall'inizio dei sintomi. I livelli di D-dimero, infine, correlano con l'estensione della trombosi, per cui pazienti con minor coinvolgimento trombotico possono avere un valore falsamente negativo. ${ }^{15}$

Le ultime linee guida AHA/ASA raccomandano l'esecuzione del D-dimero in tutti i pazienti con sospetto di TVC, tenendo ben presente che in caso di

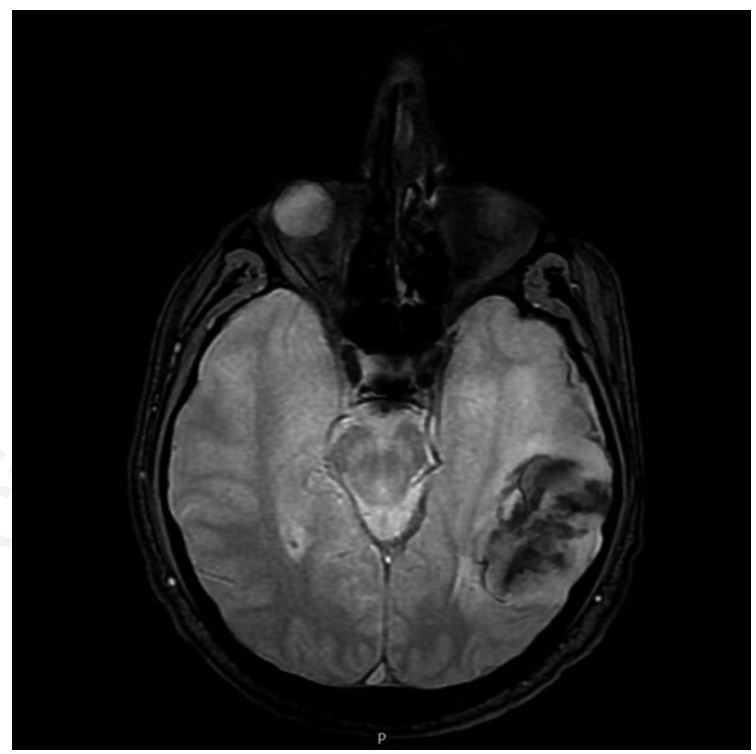

Figura 6. GRE: infarto venoso da trombosi del seno trasverso sinistro.

Tabella 3. Vantaggi e svantaggi di TC e RM nella diagnosi di TVC.

\begin{tabular}{|c|c|c|}
\hline & TC/angioTC & RM/angioRM \\
\hline Vantaggi & $\begin{array}{l}\text { - Buona visualizzazione dei seni venosi maggiori } \\
\text { - Rapidità di esecuzione } \\
\text { - Pochi artefatti da movimento } \\
\text { - Nessuna interazione con dispositivi magnetici }\end{array}$ & $\begin{array}{l}\text { - Visualizzazione del sistema venoso superficiale e } \\
\text { profondo } \\
\text { - Buona definizione del parenchima cerebrale } \\
\text { - Individuazione precoce di lesioni ischemiche } \\
\text { - Non esposizione a radiazioni } \\
\text { - Rilevamento di macro e microsanguinamenti }\end{array}$ \\
\hline Svantaggi & $\begin{array}{l}\text { - Esposizione a radiazioni ionizzanti } \\
\text { - Rischio di reazioni al mezzo di contrasto } \\
\text { - Rischio di nefropatia da contrasto } \\
\text { - Bassa risoluzione per piccole lesioni parenchimali } \\
\text { - Scarsa individuazione delle trombosi venose } \\
\text { corticali e profonde }\end{array}$ & $\begin{array}{l}\text { - Tempo di esecuzione lungo } \\
\text { - Artefatti da movimento } \\
\text { - Disponibilià } \\
\text { - Uso limitato in portatori di dispositivi metallici } \\
\text { - Basso rischio di fibrosi sistemica nefrogenica } \\
\text { gadolinio-indotta } \\
\text { - Interpretazione inficiata da stati dibasso flusso, pattern } \\
\text { di flusso complessi e variazioni anatomiche nei seni durali }\end{array}$ \\
\hline Applicazioni pratiche & $\begin{array}{l}\text { - Insorgenza acuta dei sintomi } \\
\text { - Setting di emergenza } \\
\text { - Esame iniziale se angioRM non disponibile }\end{array}$ & $\begin{array}{l}\text { - Insorgenza acuta/subacuta dei sintomi } \\
\text { - Setting di emergenza o ambulatoriale } \\
\text { - Se TC/angioTC negative ma elevato sospetto clinico }\end{array}$ \\
\hline
\end{tabular}

Riadattata da Saposnik G. Stroke 2011;42: 1158-1192. 
forte sospetto clinico un valore negativo non deve automaticamente escludere la diagnosi. ${ }^{14}$

Posta diagnosi di TVC è importante determinarne l'eziologia; è necessario pertanto eseguire esami ematochimici comprensivi di emocromo con formula, pannello metabolico completo, tempo di protrombina (PT) e di tromboplastina parziale attivata (aPTT). Se nessun fattore acquisito può essere considerato la causa della TVC è necessario eseguire lo screening trombofilico ricercando il deficit di antitrombina III, il deficit di proteina $\mathrm{C}$ e di proteina $\mathrm{S}$, la mutazione della fattore $\mathrm{V}$ Leiden e del gene della protrombina (mutazione G20210A), l'iperomocisteinemia e gli anticorpi antifosfolipidi e anticardiolipina.

\section{Key message: Diagnosi di TVC}

- La diagnosi di TVC si avvale delle neuroimmagini adiuvate sempre dal sospetto clinico.

- La TC anche se eseguita con mezzo di contrasto ha una bassa specificità ed una percentuale elevata di falsi negativi.

- La RMN consente di visualizzare segni indiretti e segni diretti di TVC; i segni diretti variano a seconda del tempo di presentazione del paziente, $\mathrm{i}$ segni indiretti sono rappresentati dall'edema cerebrale e dagli infarti venosi.

- L'angiografia cerebrale è il gold standard nella diagnosi di TVC e mostra la trombosi come difetto di riempimento.

- Il D-dimero è un utile marker di trombosi venosa, ma la sua negatività in caso di elevato sospetto clinico non deve esimere il clinico dall'eseguire ulteriori indagini volte a confermare o escludere la diagnosi.

- Lo screening trombofilico deve essere eseguito in tutti i pazienti in cui la causa di TVC non è da ricercare nei fattori di rischio acquisiti.

\section{Prognosi}

Il decorso clinico delle TVC non è prevedibile nei primi giorni dall'evento e circa $1 / 4$ dei pazienti hanno un deterioramento clinico in fase acuta, con alterazioni dello stato di coscienza, cefalea o deficit focali e in $1 / 3$ di questi pazienti si riscontra una nuova lesione parenchimale ad esami radiologici. La mortalità in tale fase è del $4 \%$ ed è generalmente dovuta ad erniazione transtentoriale secondaria ad estese lesioni emorragiche, a lesioni multiple o ad edema cerebrale. Il rischio di mortalità a lungo termine è di circa il $10 \%$. Una scarsa prognosi si riscontra nelle trombosi correlate ad infezioni del sistema nervoso centrale, a cancro e ad emorragia intracerebrale. Allo stesso modo, pazienti maschi di età superiore a 37 anni con Glasgow Coma Scale all'ingresso inferiore a 9 e alterazioni della coscienza correlano con una prognosi peggiore.

La maggior parte dei pazienti (79\%) ha regressione completa della patologia con un valore di 0-1 nella scala di Rankin modificata (nessuna disabilità/disabilità lieve).

Il tasso di ricorrenza della TVC varia tra il $2 \%$ e il $5 \%$, con una maggior ricorrenza entro un anno dal primo evento trombotico. Il sesso maschile e la policitemia o la trombocitemia sono fattori di rischio noti di ricorrenza, così come la trombofilia severa e i pregressi eventi tromboembolici venosi. Sebbene la gravidanza sia associata ad un aumentato rischio di TVC, il rischio assoluto di ricorrenza è basso ( 9 casi/1000 gravidanze), pertanto una storia di TVC non deve essere una controindicazione a gravidanze future. In tali casi è indicato il trattamento anticoagulante in profilassi.

\section{Key message: Prognosi nella TVC}

- La mortalità dei pazienti con TVC in fase acuta è di circa il $4 \%$ dovuta generalmente ad erniazione transtentoriale.

- Il rischio di mortalità a lungo termine è del $10 \%$; la maggior parte ha regressione clinica completa.

- Una scarsa prognosi si riscontra nella TVC correlata a infezioni del SNC e a cancro.

- Il tasso di ricorrenza di TVC varia tra il $2 \%$ e il 5\% con una maggior ricorrenza nel primo anno dall'evento.

\section{Trattamento}

Nella diagnosi di TVC è necessaria un'attenta valutazione da parte del neurologo ed il ricovero in Stroke Unit in quanto è dimostrato che un sistema di cure organizzato riduce la mortalità e la morbidità dopo un evento trombotico. ${ }^{17}$

Dati riportano che il ricovero in stroke unit è associato ad una riduzione del $14 \%$ del rischio di morte ad 1 anno, indipendentemente dall'età, dal sesso, dalla severità dell'ictus e dalla tipologia di ictus. ${ }^{18}$

La TVC è una causa rara di ictus ma potenzialmente seria e fatale, pertanto il management in una Stroke Unit, come da linea guida, atto a ottimizzare le cure e ridurre le complicanze è di fondamentale importanza.

\section{Terapia anticoagulante}

Gli scopi della terapia anticoagulante sono molteplici: prevenire la crescita del trombo, facilitare la ricanalizzazione del vaso e prevenire una trombosi venosa profonda. Sono stati condotti due studi randomizzati che confrontano la terapia anticoagulante versus placebo in pazienti con TVC confermata all'imaging. Il primo ha comparato l'eparina non frazionata endove- 
nosa (UFH) con placebo, dosandola in base all'aPTT (valore terapeutico 2 volte il valore normale). In questo studio veniva somministrato un bolo di $3000 \mathrm{U}$ di UFH seguito da infusione continua. L'endpoint primario era una scala di severità della TVC a 3 mesi che valutava la cefalea, i segni focali, la presenza di eventi epilettici e il livello di coscienza. L'outcome secondario erano le emorragie intracerebrali. Dopo tre mesi è stata evidenziata una differenza statisticamente significativa in favore del trattamento con UFH nel numero di pazienti con recupero clinico buono o completo. ${ }^{19}$

Il secondo trial comparava la nadroparina sottocute a dosaggio variabile in base al peso corporeo (180 $\mathrm{U} / \mathrm{kg}$ bid) vs placebo per 3 mesi, seguito poi da ulteriori 3 mesi di terapia con warfarin nei pazienti trattati con nadroparina. L'endpoint primario erano scores che valutassero le attività di vita quotidiana dei pazienti, la Oxford Stroke Handicap Scale e la mortalità. L'endpoint secondario era lo sviluppo di emorragia intracerebrale sintomatica ed altri sanguinamenti maggiori. Questo trial ha dimostrato una riduzione non significativa nel rischio di morte o dipendenza nei pazienti trattati con EBPM: mRS $>213 \%$ vs $21 \% .^{20}$

Una metanalisi dei due studi ha dimostrato come l'uso della terapia eparinica comporti una riduzione del $13 \%$ del rischio assoluto di morte o disabilità, evidenziando una sicurezza terapeutica anche in presenza di ematomi o infarti emorragici. ${ }^{21}$

Nei pazienti con TVC associata ad emorragia, anche in assenza di anticoagulazione, l'emorragia è associata ad un outcome avverso ma il sanguinamento cerebrale non è correlato alla terapia anticoagulante. Pertanto anche questi pazienti devono essere trattati con l'anticoagulazione.

In aggiunta ai suddetti trials randomizzati controllati vi sono numerosi studi osservazionali sia retrospettivi che prospettici che supportano il ruolo della terapia anticoagulante nel trattamento della TVC, nonostante la presenza di emorragia intracerebrale pretrattamento, rendendo quindi la terapia sicura ed efficace. $^{22-25}$

Non ci sono dati che supportino differenze di outcome tra l'UFH e le eparine a basso peso molecolare (LMWH), ma nel setting della trombosi venosa profonda e dell'embolia polmonare, una recente metanalisi di 22 studi mostra un minor rischio di sanguinamenti maggiori $(1,2 \%$ vs $2,1 \%)$, di complicanze trombotiche $(3,6 \%$ vs $5,4 \%)$ e di mortalità $(4,5 \%$ vs $6,0 \%$ ) nei pazienti trattati con $\mathrm{LMWH}^{26}$

Un breve cenno va fatto sull'uso degli inibitori diretti della trombina (dabigatran) e sugli antiX attivati (rivaroxaban, apixaban, edoxaban), ormai capisaldi della terapia di TVP e TEP. Allo stato attuale non vi sono ancora numerosi studi clinici randomizzati controllati del loro utilizzo nella TVC. Vi sono in letteratura alcuni studi osservazionali, uno dei quali confronta rivaroxaban versus fenprocumone dopo trattamento in fase acuta con eparina in 16 pazienti affetti da TVC. Tale studio retrospettivo mostra un beneficio clinico nel 93,8\% dei pazienti trattati nell'intera coorte (ricanalizzazione almeno parziale) senza differenze statisticamente significative tra i due gruppi. ${ }^{26} \mathrm{Un}$ altro studio retrospettivo con un totale di 18 pazienti ha analizzato l'outcome clinico a 6 mesi dall'evento andando a valutare la scala di Rankin modificata con valore di 0-1. Di questi 18 pazienti, 11 assumevano dabigatran e 7 assumevano warfarin dopo un trattamento in fase acuta con eparina; quattro dei 7 pazienti in VKA sono stati poi passati a dabigatran per eventi avversi. L' $87 \%$ dei pazienti presentava un outcome eccellente e circa $1^{\prime} 80 \%$ mostrava ricanalizzazione del vaso occluso. ${ }^{27,28}$

Entrambi gli studi non riportano incidenza statisticamente significativa di eventi emorragici maggiori nei pazienti trattati con DOAC. La qualità delle evidenze di tali studi, tuttavia, è bassa in quanto osservazionali con elevato rischio di bias.

Allo stato attuale vi è un unico trial randomizzato controllato pubblicato su JAMA Neurology a settembre 2019 in cui dabigatran $150 \mathrm{mg}$ bid è stato confrontato con warfarin in range terapeutico (RESPECT-trial). L'outcome primario era un composito di TVC ricorrente, trombosi venosa profonda degli arti inferiori, tromboembolia polmonare e trombosi delle vene splancniche o sanguinamenti maggiori durante il follow up (24 mesi). L'outcome secondario era la ricanalizzazione del vaso trombizzato e i sanguinamenti minori clinicamente rilevanti. Il trial ha dimostrato che sia i pazienti trattati con dabigatran che quelli trattati con warfarin avevano un basso rischio di eventi tromboembolici ricorrenti e che il rischio di sanguinamenti era simile tra i due gruppi, suggerendo che entrambi i farmaci sono sicuri ed efficaci nella TVC. Tuttavia,a causa del piccolo campione arruolato (120 pazienti), il trial non ha potuto dimostrare la non inferiorità o la superiorità di un trattamento rispetto ad un altro. ${ }^{29}$

Pertanto, anche se le linee guida ESO non raccomandano l'utilizzo degli inibitori diretti della trombina e del Xa in pazienti affetti da TVC, non è da escludere che in futuro nuovi studi clinici randomizzati controllati possano modificare il panorama terapeutico di tale patologia.

\section{Terapia trombolitica}

\section{Trombolisi endovenosa}

La terapia trombolitica endovenosa e/o locoregionale è una tecnica di salvataggio in quanto fino al 13\% dei pazienti con TVC hanno uno scarso outcome nonostante la terapia anticoagulante. Spesso infatti, l'anticoagulazione non riesce a dissolvere un trombo di maggior estensione, rendendo quindi la ricanalizza- 
zione del vaso incompleta anche a distanza di mesi. Al momento non ci sono trials randomizzati controllati sulla trombolisi nella TVC. Sono stati pubblicati, invece molti case reports e una successiva metanalisi degli stessi ha evidenziato il significativo incremento di sanguinamenti maggiori nei pazienti trattati con terapia trombolitica, inclusi i sanguinamenti intracranici potenzialmente fatali. Tale metanalisi conclude affermando la necessità di trials randomizzati controllati che valutino la sicurezza di tale trattamento rispetto alla terapia attualmente indicata dalle linee guida. ${ }^{30}$

\section{Terapia endovascolare}

Il successo dei trattamenti endovascolari nell'ictus ischemico acuto ha posto l'attenzione su tale trattamento anche nella TVC. L'approccio transvenoso utilizzato in quest'ultimo caso, tuttavia, è alquanto differente da quello in uso nell'ictus ischemico nonostante i devices per trombectomia siano gli stessi. Due sono i metodi utilizzati: il primo prevede l'introduzione di un microcatetere nel vaso trombizzato e l'infusione locale di un farmaco fibrinolitico (rt-PA). Il secondo è la trombectomia meccanica con angioplastica o stent retriever. I due approcci sono spesso utilizzati in combinazione. La ricanalizzazione parziale o completa dopo metodica endovascolare arriva fino al $90 \%$ in centri esperti. ${ }^{31} \mathrm{E}$ in corso il trial TO-ACT per verificare l'efficacia e la sicurezza del trattamento trombolitico endovenoso associato o meno a trombectomia meccanica rispetto alla sola terapia eparinica. I risultati preliminari presentati all'European Stroke Organization Conference nel 2017 non hanno dimostrato differenza statisticamente significativa in termini di outcome a 12 mesi tra trattamento endovascolare e trattamento convenzionale. In attesa dei risultati definitivi, al momento tale trattamento non deve essere routinariamente applicato nei pazienti affetti da TVC ma può essere preso in considerazione in pazienti che presentino un rapido deterioramento clinico nonostante l'anticoagulazione. ${ }^{32}$

\section{Trattamento e prevenzione delle complicanze precoci}

\section{Crisi epilettiche}

Le crisi epilettiche possono presentarsi fino al $40 \%$ degli adulti affetti da TVC soprattutto in fase acuta ( $7 \%$ dei pazienti entro le prime due settimane dall'evento) e in quelli che abbiano lesioni parenchimali emorragiche, in particolar modo quelle sopratentoriali. Non esistono studi clinici che abbiano indagato quale sia il farmaco antiepilettico più indicato in tali casi né tantomeno quando sia opportuno iniziare la terapia, ma le linee guida sono concordi nell'impostare una corretta terapia antiepilettica dopo il primo episodio, soprattutto in presenza di lesioni (classe I, Livello di evidenza B). Non vi è indicazione al trattamento in profilassi, in pazienti che non abbiano avuto un primo evento di natura epilettica.

\section{Idrocefalo}

Diversi gradi di idrocefalo possono svilupparsi fino al 15\% dei pazienti affetti da TVC per una compromissione del riassorbimento del liquido cefalorachidiano. L'idrocefalo ostruttivo è una complicanza rara ma potenzialmente letale della TVC, in special modo se coinvolto il sistema veonoso profondo e se sono presenti larghe lesioni emorragiche emisferiche. In questo caso l'intervento neurochirurgico di ventricolostomia o di shunt ventricolo-peritoneale può essere necessario.

\section{Ipertensione endocranica}

Nella fase acuta della TVC un'aumentata pressione intracranica è alquanto frequente (fino al $40 \%$ dei casi); nella maggior parte dei pazienti i sintomi sono limitati a cefalea con o senza papilledema ed in questo caso la terapia analgesica può migliorare il quadro sintomatologico. Non ci sono studi randomizzati su quale sia il miglior trattamento dell'ipertensione endocranica, così come sull'utilizzo di acetazolamide, un inibitore dell'anidrasi carbonica, o altri diuretici.

Allo stesso modo gli steroidi non si sono rilevati efficaci nel prevenire la mortalità o migliorare l'outcome dei pazienti con TVC, a meno che la causa della trombosi non sia una malattia infiammatoria cronica che di per sé viene trattata con cortisonici. Essi potrebbero essere addirittura dannosi (effetti protrombotici, sanguinamento gastro-enterico, infezioni, osteonescrosi avascolare, iperglicemia), pertanto andrebbero evitati soprattutto nei pazienti senza evidenza di lesione parenchimale o emorragia.

Le punture lombari seriate possono rendersi necessarie se l'ipertensione endocranica è persistente così come in casi refrattari può essere eseguito uno shunt lomboperitoneale. In pazienti con deterioramento neurologico dovuto a severo effetto massa o ad emorragia intracranica che causa ipertensione intrattabile, può essere considerato l'intervento di craniectomia decompressiva. ${ }^{14}$

\section{Key message: Trattamento della TVC}

- La terapia cardine della TVC è l'anticoagulazione con eparine a basso peso molecolare a dosaggio terapeutico (100 U/Kg bid), seguita poi da warfarin con range di INR tra 2 e 3.

- L'eparina non frazionata a parità di efficacia rispetto a quella a basso peso molecolare presenta un rischio maggiore di sanguinamenti.

- L'utilizzo dei DOAC non trova ancora spazio nella terapia della TVC in quanto mancano RCT che ne dimostrino efficacia e sicurezza. 
- Un unico RCT è stato pubblicato sul Dabigatran con buoni endpoints, ma data l'esiguità numerica del campione non è stato possibile dimostrare la non inferiorità del farmaco in studio rispetto al controllo.

- La trombolisi endovenosa e/o meccanica non sono terapie di prima linea nella TVC. Il trattamento endovascolare è suggerito in caso di deterioramento clinico del paziente nonostante l'anticoagulazione.

- È importante instaurare un pronto intervento delle complicanze precoci della TVC. Le crisi epilettiche vanno trattate con i comuni farmaci antiepilettici. Nell'ipertensione endocranica sconsigliato è l'uso dei cortisonici, mentre metodiche decompressive devono essere eseguite in caso di ipertensione endocranica intrattabile.

\section{Management post-acuzie}

Le strategie di prevenzione mirano a prevenire le TVC ricorrenti nei pazienti ad alto rischio, nonostante non vi siano degli scores validati di stratificazione del rischio. Si possono tuttavia ragionevolmente considerare ad alto rischio soggetti con trombofilie genetiche $o$ acquisite o pazienti affetti da cancro. Nonostante non ci siano studi clinici controllati randomizzati sulla prevenzione delle ricorrenze dopo un primo episodio di TVC, si stima un tasso di ricorrenza approssimativamente del 6,5\% per anno. ${ }^{33}$

Come già specificato, il trattamento in acuto con eparina deve essere embricato già dal terzo giorno con terapia anticoagulante orale con warfarin, sospendendo la terapia eparinica al raggiungimento di INR 2,0-3,0. La TAO ha una durata variabile a seconda del fattore scatenante la TVC. Pertanto, i pazienti affetti da TVC provocata con fattori di rischio modificabili necessitano un periodo di anticoagulazione variabile tra i 3 e i 6 mesi (INR 2,0-3,0). Quelli affetti da TVC unprovoked devono essere trattati con warfarin per 6-12 mesi (INR 2,0-3,0).

In quelli infine affetti da TVC ricorrenti, tromboembolismo venoso dopo TVC o primo episodio di trombosi cerebrale con severa trombofilia le linee guida consigliano TAO a lungo termine (INR 2,0-3,0). Rientrano nella definizione di trombofilia severa le mutazioni in omozigosi del gene della protrombina G20210A, la mutazione del fattore V Leiden in omozigosi, il deficit di proteina $\mathrm{C}$ e di proteina $\mathrm{S}$ o di ATIII, i deficit trombofilici combinati e la sindrome da anticorpi antifosfolipidi ${ }^{14}$ (Figura 7).

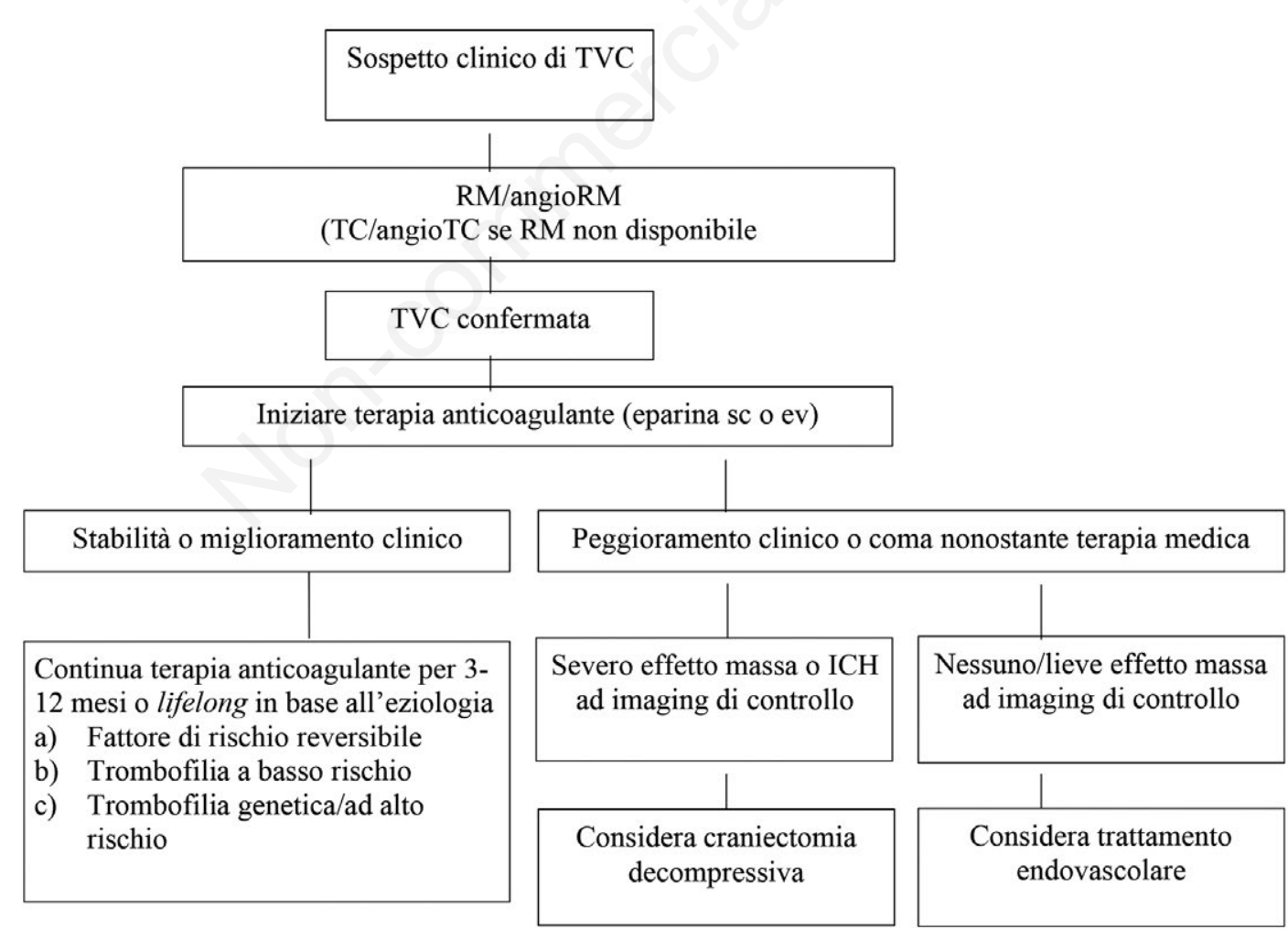

Tutti i pazienti devono ricevere il supporto per la prevenzione delle complicanze e la terapia sintomatica

Figura 7. Algoritmo proposto per il management della trombosi venosa cerebrale (TVC). ICH, emorragia intraparenchimale. Riadattato da Saposnik G. Stroke 2011;42:115. 


\section{Key message: Management post-acuzie}

- La terapia anticoagulante orale ha durata variabile a seconda dell'eziologia della TVC.

- In presenza di fattori di rischio modificabili la terapia va protratta per 3-6 mesi.

- In presenza di fattori di rischio non modificabili la terapia va protratta per 6-12 mesi.

- In caso di TVC ricorrente, tromboembolismo venoso dopo TVC o primo episodio di trombosi cerebrale con severa trombofilia la terapia anticoagulante è a lungo termine.

- In tutti i casi l'INR deve essere compreso in un range tra 2 e 3 .

\section{Trombosi venosa cerebrale in gravidanza}

La gravidanza causa un'attivazione dei meccanismi di ipercoagulabilità che si protraggono fino al puerperio, rendendo le donne maggiormente a rischio di TVC rispetto agli uomini. L'incidenza stimata per la TVC durante gravidanza varia da 1/2500 a 1/10.000 nei paesi occidentali e il maggior periodo di rischio è compreso dal terzo trimestre fino alla 4 settimana dopo il parto.

Il trattamento si basa sulla somministrazione di eparine a basso peso molecolare a dosaggio terapeutico durante tutta la gravidanza e per almeno sei settimane dopo il parto, per una durata complessiva di trattamento di almeno 6 mesi. Il warfarin è controindicato per embriopatia fetale.

Nelle donne con anamnesi di TVC è indicata in una nuova gravidanza il trattamento eparinico a dosaggio profilattico.

\section{Key message: TVC in gravidanza}

- L'incidenza di TVC in gravidanza varia da $1 / 2500$ a $1 / 10.000$.

- Il rischio maggiore si riscontra nel terzo trimestre fino a 4 settimane dopo il parto.

- Il trattamento è l'anticoagulazione con eparine a basso peso molecolare a dosaggio terapeutico.

\section{Trombosi venosa cerebrale in età pediatrica}

L'incidenza della TVC pediatrica è di 0,67/100.000 nascite/anno e la manifestazione clinica è data prevalentemente da crisi convulsive in circa i $2 / 3$ dei casi. In periodo neonatale le cause sono legate sia a forze meccaniche applicate al cranio dei neonati durante il parto che possono creare un danno alle strutture venose cerebrali con conseguente trombosi, sia all'aumentata tendenza trombotica che i piccoli pazienti hanno. Ciò è dovuto ai ridotti livelli di proteina $\mathrm{C}$ e proteina $\mathrm{S}$ circolanti, alla presenza di anticorpi antifosfolipidi trasmessi dalla madre durante la gravidanza e all'emoconcentra- zione da disidratazione relativa che si ha fisiologicamente nella prima settimana di vita. In aggiunta, fattori legati a gravidanza a rischio (pre-eclampsia/eclampsia) possono causare una TVC neonatale.

Le cause della TVC in età pediatrica, invece, sono rappresentate da malattie autoimmunitarie (lupus, sindrome nefrosica), malattie ematologiche e traumi. I disordini protrombotici in tale età possono variare dal $33 \%$ al $66 \%{ }^{34}$ Nonostante l'assenza di studi randomizzati controllati, le evidenze provenienti da studi osservazionali e case reports supportano l'efficacia dell'anticoagulazione in età pediatrica e neonatale, soprattutto dopo i 28 giorni di vita quando LMWH, UFH e warfarin devono essere somministrati a dosaggio terapeutico per almeno 3-6 mesi.

Altro elemento cardine della terapia, è il controllo delle crisi convulsive, frequenti in tale età con farmaci antiepilettici a dosaggio appropriato.

\section{Key message: TVC in età pediatrica}

- L'incidenza della TVC pediatrica è di 0,67/100.000 nascite/anno.

- La manifestazione clinica è rappresentata prevalentemente da crisi convulsive.

- In età neonatale la TVC è legata a fattori materni correlati alla gravidanza e ad alterazioni intrinseche a tale età.

In età pediatrica l'eziologia è su base autoimmunitaria o legata a malalattie ematologiche o a traumi.

- La terapia si basa sull'anticoagulazione con eparine e warfarin per almeno 3-6 mesi.

\section{Bibliografia}

1. Ferro JM, Canhã o P, Stam J, Bousser MG, Barinagarrementeria F; ISCVT Investigators. Prognosis of cerebral vein and dural sinus thrombosis: results of the International Study on Cerebral Vein and Dural Sinus Thrombosis (ISCVT). Stroke. 2004;35:664-670

2. Cantu C., Alonso E., Jara A., et al. Hyperhomocysteinemia, Low Folate and Vitamin B12 Concentrations, and Methylene Tetrahydrofolate Reductase Mutation in Cerebral Venous Thrombosis. Stroke. 2004;35:1790-1794

3. Marjot T1, Yadav S, Hasan N, Bentley P, Sharma P. Genes associated with adult cerebral venous thrombosis.Stroke. 2011 Apr;42(4):913-8

4. Canhão P, Ferro JM, Lindgren AG, Bousser MG, Stam J, Barinagarrementeria F; ISCVT Investigators. Causes and predictors of death in cerebral venous thrombosis.Stroke. 2005 Aug;36(8):1720-5

5. Dentali F1, Crowther M, Ageno W. Thrombophilic abnormalities, oral contraceptives, and risk of cerebral vein thrombosis: a meta-analysis.Blood. 2006 Apr 1;107(7): 2766-73

6. Kristoffersen ES, Harper CE, Vetvik KG, Faiz KW. Cerebral venous thrombosis epidemiology, diagnosis and treatment. Tidsskr Nor Laegeforen. 2018 Aug 20;138 
7. Silvis SM, Aguiar de Sousa D, Ferro JM, Coutinho JM. Cerebral venous thrombosis. Nat Rev Neurol. 2017 Sep;13(9):555-565

8. Devasagayam S, Wyatt B, Leyden J, Kleinig T. Cerebral Venous Sinus Thrombosis Incidence Is Higher Than Previously Thought: A Retrospective Population-Based Study. Stroke. 2016 Sep;47(9):2180-2

9. Bousser MG1, Ferro JM. Cerebral venous thrombosis: an update. Lancet Neurol. 2007 Feb;6(2):162-70

10. Cumurciuc R1, Crassard I, Sarov M, Valade D, Bousser MG. Headache as the only neurological sign of cerebral venous thrombosis: a series of 17 cases. J Neurol Neurosurg Psychiatry. 2005 Aug;76(8):1084-7

11. Selim M, Fink J, Linfante I, Kumar S, Schlaug G, Caplan LR. Diagnosis of cerebral venous thrombosis with echo-planar $\mathrm{T} 2 *$-weighted magnetic resonance imaging.Arch Neurol. 2002 Jun;59(6):1021-6

12. Cakmak S, Hermier M, Montavont A, Derex L, Mauguière F, Trouillas $\mathrm{P}$, Nighoghossian N. T2*-weighted MRI in cortical venous thrombosis. Neurology. 2004 Nov 9;63(9):1698

13. Idbaih A, Boukobza M, Crassard I, Porcher R, Bousser MG, Chabriat H. MRI of clot in cerebral venous thrombosis: high diagnostic value of susceptibility-weighted images.Stroke. 2006 Apr;37(4):991-5

14. Saposnik G, Barinagarrementeria F, Brown RD Jr, Bushnell CD, Cucchiara B, Cushman M, deVeber G, Ferro JM, Tsai FY; American Heart Association Stroke Council and the Council on Epidemiology and Prevention. Diagnosis and management of cerebral venous thrombosis: a statement for healthcare professionals from the American Heart Association/American Stroke Association. Stroke. 2011 Apr;42(4):1158-92

15. Kosinski CM, Mull M, Schwarz M, Koch B, Biniek R, Schlafer J, Milkereit E, Willmes K, Schiefer J. Do normal D-dimer levels reliably exclude cerebral sinus thrombosis? Stroke. 2004;35:2820-2825

16. Crassard I, Soria C, Tzourio C, Woimant F, Drouet L, Ducros A, Bousser MG. A negative D-dimer assay does not rule out cerebral venous thrombosis: a series of seventy-three patients. Stroke. 2005;36:1716-1719

17. Stroke Unit Trialists' Collaboration. Organised inpatient (stroke unit) care for stroke. Cochrane Database Syst Rev. 2007 Oct 17;(4) Stroke Unit Trialists' Collaboration. How do stroke units improve patient outcomes? A collaborative systematic review of the randomized trials. Stroke Unit Trialists Collaboration. Stroke. 1997;28:2139-2144

18. Saposnik G, Fang J, O’Donnell M, Hachinski V, Kapral MK, Hill MD; Investigators of the Registry of the Canadian Stroke Network (RCSN) for the Stroke Outcome Research Canada (SORCan) Working Group. Escalating levels of access to in-hospital care and stroke mortality. Stroke. 2008;39:2522-2530

19. Einha upl KM, Villringer A, Meister W, Mehraein S, Garner C, Pellkofer M, Haberl RL, Pfister HW, Schmiedek P. Heparin treatment in sinus venous thrombosis [published correction appears in Lancet. 1991; 338:958]. Lancet. 1991;338:597-600

20. de Bruijn SF, Stam J. Randomized, placebo-controlled trial of anticoagulant treatment with low-molecular-weight heparin for cerebral sinus thrombosis. Stroke. 1999;30:484-488

21. Stam J, De Bruijn SF, DeVeber G. Anticoagulation for cerebral sinus thrombosis. Cochrane Database Syst Rev. 2002;(4)

22. Preter M, Tzourio C, Ameri A, Bousser MG. Long-term prognosis in cerebral venous thrombosis: follow-up of 77 patients. Stroke. 1996;27:243-246

23. Maqueda VM, Thijs V. Risk of thromboembolism after cerebral venous thrombosis. Eur J Neurol. 2006;13:302-305

24. Breteau G, Mounier-Vehier F, Godefroy O, Gauvrit JY, Mackowiak-Cordoliani MA, Girot M, Bertheloot D, He 'non H, Lucas C, Leclerc X, Fourrier F, Pruvo JP, Leys D. Cerebral venous thrombosis 3-year clinical outcome in 55 consecutive patients. J Neurol. 2003;250:29-35

25. Cakmak S, Derex L, Berruyer M, Nighoghossian N, Philippeau F, Adeleine P, Hermier M, Froment JC, Trouillas P. Cerebral venous thrombosis: clinical outcome and systematic screening of prothrombotic factors. Neurology. 2003;60:1175-1178

26. van Dongen CJ, van den Belt AG, Prins MH, Lensing AW. Fixed dose subcutaneous low molecular weight heparins versus adjusted dose unfractionated heparin for venous thromboembolism. Cochrane Database Syst Rev. 2004 Oct 18;(4)

27. Geisbüsch C, Richter D, Herweh C, Ringleb PA, Nagel S. Novel factor xa inhibitor for the treatment of cerebral venous and sinus thrombosis: first experience in 7 patients. Stroke. 2014 Aug;45(8):2469-71

28. Mendonça MD, Barbosa R, Cruz-e-Silva V, Calado S, Viana-Baptista M. Oral direct thrombin inhibitor as an alternative in the management of cerebral venous thrombosis: a series of 15 patients. Int J Stroke. 2015 Oct;10(7):1115-8

29. Ferro JM, Coutinho JM, Dentali F, Kobayashi A, Alasheev A, Canhão P, Karpov D, Nagel S, Posthuma L, Roriz JM, Caria J, Frässdorf M, Huisman H, Reilly P, Diener HC; RE-SPECT CVT Study Group. Safety and Efficacy of Dabigatran Etexilate vs Dose-Adjusted Warfarin in Patients With Cerebral Venous Thrombosis: A Randomized Clinical Trial. JAMA Neurol. 2019 Sep 3

30. Dentali F1, Squizzato A, Gianni M, De Lodovici ML, Venco A, Paciaroni M, Crowther M, Ageno W. Safety of thrombolysis in cerebral venous thrombosis. A systematic review of the literature. Thromb Haemost. 2010 Nov;104(5):1055-62

31. Silvis SM, Aguiar de Sousa D, Ferro JM, Coutinho JM. Cerebral venous thrombosis. Nat Rev Neurol. 2017 Sep;13(9):555-565

32. Coutinho JM, Ferro JM, Zuurbier SM, Mink MS, Canhão P, Crassard I, Majoie CB, Reekers JA, Houdart E, de Haan RJ, Bousser MG, Stam J. Thrombolysis or anticoagulation for cerebral venous thrombosis: rationale and design of the TO-ACT trial.Int J Stroke. 2013 Feb;8(2):135-40

33. Ferro JM, Lopes MG, Rosas MJ, Ferro MA, Fontes J; Cerebral Venous Thrombosis Portuguese Collaborative Study Group. Long-term prognosis of cerebral vein and dural sinus thrombosis: results of the VENOPORT study. Cerebrovasc Dis. 2002;13:272-278

34. deVeber G, Andrew M, Adams C, Bjornson B, Booth F, Buckley DJ, Camfield CS, David M, Humphreys P, Langevin P, MacDonald EA, Gillett J, Meaney B, Shevell M, Sinclair DB, Yager J; Canadian Pediatric Ischemic Stroke Study Group. Cerebral sinovenous thrombosis in children. N Engl J Med. 2001;345:417-423. 


\title{
Gestione della disfagia e prevenzione delle complicanze
}

\author{
Roberta Rapetti, ${ }^{1}$ Fabio Bertoncini, ${ }^{2}$ Lucilla Vestito ${ }^{3}$ \\ ${ }^{1}$ Ospedale San Paolo, Savona; ${ }^{2}$ ASL Biella; ${ }^{3}$ IRCCS Ospedale Policlinico San Martino, Genova, Italia
}

\section{Introduzione}

La deglutizione è un atto fisiologico complesso che richiede la coordinazione di molteplici strutture anatomo-funzionali del Sistema Nervoso Centrale e Periferico, con l'obiettivo di spingere il bolo alimentare dalla cavità orale allo stomaco. Tale atto è molto rapido (circa due secondi) e prevede il coinvolgimento delle labbra, della lingua, del palato molle, della faringe, della laringe, dell'esofago e del sistema respiratorio. In particolare, la coordinazione tra vie digestive ed apparato respiratorio rappresenta un meccanismo essenziale per un processo di deglutizione sicura.

La deglutizione è generalmente suddivisa in più fasi: fase 0 (preparazione extraorale degli alimenti, ad esempio cottura, frantumazione, omogeneizzazione, etc), fase 1 (preparazione orale delle sostanze da deglutire mediante masticazione, insalivazione, detersione buccale), fase 2 (stadio orale o coinvolgimento del bolo verso l'ostio delle fauci ed elicitazione del riflesso della deglutizione faringea), fase 3 (stadio faringeo o di transito del quadrivio faringeo o incrocio della via respiratoria), fase 4 (stadio esofageo), fase 5 (stadio gastrico). ${ }^{1}$ Le prime tre fasi sono sotto il controllo volontario, mentre le ultime avvengono in modo automatico e riflesso.

Questo complesso processo sensitivo-motorio è regolato da un centro regolatore (Central Program Generator o $\mathrm{CPG}$ ) posto nel tronco encefalico e più precisamente nel bulbo a livello del nucleo del tratto solitario. Il CPG è, tuttavia, sotto il controllo di un ampio network corticale e sottocorticale, di cui i principali componenti risultano essere la corteccia sensori-

Corrispondente: Fabio Bertoncini, ASL Biella, Italia E-mail: fabio.bertoncini@hotmail.it

Articolo pubblicato secondo la Creative Commons Attribution NonCommercial 4.0 License (CC BY-NC 4.0).

${ }^{\circ}$ Copyright: the Author(s), 2020

Licensee PAGEPress, Italy

QUADERNI - Italian Journal of Medicine 2020; 8(2):168-175 motoria primaria e secondaria, la corteccia premotoria, l'insula anteriore, il giro cingolato, l'area supplementare motoria e i gangli della base. Non meno importante è il ruolo del cervelletto nella pianificazione e nel controllo esecutivo-motorio. In tale network articolato il CPG riceve input neurali dalla corteccia cerebrale, dai muscoli periferici e dai recettori della mucosa (meccanocettori, chemiocettori e termocettori) coordinando il processo di deglutizione. In particolare, gli input sensitivi provenienti da orofaringe, laringe ed esofago afferiscono al CGP e alla corteccia cerebrale sensoriale mediante fibre dei nervi cranici (V, VII, IX, $\mathrm{X}, \mathrm{XII})$. La corteccia cerebrale elabora le informazioni ottenute e le invia al CPG, a livello del quale vengono attivati i muscoli implicati nella deglutizione. ${ }^{2}$

Le tecniche di neuroimaging (Risonanza Magnetica Funzionale o fMRI) e di neurostimolazione (stimolazione magnetica transcranica o TMS) hanno consentito la mappatura della muscolatura coinvolta nella deglutizione, dimostrando una rappresentazione bilaterale della stessa (corteccia motoria e premotoria), con un'asimmetria interemisferica che non è necessariamente corrispondente alla dominanza manuale. Inoltre le stesse tecniche hanno permesso di evidenziare come esista una specializzazione emisferica per le differenti fasi della deglutizione: la fase orale vedrebbe una maggiore lateralizzazione sinistra, mentre in quella faringea prevarrebbe la funzione emisferica destra. ${ }^{3}$

\section{Disfagia}

L'alterazione della deglutizione prende il nome di disfagia (dal greco dys-phagein = difficoltà a mangiare) ed è definita come la difficoltà o l'impossibilità ad attuare un'alimentazione orale autonoma e sicura. La disfagia non è una malattia, ma un sintomo (se avvertito dal paziente) o un segno clinico (se ravvisata dal clinico). La prevalenza nella popolazione generale varia dal $2 \%$ al $16 \%$, valore che sale sino al $40 \%$ dei soggetti ospedalizzati. ${ }^{4}$ La disfagia può manifestarsi anche in soggetti sani anziani, a causa di modificazioni dell'orofaringe correlate all'età, ed è denominata presbifagia. Nella grande maggioranza dei casi, tuttavia, essa compare a seguito di quadri morbosi di varia natura, i più significativi dei quali vengono mostrati in Tabella 1. 
Se non precocemente identificata e correttamente governata, la disfagia può condurre ad un progressivo stato di malnutrizione, disidratazione, carenza vitaminica, sarcopenia e al rischio di aspirazione di materiale estraneo con conseguente broncopneumopatia ab ingestis. ${ }^{5}$

\section{Disfagia e stroke}

La difficoltà a deglutire a seguito di ictus è una complicanza comune e rappresenta la prima causa di disfagia neurogena. ${ }^{6}$ L'incidenza di disfagia post ictus è di circa il $40 \%$ dei pazienti con lesioni emisferiche corticali e di oltre il $50 \%$ nei pazienti con lesioni cortico-sottocorticali. Tali percentuali aumentano notevolmente se viene interessato il tronco encefalico, soprattutto ponte e bulbo (Figura 1).

La disfagia orofaringea (DO) post ictus può essere dovuta a diverse cause: a deficit neuromuscolari che comandano i muscoli implicati nella deglutizione, a difficoltà nella coordinazione dei movimenti, a ritardo del tempo d'innesco faringeo, ad un'alterazione della sensibilità faringo-laringea e ad una diminuzione dei livelli di coscienza. La DO espone il paziente ad un elevato rischio di aspirazione (circa il 50\% dei pazienti con stroke) e per $1 / 3$ di questi a insorgenza di polmonite $\mathrm{ab}$ ingestis. Nel caso in cui piccole quantità di alimenti o di altro materiale (es. saliva) entrino in laringe in assenza di manifestazioni cliniche, come la tosse, si parla di aspirazione silente. Diversamente, il passaggio di sostanze alimentari nelle vie aeree si può manifestare in modo evidente, con senso di soffocamento, tosse insistente, comparsa di colorito rosso o cianotico al volto.

La caduta del bolo nelle vie aeree può verificarsi in momenti diversi del suo transito. Per tale ragione si distinguono: i) aspirazione pre-deglutitoria: il bolo cade nel vestibolo laringeo e viene aspirato in trachea per mancato contenimento della cavità orale in seguito a deficit della motilità velare e/o della muscolatura linguale; ii) aspirazione intra-deglutitoria: caduta del bolo in trachea durante la fase faringea, per deficit di

Tabella 1. Le più significative cause di disfagia.

IATROGENE (es. effetti collaterali di terapie farmacologiche, radiazioni)

INFETTIVE (es. difterite, sifilide, mucosite ecc.)

METABOLICHE (es. amiloidosi, Morbo di Wilson, ecc.)

MIOPATICHE (es. dermatomiosite, miastenia grave, sarcoidosi, ecc.)

NEUROLOGICHE (es. Tumori del tronco, ictus, Sclerosi Multipla, Morbo di Parkinson, Demenza, SLA, ecc.)

STRUTTURALI (es. diverticolo di Zenker, tumori orofaringei, ecc.) adduzione glottica, ribaltamento della epiglottide, ipomobilità delle aritenoidi, retropulsione linguale, ridotta o ritardata apertura dello sfintere esofageo superiore (SES); iii) aspirazione post-deglutitoria: il bolo ristagna nei seni piriformi con successiva aspirazione in trachea, per deficit della peristalsi faringea, del movimento di innalzamento e avanzamento del complesso ioido-laringeo, e/o per mancata o ridotta apertura del SES.

Occorre tenere presente come la polmonite ab ingestis non sia causata solamente dalla presenza di disfagia orofaringea, ma i ridotti livelli di vigilanza, la presenza di materiale nel cavo orale a causa di scarsa igiene (agenti patogeni e batteri normalmente presenti), le interferenze meccaniche da posizionamento di presidi medici quali ad esempio il sondino naso gastrico o le cannule tracheali rappresentano dei fattori predisponenti alle polmoniti da aspirazione.

Come già accennato, la malnutrizione e la disidratazione nel paziente disfagico con ictus sono eventi frequenti che possono causare una riduzione delle abilità cognitive, un aumento del rischio di infezioni, una perdita di massa muscolare fino alla sarcopenia e un peggioramento delle abilità motorie. Ne consegue un aumentata incidenza di mortalità dei pazienti con ictus e disfagia. E' pertanto necessario che la prima valutazione del rischio nutrizionale venga eseguita precocemente, ossia entro $24-48$ ore dal ricovero ospedaliero. ${ }^{7,8}$

Dal punto di vista dell'evoluzione del disturbo, è noto che alcuni soggetti riprendono a deglutire spontaneamente nelle due settimane successive all'ictus (grazie ai fenomeni di plasticità cerebrale). Una discreta percentuale di soggetti, che può arrivare anche al $50 \%$, continuano però a presentare disfagia anche a distanza di alcuni mesi dall'evento ictale, rendendo necessario un approccio riabilitativo a lungo termine.

\begin{tabular}{|c|c|c|c|}
\hline \multicolumn{4}{|c|}{$\begin{array}{l}\text { Alterazioni della deglutizione per tipi di } \\
\text { ictus }\end{array}$} \\
\hline Tipo di ictus & $\begin{array}{l}\text { Tipo di } \\
\text { lesione }\end{array}$ & Gravità & $\begin{array}{l}\text { Tipo di } \\
\text { alterazione }\end{array}$ \\
\hline emisferico & $\begin{array}{l}\text { Monolaterale } \\
\text { sinistro }\end{array}$ & $++\cdots$ & $\begin{array}{l}\text { Fase orale } \\
\text { della } \\
\text { deglutizione }\end{array}$ \\
\hline emisferico & $\begin{array}{l}\text { Monolaterale } \\
\text { destro }\end{array}$ & $++\cdots$ & $\begin{array}{l}\text { Fase faringea } \\
\text { della } \\
\text { deglutizione }\end{array}$ \\
\hline emisferico & $\begin{array}{l}\text { Corticale } \\
\text { bilaterale }\end{array}$ & +++ & tutte \\
\hline tronco & $\begin{array}{l}\text { Mono o } \\
\text { bilaterale }\end{array}$ & ++++ & tutte \\
\hline
\end{tabular}

Figura 1. correlazioni tra ictus e alterazioni della deglutizione. 


\section{Segni e sintomi}

In generale, i segni che devono indurre il sospetto di disfagia sono: i) comparsa di tosse involontaria durante o dopo la deglutizione; ii) comparsa di voce velata o gorgogliante dopo la deglutizione; iii) fuoriuscita di liquidi o di cibo dal naso; iv) comparsa di odinofagia (dolore associato alla deglutizione); v) sensazione di cibo fermo in gola o presenza di residui di alimenti in misura significativa nel cavo orale dopo la deglutizione; vi) allungamento del tempo dedicato al pasto o fatica durante l'assunzione dello stesso; vii) perdita di peso senza causa apparente; viii) presenza di frequenti infezioni respiratorie.

\section{Strategie assistenziali}

La disfagia orofaringea del paziente ictato riconosce una patogenesi multifattoriale e deve pertanto essere affrontata con il contributo di un team multidisciplinare: medici, infermieri, logopedisti, fisioterapisti, dietisti, operatori socio sanitari. L'approccio diagnostico al paziente disfagico in seguito a stroke presuppone una serie di passaggi che prevedono un iniziale screening deglutitorio e, a seconda degli esiti di quest'ultimo, una valutazione clinica corredata, in situazioni ideali, da una valutazione strumentale.

\section{Lo screening}

Secondo le recenti linee guida di prevenzione e trattamento italiane e USA, ${ }^{9,10}$ tutti i pazienti con ictus devono essere avviati ad un percorso di screening prima di iniziare la somministrazione di alimenti o bevande (livello di evidenza IIA, raccomandazione forte a favore).

In modo particolare le persone con ictus acuto dovrebbero essere sottoposte ad un test di screening ad opera del personale infermieristico entro quattro ore dall'arrivo in ospedale. Lo screening è una procedura di tipo dentro/fuori con la finalità di identificare quei soggetti che necessitano di una valutazione clinica completa della funzione deglutitoria.

Gli screening devono possedere alcune importanti caratteristiche: i) rapida somministrazione (1520 minuti); ii) relativamente non invasivi; iii) a basso rischio per il paziente; iv) altamente sensibili (capacità di identificare tutti i soggetti con disfagia); v) altamente specifici (capacità di identificare ed escludere tutti i soggetti non affetti da disfagia).

Dalla disamina della letteratura scientifica emerge che esistono numerosi test di screening, ma a tutt'oggi, non è stato identificato un unico e valido test per la valutazione della disfagia nell'ictus acuto. ${ }^{11,12}$ Un denominatore comune a queste valuta- zioni è la presenza del test del bolo d'acqua (nella forma originale o modificato o in associazione con altre prove $)^{13}$ con una sensibilità discreta $(>70 \%)$, ma con specificità non ottimale (22-66\%). Vi è un accordo generale, tuttavia, ${ }^{14}$ nel sostenere come il test del bolo d'acqua debba far parte dello screening (Raccomandazione di Grado B) e che la presenza di tosse o di modifiche delle caratteristiche acustiche della voce, dopo assunzione d'acqua, rappresenta un predittore essenziale di possibile aspirazione. Il test del bolo d'acqua (Three-oz water swallow test) è una procedura semplice che viene eseguita a paziente vigile in posizione seduta e con la testa in asse. Si procede somministrando al paziente $5 \mathrm{ml}$ di acqua con un cucchiaino, per tre volte, verificando che il paziente abbia deglutito. Se il test è negativo si somministrano successivamente $50 \mathrm{~mL}$ dal bicchiere.

I segni che rendono il test di screening positivo sono: i) tosse immediata o entro 1 minuto; ii) alterazione delle qualità acustiche della voce (gorgogliante o umida); iii) multiple deglutizioni per bolo.

Occorre sottolineare come il test del bolo d'acqua è controindicato nei pazienti in cui l'aspirazione sia probabile o nota sulla base di altri segni.

Le procedure di screening devono sempre includere una valutazione preliminare che tenga in considerazione: i) la vigilanza (capacità del paziente di mantenere l'attenzione per un periodo > di $15 \mathrm{mi}-$ nuti); ii) il grado di collaborazione attiva; iii) il controllo del capo e del tronco; iv) l'igiene orale; v) il controllo delle secrezioni orali.

I pazienti che non sono in grado di effettuare lo screening dovrebbero essere rivisti nelle successive 24 ore per verificarne l'evoluzione.

$\mathrm{Al}$ termine della procedura gli operatori dovranno fornire chiare indicazioni di azione (es. visita specialistica successiva, possibilità di alimentazione per os, nulla per os) (Figura 2).

\section{La valutazione clinica}

Successivamente allo screening, la valutazione clinica al letto del paziente, o Bedside Examination, consiste nella valutazione non strumentale della deglutizione e dovrebbe essere effettuata da un professionista competente nella gestione della disfagia (di norma il logopedista). ${ }^{15}$ Essa prevede una valutazione generale (individuazione delle possibili cause di disfagia) e una valutazione specifica.

In particolare la valutazione clinica deve comprendere: i) la raccolta anamnestica; ii) la valutazione delle abilità cognitive e comunicative; iii) la valutazione delle strutture motorie orali, della sensibilità e delle loro funzioni; iv) le prove di deglutizione con liquidi ed alimenti a differente consistenza (semisolidi e solidi). 
Al termine della valutazione l'esaminatore dovrà essere in grado di stabilire: i) la gravità della disfagia; ii) l'attuazione di possibili modifiche posturali e dietetiche; iii) l'identificazione degli obiettivi di trattamento riabilitativo.

\section{La valutazione strumentale}

Secondo 1'American Speech-Language-Hearing Association (ASHA) ${ }^{16}$ la valutazione strumentale, di competenza medica (foniatra, otorinolaringoiatra, radiologo, gastroenterologo), ha l'obiettivo di stabilire l'integrità delle strutture coinvolte nella deglutizione, documentare la progressione di secrezioni o del bolo, evidenziando la presenza di fenomeni di penetrazione (progressione fino alle corde vocali) e di aspirazione (progressione al di sotto delle corde vocali), confermare una diagnosi sospetta all'esame clinico e verificare l'efficacia di manovre e posture di compenso. Tra le diverse tecniche strumentali vengono di seguito citate quelle maggiormente utilizzate: i) videofluoroscopia (VFSS): considerata il gold standard, è una metodica radiologica che si realizza mediante la somministrazione di alimenti a differente consistenza, associati al bario. Essa valuta l'intero transito del bolo (funzionalità delle strutture, tempi, episodi di penetrazione/aspirazione). Consente un'indagine in tempo reale di tutte le fasi della deglutizione, ma richiede un team di operatori specializzati e la collaborazione del paziente; ii) esame endoscopico a fibre ottiche (FEES): rappresenta la tecnica più utilizzata nella pra-

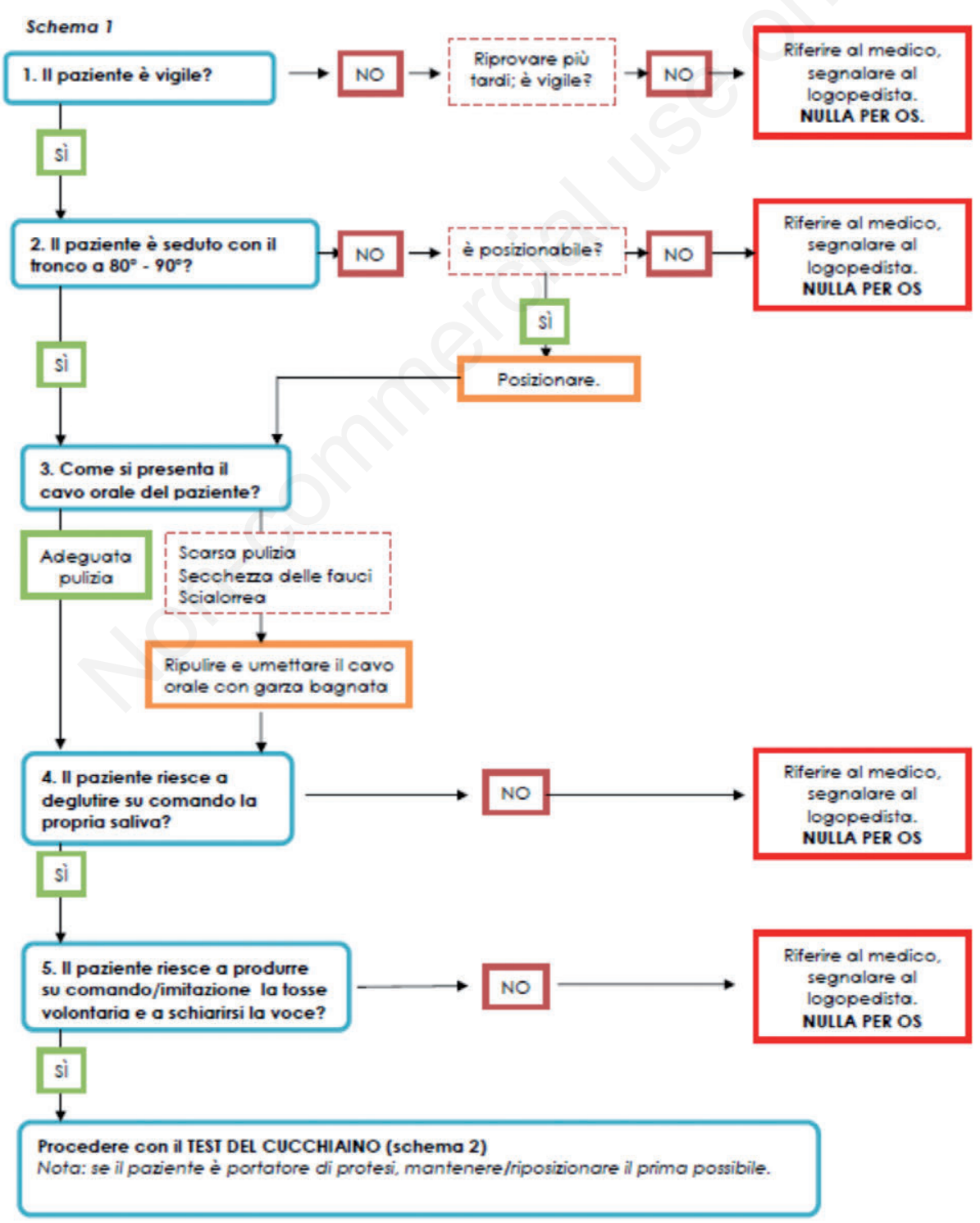

Figura 2. PDTA ASST Pavia. 
tica clinica in virtù della sua facile esecuzione, della elevata tollerabilità e dei bassi costi. Essa valuta la componente motoria e sensoriale e la gestione dei ristagni di bolo; iii) manometria faringea: valuta la forza e la velocità della peristalsi esofagea, nonché il rilassamento degli sfinteri faringei ed esofagei. In particolare, può fornire importanti informazioni sulla possibile disfunzione crico-faringea. Tale tecnica è invasiva e di costo non indifferente; iv) elettromiografia (EMG) dei muscoli coinvolti nella deglutizione condotta con l'utilizzo di elettrodi intramuscolari e/o elettrodi di superficie. Essa permette di stabilire quali muscoli sono attivi e la durata della loro attività nelle diverse fasi della deglutizione.

\section{Interventi assistenziali}

Come già sottolineato, la disfagia secondaria ad ictus vede in molti casi una risoluzione spontanea. Quando ciò non si verifica è opportuno attuare una serie di accorgimenti per garantire una adeguata nutrizione ed impedire possibili complicanze, talora fatali.

In attesa di stabilire un metodo sicuro di nutrizione ed idratazione per bocca, occorre prendere in considerazione: i) adeguata idratazione con fluidi con una via di somministrazione diversa da quella orale; ii) alimentazione con sondino nasogastrico entro 24 ore; iii) efficace somministrazione di farmaci in forme farmaceutiche diverse e vie di infusione alternative; iv) valutazioni specialistiche precoci (cliniche e clinico-strumentali).

In caso invece di disfagia accertata, dovranno essere prese in considerazioni procedure terapeutiche in base alla necessità del singolo paziente come, ad esempio, l'utilizzo di metodiche di compenso, come la manovra di deglutizione sovraglottica. Molto importante è l'utilizzo di posture facilitanti, come ad esempio la postura con capo flesso anteriormente. Questo tipo di postura viene largamente utilizzata nei pazienti con disfagia post ictus poiché determina una maggiore elevazione della laringe, uno spostamento posteriore della base lingua e l'orizzontalizzazione dell'epiglottide. Le modifiche così indotte garantiscono, in caso di ritardo d'innesco del riflesso della deglutizione, una maggiore possibilità di trattenere l'ingesto nello spazio vallecolare fino alla comparsa dell'atto deglutitorio. Di non minore rilievo è l'utilizzo di provvedimenti adattivi, come le modifiche dietetiche, l'utilizzo di ausili speciali e il counseling al paziente e al care-giver. Riguardo a quest'ultimo aspetto, si sottolinea il fatto che non solo il personale che è coinvolto nell'assistenza al paziente disfagico ma gli stessi care-giver abbiano consapevolezza dell'importanza dei disturbi della deglutizione e sappiano gestire il paziente durante l'assunzione dei pasti. A titolo di esempio, si ricordano alcuni comportamenti facilitanti l'alimentazione (salvo diverse indicazioni): i) durante il pasto controllare lo stato di vigilanza e la postura del paziente: il busto deve rimanere ben eretto con un sostegno per gli avambracci e i piedi appoggiati per terra. In caso di persona allettata il busto deve essere alzato il più possibile, almeno di $80^{\circ}$; ii) le fasi della deglutizione dovrebbero essere svolte con tempi consoni, senza fretta e distrazioni, introducendo un boccone per volta; iii) controllare a intervalli regolari la presenza di residui faringei eseguendo colpi di tosse; la ripresa dell'alimentazione può avvenire soltanto dopo completa detersione; iv) la somministrazione di acqua, quando consentita, deve avvenire previa detersione da residui faringei, secondo le modalità sopra descritte; v) terminata la consumazione del cibo, far mantenere al paziente una posizione seduta per almeno 30 minuti; vi) mantenere sempre il cavo orale pulito e le mucose idratate.

E' importante ricordare come, per quanto riguarda le indicazioni dietetiche, devono essere tenuti in considerazione i seguenti diversi criteri, ${ }^{17}$ i) consistenza; ii) grado di coesione; iii) omogeneità; scivolosità; iv) volume del bolo; temperatura; colore degli alimenti; v) sapore degli alimenti; vi) appetibilità.

$\mathrm{Al}$ fine di rendere coerente e uniforme il linguaggio utilizzato dai professionisti sanitari e fornire indicazioni precise e strutturate in termini di necessità di modificazioni dietetiche, è consigliabile utilizzare il framework Internationl Dysphagia Diet Standardization Initiative (IDDSI), ${ }^{18}$ uno strumento che categorizza livelli in cui viene definita la consistenza minima necessaria degli alimenti da assumere: i) $l i-$ quidi senza scorie: non necessitano di preparazione orale (es. acqua, the); ii) liquidi con scorie: non necessitano di preparazione orale, ma se aspirati possono causare maggiori problematiche infiammatorie a livello broncopolmonare (es. latte o succo di frutta); iii) semiliquidi: necessitano di modesta preparazione orale, possono essere bevuti da una tazza o assunti con un cucchiaio (es. frullati, passati di verdura, gelati); iv) semi-solidi: richiedono una masticazione orale poco più impegnativa rispetto alla precedente, non possono essere bevuti ma vengono assunti con un cucchiaio (es. omogeneizzati, passati densi, ricotta); v) solidi: dieta a base di alimenti di consumo abituale e diverse consistenze.

Occorre ricordare come sia possibile modificare la consistenza degli alimenti con l'utilizzo di addensanti naturali (es. gelatine, fecola), diluenti (es. acqua, brodo) e lubrificanti (es. olio, burro). Al fine di distinguere il grado di densità degli alimenti è possibile utilizzare il cosiddetto test del cucchiaino: esso consiste nell'immergere un cucchiaino nell'alimento e valutare la rapidità della caduta dello stesso: caduta 
immediata equivale a densità liquida; caduta rallentata a densità semiliquida; caduta lentissima o assente a densità semisolida.

In pazienti con rischio di aspirazione sono da evitare: i) alcolici; ii) alimenti a doppia consistenza (es. pastina in brodo, minestrone con verdure in pezzi); iii) alimenti con filamenti (es. finocchi, bollito); iv) legumi; v) frutta secca; vi) alimenti solidi friabili (es. fette biscottate, grissini); vii) cereali (es. riso, farro).

Anche la modificazione della consistenza dei liquidi, quando viene posta indicazione alla restrizione di acqua libera o altre consistenza liquide deve poter essere categorizzata in modalità univoca e facilmente identificabile a seconda del livello di densità desiderata, fornendo istruzioni chiare e dettagliate.

Due considerazioni a completamento che rivestono particolare importanza nella gestione del paziente con disfagia orofaringea post ictus di grado severo: i) la scelta di una corretta via alternativa di nutrizione; e ii) la somministrazione dei farmaci.

- Secondo le linee guida italiane sulla gestione del paziente disfagico adulto in Foniatria e Logopedia, ${ }^{15}$ nella disfagia in fase acuta deve essere preso in considerazione il più precocemente possibile il posizionamento del sondino naso gastrico (SNG) per i pazienti che non sono in grado di assumere per bocca il fabbisogno nutrizionale. ${ }^{19}$ Nei soggetti con disfagia persistente post-ictus (di durata superiore a due mesi) va presa in considerazione anche la possibilità di posizionare la gastrostomia endoscopica percutanea (PEG).

La nutrizione enterale tramite SNG e con l'ausilio di pompe peristaltiche è ritenuta più appropriata rispetto alla nutrizione parenterale per il supporto nutrizionale a breve termine in pazienti con grave disfagia dovuta a ictus.

Sia la nutrizione tramite SNG sia quella tramite PEG, consentono il mantenimento del trofismo della mucosa intestinale e della sua funzione immunitaria e di barriera, riducendo la traslocazione batterica. Inoltre hanno minori complicanze infettive e metaboliche.

- La somministrazione dei farmaci per via orale diventa un processo assistenziale estremamente complesso nei pazienti che presentano difficoltà nella deglutizione. In effetti, la manipolazione del farmaco, come ad esempio la polverizzazione delle compresse, può comportare: i) rischi per la salute del paziente; ii) alterazione della stabilità della formulazione; iii) degradazione del principio attivo per esposizione alla luce; iv) variazioni nella farmacocinetica/farmacodinamica del farmaco; v) effetto irritante sulla mucosa del tubo digerente; vi) gusto sgradevole e conseguente scarsa compliance del paziente.

In caso di difficoltà nella somministrazione della terapia farmacologica, la prima scelta, di competenza medica, sarà quella di individuare, se possibile, formulazioni alternative del farmaco, adatte al tipo di paziente.

Sono necessari, inoltre, alcuni specifici accorgimenti: i) le compresse sono frantumabili solo se hanno la dicitura compressa divisibile e se sulla loro superficie hanno la pre-divisione in parti; ii) la compressa polverizzata può essere miscelata con una piccola quantità di semisolido (frullati/ budini) o con acquagel, valutando se questo possa interferire con l'azione del farmaco; iii) alcuni farmaci possono essere inattivati se miscelati ad addensanti o acqua gel (es. Movicol); iv) e' consigliabile non somministrare insieme due farmaci, poiché ciò potrebbe rendere il farmaco sottodosato o inattivo, oppure creare dei composti dannosi per il paziente; v) pulire bene, tra una somministrazione e l'altra, il contenitore del trita pastiglie; vi) controllare il cavo orale alla fine della somministrazione dei farmaci, per constatare la presenza di residui di farmaco; se questo si verifica favorire la detersione con l'aiuto di semisolido o acqua gelificata. ${ }^{20}$

\section{Il trattamento riabilitativo}

Il trattamento riabilitativo si pone il duplice obiettivo di ridurre il rischio di complicanze e di migliorare la fiopatologia deglutitoria.

A tale scopo possono essere utilizzate metodiche compensative (precedentemente trattate) e metodiche rimediative quali le stimolazioni sensoriali, il rinforzo muscolare e la neuromodulazione.

Alcune di queste tecniche rappresentano delle metodiche riabilitative potenzialmente efficaci e sicure, raffigurandosi come delle promettenti alternative al trattamento riabilitativo tradizionale.

Tra queste tecniche ricordiamo: i) la stimolazione elettrica neuromuscolare transcutanea: tecnica non invasiva, che sfrutta correnti a bassa intensità tramite elettrodi posti nella zona anteriore del collo, indicendo la contrazione muscolare, ${ }^{21}$ ii) le tecniche di stimolazione cerebrale non invasiva: la stimolazione magnetica transcranica ripetitiva (rTMS) e la stimolazione elettrica transcranica a corrente continua (tDCS). La prima si basa sull'induzione di una corrente elettrica nel tessuto cerebrale attraverso uno stimolo magnetico generato dal coil; la seconda sfrutta correnti elettriche di bassa intensità erogate da elettrodi posti direttamente sullo scalpo. Le tecniche di stimolazione cerebrale non invasiva, favoriscono la plasticità cerebrale e se utilizzate congiuntamente a strategie di riabilitazione, sembrano potenziare i possibili effetti terapeutici; ${ }^{22}$ iii) stimolazione elettrica faringea (PES): attraverso cateteri intraluminali specifici per posizione introdotti transnasalmente consentono la stimolazione diretta della faringe. ${ }^{3}$ 


\section{Interventi educativi alla dimissione e follow-up}

Aspetto essenziale nella pianificazione delle cure da erogare a domicilio alla persona con disfagia da ictus risulta essere la stesura di progetti educativi da somministrare al paziente e ai familiari.

Le evidenze sostenute dalla letteratura sono coerenti nel considerare, come aspetto fondamentale di

primaria importanza, l'addestramento ai familiari/caregiver alla gestione sicura del paziente e lo stabilire ravvicinati follow up di valutazione clinica e assistenziale, così come consegnare ai familiari/care giver una guida con le informazioni utili su una nutrizione sicura (cibo e fluidi) e le modalità comportamentali da adottare durante queste fasi.

Il focus su cui è necessario focalizzare l'attenzione riguarda però il contenuto dei programmi di educazione terapeutica, così come delle guide cartacee da consegnare: se da una parte l'utilizzo di diete a consistenze modificate riducono il rischio di aspirazione, dall'altra le modificazioni bromatologiche necessarie a rendere sicura la consistenza, potrebbero portare a malnutrizione o sbilanciamenti nutrizionali di altra natura. ${ }^{23}$

Rimane di fondamentale importanza il sottolineare come la componente interdisciplinare nella gestione di un aspetto con un numero di esiti così elevato, assuma un ruolo cardine nella cura della persona con disfagia.

\section{Bibliografia}

1. Schindler O, Ruoppolo G, Schindler A "Deglutologia", Omega Edizioni, 2001.

2. Wilmskoetter J, Bonilha L, Martin-Harris B, et al. Mapping acute lesion locations to physiological swallow impairments after stroke. Neuroimage: Clinical 2019; 22: 101685.

3. Restivo Domenico A, Hamdy Shaheen "Dispositivo di stimolazione elettrica faringea per il trattamento della disfagia neurogena: aggiornamento tecnologico" 4 gennaio 2018, 11:21-26.

4. Care Carol Marie McGinnis, Kimberly Homan, Meghan Solomon, et al. Dysphagia: Interprofessional Management, Impact and Patient-Centered Nutrition in Clinical Practice Volume 34 Number 1February 2019 80-95, 2018 American Society for Parenteral and Enteral Nutrition.

5. Muehlemann N, Jouaneton B, de Léotoing L,et al. "Hospital costs impact of post ischemic stroke dysphagia: Database analyses of hospital discharges in France and Switzerland". PLoS One. 2019 Jan 10;14(1):e0210313. doi: 10.1371/journal.pone.0210313. eCollection 2019.

6. World Gastroenterology Organisation Global Guideli- nes, Dysphagia 2, September 2014.

7. National Cinical Guideline for stroke firth edition 2016, Royal College of Physician

8. Cairella G, Scalfi L, et al. Nutritional recommendations for stroke patients. Rivista Italiana di Nutrizione Parenterale ed Enterale 2004;22:205-26. 5.

9. Linee guida italiane di prevenzione e trattamento SPREAD VII edizione.

10. Powers WJ, Rabinstein AA, Ackerson T, et al.; on behalf of the American Heart Association Stroke Council. 2018 Guidelines for the early management of patients with acute ischemic stroke: a guideline for healthcare professionals from the American Heart Association/American Stroke Association. Stroke. 2018;49

11. Perry L, Love CP "Screening for dysphagia and aspiration in acute stroke: a systematic review" Dysphagia 2001 Winter; 16(1);7-18

12. Andrade PA, Santos CAD, Firmino HH, Rosa COB "The importance of dysphagia screening and nutritional assessment in hospitalized patients". Einstein (Sao Paulo) 2018 Jun 7;16(2):e AO4189. doi: 10.1590/ S1679-45082018AO4189.

13. De Pippo KL, Holas MA, Reding MJ (1992) Validation of the 3 oz. water swallow test for aspiration following stroke. Arch Neurol 49:1259-1261

14. Daniels SK, Anderson JA, Willson PC "Valid items for screening dysphagia risk in patients with stroke: a systematic review" Stroke 2012 Mar;43 (3):892-7

15. Linee guida sulla gestione del paziente disfagico adulto in foniatria e logopedia, Torino, 29 Gennaio 2007

16. ASHA Special interest division 13. Swallowing and swallowing disorders 1998.

17. Shimizu, K. Maeda, K. Tanaka, M. Ogawa, J. Kayashita. Texture-modified diets are associated with decreased muscle mass in older adults admitted to a rehabilitation ward. Geriatr Gerontol Int. 2018; 18: 698-700

18. Shimizu, R. Momosaki, J. Kayashita, I. Fujishima. Impact of multiplke texture-modified diets on oral intake annd nutritional status in older patient with pneumonia: a retrospective color study. Dysphagia. 2019; 34(1); 5 - 8-

19. Wirth R, Smoliner C, et al. Guideline clinical nutrition in patients with stroke. Exp Transl Stroke Med 2013;5:14.

20. Negrini G., Alterata somministrazione di farmaci: frazionamento, triturazione, camuffamento, Rischio Sanità, 2014.

21. Li L, Li Y, Huang r, Yin J, Shen Y, Shi J "The value of adding transcutaneus neuromuscular eletrical stimulation (VitalStim) to traditional therapy for post-stroke dysphagia: a randomized controlled trial.

22. Kumar S, Wagner CW, Frayne C, et al. Noninvasive brain stimulation may improve stroke-related dysphagia: a pilot study. Stroke. 2011 Apr;42(4):1035-40.

23. David L Cohen, Christine Roffe, Jessica Beavan, et al. "Post-stroke dysphagia: A review and design considerations for future trials". International Journal of Stroke 2016, Vol. 11(4) 399-411. 


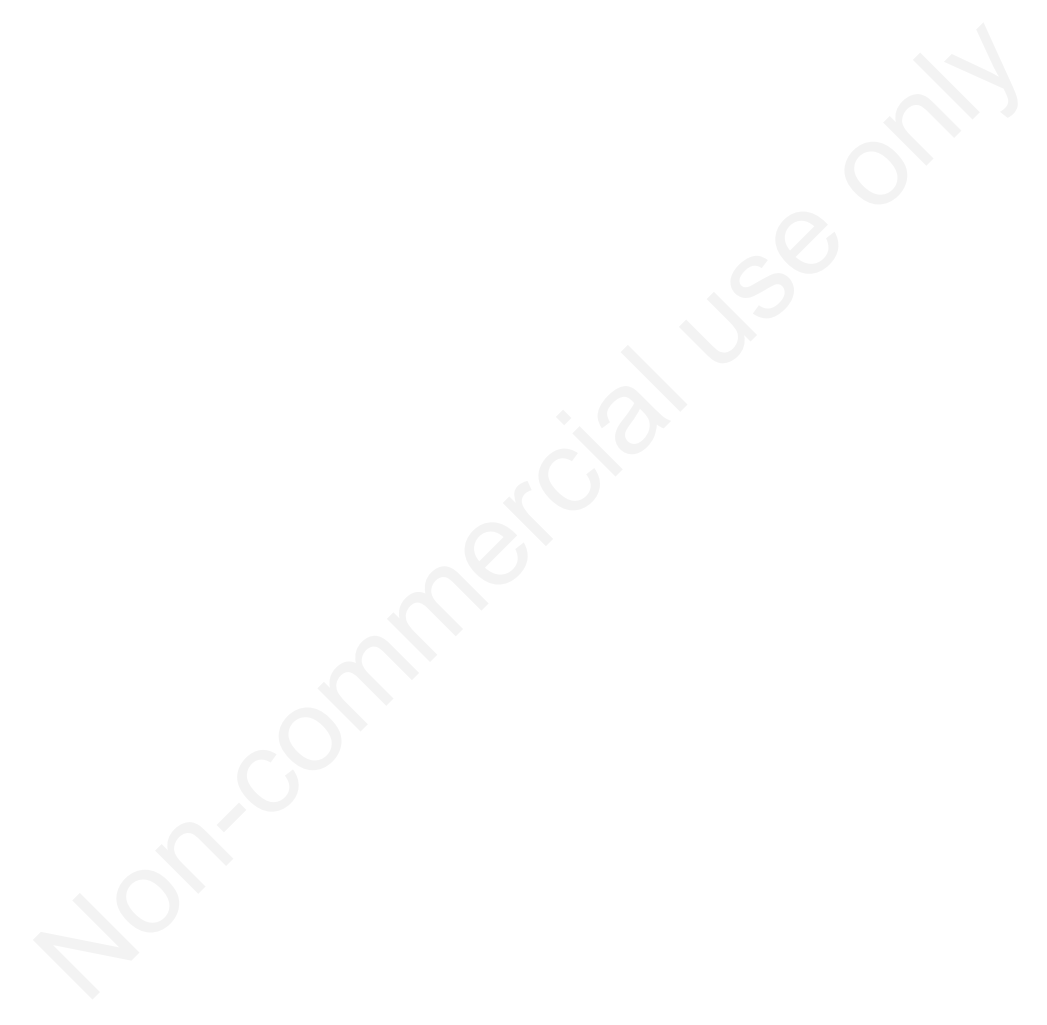




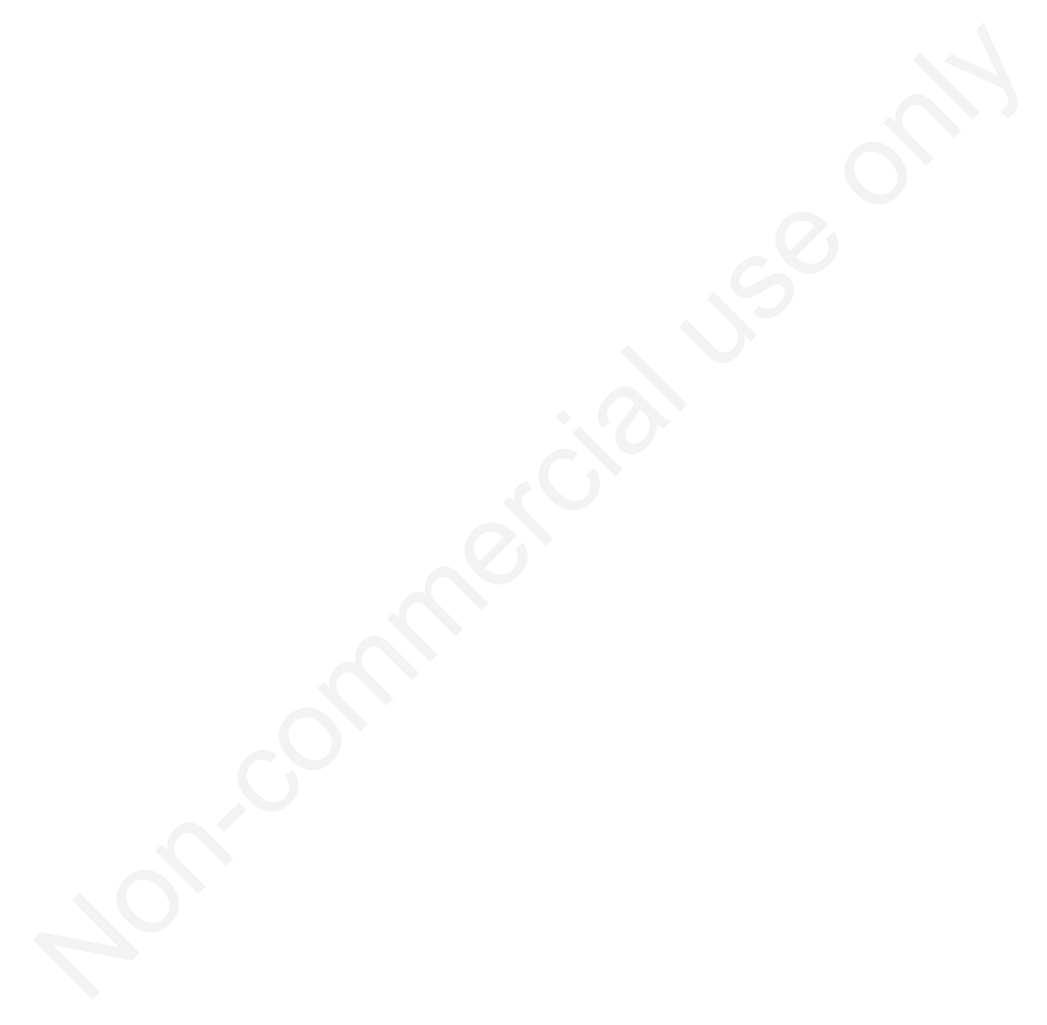




\section{LINEE GUIDA PER GLI AUTORI}

I Quaderni dell 'Italian Journal of Medicine (Quaderni ITJM), costituiscono una collana supplementare solo online annessa alla rivista Italian Journal of Medicine contenente lavori solo in lingua italiana.

I Quaderni ITJM pubblicano:

- Monografie ad hoc individuate dal Presidente FADOI, dal Consiglio Direttivo, dal Board Scientifico o dall'Editor in Chief dell'Italian Journal of Medicine, in funzione del contesto scientifico-istituzionale attuale.

- Monografie ad hoc su temi di particolare rilevanza scientifica a cura della Commissione FADOI Giovani.

- Traduzioni in italiano di alcuni lavori pubblicati sui numeri standard dell'Italian Journal of Medicine, di particolare interesse per la comunità scientifica.

\section{STESURA DEI LAVORI}

I lavori dovranno essere redatti in modo conforme alle linee guida sotto riportate:

- I manoscritti devono essere scritti interamente in lingua italiana, su documento di Word, con carattere Times New Roman/Arial, dimensione 12, formato A4, interlinea doppia e margini $2,54 \mathrm{~cm}$

Parole totali: $\max$ 4000; Sommario/Abstract: $\max 250$ parole; Bibliografia: $\min 40$ voci; Tabelle e Figure: $3 / 5$ totali (le tabelle non devono superare n. 1 pagina del documento in Word).

- La strutturazione del contenuto deve attenersi agli standard internazionali per la Rassegna (Review): i) Abstract riassuntivo dell'intero lavoro; ii) Introduzione al tema trattato; iii) Criteri e strumenti di ricerca (criteri di inclusione/esclusione, banche dati consultate, ...); iv) i successivi paragrafi devono illustrare le più recenti scoperte scientifiche nel settore; v) Conclusioni; vi) Bibliografia.

- La prima pagina deve riportare: i) titolo (in stampatello minuscolo), senza acronimi; ii) nome e cognome per esteso di ciascun autore; iii) affiliazione(i) di ciascun autore, numerate con numeri arabi; iv) eventuali ringraziamenti; v) nome e indirizzo postale completi dell'autore corrispondente, corredati da telefono, fax, e-mail; vi) da 3 a 5 parole chiave, separate da virgola. La seconda pagina può riportare: i) contributi degli autori, e.g. informazioni relative a contributi sostanziali delle persone coinvolte nello studio (http://www.icmje.org/\#author); ii) dichiarazione relativa a potenziali conflitti d'interesse; iii) ulteriori informazioni (e.g. fondi, esposizioni durante conferenze...).

- In caso di utilizzo di tabelle, queste devono essere tutte numerate con numeri arabi e citate nel testo in ordine consecutivo (e.g. NON nominare le tabelle come Tabella $1 \mathrm{~A}, 1 \mathrm{~B}, \ldots$ o $1.0,1.1, \ldots$ ). Le tabelle devono essere presentate in formato editabile. Ciascuna tabella deve essere corredata da una breve didascalia; in caso di abbreviazioni, riportare una nota a piè di CIASCUNA tabella che spieghi TUTTE le abbreviazioni presenti in ognuna.

- In caso di utilizzo di figure, queste devono essere inviate in formato tiff o .jpg, allegate al manoscritto in singoli files, secondo le seguenti specifiche:

i) a colori (salvate in modalità CMYK): minimo 300 dpi di risoluzione;

ii) in bianco e nero: minimo 600 dpi di risoluzione;

iii) minimo $17,5 \mathrm{~cm}$ di larghezza.

Ciascuna figura deve essere corredata da una breve didascalia.

$N B$ : In caso di Tabelle/Figure riprese e/o modificate da altri lavori già pubblicati, sarà cura degli autori accertarsi se tali materiali siano o meno coperti da copyright e procurarsi i permessi necessari per la riproduzione. Tali permessi dovranno essere allegati alla versione definitiva del lavoro. L'ufficio editoriale si riserva la facoltà di rimuovere Tabelle/Figure coperte da copyright, se sprovviste dei necessari permessi.

- In caso di utilizzo di abbreviazioni, la prima volta che esse sono citate è necessario scrivere per esteso la definizione+abbreviazione tra parentesi tonde [e.g. risonanza magnetica (RMN)], a seguire si dovrà riportare solo l'abbreviazione (unica eccezione: nei titoli e nelle didascalie di tabelle e figure NON si utilizzano abbreviazioni).

\section{BIBLIOGRAFIA}

Le voci bibliografiche devono essere formattate secondo lo stile Vancouver.

Nella sezione Bibliografia, le voci bibliografiche devono essere numerate consecutivamente nell'ordine in cui appaiono per la prima volta nel testo (NON in ordine alfabetico) e, nel testo, devono essere indicate con numeri arabi in apice. Voci bibliografiche riferite a comunicazioni personali o dati non pubblicati devono essere incorporate nel testo e NON inserite tra le voci numerate [e.g. (Wright 2011, dati non pubblicati) o (Wright 2011, comunicazione personale)]. Le voci bibliografiche nella sezione Bibliografia devono tassativamente essere preparate come segue:

i) più di 3 autori, citare 3 autori, et al. Se il lavoro contiene solo 4 autori, citarli tutti e 4;

ii) titolo del lavoro in stampatello minuscolo;

iii) nome della rivista, senza punti, abbreviato secondo gli standard internazionali; in caso di dubbi sulla corretta abbreviazione, fare riferimento ai seguenti siti:

a. ISI Journal Abbreviations Index (http://library.caltech.edu/ reference/abbreviations/);

b. Biological Journals and Abbreviations (http://home.ncifcrf. gov/research/bja/);

c. Medline List of Journal Titles (ftp://ftp.ncbi.nih.gov/pubmed/J_Medline.txt);

iv) inserire l'anno di pubblicazione subito dopo il nome della rivista, seguito da punto e virgola;

v) NON inserire giorno o mese di pubblicazione;

vi) citare solo il volume, seguito dai due punti (NON citare il fascicolo tra parentesi);

vii) abbreviare le pagine, e.g. 351-8

Per accertarsi di aver correttamente formattato le voci bibliografiche, confrontarle con le citazioni in PubMed (http://www.ncbi. nlm.nih.gov/pubmed).

Esempi (prestare attenzione anche alla punteggiatura):

Articolo standard su Rivista

Halpern SD, Ubel PA, Caplan AL. Solid-organ transplantation in HIV-infected patients. N Engl J Med 2002;347:284-7.

Proceedings

Christensen S, Oppacher F. An analysis of Koza's computational effort statistic for genetic programming. In: Foster JA, Lutton E, Miller J, Ryan C, Tettamanzi AG, eds. Genetic programming. EuroGP 2002: Proceedings of the 5th European Conference on Genetic Programming, 2002 Apr 3-5, Kinsdale, Ireland. Berlin: Springer; 2002. pp 182-91.

Articoli i cui autori sono Organizzazioni

Diabetes Prevention Program Research Group. Hypertension, insulin, and proinsulin in participants with impaired glucose tolerance. Hypertension 2002;40:679-86.

Libri

Murray PR, Rosenthal KS, Kobayashi GS, Pfaller MA. Medical microbiology. 4th ed. St. Louis, MO: Mosby; 2002. (CITAZIONE DEL LIBRO INTERO)

Meltzer PS, Kallioniemi A, Trent JM. Chromosome alterations in human solid tumors. In: Vogelstein B, Kinzler KW, eds. The genetic basis of human cancer. New York, NY: McGraw-Hill; 2002. pp 93113. (CITAZIONE DI UN CAPITOLO)

\section{MODALITÀ D'INVIO DEI LAVORI}

Monografie

Gli Autori dovranno fare riferimento all'Editor-in-Chief o alle persone da lui designate nelle lettere di invito a scrivere gli articoli programmati.

Per gli articoli inviati da giovani internisti, gli Autori dovranno fare riferimento al Dr. Michele Meschi (e-mail: mmeschi@ausl.pr.it)e agli eventuali altri referenti da lei designati, nelle prime fasi di stesura dei manoscritti, revisioni e correzioni. 


\section{QUADERNI}

dell'Italian Journal

of Medicine

Il Dr. Meschi raccoglierà poi le versioni definitive dei lavori di ciascuna monografia e provvederà all'invio di tutti i materiali all'ufficio editoriale.

I lavori solo nella loro versione definitiva e approvata dalla Commissione FADOI Giovani dovranno pervenire all'ufficio editoriale già pronti per l'impaginazione e immediata pubblicazione (già corredati da eventuali permessi per la riproduzione di tabelle e immagini redatti secondo le presenti linee guida).

Traduzioni

Previo invito dell'Editor-in-Chief, gli Autori dovranno far pervenire all'ufficio editoriale la versione tradotta in italiano, al seguente indirizzo e-mail: paola.granata@pagepress.org

Il file in formato Word dovrà essere formattato secondo gli standard editoriali della rivista ufficiale ed essere già pronto per impagina- zione e immediata pubblicazione (corredato da eventuali permessi per la riproduzione di tabelle e immagini).

Si prega di inviare le eventuali tabelle in formato editabile e le figure in alta definizione secondo gli standard sopra riportati.

\section{NOTA PER GLI AUTORI}

I lavori pubblicati sui Quaderni ITJM non verranno indicizzati, ma saranno liberamente disponibili in un'apposita sezione del sito FADOI (http://www.fadoi.org/) e della rivista ufficiale.

Gli Autori i cui lavori siano accettati per la pubblicazione sui Quaderni ITJM e che fossero interessati a vederli pubblicati anche sulla rivista ufficiale, dovranno sottomettere attraverso il sito dell'ITJM (www.italjmed.org) la versione (già tradotta) in inglese e redatta in modo conforme alle linee guida della rivista; seguiranno poi la procedura di selezione tramite peer review e, se accettati, saranno inseriti nel piano editoriale standard.

\section{STAFF EDITORIALE}

Paola Granata, Journal Manager

paola.granata@pagepress.org

Claudia Castellano, Production Editor

Tiziano Taccini, Technical Support
PUBBLICATO DA

PAGEPress Publications

via A. Cavagna Sangiuliani 5

27100 Pavia, Italy

T. +39.0382 .464340$

F: +39.0382 .34872$

\section{agepress}

www.pagepress.org info@pagepress.org

\section{QUADERNI - ITALIAN JOURNAL OF MEDICINE}

Tutti gli articoli pubblicati sui QUADERNI - Italian Journal of Medicine sono redatti sotto la responsabilità degli Autori. La pubblicazione o la ristampa degli articoli della rivista deve essere autorizzata per iscritto dall'editore. Ai sensi dell'art. 13 del D.Lgs 196/03, i dati di tutti i lettori saranno trattati sia manualmente, sia con strumenti informatici e saranno utilizzati per l'invio di questa e di altre pubblicazioni e di materiale informativo e promozionale. Le modalità di trattamento saranno conformi a quanto previsto dall'art. 11 del D.Lgs 196/03. I dati potranno essere comunicati a soggetti con i quali PAGEPress intrattiene rapporti contrattuali necessari per l'invio delle copie della rivista. Il titolare del trattamento dei dati è PAGEPress Srl, via A. Cavagna Sangiuliani 5 - 27100 Pavia, al quale il lettore si potrà rivolgere per chiedere l'aggiornamento, l'integrazione, la cancellazione e ogni altra operazione di cui all'art. 7 del D.Lgs 196/03.

https://www.italjmed.org/index.php/ijm/quad 
PAGEPress - Scientific Publications

Via A. Cavagna Sangiuliani, 5 - 27100 Pavia - Italy

Phone +390382 464340 - Fax +390382 34872

www.pagepress.org 